\title{
Changes in gene expression linked to Alzheimer's disease and "healthy" cognitive aging
}

\author{
Dissertation
}

\author{
for the award of the degree \\ "Doctor rerum naturalium" \\ of the Georg-August-Universität Göttingen \\ within the doctoral program Molecular Physiology of the Brain, GGNB \\ of the Georg-August University School of Science (GAUSS)
}

submitted by

\section{Magdalena Navarro Sala}

from Barcelona, Spain

Göttingen 2018 


\section{Thesis committee:}

1. Prof. Dr. André Fischer, Deutsches Zentrum für Neurodegenerative Erkrankungen (DZNE), University Medical Center, Göttingen

2. Prof. Dr. Tiago Outeiro, Department of Neurodegeneration and Restorative Research, University Medical Center, Göttingen

3. Prof. Dr. Thomas Dresbach, Director of the Department of Anatomy and Embryology, University Medical Center, Göttingen

\section{Members of the examination board:}

Referee: Prof. Dr. André Fischer, Deutsches Zentrum für Neurodegenerative Erkrankungen (DZNE), University Medical Center, Göttingen

$2^{\text {nd }}$ referee: Prof. Dr. Tiago Outeiro, Department of Neurodegeneration and Restorative Research, University Medical Center, Göttingen

Prof. Dr. Thomas Dresbach, Director of the Department of Anatomy and Embryology, University Medical Center, Göttingen

Dr. Camin Dean, European Neuroscience Institute Göttingen (ENI-G), University Medical Center and Max-Planck Society, Göttingen

Prof. Dr. Ahmed Mansouri, Molecular Cell Differentiation, Max Planck Institute for Biophysical Chemistry, Göttingen

Prof. Dr. Hubertus Jarry, Department of Research Animal Welfare Officer, University Medical Center, Göttingen

Date of oral examination: 5th of July 2018 


\section{Declaration:}

I hereby declare that I have written the dissertation

"Changes in gene expression linked to Alzheimer's disease and "healthy" cognitive aging "

entirely by myself with no other aids or sources than quoted.

Göttingen, 28.05.18

Magdalena Navarro Sala 
LIST OF FIGURES

LIST OF TABLES

1. INTRODUCTION

1.1.Learning and memory 13

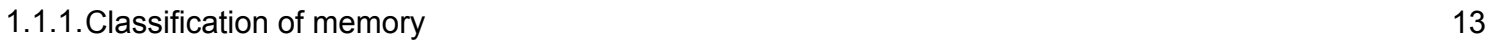

1.1.2. Hippocampus in learning and memory $\quad 15$

$\begin{array}{ll}\text { 1.1.3. Molecular mechanisms involved in learning and memory } & 17\end{array}$

$\begin{array}{ll}\text { 1.2.Alzheimer's disease } & 18\end{array}$

$\begin{array}{ll}\text { 1.2.1.Epidemiology } & 18\end{array}$

1.2.2.Pathology of Alzheimer's disease $\quad 19$

1.2.3. Molecular mechanisms of Alzheimer's disease $\quad 20$

1.2.4. Mouse models of Alzheimer's disease $\quad 22$

1.2.5.Diagnosis and treatment of Alzheimer's disease $\quad 24$

1.2.6. Risk factors for Alzheimer's disease $\quad 24$

1.2.7. Gene expression and exon usage associated with AD 25

1.3.Aging 28

1.3.1.Molecular, gene expression and mRNA processing changes along aging 29

1.4.Epigenetic factors

1.5.Intergenerational inheritance 37

1.5.1. History of evolution: Lamarck $\quad 37$

1.5.2. Mechanisms of transmission 38

1.5.3. Candidates of germ-line transmission $\quad 39$

2. OBJECTIVES 43

3. MATERIALS AND METHODS 46

3.1.Animals 46

$\begin{array}{ll}\text { 3.1.1.Housing conditions } & 46\end{array}$

3.1.2.APPPS1-21 transgenic mice and C57BL/6J wild-type mice 46

$\begin{array}{ll}\text { 3.1.3.Genotyping } & 47\end{array}$

3.1.4.Dissection of the brain, and extraction of blood and sperm 48

3.1.4.1.Extraction of the blood extraction and dissection of the liver 49

3.1.4.2.Dissection of the hippocampal subregions 49

$\begin{array}{ll}\text { 3.1.4.3.Extraction of sperm } & 49\end{array}$

3.2.Behavioral experiments $\quad 50$

$\begin{array}{ll}\text { 3.2.1. Open field test } & 50\end{array}$

$\begin{array}{lr}\text { 3.2.2. Morris water maze } & 50\end{array}$ 
3.2.3. Contextual fear conditioning

3.2.4.Barnes Maze

3.3.Molecular experiments

3.3.1.RNA isolation from brain

3.3.2. RNA isolation from the sperm

3.3.3. RNA isolation from the blood

3.3.4. RNA concentration and quality 55

3.3.5. RNA integrity 56

3.3.6.cDNA preparation for RNA detection

3.3.7.Primer design 57

3.3.8. Quantitative real time PCR to quantify mRNA levels 58

3.3.9.qPCR for RNA detection

3.4. Experimental analysis 59

3.4.1. Analysis of $\mathrm{qPCR}$ data 59

3.4.2. Next-Generation Sequencing data analysis 59

3.4.3. Differentially expression of genes and small RNAs

3.4.4. Differential exon usage

4.1.The early characteristics of Alzheimer's disease in APPPS1-21 mouse model

4.1.1. Cognitive abilities in APPPS1-21 transgenic mice at 3 months of age

4.1.2. Transcriptional profiling and functional pathway analysis in APPPS1-21 mice at $3 \mathrm{~m}$

4.1.3. Alternative exon usage and functional pathway analysis in APPPS1-21 transgenic mice at $3 \mathrm{~m}$

4.2.1. Cognitive abilities of wild-type mice born to APPPS1-21 transgenic parents

4.2.2. Accounting for group size, batch and litter effects

4.2.3. Intergenerational effect on transcriptional profiling and functional pathways

4.2.4. Alternative exon usage and pathway analysis in wild-type mice born to APPPS1-21 mice at 3m

4.2.6.APP expression in wt mice born to APP fathers

4.3.1. Increased variability in cognitive performance in aging mice: choosing good vs. bad learners 
5.1.The early stage of Alzheimer's disease in APPPS1-21 mouse model

5.1.1. Spatial cognitive deficits in APPPS1-21 transgenic mice are detectable at $3 \mathrm{~m}$

5.1.2.Age-progressive decline of spatial memory and motor abilities

5.1.3. No gender differences in behavioral phenotypes of offspring APPPS1-21 mice

5.1.4. Transcriptional profiling and pathway analysis in APPPS1-21 transgenic mice at 3m

155

5.1.5. Alternative exon usage and pathway analysis in APPPS1-21 transgenic mice at 3m

5.2.Paternal line-specific inheritance of cognitive deficits in APPPS1-21 mice via non-genetic mechanisms

163

5.2.1.Spatial cognitive behavior in wild-type mice born to one APPPS1-21 parent

5.2.2. Transcriptional profiling and pathway analysis in APPPS $1-21$ wild-type mice at $3 \mathrm{~m}$

166

5.2.3. Alternative exon usage and pathway analysis in APPPS1-21 wild-type mice at $3 \mathrm{~m}$

168

5.2.4. Transmission of non-genetic factors by germ-line

5.3.Inter-individual differences in cognitive performance and associated molecular pathways in aging mice

5.3.1. Inter-individual differences in cognitive performance

5.3.2. Differences in the transcriptional profiling and functional pathways of aged "good" and "bad" performers

5.3.3. Detection of biomarkers to screen cognitive abilities 


\section{LIST OF FIGURES}

Figure 1.1. Classification of memory.

Figure 1.2. Hippocampus subregions and circuit connections.

Figure 1.3. Incidence and prevalence of dementia cases.

Figure 1.4. Schematic representation of APP processing and $A \beta$ aggregation.

Figure 1.5. Schematic representation of the stabilization of microtubules by tau protein.

Figure 1.6. Waddington epigenetic landscape. 33

Figure 1.7. Process of inheritance.

Figure 3.1. Experimental groups.

Figure 4.1.1. Experimental groups and design.

Figure 4.1.2. Transgenic mice did not show motor impairments in the open field, but showed less anxiety levels at 3 months of age.

Figure 4.1.4. Transgenic mice showed spatial memory impairments at 3 months of age. $-64$

Figure 4.1.5. Transgenic mice did not show swimming difficulties at 3 months of age in the Morris water maze (MWM) spatial task.68 Figure 4.1.6. APPPS1-21 tg mice did not show deficits of contextual associative memory $-69$ at 3 months of age. $-69$

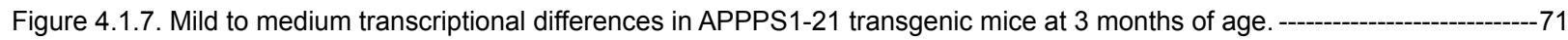

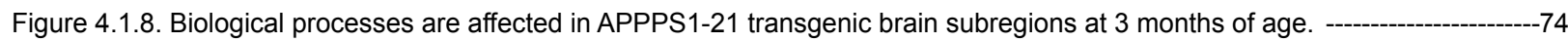

Figure 4.1.9. Molecular functions are affected in APPPS1-21 transgenic brain subregions at 3 months of age. ---15

Figure 4.1.10. Cellular components are affected in APPPS1-21 transgenic brain subregions at 3 months of age. ------------76

Figure 4.1.11. Functional pathways affected in APPPS1-21 transgenic mice, specifically in DG from males and females. -----------78

Figure 4.12. Drastic alteration in the alternative exon usage in DG and CA1 of APPPS1-21 transgenic mice. --------80

Figure 4.1.13. A large number of identical genes showed alternative exon usage in DG from males and females and CA1 from males of genes in APPPS1-21 transgenic mice.

Figure 4.1.14. Top 20 significant pathways affected by alternative exon usage in genes of CA1 and DG from APPPS1-21 transgenic mice.

Figure 4.1.15. Around $30 \%$ of genes were affected by differential expression and splicing in CA1 and DG regions of APPPS1-21 transgenic mice.

Figure 4.1.16. Several pathways were affected by those genes that were both differentially expressed and had an alternative exon usage in DG region of APPPS1-21 transgenic mice.

Figure 4.2.1. Experimental groups and design. $-85$

Figure 4.2.2. Wild-type mice born to APPPS1-21 transgenic fathers (wttgwt) did not show motor impairments, an increase of apathy or levels of anxiety, whereas wild-type born to APPPS1-21 transgenic mothers (wtwttg) showed a slight increase of apathy and reduction of anxiety levels in the open field test at 3 months of age. 
Figure 4.2.3. Only wild-type mice born to an APPPS1-21 transgenic father showed difficulties to learn a spatial task at 3 months of age.

Figure 4.2.4. Only wild-type mice born to APPPS1-21 transgenic father (wttgwt) showed spatial memory impairments at 3 months of age. $-90$

Figure 4.2.5. Wild-type mice from APPPS1-21 parents did not show motor impairments at 3 months of age in the Morris water maze task (MWM). $--91$

Figure 4.2.6. APPPS1-21 wild-type mice groups did not show deficits of contextual associative memory at 3 months of age. -------92

Figure 4.2.7. Experimental groups and design. $-93$

Figure 4.2.8. Wild-type and transgenic mice born to APPPS1-21 transgenic fathers showed a reduction of their motor abilities and only wild-type mice born to APPPS1-21 tg mother (wtwttg) showed an increase of anxiety levels opposite to what they showed at 9 months of age. $-94$

Figure 4.2.9. Only APPPS1-21 transgenic mice showed difficulties to learn the spatial task at 9 months of age. $-95$

Figure 4.2.10. Wild-type and transgenic mice born to APPPS1-21 transgenic fathers, and transgenic mice born to APPPS1-21 tg mother showed spatial memory impairments at 9 months of age.

Figure 4.2.11. Wild-type and transgenic mice born to an APPPS1-21 transgenic father showed mild motor impairments. ----------99

Figure 4.2.12. Fear conditioning in wild-type and transgenic mice born to APPPS-21 transgenic parents at 9 months of age. -----100

Figure 4.2.13. Mild reduction of locomotor abilities and increased of anxiety levels with aging in all groups, except in wtwttg and tgwttg.

Figure 4.2.14. Learning spatial task is impaired with age in all offspring groups. $--103$

Figure 4.2.15. Only young control wild-type mice (wtwtwt) and young wtwttg mice showed a preference for the target quadrant. 104 Figure 4.2.16. All old offspring groups showed a more severe spatial cognitive impairment than young equivalent groups. -------106 Figure 4.2.17. Only transgenic mice born to a transgenic father showed motor impairments with aging during MWM task. --------106

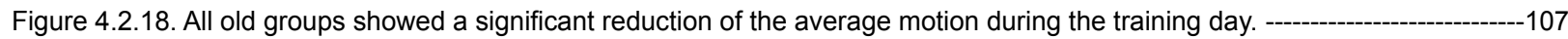

Figure 4.2.19. No litter-specific effect in Morris water maze behavioral data. 109

Figure 4.1.20. No significant differences were observed between batches regarding the time spent in the target quadrant during the second probe trial (PT2) in Morris water maze. $-111$

Figure 4.2.21. Moderate changes in transcriptional profile in brain subregions of wild-type born to an APPPS1-21 transgenic father (wttgwt) at 3 months of age.

Figure 4.2.22. Little transcriptomic differences between wild-type mice born to an APPPS1-21 tg father (wttgwt) or mother (wtwttg) and APPPS1-21 transgenic mice.

Figure 4.2.23. Around $50 \%$ of genes overlap when wild-type mice born to an APPPS1-21 transgenic father (wttgwt) and APPPS-21 transgenic mice were compared to wtwtwt. $-116$

Figure 4.2.24. Biological processes are affected in the DG and CA1 regions of wild-type mice born to an APPPS1-21 transgenic father (wttgwt) at 3 months of age. $-118$

Figure 4.2.25. Molecular functions are affected in the DG and CA1 regions of wild-type mice born to an APPPS1-21 transgenic father (wttgwt) at 3 months of age. 
Figure 4.2.26. Cellular components are affected in DG and CA1 regions of wild-type mice born to an APPPS1-21 transgenic father (wttgwt) at 3 months of age. -

Figure 4.2.27. Functions affected in CA1 and DG of wild-type mice born to APPPS1-21 transgenic fathers. ---123

Figure 4.2.28. Around 30 to $40 \%$ of pathways overlap between wt mice born to an APPPS1-21 tg father (wttgwt) and APPPS1-21 tg mice when both were compared to wt control mice (wtwtwt). -

Figure 4.2.29. Around $65 \%$ of pathways overlap overlap between wt mice born to an APPPS1-21 tg father (wttgwt) and APPPS1-21

tg mice. - ---

Figure 4.2.30. Drastic alteration in alternative exon usage in DG and CA1 of wild-type mice born to an APPPS1-21 transgenic father and almost negligible changes in wild-type offspring from APPPS1-21 transgenic mothers compared to control wild-type mice. --126

Figure 4.2.31. Small changes alternative exon usage were identified between wt mice born to an APPPS1-21 tg father (wttgwt) or mother (wtwttg) and transgenic APPPS1-21 offspring. $-127$

Figure 4.2.32. A large number of identical genes showed alternative exon usage in CA1 and DG regions of wild-type mice born to APPPS1-21 transgenic fathers.

Figure 4.2.33. Several functions were affected by alternative exon usage in genes of wild-type mice born to APPPS1-21 transgenic fathers.

Figure 4.2.34. A large number of differentially spliced genes overlap between wt mice born to APPPS1-21 tg fathers and APPPS1-21 $\operatorname{tg}$ mice when both were compared to wt control mice (wtwtwt). $-130$

Figure 4.2.35. Subregion variability regarding common genes affected by differentially 131 expressed and spliced genes in wt mice born to APPPS1-21 tg fathers (wttgwt) when were compared to wt control mice (wtwtwt). -131

Figure 4.2.36. Several networks were affected by differentially expressed and spliced genes in DG region of wild-type mice born to APPPS1-21 transgenic mice.

Figure 4.2.37. Expression of small RNAs that could play an important role in the altered phenotype, transcriptome and splicing in wild-type and transgenic offspring of APPPS1-21 transgenic fathers.

Figure 4.2.38. No expression of APP in sperm of APPPS1-21 transgenic fathers. -

Figure 4.3.1. Increase inter-individual variability in cognitive performance in aging mice. - --137

Figure 4.3.2. Establishment of good and bad performers. - -

Figure 4.3.3. No motor variability between good and bad performers. -

Figure 4.3.4. No changes in motor and abilities and anxiety levels between good and bad performers during the open field test. 140 Figure 4.3.5. Establishment of good and bad performers based on escape latency (or performance) in the Barnes maze (BM). -141

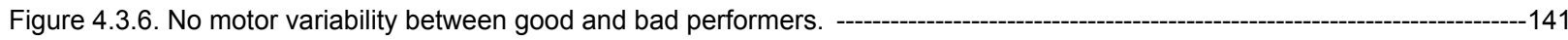

Figure 4.3.7. No anxiety variability between defined good and bad performers during the open field test. ----------------------------142

Figure 4.3.8. Changes in transcriptional profile in brain subregions of bad performers compared to good performers. ---------------143

Figure 4.3.9. Biological processes are differentially affected in good and bad performer mice. - -

Figure 4.3.10. Molecular functions were differentially affected in good and bad performer mice. - -

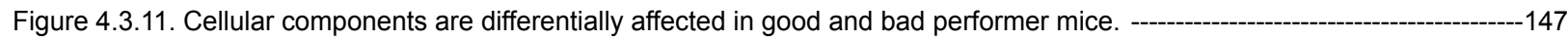

Figure 4.3.12. Blood small RNAs used as biomarkers of good and bad learners. - 
Figure 6.1. Summary of the data described in the three approaches from this thesis.

Appendix figure 1. Male and female wt and tg mice born to APPPS1-21 transgenic fathers or mothers did not show motor abilities and anxiety level differences, except for APPPS1-21 tg females that showed an slight increase in their motor abilities.

Appendix figure 2. Male and female wt and tg mice born to APPPS1-21 transgenic fathers or mothers did not show differences in learning a spatial task at 3 months of age.

Appendix Figure 4. Male and female wt and tg mice born to APPPS1-21 transgenic fathers or mothers did not show differences in fear conditioning at 3 months of age.

Appendix Figure 5. Male and female wt and tg mice born to APPPS1-21 transgenic fathers or mothers did not show differences in motor abilities and anxiety level at 9 months of age.

Appendix Figure 6. Male and female wt and tg mice born to APPPS1-21 transgenic fathers or mothers did not show differences in learning a spatial task at 9 months of age.

Appendix figure 7. Male and female wt and tg mice born to APPPS1-21 transgenic fathers or mothers did not show differences in spatial memory at 9 months of age.

Appendix figure 8. Male and female wt and tg mice born to APPPS1-21 transgenic fathers or mothers did not show differences in fear conditioning at 9 months of age.

\section{LIST OF TABLES}

Table 3.1. PCR master mix for genotyping $-48$

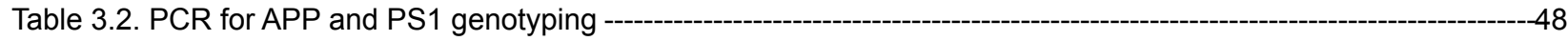

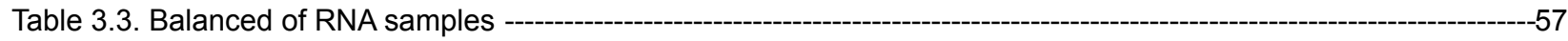

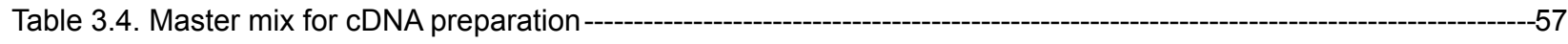

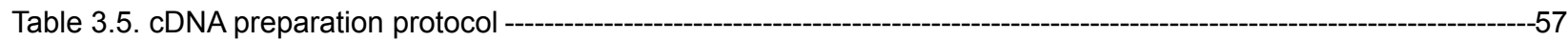

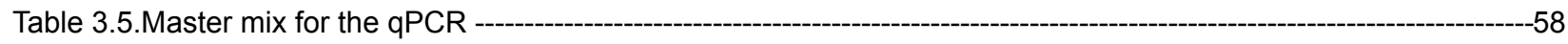

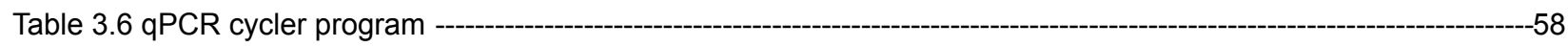

Table 4.2.1. Large group number of mice used for Morris water maze at 3 and 9 months of age. --------108

Appendix table 1. Significant alternative exon usage of App, Ps1 and Mapt genes from male and female DG and male CA1 of APPPS1-21 transgenic mice compared to control wild-types (wtwtwt). $-189$

Appendix table 2. Variability between tg mice born to an APPPS1-21 tg father or mother and between APPPS1-21 tg mice together and control wild-type (wtwtwt) mice. $-189$

Appendix table 3. Top differentially expressed genes in male and female DG and male CA1 of APPPS1-21 transgenic mice.

Appendix table 4. Top 10 genes that had a significant alternative exon usage in male and female DG and male CA1 of APPPS1-21 transgenic mice.

Appendix table 5. Top 10 genes that had a significant alternative exon usage in male and female DG and male CA1 of wild-type mice born to APPPS1-21 transgenic fathers. 
INTRODUCTION 


\section{INTRODUCTION}

\subsection{Learning and memory}

Since ancient times, humans have tried to discover how memory works. Culture, complex societies or science exist thanks to human cognitive abilities. These achievements enable us to transmit information across generations and develop a cognitive complexity that is essential for survival and distinct than other organisms. Several theories and definitions of memory have been described, but the precise mechanism and universal definition remain to be elucidated (Spear \& Riccio, 1994). Memory was considered to be a location where the information is stored (Stern, 1991). It was also defined as a process by which new experiences are encoded (learn) and stored so that the information can be retrieved (Lechner, Squire, \& Byrne, 1999; Melton, 1963; Sternberg \& Sternberg, 2017; Tulving \& Thomson, 1973). Memory was also defined as a group of activated cells during learning that persistently change their conditions to retain the information and can be reactivated it (memory engrams) (Semon, 1911).

\subsubsection{Classification of memory}

Memory can be classified following different criteria. The Atkinson-Shiffrin model postulates that the memory can be classified by time of retention as short-term and long-term memory (James, 1950). Short-term memory (STM) retains the information for a short time, from seconds to minutes. The time is variable between subjects and studies (Cowan, 2008). It was suggested that STM could retain information of up to 7 items (G. A. Miller, 1956) and was considered to be part of working memory, but nowadays this is an independent type. Working memory participates in the manipulation of information for complex tasks (Baddeley, 2010; Chai, Abd Hamid, \& Abdullah, 2018). During the consolidation phase, STM is converted to long-term memory (LTM) (Lechner et al., 1999). This phase consists of two principal mechanisms, synaptic and system consolidation (Dudai, 2004). Synaptic consolidation is a short process of stabilization (minutes to hours), whereas the system consolidation is a long process (weeks or more). During this last step, neurons in the cortical areas increase the number of signals and connections, till different neurons fire together (Dudai, 2004). It is possible with a process known as long-term potentiation (LTP). Long-term memory (LTM) can be stored for long periods of time inducing changes on synaptic connections (Atkinson, 1967; Brashers-Krug, Shadmehr, \& Bizzi, 
1996; Dudai, 2004; Jonides et al., 2008; McGaugh, 2000). Long-term memory can be distinguished as declarative (similar as explicit) and non-declarative (similar as implicit) (Squire, 1987; E. Tulving, 1985). Tulving introduced these new terms of declarative and non-declarative memories (E. Tulving, 1985; Tulving \& Thomson, 1973). Graff and Schacter described for the first time implicit and explicit terms in 1985 (Graf \& Schacter, 1985). Declarative or explicit memory is based on daily memories, which can be semantic memories, when are relate to facts, and episodic memories, when are related to an event on specific space and time (Endel Tulving, 1985). One subtype of episodic memory is the autobiographical memory, and it refers to the retention of experiences of our own life (Conway \& Pleydell-Pearce, 2000; Fivush, 2011). Nondeclarative or implicit memories generally are acquired without the intention to be learned by repetition and practice. These memories are unforgettable allowing humans to develop daily activities. There are different subtypes of non-declarative memories related to different conditions: priming, habits, skills, emotional response, skeletal musculature and habituation (Figure 1.1) (N. J. Cohen \& Squire, 1980; Eichenbaum \& Cohen, 2004; Gazzaniga, Ivry, \& Mangun, 2002).

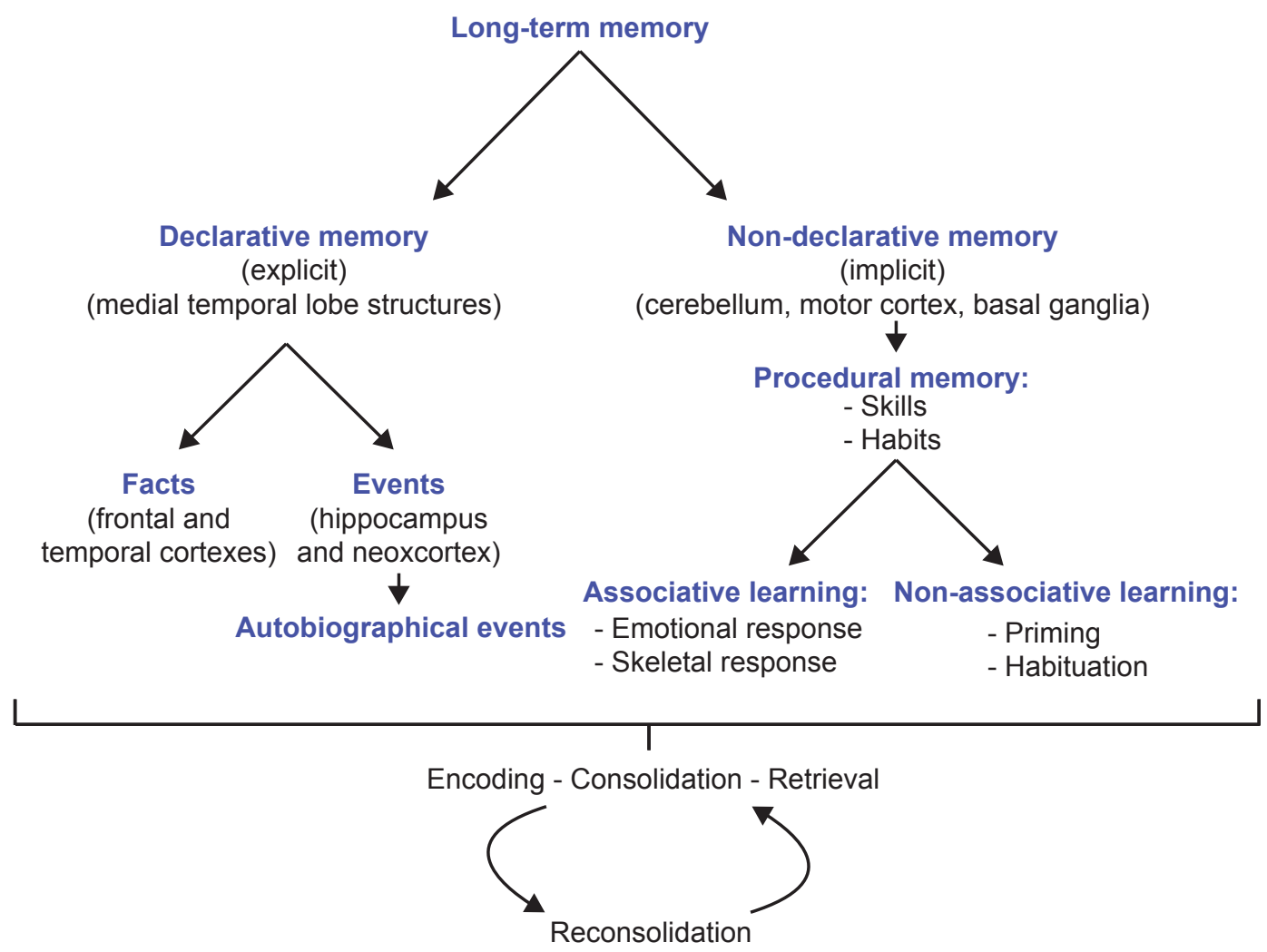

Figure 1.1. Classification of memory.

Classification of long-term memory and brain regions involved. Figure adapted from (Brem, Ran, \& Pascual-Leone, 2013). 


\subsubsection{Hippocampus in learning and memory}

Several clinical, brain imaging and brain region lesion studies indicate that many brain regions play a different role in distinct types of memory (Binder \& Desai, 2011; N. J. Cohen, Eichenbaum, Deacedo, \& Corkin, 1985; N. J. Cohen \& Squire, 1980; Doyon et al., 2009; Eichenbaum, 2001; Eichenbaum \& Cohen, 2004; Gazzaniga et al., 2002; Hikosaka, Nakamura, Sakai, \& Nakahara, 2002; Wixted et al., 2018). Memories defined as non-declarative are more likely to be conducted for the cerebellum, basal ganglia, amygdala and motor cortex (N. J. Cohen \& Squire, 1980; Doyon et al., 2009; Eichenbaum \& Cohen, 2004; Gazzaniga et al., 2002; Hikosaka et al., 2002; LeDoux, 2003), whereas the medial temporal lobe (hippocampus) and the neocortex have been more associated with declarative memories (Binder \& Desai, 2011; Eichenbaum, 2001; Wixted et al., 2018). Several studies in rodents showed the alteration of the amygdala in implicit fear memory (LeDoux, 2003; Rogan, Leon, Perez, \& Kandel, 2005). One of the first studies that demonstrated the importance of temporal lobe (hippocampus) in declarative memory was the study of the patient Henry Molaison (H.M.) in 1957 (Scoville \& Milner, 1957). To treat his epilepsy, the medial temporal lobe, the hippocampus and the amygdala were removed. After this surgery, his epilepsy was reduced, but he could not retain memories for long-term, only for short-term (Scoville \& Milner, 1957). These findings suggest that temporal lobe (hippocampus) play an important role in the consolidation of declarative memories (Poldrack et al., 2001; Squire, 1986; Squire, Stark, \& Clark, 2004). Hippocampus brain region is part of the medial temporal lobe in humans and is essential for acquisition and consolidation of memory 44. The specific hippocampal function in semantic (familiarity) or episodic (recollection) memory is still controversial (Jacoby \& Dallas, 1981; O'Reilly \& Norman, 2002), due to the complexity to identify specific neuron activation involved in new connections. Hippocampal neurons, known as place cells, are involved in spatial processing (Ekstrom et al., 2003; Moser, Kropff, \& Moser, 2008; O'Keefe, 1976). The activation of these cells provide information of the spatial position of the animal in the brain (O'Keefe \& Dostrovsky, 1971; Saleem, Ayaz, Jeffery, Harris, \& Carandini, 2013), replay experiences of the past (A. K. Lee \& Wilson, 2002; Skaggs \& McNaughton, 1996) and build new trajectories (Gupta, van der Meer, Touretzky, \& Redish, 2010). The stability of this spatial memory depends on the attention level (Frank, Brown, \& Wilson, 2000; Kentros, Agnihotri, Streater, Hawkins, \& Kandel, 2004; Muzzio, Kentros, \& Kandel, 2009). Cell grids from entorhinal cortex also play an important role in spatial representation, providing information about the position, direction and distance (Fyhn, Molden, Witter, Moser, \& Moser, 2004; Moser et al., 2008). The memory activity of entorhinal cortex (EC) has also been investigated in imaging studies (Suzuki, 2009). There are at least two pathways that connect the hippocampus with EC. 
The hippocampus brain region from humans is divided into Cornu Ammonis (CA1, CA2 and CA3) and Dentate Gyrus (DG), which are interconnected. An indirect pathway, known as the perforant path, connect the EC (layer II) with the DG, then the DG is connected to the CA3 by mossy fibers and finally to CA1 by Schaffer collateral connections (Amaral \& Witter, 1989). A direct pathway, known as temporoammonic pathway, connects EC (layer III) to CA1 (Witter, Groenewegen, Lopes da Silva, \& Lohman, 1989) (Figure 1.1.). It seems that part of the hippocampal memory role is due to the adult neurogenesis in DG, but the precise mechanism remains to be elucidated (Dupret et al., 2007; Kropff, Yang, \& Schinder, 2015; Lazarov \& Hollands, 2016). Moreover, hippocampus region is also highly connected with other cortical and subcortical brain regions, such as basal ganglia (Atallah, Frank, \& O'Reilly, 2004), cerebellum (Onuki, Van Someren, De Zeeuw, \& Van der Werf, 2015), prefrontal cortex (J. Jin \& Maren, 2015), hypothalamus (Aggleton et al., 2010) and the amygdala (Hill, Sahay, \& Hen, 2015; Sahay \& Hen, 2007). Furthermore, hippocampus subregions also participate in spatial working memory (Olton, Walker, \& Gage, 1978; Squire \& Cave, 1991), navigation to a specific item (Broadbent, Squire, \& Clark, 2006; R. Morris, 1984) and contextual fear memory (Anagnostaras, Maren, \& Fanselow, 1999; S. H. Lee et al., 2012). Finally, the hippocampus and its connections are affected at early stages of Alzheimer's disease and during the process of aging. Several studies found amyloid plaque deposition, neuronal loss and reduction of neuronal density in the hippocampus of human brain and mouse models of AD. The gradual loss of memory in AD cases was directly associated with alterations in the hippocampus (Arlt et al., 2013; BonnerJackson, Mahmoud, Miller, \& Banks, 2015; Padurariu, Ciobica, Mavroudis, Fotiou, \& Baloyannis, 2012).

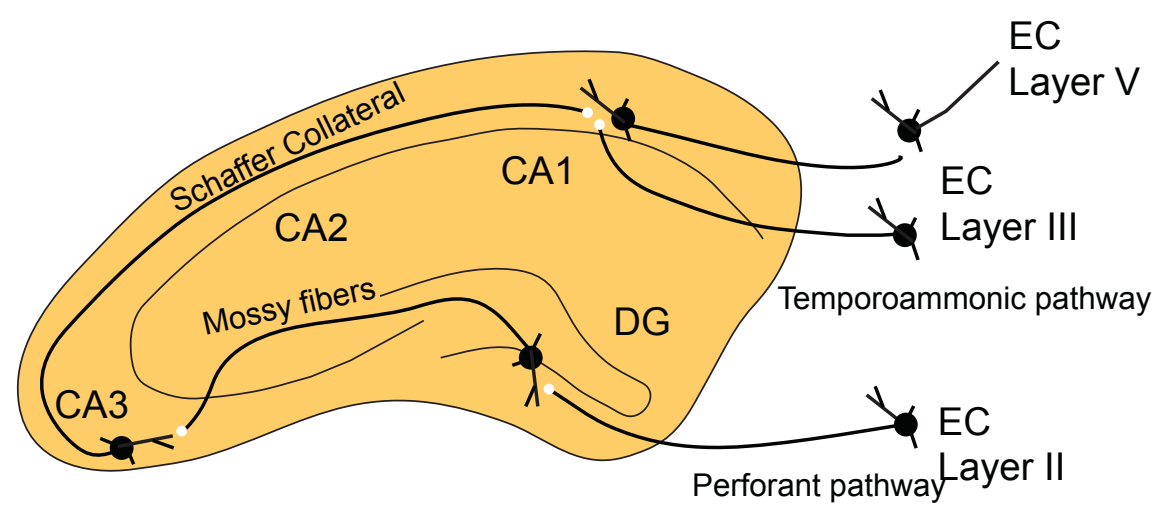

Figure 1.2. Hippocampus subregions and circuit connections.

Hippocampus is formed by cornus ammonis (CA1, CA2 and CA3) and dentate gyrus (DG). The entorhinal cortex (EC) is connected to the CA1 via temporoammonic pathway and DG via the perforant pathway. Then, DG connects to CA through mossy fibers. Representation adapted from (E. R. Kandel, Dudai, \& Mayford, 2014; Neves, Cooke, \& Bliss, 2008). 


\subsubsection{Molecular mechanisms involved in learning and memory}

Cellular and molecular mechanisms involved in memory and learning process have been actively investigated; however, the precise mechanisms remain to be elucidated. In 1949, Hebb theories established the bases of current theories of learning and memory. He considered that continuous presynaptic neuron stimulations excite the postsynaptic neuron inducing metabolic changes that increase the strength of the synapse during learning and memory (Hebb, 1949). From his postulations and based on an experimental approach in rabbit hippocampus, it was discovered that high-frequency synapse stimulations induce changes in the strength of these synapses that persist different days. This process was defined as long-term potentiation (LTP) (Bliss \& Lomo, 1973; Lomo, 1966). LTP process is complemented with long-term depression (LTD) (Dudek \& Bear, 1992). These processes of synaptic strength (synaptic plasticity) are the bases of memory engram formation. The induction of LTP varies in different brain regions and some subregions, such as CA1, which depends on N-methyl-D-aspartate receptors (NMDA). One of the first pharmacological investigations about spatial learning and LTP induction confirm that the inhibition of NMDA receptors block LTP induction and promote spatial cognitive impairments in Morris Water Maze task (Butcher, Davis, \& Morris, 1990). The activation of NMDA receptors induces a postsynaptic Ca2+ influx necessary to produce LTP (E. R. Kandel, 2001; Malenka \& Bear, 2004). The proportion of Ca2+ transferred determines the strength of the synaptic connections (Madison, Malenka, \& Nicoll, 1991; Malenka \& Nicoll, 1999). These changes promote the activation of protein kinases, such as CaMKII, PKC, PKA, MAPK (Malenka \& Bear, 2004), phosphatases (PP1 and Calcineurin) and the incorporation of AMPA receptors into the synapse membrane (Soderling \& Derkach, 2000). The activation of AMPA receptor increases the amplitude of excitatory postsynaptic potentials (fEPSs). LTP is maintained for days and requires the synthesis of new proteins, induced by different protein kinases (PKA and MAPK) that stimulate different transcription factors (TFs), such as CREB-1 (Abel et al., 1997; Frey, Huang, \& Kandel, 1993). The activation of TFs promotes the activation of several RNA polymerases and therefore the synthesis of new proteins, which is a crucial step for the formation of new synaptic connections. Long-term memories require the synthesis of new proteins (H. P. Davis \& Squire, 1984), whereas short memories require only posttranslational modifications (Routtenberg \& Rekart, 2005). Moreover epigenetic factors regulate protein synthesis, such as histone modifications during the formation of long-term memory (Guan et al., 2002). In summary, synaptic plasticity, gene expression and protein synthesis are essential mechanisms for long-term memory. 


\subsection{Alzheimer's disease}

\subsubsection{Epidemiology}

Alzheimer's disease (AD) is a neurodegenerative disorder that affects millions of people around the world. According to the World Alzheimer's Report 2015, 47.47 millions of people worldwide showed symptoms of dementia in 2015 , and it is expected to increase to 131.5 million of cases in the next 30 years (Figure 1.3A) (Prince et al., 2015). In the last decades, the expectancy of life and also the incidence of age-related diseases have raised. The main characteristic of agerelated diseases is that the number of cases rises exponentially along age. Alzheimer's disease is part of this group of pathologies. According to the World Alzheimer's Report 2015 (Prince et al., 2015), the number of cases in Europe in 2015 at the age of $65-74$ was around $0.9 \%$, from 75 to 79 was $5.8 \%$ and more than 85 years old was $24.5 \%$ (Figure $1.3 \mathrm{~B}$ ). The prevalence of $A D$ cases in women is higher than in men (Mielke, Vemuri, \& Rocca, 2014; Vina \& Lloret, 2010).
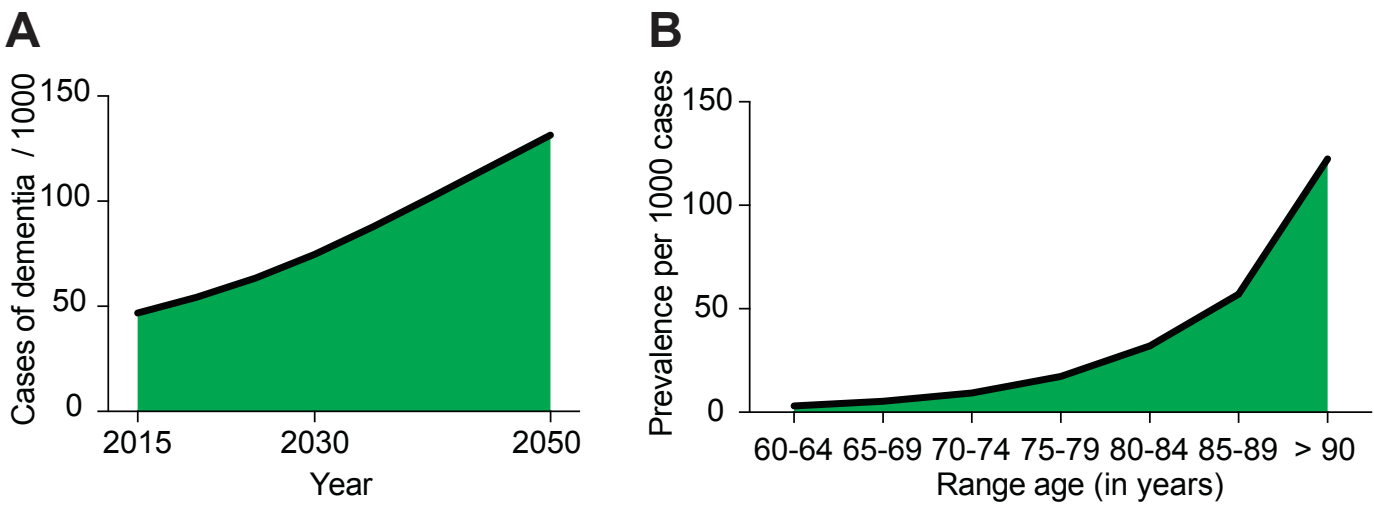

Figure 1.3. Incidence and prevalence of dementia cases.

(A) Number of dementia cases worldwide reported in 2015 and estimated in 2050. (B) Prevalence of cases of dementia in an age-specific range in Europe. Graphics were adapted from World Alzheimer's Report 2015 (Prince et al., 2015). 


\subsubsection{Pathology of Alzheimer's disease}

Alzheimer's disease (AD) is the most common form of dementia. The most common alterations in $A D$ are the loss of neurons and synaptic connections, neuroinflammation, reduction of the volume of the brain and oxidative stress (Bradshaw et al., 2013; Fox et al., 1996; Guerreiro et al., 2013; Hamos, DeGennaro, \& Drachman, 1989; Jack et al., 2004; Katzman \& Saitoh, 1991; C. Y. Lee \& Landreth, 2010; Markesbery, 1997; Scheff, Price, Schmitt, \& Mufson, 2006). The main visible signs are the loss of memory, difficulties in planning and solving problems, challenges to conclude daily activities, time and space confusion, difficulties with language, agitation and alterations of mood and personality (Reisberg, Ferris, de Leon, \& Crook, 1988). The external symptoms advance dramatically from Mild Cognitive Impairment (MCl) to severe stages during the progression of the pathology and can be categorized into six Braak stages (I$\mathrm{VI}$ ) depending on the deposition of tau protein in postmortem brain. At early AD stages, tau protein is detected in transentorhinal and entorhinal regions (I-II). Then, it is localized in limbic allocortex and neocortex at III-IV stages. Lastly, during V-VI stages, tau protein is spread throughout the neocortex (Braak, Alafuzoff, Arzberger, Kretzschmar, \& Del Tredici, 2006; Braak \& Braak, 1991).

There are two forms of Alzheimer's, the familiar or early-onset (eoAD) and the sporadic or lateonset (IOAD). The first form normally appears at early age, before 65 years, and accounts for small percentages of cases. The first form is linked to genetic mutations that affect gene for presenilin 1 or 2 (PS1 or 2) and amyloid precursor protein (APP) (Goate et al., 1991; Levy-Lahad et al., 1995; Rogaev et al., 1995; Sherrington et al., 1995). These genes show multiple mutations in different codons. For example, APP showed several mutations at codon 670/671, 692 or 717 (Goate et al., 1991; L. Hendriks et al., 1992; Levy et al., 1990). Since these mutations have been directly connected to one form of Alzheimer's disease, several groups have used these mutations to produce models of AD (Andorfer et al., 2003; Duff et al., 1996; Hsiao et al., 1996; Oakley et al., 2006; Pearson \& Choi, 1993; Quon et al., 1991; Sandhu, Salim, \& Zain, 1991). The second form of $A D$, sporadic form, is the most frequent and appears typically after 65 years old. Several factors cause the pathogenesis of LoAD, and it does not exist a direct connection with previously described genetic mutations, but it is strongly influenced by genetic components, such as $\varepsilon 4$ allele from the apolipoprotein $E$ gene (APOE- $\varepsilon 4)$ (Strittmatter et al., 1993). 


\subsubsection{Molecular mechanisms of Alzheimer's disease}

Dr. Alois Alzheimer characterized "presenile dementia" for the first time in 1906. One of his patients was Auguste Deter, who suffered from memory loss, confusion and language difficulties. Once she died in 1906, he discovered that the autopsied brain contained dense formations outside the neurons and internal accumulations of filaments or tangles (Alzheimer, Stelzmann, Schnitzlein, \& Murtagh, 1995; Hippius \& Neundorfer, 2003). Afterward, these aggregations were considered to play an essential role in the pathology. The extracellular aggregation of protein beta-amyloid $(A \beta)$ is currently known as $A \beta$ plaques, and the intracellular aggregation of hyperphosphorylated protein tau is named as neurofibrillary tangles. In 1985 and 1987 amyloid precursor protein (APP) and microtubule-associated protein tau (MAPT, coding for tau protein) genes and proteins were identified (Goldgaber, Lerman, McBride, Saffiotti, \& Gajdusek, 1987; Kang et al., 1987; Masters et al., 1985; Tanzi et al., 1987; Weingarten, Lockwood, Hwo, \& Kirschner, 1975). Since 1987 several studies have been investigated the physiological functions of APP, an integral type I transmembrane protein; however, the main function remains unclear. APP coding protein has been linked to cell and synaptic adhesion (Beher, Hesse, Masters, \& Multhaup, 1996; Soba et al., 2005), trophic and synaptic activity (Bell, Zheng, Fahrenholz, \& Cuello, 2008), neurogenesis (Rohe et al., 2008) and iron export (Duce et al., 2010).

The APP is cleaved by several secretases in different positions ( $\alpha-, \beta$ - and $\gamma$-secretase) to obtain different amyloid peptides. Specifically, $A \beta$ peptide is formed by cleavage of $\beta$-secretase (BACE-1) and then Y-secretase (Haass, Hung, Schlossmacher, Teplow, \& Selkoe, 1993). Ysecretase cleaves at different levels of the $\mathrm{C}$-terminal, producing different isoforms with distinct lengths (Figure 1.4). Around 34 to 50 forms of APP have been identified (Kummer \& Heneka, 2014; Takami et al., 2009). To note, these distinct forms show different levels of amyloidogenesis. The specific role of these peptides or aggregation forms at physiological or pathological state remains to be elucidated. There is new evidence confirming the role of these amyloid forms in AD pathology (Selkoe \& Hardy, 2016), and therefore amyloid forms are still therapeutic targets (Hardy \& Selkoe, 2002). The most common isoforms are $A \beta 40$ and $A \beta 42$. At physiological state, $A \beta 40$ isoform is higher expressed than $A \beta 42$. In contrast, $A \beta 42$ isoform is linked to the pathological state, probably because this last isoform is more hydrophobic and is more prone to aggregate than A 340 (Haass \& Selkoe, 2007; Jarrett, Berger, \& Lansbury, 1993). Mutations in PS1 or APP genes increase the proportion of A 42 peptide (Scheuner et al., 1996; Y. I. Yin et al., 2007). Following cleavage steps, all isoforms of the APP are released to the extracellular environment. Mature peptides can accumulate inside the neurons forming from soluble oligomers till fibrils that aggregate in insoluble plaques (Serpell, 2000). This plaque formation is correlated with a decrease of intraneuronal A $\beta$ levels (Mori et al., 2002; Oddo, 
Caccamo, Smith, Green, \& LaFerla, 2006). Although there is an apparent increased of betaamyloid plaques in $\mathrm{AD}$ patients, it is still not clear the impact of oligomers and plaques on the progression of the disease (Hardy \& Selkoe, 2002; Puzzo \& Arancio, 2013). Some studies revealed that $A \beta$ oligomers promote LTP deficits in the hippocampus (Gong et al., 2006) and cognitive decline (Cleary et al., 2005).

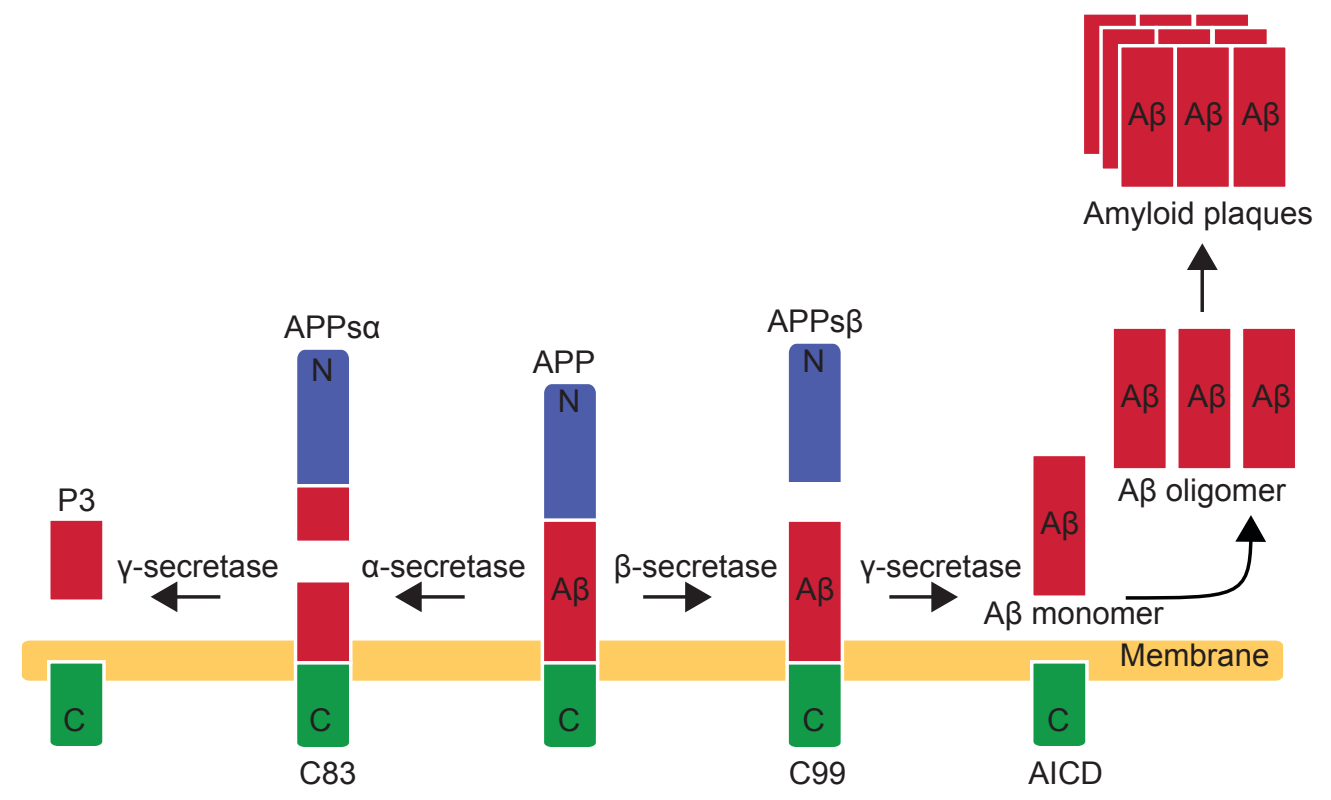

Figure 1.4. Schematic representation of APP processing and $A \beta$ aggregation.

The APP is cleaved by $\alpha$-, $\beta$ - and $\gamma$-secretases in different steps synthesizing different amyloid peptides that can form oligomers and plaques. Figure was adapted from (Eric R. Kandel, 2013).

Alois Alzheimer also identified an intracellular structure known as neurofibrillary tangles (NFT). In 1980 , it was described that NFT were composed of aggregates of tau proteins that were hyperphosphorylated (Goedert, Wischik, Crowther, Walker, \& Klug, 1988; Grundke-lqbal et al., 1986; Ihara, Nukina, Miura, \& Ogawara, 1986; Kosik, Joachim, \& Selkoe, 1986). Tau protein belongs to the family of microtubule-associated protein (MAP), and it is abundant in the central nervous system, where it participates in the assembly of tubulin into microtubules and stabilization of their structure (Grundke-lqbal et al., 1986; Mietelska-Porowska, Wasik, Goras, Filipek, \& Niewiadomska, 2014; Weingarten et al., 1975). There are different isoform variants of tau that come from the same gene, but different alternative splicing forms (Niblock \& Gallo, 2012). These isoforms showed different tubulin-binding repeats (Goode, Chau, Denis, \& Feinstein, 2000; M. Lu \& Kosik, 2001). Under a pathological state, there is an unbalance of dephosphorylated and phosphorylated levels of tau, increasing the number of hyperphosphorylated tau, promoting the detachment from the microtubule (Mandelkow \& 
Mandelkow, 2012). These alterations increase the number of single tau fractions and posterior lead to fibrillation and aggregation of tau (Figure 1.5) (Kuret et al., 2005). From Alois Alzheimer, the relation between tau-pathology and Alzheimer's disease has repeatedly been investigated (Blurton-Jones \& Laferla, 2006; Oddo, Caccamo, Tran, et al., 2006). However, the mechanism involved remains to be fully characterized. An alteration of the physiological status of tau protein promotes changes in axonal transport leading to a synaptic dysfunction and neurodegeneration (Roy, Zhang, Lee, \& Trojanowski, 2005; Trojanowski, Smith, Huryn, \& Lee, 2005). A previous study has shown that a reduction of physiological tau protein improves $A \beta$-induced deficits in a mouse model of AD (Rapoport, Dawson, Binder, Vitek, \& Ferreira, 2002).
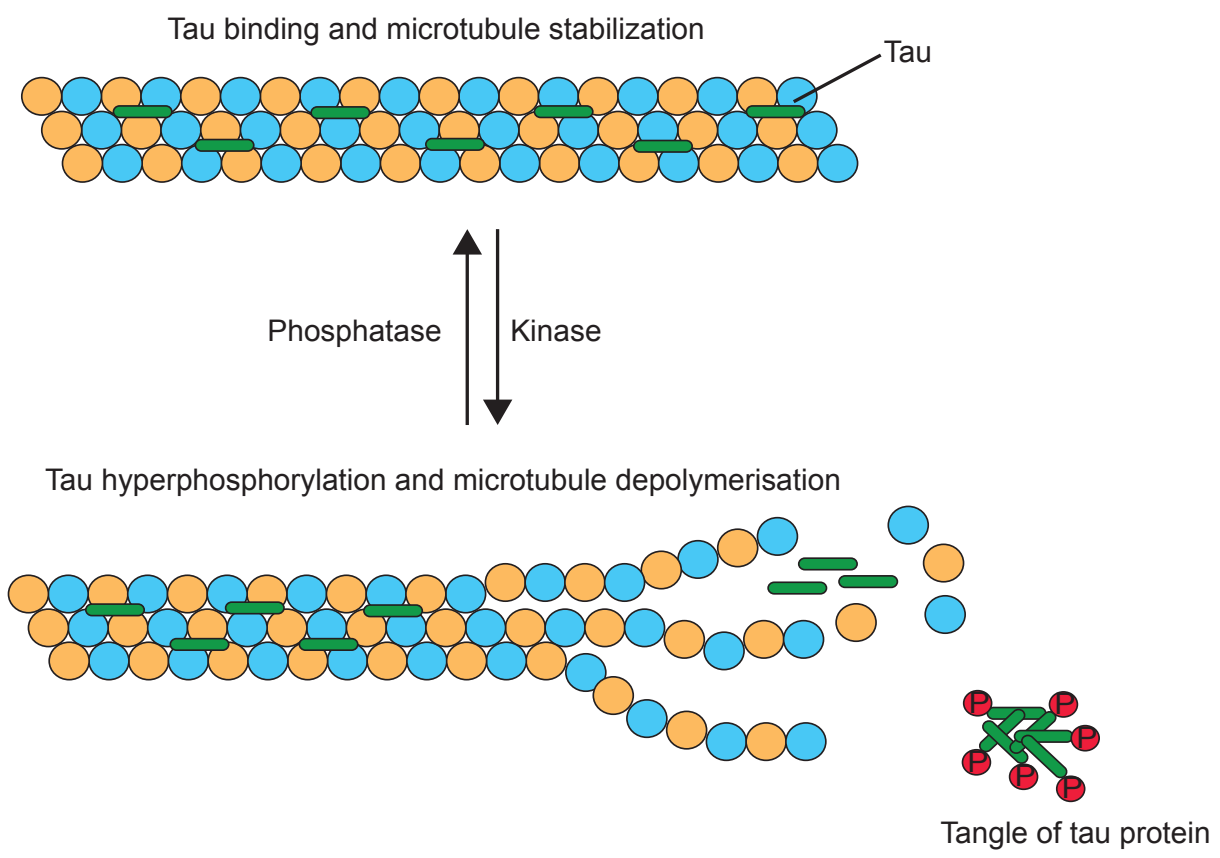

Figure 1.5. Schematic representation of the stabilization of microtubules by tau protein. Hyperphosphorylation of tau induces aggregation and formation of neurofibrillary tangles. Figure adapted from (Brunden, Trojanowski, \& Lee, 2009).

\subsubsection{Mouse models of Alzheimer's disease}

The selection of which animal model will be used depend on several factors. Among these factors are taxonomy shared with humans, life expectancy, genome, level of manipulation, size and economic costs. In the last decades, the number of animal models increased exponentially. One of the most commonly used animals is mouse species, more precisely Mus musculus. Animal models are necessary to investigate the pathology and molecular mechanisms of several 
human diseases. Specifically, these models are essential for studying human regions that are inaccessible, such as brain region, which can be only investigated in human postmortem tissues. For the last decades, different types of $A D$ models have been generated to mimic certain pathological symptoms and to further elucidate the biological basis of $A D$ and the potential targets for treatments (Allen et al., 2002; Oakley et al., 2006; Radde et al., 2006).

First transgenic mice were based on the early-onset $A D$, overexpressing one or multiple mutations of human genes involved in the familial $A D$, such as amyloid precursor protein (APP) and presenilin 1 (PS1). In 1991, first models expressed high levels of $A \beta$, overexpressing the isoform $\beta$-APP751, C-100 fragment of the human APP or the entire human APP (Pearson \& Choi, 1993; Quon et al., 1991; Sandhu et al., 1991). These models showed high levels of A , but they did not show other characteristics from AD brains. After these first models, new models were designed for expressing human mutated genes associated with specific promoters. Mutations such as London (V717I) (Goate et al., 1991; "Molecular classification of Alzheimer's disease," 1991), Swedish (K670N and M671L) (Mullan et al., 1992) or Arctic (E693G) (Kamino et al., 1992) have been used to produce several models of $A D$. Mutations of PS1 were also used to design models of AD, such as L286V (Sherrington et al., 1995) or M146L (Duff et al., 1996). Models of AD with single (APP, PS1 or TAU) (Andorfer et al., 2003; Duff et al., 1996; Hsiao et al., 1996), double (APP and PS1) (Holcomb et al., 1998; Jensen, Mottin, Cracchiolo, Leighty, \& Arendash, 2005; Radde et al., 2006), triple mutated-genes (APP, PS1 and TAU) (Oddo et al., 2003) or even more mutations were generated (Oakley et al., 2006). For example, one model currently used is 5XFAD that carries 5 FAD mutations in the APP (K670N/M671L/I716V/V717I) and PS1 (M146L/L286V) regulated by Thy1 promoter (Oakley et al., 2006). The 5XFAD model shows an increase of intraneuronal $A \beta$ content and extracellular plaque deposition, neuronal loss in cortical layer 5 (not in the hippocampus), age-dependent motor impairments and working memory decline (Oakley et al., 2006). Another well-established mouse model of AD is APPPS1-21, which contains a double Swedish mutation in APP and L166P mutation in PS1 and is regulated by Thy1 promoter (Radde et al., 2006). This model exhibits human APP protein, neuronal loss, alterations in LTP and amyloid plaque deposition (Gengler, Hamilton, \& Holscher, 2010; Radde et al., 2006). Formation of plaques was firstly observed in neocortex after 2 months, DG at 3 months and CA1 at 5-6 months. Different studies tested this model in different tasks and they observed cognitive deficits at different ages and behavior tasks (Govindarajan, Agis-Balboa, Walter, Sananbenesi, \& Fischer, 2011; Radde et al., 2006; Serneels et al., 2009). It was reported that these mice show impairments in reversal learning of a food-rewarded four-arm spatial maze at 8-months of age (Radde et al., 2006). Besides, APPPS1-21 mice showed spatial learning and memory deficits in Morris water maze at 7-8 months and alterations of associative 
memory in fear conditioning paradigm at 12-months of age (Govindarajan et al., 2011; Serneels et al., 2009; Zovoilis et al., 2011).

\subsubsection{Diagnosis and treatment of Alzheimer's disease}

One of the main difficulties to treat or diagnose Alzheimer's disease (AD) is that is more likely a multifactorial process, which starts years before the external symptoms are detectable (Bateman et al., 2012). From 1984, Alzheimer's disease cases have been diagnosed based on clinical observations, conduct test of memory, patient record and information from family members (G. McKhann et al., 1984). Nowadays, the criteria established in 1984 have been redefined, and new tools are used to detect the pathology, performing medical tests (blood and urine tests) or using brain scans such as magnetic resonance imaging, computed tomography or positron emission tomography (Frisoni, Fox, Jack, Scheltens, \& Thompson, 2010; Hort et al., 2010; G. M. McKhann et al., 2011; Sen, Majumder, Arora, Yadu, \& Chakrabarti, 2017; X. Y. Zhang, Yang, Lu, Yang, \& Zhang, 2017). However, it does not exist a fix protocol for AD diagnosis, since the results using different techniques show controversial values. More studies are necessary to obtain a precise diagnostic (Dolci et al., 2017; Sperling \& Johnson, 2013). Besides, it does not exist an effective treatment to prevent or cure AD. Currently, the only pharmacological treatments that exist try to reverse symptoms associated with the pathology of $A D$, but not to reduce or stop the neuronal loss responsible for the progression of Alzheimer's symptoms (Lane, Hardy, \& Schott, 2017). Thus, it is important to investigate further the molecular mechanisms involved in the pathology and redefine the treatment approach based on the fact that $A D$ is a multifactorial disease (Hampel et al., 2018; Weinstein, 2018). Another system to prevent the pathology is reducing daily habits that promote specific modifiable risk factors associated with Alzheimer's disease (D. E. Barnes \& Yaffe, 2011).

\subsubsection{Risk factors for Alzheimer's disease}

Several risk factors were linked to Alzheimer's disease including, aging, genetic factors, heart diseases, diabetes, stroke, hypertension, high cholesterol, smoking, diet, exercise or level of education (Appleton, Scutt, Sprigg, \& Bath, 2017; Moonga et al., 2017; Profenno, Porsteinsson, \& Faraone, 2010; Sah, Lee, Jang, \& Park, 2017; Saito, Diaz, Chung, \& McMurtray, 2017; Sharp $\&$ Gatz, 2011). There are intrinsic risk factors that cannot be modified, such as aging, familial predisposition or genetic factors. One of the most relevant factors is aging. The probability to develop Alzheimer's disease increases dramatically with age (Prince et al., 2015). Regarding genetic factors, one example is APOE- $\varepsilon 4$ protein, which is part of a cholesterol transporter 
family. There are three variants of APOE protein; the most frequent is APOE- $\varepsilon 3$, but individuals that carry $A P O E-\varepsilon 4$ showed major risk to develop $A D$ and number of plaque deposition than APOE-ع3 (Bales et al., 1997; Corder et al., 1993; Strittmatter et al., 1993). The modifiable risk factors are related to cardiovascular diseases (hypertension, high cholesterol, diabetes, obesity) (Appleton et al., 2017; Moonga et al., 2017; Profenno et al., 2010) and quotidian habits (smoking, diet, exercise and education) (Sah et al., 2017; Saito et al., 2017; Sharp \& Gatz, 2011). For example, an increase of insulin levels has been linked to increased levels of APP (Farris et al., 2003). Moreover, epidemiological studies showed that exercise reduces the risk of AD by half (Rolland, Abellan van Kan, \& Vellas, 2008).

\subsubsection{Gene expression and exon usage associated with $A D$}

The expression of specific genes at specific time establishes the full functions of a cell by encoding proteins. Thus, mRNA molecules reflect the cellular state at a specific time. The process of gene expression is extremely regulated and this regulation determines the production of a certain amount of specific proteins (Neaves, 2002). François Jacob and Jacques Monod were the firsts to show a model for regulation of protein synthesis in 1961. They investigated how a bacteria regulate the synthesis of an enzyme called beta-galactosidase (Jacob \& Monod, 1961). The process of gene expression comprises two main parts: transcription and translation (Djebali et al., 2012; Hahn, 2004; Hampsey, 1998; T. I. Lee \& Young, 2000; Neaves, 2002). In the last decades, specific gene mutations and alterations of gene expression have been related to Alzheimer's disease (AD). Mutations in Amyloid Precursor Protein (APP), Presenilin 1 and 2 (PS1 and PS2) are the cause of AD familiar form (Goate et al., 1991; Levy-Lahad et al., 1995; Rogaev et al., 1995; Sherrington et al., 1995). Increase levels of certain variants of genes promote a higher susceptibility to develop AD, such as APOE- $\varepsilon 4$ gene (Strittmatter et al., 1993), TOMM40 (translocase of outer mitochondrial membrane 40 homolog) (Roses et al., 2010), CR1 (complement C3b/C4b receptor 1) (Lambert et al., 2009), CLU (Clusterin protein), ABCA7 (ATP binding cassette subfamily A member 7) (Hollingworth et al., 2011), BIN1 (bridging integrator 1) (Seshadri et al., 2010) or PICALM (phosphatidylinositol binding clathrin assembly protein) (Harold et al., 2009). Moreover, gene expression studies performed in cell lines, mouse model brains and human postmortem brain regions found different transcriptional profiles during AD progression (Bossers et al., 2010; Matigian et al., 2010; Pasinetti, 2001). Some of these studies investigated a specific period of the pathology, early or late AD stages (Gatta, D'Aurora, Granzotto, Stuppia, \& Sensi, 2014; Pasinetti, 2001; Twine, Janitz, Wilkins, \& Janitz, 2011). A recent study in human postmortem brain regions identified gene expression and splicing changes in the temporal and frontal lobes. In the frontal lobe of $A D$, there was an increase of 
expression of genes involved in synaptic transmission and regulation, neurotransmitter transport and negative regulation of neuronal apoptosis. Contrarily, the expression of genes associated with axon transport and neurotransmission was increased in the temporal lobe of AD brain (Twine et al., 2011). Another study investigated in which level genes linked to immune or inflammatory pathways are expressed in different areas of the human brain such as entorhinal cortex, superior frontal gyrus and hippocampus during aging and AD (Cribbs et al., 2012). They found that genes related to immune and inflammatory pathways showed more alterations during aging than $A D$ progression. Furthermore, other studies have screened the expression at different stages of AD (Blalock et al., 2004; Bossers et al., 2010; Katsel, Li, \& Haroutunian, 2007). Gene expression analysis in the human medial frontal brain region from different Braak stages showed that 922 genes have a direct correlation with Braak stages (Bossers et al., 2010). Geneexpression patterns from early stages were distinct than from late stages, suggesting a direct connection between the transcriptional alterations and the neurofibrillary tangle changes. Genes that increased during the progression of $A D$ were linked to differentiation and proliferation, metal ion binding and inflammation. Genes that decreased during the progression of the pathology were associated with synaptic activity and cell adhesion, such as genes linked to exocytosis of neurotransmitters (VAMP7, SYT4, SNAP25) and potassium voltage-gated channels (KCNS3, KCNB1) (Bossers et al., 2010). In addition to human studies, the transcriptional profile of AD mouse models has also been investigated (Blalock et al., 2004; Gatta et al., 2014; J. A. Miller, Oldham, \& Geschwind, 2008; Parra-Damas et al., 2014; Seshadri et al., 2010; Twine et al., 2011). Gene expression in the hippocampal brain subregion of 3xTg-AD mouse model showed multiple changes at 3 and 12 months of age. During early stages of AD genes related to mitochondrial activity, regulation of $\mathrm{Ca} 2+$, inflammatory response and synaptic activity were altered. In contrast, at late stages, AD hippocampus showed expression changes in genes related to synaptic activity, differentiation, cell division, neuronal proliferation and cognitive decline (Gatta et al., 2014). Although the transcriptional profile of brain regions of AD humans and mouse models has been intensively researched, the precise role of transcriptional changes remain poorly understood and some results are still controversial.

In addition to transcriptional changes, recent studies indicate that alterations in mRNA processing contribute to AD (M. K. Lai, Esiri, \& Tan, 2014; Mills et al., 2013; Stilling et al., 2014; Tollervey et al., 2011; Twine et al., 2011). The last step of transcription is RNA processing, in which introns are removed from the RNA structure, and exons are spliced together. Multiple small nuclear ribonucleoproteins rich with Us (snRNPs: U1, U2, U4, U5 and U6) drive the mRNA processing step (Busch, Reddy, Rothblum, \& Choi, 1982; Weinberg \& Penman, 1968). The combination of these 5 snRNPs and specific proteins forms the spliceosome. In conjunction with 
the rest of transcription steps, it is highly regulated by exonic splicing enhancers and silencers, intronic splicing silencers and heterogeneous nuclear ribonucleoproteins (hnRNPs) (Neaves, 2002; Qian \& Liu, 2014). Alternative splicing is an essential process for complex organisms providing a high level of proteomic complexity since this promotes an exponential increase of mRNA variants from a single gene (Neaves, 2002; C. W. Smith \& Valcarcel, 2000). A previous study indicated that 92 to $94 \%$ of the human genes showed alternative splicing forms (E. T. Wang et al., 2008). Deregulation of alternative splicing can play an important role in the progression of a disease (Buratti, Brindisi, Pagani, \& Baralle, 2004; Disset et al., 2006; Sebestyen, Zawisza, \& Eyras, 2015). Genes that participate in the progression of AD pathology have alternative splicing forms, such as APP, PS1, PS2, TAU and APOE genes. For example, APP has an alternative exclusion in exon 8 (Penna et al., 2013), PS1 gene in exon 4 (De Jonghe et al., 1999), APOE gene in exon 5 (Mills et al., 2013) or TAU present an exon 10 inclusion (Andreadis, Brown, \& Kosik, 1992). These alternative variants might play an important role in $A D$. For example, APP variant with exon 7 has higher levels in the AD than the control group and is associated with beta-amyloid production (Alam, Suzuki, \& Tsukahara, 2014). Moreover, global changes in splicing events have been investigated for the AD pathology. In some cases, these changes correlate to gene expression, in others not (Stilling et al., 2014; Tollervey et al., 2011; Twine et al., 2011). For example, a recent study identified alterations of distinct pathways associated with gene expression and splicing changes in an AD mouse model. Gene expression changes affected pathways related to inflammation, whereas genes with different splicing forms were linked to neuronal plasticity. Similar to the transcriptomic data, further investigations are required to fully understand the role of alternative splicing during the progression of $A D$. 


\subsection{Aging}

The major risk factor for developing Alzheimer's disease is aging. Aging is a complex process based on the progressive dysfunction of almost every tissue and cells from the most living organism at different times. Furthermore, there is high variability between old individuals; some individuals exhibit normal cognition, whereas others show memory loss or physical problems (Koivisto et al., 1995; Montesanto, Dato, Bellizzi, Rose, \& Passarino, 2012). Thus, aging is a complex process to study. Multiple theories postulated different causes of aging, such as the formation of mitochondrial free radicals (Harman, 2003), inflammatory response (Chung, Kim, Kim, \& Yu, 2001), activation of immune system (Franceschi, 1989), mitochondrial dysfunction (Cadenas \& Davies, 2000) or telomere shortening (Martin-Ruiz et al., 2006). There is a global agreement that aging is caused by a progressive accumulation of molecular damage that at a certain time cannot be repaired or removed it (Harman, 2003; Kirkwood et al., 2003; Vijg \& Campisi, 2008). Several external factors can contribute to this damage, such as stress, irradiation, UV light, toxins, genetics, epigenetics or exercise (Jansen-Durr \& Osiewacz, 2002). However, the precise causes and mechanisms involved remain unclear. Nowadays, there is a global consensus that multiple molecular mechanisms cause aging (Kirkwood, 2005; Kowald \& Kirkwood, 1996; Weinert \& Timiras, 2003). Aging process affects almost every tissue, including the brain. The process of aging in mammalian brains affects the structure and activity of neurons, promoting alterations in dendritic patterns (Burke \& Barnes, 2006), reduction of white matter density (Bartzokis et al., 2003; Hedden \& Gabrieli, 2004), alteration of frontal cortex connections with the hippocampus (Hedden \& Gabrieli, 2004) and reduction synapse number (X. Liu, Erikson, \& Brun, 1996). These changes in brain structure induce cognitive deficits and motor impairments 252,253 . Specifically in human brains, aging promotes a delay of verbal information recalling, deficits in working and spatial memory, reductions of the processed speed (Albert, Duffy, \& Naeser, 1987; Craik, Moscovitch, \& McDowd, 1994; Petersen, Smith, Kokmen, Ivnik, \& Tangalos, 1992; Zelinski \& Burnight, 1997). Imaging studies of human brains found a reduction of brain region connections, reduce activations and poorly organized activations in regions involved in cognition (Andrews-Hanna et al., 2007; Logan, Sanders, Snyder, Morris, \& Buckner, 2002; Persson et al., 2004). One of the most conserved aged features between mammalian species is spatial cognitive impairment (Govindarajan et al., 2013; Head et al., 1995; Z. C. Lai, Moss, Killiany, Rosene, \& Herndon, 1995). It is difficult to separate cognitive decline and functional changes originated from neurodegenerative diseases and aging. Previous studies using functional magnetic resonance imaging correlated a reduction of metabolic activities in the 
subiculum and dentate gyrus with normal aging, whereas a reduction of activity in the entorhinal cortex was correlated with Alzheimer's disease (Small, Tsai, DeLaPaz, Mayeux, \& Stern, 2002).

\subsubsection{Molecular, gene expression and mRNA processing changes along aging}

Aging promotes deregulation of the expression of specific genes, affecting pathways that are well conserved between species. For example, changes in genes related to stress response or mitochondrial functions during aging were observed in Caenorhabditis elegans (McCarroll et al., 2004), Drosophila (McCarroll et al., 2004; Pletcher et al., 2002; Zou, Meadows, Sharp, Jan, \& Jan, 2000), rats (Blalock et al., 2003), mice (Jiang, Tsien, Schultz, \& Hu, 2001; C. K. Lee, Weindruch, \& Prolla, 2000) and humans (T. Lu et al., 2004). Aged-animals show alterations to genes related to similar pathways, which were defined as the gene hallmarks of aging. The majority of genes altered in aging are involved in stress response and DNA damage, immune system, protein synthesis, mitochondrial function, gene expression, mRNA processing and growth factor signaling. The deregulation of these genes is in agreement with previous aging theories that described alteration of certain of these pathways. There is a close relation between transcriptional changes and functional alterations.

Genes related to oxidative stress are strongly up-regulated in aged mice, including genes involved in DNA damage, cellular defense and immune system (Fraser, Khaitovich, Plotkin, Paabo, \& Eisen, 2005; C. K. Lee et al., 2000). Caloric restriction in aged-mice reverses certain age-associated changes in gene expression and delays oxidative stress (Hyun, Emerson, Jo, Mattson, \& de Cabo, 2006; C. K. Lee et al., 2000). DNA damage has been extensively associated with aging. Internal and external factors, such as reactive oxygen species (ROS), chemicals or spontaneous mutations, can alter the integrity of DNA promoting telomere shortening, translocations and gene mutations (Faggioli, Wang, Vijg, \& Montagna, 2012; Hoeijmakers, 2009). In case of Werner syndrome, mutations in DNA helicases induced accelerated-aged pathology (Burtner \& Kennedy, 2010).

Furthermore, aged-brain shows changes in immune and inflammatory systems (Salminen, Kaarniranta, \& Kauppinen, 2012; Weiskopf, Weinberger, \& Grubeck-Loebenstein, 2009). There is a reduced reaction against pathogens and alter over-activity in physiological stages (Franceschi \& Campisi, 2014; Salminen et al., 2012). This over-activity of inflammation, known as "inflammaging", can be induced by tissue damage, increased of senescent cells or enhancement of NF-kB transcription factor (Salminen et al., 2012). Inhibition of NF-kB transcription factor activity in models of accelerated aging delays aged-associated characteristics (Osorio et al., 2012). Genes related to inflammatory responses and immune 
systems were up-regulated in aged human blood and brain (Berchtold et al., 2008; de Magalhaes, Curado, \& Church, 2009; Mirza, Pollock, Hoelzinger, Dominguez, \& Lustgarten, 2011; Peters et al., 2015).

There is a significant reduction of synaptic connections and genes linked to synaptic activities, such as genes related to glutamate receptors, calcium signaling pathway, synaptic vesicles and signal transduction in the human frontal cortex during aging (T. Lu et al., 2004). Reduction of genes related to synaptic activities was also observed in other brain regions of aged-organisms (Blalock et al., 2003; Fraser et al., 2005; Loerch et al., 2008).

There is evidence confirming that mitochondrial dysfunction contributes to aging (Cadenas \& Davies, 2000; Green, Galluzzi, \& Kroemer, 2011; Harman, 2003). These alterations affect mainly mitochondrial DNA (mtDNA) and respiratory chain. The mitochondrial respiratory chain provides the energy (ATP) to rest of tissues, including the brain. Alteration in this process can stimulate the formation of reactive oxygen species (ROS). Several mechanisms protect the mitochondria against ROS. However, these defenses are reduced during aging process. In the past, the mitochondrial free radical theory postulated that an increase of ROS induces macromolecular damage, causing the process of aging (Harman, 1965). In contrast, recent studies suggested that an increase of ROS might extend the lifespan of yeast and Caenorhabditis elegans (Doonan et al., 2008; Mesquita et al., 2010) and do not accelerate aging (Van Remmen et al., 2003; Y. Zhang et al., 2009). Nowadays, the connection between aging and ROS involve stress response (Hekimi, Lapointe, \& Wen, 2011). Other alterations in mitochondrial functions have been associated with aging. Mitochondrial DNA (mtDNA) is more vulnerable to suffer mutations, due to the oxidative condition and reduced efficiency of mtDNA reparation (Linnane, Marzuki, Ozawa, \& Tanaka, 1989). Mutations in mtDNA and increase of oxidative DNA damage have been identified in aging and Alzheimer's disease brains (Honda et al., 2005; T. Lu et al., 2004; Mecocci et al., 1993). These mutations in mtDNA decrease the activity of respiratory chain enzymes (Lin, Simon, Ahn, Kim, \& Beal, 2002). Removing mitochondrial DNA polymerase promote a decrease in lifespan, accelerated aging and mitochondrial dysfunction (Trifunovic et al., 2004; Vermulst et al., 2008). Moreover, genes related to mitochondria were down-regulated in different animals with aging (Blalock et al., 2003; T. Lu et al., 2004).

Moreover, genes related to protein synthesis are also altered in different species during aging, such as genes related to ribosome biogenesis and translation. Both functions play an important role in mechanistic target of rapamycin pathway (mTOR), which has a crucial effect in stimulation of translation, inhibition of autophagy and longevity (ladevaia, Liu, \& Proud, 2014; S. C. Johnson, Rabinovitch, \& Kaeberlein, 2013; Lamming, 2016). This down-regulation of ribosome-associated genes have been observed in yeasts (Janssens et al., 2015; Kamei, Tamada, Nakayama, Fukusaki, \& Mukai, 2014), Caenorhabditis elegans (Ma et al., 2016), mice 
(W. Zhang et al., 2015) and humans (Berchtold et al., 2008; Kumar et al., 2013). Furthermore, genes related to the process of gene transcription were altered during aging. Alterations in transcription during aging enhance mRNA heterogeneity (Martinez-Jimenez et al., 2017; Wiley et al., 2017).

There is an alteration post-transcriptional processing during aging. Moreover, genes related to splicing and polyadenylation were deregulated in human aging (Harries et al., 2011; Meshorer \& Soreq, 2002) and mice (S. A. Rodriguez et al., 2016). Over-expression of splicing factor 1 (SFA-1) increases lifespan in Caenorhabditis elegans (Heintz et al., 2017). Several genes show a differential exon usage in the skin, muscle, bone, white adipose, thymus and hippocampus brain region of aging mice (S. A. Rodriguez et al., 2016; Stilling et al., 2014). In summary, aging is a complex process that is affected at different levels for multiple dysfunctions in the organism. 


\subsection{Epigenetic factors}

Conrad Waddington firstly defined the epigenetic concept in 1939. He defined it as "the causal interactions between genes and their products, which bring the phenotype into being" (Waddington, 1939; C. H. Waddington, 1957; Waddington, 1959, 2012). In 1957, he published the book "The strategy of genes" where he defined how cells with an exact genetic background could differentiate to a distinct tissue (Figure 1.6) (C.H. Waddington, 1957). The epigenetic process is a mechanism that promotes reversible changes in gene expression without altering the sequence of DNA itself (epigenetic, above genes) (Holliday, 1987; V. E. A. Russo, Robert A. Martienssen, \& Arthur D. Riggs, 1996). It is considered to be the bridge between the external environment and internal integration of this information, playing an essential role in the adaptation to different external or internal conditions (Van Speybroeck, 2002). Epigenetic factors are related to several physiological functions and also linked to several disease pathologies (Cao \& Sudhof, 2001; Crosio, Heitz, Allis, Borrelli, \& Sassone-Corsi, 2003; Dompierre et al., 2007; Fischer, Sananbenesi, Wang, Dobbin, \& Tsai, 2007; Guan et al., 2002; Neumann et al., 2006; Tsankova, Kumar, \& Nestler, 2004). These epigenetic changes can be reversed or persist during the whole life and even be transmitted to the next generations. Recent studies found that different types of epigenetic factors can be transmitted to the offspring promoting changes of physiological conditions (Anway, Cupp, Uzumcu, \& Skinner, 2005; Byrnes, 2005; Carone et al., 2010; Chamorro-Garcia et al., 2013; de Castro Barbosa et al., 2016b; Dias \& Ressler, 2014b; Dunn \& Bale, 2009; Gapp et al., 2014; Ng et al., 2010; Ost et al., 2014; Ribeiro et al., 2017; Schmauss, Lee-McDermott, \& Medina, 2014; Vassoler, White, Schmidt, Sadri-Vakili, \& Pierce, 2013; Wei et al., 2014; S. Zhang et al., 2017). This inherited transmission can occur for one generation (intergenerational) or more (transgenerational). 


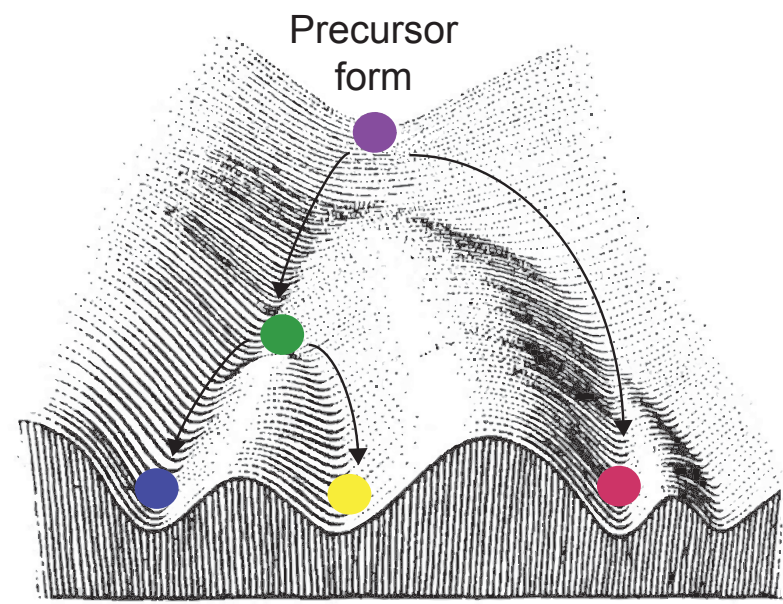

Astrocyte Oligodendrocyte Neuron

Figure 1.6. Waddington epigenetic landscape.

Model of Waddington to describe the cellular decisions during development. The cell with unique genetic information is represented by a ball that rolls down to different cell fates (colors) controlled by epigenetic factors till reaches one of the valleys (maturation form). Representation was adapted from Waddington, 1957 (C.H. Waddington, 1957).

Epigenetic changes involve different factors, including post-translational histone modifications, DNA methylation and non-coding RNAs. Histone modifications bind to the tail domain of histones. Histone proteins constitute the structure where the DNA is wrapped around forming the nucleosomes. This structure (histone octamer) is formed for two of each histone: H2A, H2B, H3 and H4 and the histone linker H1 (Luger, Mader, Richmond, Sargent, \& Richmond, 1997). Transcription depends on the level of DNA condensation. Multiple histone tail modifications, such as the addition or removal of acetyl, methyl, phosphoryl and ubiquitin groups regulate the level of DNA compaction. These incorporations promote different levels of compaction and therefore distinct stimulation of transcription process (Bradbury, Inglis, Matthews, \& Sarner, 1973; Goldknopf et al., 1975; Murray, 1964; Tanner et al., 1999; Verdin \& Ott, 2015). Several genes participate in those processes of adding or removing groups from the tails, such as acetyltransferases (HATs), deacetylases (HDAC), methyltransferases (HMT) or demethylases (Allfrey, Faulkner, \& Mirsky, 1964). These histone tail modifications alter distinct process associated with aging and Alzheimer's disease (AD). For example, H4K12 acetylation and H4K16 acetylation proportion increases with aging, whereas as H3K9 methylation proportion decreases with aging (Benito et al., 2015; Fraga \& Esteller, 2007; Han \& Brunet, 2012). Inhibition of HDAC6 in a mouse model of AD restores memory cognition (Govindarajan et al., 2013). 
Moreover, changes in DNA methylation or hydroxymethylation also regulate the gene expression process. DNA methyltransferases (DNMTs) transfer a methyl group specifically to cytosine residues in regions enriched with cytosine and guanine nucleotides, called CpG islands, silencing gene transcription (B. Jin, Li, \& Robertson, 2011; Okano, Xie, \& Li, 1998). There is a reduction of levels of methylation and hydroxymethylation in samples of $A D$ patients or $A D$ mouse models (Mastroeni et al., 2010; Shu et al., 2016). In 2010, there was a study that described a reduction of 5-methylcytosine in neurons of postmortem cortical tissue of AD patients (Mastroeni et al., 2010).

Lastly, non-coding RNAs (ncRNAs) regulate gene expression at different levels, transcriptional and post-transcriptional levels. The first described RNAs that play a role in transcription and translation were the ribosomal RNA ( $R R N A$ ) and transfer RNA (tRNA), which were discovered in the 1950s (Roberts, 1958). Thirty years later, the first non-coding RNA structures were described in Caenorhabditis elegans (R. C. Lee, Feinbaum, \& Ambros, 1993). NcRNAs are transcribed RNAs from non-coding regions of the genome, which do not translate into proteins. These noncoding regions represent more than $90 \%$ of the genome (Carninci et al., 2005; Mehler \& Mattick, 2006). NcRNAs regulate several physiological functions of the organism, such as development, metabolism, memory formation, synaptic plasticity, homeostasis and cell death (Bernard et al., 2010; Mehler \& Mattick, 2006; Rani et al., 2016). In contrast, ncRNAs also participated in the pathology of several diseases, including neurodegenerative diseases (Esteller, 2011; Gangwar, Rajagopalan, Natarajan, \& Deiuliis, 2018; Hebert et al., 2010; Qureshi \& Mehler, 2013; Salta \& De Strooper, 2017; Tan et al., 2014). Furthermore, several studies indicate that ncRNAs are tissue-specific over a specific time (Belgard et al., 2011; Credendino et al., 2017; Krichevsky, Sonntag, Isacson, \& Kosik, 2006; Ludwig et al., 2016; Mercer, Dinger, Sunkin, Mehler, \& Mattick, 2008). Depending on the length, ncRNAs are classified in small non-coding RNAs (sncRNAs) with less of 200 base pairs or long non-coding RNAs (IncRNAs) with more than 200bp. The most studied sncRNAs are the microRNAs (miRNAs), which are small RNA structures formed by 19-25 nucleotides. These structures are synthesized from a primary form of miRNA (pri-miRNA) that is first cleaved by DROSHA ribonuclease in the nucleus and then by DICER1 ribonuclease in the cytoplasm (Lagos-Quintana, Rauhut, Lendeckel, \& Tuschl, 2001; Mattick \& Makunin, 2005; Qureshi \& Mehler, 2012). The mature miRNA is combined with Ago proteins forming the RNA-induced silencing complex (RISC) (Qureshi \& Mehler, 2012). The miRNAs bind to the complementary mRNA repressing or deleting the expression (Mattick \& Makunin, 2005). Interestingly, one miRNA can bind to multiple mRNA targets, and multiple miRNAs can bind to the same mRNA. Thus, one or few miRNAs can regulate complex networks (Qureshi \& Mehler, 2012). A wide variety of miRNAs are expressed in mouse (Bak et al., 2008; He et al., 2012) and human brains (Ludwig et al., 2016; Lukiw, 2012). MiRNAs participate in nervous system 
physiological functions, such as synaptic plasticity and neurogenesis (Kawasaki \& Taira, 2003; Shibata, Nakao, Kiyonari, Abe, \& Aizawa, 2011; Smirnova et al., 2005; Tognini, Putignano, Coatti, \& Pizzorusso, 2011). Moreover, miRNAs are involved in memory and learning process (Konopka et al., 2010; W. Wang, Kwon, \& Tsai, 2012). In contrast, miRNAs also participate in neurological pathologies, such as Alzheimer's or Parkinson diseases (W. Wang et al., 2012). Changes in miRNAs expression have also been detected during the process of aging (reference). Since miRNA expressions have an impact in several pathological processes and are stable in organism fluids, these non-coding structures have been proposed and used as disease biomarkers. Moreover, extracellular miRNAs can reflect changes from other internal tissues, facilitating the diagnosis of pathologies associated to tissues of difficult access, such as the brain (Rao, Benito, \& Fischer, 2013). MicroRNAs from the brain are secreted to the extracellular spaces inside exosomes (Valadi et al., 2007), bind to high-density lipoproteins (Vickers, Palmisano, Shoucri, Shamburek, \& Remaley, 2011) and forming a complex with Ago proteins (Arroyo et al., 2011; Turchinovich, Weiz, Langheinz, \& Burwinkel, 2011). Detection of circulating miRNAs in the blood could provide information for the diagnosis of pathology (X. Chen et al., 2008; S. J. Cohen et al., 2008; Y. K. Kim, 2015; Rao et al., 2013).

Furthermore, there are other forms of small RNAs, which are less known, the small nucleolar RNAs (snoRNAs) and Piwi-interacting RNAs (piRNAs). Small nucleolar RNAs (snoRNAs) are small structures of 60-170 nucleotides expressed in the nucleolus. There are two classes of snoRNAs, the box C/D snoRNAs and box H/ACA snoRNAs. The most known function of snoRNAs is the posttranscriptional maturation of ribosomal RNAs (rRNAs) (Cavaille, Nicoloso, \& Bachellerie, 1996; Ganot, Bortolin, \& Kiss, 1997). These structures also participate in the guidance of RNA modifications, regulation of splicing (Kishore et al., 2010; Scott et al., 2012; Q. F. Yin et al., 2012), regulation of chromatin structure (Schubert et al., 2012) and mediation of oxidative stress (Michel et al., 2011; Scott et al., 2012). Some of the snoRNAs are exclusively identified in the brain (Cavaille et al., 2000; Huttenhofer et al., 2001; Rogelj, Hartmann, Yeo, Hunt, \& Giese, 2003), and others were identified in other tissues (Jorjani et al., 2016). SnoRNAs have also been related to neurological pathologies and process of aging (Sridhar, Gan, \& Schlick, 2008; Steinbusch et al., 2017). Specific snoRNAs also play a role in associative memory (Rogelj et al., 2003). Furthermore, snoRNAs can be cleaved into small fragments (20-24 nucleotides), known as snoRNA-derived RNA (sdRNAs) (Scott, Avolio, Ono, Lamond, \& Barton, 2009; Taft et al., 2009). Multiple studies showed that these fragments are precursors of miRNAs or have similar roles than miRNAs (Brameier, Herwig, Reinhardt, Walter, \& Gruber, 2011; Ender et al., 2008; Falaleeva \& Stamm, 2013; Garcia-Lopez et al., 2015; Ono et al., 2011; Scott et al., 2009). However, the precise mechanism of synthesis and functional activity remains to be elucidated. Piwi-interacting RNAs (piRNAS) are small structures of 20-35 nucleotides that are 
detected in large proportion in germline cells of different species, such as Drosophila, Caenorhabditis elegans, Xenopus tropicalis, mice and rats (A. A. Aravin et al., 2003; A. A. Aravin et al., 2001; A. A. Aravin et al., 2008; A. Aravin et al., 2006; Armisen, Gilchrist, Wilczynska, Standart, \& Miska, 2009; Batista et al., 2008; Lau et al., 2006). PiRNAs play an essential role in the development of germ-line cells, silencing the transposable elements (TE). For that, piRNAs bind to PIWI proteins (A. A. Aravin, Sachidanandam, Girard, Fejes-Toth, \& Hannon, 2007; Zheng \& Wang, 2012). PiRNAs have also been identified in somatic cells of the nervous system, liver, kidney, spleen and lung (E. J. Lee et al., 2011; Yan et al., 2011). However, the piRNA function in somatic cells remains unknown. Recent studies suggest that piRNAs could be involved in spine formation (E. J. Lee et al., 2011) and could participate in Alzheimer's disease (Qiu et al., 2017). 


\subsection{Intergenerational inheritance}

\subsubsection{History of evolution: Lamarck}

For almost two centuries the theory of evolution from Lamarck was forgotten. However, in the last decades, it gained importance again. Jean-Baptiste Pierre Antoine de Monet, Chevalier de Lamarck published the "Philosophie zoologique ou exposition des considerations relatives à I'histoire naturelle des animaux" in 1908. In this publication, he mainly formulated two concepts. First, he postulated that organisms change their characteristics according to external conditions and repetitive use of specific parts of the body. Secondly, these changes can be transmitted to the next generations (Lamarck, 1809). Moreover, Conrad Waddington described for the first time the concept of epigenetics in 1939 (Waddington, 1939). Nowadays, the definition of epigenetic factors is the combination of heritable factors that promote changes in gene activity without altering the DNA sequence (Bird, 2007). There is emerging evidence that phenotypic changes induced by paternal exposition to specific external conditions can alter the phenotype of the offspring through the transmission of certain epigenetic factors 219,(Danchin et al., 2011). This new concept of epigenetic transmission is closely related to Lamarck postulations and do not contradict Darwin theory of evolution. For the last decades, there is an exponential increase of studies of epigenetic inheritance. Despite this increase of research, it does not exist an official and universal definition of this kind of transmission (Bird, 2007; Griesemer, 2002; Jablonka \& Raz, 2009; Ptashne, 2007; Vincenzo E. A. Russo, Robert A. Martienssen, \& Arthur D. Riggs, 1996). In general, epigenetic transmission or epigenetic inheritance refers to the transmission of specific phenotype to the next generation potentially through epigenetic factors. The existence of epigenetic marks in germ-line cells suggests that these factors might participate in the transmission of certain information to the next generations 98-112. The exact mechanism is poorly understood. This process of transmission can pass to one generation or several, according to that it will be an intergenerational or transgenerational inheritance (Figure 1.7A-B). The intergenerational inheritance consists in the transmission of changes only to the first generation (F1), which is also indirectly in contact with the external condition (F1 germ cells) (Dias \& Ressler, 2014a; Franklin et al., 2010; Rodgers, Morgan, Bronson, Revello, \& Bale, 2013; Roth, Lubin, Funk, \& Sweatt, 2009). Moreover, it is also the transmission of changes between $\mathrm{F} 1$, who is exposed to a condition in the uterus or after birth, and their offspring (F2). On the other hand, the process of transgenerational inheritance consists in the transmission of nongenetic information for at least three generations. The third generation is the first one that is not exposed to the external condition (Anway et al., 2005; Newbold, Padilla-Banks, \& Jefferson, 
2006; Skinner, 2008). This transmission is probably due to a stable modification of the epigenome profile in germ cells (Anway et al., 2005; Skinner, Anway, Savenkova, Gore, \& Crews, 2008). F3 generation from F0 exposed to vinclozolin exhibited changes in anxiety behaviors and gene expression in the hippocampus and amygdala (Skinner et al., 2008).

\subsubsection{Mechanisms of transmission}

The epigenetic phenotypic changes can mainly be transmitted independently or through the germ-line (Youngson \& Whitelaw, 2008). The early development and adult behavior depend on the maternal and paternal care (Figure 1.7C) (I. C. Weaver, 2007). Licking, grooming and nursing behaviors can have a substantial impact in the offspring, promoting changes that can remain till adulthood. Multiple animal species showed altered behavior phenotypes due to different maternal or paternal cares (Gerra et al., 2007; Kikusui, Isaka, \& Mori, 2005; Lovic, Gonzalez, \& Fleming, 2001; Maestripieri, 2005). For example, female rat offspring who was subjected to maternal care deprivation showed deficits in maternal care in adulthood (Gonzalez, Lovic, Ward, Wainwright, \& Fleming, 2001). These phenotype alterations commonly do not pass to the second or third generations, since the transmission depends on maternal and paternal care in each generation. In some cases, this maternal or paternal care promotes changes in gene expression and epigenetic factors that might induce alterations in the adult pattern and be transmitted to the next generations (Champagne, 2008; Champagne et al., 2006; Franklin et al., 2010; Gapp et al., 2014; Meaney, 2001; I. C. Weaver et al., 2004; I. C. Weaver et al., 2005). Offspring from males that suffer maternal deprivation showed behavioral changes and alteration of DNA methylation profile in the brain (Franklin et al., 2010). Moreover, the interaction of pregnant female with specific external conditions could alter the embryonic epigenetic profile, inducing phenotypic changes during development that can persist till adult stages. This type of exposition can even modify the molecular profile of F1 germ-line. Stress, drug administration or changes in the diet during pregnancy can alter several factors of the embryo, promoting changes in the adult phenotype (Anway et al., 2005; M. Chen \& Zhang, 2011; Noorlander, Visser, Ramakers, Nikkels, \& de Graan, 2008; Plagemann, 2005). For example, exposure of pregnant females to an estrogenic or antiandrogenic compound promotes a reduction of spermatogenesis and fertility in the next generation (Anway et al., 2005). Cocaine intake during pregnancy stimulates dopaminergic system and stress reactivity in the offspring (Malanga, Pejchal, \& Kosofsky, 2007; W. Wang et al., 2013). Furthermore, exposure to distinct environmental factors might alter the phenotype and the epigenetic profile of F0 and corresponding germ-line (F1), transferring changes to the next generations (Anway et al., 2005; Byrnes, 2005; Carone et al., 2010; Chamorro-Garcia et al., 2013; de Castro Barbosa et al., 2016b; Dias \& Ressler, 2014b; 
Dietz et al., 2011; Dunn \& Bale, 2009; Gapp et al., 2014; Ng et al., 2010; Ost et al., 2014; Ribeiro et al., 2017; Schmauss et al., 2014; Vassoler et al., 2013; Wei et al., 2014; S. Zhang et al., 2017). In some cases, it represents a protective change for the offspring (Ribeiro et al., 2017; Vassoler et al., 2013; S. Zhang et al., 2017). For example, offspring born to fathers that train in spatial tasks show an increase in cognitive performance and synaptic plasticity 98 . In contrast, these changes can have a negative repercussion to the offspring, promoting stress, reduction of fertility or increasing the range of obesity (Anway et al., 2005; Byrnes, 2005; Chamorro-Garcia et al., 2013; de Castro Barbosa et al., 2016b; Dunn \& Bale, 2009; Gapp et al., 2014; Ng et al., 2010; Ost et al., 2014; Schmauss et al., 2014; Wei et al., 2014). Some of these positive or negative phenotypic changes are gender-specific or at least they have been investigated exclusively in one gender (Byrnes, 2005; Carone et al., 2010; Dunn \& Bale, 2009; Ng et al., 2010). Few studies have examined the phenotypic alterations in the offspring born to a father or a mother exposed to the same condition (Madathil et al., 2015; Qvarnstrom \& Price, 2001; Whitaker, Jarvis, Beeken, Boniface, \& Wardle, 2010). A recent study showed that maternal weight has a major impact on the children obesity than paternal weight (Whitaker et al., 2010). Moreover, this intergenerational inheritance can alter the phenotype of both gender offspring (de Castro Barbosa et al., 2016b; Dietz et al., 2011) or exclusively affect one sex (Alter et al., 2009; de Castro Barbosa et al., 2016b; Vassoler et al., 2013). For example, adult females and not males from fathers fed with high-fat diet (HFD) suffer glucose intolerance and resistance to gain weight when they are fed with HFD (de Castro Barbosa et al., 2016b).

\subsubsection{Candidates of germ-line transmission}

Multiple epigenetic factors have been found in germ-line, specifically in sperm, suggesting that they might play a crucial role in the transmission of specific non-genetic phenotypes to the offspring (Kobayashi et al., 2012; Rodgers, Morgan, Leu, \& Bale, 2015; Vassoler et al., 2013). At the same time, there is emerging evidence that an epigenetic resetting process occurs during gametogenesis, to ensure a totipotent zygote (Hajkova, 2011; J. R. Weaver, Susiarjo, \& Bartolomei, 2009). For example, during spermatogenesis histone modifications are removed, and histones are replaced to protamines (Fischer, Sananbenesi, Mungenast, \& Tsai, 2010). However, it seems that the reprogramming does not erase all the epigenetic factors allowing the transmission of specific information through epigenetic factors (G. D. Johnson et al., 2011; Kobayashi et al., 2012; Krawetz, 2005; Rodgers et al., 2015; Vassoler et al., 2013). Furthermore, specific non-coding RNAs are expressed in germ-line and might participate in this epigenetic transmission (G. D. Johnson et al., 2011; Krawetz, 2005; Rodgers et al., 2015). 
One of the candidates for transmission is DNA methylation. In certain conditions, DNA methylation can escape reprogramming and transmit information to the next generations (Denham, O'Brien, Harvey, \& Charchar, 2015; Kobayashi et al., 2012; Radford et al., 2014) (Jenkins et al., 2017). For example, humans that train for 3 months have different levels of DNAmethylation in the sperm, and this might alter the features of the next generation (Denham et al., 2015). Moreover, DNA methylation participates in genomic imprinting, a process by which one of the parental alleles of a specific gene is selected by silencing the other with DNA methylation (Bartolomei \& Ferguson-Smith, 2011; S. Feng, Jacobsen, \& Reik, 2010; Paoloni-Giacobino \& Chaillet, 2006; Reik \& Walter, 2001; Sha, 2008; J. R. Weaver et al., 2009).

Secondly, some histone modifications can also transmit certain changes by the germ-line. The probability is lower since $98 \%$ of histones in mice and $85 \%$ in humans are replaced by protamines in sperm cells (Balhorn, Brewer, \& Corzett, 2000; Hammoud et al., 2009; G. D. Johnson et al., 2011; Ward \& Coffey, 1991). The remainder of histones in the sperm might transmit some information (Ben Maamar, Sadler-Riggleman, Beck, \& Skinner, 2018; Brykczynska et al., 2010; Hammoud et al., 2009; Terashima et al., 2015). A recent study found different H3-occupancy at genes associated with the process of embryogenesis and higher levels of H3K4me1 at transcription factor genes in the sperm of fathers fed with high-fat diet (Terashima et al., 2015).

Finally, different forms of non-coding RNAs have been found in the mature sperm or oocytes cells. Different studies indicate that these structures can play an important role in the transmission of information from parents to the offspring by affecting the transcriptional and translational process, and consequently changing the phenotype of the next generation (Gapp et al., 2014; Hamatani, 2012; Krawetz et al., 2011; K. V. Morris, Chan, Jacobsen, \& Looney, 2004; Rodgers et al., 2013; Suh \& Blelloch, 2011; Younger \& Corey, 2011). The injection of sperm RNA (combination of miRNAs and piRNAs) from males with induced trauma into a naïve-fertilized oocyte alters the behavioral phenotype and metabolism of the offspring. This behavioral phenotype is similar to the offspring from maternal separation combined with unpredictable maternal stress parents (MSUS) (Gapp et al., 2014). Interestingly, some studies suggest that alter patterns of non-coding RNAs originated in different parts of the organism can be transported inside exosomes to the sperm and then transmitted to the next generations (Blake \& Watson, 2016; Cossetti et al., 2014; Vojtech et al., 2014). However, the precise mechanism is poorly understood, and further investigations are required. 

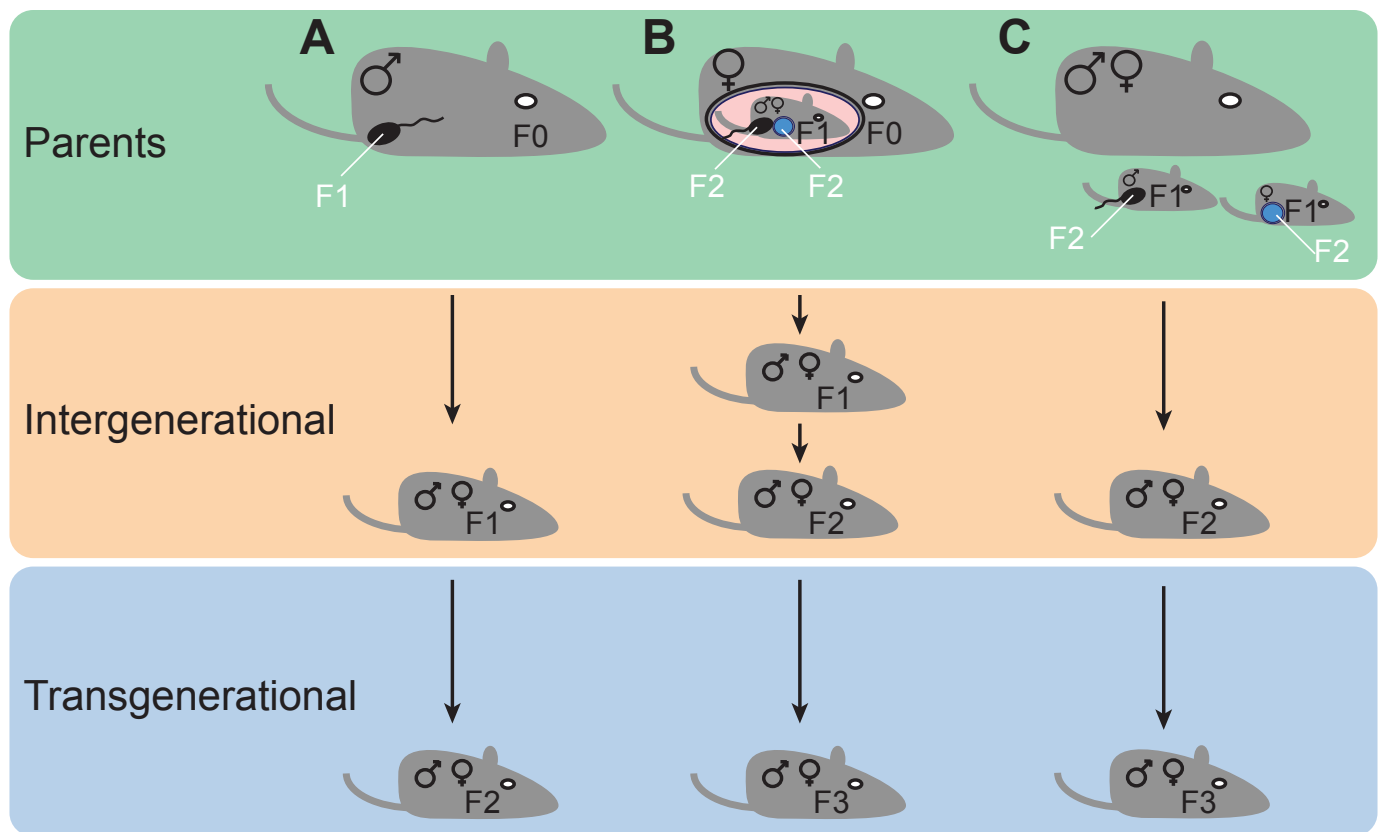

Figure 1.7. Process of inheritance.

(A) Paternal transmission (B) Maternal transmission (C) Maternal or paternal care transmission. F0 = parents; F1,2,3 = first, second and third generation. Figure was adapted from (Dias \& Ressler, 2014a). 
OBJECTIVES 


\section{OBJECTIVES}

Alzheimer's disease (AD) is the most common form of dementia. According to the World Alzheimer Report from 2015, around 50 million people suffered from AD (Prince et al., 2015). Despite the large number of cases, no cure exists to date and the diagnosis of the pathology is too late. This difficulty to identify an effective drug is probably due to the complexity of the pathology, which is caused by several factors, such as genetic and environmental factors. Such genome-environment interactions are mediated by epigenetic processes that control gene expression at the systems level. There is accumulating evidence that such changes in gene expression are key processes underlying the etiology of $A D$ and age-associated dementia (Annese et al., 2018; Blalock et al., 2004; Bossers et al., 2010). Thus, it is crucial to evaluate gene expression changes at different stages of $A D$ pathology and in aged individuals. The overall aim of my PhD work was to better understand these gene expression changes in the context of memory decline. I used three complementary approaches and tested:

1. if gene expression changes at early AD stages affected the pathology and cognitive deficits.

2. if intergenerational effects have an impact on AD-related memory decline and the interpretation of results obtained from commonly used mouse models for amyloid deposition, a key hallmark of AD.

3. if gene expression changes might play a role in inter-individual differences during cognitive aging.

1. Regarding the first aim, I performed a thorough analysis of memory function in the APPPS1-21 mouse model for amyloid deposition at early stages. I also tested hippocampal gene-expression in these mice in order to identify candidate genes and pathways for further investigations of early treatments and diagnosis.

2. To assay transgenerational effects I decided to specifically analyze at the behavior and molecular levels the wild-type offspring originating from either a transgenic APPPS1-21 father or mother. I reasoned that the corresponding data would allow me to test if the breeding scheme could explain a well-known phenomenon, namely that phenotypes of mutant mice can change over time (Casellas, 2011; Wahlsten, Bachmanov, Finn, \& Crabbe, 2006) and if genetic factors present in parents may also impact on the offspring cognitive health via epigenetic inheritance. 


\section{OBJECTIVES}

3. In the third objective, I decided to study another well-known observation, namely that some individuals develop age-associated cognitive decline, while others remain cognitively intact even at an advanced age. Thus, I used a mouse model for age-associated memory decline to define such groups of individuals and subsequently analyze their gene expression profile. Finally, I tried to detect biomarkers that would allow for the assessment of cognitive levels in a non-invasive manner. These would provide a marker to identify first changes of memory and targetmechanisms for drug treatment of memory loss. 
MATERIAL AND METHODS 


\section{MATERIALS AND METHODS}

\subsection{Animals}

\subsubsection{Housing conditions}

All mice were housed in IVC ventilated cages $(365 \times 207 \times 140 \mathrm{~mm})$ with free access to food and water under standard light/dark cycle (12/12h), fix room temperature (RT) at $22^{\circ} \mathrm{C}$ and air humidity of $58 \%$. Food and water were provided ad libitum. Housing, breeding and experimental procedures were conducted in accordance of the German Federal Act on the Protection of Animals. APPPS1-21 mice were housed in groups of maximum 5 mice per cage and were single caged in the holding room one week before starting with the behavioral battery. In case of the C57BL/6J wild-type mice used for the aging project, the first batch of mice was ordered at 16 months of age, singled cage and after 1 month used for the behavioral experiments. The second batch of wild-type mice was ordered at 13 months of age, kept in groups of maximum 5 mice and singled cage one month before the behavioral experiments.

\subsubsection{APPPS1-21 transgenic mice and C57BL/6J wild-type mice}

All transgenic and wild-type mice used for the early AD genetic and intergenerational inheritance projects were obtained from APPPS1-21 mouse line B6-Tg (Thy1-APPswe; Thy1-PS1 L166P) that comes from C57BL/6J strain background (Radde et al., 2006). This mouse line was first established by Mathias Jucker (German Center for Neurodegenerative Diseases; Tübingen, Germany) (Radde et al., 2006). Transgenic animals are heterozygous for both human amyloid precursor protein (APP) gene and human presenilin 1 (PS1) gene. APP gene contained a Swedish mutation (K670N (lysine to asparagine) and M671L (methionine to leucine) and PS1 contain a L166P mutation (leucine to proline). Thy1 promoter regulates the transgene. All APPPS1-21 experimental and control groups were obtained from a colony maintained in-house by crossing male and female described in the figure below (Figure 3.1). APPPS1-21 male and female transgenic and wild-type mice at 3 and 9 months of age were used for the behavioral experiments.

Wild-type mice used for the aging project were C57BL/6J mice purchased from Janvier Labs. 
A

3-month

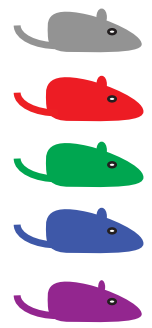

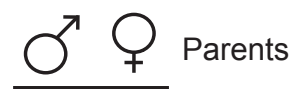

wt wtwt (wt $x$ wt)

$w^{\text {tgwt }}(\operatorname{tg} \times w t)$

$\operatorname{tg}^{\text {tgwt }}(\operatorname{tg} \times w t)$

wt wttg (wt $\times$ tg)

$\operatorname{tg}^{\text {wtg }}$ (wt $\times \operatorname{tg}$ )
B

9-month

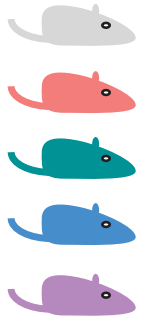

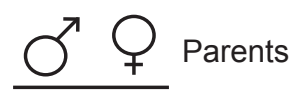

wt wtwt (wt $x$ wt)

$w^{\text {tgwt }}(\operatorname{tg} \times w t)$

$\operatorname{tg}^{\text {tgwt }}(\operatorname{tg} \times w t)$

$\operatorname{tg}{ }^{\text {tgwt }}(\operatorname{tg} x w t)$

$\operatorname{tg}^{\text {wttg }}$ (wt $\times \operatorname{tg}$ )

Figure 3.1. Experimental groups.

(A) 3-month old wild-type or transgenic APPPS1-21 animals born to transgenic father (wttgwt and tgtgwt) or mother ( $w^{t^{w t t g}}$ and tg $\left.g^{w t t}\right)$ vs. control wild-type animals born to wild-type parents (wtwtwt). (B) 9-month old APPPS1-21 wild-type or transgenic animals born to transgenic father ( $\mathrm{t}^{\mathrm{tg} w \mathrm{t}}$ and $\mathrm{tg}^{\mathrm{tg} w \mathrm{t}}$ ) or mother ( $\mathrm{wt}^{\mathrm{wttg}}$ and $\operatorname{tg}^{\mathrm{wttg}}$ ) vs. control wild-type animals born to wild-type parents (wtwtwt).

\subsubsection{Genotyping}

To identify the animals genotypes, mouse tail biopsies were lysed in $300 \mu$ l of DirectPCR tail-lysis

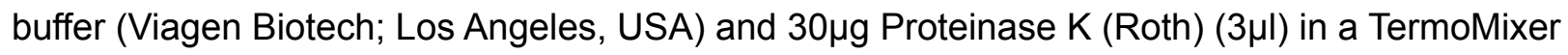
(Eppendorf; Hamburg, Germany) at $1400 \mathrm{rpm}$ and $55^{\circ} \mathrm{C}$ for $3 \mathrm{~h}$ and then inactivated at $85^{\circ} \mathrm{C}$ for 25 minutes.

Primers used for the PCR were:

- APP (3': AATTCCGACATGACTCAGG, 5': GTTCTGCTGCATCTTGGACA)

- PS1 (5': AATAGAGAACGGCAGGAGCA, 3':GCCATGAGGGCACTAATCAT)

Then, the PCR was performed mixing the PCR master mix and DNA samples according to the table 3.1 and using the Cycler according to table 3.2. Genotypes were checked via gel electrophoresis on $1.5 \%$ agarose gel containing $0.1 \%$ ethidium bromide. The gel was run for 45-90 minutes at 70-130V on TAE $1 x$ buffer. DNA bands were detected using a UV light device and observing the bands under $300 \mathrm{~nm}$ illumination.

During the last months, a second protocol with a safer and non-carcinogenic DNA stain was used. Samples were evaluated via $1.5 \%$ agarose gel containing $0.06 \mu \mathrm{l} / \mathrm{ml}$ Midori green (Takara Clone Tech; Japan) and bands were detected using the FAS V Gel Documentation System (Nippon genetics; Japan). 
Table 3.1. PCR master mix for genotyping.

\begin{tabular}{|l|l|}
\hline Component & $\begin{array}{l}\text { Volumel } \\
\text { reaction } \\
18.54 \mu \mathrm{l}\end{array}$ \\
\hline $\mathrm{H}_{2} \mathrm{O}$ & $2,5 \mu \mathrm{l}$ \\
\hline $\begin{array}{l}\text { 10x DreamTaq } \\
\text { Green buffer } \\
\text { dNTP Mix, }\end{array}$ & $2,0 \mu \mathrm{l}$ \\
\hline 2.5mM & $0.25 \mu \mathrm{l}$ \\
\hline Primer APP 5' & $0.25 \mu \mathrm{l}$ \\
\hline $\begin{array}{l}\text { Primer APP 3' } \\
\text { Primer PS1 5' }\end{array}$ & $0.13 \mu \mathrm{l}$ \\
\hline Primer PS1 3' & $0.13 \mu \mathrm{l}$ \\
\hline $\begin{array}{l}\text { DreamTaq } \\
\text { Polymerase }\end{array}$ & $0.20 \mu \mathrm{l}$ \\
\hline
\end{tabular}

Table 3.2. PCR for APP and PS1 genotyping

\begin{tabular}{|c|c|c|}
\hline Step & Temp. $\left({ }^{\circ} \mathbf{C}\right)$ & Time \\
\hline Denaturing & 95 & $2 \min$ \\
\hline \multirow{3}{*}{$\begin{array}{l}\text { Amplification } \\
(\times 30)\end{array}$} & 95 & $30 \mathrm{sec}$ \\
\hline & 58 & $1 \mathrm{~min}$ \\
\hline & 72 & $1 \mathrm{~min}$ \\
\hline Elongation & 72 & $5 \min$ \\
\hline
\end{tabular}

\subsubsection{Dissection of the brain, and extraction of blood and sperm}

Mice were sacrificed one week (offspring project) and one month (aging project) after the behavioral battery using an overdose of Ketamine/Xylazine. The dose used per each mouse was $0.3 \mu \mathrm{l}$ of the combination of $20 \mu \mathrm{l}$ of Ketamine, $150 \mu \mathrm{l}$ of Xylazine and $30 \mu \mathrm{l} 0.9 \% \mathrm{NaCl}$.

After death confirmation, the abdominal cavity was carefully opened, a needle with a syringe (22 gauges/ $1 \mathrm{ml}$ syringe) was inserted into the heart, and then the blood was extracted and collected in a specific RNAprotect Animal Blood Tubes (Qiagen; Hilden, Germany). After this step, a small piece of liver was cut. Both samples were directly frozen with liquid nitrogen. 


\subsubsection{Extraction of the blood extraction and dissection of the liver}

After death confirmation, the abdominal cavity was carefully opened, a needle with a syringe (22 gauges/ 1ml syringe) was inserted into the heart, and then the blood was extracted and collected in a specific RNAprotect Animal Blood Tubes (Qiagen; Hilden, Germany). After this step, a small piece of liver was cut. Both samples were directly frozen with liquid nitrogen.

\subsubsection{Dissection of the hippocampal subregions}

To remove the brain, the scalp was opened; the skull was carefully cut on the middle incision, laterals and the most anterior part between the eyes; and then the brain was placed into a metal plate on ice to cool down the brain immediately.

For the hippocampal subregions, the brain was inserted inside a cold PBS Petri dish and cut along the medial longitudinal fissure and the cerebral hemisphere was medial sided up. The Dentate Gyrus (DG) was dissected from the hippocampus by detaching its borders sliding superficially with a needle. The remaining hippocampal parts were pulled off the cortex using a surgical scoop and split into Cornu Ammonis 1 (CA1) and 3 (CA3). All the dissection steps were performed using a stereoscope.

\subsubsection{Extraction of sperm}

For the sperm extraction, cauda epididymis was cut from the mice, placed in a Petri dish with $1 \mathrm{ml}$ of PBS and cut it in several parts. To allow the sperm to swim out, the sample was incubated at $37^{\circ} \mathrm{C}$ and $5 \% \mathrm{CO} 2$ for 15 minutes. PBS with the spermatozoids was transferred to a new tube and centrifuged at $13000 \mathrm{rpm}$ for 15 minutes at $4^{\circ} \mathrm{C}$.

All dissected regions and extractions were directly frozen in liquid nitrogen and afterward stored at $-80^{\circ} \mathrm{C}$ freezer until the tissue is ready for processing. 


\subsection{Behavioral experiments}

Mice from both projects were subjected to a battery of behavioral tests. For the offspring project, both females and males were used for behavioral testing. Behavioral experiments were carried out by an experimenter blinded to treatment and always by the same experimenter. All tests were recorded using a camera setup and Videomot2 tracking system (Version 7.02, TSE monitoring system; Bad Homburg, Germany). All behavior devices were cleaned with $70 \%$ ethanol before every experimental test.

\subsubsection{Open field test}

Open Field (OF) test is a common behavioral test to evaluate exploratory behavior, anxiety levels and general motor abilities. This test consists of an open arena $(50 \times 50 \mathrm{~cm})$ with surrounding walls $(20 \mathrm{~cm}$ high walls) that prevent the mouse from escaping. A mouse was placed into the central area and was allowed to explore the arena for 5 minutes freely. Time and distance spent in the center and periphery, average speed and the total distance were recorded. For the data analysis, the open field was virtually divided into 9 identical squares.

\subsubsection{Morris water maze}

Richard G. Morris first established Morris Water Maze test in 1981 to test hippocampaldependent spatial learning and memory (R., 1981). This test consists of a circular pool (1.2m in diameter) filled with opaque water at $22^{\circ} \mathrm{C}$, a submerged platform $(10 \times 10 \mathrm{~cm})$ and four signs around the pool. The pool was virtually divided into 4 equally sections and the platform was located in the center of one of the quadrants and right underneath the water surface $(1 \mathrm{~cm})$. Each mouse was placed inside the pool four times every training day for 1 minute, every time from a different position. Mice that did not find the platform were guided to the platform and let stay there for 15 seconds. During training days, the time and distance (latency) to find the platform every day was recorded. During the probe trials (PT), the platform was removed from the pool and mouse was placed inside the pool from the opposite side of the original platform position for 1 minute. Percentage of time and distance spent in the target quadrant, percentage of time and distance spent in the control region, average/cumulative distance to the platform, number of visits to the platform position and average speed were quantified. Mice that require 55 seconds 
or more to find the platform during 3 or more training days or with speed less of 3 a.u./sec during both probe tests were removed from the analysis.

\subsubsection{Contextual fear conditioning}

Fear conditioning (FC) is a standard test used to study an animal ability to learn basic associations. This test consists of a chamber protected from the external sound $(58 \mathrm{~cm} \mathrm{x} 30 \mathrm{~cm}$ $x 27 \mathrm{~cm}$ ) with white noise, white light and a grid on the floor. NIR Video Fear Conditioning software controlled the light, noise and video record. During the training day, mice were introduced inside the chamber and were allowed to explore the novel context for 3 minutes till the last two seconds when they received a foot-shock $(0.5 \mathrm{mV}$, during 2 seconds) and then were returned to the standard cage. After 24 hours (recalling day), mice were introduced inside the same chamber, but without receiving any shock. The percentage freezing during training and test days was automatically tracked, considering freezing if the motion index was below a threshold of 50 for at least 30 frames. The complete equipment and analysis system used was from Med Associates; St. Albans, USA.

\subsubsection{Barnes Maze}

Barnes Maze was established by Carol Barnes for testing spatial learning and memory in 1979 (C. A. Barnes, 1979). This maze consists of a circular open surface with 20 possible escape holes symmetrically distributed around the perimeter of the circle and 4 visual cues. Only one of the holes has an escape box below. A bright light and different white noises were used to stimulate the mouse to enter the escape cage. This test was performed according to Attar with some modifications (Attar et al., 2013). On the habituation day, a mouse was placed in the middle of the maze underneath a clear glass beaker for 30 seconds while the light was switched on. Then, the mouse was guided to the target hole, where it was allowed to enter the escape cage and stayed inside for a 1 minute. During training days, the mouse was placed inside an opaque plastic cylinder in the center for 15 seconds. Then, it was allowed to explore for 2 minutes freely. In case that the mouse entered the dark chamber, it was allowed to stay inside for 1 minute and then returned to the regular cage. Mice that did not enter the dark chamber were guided to the target hole using a glass beaker and were let them enter in the next 3 minutes. If they did not enter the escape cage during the 3 minutes, they were nudged with the beaker until they entered the cage and were allowed to stay 1 minute inside the cage. On the probe test day, the escape cage was removed and mouse was placed in the middle underneath the opaque cylinder for 15 seconds. Then, each mouse was allowed to move for 2 minutes 


\section{MATERIALS AND METHODS}

freely. The time spent in the target quadrant and control region were recorded. During each training and test days, type of white noise was changed. First training day only an extremely bright switched was switched on. Further training and test days, the light was switched on and different noises were played and stopped as soon as the mouse entered the cage. 


\subsection{Molecular experiments}

\subsubsection{RNA isolation from brain}

The method selected for RNA isolation was an organic extraction method. All protocol was done in a sterile fume hood and all the equipment used was previously cleaned with $70 \%$ ethanol and RNaseZap to avoid contamination of the samples. Brain tissue was defrosted and kept on ice during all the protocol to avoid degradation of samples. Brain samples were homogenized in TRIzol Reagent (Sigma-Aldrich; St. Louis, USA) $(400 \mu$ l for CA and $200 \mu l$ for DG) using 10 ceramic beads and the Omni Bead-Ruptor 24 (Omni International; Kennesaw, USA) at a speed of 2.6 for 5 seconds. The same amount of TRIzol was added for the second time and samples were incubated at room temperature (RT) for 5 minutes to lyse the tissue and then were shortly vortexed.

The solution was separated into an aqueous phase and an organic phase, chloroform was added (160 $\mu$ l for CA and $80 \mu \mathrm{l}$ for DG), tubes were shaken manually for 15 seconds and were incubated at RT for 15 minutes. The aqueous phase, which contains the RNA, was transferred to a fresh tube and the interphase (DNA) and organic phase (proteins and phenol) were kept for future experiments. The RNA was obtained from the aqueous phase by precipitation adding $500 \mu$ l of isopropanol for $1 \mathrm{ml}$ TRIzol reagent and $1 \mu \mathrm{l}$ of GlycoBlue (Thermo Fischer Scientific; Waltham, USA), vortexing the tube and incubating at $-20^{\circ} \mathrm{C}$ overnight. To obtain the pellet of RNA, samples were shortly vortex and centrifugated at 12000 rpm for 30 minutes at $4^{\circ} \mathrm{C}$. Then the supernatant was discarded.

To remove any residual contaminating DNA and ensure a high quality of RNA, RNA was treated with $1 \mu \mathrm{l}$ DNasel (Invitrogen) (10U/ $\mu \mathrm{l}), 5 \mu \mathrm{l}$ of 10x Incubation Buffer (Thermo Fischer Scientific; Waltham, USA), $0.5 \mu$ l of RNase OUT (40U/ $\mu \mathrm{l})$ (Thermo Fischer Scientific; Waltham, USA) and 43.5 $\mu \mathrm{l}$ RNase free water; and was incubated for 20 minutes at $37^{\circ} \mathrm{C}$. RNA was purified by adding 1 volume $(200 \mu \mathrm{l})$ of phenol/chloroform/isoamyl alcohol (25:24:1) and 150 $\mu$ R Rase free water, vortexing and centrifuging at $13000 \mathrm{rpm}$ for 2 minutes at RT. Then, the aqueous phase with RNA was transferred to a new tube and the RNA was precipitated by including 1/10 of the volume of the aqueous phase $3 \mathrm{M} \mathrm{Na}$-acetate $\mathrm{pH} 4.8,1$ volume of isopropyl alcohol and $1 \mu \mathrm{l}$ of GlycoBlue (Thermo Fischer Scientific; Waltham, USA); vortexing and centrifuging samples at 13000rpm for 30 minutes at $4^{\circ} \mathrm{C}$.

For the washing steps, the supernatant from the samples was removed, $1 \mathrm{ml}$ of $75 \%$ ethanol was added and the sample was centrifuged at 13000 rpm for 5 minutes at $4^{\circ} \mathrm{C}$ three times. 
To dissolve the RNA, the supernatant was removed, samples were air-dried for 10 minutes to clean the RNA from ethanol, the pellet of RNA was redissolved in 50-100 $\mu$ l RNase free water and mixed it with the thermomixer at $42^{\circ} \mathrm{C}$ for 10 minutes.

RNA concentration and purity were measured using Nanodrop 2000 (Thermo Fischer Scientific;

Waltham, USA) and the integrity was checked by using Agilent 2100 Bioanalyzer (Agilent Technologies; Santa Clara, USA). Samples with a lower value of 1.8 for 260/280 ratio of absorbance or an RNA integrity number (RIN) below 8.0 were treated again with DNase treatment and re-purified following previous steps described.

\subsubsection{RNA isolation from the sperm}

The spermatozoa-containing solution was treated with a hypotonic buffer and a somatic cell lysis buffer (SCLB; $0.1 \%$ SDS, $0.5 \%$ Triton X; $100 \%$ solved in deionized water) to remove remaining somatic cells. This procedure was based on the method previously described by Goodrich (Goodrich, Johnson, \& Krawetz, 2007). Sperm pellet was thawed and was resuspended by adding $1 \mathrm{ml}$ SCLB and pipetting. $4 \mathrm{ml}$ more of SCLB were added before incubation on ice for 30 minutes. The sample was centrifuged at $600 \mathrm{rpm}$ for 15 minutes at $4^{\circ} \mathrm{C}$. To remove traces of SCLB, the supernatant was removed and washed twice by adding $1 \mathrm{ml} \mathrm{PBS}$ and centrifuging at $600 \mathrm{rpm}$ for 5 minutes at $4^{\circ} \mathrm{C}$. To isolate the RNA from the sperm, the standard protocol of RNA isolation explained above was followed.

\subsubsection{RNA isolation from the blood}

RNA from the blood was extracted using the RNeasy Protect Animal Blood Kit (RNeasy silicamembrane technology) from Qiagen (Hilden, Germany). RNAprotect blood tubes were incubated at least for $2 \mathrm{~h}$ at $\mathrm{RT}$ to ensure complete lysis of blood cells.

Afterward, these tubes were centrifuged at 5000rpm at RT for 3 minutes and the supernatant was removed by decanting or pipetting. RNA pellet was dissolved by adding $1 \mathrm{ml}$ of RNase free water, vortexing and centrifuging at 5000rpm for 3 minutes at RT. To resuspend the pellet, the supernatant was removed, 240 $\mu$ l of RSB (resuspension buffer) were added, and then everything was vortexed till the pellet was dissolved and transferred to a fresh tube. Blood samples were digested using $200 \mu \mathrm{l}$ of RBT (binding buffer) and $20 \mu \mathrm{l}$ of proteinase $\mathrm{K}$, mixing shortly by vortexing for 5 seconds and incubating in a shaker-incubator at 400-1400rpm for 10 minutes at $55^{\circ} \mathrm{C}$. Samples were homogenized by pipetting the product to a QIAshredder spin column placed in a $2 \mathrm{ml}$ collection tube and centrifuge at 19000rpm for 3 minutes at RT. Then the supernatant was placed to a new $1.5 \mathrm{ml}$ collection tube without touching the pellet, 1.5 volume 
(generally $690 \mu \mathrm{l}$ ) of $100 \%$ ethanol was added (to optimize binding conditions) and was mixed by vortexing. RNA was pipetted into an RNeasy MinElute spin column placed in $2 \mathrm{ml}$ collection tube and centrifuge at 10000rpm for 1 minute at RT. The RNA was bound to the silica membrane and the supernatant was removed.

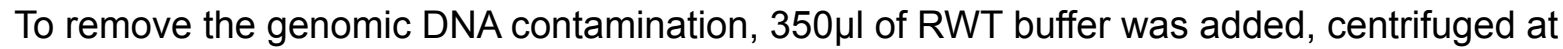
10000 rpm for 15 seconds, discarded the flow-through, treated with $80 \mu \mathrm{l}$ of DNasel incubation

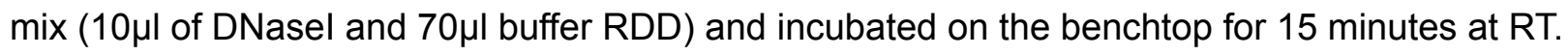
Then, 350 $\mu$ l of RWT buffer was added again, centrifuged at 10000rpm for 15 seconds and

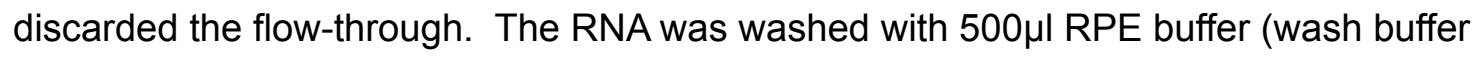
concentrate), centrifuged at 10000rpm for 15 seconds and discarded the flow-through. Afterward, $500 \mu \mathrm{l}$ of $80 \%$ ethanol was added and centrifuged at 10000rpm for 2 minutes. The spin column was placed in a new $2 \mathrm{ml}$ tube, opened centrifuged at full speed for 5 minutes and discarded the flow-through and the tube.

To elute the RNA, the column was placed in a new $1.5 \mathrm{ml}$ tube, pipetted $14-30 \mu \mathrm{l}$ REB buffer (elution buffer), centrifuged at 10000rpm for 1 minute, incubated the RNA for 5 minutes at $65^{\circ} \mathrm{C}$ in the shaker-incubator without shaking and chilled immediately on ice.

RNA concentration and purity were quantified using Nanodrop 2000 (Thermo Fischer Scientific;

Waltham, USA) and the integrity was tested by using Agilent 2100 Bioanalyzer (Agilent Technologies; Santa Clara, USA).

\subsubsection{RNA concentration and quality}

The RNA quantity and purity were controlled with Nanodrop 2000 (Thermo Scientific). Nanodrop 2000 is a microvolume UV-Vis spectrophotometer that allows the measurement of the RNA concentration and purity. Previous to sample measurement, settings were adjusted for RNA samples, and the blank (RNase free water) was measured. RNA quantity and purity were checked by adding $1 \mu$ l of the sample onto the pedestal, closing the arm and measuring by clicking measure. The absorbance was measured.

The concentration is based on $260 \mathrm{~nm}$ absorbance. The purity of DNA and RNA are evaluated observing the ratio $260 / 280 \mathrm{~nm}$. The DNA is pure when the value of $260 / 280 \mathrm{~nm}$ ratio is around 1.8. The RNA is pure when the value of $260 / 280 \mathrm{~nm}$ ratio is around 2.0. A low ratio might be an indication of phenol or protein contamination. 


\subsubsection{RNA integrity}

RNA integration is analyzed using Agilent Bioanalyzer. This equipment allows confirming that the ratio of $18 \mathrm{~S}: 28 \mathrm{~S}$ RNA is closed to the theoretical maximum of 2.5 using electrophoretic separation and subsequent detection via laser-induced fluorescence and representation in an electropherogram. The electropherogram allows the detection of the quality of an RNA sample. Profiles generated on the Agilent 2100 Bioanalyzer provide information about the concentration and the RNA integrity. The RNA Integrity Number (RIN) is used to screen the integrity of the total RNA and is ranged from 1 to 10 , from more to less degraded, respectively.

Previous to the measurement, electrodes were cleaned with RNase free water and RNaseZap and the priming station and the program were set for RNA measuring. A new RNA Nano LabChip was introduced on the chip priming station. $9 \mu$ l of gel-dye (previously prepare the mix of $1 \mu l$ of RNA dye concentrate and $65 \mu$ l of filtered gel, vortexing and centrifuging at $13000 \mathrm{rpm}$ at RT for 10 minutes) was added in a specific well marked with white $G$ and the plunger was pushed until it was subjected by the clip for 30 seconds and then it was released for 5 seconds. $9 \mu$ of gel-dye was also added in two wells marked with black $G$.

The loading RNA marker was added in all 12 samples, $1 \mu$ of the prepared ladder was pipetted in its specific place and $1 \mu \mathrm{l}$ of samples were pipetted in each of the 12 sample wells. The chip was vortexed in the IKA vortexer at 2400rpm for 1 minute, it was inserted inside the Bioanalyzer and the program was run. Results were analyzed on the computer. Samples with an RNA integrity number (RIN) below 8.0 were treated again with DNase treatment and re-purified as described. Samples with RIN below 6.0 were not used for further analysis.

\subsection{6.cDNA preparation for RNA detection}

The complementary DNA (cDNA) was prepared using the reverse transcription protocol from Roche, Transcriptor First Strand cDNA Synthesis Kit (Roche; Basel, Switzerland).

RNA concentrations were measured with the Nanodrop and all samples were equally balanced at the same concentration adding RNase free water (Table 3.3). RNA samples were mixed with primers and RNase free water (Table 3.4). The mixer was denaturated by incubating for 10 minutes at $65^{\circ} \mathrm{C}$ in a PCR cycler. Afterward, the concentration of cDNA was diluted to 3-5ng/ $\mu$. The qPCR mixture and template-primer mix were combined and incubated as described in the PCR cycler (Table 3.5). 
Table 3.3. Balanced of RNA samples

\begin{tabular}{|l|l|l}
\hline Component & $\mathbf{1 1 \mu l}$ & $\mathbf{5 . 5 \mu l}$ \\
\hline Random hexamer primer $(0.6 \mathrm{mM})$ & $2 \mu \mathrm{l}$ & $1 \mu \mathrm{l}$ \\
\hline Template RNA (500ng) & $\mathrm{x} \mu \mathrm{l}$ & $\mathrm{x} \mu \mathrm{l}$ \\
\hline RNase free water & $11-\mathrm{x} \mu \mathrm{l}$ & $5.5-\mathrm{x \mu l}$
\end{tabular}

Table 3.4. Master mix for cDNA preparation

\begin{tabular}{|l|l|l|}
\hline Component & $\mathbf{1 1 \mu l}$ & $\mathbf{5 . 5 \mu l}$ \\
\hline $5 x$ Reaction buffer & $4 \mu \mathrm{l}$ & $2 \mu \mathrm{l}$ \\
\hline RNase Inhibitor $(40 \mathrm{U} / \mu \mathrm{l})$ & $0.5 \mu \mathrm{l}$ & $0.25 \mu \mathrm{l}$ \\
\hline dNTP Mix $(10 \mathrm{mM}$ each) & $0.5 \mu \mathrm{l}$ & $0.25 \mu \mathrm{l}$ \\
\hline Reverse Transcriptase & $2 \mu \mathrm{l}$ & $1 \mu \mathrm{l}$ \\
\hline Template-primer mix & $13 \mu \mathrm{l}$ & $6.5 \mu \mathrm{l}$
\end{tabular}

Table 3.5. cDNA preparation protocol

\begin{tabular}{|l|l|}
\hline Temperature & Time \\
\hline $25^{\circ} \mathrm{C}$ & $10 \mathrm{~min}$ \\
\hline $55^{\circ} \mathrm{C}$ & $30 \mathrm{~min}$ \\
\hline $85^{\circ} \mathrm{C}$ & $5 \mathrm{~min}$ \\
\hline $4^{\circ} \mathrm{C}$ & $\infty$ \\
\hline
\end{tabular}

\subsubsection{Primer design}

PCR primer design depends heavily on several factors, including melting temperature (Tm), length, GC content, secondary structure and specificity. A good primer contains from 18-22 bases, $50-55 \%$ GC content and similar melting temperature between both primers. Primers for qPCR were designed using the "UPL Assay Design Center"(http:// www.universalprobelibrary.com) from Roche and ordered from Sigma Aldrich (St. Louis, USA). 


\subsubsection{Quantitative real time PCR to quantify mRNA levels}

The LightCycler 480 instrument (Roche; Basel, Switzerland) is a quantitative real-time PCR (qPCR) and was used to quantify the expression of transcripts and microRNAs.

\subsection{9.qPCR for RNA detection}

\subsubsection{SYBR Green qPCR}

SYBR Green I Mastermix (2x), water and primers were mixed according to the table 3.5 and they were added to each well from the plate. Besides, the cDNA was introduced inside the plate. The plate was sealed with Heat-Sealing film and was shortly centrifuged at 10000rpm at RT for 1 minute. Afterward, the thermal cycling conditions were set as described in table 3.6, the plate was placed inside the real-time cycler, and the cycling program was started.

Table 3.5.Master mix for the qPCR

\begin{tabular}{|l|l|}
\hline Component & Volume/reaction \\
\hline SYBR green I Master Mix $(2 \mathrm{x})$ & $7.50 \mu \mathrm{l}$ \\
\hline Primer Reverse & $0.75 \mu \mathrm{l}$ \\
\hline Primer Forward & $0.75 \mu \mathrm{l}$ \\
\hline PCR-graded water & $5.00 \mu \mathrm{l}$ \\
\hline cDNA sample (1:10 diluted) & $1 \mu \mathrm{l}$ \\
\hline
\end{tabular}

Table 3.6 qPCR cycler program

\begin{tabular}{|l|l|l|}
\hline Step & Temp. $\left({ }^{\circ} \mathrm{C}\right)$ & Time \\
\hline PCR initial activation step & $95^{\circ} \mathrm{C}$ & $5 \mathrm{~min}$ \\
\hline Denaturation & $95^{\circ} \mathrm{C}$ & $10 \mathrm{sec}$ \\
\hline Annealing & $60^{\circ} \mathrm{C}$ & $15 \mathrm{sec}$ \\
\hline Extension & $72^{\circ} \mathrm{C}$ & $10 \mathrm{sec}$ \\
\hline Cooling & $40^{\circ} \mathrm{C}$ & $10 \mathrm{sec}$ \\
\hline Melting & $95^{\circ} \mathrm{C}$ & $15 \mathrm{sec}$ \\
\hline & $67^{\circ} \mathrm{C}$ & $30 \mathrm{sec}$ \\
\hline Cycle number & $95^{\circ} \mathrm{C}$ & Acquisition \\
\hline & $40^{\circ} \mathrm{C}$ & $10 \mathrm{sec}$ \\
\hline
\end{tabular}




\subsection{Experimental analysis}

\subsubsection{Analysis of qPCR data}

The qPCR data was analyzed following the double delta Ct method ( $\Delta \Delta \mathrm{Ct})$ (Livak \& Schmittgen, 2001). Relative quantification of gene expression and microRNAs was expressed as a fold change (FC). To apply this method, control and experimental samples were tested with genes of interest and housekeeping genes (endogenous controls) (Hprt, Gapdh, Tubb3). In case of microRNA analysis, the "housekeeping" smallRNA used is RNU6B (miScript SYBR Green PCR kit) and SnoRNA202 (Taqman Universal PCR Master Mix). The average of the same sample replicates was taken, and the CT value from the gene of interest was normalized by the CT values from the same sample but with the housekeeping gene.

\subsubsection{Next-Generation Sequencing data analysis}

Sequencing data was processed using software pipeline, which was customized in-house. Base calls from BCL were converted to FASTQ files using Illumina bcl2fastq (v1.8.4). Then the bases were assigned (base calling); the adapters were remove (adapter trimming) and the read sequence were divided (demultiplexing). Afterward, the quality of the raw sequence data was evaluated using FastQC (v 0.11.5). The mouse transcriptome (Mus_musculus.GRCm38.86) was used to map the reads. The unmapped reads were the mapped to the mouse genome (mm10). RNA-STAR 2.5.2b was used for the entire mapping. All the default specifications were used, except for the mismatches. No mismatches for the reads $<19 \mathrm{~b}$, one mismatch for reads between $20 \mathrm{~b}$ to $39 \mathrm{~b}$, two mismatches for reads between $40 \mathrm{~b}$ to $59 \mathrm{~b}$ and so on were allowed. The number of aligned reads overlapping the exons for each gene was counted using HTSeq 0.9.1 (intersection-non-empty mode). The first analysis of differentially expressed genes was done observing changes in a scatter plot, known as volcano plot. The cluster of samples was evaluated using principal component analysis (PCA). 


\subsubsection{Differentially expression of genes and small RNAs}

To identify differentially expressed genes and microRNAs, the unwanted sources of variation (RUVs) were identified and corrected using RUVSeq (v. 1.8.0). The differential expression analysis was performed using DESeq2 (v. 1.14.1) (Love, Huber, \& Anders, 2014). For further analyses, significant thresholds were established according to basemean $\geq 1, \log _{2} \mathrm{FC} \pm 0.25$ and padj $\leq 0.05$ (Benjamini-Hochberg). Heatmaps and Venn diagrams with these thresholds were generated. Bioinformatics \& evolutionary genomics (http://bioinformatics.psb.ugent.be/intranet/) and VennDiagrams (http://www.venndiagrams.net/) web tools were used to measure the overlap of genes and generate venn diagrams.

\subsubsection{Differential exon usage}

To identify differentially expressed exons, a customized in-house software pipeline was designed, and DEXSeq 1.22 was performed following the standard instructions and using the mm10 reference genome (Anders, Reyes, \& Huber, 2012). Exons with an adjusted p-value $\leq 0.05$ and $\log _{2} \mathrm{FC} \pm 0.25$ were considered as significantly differentially expressed exons. The measurement of the overlap of gene-set and the Venn diagram illustrations were generated using Bioinformatics \& evolutionary genomics (http://bioinformatics.psb.ugent.be/intranet/) and VennDiagrams (http://www.venndiagrams.net/) web tools.

\subsubsection{Pathway analysis}

Two different approaches were used for the functional analysis. First, Gene Set Enrichment Analysis (GSEA) (Subramanian et al., 2005), which allows the study of whole dataset functions without restricting the study to only genes with a specific threshold. This analysis was performed using the standard protocol and selecting following settings, permutation type: gene_set (sample size was lower than 7 per group); number permutations: 1000 and chip platform: gseaftp.broadinstitute.org://pub/gsea/annotations/GENE_SYMBOL.chip. All three GO term categories (biological process, molecular function and cellular component) were evaluated independently. The top 50 and 20 significant pathways (nominal pval $\leq 0.05$; false discovery rate $($ FDR $) \leq 0.1$ ) from each category (biological process, molecular function and cellular component) were selected and ranked by Normalized Enrichment Score (NES). The top 25 overrepresented 
and underrepresented were categorized in a broader biological process and were represented in pie charts. GSEA was only used for the transcriptome functional analysis.

The second approach used for the functional analysis was Cytoscape 3.0 and Cluego 2.3.3 (Bindea et al., 2009; Shannon et al., 2003), which were used to find the significantly enriched pathways in differentially expressed genes, using a cut-off based on padj $\leq 0.05$ and $\log _{2} \mathrm{FC} \pm$ 0.25. Significant pathways (Benjamini-Hochberg, pval $\leq 0.05$ ) were displayed with Cytoscape plotting tool and also the top 20 were displayed in contingency bars. Up-regulated and downregulated genes were separated in 2 clusters. All three GO term categories (biological process, molecular function and cellular component) were evaluated together. This approach was used for the functional analysis of differentially expressed genes, exons and microRNA target mRNAs.

\subsubsection{Statistics}

Basic analysis and table preparation were done using Microsoft Excel 2010 (Microsoft; Redmond, USA). Statistic studies were mainly performed using Prism7 (GraphPad Software; La Jolla, USA). Unpaired T-test was used to analyze the significant differences between groups. To screened whole dataset one-way or two-way ANOVA was used. To identify outliers inside a group the ROUT test $(\mathrm{Q}=10 \%)$ was performed. Differences with a $p$-value $\leq 0.05$ were considered significant $\left({ }^{*}\right)$, p-values $\leq 0.01\left(^{* *}\right)$ and $p$-values $\leq 0.001\left(^{* * *}\right)$. In order to evaluate the litter and batch effects, a linear mixed model was used. This model was specifically designed in-house to evaluate these parameters (random factors) using $\mathrm{R}$. The treatment and gender were considered as fixed factors (Oberg \& Mahoney, 2007). 
RESULTS

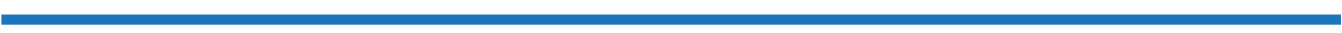


RESULTS

\section{RESULTS}

\subsection{The early characteristics of Alzheimer's disease in APPPS1-21 mouse model}

\subsubsection{Cognitive abilities in APPPS1-21 transgenic mice at 3 months of age}

In order to study the cognitive abilities at early stages of Alzheimer's disease pathology, I decided to employ a model for AD, namely the APPPS1-21 mice. Previous studies demonstrated that different mouse models of AD showed learning and memory impairments at different ages (Gengler et al., 2010; Radde et al., 2006; Serneels et al., 2009). However, this specific model of $A D$ has not been characterized at early stages. I characterized cognitive performance in APPPS1-21 mice at 3 months of age as compared to wild-type (wt) controls. Moreover, I analyzed the parental-specific effect of the APP and PS1 mutations on behavior, testing APPPS1-21 transgenic mice obtained from a transgenic father $\left(\mathrm{tg}^{\mathrm{tg} w \mathrm{t}}\right)$ or a transgenic mother (tg ${ }^{\mathrm{wttg}}$ ) (Figure 4.1.1A). APPPS1-21 mice were tested in the following battery of behavior tests: open field (OF), Morris water maze (MWM) and fear conditioning (FC) (Figure 4.1.1B). Both female and male mice were used in the experiment and were pooled together since no statistically significant differences in cognitive abilities were observed between them (Appendix figure 1-5).
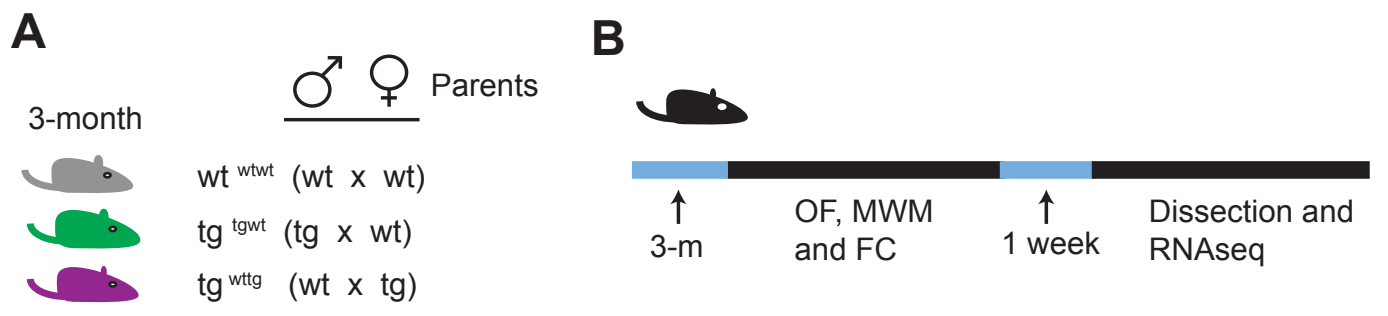

Figure 4.1.1. Experimental groups and design.

(A) 3 months old APPPS1-21 transgenic mice born to transgenic fathers (tgtgwt) or mothers (tgwttg) were compared to control wild-type mice born to wild-type parents (wtwtwt). (B) 3 months old mice were subjected to a battery of behavioral experiments, open field (OF), Morris water maze (MWM) and fear conditioning (FC). A week later, brain subregions were dissected, RNA was isolated and RNA sequence was ran and analyzed. 
I first tested mice in the open field, a commonly used task to measure exploratory behavior, general locomotor activity and anxiety. Total distance and speed were comparable between groups (Figure 4.1.2A-B), indicating no motor deficit. Moreover, both transgenic groups spent significantly more time and traveled longer distances exploring the central area and visited the center more often than wild-type mice (Figure 4.1.2C-E). These might suggest that transgenic mice at 3 months of age are less anxious compared to wild-type controls.
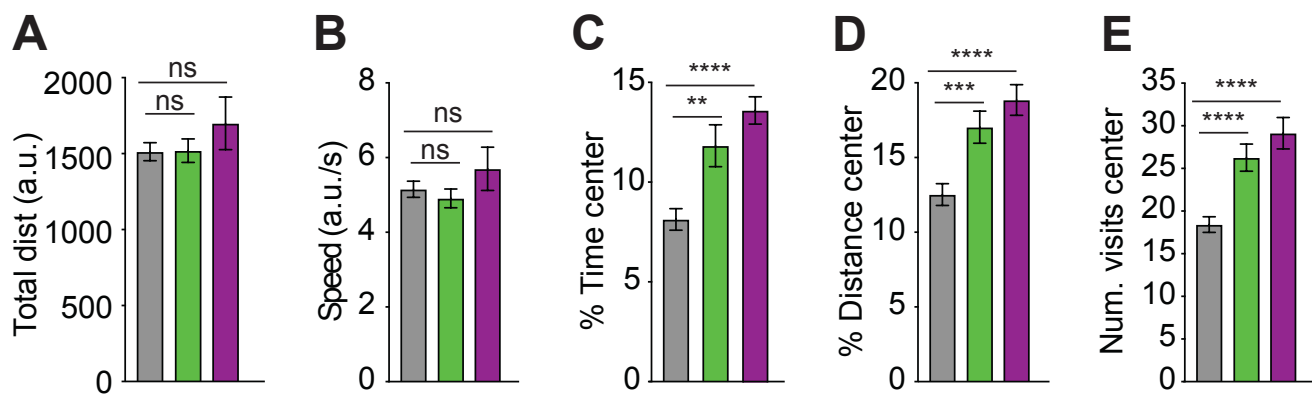

Figure 4.1.2. Transgenic mice did not show motor impairments in the open field, but showed less anxiety levels at 3 months of age.

Open field test in APPPS1-21 transgenic mice born to a transgenic father (tg ${ }^{\text {tgwt }}, \mathrm{n}=27$ ) or mother (tgittg, $\mathrm{n}=16$ ) or pure wild-type mice ( $\mathrm{tt}^{\mathrm{wtwt}}, \mathrm{n}=34$ ) at 3 months of age. (A and $\left.\mathbf{B}\right)$ Total distance traveled $(\mathbf{A})$ and average speed (B) during the open field test. (C, D and E) Percentage of time (C) and distance (D) spent on the central area of the field and number of visits to the center $(E)$. Student's two-tailed T-test, ns = no significant differences; ${ }^{* *} \mathrm{P}<0.01 ;{ }^{* \star *} \mathrm{P}<0.001 ;{ }^{* \star *} \mathrm{P}<0.0001$. Error bars indicated SEM.

Next, I tested mice for spatial memory in the Morris water maze (MWM). MWM consists of 2 parts. The first part is the training period, where mice are trained to navigate a pool to find an escape platform submerged under water. The second part is the test period, where the mice' memory abilities are evaluated by removing the platform and recording the time mice spend searching for the platform in the quadrant where the platform was previously located (the target quadrant), indicating their ability to navigate through the maze and recall the target position. During the training phase, APPPS1-21 transgenic mice from a mutant father ( $\operatorname{tg}^{\text {tgwt }}$ ) or mother (tgwttg) required significantly more time to find the platform than controls ( $w^{\text {twtwt }}$ ) at 3 months of age, which was reflected both in the repeated measures ANOVA and the areas under the latency curve (AUC) analysis (Figure 4.1.3A-C). AUC in this data represents the total latency time over the training days and it is measured by the trapezoidal rule (Bailer, 1988; Gagnon \& Peterson, 1998; Jaki \& Wolfsegger, 2009). Nevertheless, all groups presented a daily learning improvement as reflected in the decrease in the latency to find the platform. 


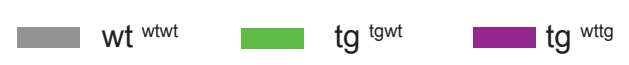

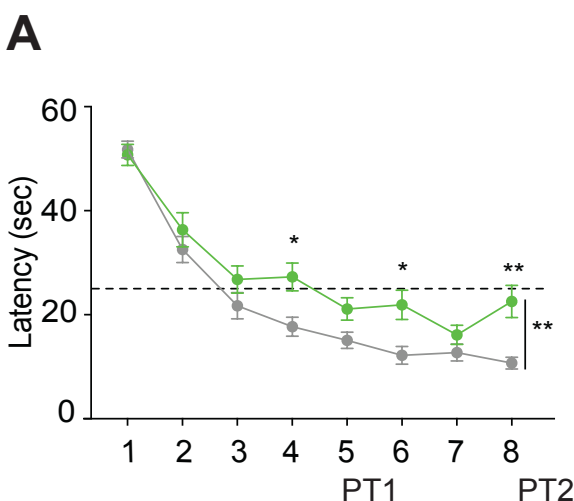

Day
B

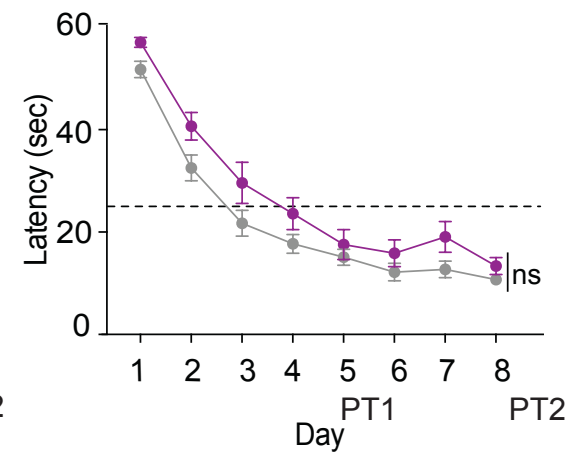

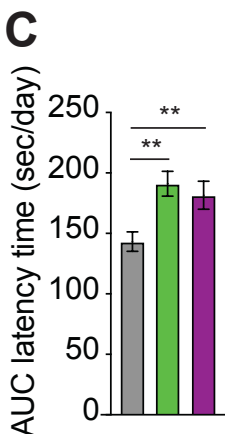

Figure 4.1.3. APPPS1-21 $\mathrm{tg}$ mice showed difficulties to learn a spatial task at 3 months of age.

Morris water maze (MWM) in APPPS1-21 transgenic mice born to a transgenic father (tg ${ }^{\text {tgwt }}, \mathrm{n}=25$ ) or

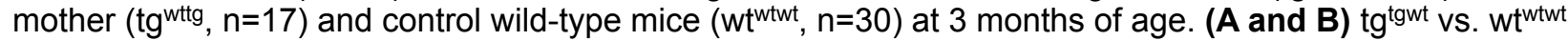
(A) and tgwttg vs. wtwtwt (B) group-average time to find the target platform on each consecutive training day (Day 1-8). Two-way ANOVA after Sidak correction, ${ }^{*} P<0.05 ;{ }^{* *} P<0.01$. The dashed line represents the chance level of $25 \%$. First probe trial (PT1, day 6) and second probe trial (PT2, day 10). (C) Comparison

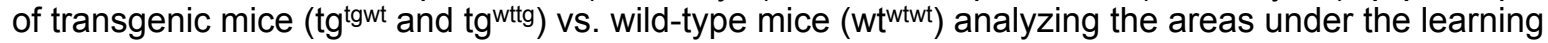
curves (AUC) of escape latency for each group. Student's two-tailed T-test, ns = no significant differences; ${ }^{*} \mathrm{P}<0.05 ;{ }^{* *} \mathrm{P}<0.01$. Error bars indicated SEM.

I carried out 2 probe tests to analyze spatial memory. Probe test 1 (PT1) was performed after 5 days of training and showed that after these days of training none of the mice in any group had learned the task. This suggests that 5 days were not enough to consolidate the platform's position in this mouse model and with this specific protocol. None of the mice in any group spent significantly more than $25 \%$ of the time and traveled distance in the target quadrant. Despite this, wild-type control mice ( $w^{t^{w t w t}}$ ) showed a clear preference for and traveled longer distances in the target quadrant than both APPPS1-21 transgenic groups (tgtgwt and tg $^{\text {wttg) }}$ (Figure 4.1.4AC).

Based on these results, I trained the mice for 3 additional days before I subjected them to a second probe test (PT2) after 8 total days of training. At this point, wt ${ }^{\text {wtwt }}$ mice spent more than $25 \%$ of the time and traveled distance in the target quadrant meaning that they had learned the task, whereas both transgenic groups spent similar amounts of time and traveled distance in the target and other quadrants. These results indicate that APPPS1-21 transgenic groups did not learn the position of the platform at 3 months of age. It is interesting to note, that although the learning impairment was apparent for both transgenic groups, the direct comparison for time, distance and platform visits of $w^{t^{w t w t}} v s$. $\operatorname{tg}^{\text {wttg }}$ mice did not reach significance. This probably 
indicates a slightly less severe manifestation of the behavioral phenotype in Morris water maze (MWM) in mice born to a transgenic mother (Figure 4.1.4D-F).

As a refinement to the classical MWM memory parameters, I included a more sensitive analysis consisting of recording the distance between the position of the mouse and the position of the platform during the PT every two seconds. This parameter confirms the significant difference in performance between groups during first probe trial for both $\operatorname{tg}^{\text {tgwt }}$ and tg $^{\text {witg }}$ mice (Figure 4.1.4GH). In PT2, only tg ${ }^{\text {tgwt }}$ mice showed a significant difference to wt controls (wtwtwt) (Figure 4.1.4I-J).

wt wtwt

B PT1

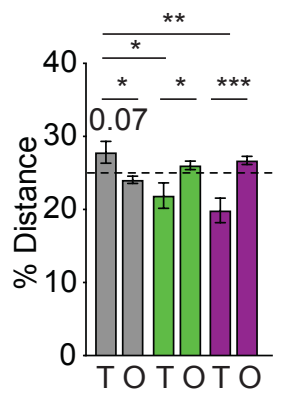

D

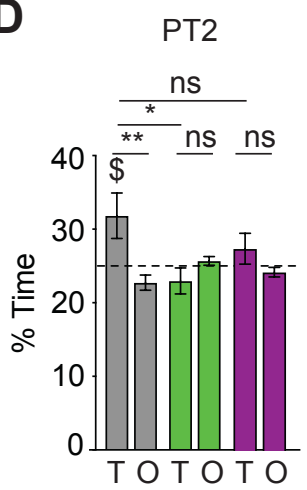

A

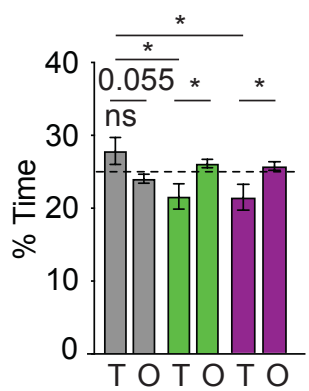

E

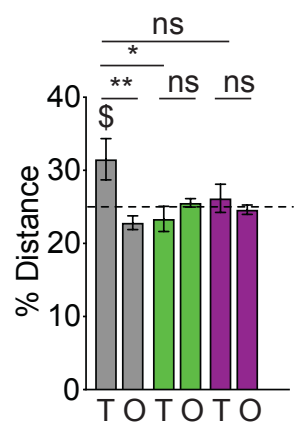

$\operatorname{tg}$ tgwt

tg wttg

C PT1

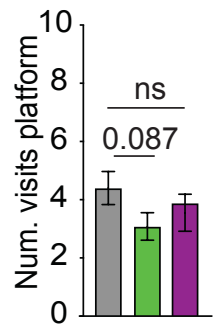

F

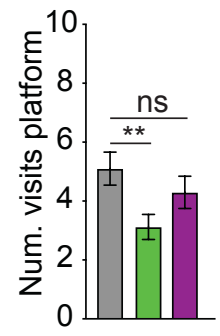

H PT1

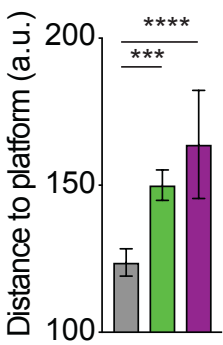

J

PT2

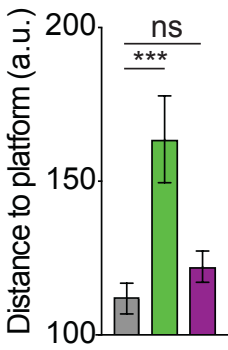


RESULTS
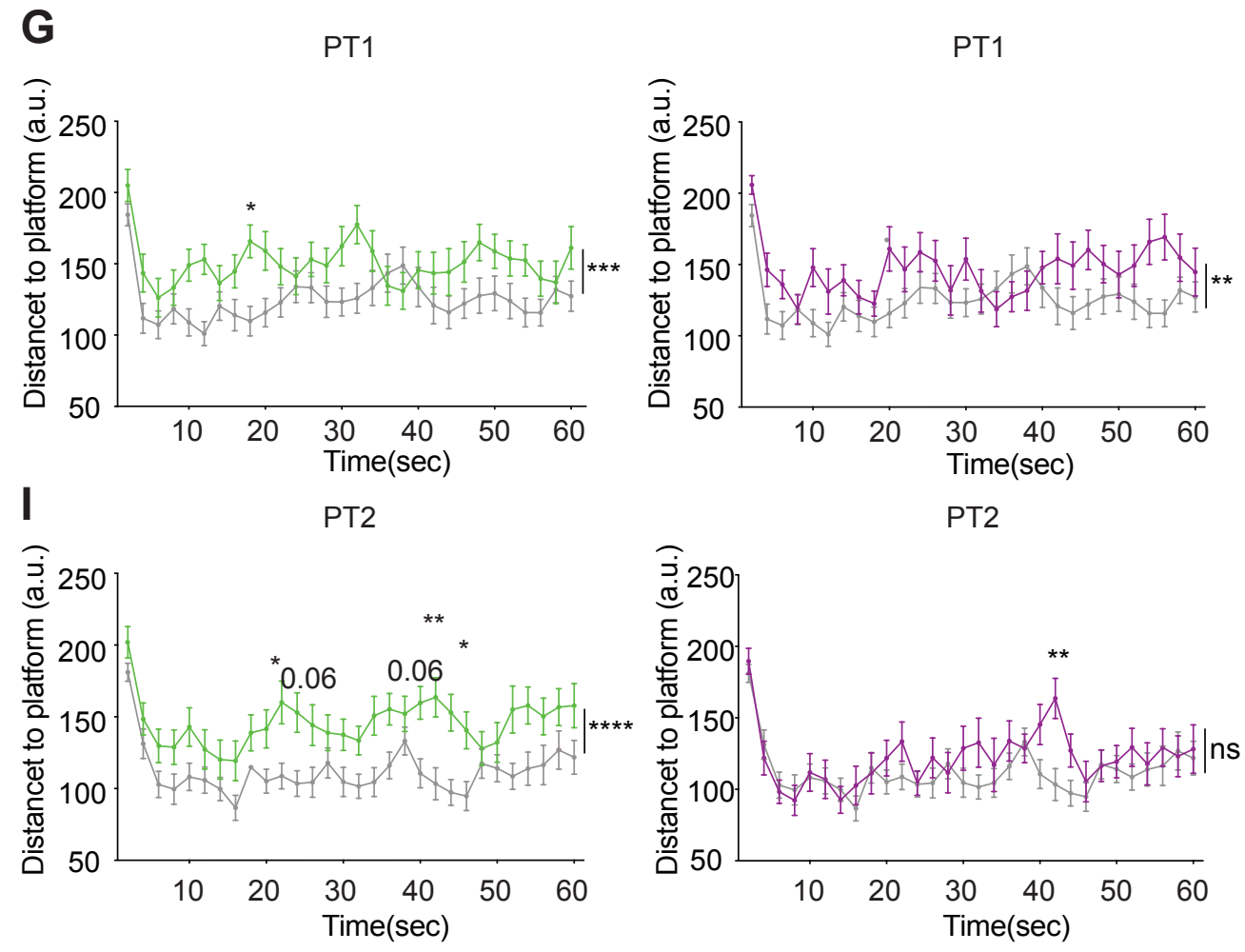

Figure 4.1.4. Transgenic mice showed spatial memory impairments at 3 months of age.

Morris water maze (MWM) test in APPPS1-21 transgenic mice born to a transgenic father $\left(\operatorname{tg}^{\text {tgwt }}, \mathrm{n}=25\right)$ or mother (tgwttg, $n=17$ ) and control wild-type mice (wtwtwt, $n=30$ ) at 3 months of age. (A-F) Percentage of time (A) and distance (B) spent in the target quadrant (T) vs. average of other quadrants $(\mathrm{O})$ and number of visits to the platform(C) during first probe trial (PT1, day 6) (A-C) and second probe trial (PT2, day 10) (DF). Student's two-tailed T-test, $n s=$ no significant differences; ${ }^{*} P<0.05$; ${ }^{* *} P<0.01$; ${ }^{* * *} P<0.001$. The dashed line represents the chance level of $25 \%$. (G-J) The distance between mouse and the platform position every two seconds during PT1 (G) and PT2 (I) and the average of total distance during PT1 (H) and PT2 (J). Two-way ANOVA after Sidak correction and student's two-tailed T-test, ${ }^{*} \mathrm{P}<0.05$; ${ }^{* *} \mathrm{P}<0.01$; ${ }^{* * *} \mathrm{P}<0.001 ;{ }^{* * * *} \mathrm{P}<0.0001$. Error bars indicated SEM.

Importantly, none of the mice in any group presented a reduction of the motor skills during all training and test phases. During the first day of training and the first probe trial (PT1) all mice swam at similar speeds (Figure 4.1.5A-B). Only during the last probe trial (PT2), transgenic mice swam significantly faster than wild-type mice (Figure 4.1.4C). 


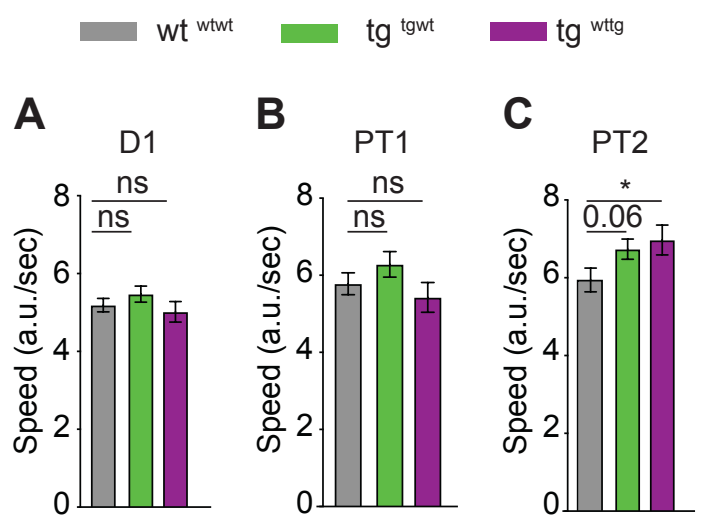

Figure 4.1.5. Transgenic mice did not show swimming difficulties at 3 months of age in the Morris water maze (MWM) spatial task.

Morris water maze (MWM) test in APPPS1-21 transgenic mice born to a transgenic father (tgtgwt, $\mathrm{n}=25$ ) or mother (tgwttg, $n=17$ ) and control wild-type mice ( $w^{\text {twtwt }}, n=30$ ) at 3 months of age. (A) Average speed during the first training day of the MWM. (B and C) Average speed during the first probe trial (PT1) (B) and second probe trial (PT2) (C). Student's two-tailed T-test, ns = no significant differences; ${ }^{*} P<0.05$. Error bars indicated SEM.

Together, these results confirm that transgenic APPPS1-21 mice exhibit memory impairment already at 3 months of age. This effect is comparatively stronger in case of a transgenic father $\left(\mathrm{tg}^{\text {tgwt }}\right)$, whereas APPPS1-21 offspring from a transgenic mother (tg $\left.{ }^{\text {wttg }}\right)$ show a milder - yet significant - impairment. These impairments in APPPS1-21 offspring are not associated with one gender since there were no remarkable gender-dependent differences in the MWM in any group (Appendix figure 2 and 3 ).

Finally, I tested mice in the contextual fear-conditioning (FC) paradigm, a test for associative memory. During the training day, wild-type and tgwttg mice showed similar levels of motility. Only tg ${ }^{\text {tgwt }}$ mice presented significantly lower motility and significantly higher levels of freezing (Figure 4.1.6A). During the test, all groups showed similar levels of freezing (Figure 4.1.6B-C). These data suggest that at 3 months of age there is no obvious effect of the APP and PS1 transgene on contextual associative memory. This reduction of the motility was not associated to one gender, since there were no remarkable gender-dependent differences in any of the groups in FC (Appendix figure 4). 


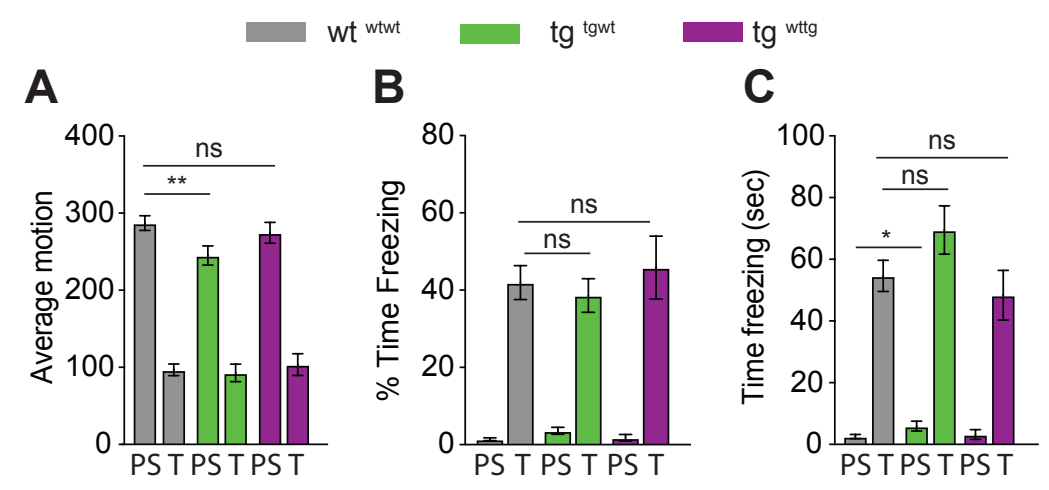

Figure 4.1.6. APPPS1-21 tg mice did not show deficits of contextual associative memory at 3 months of age.

Fear conditioning in APPPS1-21 transgenic mice born to a transgenic father ( $\operatorname{tg}^{\text {tgwt }}, n=28$ ) or mother (tg ${ }^{w 1 t g}$, $n=19)$ and control wild-type mice ( $\left.w^{\text {twtwt }}, n=34\right)$ at 3 months of age. (A) Average of motion during the training day. (B and C) Percentage of time (B) and time (C) freezing during training (first column) and testing day (second column). $\mathrm{PS}=$ pre-shock. $\mathrm{T}=$ test $(24 \mathrm{~h}$ later). Student's two-tailed T-test, ns $=$ no significant differences; ${ }^{*} P<0.05 ;{ }^{* *} P<0.01$. Error bars indicated SEM.

\subsubsection{Transcriptional profiling and functional pathway analysis in}

\section{APPPS1-21 mice at $3 \mathrm{~m}$}

So far, I have established that APPPS1-21 transgenic mice show spatial cognitive deficits already at 3 months of age. I also identified that there are differences in the cognitive performance of APPPS1-21 transgenic mice depending on whether they come from a mutant mother or father. These data suggest that APPPS1-21 transgenic mice might present changes in the cellular state at 3 months of age. Thus, I decided to use the transcriptome as a readout of the cellular state and wondered whether I would be able to dissect molecular pathways already affected at 3 months of age.

\subsubsection{Transcriptional profiling of APPPS1-21 transgenic mice at 3 months of age}

I first concentrated on the analysis of spatial-memory relevant regions of APPPS1-21 transgenic mice that originated from an APPPS1-21 $\mathrm{tg}$ father or mother ( $\operatorname{tg}^{\text {tgwt }}$ and $\mathrm{tg}^{\mathrm{wt}}{ }^{\mathrm{tt}}$ ) at 3 months of age. Specifically, I analyzed two specific subregions of the hippocampus, the Cornu Ammonis (CA1) and the Dentate Gyrus (DG). Both of these regions were involved in spatial memory and were one the first regions affected in APPPS1-21 tg mice, by the deposition of amyloid plaques, DG at 3 months and CA1 at 5 months of age (Radde et al., 2006). I compared the CA1 and DG transcriptome from $\operatorname{tg}^{\text {tgwt }}$ and $\operatorname{tg}^{\text {wttg }}$ mice to that of control wt mice (wt $\left.{ }^{\text {twt }}\right)$. I also stratified samples 
from male and female APPPS1-21 tg mice. Although I previously defined no significant genderassociated differences in the cognitive performance for these groups (Appendix figure 1-5), I wondered whether there would be gender-specific differences at the transcriptomic levels as there are gene expression regulatory mechanisms that are known to be gender-specific (Khor, Tong, Qian, \& Song, 2008; Manoli \& Tollkuhn, 2018). The quality of CA1 female samples was rather poor and thus results from these samples will be omitted in this report.

As seen previously, tg mice born to APPPS1-21 tg fathers (tg ${ }^{\text {tgwt }}$ ) showed strong spatial cognitive deficits. These deficits were also observed in tg mice born to APPPS1-21 $\operatorname{tg}$ mother (tg ${ }^{\text {wttg}}$ ), albeit in a milder form. Despite the mild cognitive differences, $\operatorname{tg}^{\text {tgwt }}$ and tg ${ }^{\text {witg }}$ were mostly undistinguishable at transcriptomic level, so they were combined in order to identify stronger effects linked to the APPPS1-21 phenotype. At the transcriptomic level, the variability of $\operatorname{tg}^{\text {tgwt }}$ vs. tg ${ }^{\text {wttg }}$ mice $(0.4 \%$ for $\mathrm{DGm}, 0.06 \%$ for $\mathrm{DGf}$ and $0.05 \%$ for $\mathrm{CA} 1 \mathrm{~m})$ was almost negligible compared to the combination of APPPS1-21 tg groups vs. control wtwtwt $(2.8 \%$ for DGm, 3.3\% for DGf and 0.11 for CA1m) (Appendix table 2).

The transcriptomic profile was evaluated based on significant differentially expressed genes (padj $\leq 0.05$ ) and observing level of changes defined by $\log _{2} \mathrm{FC}$ cutoff as severe $( \pm 1.00)$, medium ( \pm 0.50$)$ and mild $( \pm 0.25)$ (Figure 4.1.7A). The majority of genes fell into mild to medium changes in APPPS1-21 tg mice, indicating a moderate alteration of the transcriptome, maybe affected by certain compensatory mechanisms. Because the main changes in the transcriptome were observed for genes at the mild level (padj $\leq 0.05$; $\log _{2} \mathrm{FC} \pm 0.25$ ), further differentially expressed and signaling pathway analyses were focused on these genes.

Analyzing specifically genes associated with mild changes, around $54-60 \%$ of total genes were up-regulated in all three regions of the hippocampus of APPPS1-21 transgenic mice. Compared to DG from males and females (1374 for DG from males and 1595 for DG from females), the CA1 region from APPPS1-21 tg males was only mildly affected regarding gene expression all compared to wild-type controls ( $w^{t^{\text {twtt }}}$ ) (only 55 for CA1 from males) (Figure 4.1.7A-B and D-F). This might be connected to the fact that at 3 months of age only DG and neocortex were affected by plaque formation, whereas CA1 will not be affected until 2 or 3 months later in this model of AD (Radde et al., 2006). From the total number of significant differentially expressed genes (padj $\leq 0.05$ and $\log _{2} F C \pm 0.25$ ) in transgenic mice, around $25 \%$ of genes overlapped between male and female mice in DG regions (Figure 4.1.7C). This percentage suggests that male and female tg mice exhibited different transcriptomic profile, although cognitive performance was similar between both genders. Thus, as I hypothesized, there were in fact gender-specific transcriptomic states despite similar behavioral output. $75 \%$ of genes affected by the double mutation depend on the gender. These differences might indicate a genderdependent gene expression regulation. 
$\operatorname{tg}$ vs. wtwtwt

\begin{tabular}{|l|l|l|l|}
\hline padj $<=0.05$ & Severe & Medium & Mild \\
\hline Total $=$ up + down & Log $_{2} \mathrm{FC} \pm 1.00$ & $\log _{2} \mathrm{FC} \pm 0.50$ & Log $_{2} \mathrm{FC} \pm \mathbf{0 . 2 5}$ \\
\hline DGm & $23=21+1$ & $592=477+115$ & $\mathbf{1 3 7 4 = 8 9 9 + 4 7 5}$ \\
\hline DGf & $17=5+12$ & $600=368+232$ & $\mathbf{1 5 9 5 = 8 5 4 + 7 4 1}$ \\
\hline CA1m & $1=1+0$ & $5=3+2$ & $\mathbf{5 5}=\mathbf{3 0 + 2 5}$ \\
\hline
\end{tabular}

B

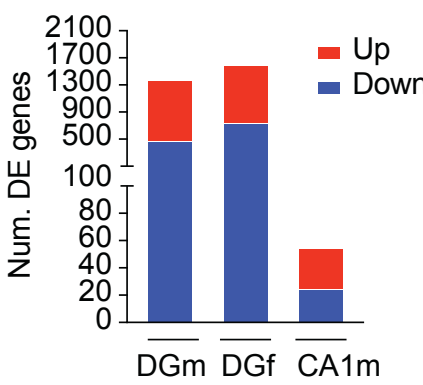

C

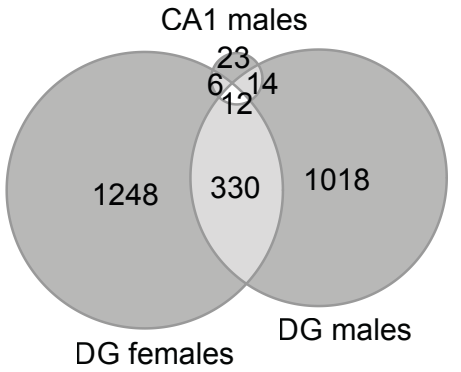

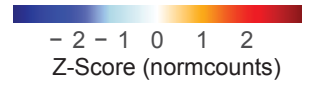

D

DG males

(1374)

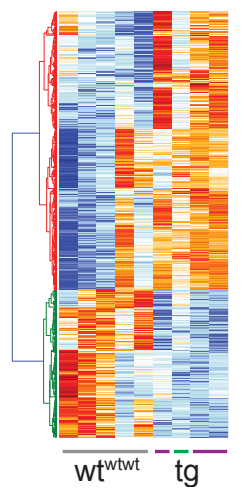

wt wtwt

E

DG females

(1595)

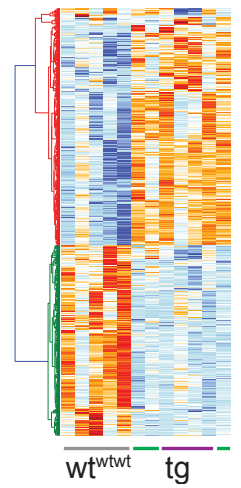

$\operatorname{tg}$ tgwt

tg witg

F

CA1 males

( 55 )

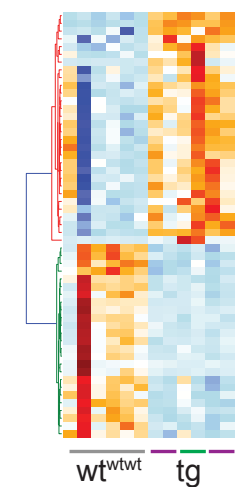

Figure 4.1.7. Mild to medium transcriptional differences in APPPS1-21 transgenic mice at 3 months of age.

(A) Number of significant differentially expressed genes with different level of changes (severe $( \pm 1.00)$, medium $( \pm 0.50)$ and mild $( \pm 0.25)$, established by the $\left.\log _{2} F C\right)$ between transgenic $\left(\operatorname{tg}=\operatorname{tg}^{\text {tgwt }}+\operatorname{tg}^{\text {wttg }}\right)$ and control wild-type ( $w^{\text {twtwt }}$ mice. Padj, P value adjusted and $\log _{2} F C$, logarithm base 2 of Fold Change. (B) Number of significant differentially expressed up- and down-regulated genes for DG males (DGm), DG females (DGf) and CA1 males (CA1m) in tg vs. wtwtwt comparison. Padj $\leq 0.05, \log _{2} F C \pm 0.25$. (C) Venn diagram showing overlap between significant genes in different subregions (DGm, DGf and CA1m). Padj $\leq$ $0.05, \log _{2} F C \pm 0.25$. (D-F) Heatmaps of differentially expressed genes in 3 months old tg vs. $w^{w t w t}$. High expression is indicated by the red color spectrum and low expression by the blue color spectrum. Padj $\leq$ $0.05, \log _{2} F C \pm 0.25$. For DG males: transgenic $\operatorname{tg} n=4$ and $w^{t w t w t} n=5$; for $D G$ females $\operatorname{tg} n=7$ and wtwtwt $n=5$ and for CA 1 males transgenic $\operatorname{tg} n=6$ and $w^{t^{w t w t}} n=6$. 
4.1.2.2. Functional pathway analysis of APPPS1-21 transgenic mice at 3 months of age

Having established the transcriptomic signature of transgenic APPPS1-21 mice in DG and CA1 at 3 months of age, I next focused on the functional pathways linked with those genes. For that, I used the analytical method called Gene Set Enrichment Analysis (GSEA) (Mootha et al., 2003; Subramanian et al., 2005) that allows me to study the whole dataset functions without restricting the study to only genes with a specific threshold.

In order to further investigate the molecular state in DG and CA1 of transgenic mice at 3 months of age, I ran the full list of normalized counts in control wt mice ( $\left.w^{\text {twtwt }}\right)$ and APPPS1-21 tg mice through GSEA. To obtain a first idea of the mechanisms involved, I selected the top 50 significant pathways (nominal pval $\leq 0.05$; FDR $\leq 0.1$ ) from each category (biological process, molecular function and cellular component) ranked by Normalized Enrichment Score (NES). The NES indicates the level to which a group of genes is represented in the upper or lower part of the ranked list of genes in the expression dataset (Subramanian et al., 2005). Positive pathways mean enrichment in wild-type mice, whereas negative pathways are enriched in transgenic mice. From these top 50 pathways, I screened in detail which broader term could be used to summarize the pathways, in order to get a first idea of the most important mechanisms associated with tg mice at 3 months of age.

Analyzing the broader biological processes associated with the top 50 preselected pathways, similar functions were affected in male and female DG regions (Figure 4.1.8B, C, E and F). Even though these regions shared only $25 \%$ of common genes; similar functional pathways were affected in both genders. The majority of enriched GO terms in DG of APPPS1-21 tg mice were linked to synapse and nervous system development, an expected finding since DG is an important area of adult neurogenesis (Ming \& Song, 2011) (Figure 4.1.8A, C,D and F). On the contrary, pathways related to metabolic process, mitochondria complex and respiratory chain, localization, transport and translation were underrepresented in APPPS1-21 tg mice (Figure 4.1.8A, B, D and E). Taking into account that APPPS1-21 tg mice previously manifested spatial cognitive impairments, this overrepresentation of genes related to behavior, synapse or nervous system development might be part of a compensatory network that counteract acted AD pathology changes. Apart from these similar categories in male and female DG regions, there were gender-specific changes in certain gene-sets. In DG from males, several genes were involved in RNA metabolism and cellular component biogenesis (Figure 4.1.8A-C), whereas in DG from females, pathways were related to cell component and organelle organization (Figure 4.1.8D-F). 
In the CA1 male region of APPPS1-21 transgenic mice, genes related to metabolic processes, mitochondria complex and respiratory chain, synapse, transport and localization were altered similarly as in male and female DG regions (Figure 4.1.8G-I). However, pathways linked to lipid metabolism, secretion, inflammation and coagulation were exclusively affected in the CA1 hippocampus subregion (Figure 4.1.8G-I).

DG males

A

Establishment of protein localization to ER Nuclear transcribed mRN NA catab process nonsense mediated decay Protein localization to endoplasmic reticulum Ribosome biogenesis Ribosome biogenesis RRNA metabolic process xidative phosphorylation Mitochondrial respiratory chain complex assembly Ribonucleoprotein complex biogenesis Camp mediated signaling Neuron projection guidance Cyclic nucleotide mediated signaling Hindlimb morphogenesis Cell morphogenesis involved in neuron differentiation Central nervous system neuron differentiation Neuron projection morphogenesis Cell cell adhesion of neuron migration

DG females philic cell adhesion via plasma membrane adhesion molec

D

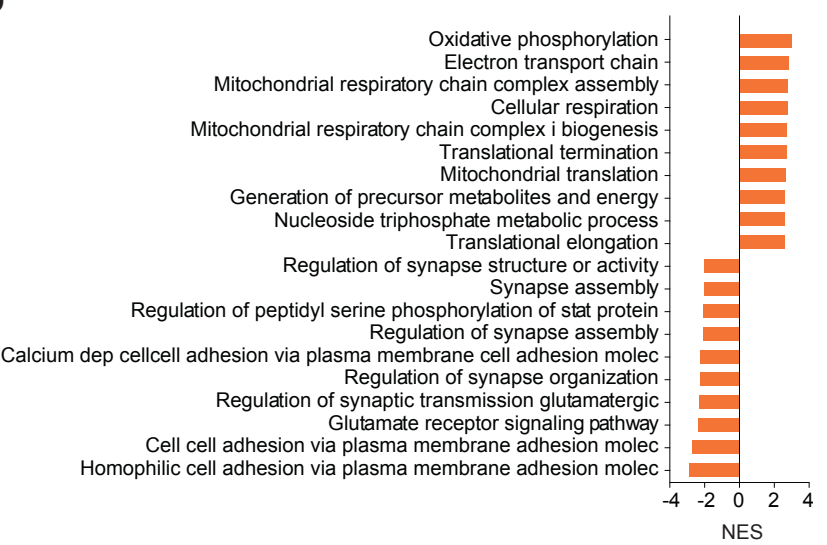

CA1 males

G

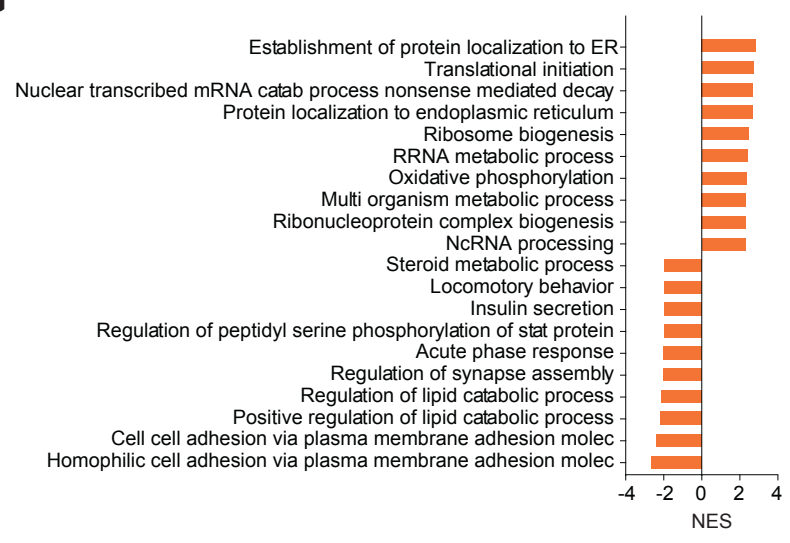

B Negative in tg mice

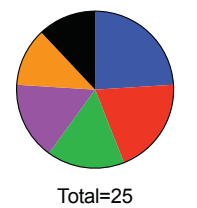

RNA metabolic process Mitochondria complex/ Respiratory chain Cellular metabolic process Translation biogenesis and assembly Protein localization

C Positive in tg mice

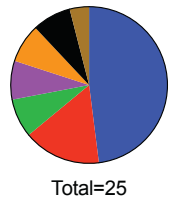

Nervous system development and migration Tissue developmen Synapse Sehavior/Cognition Cell-cell adhesion Mithocondria / respiratory chain / Cell metabolic process
Mello

E Negative in tg mice

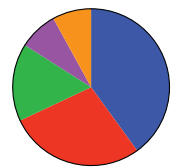

Total $=25$

F

Positive in tg mice

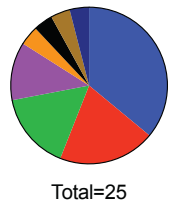

H Negative in tg mice

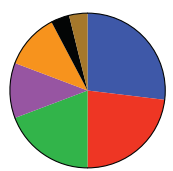

Total $=26$

I

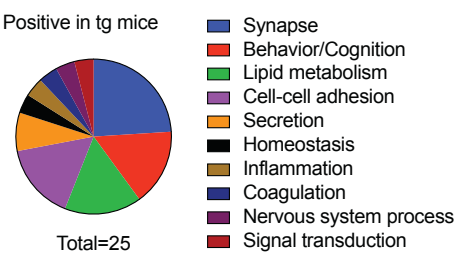

Transport and localization
- Mitochondria complex/ Respiratory chain

Cellular metabolic process

$\square$ Translation

Cellular component and organelle organization

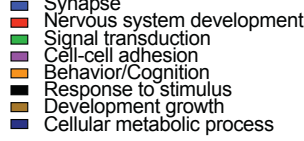

.


Figure 4.1.8. Biological processes are affected in APPPS1-21 transgenic brain subregions at 3 months of age.

(A, D and G) Top 10 preselected biological processes over or underrepresented within the whole dataset of genes in tg or control (wtwtwt) brain subregions. DGm (A), DGf (D) and CA1m (G). NES= Normalized Enrichment Score. Positive NES means underrepresentation in tg mice, whereas negative NES means overrepresentation in tg mice. (B, C, E, F, H and I) Broader term summary of the top 25 overrepresented (C, $\mathbf{F}$ and $\mathrm{I})$ and 25 underrepresented (B, E and F) pathways in transgenic brain subregions. Nominal pval $\leq 0.05$; FDR $\leq 0.25$. For DG males: transgenic $\operatorname{tg} n=4$ and $w^{t w t w t} n=5$; for $D G$ females $\operatorname{tg} n=7$ and wtwt $\mathrm{n}=5$ and for CA 1 males transgenic $\operatorname{tg} \mathrm{n}=6$ and $\mathrm{wt}^{\mathrm{wtwt}} \mathrm{n}=6$.

Interestingly, genes associated with the top 50 significant molecular function ontologies were highly compatible with the observations in the biological process ontology. Genes associated with oxidoreductase activity, a critical activity in the mitochondrial complex, were altered in CA1 and DG regions of APPPS1-21 tg mice. Moreover, genes associated with transcription regulatory activity, an activity essential for signal transduction, were as well altered in the CA1 and DG regions (Figure 4.1.9A-I).

DG males

A

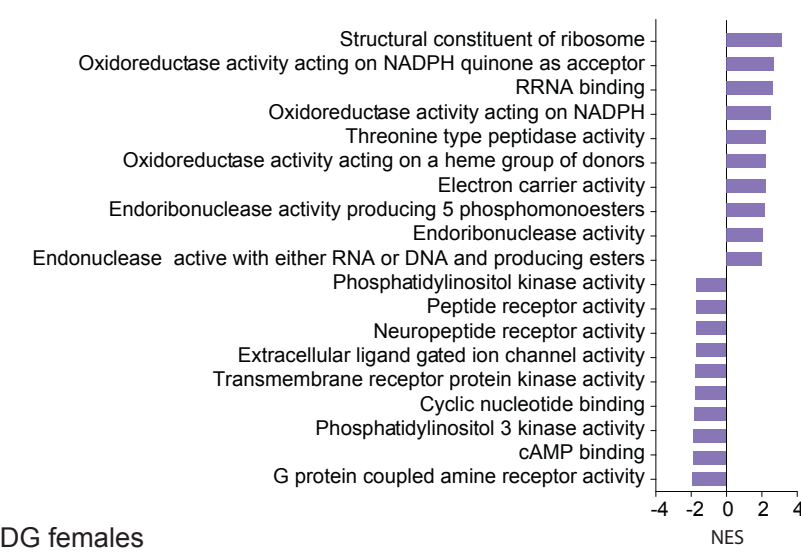

D

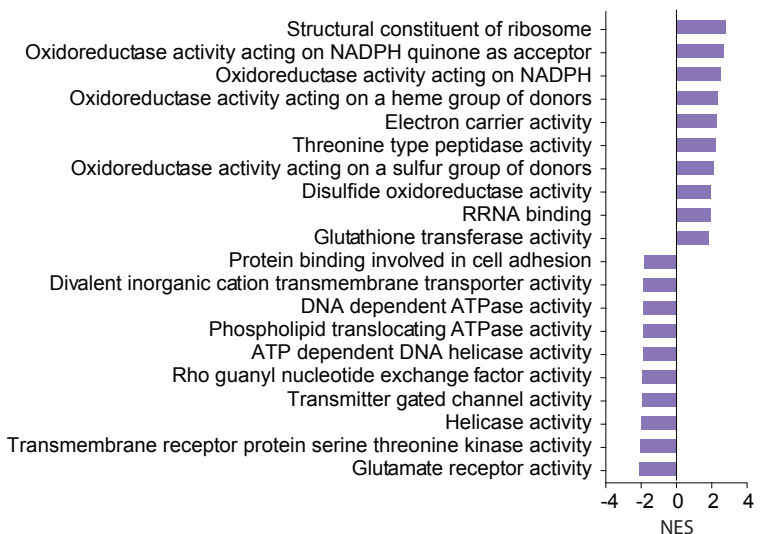

B

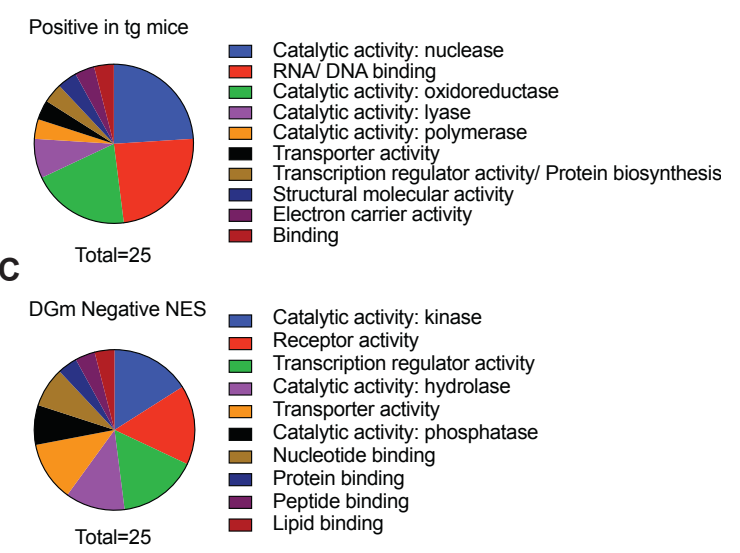

E

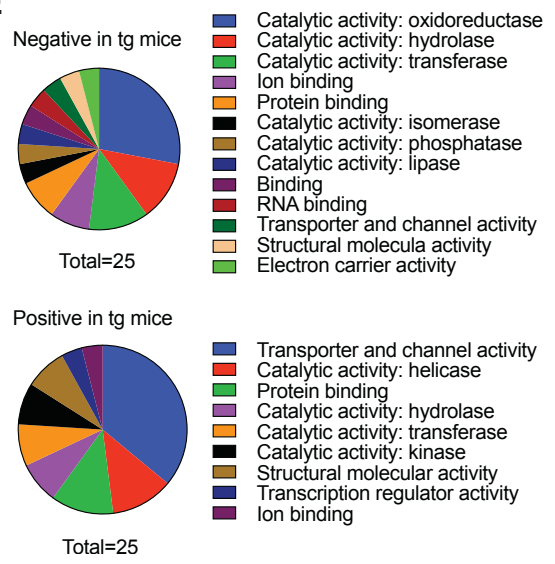




\section{CA1 males}

G

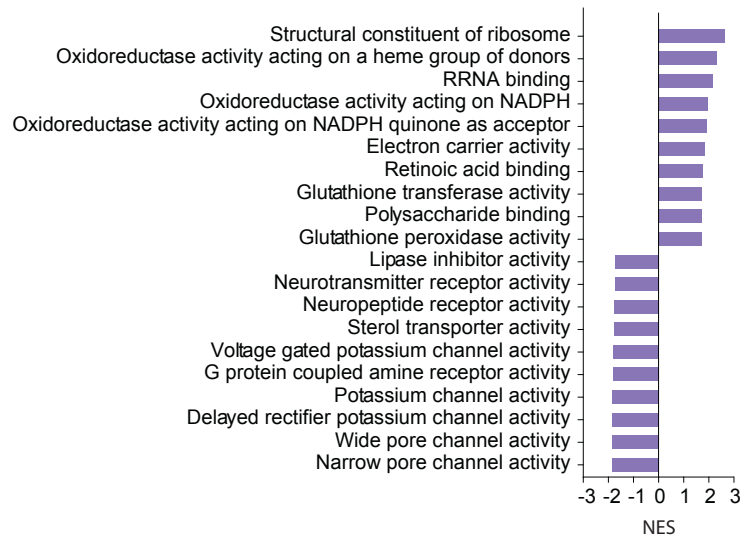

$\mathrm{H}$

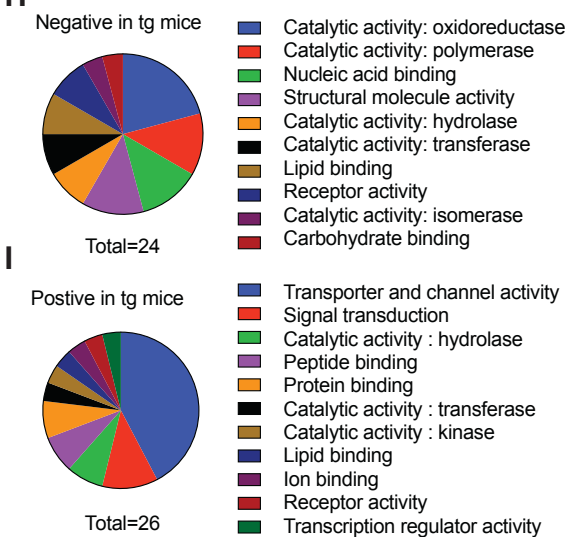

Figure 4.1.9. Molecular functions are affected in APPPS1-21 transgenic brain subregions at 3 months of age.

(A, D and G) Top 10 preselected molecular functions over/underrepresented within the whole dataset of genes in tg or control (wtwtwt) brain subregions: DGm (A), DGf (D) and CA1m (G). NES= Normalized Enrichment Score. Positive NES means underrepresentation in tg mice, whereas negative NES means overrepresentation in tg mice. (B, C, E, F, H and I) Broader term summary of the top 25 overrepresented (C, F and I) and 25 underrepresented (B, E and F) pathways in transgenic brain subregions. Nominal pval $\leq 0.05$; FDR $\leq 0.25$. For DG males: transgenic $\operatorname{tg} n=4$ and $w^{w}{ }^{w t w t} n=5$; for $D G$ females $\operatorname{tg} n=7$ and wtwtwt $\mathrm{n}=5$ and for CA1 males transgenic $\operatorname{tg} \mathrm{n}=6$ and $\mathrm{wt}^{\mathrm{wtwt}} \mathrm{n}=6$.

The cellular component ontology revealed genes related to synapse, ribosome and mitochondria in DG and CA1 regions of APPPS1-21 tg mice compared to control wt mice (Figure 4.1.8A-I). In DG from males and females and CA1 from males, cellular components associated with mitochondria complex, respiratory chain, ribosome and splicing were underrepresented in APPPS1-21 tg mice (Figure 4.1.10A, B, D, E, G and H). In contrast, genes linked to synapse, neuronal parts and cytoskeleton were up-regulated in APPPS1-21 tg mice (Figure 4.1.10A, C, D, $\mathrm{F}, \mathrm{G}$ and I). This data from the cellular components is in agreement with the previous results seen for biological process and molecular functions (Figure 4.1.8A-I and Figure 4.1.9A-I). 
DG males
A

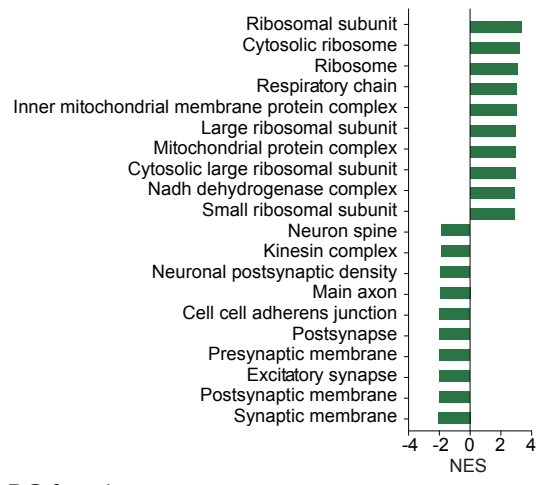

B

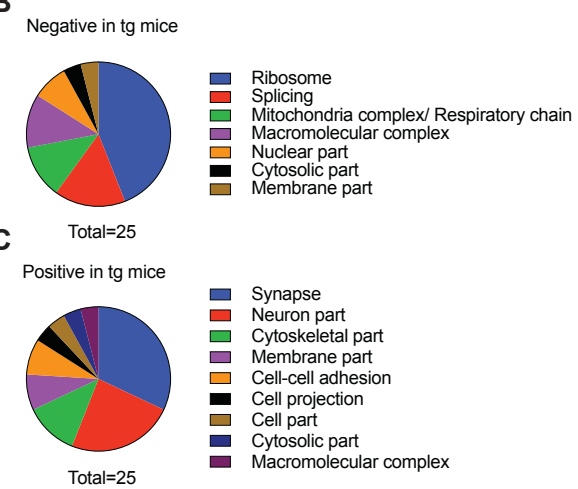

DG females
D

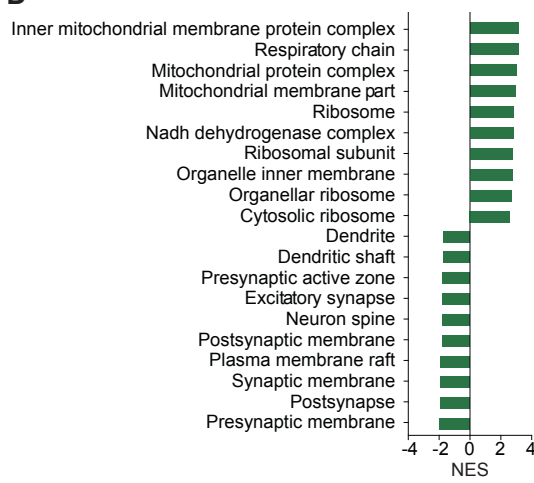

CA1 males

G

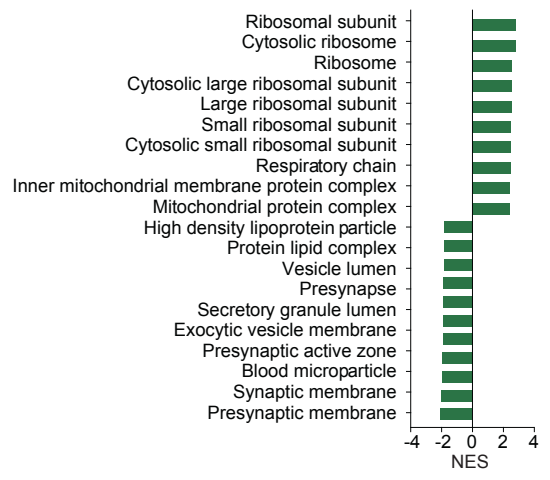

E

Negative in tg mice

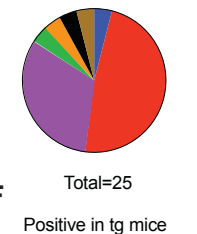

Positive in tg mice
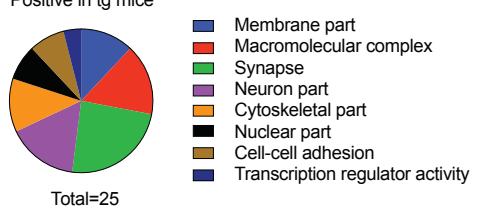
$\square$ Membrane part $\square$ Macromolecular complex - Cytoskeletal part Cytoplasmic part
H Negative in tg mice

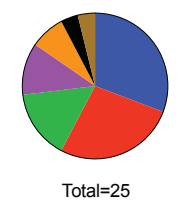
Ribosome Positive in tg mice
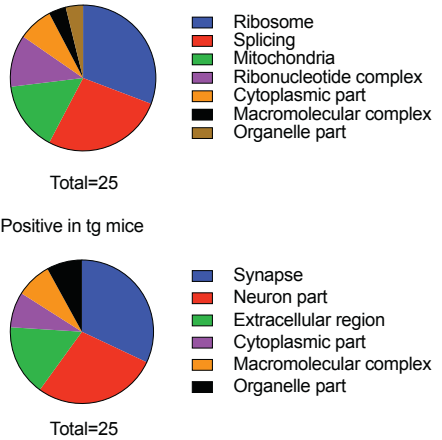
Organelle part

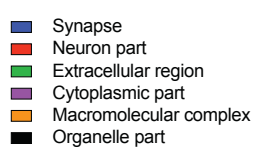

Figure 4.1.10. Cellular components are affected in APPPS1-21 transgenic brain subregions at 3 months of age.

(A, D and G) Top 10 preselected cellular components over/underrepresented within the whole dataset of genes in tg or control (wtwtwt) brain subregions: DGm (A), DGf (D) and CA1m (G). NES= Normalized Enrichment Score. Positive NES means underrepresentation in tg mice, whereas negative NES means overrepresentation in tg mice. (B, C, E, F, H and I) Broader term summary of the top 25 overrepresented (C, F and I) and 25 underrepresented (B, E and F) pathways in transgenic brain subregions. Nominal pval $\leq 0.05$; FDR $\leq 0.25$. For DG males: transgenic tg $n=4$ and $w^{\text {twtwt }} n=5$; for DG females $\operatorname{tg} n=7$ and $w^{\text {twtwt }}$ $\mathrm{n}=5$ and for CA1 males transgenic $\operatorname{tg} \mathrm{n}=6$ and $\mathrm{wt}^{\mathrm{wtwt}} \mathrm{n}=6$. 
Taken together, these data indicate that APPPS1-21 transgenic mice show moderate but consistent changes in gene expression variation that accompany cognitive deficits already at 3 months of age. These findings confirm that the genetic alteration from transgenic offspring promotes changes at the global molecular state of the hippocampus of these mice, affecting networks linked to synapse, nervous system development, energy metabolism and protein biosynthesis.

As an alternative method to find significantly enriched pathways in differentially expressed genes in APPPS1-21 transgenic vs. control wild-type mice, I used a cutoff-based approach. In contrast to GSEA analyses, classical Gene Ontology (GO) analyses make use of only a partial set of the dataset, usually genes that show significant deregulation at a certain cutoff. I screened exclusively the differentially expressed genes (padj $\leq 0.05$; $\log _{2} \mathrm{FC} \pm 0.25$ ) for $\mathrm{GO}$ terms using a tool called ClueGO that uses Cytoscape to represent associated networks (Bindea et al., 2009; Shannon et al., 2003). All pathways represented in figure 4.1.11 exhibited a significant pvalue of $\leq 0.05$. A substantial shift towards pathways related to up-regulated genes can be observed in all three regions (Figure 4.1.11A-C).

The most significantly affected pathways in DG regions, indicated by an intense red or blue color in figure 4.1.11A-B, are linked to nervous system development, synapse, RNA metabolic process, mitochondrial complex and ion transport. In particular, analyzing only the top 20 significant pathways, around $75 \%$ of the pathways were related to nervous system development and neurogenesis (Figure 4.1.11A-B and D-E). Interestingly transgenic mice showed strong changes in one of the most relevant functions of the DG, neurogenesis process (Ming \& Song, 2011). To a lesser extent, pathways related to signal transduction, metabolic process, cell-cell adhesion, component biogenesis, chromatin modification and regulation membrane potential were altered in transgenic mice (Figure 4.1.11A-B and D-E). Interestingly, several biological processes were shared between males and females, in agreement with the results previously seen in the GSEA analysis (Figure 4.1.11A-B and D-E). Particularly, in CA1 from male mice only cell-cell adhesion and cell projection, pathways associated with up-regulated genes, were significantly different (Figure 4.1.8C and F). These results confirm that the CA1 transcriptome is almost not altered compared to the DG region at 3 months of age in this model of AD. 


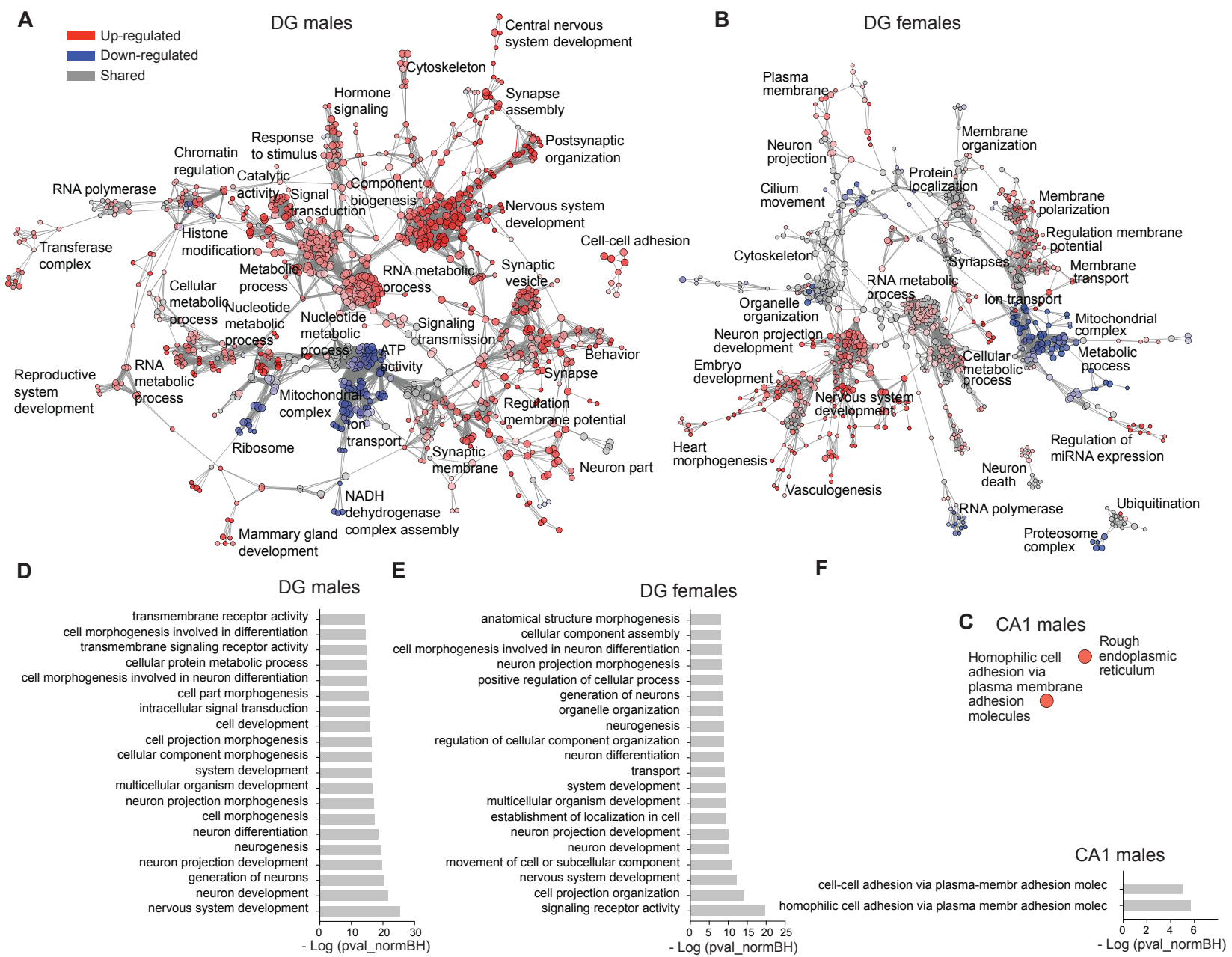

Figure 4.1.11. Functional pathways affected in APPPS1-21 transgenic mice, specifically in DG from males and females.

(A-C) Network of functional categories (biological process, molecular function and cellular component ontologies) of significant differentially expressed genes in brain subregions of transgenic APPPS1-21 mice. The size of the nodes correlates inversely with statistical significance. Categories with a red color spectrum correspond to up-regulated genes in tg mice and blue correspond to down-regulated genes. Categories that shared up- and down-regulated genes are represented in grey. Genes, padj $\leq 0.05$; $\log _{2} \mathrm{FC} \pm 0.25$ and pathways, pval $<0.05$. (D-F) Top 20 significant functional pathways ranked by $-\log \mathrm{pVal}$ after Benjamini-Hochberg correction of significant differentially expressed genes in brain subregions of wt $^{\text {tgwt }}$ vs. wt ${ }^{\text {twt }}$ mice. Genes, padj $\leq 0.05 ; \log _{2} \mathrm{FC} \pm 0.25$ and pathways, pval $<0.05$.

These findings with ClueGO/Cytoscape are in agreement with GSEA data and reflect the important role that genes linked to synapse, nervous system development, cell-cell adhesion, protein localization, metabolism and gene expression play on the molecular state of transgenic mice at 3 months of age. 


\subsubsection{Alternative exon usage and functional pathway analysis in APPPS1-21 transgenic mice at 3m}

So far, I have analyzed the variability concerning cognitive performance and transcriptome from APPPS1-21 transgenic mice at 3 months of age compared to control wt mice. I used the transcriptome as a readout of cellular state of APPPS1-21 tg mice, and I was able to identify different functional pathways associated with the APP/PS1 transgene. And I found that pathways related to splicing were altered in tg mice. Thus, I decided to study the alternative exon usage in APPPS1-21 tg mice.

\subsubsection{Alternative exon usage in APPPS1-21 transgenic mice}

To analyze the differences of exon usage between transgenic and control wild-type mice, I screened the alternative exon usage in previously analyzed hippocampus subregions (CA1 and DG) of APPPS1-21 tg mice with DEXSeq (Anders et al., 2012). Only statistically significant genes (padj $\leq 0.05$ ) were evaluated and changes in levels of exon usage were analyzed by $\log _{2} \mathrm{FC}$ cutoff as severe $( \pm 1.00)$, medium $( \pm 0.50)$ and mild $( \pm 0.25)$.

Notably, a large number of genes showed significant (padj $\leq 0.05$ ) alternative exon usage in CA1 and DG from APPPS1-21 tg mice. From 150 for CA1m to 2330 for DGf genes showed severe changes and thousands of them (from 3659 for DGm to 9605 for DGf) showed a mild change in $\operatorname{tg}$ mice (Figure 4.1.12A), indicating a strong alteration of the exon usage. The CA1 region of APPPS1-21 tg mice was also strongly affected and a substantial shift towards the inclusion of exons can be observed in CA1 and DG of the experimental groups (Figure 4.1.12A-B)

A

\begin{tabular}{|l|l|l|l|}
\hline padj $\leq 0.05$ & Severe & Medium & Mild \\
\hline Total $=$ incl + excl & Log $_{2}$ FC \pm 1.00 & $\log _{2} \mathrm{FC} \pm 0.50$ & $\log _{2} \mathrm{FC} \pm 0.25$ \\
\hline DGm & $742=531+211$ & $2896=1852+1044$ & $3659=2236+1423$ \\
\hline DGf & $2330=1944+386$ & $7455=4311+3144$ & $9605=5155+4450$ \\
\hline CA1m & $150=109+41$ & $2572=1843+729$ & $5006=3264+1742$ \\
\hline
\end{tabular}

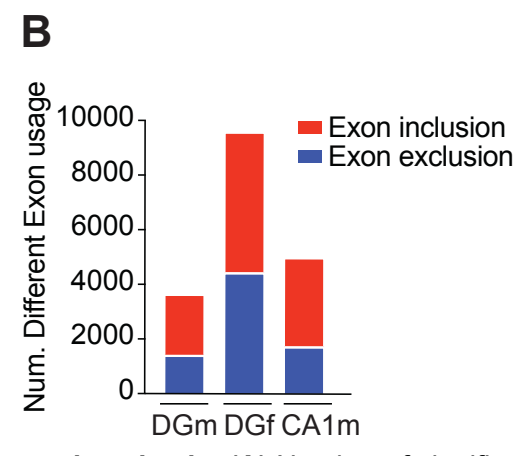


Figure 4.12. Drastic alteration in the alternative exon usage in DG and CA1 of APPPS1-21 transgenic mice.

(A) Number of significant alternative exon usage with different levels of changes (severe $( \pm 1.00)$, medium $( \pm 0.50)$ and mild $( \pm 0.25)$, established by the $\left.\log _{2} F C\right)$ in tg mice compared to wtwtwt mice. Exon usage, padj $\leq 0.05$. (B) Number of significant included and excluded exons for DG males (DGm), DG females (DGf) and CA1 males $(\mathrm{CA} 1 \mathrm{~m})$ in tg vs. wtwtwt mice. Red columns represent significant alternative included exons and blue columns represent significant alternative excluded exons. Exon usage, padj $\leq 0.05$, $\log _{2} F C \pm 0.25$. For $D G$ males: $\operatorname{tg} n=4$ and $w^{t^{w t w t}} n=5$; for $D G$ females $\operatorname{tg} n=7$ and $w^{t^{w t w t}} n=5$ and for $C A 1$ males tg $n=6$ and $w^{\text {twtwt }} n=6$.

Based on significant differential exon usage (padj $\leq 0.05$ ), I compared DG from males and females and CA1 from males of APPPS1-21 tg mice, all of them compared to control wt mice (wtwtwt). I found that a large number of identical genes (1422) showed alternative exon usage in all three regions. In DG from males, this represented a $46.4 \%$ from the total alternative exon usage, $21.8 \%$ in DG from females and $35 \%$ in CA1 from males (Figure 4.1.13A-C).

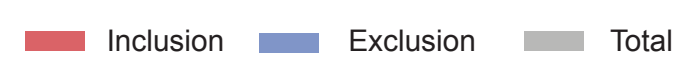

A

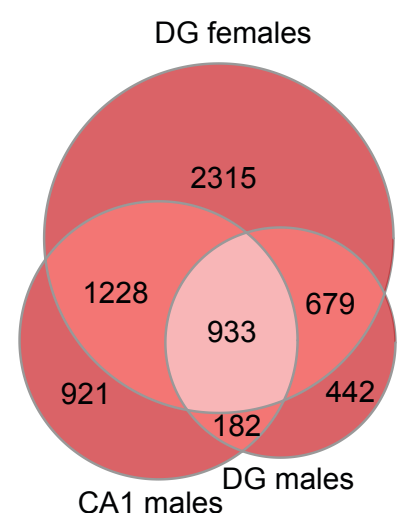

B

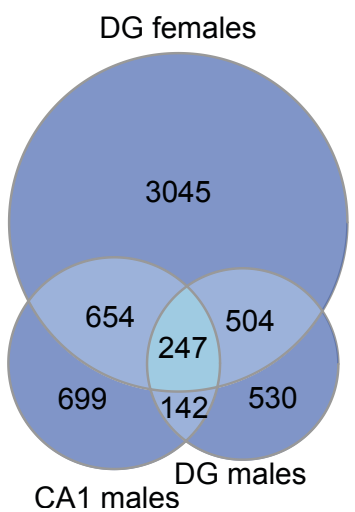

C

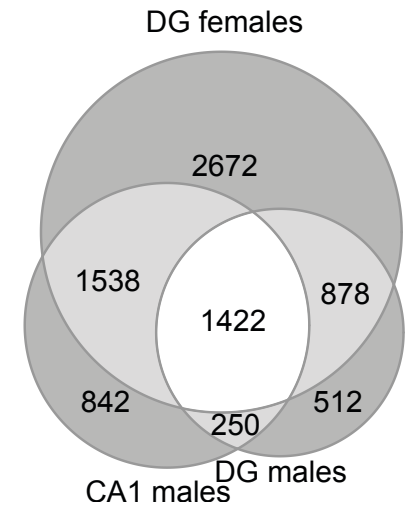

Figure 4.1.13. A large number of identical genes showed alternative exon usage in DG from males and females and CA1 from males of genes in APPPS1-21 transgenic mice.

(A-C) Venn diagrams illustrating overlap of spliced genes in DG from males and females and CA1 from males in tg vs. wtwtwt comparison. Genes, padj $\leq 0.05$; $\log _{2} F C \pm 0.25$. For $D G$ males: $\operatorname{tg} n=4$ and $w^{t w t w t} n=5$; for DG females tg $n=7$ and $w^{\text {twtwt }} n=5$ and for CA1 males tg $n=6$ and $w^{t^{w t w t}} n=6$.

Taken together, these results indicate that APPPS1-21 transgenic mice show more drastic alternative exon usage changes than transcriptomic changes in DG and CA1 regions. A large number of genes that had alternative exon usage were shared between CA1 and DG regions of APPPS1-21 tg mice. 
4.1.3.2. Functional pathways linked to alternative exons in APPPS1-21 transgenic mice at 3 months of age

In order to further investigate the molecular state affected by alternative exon usage, I performed a functional analysis using ClueGO/Cytoscape (Bindea et al., 2009) (Shannon et al., 2003). I analyzed the genes with a significantly altered exon usage (padj $\leq 0.05$ and $\log _{2} F C \pm 0.25$ ) in APPPS1-21 tg mice compared to control wt mice (wtwtwt). Thousands of signaling pathways were disrupted in male and female DG and male CA1 of APPPS1-21 tg mice (2460 for DGm, 3556 for DGf and 2887 for CA1m). These dramatic changes suggest that the combination of inherited genetic and non-genetic factors promote an extreme alteration in the global translational processes, affecting genes associated to a very wide spectrum of functions. To focus on the most relevant pathways, I screened the top 20 most significantly affected biological processes and molecular functions ranked by corrected p-value (Benjamini-Hochberg). Differentially exon usage was observed in many genes involved in the system and nervous system development, cell and neuronal projection, signaling receptor activity, transport and localization (mainly of proteins) and metabolic process (mainly protein) (Figure 4.1.14A-C).

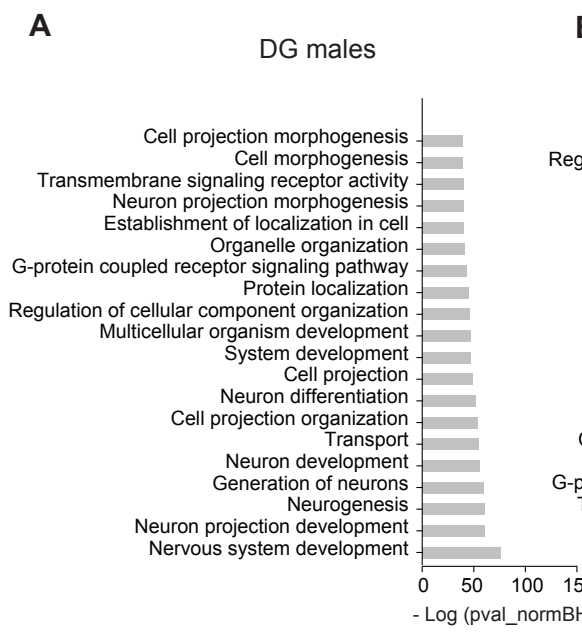

B

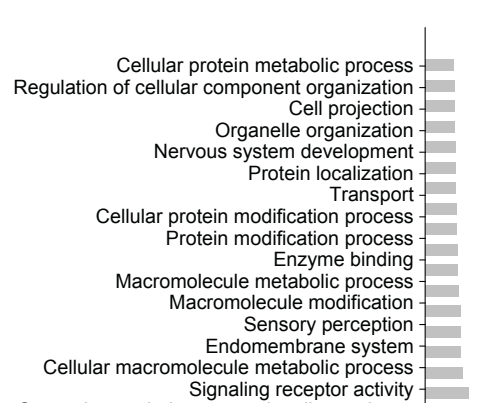

-protein coupled receptor signaling pathway

Transmembrane signaling receptor activity -

Transmembrane receptor activity
G-protein coupled receptor activity
C

CA1 males

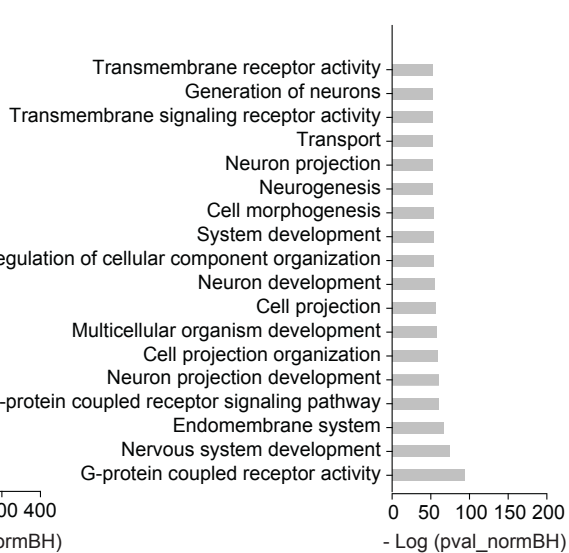

Figure 4.1.14. Top 20 significant pathways affected by alternative exon usage in genes of CA1 and DG from APPPS1-21 transgenic mice.

(A-C) Top 20 significant functional pathways ranked by -log pval after Benjamini-Hochberg correction of significant differentially expressed genes in brain subregions of tg vs. wtwtwt comparison. Genes, padj $\leq$ 0.05 ; $\log _{2} F C \pm 0.25$ and pathways, pval $<0.05$. For $D G$ males: $\operatorname{tg} n=4 ; w^{t^{\text {tgwt }}} n=7$ and $w^{\text {twtwt }} n=5$; for $D G$ females tg $n=7$; wt tgwt $n=5$ and $w^{t^{\text {twt }}} n=5$ and for CA1 males tg $n=6$; $w^{\text {tgwt }} n=7$ and $w^{t^{w t w t}} n=6$. 
Notably, several of these biological functions were also affected in the transcriptome of APPPS1-21 tg mice (section), such as nervous system development, transport and localization and metabolic process. Thus, I wondered whether these genes affected by alternative exon usage corresponded to the significant differentially expressed genes in APPPS1-21 tg mice. To this end, I compared the up-/down-regulated and the total number of differentially expressed and alternatively spliced genes in APPPS1-21 tg mice compared to wt control mice. Around $30 \%$ of the differential expressed genes in CA1 and DG regions of tg mice also showed modifications on the splicing events (Figure 4.1.15A-C). These results indicate that only $30 \%$ of the transcriptome from APPPS1-21 tg mice is also affected by an alternative splicing process, whereas $70 \%$ of the differentially expressed genes in APPPS1-21 tg mice did not show an alteration of their splice events.

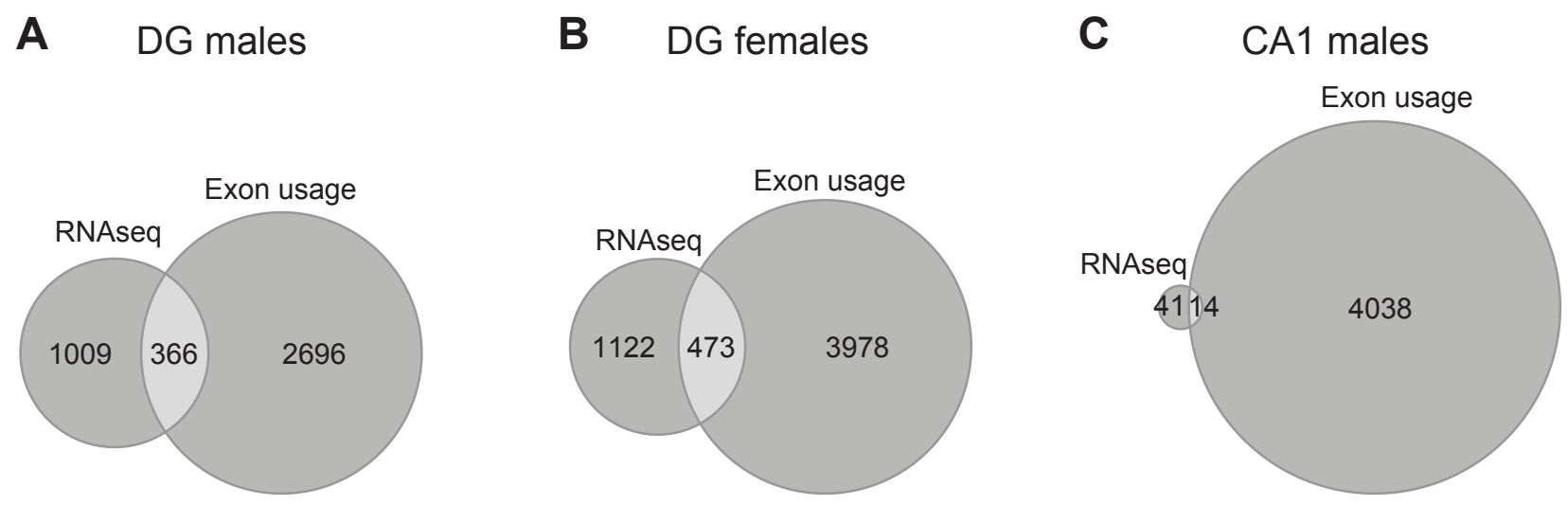

Figure 4.1.15. Around 30\% of genes were affected by differential expression and splicing in CA1 and DG regions of APPPS1-21 transgenic mice.

(A-C) Venn-diagrams are showing the overlap of genes affected by differentially expression and splicing in CA1 and DG regions of APPPS1-21 tg mice. Genes, padj $\leq 0.05$; $\log _{2} F C \pm 0.25$ and pathways, pval < 0.05 . For DG males: $\operatorname{tg} n=4$ and $w^{t^{t t w t}} n=5$; for $D G$ females $\operatorname{tg} n=7$ and $w^{\text {twtwt }} n=5$ and for $C A 1$ males tg $\mathrm{n}=6$ and $\mathrm{wt}^{\mathrm{t} \text { twt }} \mathrm{n}=6$.

Next, I analyzed the pathways of those genes that were both differentially expressed and had an alternative exon usage in APPPS1-21 tg mice. Along these genes in DG from male and female APPPS1-21 tg mice, I could find genes related to nervous system development, embryo development, synapse, microtubule and cytoskeleton, cell metabolic process, chromatin modification, cell death, transmembrane transport, RNA metabolic process and response to stimulus (Figure 4.1.16A-C). Almost all pathways were associated with up-regulated genes, except pathways connected with mitochondria, ATPase activity and ribonucleotide metabolism, which were associated with down-regulated genes (Figure 4.1.16A-C). The overlap between differentially expressed and spliced genes in CA1 from males was too small to observe any network associated with it (Figure 4.1.15C). 


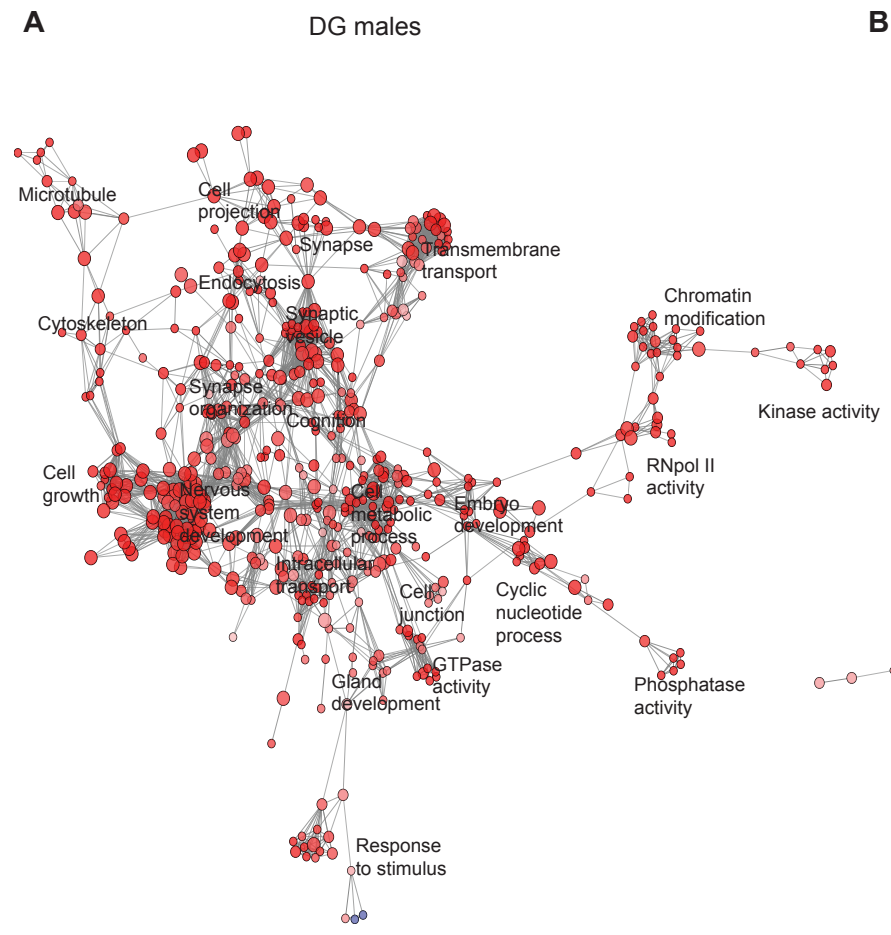

B

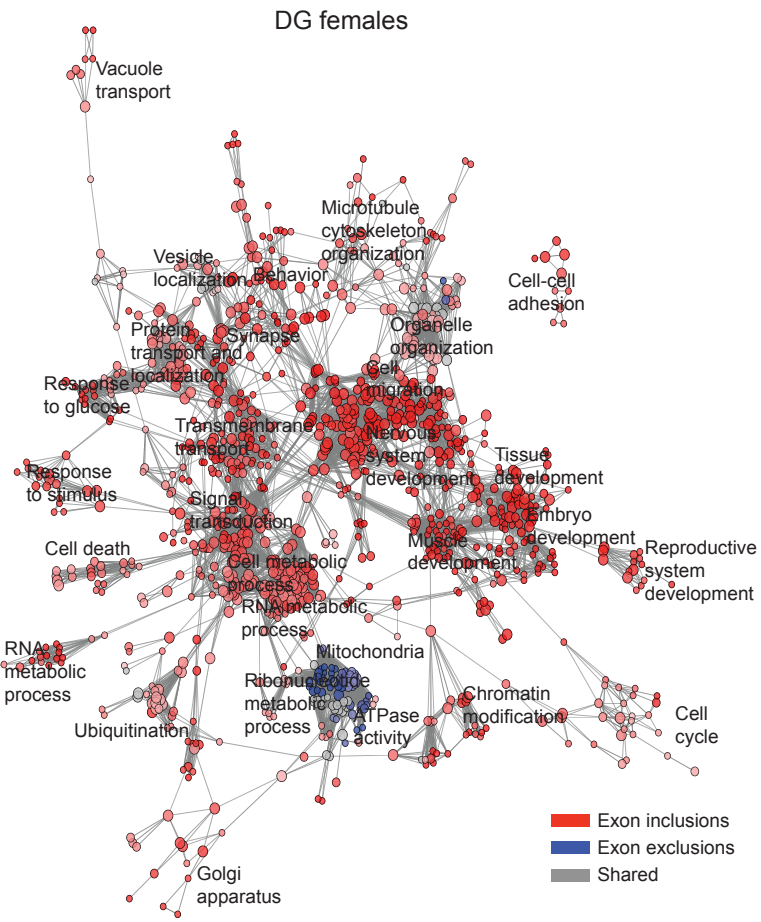

Figure 4.1.16. Several pathways were affected by those genes that were both differentially expressed and had an alternative exon usage in DG region of APPPS1-21 transgenic mice.

(A-B) Network of functional categories (biological process, molecular function and cellular component ontologies) of significant differentially expressed and spliced genes in DG of APPPS1-21 transgenic mice. The size of the nodes correlates inversely with statistical significance. Categories with a red color spectrum correspond to pathways associated with genes with inclusion of exons and up-regulated genes in transgenic mice and with blue correspond to exclusion of exons and down-regulated genes. Genes, padj $\leq 0.05$; $\log _{2} F C \pm 0.25$ and pathways, pval $<0.05$. For DG males: tg $n=4$ and $w^{t^{w t w t}} n=5$; for DG females $\operatorname{tg} n=7$ and $w^{\text {twtwt }} n=5$ and for CA1 males tg $n=6$ and $w^{w{ }^{t w t}} n=6$.

These findings suggest the existence of a clear connection between the altered expressed genes and the alternative exon usage in APPPS1-21 tg mice and therefore of the pathways associated with them. On the other hand, a high proportion of differentially expressed or differentially spliced genes did not overlap (around 70\%), indicating alteration of distinct signaling pathways that directly or indirectly can be connected in similar networks associated with those pathways described earlier. 


\title{
4.2.Intergenerational effect on wild-type mice born to an APPPS1-21 transgenic father or mother
}

\author{
4.2.1.Cognitive abilities of wild-type mice born to APPPS1-21 \\ transgenic parents
}
4.2.1.1. Cognitive abilities in wild-type mice born to APPPS1-21 transgenic fathers or mothers at 3 months of age

Having established the cognitive performance, the transcriptome and the exon usage of APPPS1-21 tg mice, I next focused on the potential intergenerational transmission of nongenetic factors from tg parents to their wild-type offspring. This paternal transmission might impact on memory function. Several studies have shown a substantial effect of parental lifestyle on offspring phenotypes via epigenetic inheritance (Anway et al., 2005; Rodgers et al., 2015; Siklenka et al., 2015; Wei et al., 2014). I wondered whether a genetic factor, such as a specific genotypic composition, could also influence the next generation through non-genetic mechanisms. Concretely, I posed the question of whether wt offspring from APPPS1-21 tg parents would show any cognitive differences as compared to control wild-type mice born to two wt parents, i.e. whether there is any non-genetic trace of the parent's genotype that gets transmitted to the next generation. To study this idea, I tested the following groups: wt mice born to an APPPS1-21 tg father and a wt mother (wtgwt), wt mice born to a wt father and an APPPS1-21 tg mother ( $w^{t^{w t g}}$ ), as the main experimental groups. As control groups I used wt mice born to wt parents ( $w t^{w t w t)}$, as well as $\operatorname{tg}$ mice born to a tg father (tg ${ }^{\operatorname{tg} w t)}$, and $\operatorname{tg}$ mice born to a tg mother ( $\operatorname{tg}^{\text {wttg}}$ ) (Figure 4.2.1A). Both male and female were evaluated together since independently did not show statistically significant differences in learning tasks (Appendix figure 1-5). Both gender offspring were tested through the same behavioral battery delineated earlier: open field (OF), Morris water maze (MWM) and fear conditioning (FC) (Figure 4.2.1B). I always carried out two groups of comparisons. First, I compared wtgwt mice to control wt (wtwtwt) mice and $\operatorname{tg}^{\text {tgwt }}$ mice. Second, I compared wt $t^{w t t g}$ mice to control wt (wt $\left.{ }^{w t w t}\right)$ mice and $\operatorname{tg}^{\text {wttg }}$ mice. 
A

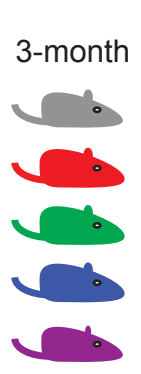

B

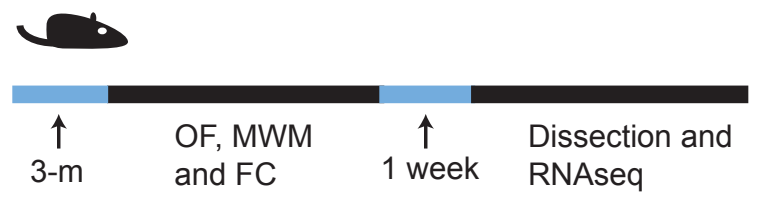

Figure 4.2.1. Experimental groups and design.

(A) 3-month old APPPS1-21 wild-type or transgenic mice born to transgenic fathers (wtgwt and tg ${ }^{\text {tgwt }}$ ) or mothers ( $w^{t^{w t t g}}$ and $\left.t^{w t t g}\right)$ vs. control wild-type mice born to wild-type parents ( $\left.w^{t^{w t w t}}\right)$. (B) Mice were subjected to a battery of behavioral experiments, open field (OF), Morris water maze (MWM) and fear conditioning (FC). A week later, brain subregions were dissected, RNA was isolated and RNA sequence was ran and analyzed.

I first tested mice in the open field test (OF). Wt and tg mice born to an APPPS1-21 tg father did not show any differences in distance or speed in the OF test, indicating no motor impairments or apathy (Figure 4.2.2A-B). In contrast, $\mathrm{w}^{\mathrm{t}}{ }^{\mathrm{wtg}}$ mice showed a mild but significant reduction in the speed and traveled distance (Figure 4.2.2F-G). This reduction is probably due to an increase of apathy more than an impairment of their motor abilities, since $w^{\text {twttg }}$ also traveled more distance in the center compared to control wt mice (Figure 4.2.2I). Importantly, wt ${ }^{\text {tgwt }}$ did not show the decrease anxiety phenotype described for tg ${ }^{\text {tgwt }}$ (Figure 4.2.2C-E). Thus, wt ${ }^{\text {tgwt }}$ resembled control wt mice more than they did tg mice in the open field parameters. For $w^{t w t t g}$, the situation was similar, although there was a slight trend towards tg ${ }^{\text {wttg }}$ showing a slightly less anxious phenotype as compared to control wt mice (Figure 4.2.2H-J). 


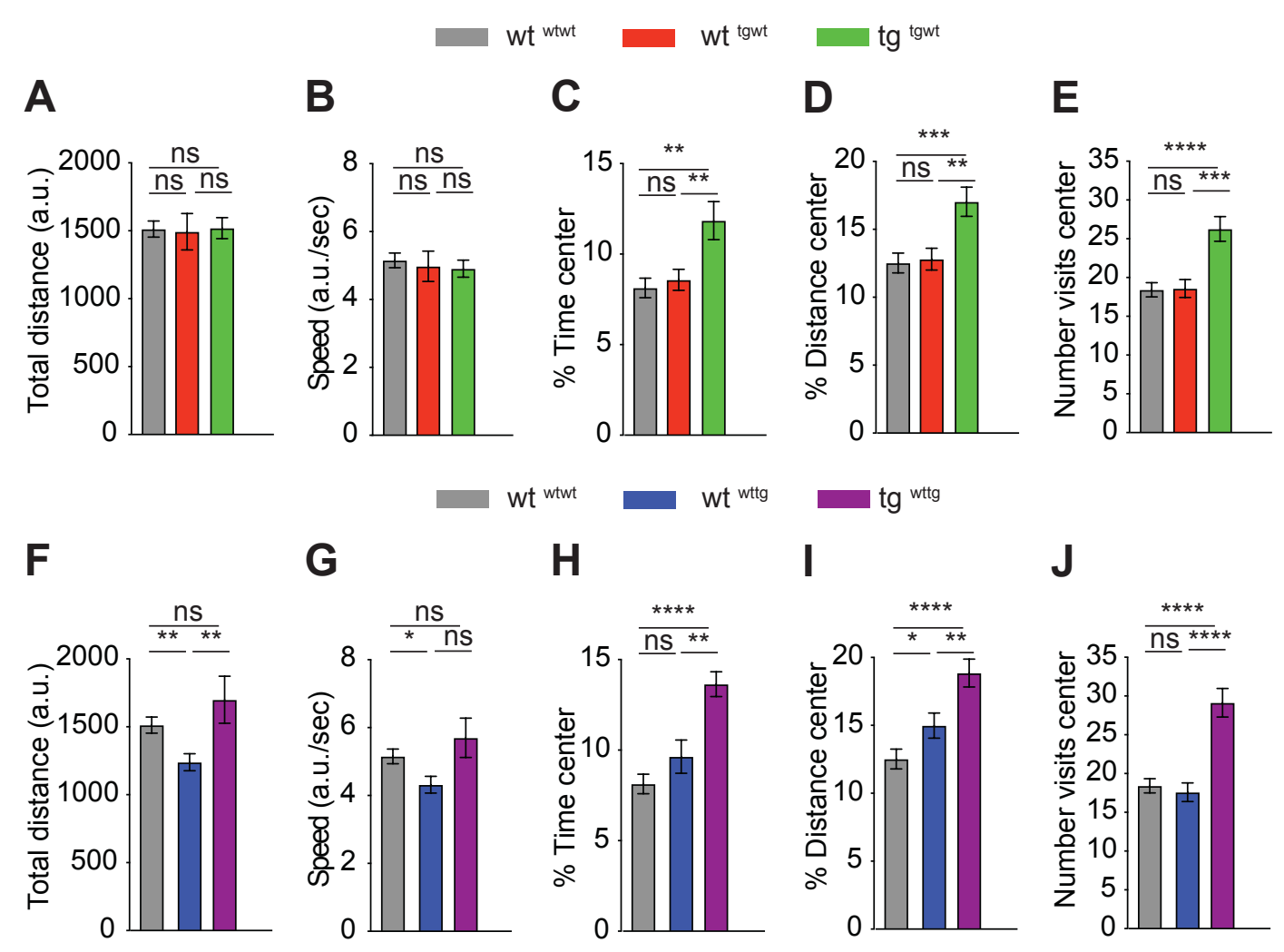

Figure 4.2.2. Wild-type mice born to APPPS1-21 transgenic fathers (wt $t^{\text {tgwt }}$ ) did not show motor impairments, an increase of apathy or levels of anxiety, whereas wild-type born to APPPS1-21 transgenic mothers (wtwttg) showed a slight increase of apathy and reduction of anxiety levels in the open field test at 3 months of age.

Open field test in APPPS1-21 wild-type and transgenic mice born to transgenic father ( $\mathrm{wt}^{\mathrm{tgwt}}, \mathrm{n}=32$; $\mathrm{tg}^{\text {tgwt }}$, $n=27)$ or mother ( $w^{\text {twttg }}, n=24$; tg $\left.{ }^{\text {wttg }}, n=16\right)$ and control wild-type mice $\left(w^{t w t w t}, n=34\right)$ at 3 months of age: $(A$ and $B$ ) Total traveled distance (A) and average speed (B) during the open field test. (C-E) Percentage of time (C) and distance (D) spent in the central area of the field and number of visits center (E). Student's two-tailed T-test, ns = no significant differences; ${ }^{*} \mathrm{P}<0.05 ;{ }^{* *} \mathrm{P}<0.01$; ${ }^{* * *} \mathrm{P}<0.001 ;{ }^{* * * *} \mathrm{P}<0.0001$. Error bars indicated SEM.

In conclusion, OF revealed no major motor impairments in wild-type mice born to either APPPS1-21 tg mothers or fathers and indicated no overall significant differences in anxiety behavior.

Next, I tested spatial memory in the Morris water maze (MWM). During the spatial learning process in MWM task, wild-type born to APPPS1-21 transgenic fathers (wttwt) presented different spatial behavior compared to wild-type controls ( $w^{\text {twtwt }}$ ). Wt ${ }^{\text {tgwt }}$ required significantly more time than $w^{\text {twtwt }}$ to find the platform each day and overall during the total training process (Figure 4.2.3A-B). Thus, their cognitive performance resembles that of $\operatorname{tg}^{\text {tgwt }}$, indicating that there is in fact a non-genetic inheritance or trace of the genetic makeup of the male parent. On the other hand, wtwtt showed similar behavior to the $w^{t^{w t w t}}$ controls, traveling similar time to find the 
platform (Figure 4.2.3C-D). This is in line with the previous observation that tgwttg mice show a milder cognitive impairment as compared to that of tg ${ }^{\text {tgwt }}$ (Figure 4.1.4I-J).

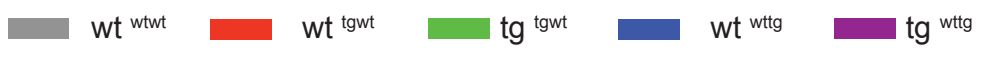

A

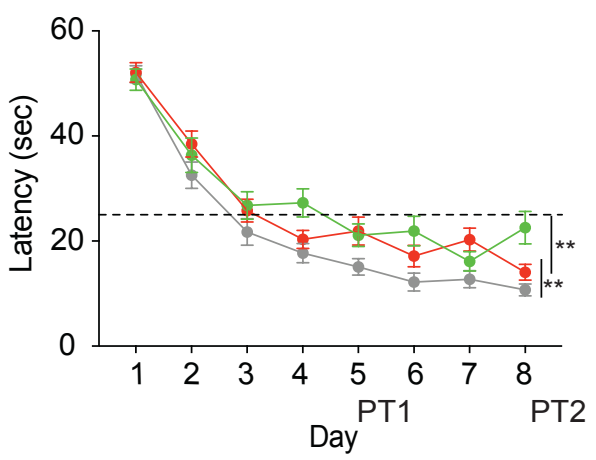

C

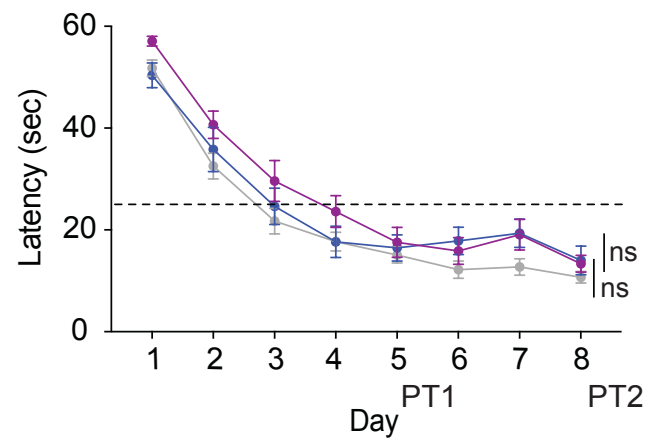

B

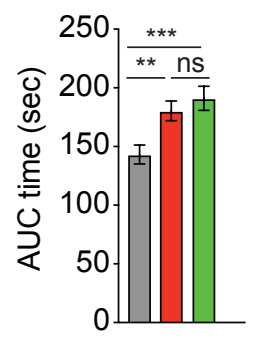

D

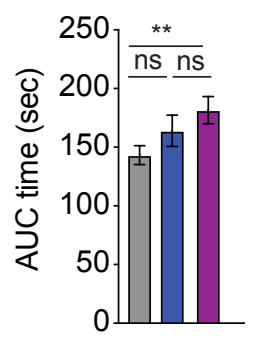

Figure 4.2.3. Only wild-type mice born to an APPPS1-21 transgenic father showed difficulties to learn a spatial task at 3 months of age.

Morris water maze (MWM) test in APPPS1-21 wild-type and transgenic mice born to a transgenic father

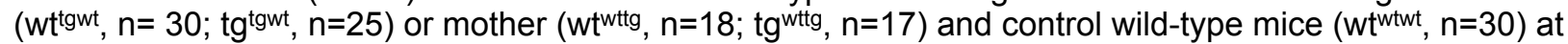
3 months of age. (A and B) $w^{\text {tgwt }} v$ s. $w^{t^{w t w t}}$ and $\operatorname{tg}^{\text {tgwt }}(\mathbf{A})$ and $w^{t^{w t t g}} v s$. $w^{t^{w t w t}}$ and tg ${ }^{\text {wttg }}$ (B) group-average time to find the target platform on each consecutive training day (Day 1-8). Two-way ANOVA after Sidak correction, $\mathrm{ns}=$ no significant differences; ${ }^{* *} \mathrm{P}<0.01$. The dashed line represents the chance level of $25 \%$. First probe trial (PT1, day 6) and second probe trial (PT2, day 10). (C) Comparison of wild-type experimental groups ( $w^{\text {tgwt }}$ and $\left.w^{w t t g}\right)$ vs. wild-type controls ( $\left.w^{t^{w t w t}}\right)$ and transgenic groups $\left(\mathrm{tg}^{\text {tgwt }}\right.$ and tg ${ }^{\text {wttg}}$ ) analyzing the areas under the learning curves (AUC) of escape latency for each group. Student's twotailed T-test, ns $=$ no significant differences; ${ }^{* *} \mathrm{P}<0.01 ;{ }^{* * *} \mathrm{P}<0.001$. Error bars indicated SEM.

Similarly to the tests presented for APPPS1-21 transgenic mice, I carried out a first probe test after 5 days of training (PT1). None of the control wild-type and experimental mice spent significantly more time in the target quadrant than in the rest of the pool. So, I conclude that for this specific colony and this specific protocol, 5 days of training were not enough to learn the task (Figure 4.2.4A-F).

I then performed a second probe trial (PT2) after 3 additional days of training, at day 8 . At this point, $w^{\text {twtwt }}$ mice spent significantly more time and traveled significantly longer distances in the 
target quadrant than in the other quadrants. Wtwtwt spent more than $25 \%$ of the traveled time and distance in the target quadrant, indicating a solid memory for the platform location (Figure 4.2.4G-H and J-K). In contrast, neither w ${ }^{\text {tgwt }}{ }^{\text {nor }}$ tg $^{\text {tgwt }}$ showed a preference for the target quadrant (Figure 4.2.4G-H). Wtwtwt visited the platform more often than the other wild-type groups, although these differences failed to reach statistical significance (Figure 4.2.4I and L). Taken together, these results support my initial observation during training that wt mice born to an APPPS1-21 tg father behaviorally resembled a tg mice more than they do wt mice (Figure 4.2.3A-B). This supports a non-genetic form of inheritance of a cognitive deficit or a predisposition to perform worse than control wt counterparts. In contrast to this and in agreement with PT1, $w^{t^{w t t g}}$ behaved more similarly to $w^{t^{\text {twt }}}$ and showed borderline memory for the platform location (Figure 4.2.4D-F and J-L).

As before, checking the distance between the mouse's trajectory and that of the platform every two seconds during the test greatly accentuated the differences between the groups. As can be observed in figure $4.2 .4 \mathrm{M}, \mathrm{O}, \mathrm{Q}$ and $\mathrm{R}$ there were big differences between $\mathrm{w}^{\mathrm{t}}{ }^{\mathrm{tgwt}}$ and control wt controls in the distance per time and in the global distance parameters. These effects were detected in both probe trials, PT1 and PT2 (Figure 4.1.10M, O, Q and R). In contrast, wt wttg mice traveled a similar distance to control mice during both probe trials, although there was a clear trend to traveled longer distances for $w^{\text {twttg }}$ and tg $^{\text {wttg }}$ (Figure 4.1.10N, P, S and T). 


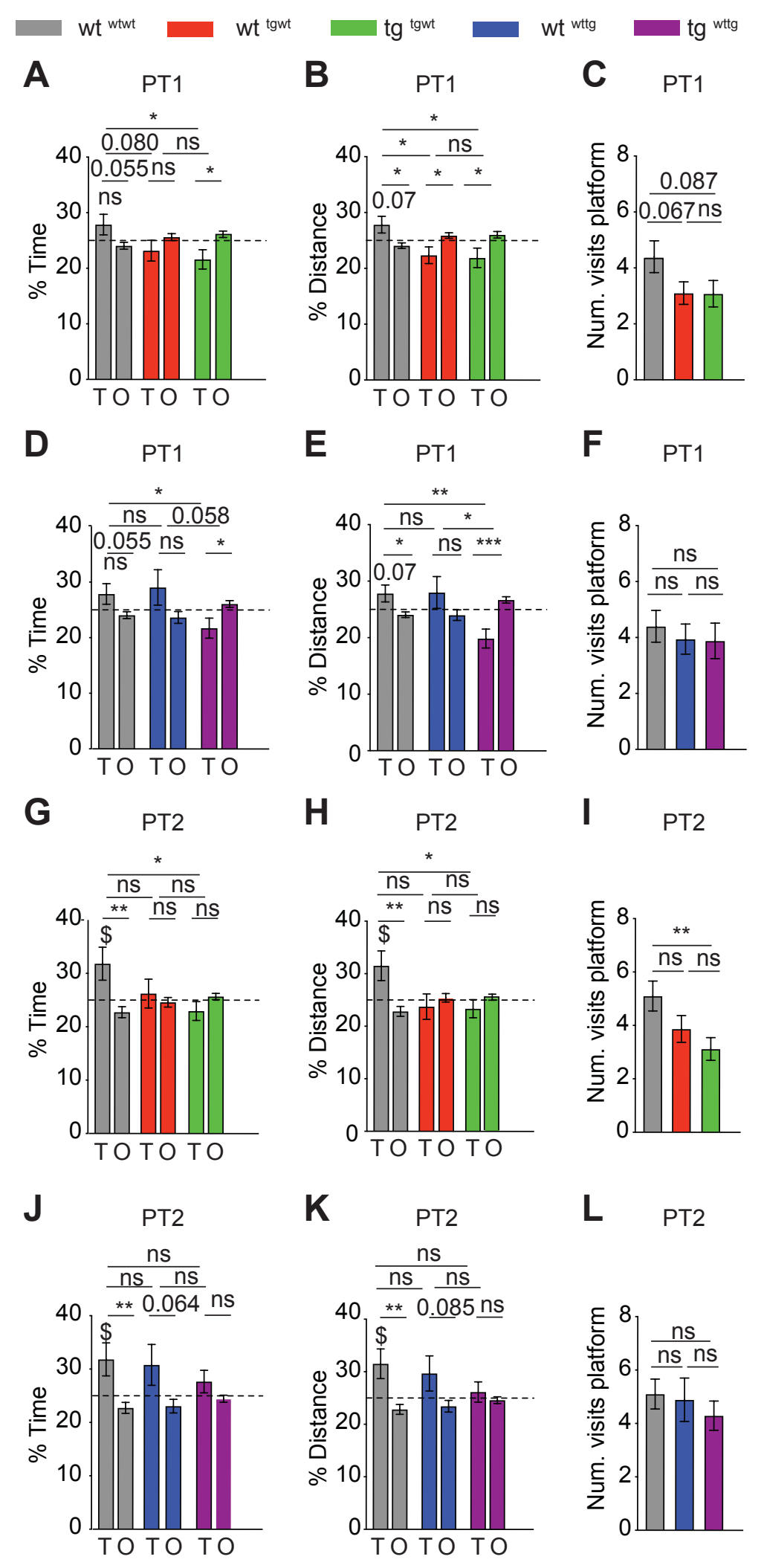



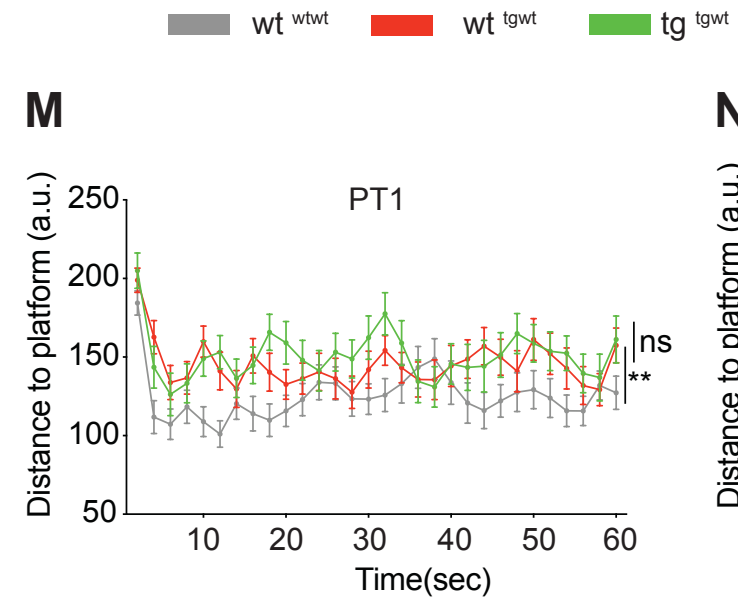

wt ${ }^{\text {wtg }}$

tg witg
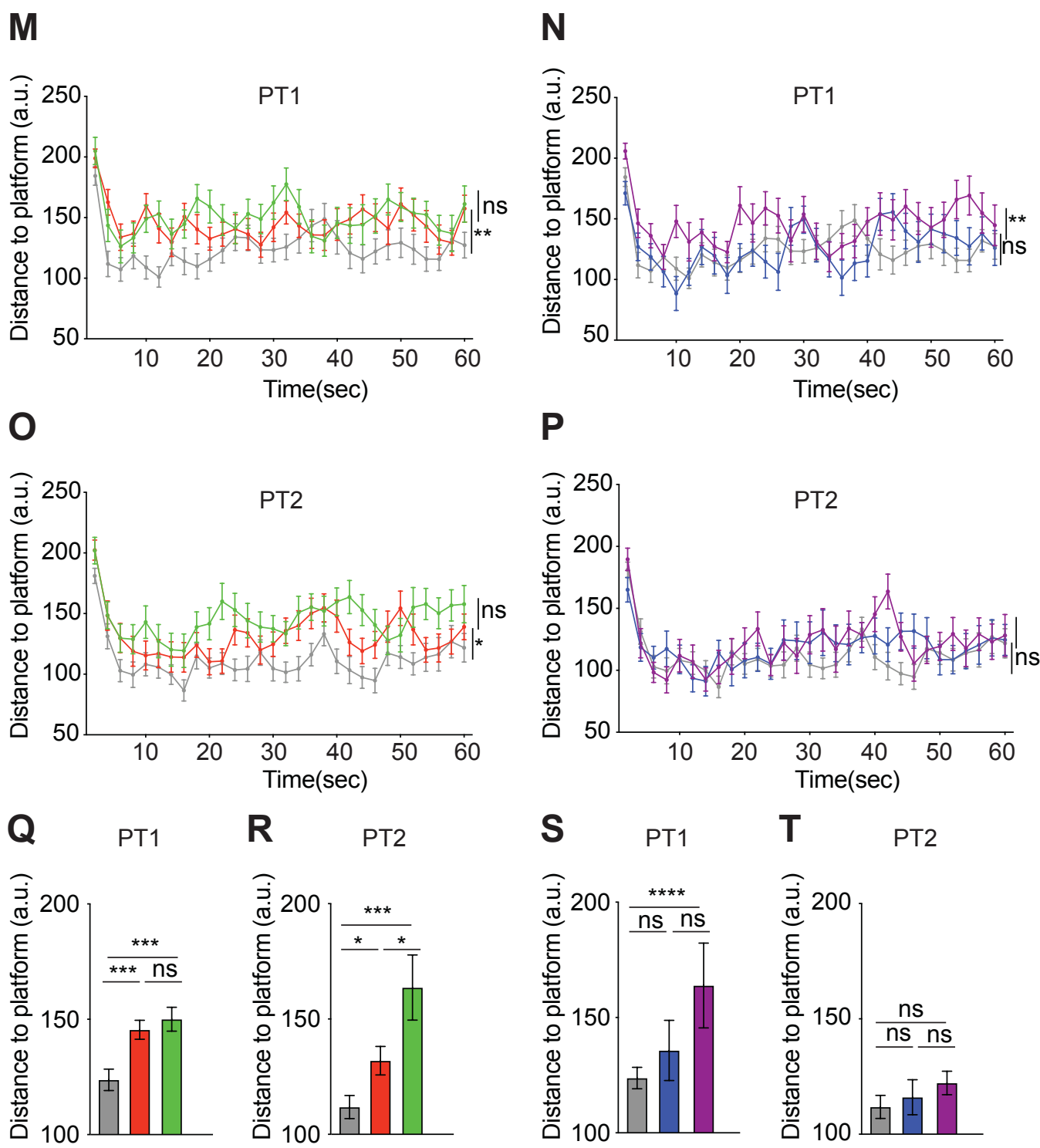

Figure 4.2.4. Only wild-type mice born to APPPS1-21 transgenic father (wttgwt) showed spatial memory impairments at 3 months of age.

Morris water maze (MWM) test in APPPS1-21 wild-type and transgenic mice born to a transgenic father

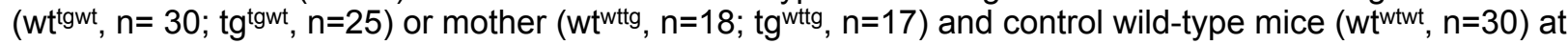
3 months of age. (A-L) Percentage of time (A, D, G and J) and distance (B, E, H and K) spent in the target quadrant $(\mathrm{T})$ vs. average other quadrants $(\mathrm{O})$ and number of visits to the platform $(\mathbf{C}, \mathbf{F}, \mathbf{I}$ and $\mathrm{L})$ during first probe trial (PT1, day 6) (A-F) and second probe trial (PT2, day 10) (G-L). Student's two-tailed T-test, ns = no significant differences; ${ }^{*} \mathrm{P}<0.05 ;{ }^{* *} \mathrm{P}<0.01$; ${ }^{* * *} \mathrm{P}<0.001$; ${ }^{* * *} \mathrm{P}<0.0001$. Column statistics, one-sample T-test, hypothetical value 25 . ns = no significant differences; $\$ \mathrm{P}<0.05$. The dashed line represents the chance level of 25\%. (M-T) The distance between mouse and the platform position every two seconds during PT1 ( $\mathbf{M}$ and $\mathbf{N}$ ) and PT2 ( $\mathbf{O}$ and $\mathbf{P})$ and the average of total distance during PT1 $(\mathbf{Q}$ and $\mathbf{S})$ and PT2 (R and T). Two-way ANOVA after Sidak correction and student's two-tailed T-test, * $P$ < $0.05 ;{ }^{* *} \mathrm{P}<0.01 ;{ }^{* * *} \mathrm{P}<0.001$. Error bars indicated SEM. 
Importantly, the swimming speed did not differ between groups during the first day of training and during the probe tests. All wild-type mice swam at similar speeds, and none of them presented alterations of the motor functions (Figure 4.2.5A-F).

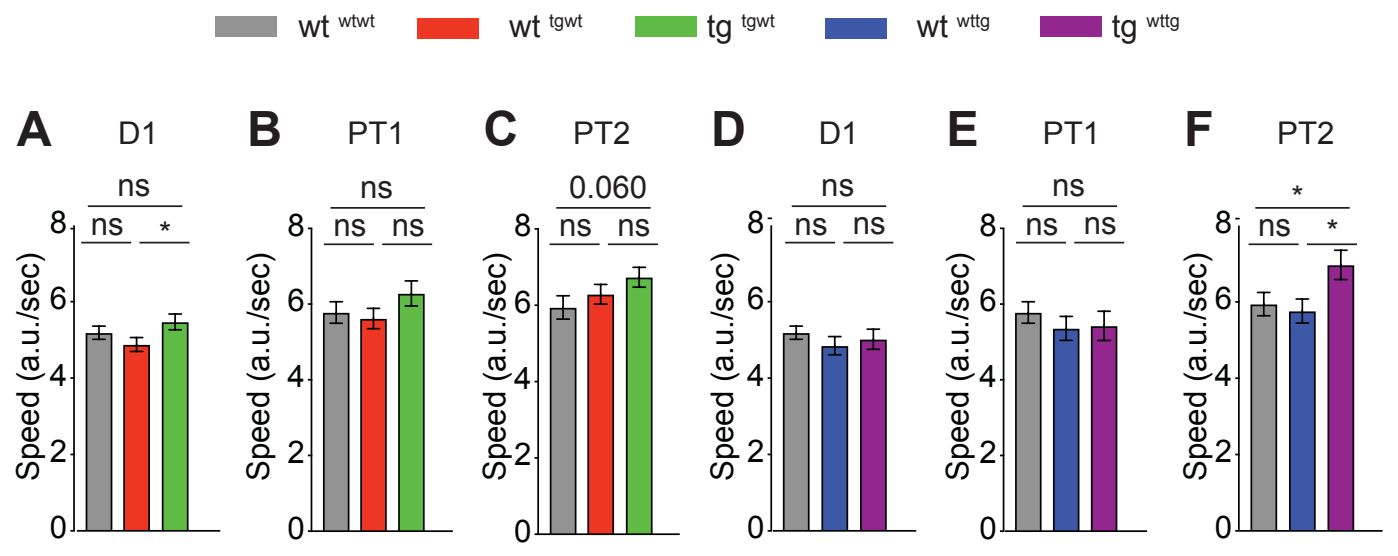

Figure 4.2.5. Wild-type mice from APPPS1-21 parents did not show motor impairments at 3 months of age in the Morris water maze task (MWM).

Morris water maze (MWM) test in APPPS1-21 wild-type and transgenic mice born to a transgenic father (wtgwt, $n=30$; tg ${ }^{\text {tgwt }}, n=25$ ) or mother ( $w^{\text {twttg }}, n=18$; tgwttg, $n=17$ ) and control wild-type mice ( $\left.w^{\text {twtwt }}, n=30\right)$ at 3 months of age. (A and D) Average speed during first training day of MWM task. (B, C, E and F) Average speed during first probe trial (PT1) (B and E) and second probe trial (PT2) (C and F). Student's two-tailed T-test, ns $=$ no significant differences; ${ }^{*} P<0.05$. Error bars indicated SEM.

These results suggest that the parenteral genotype indeed has an impact on the performance of their offspring (wild-type or transgenic mice), even if the genotype itself is not transmitted as in the case of wild-type offspring. Mice from parents with distinct genotypes presented different cognitive abilities in a spatial memory task despite having the same genotype. Specifically, wildtype mice from APPPS1-21 transgenic fathers ( $w^{\text {tgwt }}$ ) showed spatial learning and memory impairments that were similar to transgenic mice (tg $\left.{ }^{\text {tgwt }}\right)$. This parental influence seems to have a strong effect when the transgene of APP and PS1 comes from the father and a milder when it comes from the mother suggesting that a factor transmitted via sperm could be causative for the observed phenotype. Wild-type mice from transgenic mother ( $w^{\text {twttg }}$ ) performed similarly to wildtype mice from wild-type parents ( $w^{\text {twtwt }}$ ) at 3 months of age. These similarities and dissimilarities were observed at a similar level in males and females (Appendix figure 1-4).

Finally, I also tested mice in the fear-conditioning paradigm. During the training day, all wild-type groups presented similar average mobility (Figure 4.2.6A and D). During the test, all groups showed similar levels of freezing. Both wild-type mice, $w^{\text {tgwt }}$ and wt ${ }^{\text {thtg, }}$, froze similarly to the 
control group, around 40\% (Figure 4.2.6B-C and E-F). This data suggests that at 3 months of age all wild-type groups ( $w^{\text {twtwt }}$, $w^{\text {tgwt }}$ and $w^{t^{w t t g}}$ ) can acquire the associative memory for the aversive context regardless of the genetic background of the parents. Thus, the intergenerational non-genetic inheritance of cognitive deficits seems to be restricted to spatial navigation tasks.

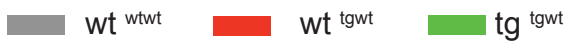

A

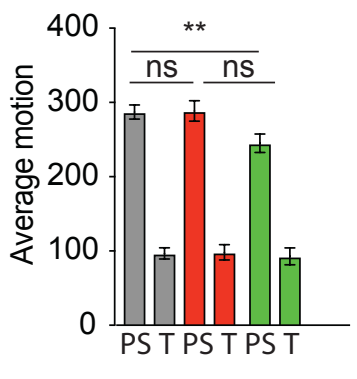

wt wtwt

B

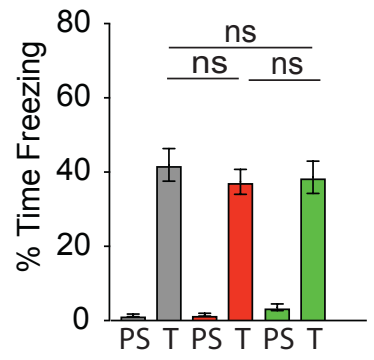

C

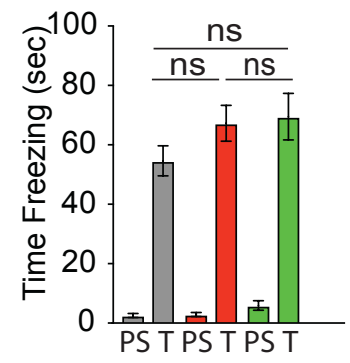

tg witg

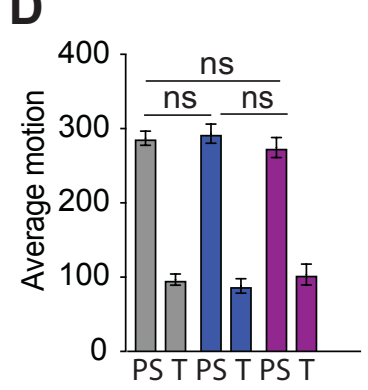

E

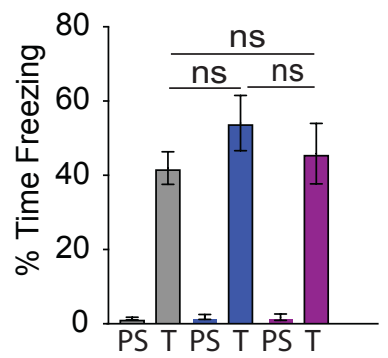

$\mathbf{F}$

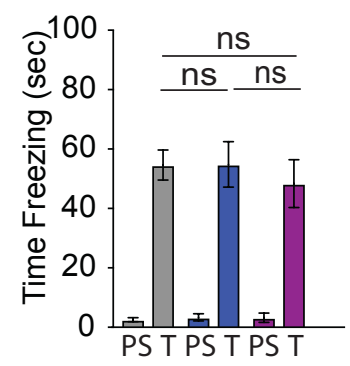

Figure 4.2.6. APPPS1-21 wild-type mice groups did not show deficits of contextual associative memory at 3 months of age.

Fear-conditioning in APPPS1-21 wild-type and transgenic mice born to a transgenic father ( $\mathrm{tt}^{\mathrm{tgwt}}, \mathrm{n}=35$; tg ${ }^{\text {tgwt }}, n=28$ ) or mother ( $w^{t^{w t t g}}, n=24$; $\operatorname{tg}^{\text {wttg }}, n=19$ ) and control wild-type mice ( $w^{\text {twtwt }}, n=34$ ) at 3 months of age. (A and D) Average of motion during the training day. (B, C, E and F) Percentage of time (B and E) and time (C and F) freezing during training (first column) and testing day (second column). PS= preshock. $\mathrm{T}=$ test $\left(24 \mathrm{~h}\right.$ later). Student's two-tailed T-test, $\mathrm{ns}=$ no significant differences; ${ }^{* *} \mathrm{P}<0.01$. Error bars indicated SEM.

4.2.1.2.Persistence of cognitive decline in wild-type and transgenic mice born to APPPS1-21 transgenic fathers or mothers at 9 months of age

Previously, I characterized the cognitive performance of transgenic and wild-type mice born to APPPS1-21 tg parents at 3 months of age, showing restricted cognitive deficits to spatial behavior tasks. Interestingly, tg and wt mice born to APPPS1-21 tg fathers showed a more severe cognitive impairment compared to offspring from APPPS1-21 tg mothers. This supports 
the idea that the factor transmitted via sperm could be causative for the observed phenotype. Previous findings showed that intergenerational phenotypes could persist into later adulthood (Dias \& Ressler, 2014b; Vassoler et al., 2013; S. Zhang et al., 2017). I posed the question of whether wt offspring born to APPPS1-21 tg parents would show cognitive differences as compared to control wt mice at late adulthood. Moreover, I evaluated the cognitive decline progression linked to $A D$ pathology, from 3 to 9 months of age, in this mouse model of AD. To test these ideas, wt and tg mice born to APPPS1-21 tg parents at 9 months of age were tested through the same behavioral battery delineated earlier: open field (OF), Morris water maze (MWM) and fear conditioning (FC) (Figure 4.2.7B). To avoid the effect of previous memories, new batches of mice were used in this section. I carried out the behavior tests to the next comparisons: control wt ( $w^{\text {twtwt }}$ ) vs. wt born to a tg father (wtgwt) vs. tg born to a tg father (tg ${ }^{\text {tgwt}}$ ); and $w^{t^{w t w t}}$ vs. wt born to a tg mother ( $w^{t w t t g}$ ) vs. $\operatorname{tg}$ born to a tg mother (tgwttg) (Figure 4.2.7A). Both female and male mice were used in the experiment and were pooled together since no statistically significant differences in cognitive abilities were observed between them (Appendix figure 6-9).

A

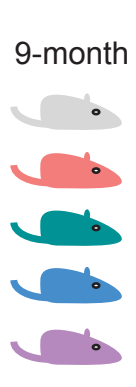

B

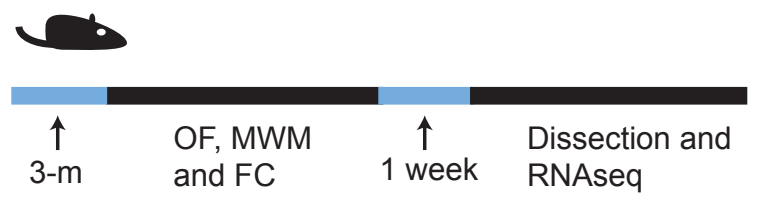

$w^{\text {wtwt }}$ (wt $\times$ wt)

$w^{\text {tgwt }}(\operatorname{tg} \times w t)$

$\operatorname{tg}^{\text {tgwt }}(\operatorname{tg} x w t)$

$\operatorname{tg}^{\text {tgwt }}(\operatorname{tg} \times w t)$

$\operatorname{tg}{ }^{w t g}(w t \times \operatorname{tg})$ Parents

Figure 4.2.7. Experimental groups and design.

(A) 9-month old APPPS1-21 wild-type or transgenic mice born to transgenic fathers (wtgwt and tg ${ }^{\text {tgwt }}$ ) or mothers ( $t^{\text {wttg }}$ and $\left.t^{w t t g}\right)$ vs. control wild-type mice born to wild-type parents (wtwtwt). (B) Mice were subjected to a battery of behavioral experiments, Open field (OF), Morris Water Maze (MWM) and Fear Conditioning (FC). A week later, brain subregions were dissected, RNA was isolated and RNA sequence was ran and analyzed.

I first tested wt and tg mice born to APPPS1-21 parents in the open field (OF). None of the offspring mice born to a tg mother ( $w^{t^{w t t g}}$ and $t^{w t t g}$ ) showed motor deficits (Figure 4.2.8F-G). In contrast, wt and tg offspring from tg father ( $w^{t^{\text {tgwt }}}$ and $\operatorname{tg}^{\text {tgwt }}$ ) showed a clearly decreased of the speed and mild reduction of the total traveled distance, indicating a reduction of their motor abilities (Figure 4.2.8A-B). Importantly, both offspring from tg father ( $\mathrm{wt}^{\mathrm{tg} w \mathrm{t}}$ and $\mathrm{tg}^{\text {tgwt}}$ ) and $\mathrm{tg}^{\mathrm{wtg}}$ mice did not show alteration of anxiety levels. In contrast, wt mice born to an APPPS1-21 tg mother ( $w^{t^{w t g}}$ ) showed an increase of anxiety, opposite to the phenotype described for this group 
at 3 months of age (Figure 4.2.8C-D and H-J). Thus, wt born to an APPPS1-21 tg father (wtgwt) at 9 months of age resembled tg mice more than they did wt control mice in the open field parameters.

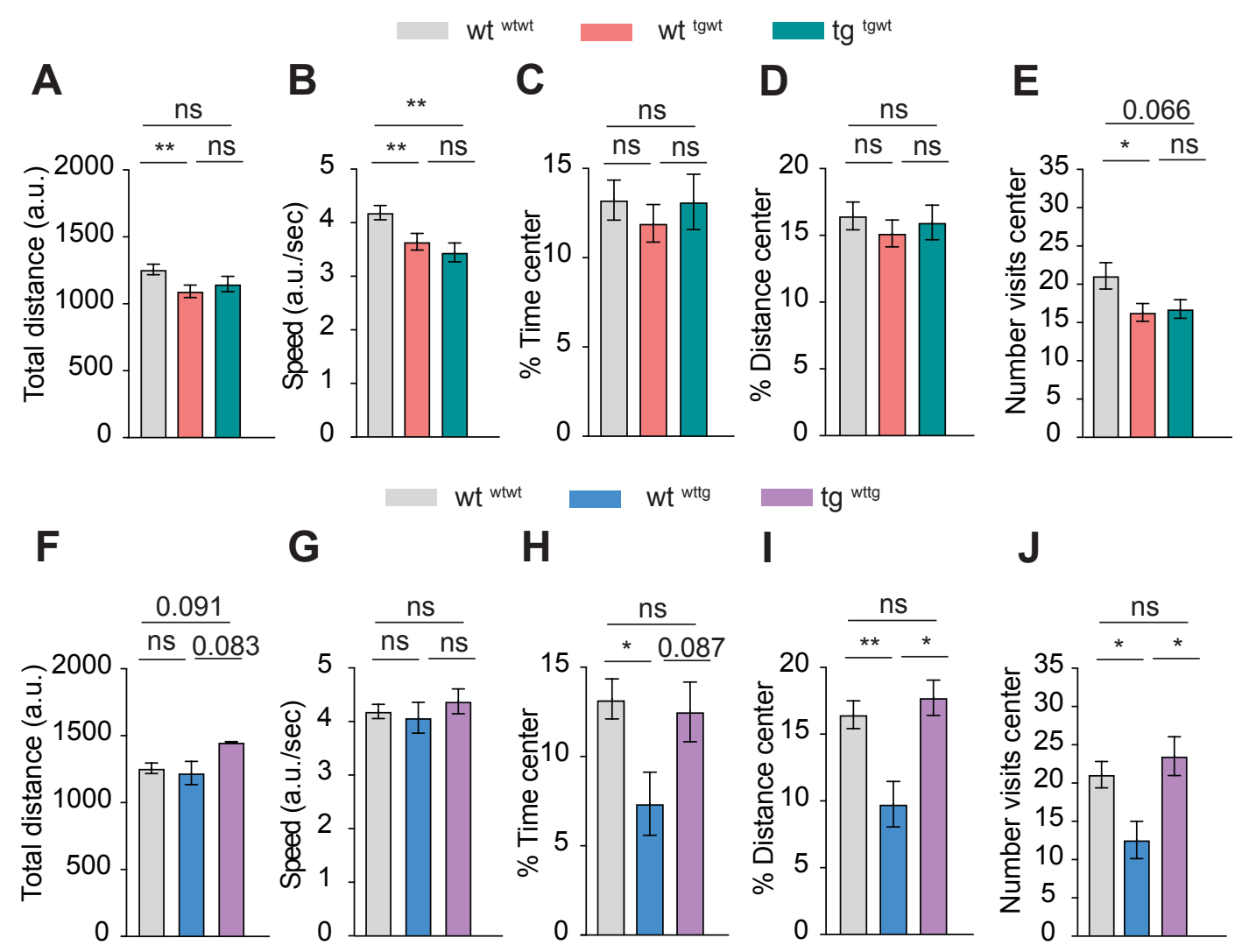

Figure 4.2.8. Wild-type and transgenic mice born to APPPS1-21 transgenic fathers showed a reduction of their motor abilities and only wild-type mice born to APPPS1-21 tg mother (wtwtt) showed an increase of anxiety levels opposite to what they showed at 9 months of age.

Open field test in APPPS1-21 wild-type and transgenic mice born to a transgenic father ( $\mathrm{wt}^{\mathrm{tgwt}}, \mathrm{n}=31$; tg ${ }^{\text {tgwt }}, n=22$ ) or mother ( $w^{\text {wttg }}, n=7$; tg $\left.{ }^{\text {wttg }}, n=4\right)$ and control wild-type mice (wtwtwt,$n=31$ ) at 9 months of age. $(A$ and $B)$ Total traveled distance $(A$ and $F)$ and average speed $(B$ and $G)$ during the open field test. (C-E and $\mathrm{H}-\mathrm{J})$ Percentage of time $(\mathrm{C}$ and $\mathrm{H})$ and traveled distance (D and $\mathrm{I})$ spent on the central area of the field and number of visits center ( $E$ and $J)$. Student's two-tailed T-test, ns = no significant differences; ${ }^{*} \mathrm{P}<$ $0.05 ;{ }^{* *} \mathrm{P}<0.01$. Error bars indicated SEM.

Next, I trained all groups for spatial memory in Morris water maze (MWM). During the training phase, none of the wild-type mice born to one transgenic parent ( $w^{t \text { tgwt }}$ and $w^{\text {twttg) }}$ showed differences in the time traveled to find the platform compared to control wt mice (wtwtwt). In contrast, tg $^{\text {tgwt }}$ and tg ${ }^{\text {wtt }}$ showed more difficulties to learn the spatial task, spending more time to daily reach the platform (Figure 4.2.9A-D). 

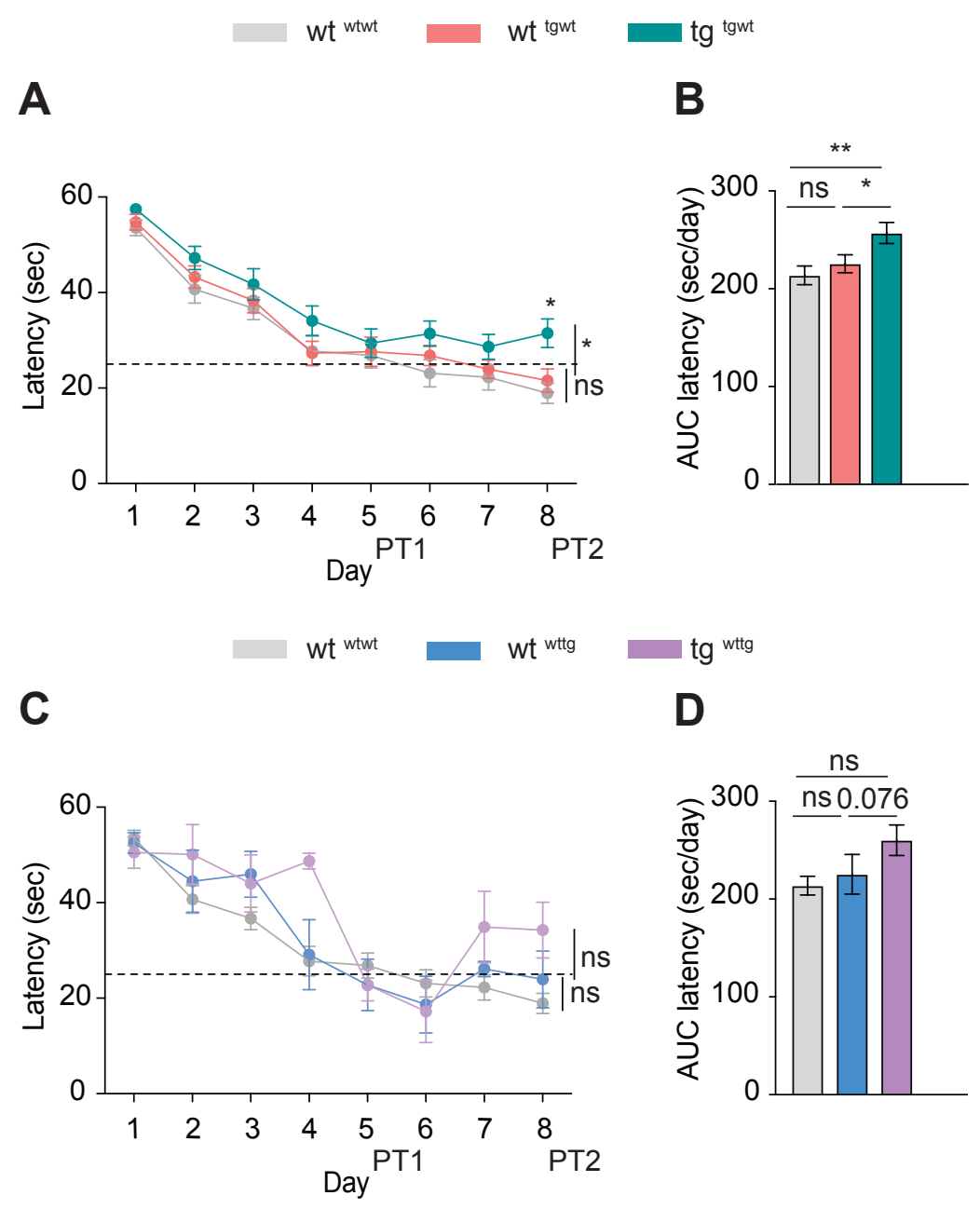

Figure 4.2.9. Only APPPS1-21 transgenic mice showed difficulties to learn the spatial task at 9 months of age.

Morris water maze (MWM) test in APPPS1-21 wild-type and transgenic mice born to a transgenic father ( t $^{\text {tgwt }}, n=26$; tg tgwt,$\left.n=20\right)$ or mother ( $w^{\text {twttg }}, n=6$; $\left.\operatorname{tg}^{\text {wttg }}, n=4\right)$ and control wild-type mice $\left(w^{\text {twtwt }}, n=26\right)$ at 9

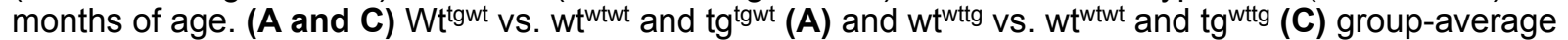
time to find the target platform on each consecutive training day (Day 1-8). Two-way ANOVA after Sidak correction, ns = no significant differences; ${ }^{*} \mathrm{P}<0.05$. The dashed line represents the chance level of $25 \%$. First probe trial (PT1, day 6) and second probe trial (PT2, day 10). (B and D) Comparison of $w^{t^{\text {tgwt }}}$ vs. $w^{\text {wtwt }}$ and $\operatorname{tg}^{\text {tgwt }}(B)$ and $w^{\text {twtg }}$ vs. $w^{t^{w t w t}}$ and $\operatorname{tg}^{\text {wttg }}$ (D) analyzing the areas under the learning curves (AUC) of escape latency for each group. Student's two-tailed T-test, ns = no significant differences; ${ }^{*} P<0.05 ;{ }^{* *} P$ $<0.01$. Error bars indicated SEM.

I carried out a first probe test after 5 days of training (PT1). Similarly to the tests presented for mice at 3 months of age, none of the mice in any group learn the task during the first probe test 1 (PT1), spending less than $25 \%$ of the time and traveled distance in the target quadrant (Figure 4.2.10A-F). Despite this, $\mathrm{wt}^{\mathrm{tg} w \mathrm{t}}$ and $\mathrm{wt}^{\mathrm{w}}{ }^{\mathrm{ttg}}$ mice showed a certain preference for travel longer distances in the target quadrant than both tg groups (Figure 4.2.10A-F). However, this tendency 
was not observed during the second probe test (Figure 4.2.10G-L). Probably the preference for the target quadrant during the first PT was a random swim traveling rather than a learning preference.

I then performed a second probe trial (PT2) after 3 additional days of training, i.e. at day 8. At this point, $\mathrm{wt}^{\mathrm{w} \text { twt }}$ spent more time traveling in the target quadrant than the other quadrants compared to other groups, although these differences were borderline (pval=0.0521). In contrast, neither wt nor tg born to APPPS1-21 tg parents showed a preference for the target quadrant (Figure 4.2.10G-L). There were no remarkable gender-dependent differences in spatial cognitive performance in any of the groups (Appendix figure 7 and 8).

As before, I included a more sensitive analysis consisting of recording the distance between the position of the mouse and the platform position during the PT every two seconds. In PT1, none of the APPPS1-21 offspring showed differences compared to controls (Figure 4.2.10M and N). In PT2, wt and tg mice born to APPPS1-21 tg fathers ( $w^{\text {tgwt }}$ and tg $^{\text {tgwt }}$ ) showed significant differences $\left(p<0.05\right.$ and $p=0.063$ ) compared to controls, whereas wt ${ }^{\text {wtg }}$ traveled similar distance than controls (Figure 4.2.100 and P). Wild-type mice born to APPPS1-21 tg fathers (wtgwt) showed spatial learning and memory impairments that were similar to transgenic mice (tg $g^{\text {tgwt }}$ ) as well at 9 months of age. 
wt wtwt $\quad$ wt tgwt $\quad \operatorname{tg}^{\text {tgwt }}$
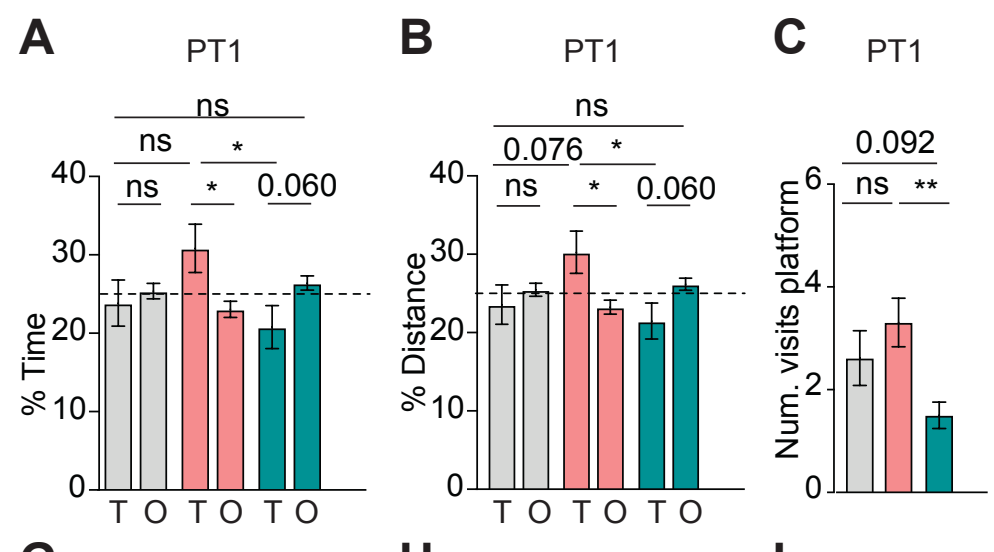

G PT2

H

PT2

PT2
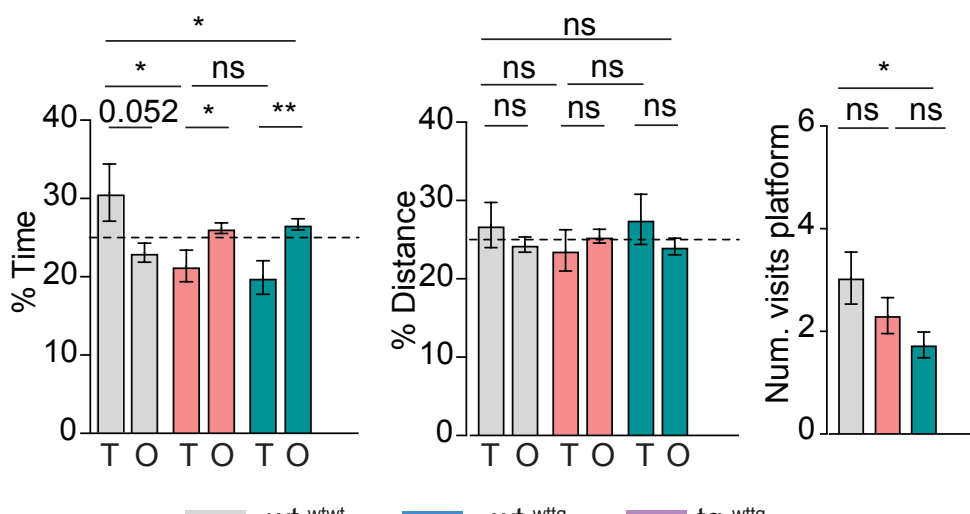

D PT1

E
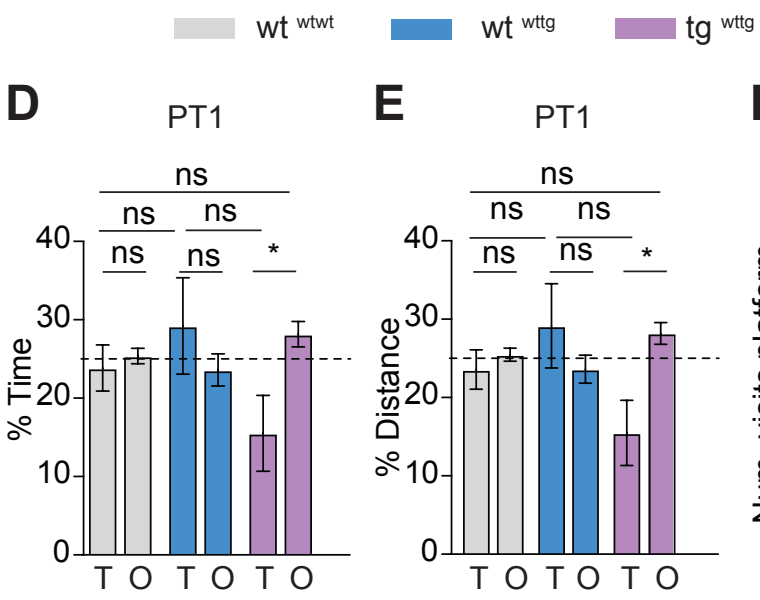

E $\quad$ PT1

F PT1

J
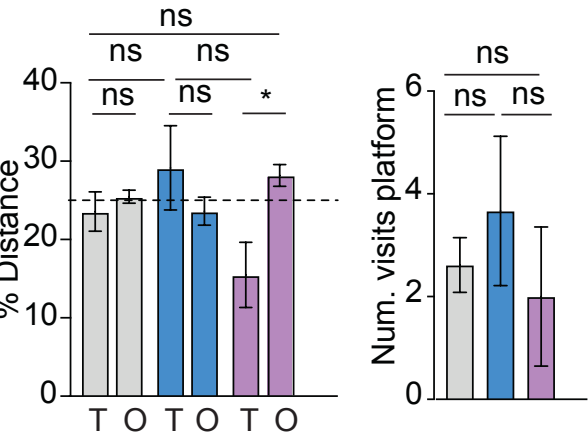

PT2

K PT2

L $\quad$ PT2
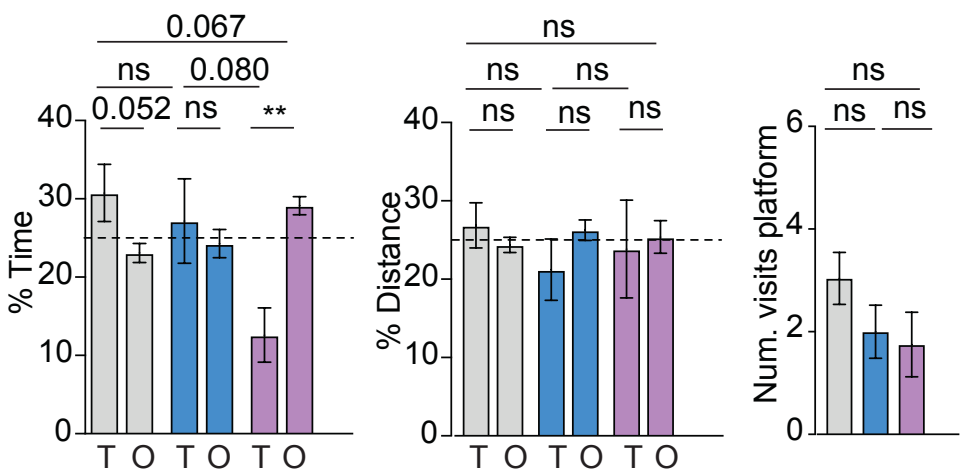

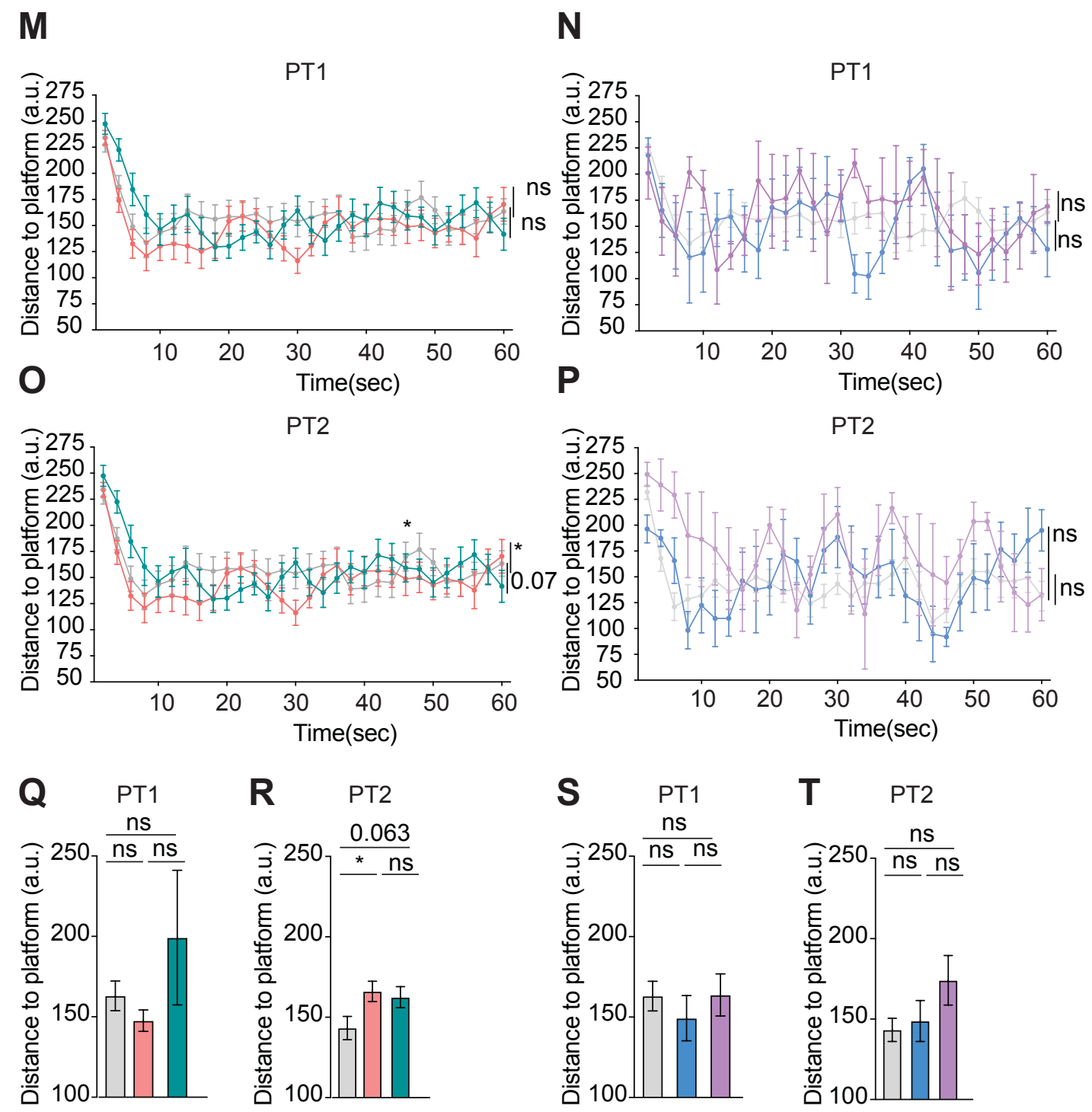

Figure 4.2.10. Wild-type and transgenic mice born to APPPS1-21 transgenic fathers, and transgenic mice born to APPPS1-21 tg mother showed spatial memory impairments at 9 months of age.

Morris water maze (MWM) test in APPPS1-21 wild-type and transgenic mice born to a transgenic father

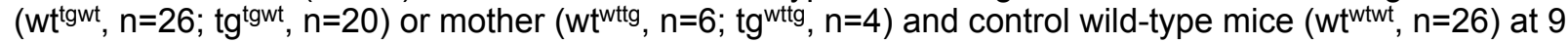
months of age. (A-L) Percentage of time (A, D, G and J) and distance (B, E, H and K) spent on the target quadrant $(T)$ vs. average other quadrants $(O)$ and number of visits to the platform $(\mathbf{C}, \mathbf{F}, \mathbf{I}$ and $\mathbf{L})$ during first probe trial (PT1, day 6) (A-F) and second probe trial (PT2, day 10) (G-L). Student's two-tailed T-test, ns $=$ no significant differences; ${ }^{*} P<0.05 ;{ }^{* *} P<0.01$. The dashed line represents the chance level of $25 \%$. (M-T) The distance between mouse and the platform position every two seconds during PT1 (M and N) and PT2 ( $\mathbf{O}$ and $\mathbf{P})$ and the average of total distance during PT1 ( $\mathbf{Q}$ and $\mathbf{S})$ and PT2 (R and T). Two-way ANOVA after Sidak correction and student's two-tailed T-test, ns = no significant differences; ${ }^{*} \mathrm{P}<0.05$. Error bars indicated SEM. 
Interestingly, the swimming speed was only reduced in wt and tg mice born to APPPS1-21 tg fathers ( $w^{\text {tgwt }}$ and tg $^{\text {tgwt}}$ ) during the first day of training, similarly to the open field test (Figure 4.2.11A). Although a motor impairment was apparent for both groups during day $1, w^{\text {tgwt }}$ and tg ${ }^{\text {tgwt }}$ swam at similar speeds than control wt mice during both probe trials, indicating a mild impairment of their motor abilities (Figure 4.2.11B-C). Wt and tg mice born to a tg mother ( $w t^{\text {wttg }}$ and tg ${ }^{\text {wtg) }}$ did not present motor impairments (Figure 4.2.11D-F).

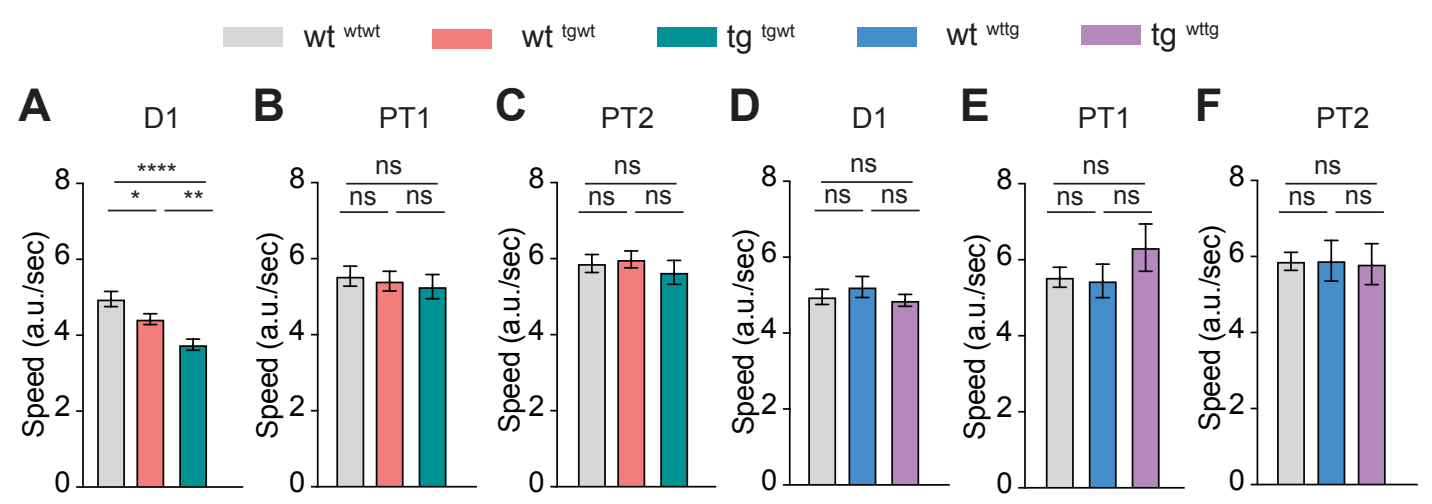

Figure 4.2.11. Wild-type and transgenic mice born to an APPPS1-21 transgenic father showed mild motor impairments.

Morris water maze (MWM) test in APPPS1-21 wild-type and transgenic mice born to a transgenic father

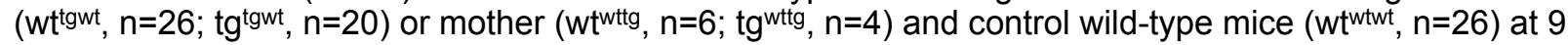
months of age. (A and D) Average speed during first training day of MWM. (B,C, E and F) Average speed during first probe trial (PT1) (B and E) and second probe trial (PT2) (C and F). Student's two-tailed T-test, ns $=$ no significant differences; ${ }^{*} \mathrm{P}<0.05 ;{ }^{* *} \mathrm{P}<0.01 ;{ }^{* * *} \mathrm{P}<0.0001$. Error bars indicated SEM.

These results suggest that all old mice show more difficulties to learn a spatial task._While control wild-type mice managed to learn the task, wt and tg mice born to APPPS1-21 tg fathers and transgenic mice born to APPPS-21 tg mothers showed learning and memory impairments. This data confirms that the parental genotype indeed has an impact on the performance of their offspring, which persist in late adulthood. It is important to highlight that the number of tg ${ }^{\text {wttg }}$ mice was limited and the results for this specific group are to be interpreted with caution.

Finally, mice were subjected to fear conditioning paradigm. During the training day, $\operatorname{tg}^{\text {tgwt }}$ and wttowt showed a reduction of their mobility, as I previously described for OF and MWM tasks (Figure 4.2.12A and D). During the test, wt and tg mice born to an APPPS1-21 tg mother did not show alterations of associative memory, whereas wt and tg mice born to an APPPS1-21 transgenic father showed an increase of freezing levels. This could have two meanings, first that they have a better associative memory than control wt mice. Second, this could mean that their 
reduction of mobility is translated as freezing periods, giving a high number of freezing events at the end of the test. Since these groups showed previously motor impairments in open field, Morris water maze and average motion during the training day, I assumed that the percentage of freezing is an artifact from their motor impairments. In order to use next time this test for this mouse line, I will need to change the analyses and extract more sensitive parameters.

A
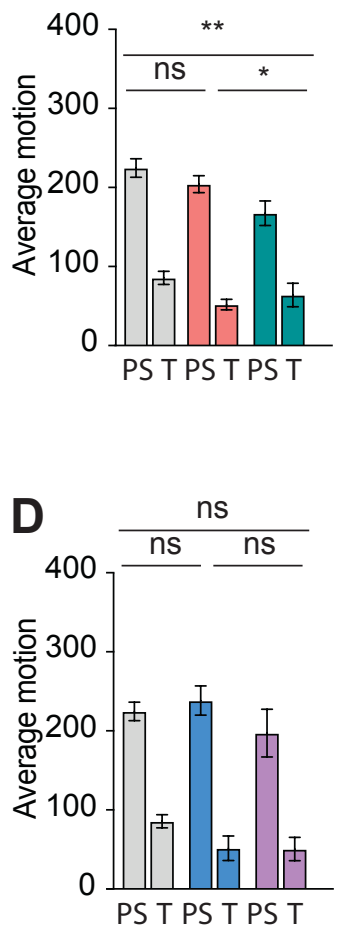

$$
\text { wt wwt }
$$
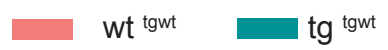

B

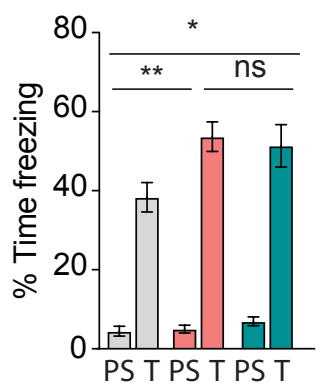

\section{C}
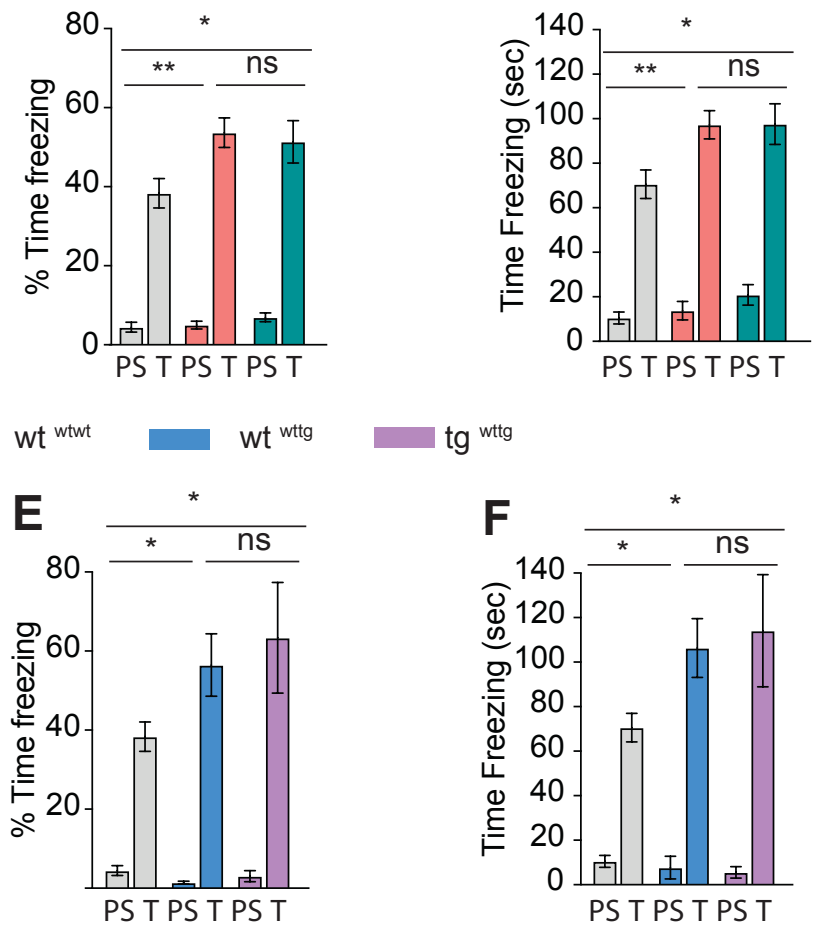

$\operatorname{tg}$ wttg

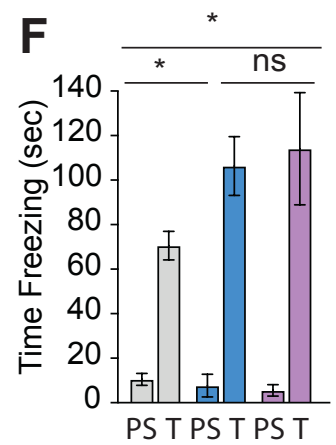

Figure 4.2.12. Fear conditioning in wild-type and transgenic mice born to APPPS-21 transgenic parents at 9 months of age.

Fear conditioning (FC) in APPPS1-21 wild-type and transgenic mice born to a transgenic father ( $\mathrm{wt}^{\mathrm{tgwt}}$, $\mathrm{n}=$ 31; $\operatorname{tg}^{\text {tgwt }}, n=18$ ) or mother ( $w^{t^{w t g}}, n=7$; tg $^{\text {wttg }}, n=4$ ) and control wild-type mice ( $w^{\text {twtwt }}, n=29$ ) at 9 months of age. (A and D) Average of motion during the training day. (B, C, E and F) Percentage of time (B and E) and time (C and F) freezing during training (first column) and testing day (second column). PS= preshock. $\mathrm{T}=$ test $\left(24 \mathrm{~h}\right.$ later). Student's two-tailed T-test, $\mathrm{ns}=$ no significant differences; ${ }^{*} \mathrm{P}<0.05 ;{ }^{* *} \mathrm{P}<0.01$. Error bars indicated SEM. 
4.2.1.3.Progressive cognitive and motor decline in wild-type and transgenic mice born to APPPS1-21 transgenic fathers or mothers

In order to study differences between young and older mice, I analyzed the previous data together for all groups of offsprings and looked for young (3 months) vs. old (9 months) differences in the open field (OF), Morris water maze (MWM) and fear conditioning (FC) behavior tasks.

During the OF, old mice, in general, showed an evident reduction in the total distance and speed, even controls groups, reflecting a slight decrease of their motor functions associated with aging. The only exception was wtwttg, traveling similar distances and with speed at both ages (Figure 4.1.22A-B). While the vast majority of groups showed a decrease of anxiety corresponding with aging, wild-type (wt) and transgenic (tg) mice born to APPPS1-21 tg mothers showed a decrease of time and distance spent in the center linked to an increased of anxiety (Figure 4.1.22C-E).

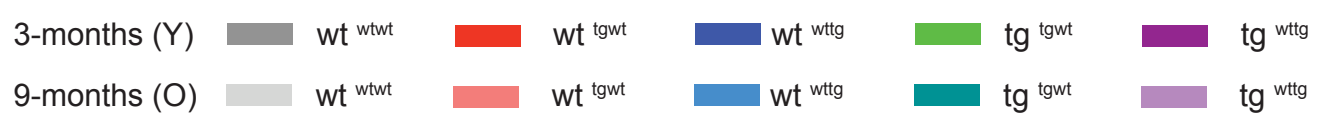

A

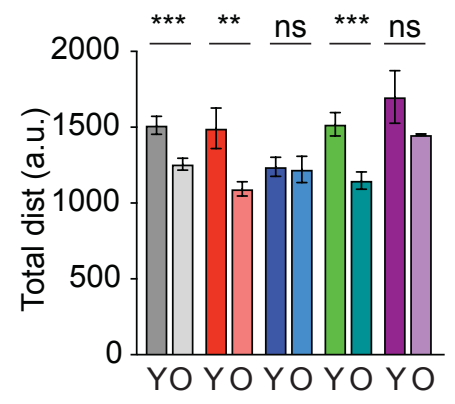

C

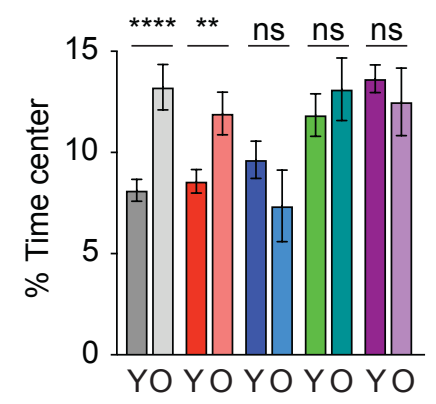

B

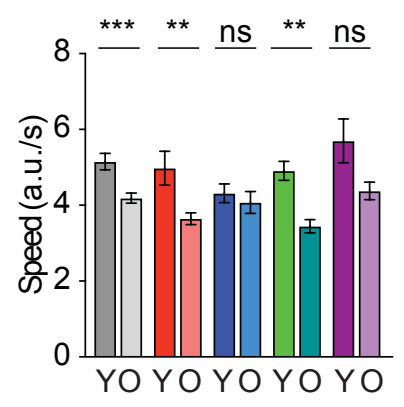

D

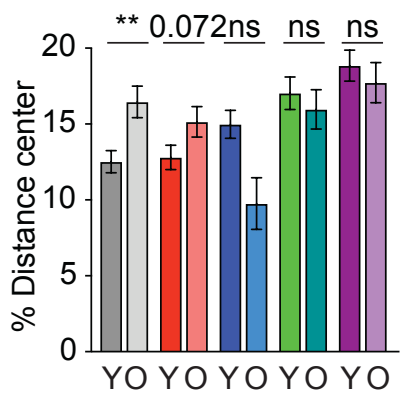

E

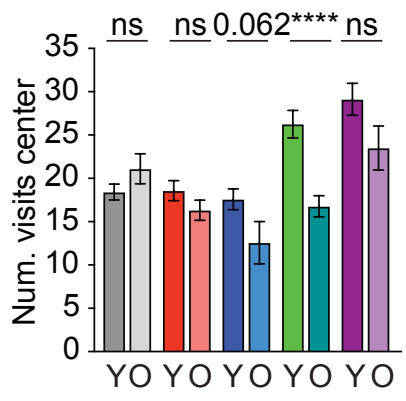


Figure 4.2.13. Mild reduction of locomotor abilities and increased of anxiety levels with aging in all groups, except in wtwttg and tgwttg.

Open field test in APPPS1-21 wild-type and transgenic mice born to a transgenic father ( $w \mathrm{t}^{\mathrm{tg} w \mathrm{t}} 3 \mathrm{~m} \mathrm{n}=32$, $9 m n=31$; tg ${ }^{\text {tgwt }}, 3 m n=27,9 m n=22$ ) or mother ( $w^{\text {wttg }} 3 m n=24,9 m n=7 ; \operatorname{tg}^{\text {wttg }} 3 m n=16,9 m n=4$ ) and control wild-type mice ( $w^{\text {twtwt }} 3 m n=34,9 m n=$ ) at 3 and 9 months of age. (A and B) Total distance traveled (A) and average speed (B) during open field test. (C-E) Percentage of time (C) and distance (D) spent on the central area of the field and number of visits to the center $(E)$. $Y=$ young (3-months) and $\mathrm{O}=$ old (9months). Student's two-tailed T-test, ns = no significant differences; ${ }^{* *} \mathrm{P}<0.01$; ${ }^{* \star *} \mathrm{P}<0.001$; ${ }^{* \star * *} \mathrm{P}<$ 0.0001 . Error bars indicated SEM.

In the MWM, all 5 groups of old mice showed difficulties to learn the spatial task, requiring significantly more time to find the platform, which was reflected in the repeated measures ANOVA and the areas under the curve (AUC) (Figure 4.2.14A-J).
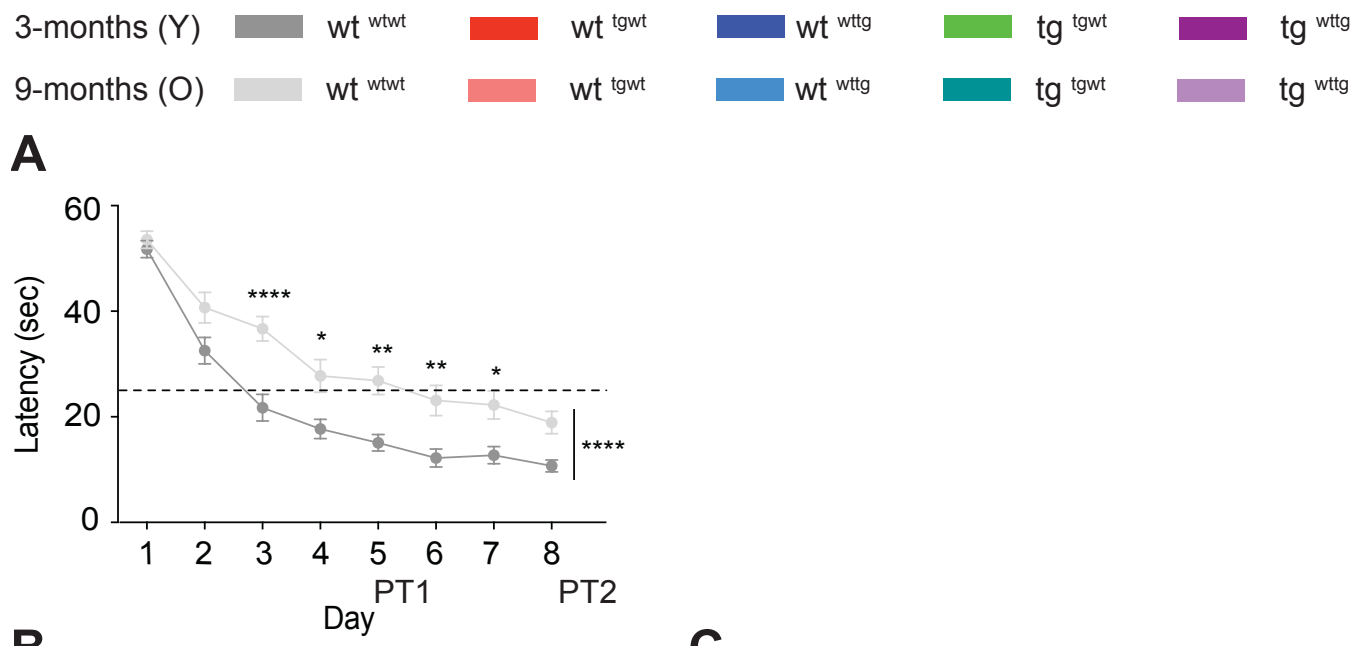

B

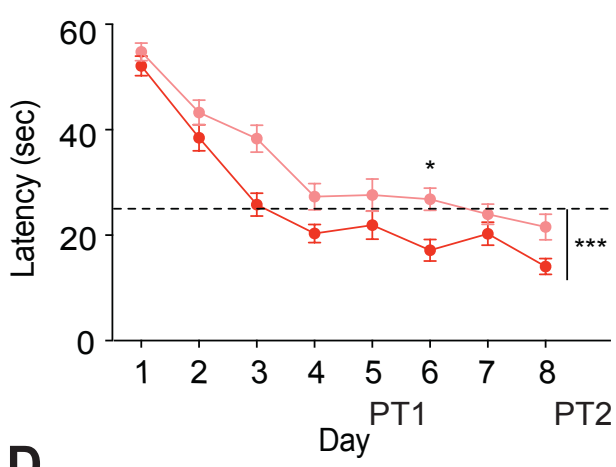

D

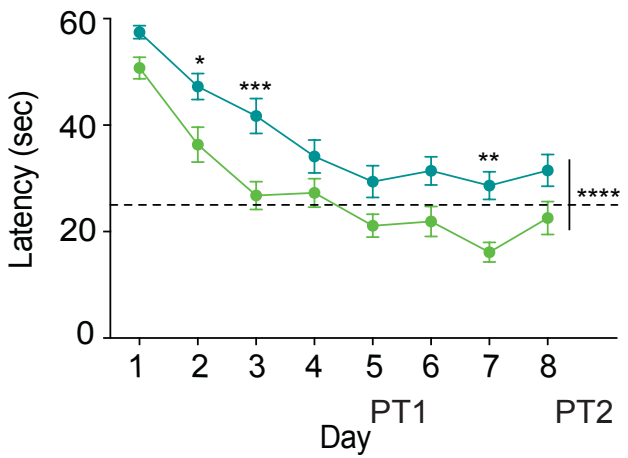

C
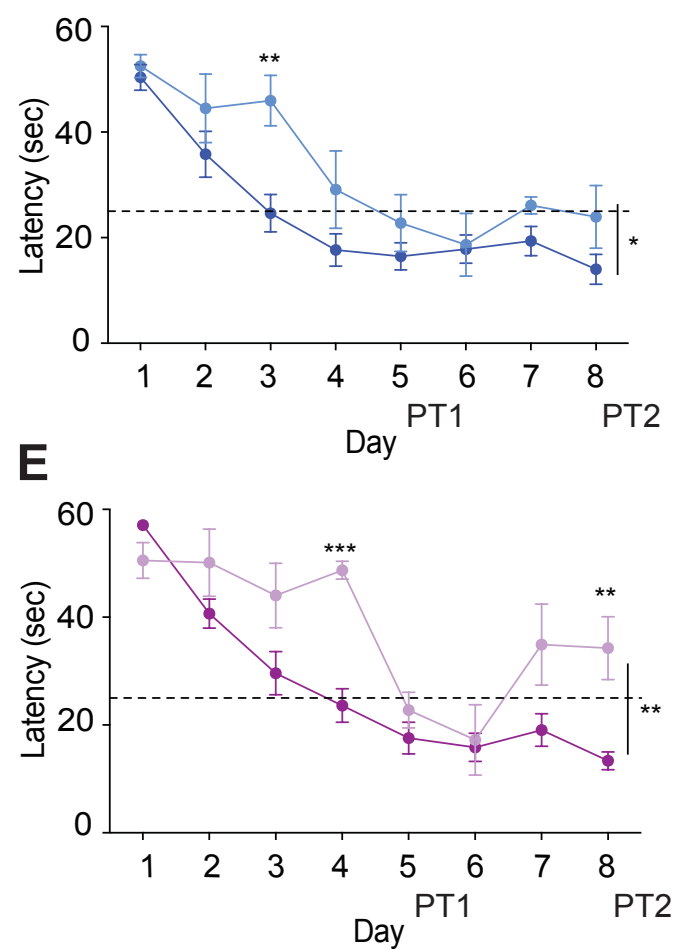

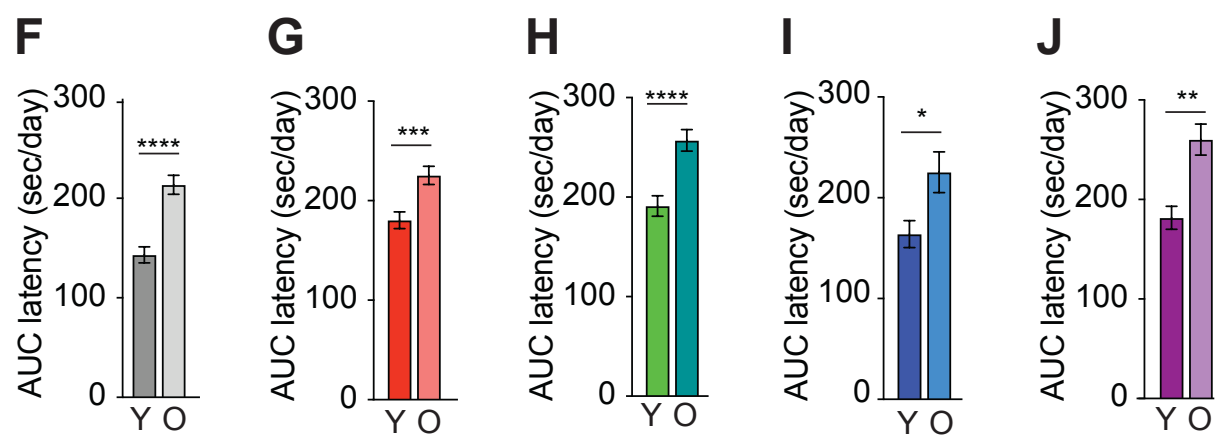

Figure 4.2.14. Learning spatial task is impaired with age in all offspring groups.

Morris water maze (MWM) test in APPPS1-21 wild-type and transgenic mice born to a transgenic father ( $w^{\text {tgwt }} 3 m n=30,9 m n=26$; tg tgwt $3 m n=25,9 m n=20$ ) or mother ( $w^{\text {twttg }} 3 m n=18,9 m n=6$; tgwttg $3 m n=17$, $9 m n=4)$ and control wild-type mice ( $\left.w^{\text {wtwt }} 3 m n=30,9 m n=26\right)$ at 3 and 9 months of age. (A-E) Young vs. old group-average time to find the target platform on each consecutive training day (Day 1-8). Two-way ANOVA after Sidak correction, ns = no significant differences; ${ }^{*} \mathrm{P}<0.05$; ${ }^{* *} \mathrm{P}<0.01$; ${ }^{* * *} \mathrm{P}<0.001$; ${ }^{* * * *} \mathrm{P}<$ 0.0001 . The dashed line represents the chance level of $25 \%$. First probe trial (PT1, day 6 ) and second probe trial (PT2, day 10). (G-J) Comparison of young and old group equivalents analyzing the areas under the learning curves (AUC) of escape latency for each group. $Y=$ young (3-months) and $\mathrm{O}=$ old (9months). Student's two-tailed T-test, ns = no significant differences; ${ }^{* *} \mathrm{P}<0.05 ;{ }^{* *} \mathrm{P}<0.01 ;{ }^{* * *} \mathrm{P}<0.001$; ${ }_{* * * *} \mathrm{P}<0.0001$. Error bars indicated SEM.

Not many changes were observed during both probe trials (PT) between the two age periods. During the first probe trial (PT1), none of the mice in any group learned the position of the platform (Figure 4.2.15A-B). During the second probe trial (PT2), only young wt controls and wt from tg mother ( $\mathrm{wt}^{\mathrm{t}} \mathrm{wtg}^{\mathrm{f}}$ ) showed a preference for the target quadrant confirming the close similarity between these groups (Figure 4.2.15C-D). 
RESULTS
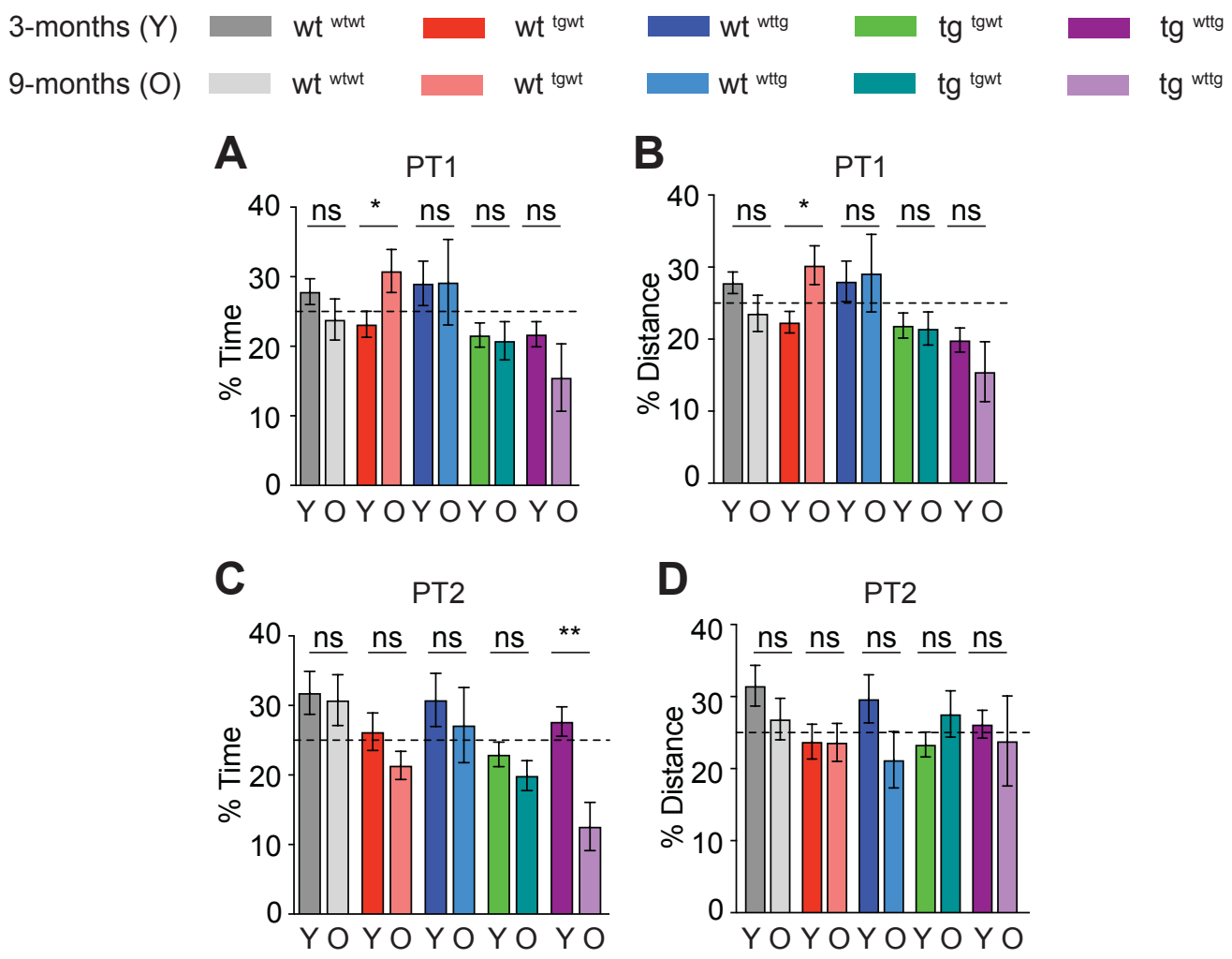

Figure 4.2.15. Only young control wild-type mice ( $\left.w t^{w t w t}\right)$ and young $w^{\text {thtg }}$ mice showed a preference for the target quadrant.

Morris Water Maze (MWM) test in APPPS1-21 wild-type and transgenic mice born to a transgenic father (wtgwt $3 m n=30,9 m n=26$; tg tgwt $3 m n=25,9 m n=20$ ) or mother ( $w^{\text {twtg }} 3 m n=18,9 m n=6$; tgwtg $^{\text {th }} 3 m n=17,9 m$ $n=4)$ and control wild-type mice (wtwtwt $3 m n=30,9 m n=26)$ at 3 and 9 months of age. (A-D) Percentage of time ( $\mathbf{A}$ and $\mathbf{C}$ ) and distance $(\mathbf{B}$ and $\mathbf{D})$ spent on the target quadrant between young $(\mathrm{Y})$ and old $(\mathrm{O})$ mice during first probe trial (PT1, day 6) (A and B) and second probe trial (PT2, day 10) (C and D). Y = young (3-months) and $\mathrm{O}=$ old (9-months). Student's two-tailed T-test, ns $=$ no significant differences; ${ }^{*} \mathrm{P}<0.05$; ${ }^{* *} \mathrm{P}<0.01$. The dashed line represents the chance level of $25 \%$.

As before, checking the distance between the position of the mouse and platform position, every two seconds during both probe trials (PT1 and 2) revealed the differences between old and young mice. While none of the young and old mice learned the task during PT1, old mice from all experimental groups and controls significantly differed from young mice counterparts during PT2 (Figure 4.2.16A-T). 


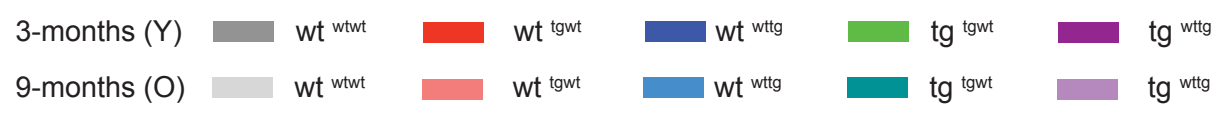

A B

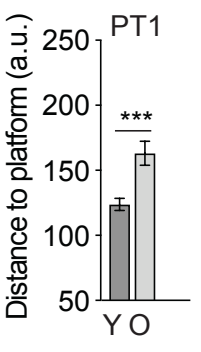

D

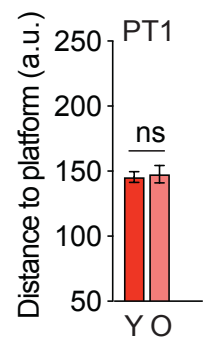

G

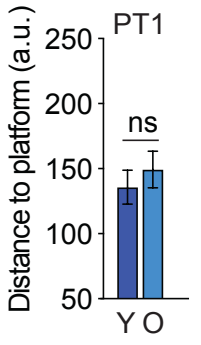

J

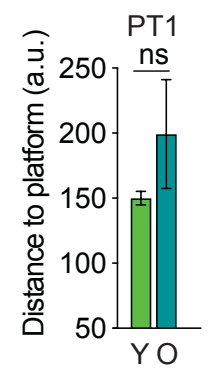

M

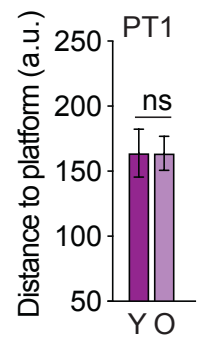

E

H

\section{K}

N
B
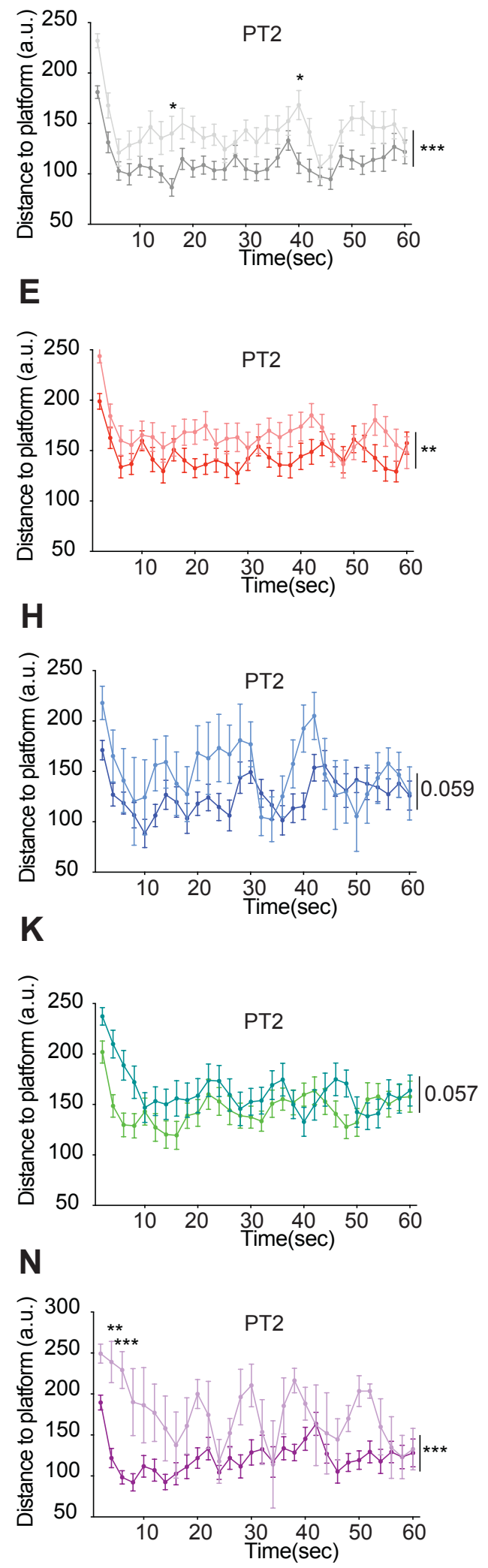

C

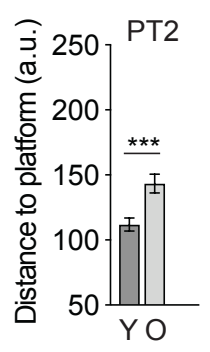

F

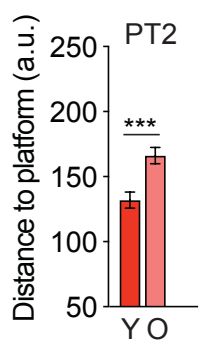

I

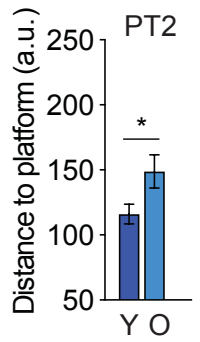

L

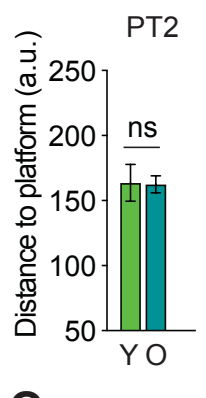

0

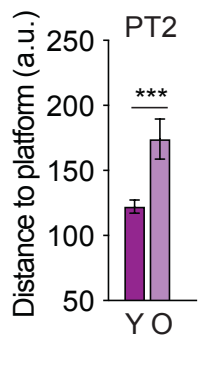


Figure 4.2.16. All old offspring groups showed a more severe spatial cognitive impairment than young equivalent groups.

Morris Water Maze (MWM) test in APPPS1-21 wild-type and transgenic mice born to a transgenic father ( $w^{\text {tgwt }} 3 m n=30,9 m n=26$; tgtgwt $3 m n=25,9 m n=20$ ) or mother ( $w^{\text {twtg }} 3 m n=18,9 m n=6$; tgwttg $3 m n=17$, $9 m n=4)$ and control wild-type mice (wtwtwt $3 m n=30,9 m n=26)$ at 3 and 9 months of age. (A-O) The distance between mouse and the platform position every two seconds during second probe trial (PT2, day 10) (B, E, H, K and N) and the average of total distance during PT1 (A, D, G, J and M) and PT2 (C, F, I, L and $\mathrm{O})$. $\mathrm{Y}=$ young (3-months) and $\mathrm{O}=$ old (9-months). Two-way ANOVA after Sidak correction and student's two-tailed T-test, ns = no significant differences; ${ }^{*} \mathrm{P}<0.05 ;{ }^{* *} \mathrm{P}<0.01 ;{ }^{* *} \mathrm{P}<0.001$. Error bars indicated SEM.

In contrast to open field task, only old transgenic mice born to an APPPS1-21 $\mathrm{tg}$ father (tg ${ }^{\text {tgwt}}$ ) showed a significant reduction of swimming speed during the first training and both probe trials (Figure 4.2.17A-C).
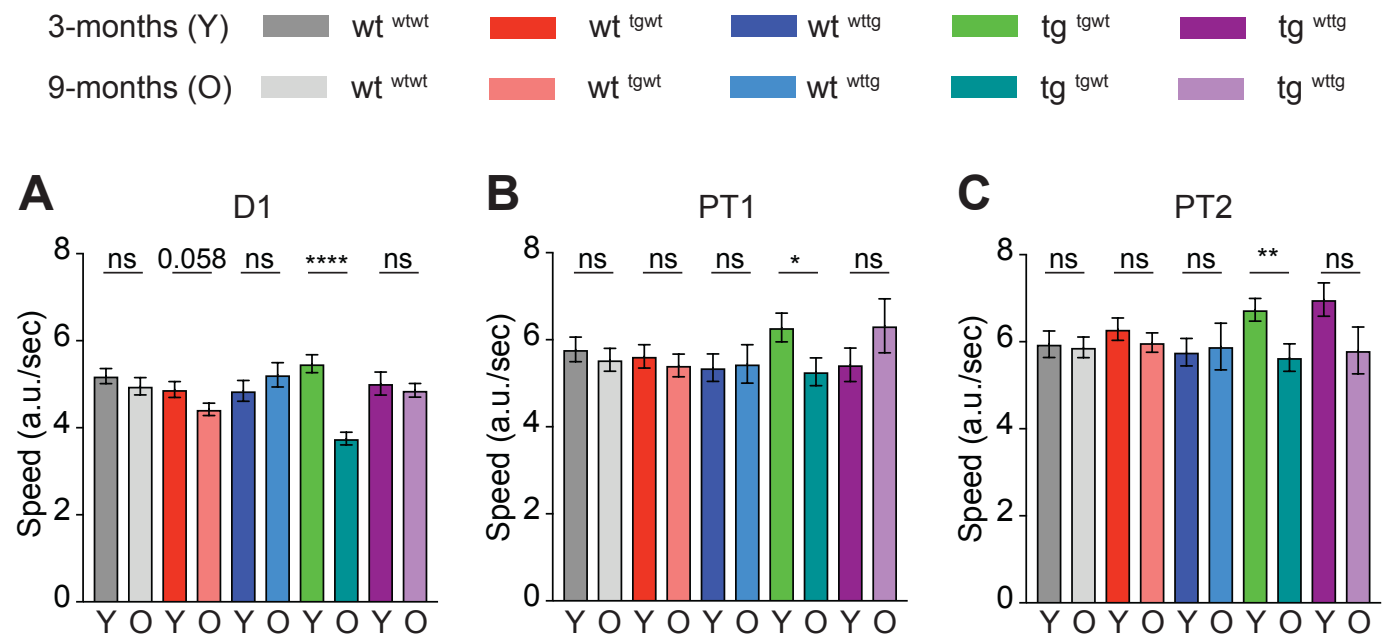

Figure 4.2.17. Only transgenic mice born to a transgenic father showed motor impairments with aging during MWM task.

Morris Water Maze (MWM) test in APPPS1-21 transgenic mice born to a transgenic father ( $w t^{\text {tgwt }} 3 m n=30$, $9 m n=26$; $\operatorname{tg}^{\text {tgwt }} 3 m n=25,9 m n=20$ ) or mother ( $w^{\text {twtg }} 3 m n=18,9 m n=6$; tg $^{\text {wttg }} 3 m n=17,9 m n=4$ ) and control wild-type mice (wtwtwt $3 m n=30,9 m n=26$ ) at 3 and 9 months of age. (A) Averaged speed during first training day of MWM. (B and C) Averaged speed during first probe trial (PT1) (B) and second probe trial (PT2) (C). Y = young (3-months) and $\mathrm{O}=$ old (9-months). Student's two-tailed T-test, ns = no significant differences; ${ }^{*} \mathrm{P}<0.05 ;{ }^{* *} \mathrm{P}<0.01$ and ${ }^{* * *} \mathrm{P}<0.0001$. Error bars indicated SEM.

Lastly, during the training day of fear conditioning I observed a significant reduction of the mobility of almost all studied groups (Figure 4.2.18A). During the test, only $w^{t^{t g w t}}$ and tg $^{\text {wttg }}$ showed an increase of freezing with aging. Taking into account the significant reduction of their mobility, I assume that this increase of freezing only reaffirms their progressive degeneration of their motor abilities (Figure 4.2.18B-C). 


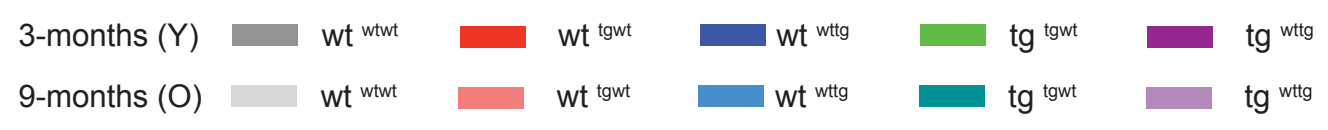

A

B
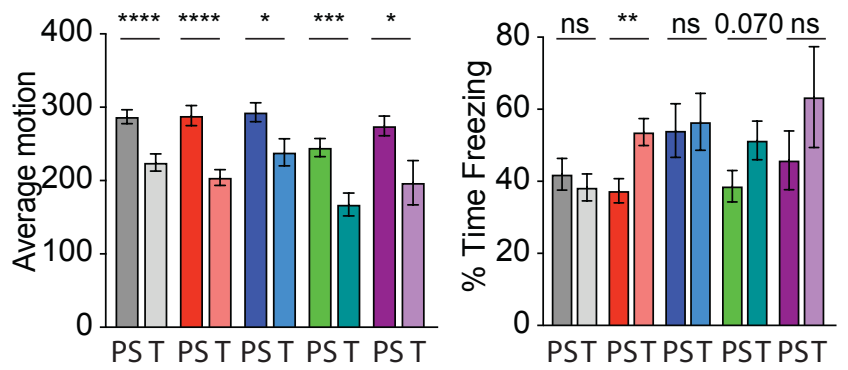

C

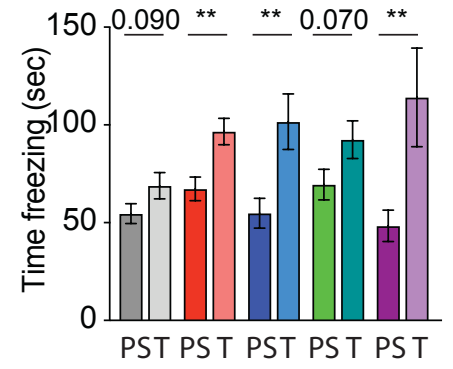

Figure 4.2.18. All old groups showed a significant reduction of the average motion during the training day.

Fear conditioning in APPPS1-21 wild-type and transgenic mice born to transgenic father ( $w^{\text {tgwt }} 3 \mathrm{~m} \mathrm{n}=35$, $9 m n=31$; tg tgwt $3 m n=28,9 m n=18$ ) or mother ( $w^{\text {twtg }} 3 m n=24,9 m n=7$; tgwttg $3 m n=19,9 m n=4$ ) and control wild-type mice ( $w^{\text {twtwt }} 3 m n=34,9 m n=29$ ) at 3 and 9 months of age. (A) Average of motion during the training day. (B and $\mathbf{C}$ ) Percentage of time (B) and time (C) freezing during the testing day at 3 (first column) and 9 (second column) months of age. $\mathrm{Y}=$ young (3-months) and $\mathrm{O}=$ old (9-months). $\mathrm{PS}=$ preshock. $\mathrm{T}=$ test $\left(24 \mathrm{~h}\right.$ later). Student's two-tailed T-test, $\mathrm{ns}=$ no significant differences; ${ }^{*} \mathrm{P}<0.05$; ${ }^{* * *} \mathrm{P}<$ $0.001 ;{ }^{* * *} \mathrm{P}<0.0001$. Error bars indicated SEM.

Taken together, this specific model of $A D$ also showed a worsening behavior with age, similar to other models (Kelly et al., 2003; Sadowski et al., 2004; Van Dam et al., 2003). 9 month-old mice showed more severe cognitive deficits than mice at 3 months of age. To note, control wild-type mice born to wt parents also showed difficulties in acquiring and learning spatial task at 9 months of age, although this age is not yet considered "old" for a wild-type mouse. Specifically, APPPS1-21 wild-type mice born to APPPS1-21 tg fathers ( $\mathrm{wt}^{\mathrm{tgwt}}$ ) showed stronger alteration of their cognitive capabilities during aging compared to wt mice born to APPPS1-21 tg mothers (wt $\left.{ }^{\text {wttg }}\right)$. This variation could be attributed to a reduction of the motor abilities of $w^{t^{\text {tgwt }}}$ and tg $^{\text {tgwt }}$ mice at 9 months of age. Nonetheless, this variability in learning and motor abilities suggests that non-genetic inheritance of an APP/PS1 phenotype from a father or mother have a different repercussion on the process of aging. 


\subsubsection{Accounting for group size, batch and litter effects}

An important technical aspect to consider in an intergenerational inheritance of any kind is group sizes, litter and batch effects. It is crucial to carry out behavior experiments in the offspring from multiple mating to verify that the effects are not litter or batch-specific. Although these factors have not always been accounted for (Bohacek \& Mansuy, 2017), they can play critical roles in the final outcome of the analysis. I took great care firstly to design our mating strategy. Secondly, I account for litter and batch effects by using standard statistical analyses and linear mixed models. Mixed linear models is a statistical a take into account fixed factors (treatment and gender) and confounding factors (litter and batch).

In order to use an appropriate group size, I used a large number of males and females from each experimental and control groups at $3(n \geq 16)$ and $9(n \geq 20$, except in wtwttg and tgwttg) months of age (Table 4.2.1). Numbers of mice per groups are defined on the table 4.1.19A-B. Due to spatial limitations and a reduced number of births respect to other groups, the numbers of wild-type and transgenic mice born to APPPS1-21 tg mothers were always lower than the other groups (18 and 16 at $3 \mathrm{~m} ; 6$ and 4 at $9 \mathrm{~m}$ ). Thus, behavior results from $w^{t^{w t g}}$ and $^{\text {towtg }}$ at 9 months of age are only illustrative. It is important to highlight that the number of $w^{t^{w t t g}}$ and $\operatorname{tg}^{\text {wttg }}$ mice was limited and the results for this specific group are to be interpreted with caution.

A

3 months

\begin{tabular}{|c|c|c|c|}
\hline Group & Male $(\mathrm{n}=)$ & Female $(\mathrm{n}=)$ & Total $(\mathbf{n}=)$ \\
\hline wt $^{\text {wtwt }}$ & 16 & 14 & $\mathbf{3 0}$ \\
\hline wt $^{\text {tgwt }}$ & 18 & 12 & $\mathbf{3 0}$ \\
\hline tg $^{\text {tgwt }}$ & 13 & 12 & $\mathbf{2 5}$ \\
\hline wt $^{\text {wtg }}$ & 11 & 7 & $\mathbf{1 8}$ \\
\hline tg $^{\text {wttg }}$ & 10 & 7 & $\mathbf{1 6}$ \\
\hline
\end{tabular}

B

9 months

\begin{tabular}{|c|c|c|c|}
\hline Group & Male $(n=)$ & Female $(n=)$ & Total $(\mathbf{n}=)$ \\
\hline wt $^{\text {wtwt }}$ & 14 & 12 & $\mathbf{2 6}$ \\
\hline wt $^{\text {tgwt }}$ & 16 & 10 & $\mathbf{2 6}$ \\
\hline tg $^{\text {tgwt }}$ & 13 & 7 & $\mathbf{2 0}$ \\
\hline wt $^{\text {wtg }}$ & 5 & 1 & $\mathbf{6}$ \\
\hline tg $^{\text {wtg }}$ & 1 & 3 & $\mathbf{4}$ \\
\hline
\end{tabular}

Table 4.2.1. Large group number of mice used for Morris water maze at 3 and 9 months of age. ( $A$ and $B$ ) Number of mice, males and females, used for behavior experiments at (A) 3 and (B) 9 months of age.

In addition to a large number of mice per group, I used mice from different mating and statistically tested specific litter repercussion by one-way ANOVA. The numbers of mating from same father and mother genotype are defined in figure 4.1.20A and D. Firstly I analyzed the effect of different litters within each group, and no significant differences were identified for any litter in any experimental and control groups (Figure 4.1.20B and E). Secondly, I averaged the mice from the same litter and evaluated their percentage of time in the target quadrant during the second probe trial in the Morris water maze (MWM) task (Figure 4.1.20C and F). Similar 
results as the ones obtained with individual mice were obtained, suggesting that the performance variability seen in the MWM is not litter-specific.

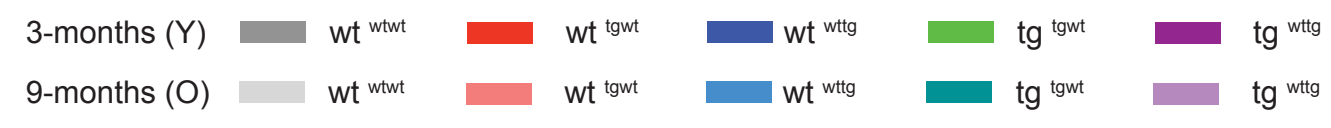

A

B

\begin{tabular}{|l|c|}
\hline Matings & Litter $3 \mathrm{~m}$ \\
\hline wt $\mathrm{x}$ wt & 7 \\
\hline $\mathrm{tg} \times \mathrm{wt}$ & 10 \\
\hline $\mathrm{wt} \times \mathrm{tg}$ & 5 \\
\hline
\end{tabular}

\begin{tabular}{|l|c|}
\hline Matings & Litter $9 \mathrm{~m}$ \\
\hline $\mathrm{wt} \mathrm{x} w \mathrm{wt}$ & 4 \\
\hline $\mathrm{tg} \mathrm{x} \mathrm{wt}$ & 6 \\
\hline $\mathrm{wt} \mathrm{x} \mathrm{tg}$ & 2 \\
\hline
\end{tabular}

C

PT2

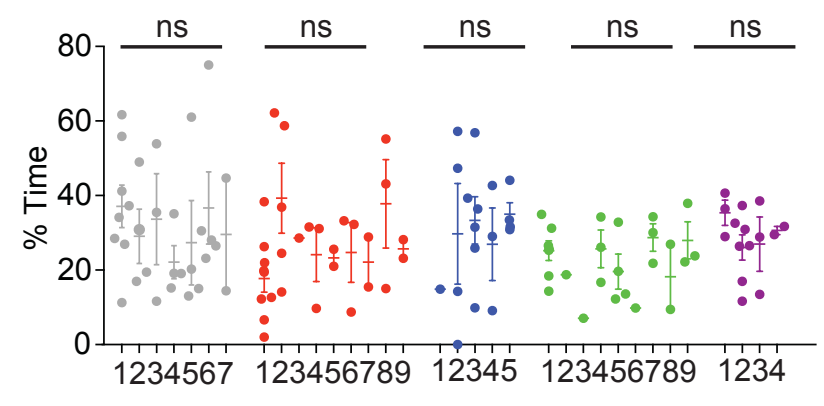

D PT2

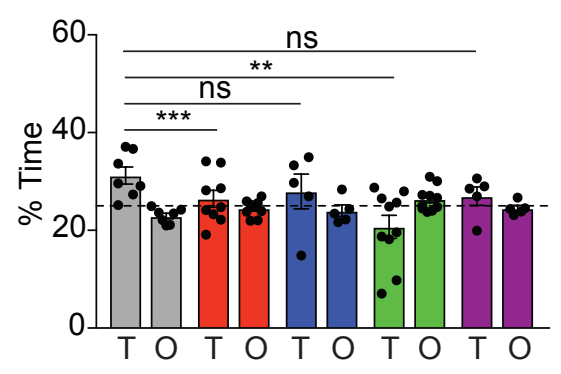

Litter number

E

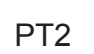

$\mathbf{F}$
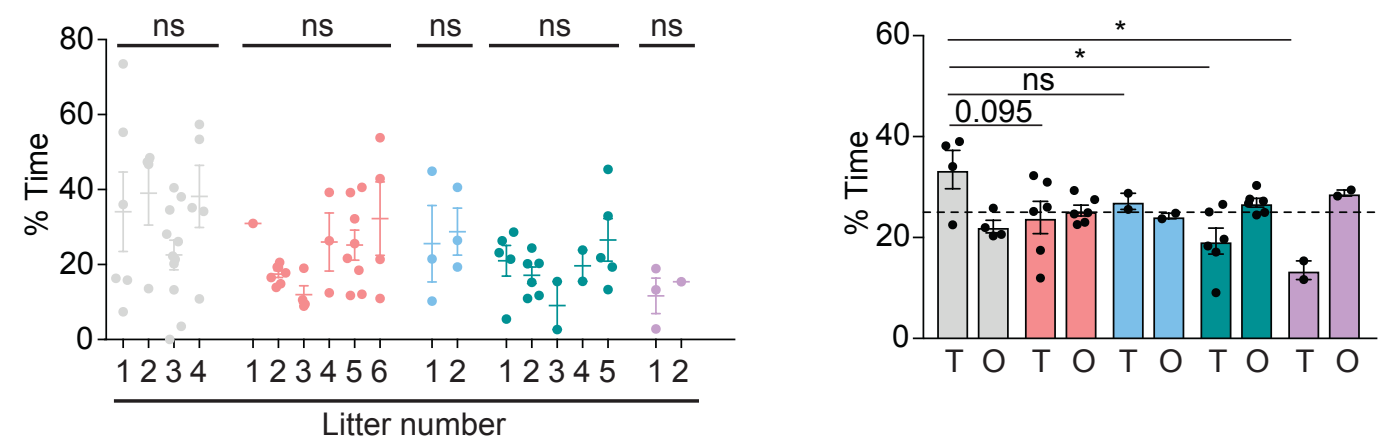

Figure 4.2.19. No litter-specific effect in Morris water maze behavioral data.

(A and B) Number of litters used to obtain enough offspring mice at (A) 3 and (B) 9 months of age to perform behavior experiments. (C and E) Variability between litter-specific mice at (C) 3 and (E) 9 months of age regarding time spent in the target quadrant during the second probe trial (PT2). One-way ANOVA after Tukey correction, $n s=$ no significant differences; ${ }^{*} \mathrm{P}<0.05 ;{ }^{* *} \mathrm{P}<0.01$. Error bars indicated SEM. (D and F) Percentage of time spent in the target quadrant ( $T$ ) vs. average time spent in other quadrants (O) between groups of (C) 3 and (F) 9 months of age during second probe trial (PT2, day 10). All mice from the same litter were previously averaged. Student's two-tailed T-test, ${ }^{*} P<0.05 ;{ }^{* *} P<0.01 ;{ }^{* * *} P<0.001$. Error bars indicated SEM. 
Taking into account the large number of mice to test and the repercussion of the day cycle might have had on performance, behavioral experiments were conducted in different batches.

Specifically, mice were split into 4 batches of mice at 3 months of age and 4 batches of mice at 9 months of age. To confirm that the behavior data previously presented was not confounded by batch-specific effects, I performed first a standard statistical analysis between batches from each group. These analyses showed that batch-specific effect did not affect the data_(Figure 4.1.21AB).

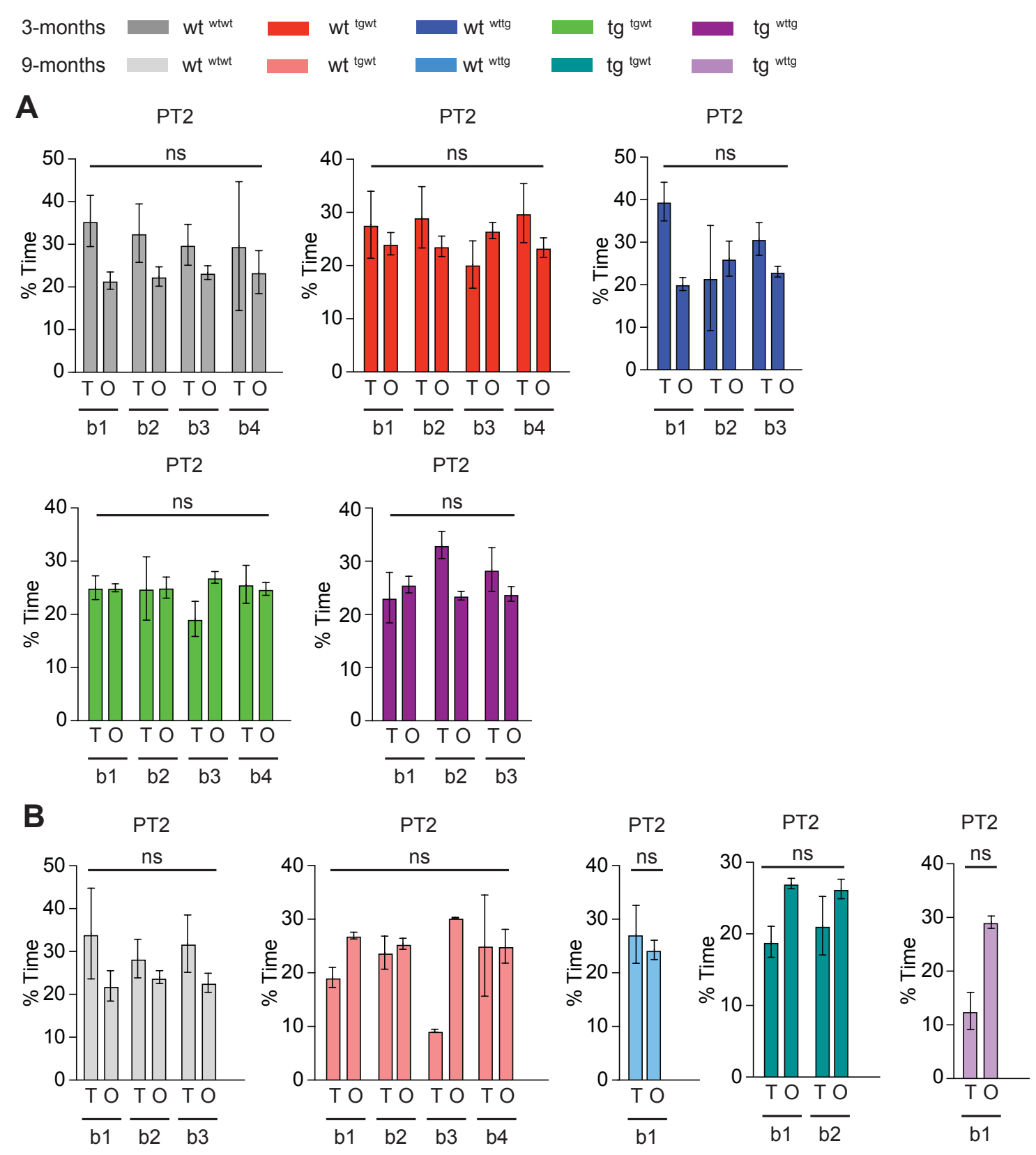


Figure 4.1.20. No significant differences were observed between batches regarding the time spent in the target quadrant during the second probe trial (PT2) in Morris water maze.

(A and B) Number of batches for each group at (A) 3 and (B) 9 months of age used for behavior experimental tests. One-way ANOVA after Tukey correction, ns $=$ no significant differences. Error bars indicated SEM.

To verify the batch- and litter-specific effect I used a more sensitive approach, the linear mixed model, taking into account fixed factors (treatment and gender) and confounding factors (litter and batch) for spatial cognitive performance (Distance to the platform during PT2). In this model, all wt and tg mice born to APPPS1-21 tg fathers ( $w^{\text {tgwt }}$ and $\operatorname{tg}^{\text {tgwt }}$ ) and mothers ( $w^{w}{ }^{\text {wtg }}$ and tg $^{\text {wttg }}$ ) were compared to control wt ( $w^{\text {twtwt}}$ ). I observed significant differences between $w^{\text {tgwt }}$ and $\operatorname{tg}^{\text {tgwt }}$ mice compared to wild-type control (wtwtwt) at 3 months of age, as I previously observed using standard statistical analysis (Table 4.2.2). This confirms that the random factors are not affecting the results obtained. However, the evaluation of mice at 9 months of age did not show the spatial cognitive differences previously detected using standard analysis. Since the linear mixed model account for random factors, there is an underlying bias or potentially a litter effect, which account for the effect not being significant. Besides, the individual variability along aging is higher than at 3 months of age.

A

\begin{tabular}{|l|l|l|}
\hline $3 \mathrm{~m}$ & $\mathrm{t}$-value & $\mathrm{p}$-value \\
\hline $\mathrm{wt}^{\mathrm{tgwt}}$ & 2.161580 & $\mathbf{0 . 0 3 3 3}$ \\
\hline $\mathrm{wt}^{\mathrm{wtg}}$ & 0.303245 & 0.7624 \\
\hline $\mathrm{tg}^{\text {tgwt }}$ & 3.427519 & $\mathbf{0 . 0 0 0 9}$ \\
\hline $\mathrm{tg}^{\text {wtg }}$ & 0.573621 & 0.5677 \\
\hline
\end{tabular}

B

\begin{tabular}{|l|l|l|}
\hline $9 \mathrm{~m}$ & $\mathrm{t}$-value & $\mathrm{p}$-value \\
\hline $\mathrm{wt}^{\mathrm{tgwt}}$ & 1.136334 & 0.8091 \\
\hline $\mathrm{wt}^{\mathrm{wtg}}$ & 1.136334 & 0.2597 \\
\hline $\mathrm{tg}^{\text {tgwt }}$ & 1.617813 & 0.1103 \\
\hline $\mathrm{tg}^{\text {wig }}$ & 0.582704 & 0.5620 \\
\hline
\end{tabular}

Table 4.2.2. Evaluation of batch- and litter-effect using a linear mixed model for statistical analysis of distance to the platform in Morris water maze (MWM) at 3 and 9 months.

(A-B) Statistical evaluation of the distance to the platform in MWM of wild-type and transgenic mice born to one APPPS1-21 parent at (A) 3 and (B) 9 months of age. Fixed factors $=$ gender and treatment. Confounding factors $=$ litter and batch. ns $>0.05{ }^{*} \mathrm{P}<0.05,{ }^{* *} \mathrm{P}<0.01 ;{ }^{* * *} \mathrm{P}<0.001$.

I am therefore confident that my results are representative of a biologically relevant factor (i.e.. parental origin of the transgene or even just paternal non-genetic heritage). 


\subsubsection{Intergenerational effect on transcriptional profiling and functional pathways}

So far, I have established that there are differences in the cognitive performance of APPPS1-21 wild-type mice depending on whether they come from a mutant mother or father at 3 and 9 months of age. These data suggest that there are lineage-specific non-genetic modes of phenotypic inheritance. Intergenerational inheritance has been associated with small non-coding RNAs and epigenetic-mediated transmission of acquired traits, implying mechanisms that promote changes at transcription level (de Castro Barbosa et al., 2016a; Herman \& Sultan, 2016). Thus, I decided to use the transcriptome as a readout of the cellular state and wondered whether I would be able to dissect molecular pathways associated with the parental-specific mode of non-genetic inheritance of the cognitive deficit. In order to obtain a cleaner signature and most robust effects, only 3 months mice were evaluated. Mice at 9 months of age start to get a mixture of aging-related effects that might confound the factor of interest that is only the non-genetic inheritance of a phenotype caused by a genetic mutation.

4.2.3.1.Transcriptional profiling of wild-type mice born to an APPPS1-21 transgenic mother or father

As before, I analyzed the transcriptome of two subregions of the hippocampus, CA1 and DG, which were involved in spatial memory. I compared the CA1 and DG transcriptome from wildtype (wt) mice born to an APPPS1-21 tg father or mother ( $w t^{\text {tgwt }}$ and $w t^{\text {wttg }}$ ) to the transcriptome of control wild-type mice ( $w^{w t w t)}$. The transcriptomic profile was analyzed based on significant differentially expressed genes (padj $\leq 0.05$ ) with distinct levels of expression defined by the $\log _{2} \mathrm{FC}$ cutoff as severe $( \pm 1.00)$, medium $( \pm 0.50)$ and mild $( \pm 0.25)$ (Figure 4.1.33A). Interestingly, only a few genes in the hippocampus subregions from wt mice born to an

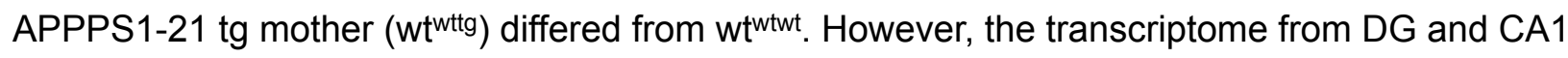
from wt mice born to an APPPS1-21 tg father ( $w^{t}{ }^{\text {tgwt}}$ ) showed several changes in gene expression compared to control wt mice ( $\left.w^{t^{w t w t}}\right)$, specifically in the DG from males and females (Figure 4.2.21A-I). This is particularly interesting since both groups are in principle wild-type mice grown under the same conditions. As previously described for transgenic mice, the majority of genes fell into mild to medium levels, and the number of differential up-regulated genes was higher in wtgwt than in controls (Figure 4.2.21A-D). Because the main changes in the transcriptome were observed for genes at the mild level of change (padj $\leq 0.05$; $\log _{2} \mathrm{FC} \pm 0.25$ ), I next analyzed these in further detail (Figure 4.2.22A and C). 
A

A $t^{\text {tgwt }}$ vS. wt $^{\text {wtwt }}$
\begin{tabular}{|l|l|l|l|}
\hline padj $\leq 0.05$ & Severe & Medium & Mild \\
\hline Total $=$ up + down & Log $_{2} \mathrm{FC} \pm 1.00$ & Log $_{2} \mathrm{FC} \pm 0.50$ & Log $_{2} \mathrm{FC} \pm 0.25$ \\
\hline DGm & 0 & $44=30+14$ & $152=92+60$ \\
\hline DGf & 0 & $169=139+30$ & $866=560+306$ \\
\hline CA1m & 0 & $2=2+0$ & $30=23+7$ \\
\hline
\end{tabular}

C

Wtwttg vs. wtwtwt
\begin{tabular}{|l|l|l|l|}
\hline padj $\leq 0.05$ & Severe & Medium & Mild \\
\hline Total $=$ up + down & Log $_{2} \mathrm{FC} \pm 1.00$ & Log $_{2} \mathrm{FC} \pm 0.50$ & Log $_{2} \mathrm{FC} \pm 0.25$ \\
\hline DGm & 0 & $3=2+1$ & $3=2+1$ \\
\hline DGf & 0 & $27=16+11$ & $45=25+20$ \\
\hline CA $1 \mathrm{~m}$ & 0 & 0 & $1=1+0$ \\
\hline
\end{tabular}

B

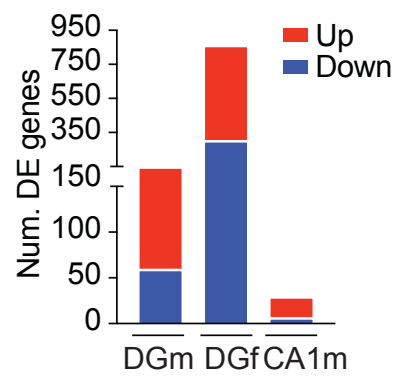

D

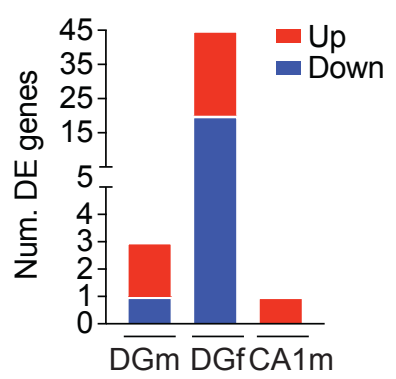

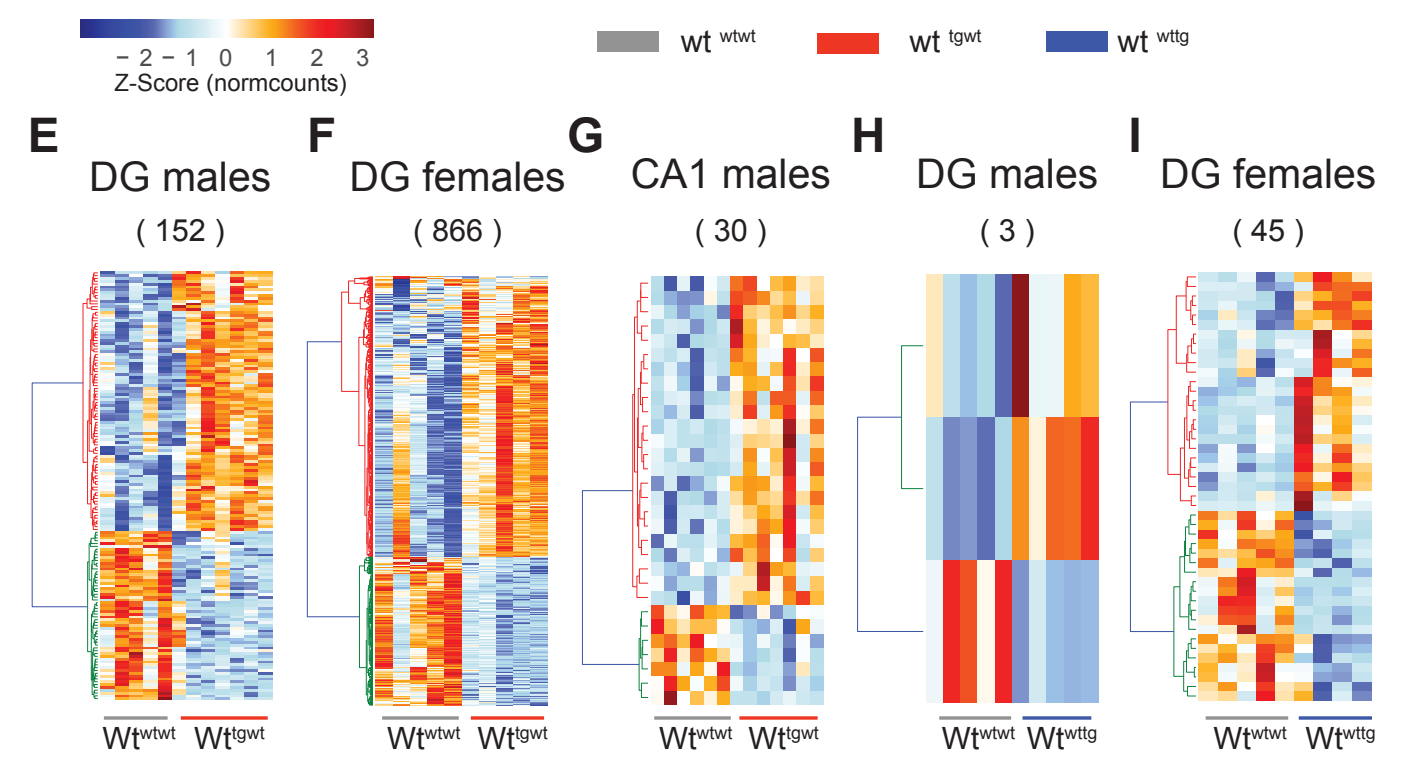

Figure 4.2.21. Moderate changes in transcriptional profile in brain subregions of wild-type born to an APPPS1-21 transgenic father ( $w^{\text {tgwt }}$ ) at 3 months of age.

(A and C) Number of significant differentially expressed genes with different level of changes (severe $( \pm$ $1.00)$, medium ( \pm 0.50$)$ and mild $( \pm 0.25)$, established by the $\left.\log _{2} F C\right)$ in $w^{\text {tgwt }} v s$. wt ${ }^{\text {wtwt }}(A)$ and $w^{\text {twttg }}$ vs. wt $^{\text {wtwt }}$ comparisons (C). Padj, P value adjusted and $\log _{2} F C$, logarithm base 2 of Fold Change. (B and D) Number of significant differentially expressed up- and down-regulated genes in the DG from males (DGm), DG from females (DGf) and CA1 from males (CA1m) in wt'twt vs. $w^{\text {twtwt }}$ (B) or wtwttg vs. wt ${ }^{\text {twt }}$ mice (D). Padj $\leq 0.05, \log _{2} F C \pm 0.25$. (E-I) Heatmaps of differentially expressed genes in 3 month-old wtgwt $v s$. wtwtwt (E-G) or $w^{t^{t t g}}$ vs. $w^{t^{t t w t}}$ mice (H and I). Padj $\leq 0.05, \log _{2} F C \pm 0.25$. For DG males: $w^{\text {tgwt }} n=7$; $w^{t^{t t g}} n=5$ and $w^{\text {wtwt }} n=5$; for DG females wt ${ }^{\text {tgwt }} n=5$; wt ${ }^{\text {wttg }} n=4$ and $w^{w t w t} n=5$ and for CA1 males $w^{\text {tgwt }} n=7$; w ${ }^{\text {wttg }}$

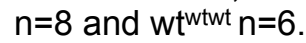


Furthermore, I compared the transcriptome of wt mice born to an APPPS1-21 tg father (wtgwt) or mother ( $w^{t^{w t}}{ }^{w}$ ) to the transcriptome of APPPS1-21 tg mice. Previously, I described the strong alteration of the transcriptome signature in the hippocampal subregions of transgenic APPPS1-21 mice at 3 months of age compared to control wt mice (wtwtwt). Surprisingly, wt mice born to a tg father ( $w^{\text {tgwt }}$ ) or mother ( $w^{\text {twttg }}$ ) showed a little amount of differentially expressed genes compared to APPPS1-21 tg mice. Wttgwt mice showed a maximum of 75 differentially expressed genes in DG region from females compared to tg mice, and wtwttg showed a maximum of 58 in DG region from females. These numbers suggest that wt mice born to APPPPS1-21 tg fathers or mothers showed minor changes in the transcriptome when they were compared to tg mice (Figure 4.2.22A-C).
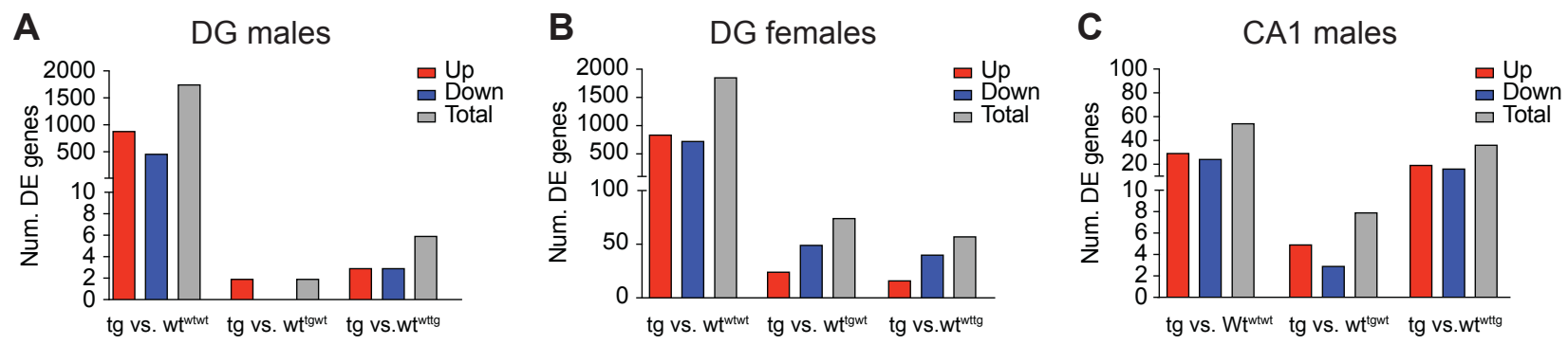

Figure 4.2.22. Little transcriptomic differences between wild-type mice born to an APPPS1-21 $\mathrm{tg}$ father (wt $\left.\mathbf{w}^{\mathrm{tg} w \mathrm{t}}\right)$ or mother (wtwtt) and APPPS1-21 transgenic mice.

(A-C) Number of significant differentially expressed up- and down-regulated genes in tg vs. wtwtwt, $t g$ vs. $w^{t^{\text {tgwt }}}$ or tg vs. $w^{\text {tttg }}$ mice. Padj $\leq 0.05$, $\log _{2} F C \pm 0.25$. For DG males: $\operatorname{tg} n=4 ; w^{\text {tgwt }} n=7$; wt ${ }^{\text {wttg }} n=5$ and $w^{\text {wtwt }} n=5$; for DG females tg $n=7$; wt ${ }^{\text {tgwt }} n=5$; $w^{t^{w t t g}} n=4$ and $w^{t^{\text {twt }}} n=5$ and for CA1 males tg $n=6$; $w^{t^{\text {tgwt }}}$ $n=7 ; w^{w t t g} n=8$ and $w^{\text {twtwt }} n=6$.

Taken together, wt mice born to an APPPS1-21 tg father (wt tgwt) exhibited a distinct transcriptome compared to that of wt controls ( $\left.w^{w^{w t w t}}\right)$. Thus, their transcriptome seemed to resemble that of tg mice more than that of the wt control mice. On the contrary, wt mice born to an APPPS1-21 tg mother ( $w^{t^{w t t g}}$ ) mice, which did not show a cognitive impairment at 3 months of age, did not show many transcriptomic differences when compared to $w^{t^{w t w t}}$ or APPPS1-21 tg mice.

In order to investigate the level of similarity between wtgwt and tg mice, I evaluated the number of common genes. DG brain subregions from male and female wt born to an APPPS1-21 tg father (wt ${ }^{\text {tgwt }}$ ) shared almost $52 \%$ of genes with APPPS1-21 tg mice when both were compared to wt control mice (wtwtwt). In the case of the CA1 region from wt ${ }^{\text {tgwt }}$ and tg mice, which showed 
less transcriptomic changes at 3 months of age, from the 30 differentially expressed genes between $w^{\text {tgwt }}$ and $w^{t w t w t}$ only two were commonly expressed among $w^{\text {tgwt }}$ and tg mice (Figure 4.2.23G-I).

Additionally, only 2 in DG from males, 71 in DG from females and 8 in CA1 from males were statistically differentially expressed between $w^{\text {tgwt }}$ and tg mice (Figure 4.2.23A-I). From these genes, only a few were significantly different in both wt groups ( $w^{\text {twttg }}$ and $w^{\text {tgwt }}$ ) comparing with tg mice (4 for DGm and 29 for DGf and 2 for CA1m) (Figure 4.2.23G-I). This data supports the conclusion that wtgwt mice indeed exhibited more transcriptional similarities with tg mice than with wt control mice ( $\left.w^{t^{\text {twt }}}\right)$.

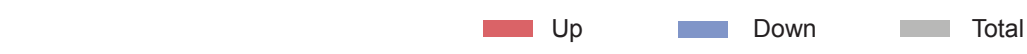

DG males

A

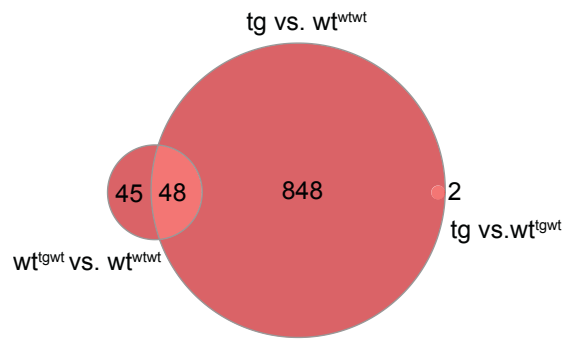

DG females

D

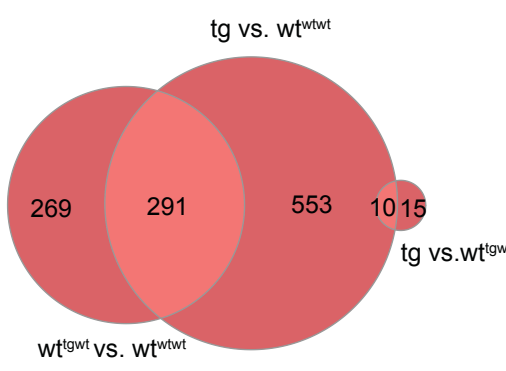

CA1 males

G

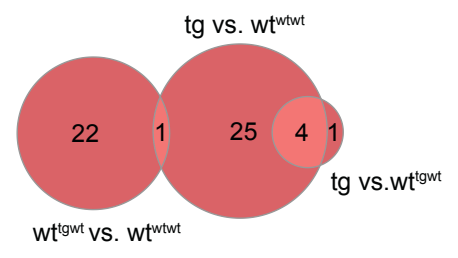

B

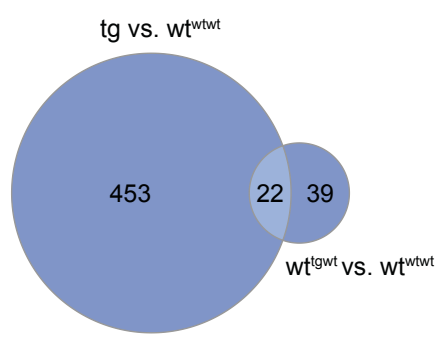

E

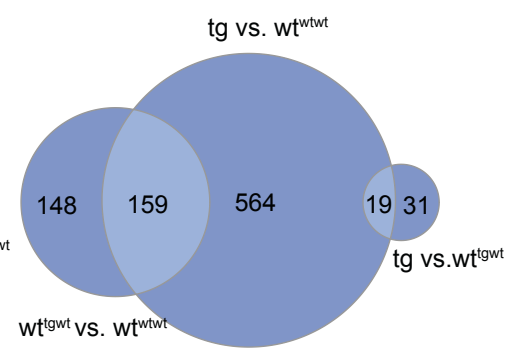

C

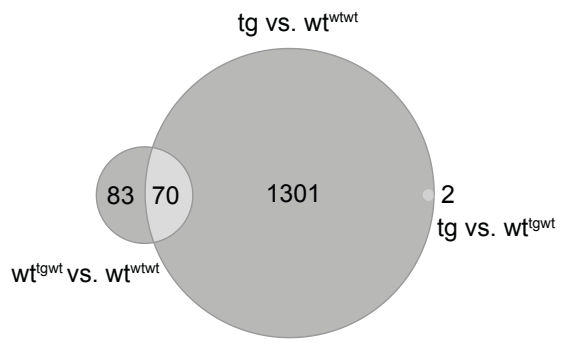

F

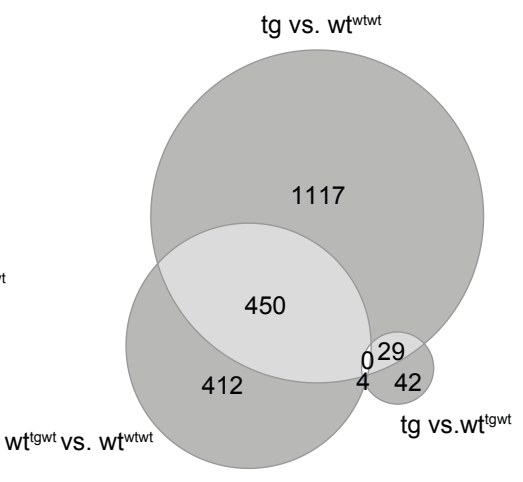

H $\operatorname{tg} v s . w t^{w t w t}$

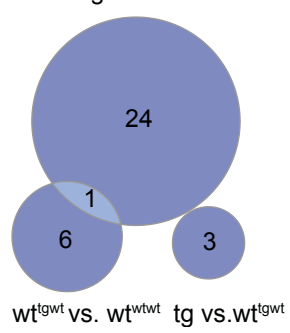

$\operatorname{tg}$ vs. $w t^{w t w t}$

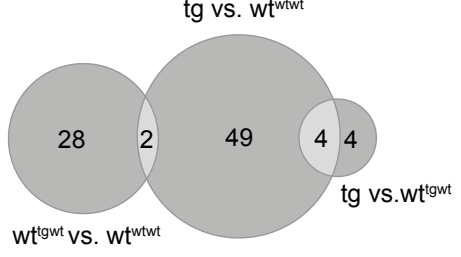


Figure 4.2.23. Around $50 \%$ of genes overlap when wild-type mice born to an APPPS1-21 transgenic father (wt $t^{\text {tgwt}}$ ) and APPPS-21 transgenic mice were compared to wtwtwt.

(A-I) Venn diagrams illustrating the overlap between up-/down-/total regulated genes in DG from males (DGm), in DG from females (DGf) and in CA1 from males (CA1m) from tg vs. wtwtwt, tg vs. $w^{t^{\text {tgwt }}}$ and $w^{\text {tgwt }}$ vs. $w^{\text {twtwt }}$ mice. Padj $\leq 0.05$, $\log _{2} F C \pm 0.25$. For $D G$ males: $\operatorname{tg} n=4 ; t^{\text {tgwt }} n=7$; $w^{\text {wttg }} n=5$ and $w t^{\text {wtwt }} n=5$; for

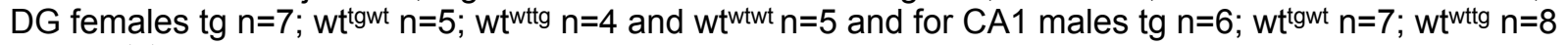
and $w^{t^{w t w t}} n=6$.

\subsubsection{Functional pathway analysis of wt mice originating from an APPPS1-21} transgenic mother or father

To further investigate the molecular state of wt mice born to APPPS1-21 tg parents I studied the functional pathways using first the whole dataset with GSEA (Subramanian et al., 2005) and then limiting to significant thresholds with ClueGO/Cytoscape (Shannon et al., 2003) (Bindea et al., 2009). Taking into account that the major transcriptomic changes were observed between wt born to an APPPS1-21 tg father (wttgwt) compared to wt control mice ( $\left.w^{\text {twtwt }}\right)$, I next analyzed these in functional analyses (Figure $4.1 .33 \mathrm{~A}$ and $\mathrm{C}$ ). Since $52 \%$ of differentially expressed genes were shared between wttgwt and tg mice, I expected to identify a certain overlap in the functional pathways affected in these mice. To achieve a first global notion of the biological processes, molecular functions and cellular components, I selected the top 25 most significant under and overrepresented pathways (nominal pvalue $\leq 0.05$ and FDR $\leq 0.25$ ) and ranked them by normalized enrichment score (NES). Positive pathways mean enrichment in wt controls ( $w^{t^{w t w t}}$ ), whereas negative pathways are enriched in wt ${ }^{\text {tgwt }}$ mice. Similarly as previously presented for $t g$ mice, these preselected pathways were manually reduced to main category groups.

Regarding biological processes, enriched genes in DG regions of wtgwt males and females compared to wt control mice were mainly linked to tissue and nervous system development, synapse and cell-cell adhesion (Figure 4.2.24A, C, D and F). On the contrary, genes underrepresented in DG from male and female wttgwt mice were mainly associated to mitochondrial process and respiratory chain, metabolic process, splicing and translation (Figure 4.2.24A, B, D and E). These were similar broader processes that were also affected in APPPS1-21 transgenic mice (Figure 4.1.8). Besides, several genes related to splice process were altered in wt mice born to an APPPS1-21 tg father (wtgwt) (Figure 4.2.24A-B). Thus, I decided to further investigate the alteration of splicing processing by analyzing the alternative exon usage in wt ${ }^{\text {tgwt }}$ and transgenic mice (Section 4.2.4). 
In the CA1 hippocampal subregion of wt ${ }^{\text {tgwt }}$ mice, enriched genes were explicitly connected with inflammation and immune process (Figure 4.2.24G and $\mathrm{H}$ ). In contrast, pathways associated with lipid metabolism and transport were underrepresented in this group (Figure 4.2.24G and I). Similar underrepresented pathways were found in CA1 subregion from APPPS1-21 transgenic mice (Figure 4.1.8G-I).

DG males

A

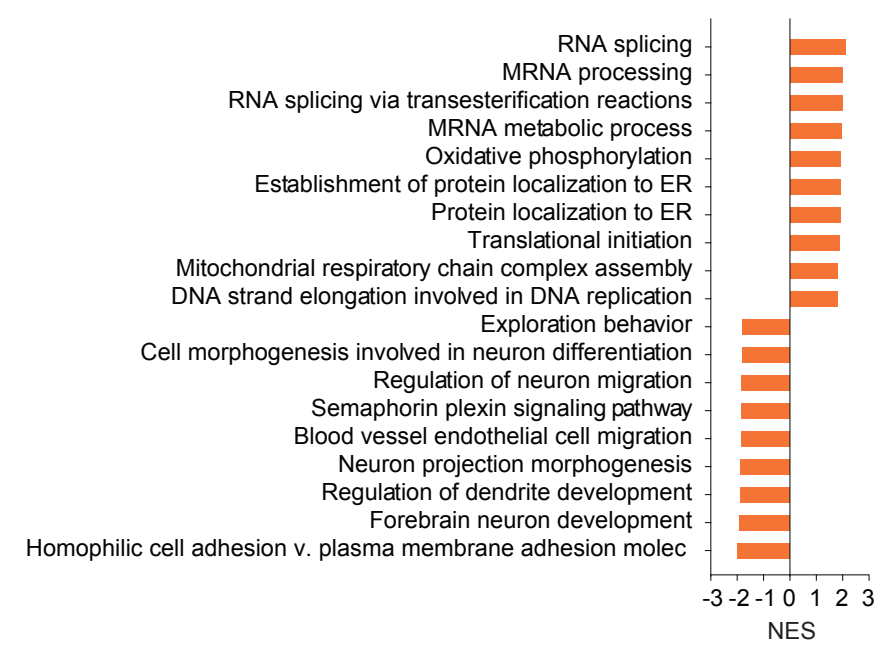

DG females

D

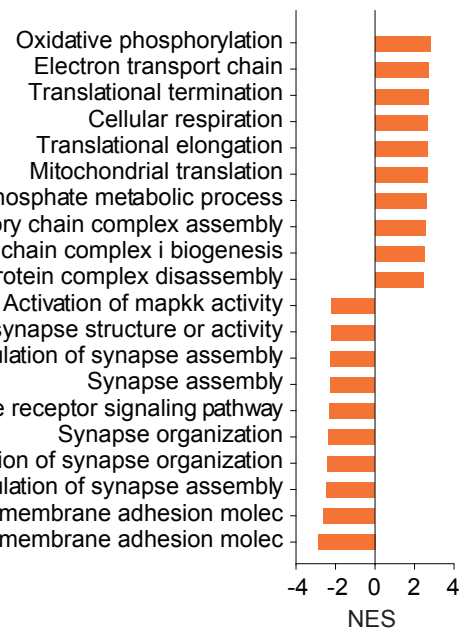

B

Negative in $\mathbf{w t}^{\mathrm{tg} w t}$

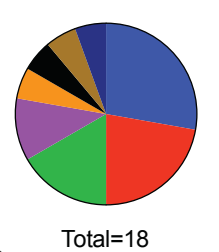

C Positive in $w^{\text {tgwt }}$

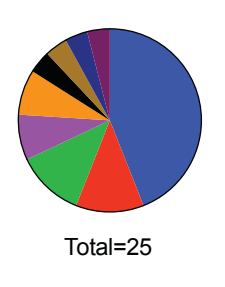
Cell cell adhesion via pla Homophilic cell adhesion via plasma membrane adhesion molec
$E$ Negative in $w^{t^{\text {tgwt }}}$

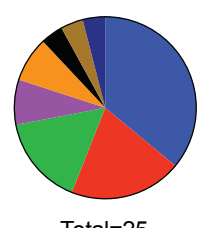

F Positive in $w^{t g w t}$ Regulation of synapse structure or activity Positive regulation of synapse assembly Glutamate receptor signaling pathway Synapse organization Regulation of synapse organization Mitochondrial respiratory chain complex assembly Mitochondrial respiratory che lood vessel endothelial cell migration

Neuron projection morphogenesis gulation of dendrite development

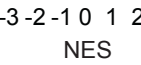

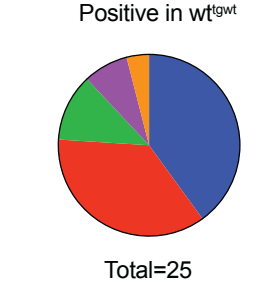$$
\text { Cellular protein complex disassembly - }
$$

RNA/DNA metabolic process

Mitochondria complex/ Respiratory chain Splicing

$\square$ Protein localization

$\square$ Organelle organization

Cellular component biogenesis

Translation

Cell metabolic process 

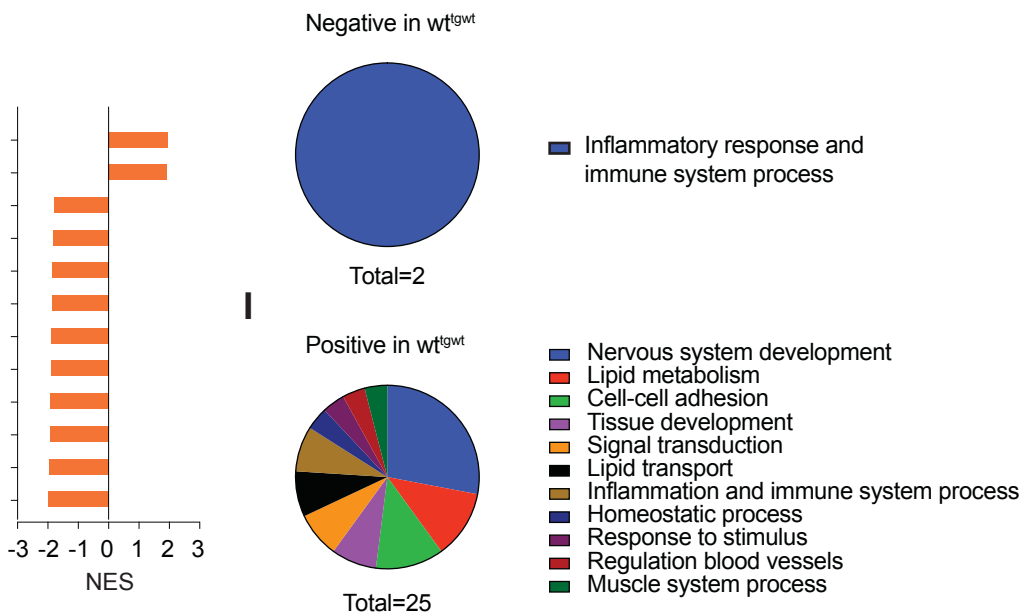

Figure 4.2.24. Biological processes are affected in the DG and CA1 regions of wild-type mice born to an APPPS1-21 transgenic father (wt ${ }^{\text {tgwt }}$ ) at 3 months of age.

(A, D and G) Top 10 preselected biological processes over or underrepresented within the whole dataset of genes in brain subregions of wt ${ }^{\text {tgwt }}$ or control (wtwtwt): DGm (A), DGf (D) and CA1m (G). NES= Normalized Enrichment Score. Positive NES means underrepresentation in wt $t^{\text {tgwt }}$ mice, whereas negative NES means overrepresentation in wt'tgwt mice. (B, C, E, F, H and I) Broader term summary of the top 25 overrepresented (C, F and I) and 25 underrepresented (B, E and F) pathways in brain subregions of wtgwt mice. Nominal pval $\leq 0.05$; FDR $\leq 0.25$. For DG males: wttgwt $n=7$ and $w^{t w t w t} n=5$; for $D G$ females $w^{t \text { tgwt }} n=5$ and $w^{t^{w t w t}} n=5$ and for CA1 males $w^{t^{t g w t}} n=7$ and $w^{t w t w t} n=6$.

Interestingly, genes associated to molecular functions showed a direct connection to the biological processes previously described. For example, mitochondrial complex and respiratory chain (oxidoreductase and hydrolase activity) or synapse (transporter and channel activity and receptor activity) were affected (Figure 4.2.25A-I). Other molecular functions were also remarkable in the DG of male and female from wt mice born to $t g$ father ( $\left.w^{t g w t}\right)$, such as protein binding and transcription regulator activity (Figure 4.2.25A-F). In the CA1 region from wtgwt, only pathways linked to translation and peptidase and transferase activity were significantly altered (Figure 4.2.25G-I). 
DG males

A

Oxidoreductase activity acting on NADPH quinone compound as acceptor Translation initiation factor binding Beta amyloid binding Transmembrane receptor protein phosphatase activity Neurotransmitter binding Excitatory extracellular ligand gated ion channel activity Notch binding TF activity direct ligand regulated sequence specific DNA binding Glutamate receptor activity Glucuronosyltransferase activity Retinoid $x$ receptor binding Retinoic acid receptor binding

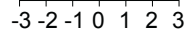

Negative in wtgw

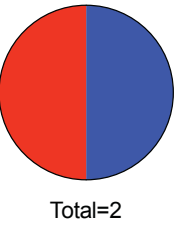

C

Positive in wt ${ }^{\text {tgwt }}$

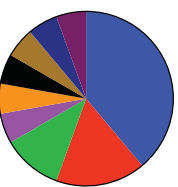

Total $=18$
Catalytic activity: oxidoreductase Transcription regulator activity

\section{DG females}

D

Structural constituent of ribosome Oxidoreductase activity acting on NADPH quinone compound as acceptor Oxidoreductase activity acting on a heme group of donors Oxidoreductase activity acting on NADPH Electron carrier activity Glutathione transferase activity Oxidoreductase activity acting on peroxide as acceptor

Glutathione peroxidase activity

Threonine type peptidase activity RRNA binding Transcription factor activity RNApol II transcription factor binding
Smad binding TF activity RNApol II core promoter proximal region sequence specific binding Divalent inorganic cation transmembrane transporter activity Map kinase kinase kinase activity Transmitter gated channel activity Transmembrane receptor protein kinase activity Glutamate receptor activity Transcriptional repressor activity RNApol II activating TF binding Transmembrane receptor protein serine threonine kinase activity

CA1 males

\section{G}

E

Negative in wtgwt

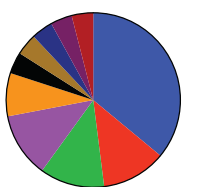

Total $=25$

F

Positive in wt ${ }^{\text {tgw }}$

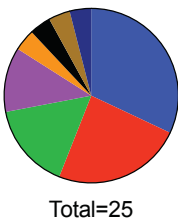

Transporter and channel activity

$\square$ Protein binding

므 Catalytic activity: kinase

Cofactor binding

- Amide binding

Catalytic activity: hydrolase

Receptor activity

H

Negative in $\mathbf{w t}^{\mathrm{tgwt}}$

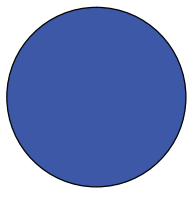

Translation

Translation initiation factor activity Transferase activity transferring nitrogenous group Aminopeptidase activity

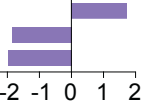

NES

Positive in wt $^{\mathrm{tg} w}$

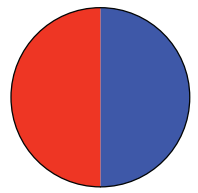

Catalytic activity: peptidase

Catalytic activity: transferase

Total $=2$ 
Figure 4.2.25. Molecular functions are affected in the DG and CA1 regions of wild-type mice born to an APPPS1-21 transgenic father ( $\mathrm{wt}^{\mathrm{tgwt}}$ ) at 3 months of age.

(A, D and G) Top 10 preselected molecular functions over or underrepresented within the whole dataset of genes in brain subregions of wtgwt or control (wtwtwt): DGm (A), DGf (D) and CA1m (G). NES= Normalized Enrichment Score. Positive NES means underrepresentation in $w^{t^{t g w t}}$ mice, whereas negative NES means overrepresentation in wtgwt mice. (B, C, E, F, H and I) Broader term summary of the top 25

overrepresented (C, $\mathbf{F}$ and $\mathrm{I})$ and 25 underrepresented $(\mathrm{B}, \mathrm{E}$ and $\mathbf{F})$ pathways in brain subregions of wt $\mathrm{t}^{\text {tgwt }}$ mice. Nominal pval $\leq 0.05$; FDR $\leq 0.25$. For DG males: $w^{\text {tgwt }} n=7$ and $w^{t w t w t} n=5$; for DG females $w^{t^{\text {tgwt }}} n=5$ and $w^{t w t w t} n=5$ and for CA1 males $w^{t g w t} n=7$ and $w^{t w t w t} n=6$.

Lastly, when analyzing cellular components, several genes related to components such as neuronal part, synapse and cytoskeletal part were enriched in DG and CA1 from male and females of wtgwt mice (Figure 4.2.26A, C, D, F, G and I). In contrast, genes linked to the ribosome, splicing and nuclear part were underrepresented in CA1 and DG of wt ${ }^{\text {tgwt }}$ mice (Figure 4.2.26A, B, D, E, G and H). These results are in accordance with the molecular functions and biological processes previously described for $w^{\text {tgwt }}$ mice (Figure 4.2.24 and 25).

DG males

A

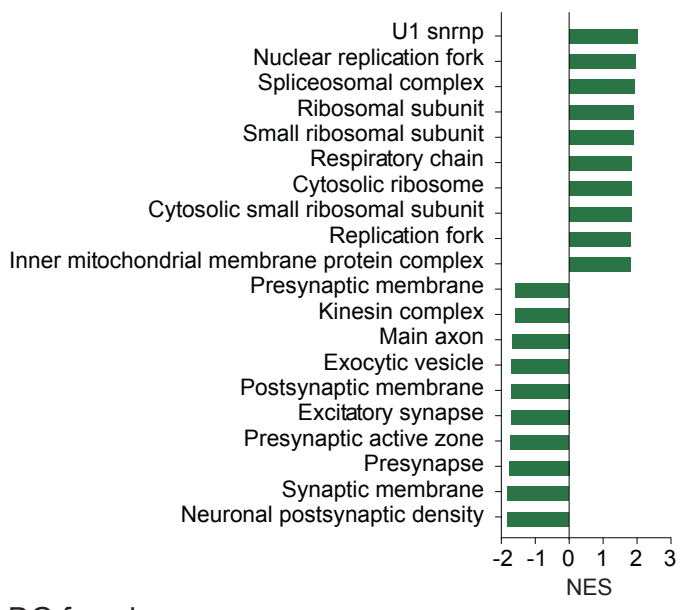

DG females

D

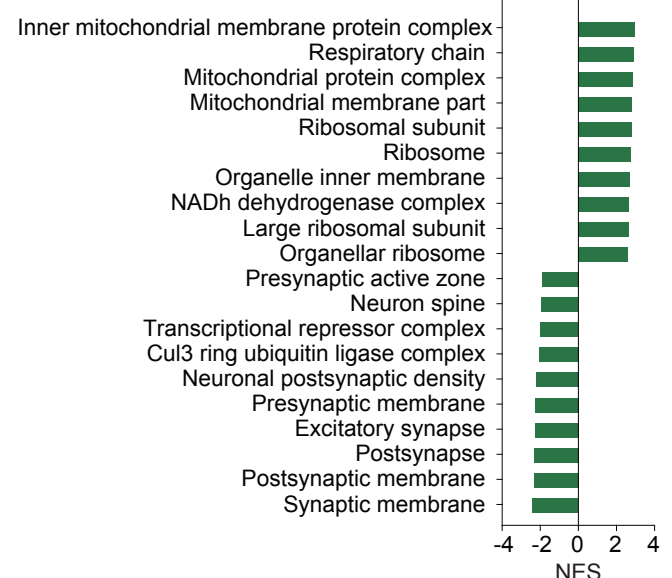

B

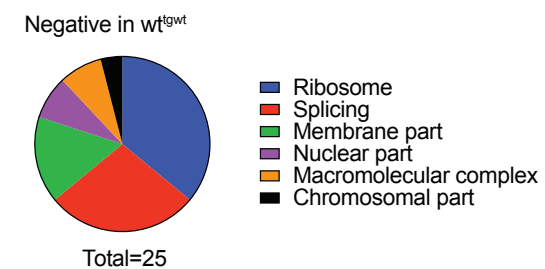

C

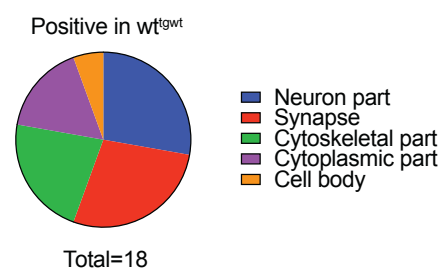

E

Negative in wt ${ }^{\text {tgmt }}$

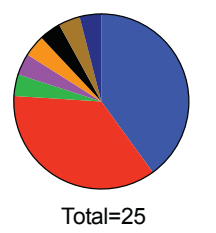

$\square$ Ribosome

Mitochondria complex/ Respiratory chain

$\square$ Membrane part

$\square$ Cytosolic part

Extracellular space

- Macromolecular complex

Organelle part

Splicing

$\mathbf{F}$

Positive in $w^{\text {tgwt }}$

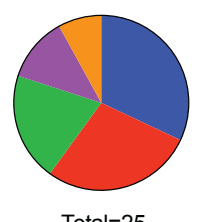

Synapse

Membrane part

$\square$ Neuron part

Macromolecular complex

$\square$ Cytoskeletal part 
CA1 males

G

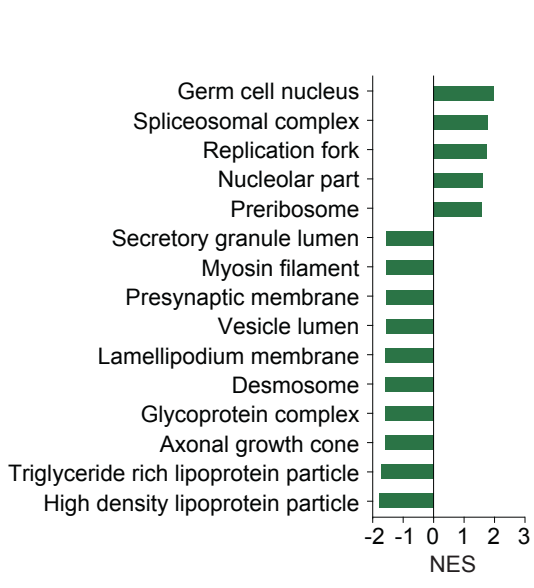

H

Negative in wtgnt

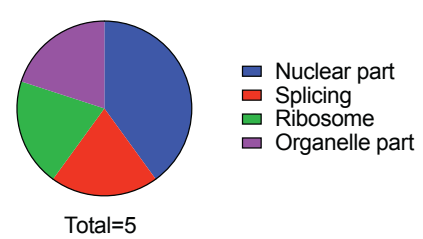

I

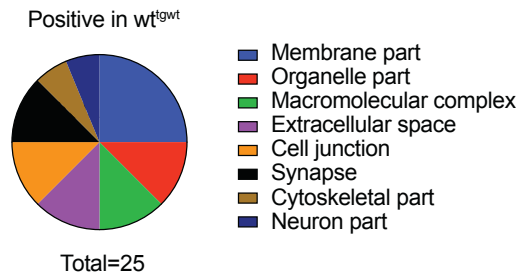

Figure 4.2.26. Cellular components are affected in DG and CA1 regions of wild-type mice born to an APPPS1-21 transgenic father ( $\left.w^{\text {tgwt}}\right)$ at 3 months of age.

(A, D and G) Top 10 preselected cellular components over or underrepresented within the whole dataset of genes in brain subregions of wt $\mathrm{t}^{\mathrm{tgwt}}$ or control (wtwtwt): DGm (A), DGf (D) and CA1m (G). NES=

Normalized Enrichment Score. Positive NES means underrepresentation in wt tgwt mice, whereas negative NES means overrepresentation in wtgwt mice. (B, C, E, F, H and I) Broader term summary of the top 25 overrepresented (C, F and I) and 25 underrepresented (B, E and F) pathways in brain subregions of wtgwt mice. Nominal pval $\leq 0.05$; FDR $\leq 0.25$. For DG males: wt $t^{\text {tgwt }} n=7$ and $w^{w t w t} n=5$; for DG females $w^{t^{\text {tgwt }} n=5}$ and $w^{\text {ttwt }} n=5$ and for CA 1 males $w^{\text {tgwt }} n=7$ and $w^{\text {twtwt }} n=6$.

Taken together, CA1 and DG subregions form wt mice born to APPPS1-21 tg fathers (wtgwt) showed moderate changes in gene expression highly linked to the cellular state earlier described for APPPS1-21 tg mice. CA1 and DG regions of $w^{t^{t g w t}}$ mice showed mainly changes in pathways related to nervous system development, synapse, splicing, energy metabolism, metabolic process and cell-cell adhesion. This data indicates that wt ${ }^{\text {tgwt }}$ mice present a different cellular state in the hippocampus than control wt mice ( $\left.w^{t w t w t}\right)$, probably due to a non-genetic transmission from their APPPS1-21 tg father. This is particularly interesting since both groups are wild-type mice.

To validate the relevance of the previously identified pathways, I used a second method based on a cutoff approach (ClueGO/Cytoscape) (Bindea et al., 2009) (Shannon et al., 2003) and submitted only differentially expressed genes to the analysis (padj $\leq 0.05$; $\log _{2} \mathrm{FC} \pm 0.25$ ). As previously described between $\mathrm{tg}$ and $\mathrm{w}^{\mathrm{t}}{ }^{\mathrm{w} w \mathrm{w}}$ mice, the number of pathways associated with upregulated genes was higher compared to the pathways linked to down-regulated genes in wtgwt compared to $w^{t^{w}{ }^{w t}}$ mice (Figure 4.2.27A-C). Furthermore, the DG $w^{t^{\text {tgwt }}}$ from male and female mice were more severely affected than the CA1 region (Figure 4.2.27A-C). Only genes related to 
regulation of gene expression were altered in the CA1 (Figure 4.2.27C and F). Notably, male $w^{t \text { tgwt }}$ mice were less affected than females, showing less altered pathways associated with significantly expressed genes. Despite this minor alteration in the DG of male wtgwt, some pathways in the DG region from both genders overlap with functional pathways affected in tg mice (Figure 4.1.11). Pathways related to nervous system development, synapse and ion transport were changed in both genders (Figure 4.2.27A-B and D-E). The molecular state of female DG was hugely affected, pathways linked to ribonucleotide metabolism, metabolic process, gene expression or chromatin modification were drastically affected in this region (Figure 4.2.27B and E).

A

DG males

B

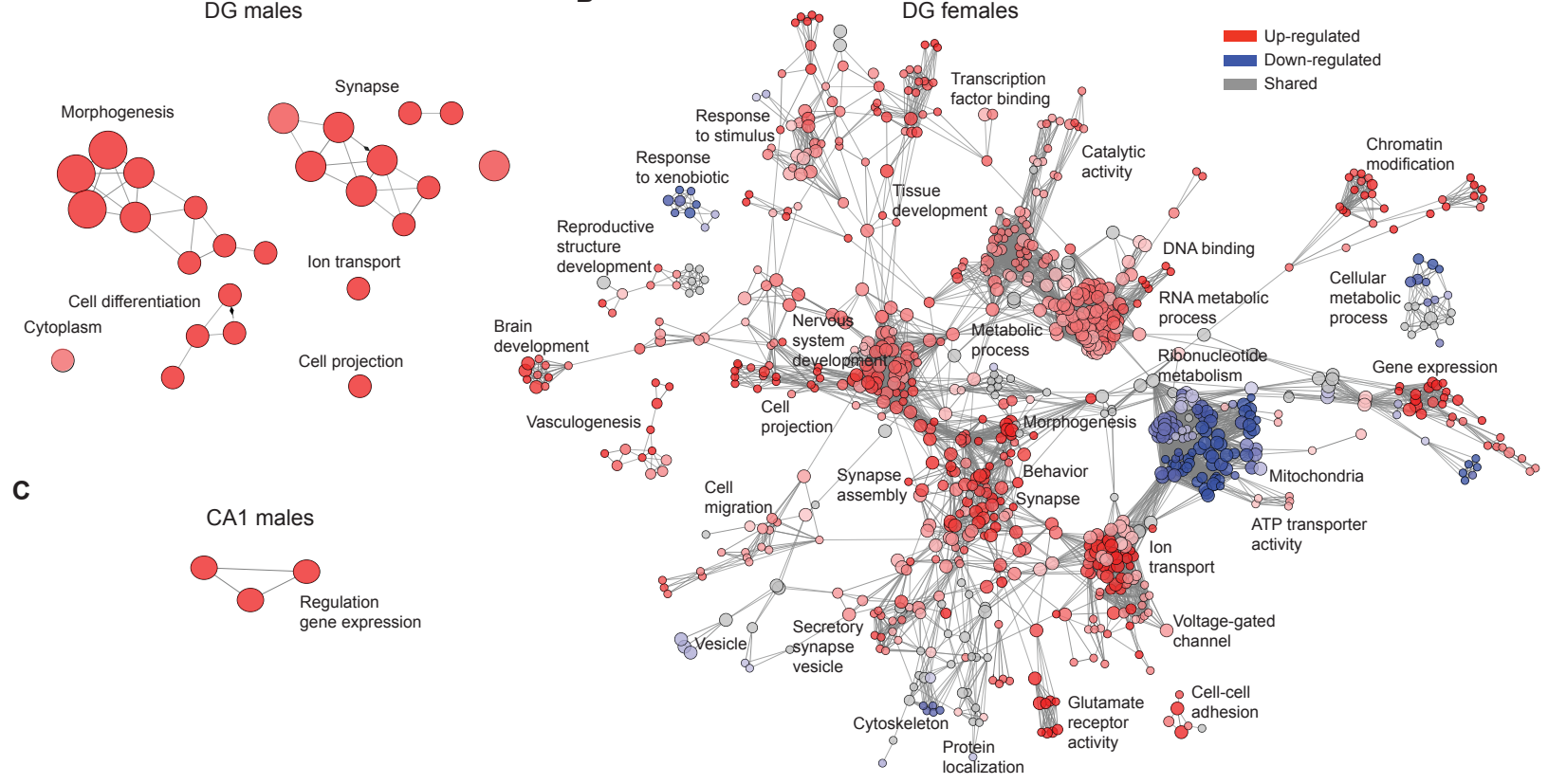

D

E DG females

F

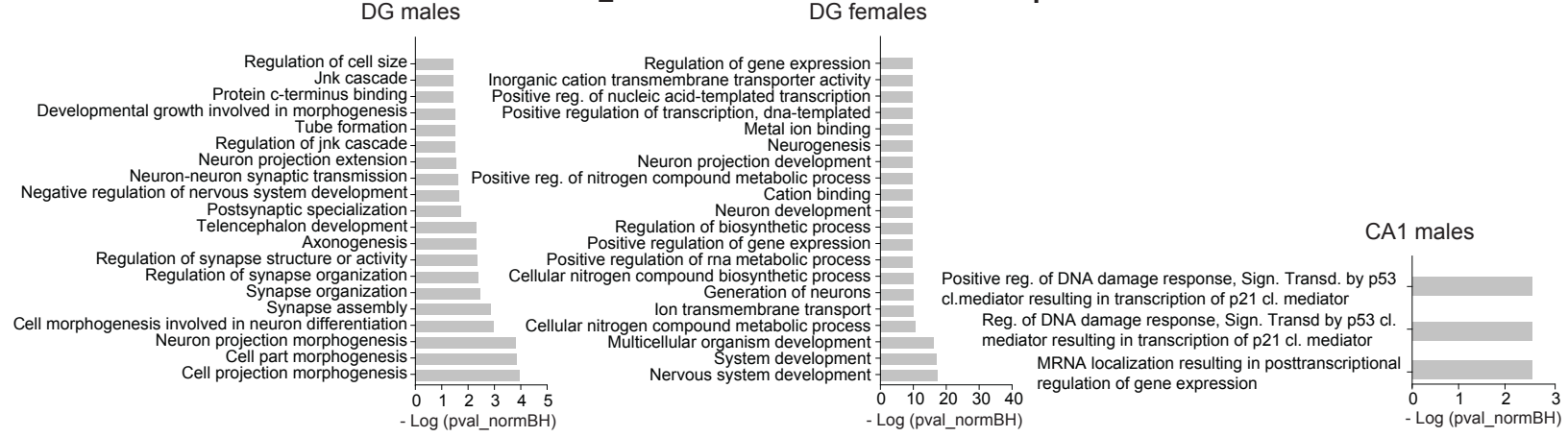


Figure 4.2.27. Functions affected in CA1 and DG of wild-type mice born to APPPS1-21 transgenic fathers.

(A-C) Network of functional categories (biological process, molecular function and cellular component ontologies) of significant differentially expressed genes in CA1 and DG from wild-type mice born to an APPPS1-21 transgenic father ( $\left.w^{t \text { tgwt }}\right)$. The size of the nodes correlates inversely with statistical significance. Categories with a red color spectrum correspond to up-regulated genes in wt tgwt mice and blue correspond to down-regulated genes. Categories that shared up- and down-regulated genes are represented in grey. Genes, padj $\leq 0.05$; $\log _{2} F C \pm 0.25$ and pathways, pval $<0.05$. (D-F) Top 20 significant functional pathways ranked by -log pval after Benjamini-Hochberg correction of significant differentially expressed genes in brain subregions of wt ${ }^{\text {tgwt }} v s$. wtwtwt mice. Genes, padj $\leq 0.05$; $\log _{2} \mathrm{FC} \pm 0.25$ and

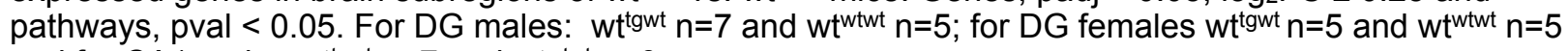
and for CA1 males $w^{t+g w t} n=7$ and $w^{t w t w t} n=6$.

These analyses reveal that several pathways defined by GSEA analysis also represented the top affected pathways since they also appeared in the more restrictive ClueGO/Cytoscape analyses (Figure 4.2.27A-F).

In addition to the number of shared genes between DG of wt ${ }^{\text {tgwt }}$ and $\operatorname{tg}$ mice (around $52 \%$ of genes overlapped) (Figure 4.2.23A-F), I analyzed the proportion of common pathways between these two groups compared to $w^{t^{w t w t}}$ mice. According to GSEA pathways, all three regions (DG from males and females and CA1from males) of wtgwt in almost all three GO terms categories showed approximately 30-40\% concordance with tg mice (Figure 4.2.28A-I). Importantly, the overlapped pathways increased extremely when only significant differentially expressed genes were analyzed using Cytoscape/ClueGO, around $65 \%$ of pathways overlapped between male and female in DG regions from w ${ }^{\text {tgwt }}$ and tg mice (Figure 4.2.29A-B). In the case of CA1 region, there was not any common pathway between wtgwt and tg mice (Figure 4.2.29C). It is important to indicate that this mechanism of overlapping pathways is limited to the name of the pathway and not the number of genes in that pathway. Therefore, it only offers a partial view of the overlapping. 
GSEA:

Biological process

A DG males

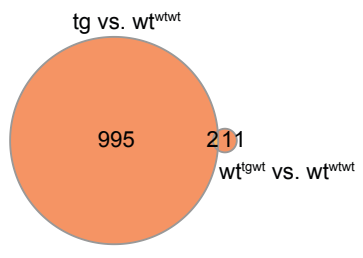

D DG males tg vs. $w t^{\text {wtwt }}$

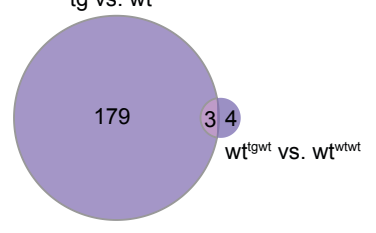

G DG males tg vs. wtwtwt

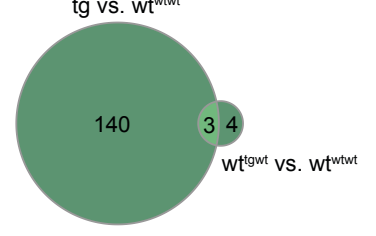

B

DG females

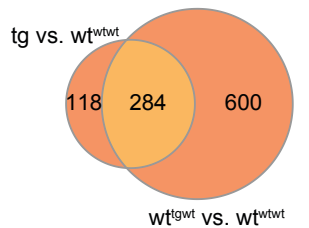

E DG females

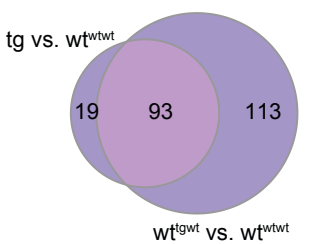

H DG females

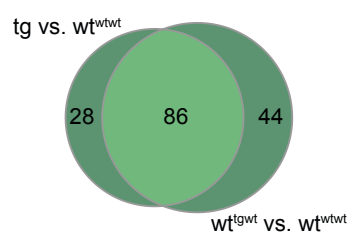

C CA1 males

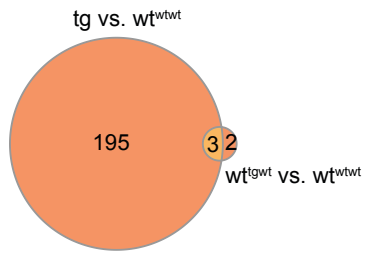

F CA1 males

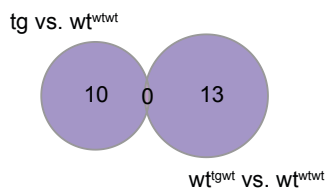

I CA1 males

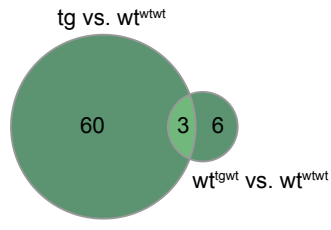

Figure 4.2.28. Around 30 to $40 \%$ of pathways overlap between wt mice born to an APPPS1-21 tg father (wttgwt) and APPPS1-21 tg mice when both were compared to wt control mice (wtwtwt).

(A-I) Venn diagrams illustrating overlap of biological processes (A-C), molecular functions (D-F) and cellular components (G-I) in tg vs. wtwtwt and wttgwt vs. wttwt comparisons. Pathways, padj $\leq 0.05$. For DG males: $\operatorname{tg} n=4$; $w^{\text {tgwt }} n=7$ and $w^{\text {twtwt }} n=5$; for $D G$ females $\operatorname{tg} n=7$; $w^{\text {tgwt }} n=5$ and $w^{\text {wtwt }} n=5$ and for CA1 males $\operatorname{tg} n=6 ; w^{\text {tgwt }} n=7$ and $w^{\text {twtwt }} n=6$.

ClueGO/ Cytoscape analysis:

A DG males

$\operatorname{tg}$ vs. $w^{\text {twtwt }}$

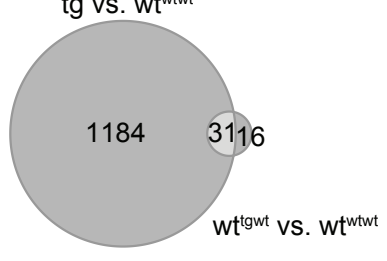

B DG females

tg vs. wtwtwt

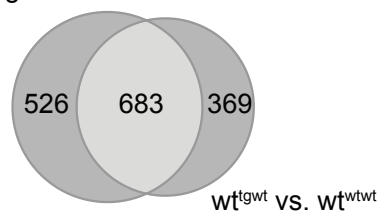

C CA1 males

$\operatorname{tg}$ vs. $w^{\text {twtwt }}$

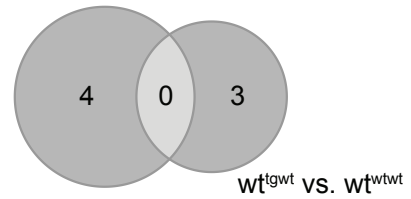


Figure 4.2.29. Around $65 \%$ of pathways overlap overlap between wt mice born to an APPPS1-21 $\mathrm{tg}$ father ( $w^{\text {tgwt}}$ ) and APPPS1-21 tg mice.

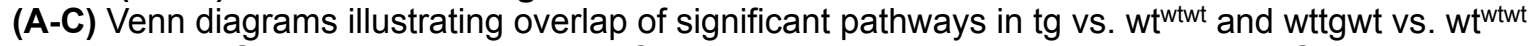
comparisons. Genes, padj $\leq 0.05$; log2FC \pm 0.25 and pathways, pval $<0.05$. For DG males: $\operatorname{tg} n=4$; wt $\mathrm{t}^{\text {tgwt }}$ $n=7$ and $w^{\text {twtwt }} n=5$; for DG females tg $n=7$; t $^{\text {tgwt }} n=5$ and $w^{t^{w t w t}} n=5$ and for CA1 males tg $n=6$; wt ${ }^{\text {tgwt }} n=7$ and $w^{t^{w t w t}} n=6$.

Overall, wild-type mice born to an APPPS1-21 tg father (wt ${ }^{\text {tgwt }}$ ) resembled APPPS1-21 tg mice to a greater extent than they did wtwtwt on cognitive performance, transcriptome and cellular state. Nonetheless, around $60 \%$ of whole dataset pathways (GSEA) and $40 \%$ from significant differentially expressed genes (ClueGO/Cytoscape) still differed between wt ${ }^{\text {tgwt }}$ and $t g$ mice (Figure 4.1.41A-I and Figure 4.1.42A-C). Not surprisingly, hippocampus subregions from tg mice showed different affected pathways when compared to $\mathrm{wt}^{\mathrm{tgwt}}$ and more altered pathways than $w^{\text {tgwt }}$. This variation could be attributed to the fact that transgenic mice are affected by the direct genetic alteration (induced APP and PS1 mutations) and the non-genetic transmission. However, the $w^{t^{\text {tgwt }}}$ mice are only affected by non-genetic transmission.

\title{
4.2.4.Alternative exon usage and pathway analysis in wild-type mice born to APPPS1-21 mice at $3 \mathrm{~m}$
}

\author{
4.2.4.1. Alternative exon usage in wild-type mice born to an APPPS1-21 tg father or \\ mother
}

Having established the transcriptome of wild-type mice born to APPPS1-21 tg parents and the genes that had an alternative exon usage of APPPS1-21 tg mice, I next focused on genes with alternative exon usage in wt mice born to APPPS1-21 tg parents. Taking into account that wt born to $t g$ fathers ( $w^{\text {tgwt}}$ ) showed changes in gene expression related to splicing process, I expected to identify significant alterations of exon usage. Thus, I analyzed the differentially expressed exons (padj 50.05 ) in wt mice born to an APPPS1-21 tg father ( wt $^{\text {tgwt}}$ ) or mother ( $w t^{w t t g)}$ both compared to wt control mice ( $\left.w t^{w t w t}\right)$.

The majority of exon usage changes fell into the mild $\left(\log _{2} \mathrm{FC} \pm 0.25\right)$ to medium $\left(\log _{2} \mathrm{FC} \pm 0.50\right)$ categories in $w^{t g w t}$ and $w^{t w t g}$ compared to wt control mice (wtwtwt) (Figure 4.2.30A-B). Moreover, some genes showed severely $\left(\log _{2} \mathrm{FC} \pm 1.00\right)$ exon changes as well in wt ${ }^{\text {tgwt }}$ mice (154 (total) for DGm, 2280 (total) for DGf and 2 (total) for CA1m) and wtwttg mice (14 for DGm, 1329 for DGf and 0 for $\mathrm{CA} 1 \mathrm{~m}$ ) compared to wt control mice $w^{t^{w t t g}}$ (Figure 4.2.30A-B). Figure 4.2.30C-E shows the 
number of genes with significant alternative exon usage that differed between control wt mice and experimental groups ( $\mathrm{wt}^{\mathrm{tg} w \mathrm{t}}$ and $\mathrm{wt}^{\mathrm{t}}{ }^{\mathrm{ttg}}$ ) at the mild category (padj $\leq 0.05$ and $\log _{2} \mathrm{FC} \pm 0.25$ ). The greatest difference regarding alternative exon usage occurs in the DG from males and females of wt mice born to APPPS1-21 tg fathers (Figure 4.2.30A-D). Here, I found thousands of genes using alternative exons (1876 (total) for DG from males and 7336 (total) for DG from females). Changes in alternative exon usage in the CA1 of males are only 106 (total). While changes in wtwttg mice compared to control wt mice were mild in the DG or negligible in the CA1 region, a vast number of alternative exon usage was observed in $w^{t^{\text {tgwt }}}$, specifically in DG regions. These changes are mainly associated with a shift towards inclusion of exons (Figure 4.2.30A-E).

Exon inclusions Exon exclusions Total

A

\begin{tabular}{|l|l|l|l|}
\hline padj $\leq 0.05$ & Severe & Medium & Mild \\
\hline Total $=$ incl + excl & Log $_{2} \mathrm{FC} \pm 1.00$ & $\log _{2} \mathrm{FC} \pm 0.50$ & Log $_{2} \mathrm{FC} \pm 0.25$ \\
\hline DGm & $154=132+22$ & $839=682+157$ & $1876=1377+499$ \\
\hline DGf & $2280=1991+289$ & $5910=3946+1964$ & $7336=4513+2823$ \\
\hline CA1m & $2=2+0$ & $25=15+10$ & $106=59+47$ \\
\hline
\end{tabular}

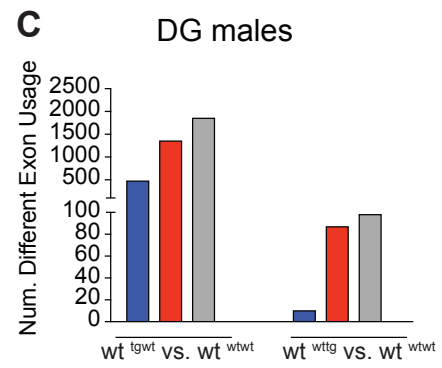

B

\begin{tabular}{|l|l|l|l}
\hline padj $\leq 0.05$ & Severe & Medium & Mild \\
\hline Total $=$ incl + excl & $\log _{2} \mathrm{FC} \pm 1.00$ & $\log _{2} \mathrm{FC} \pm 0.50$ & $\log _{2} \mathrm{FC} \pm 0.25$ \\
\hline DGm & $14=10+4$ & $47=42+5$ & $99=88+11$ \\
\hline DGf & $1329=1250+79$ & $2200=1834+366$ & $2553=1966+587$ \\
\hline CA1m & $0=0+0$ & $0=0+0$ & $0=0+0$ \\
\hline
\end{tabular}

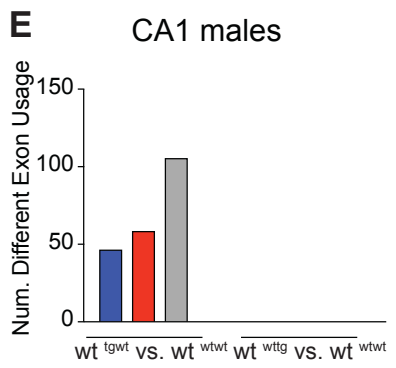

Figure 4.2.30. Drastic alteration in alternative exon usage in DG and CA1 of wild-type mice born to an APPPS1-21 transgenic father and almost negligible changes in wild-type offspring from APPPS1-21 transgenic mothers compared to control wild-type mice.

(A-B) Number of significant alternative exon usage with different levels of changes (severe $( \pm 1.00)$, medium ( \pm 0.50$)$ and mild $( \pm 0.25)$, established by the $\log _{2} F C$ ) in wt ${ }^{\text {tgwt }}$ and wtwttg mice compared to wtwtwt mice. Exon usage, padj $\leq 0.05$. (C-E) Number of significant alternative exon usage in DG from males (C) and females (D) and CA1 from male (E) $w^{t^{t g w t}}$ and $w^{\text {twtg }}{ }^{\text {mice }}$ compared to $w^{\text {twtwt }}$ mice. Red columns represent significant alternative inclusion exons; blue columns represent significant alternative exclusion exons and greys are the sums of previous two. Exons, padj $\leq 0.05$; $\log _{2} F C \pm 0.25$. For DG males: $w^{t \text { tgwt }}$

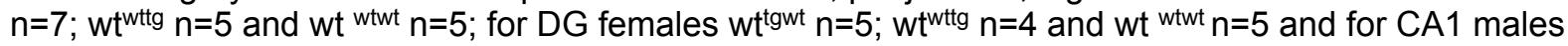

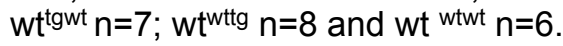

In contrast, wt mice born to an APPPS1-21 tg father (wt'tgt) or mother (wtwttg) showed less alternative exon usage variability than APPPS1-21 $\mathrm{tg}$ mice all of them compared to wt control 
mice. Comparing the wt tgwt males to tg mice, only a maximum of 31 differentially expressed exons in CA1 region were detected (Figure 4.2.31A-C). In the case of CA1 and DG regions of $w^{\text {wttg }}$ mice, some changes in exon usage were detected, but these changes were lower than those between $w^{w t w t}$ and tg mice in CA1 and DG regions (Figure 4.2.31A-C).
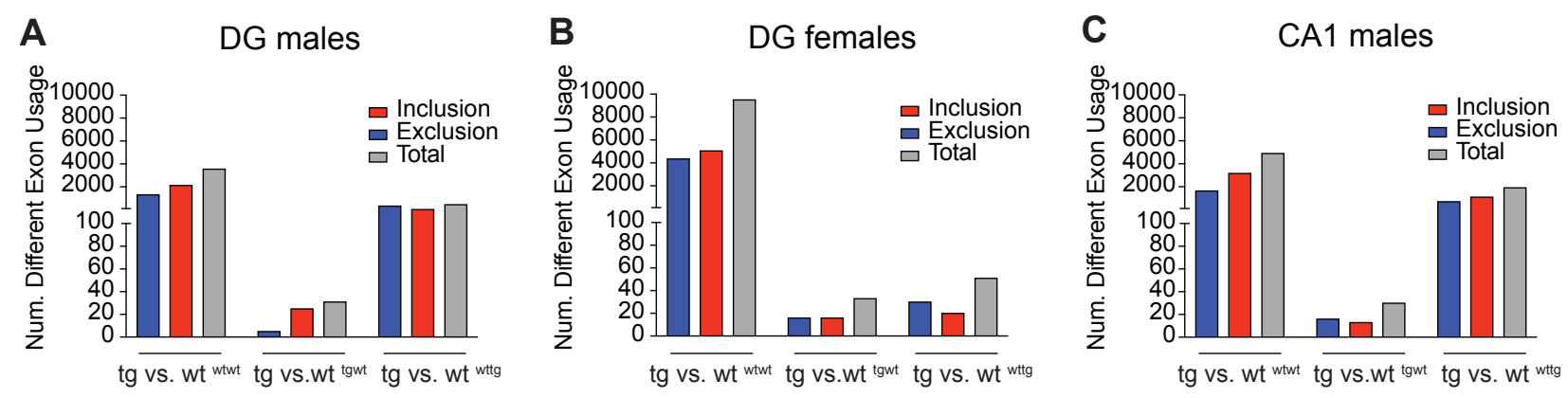

Figure 4.2.31. Small changes alternative exon usage were identified between wt mice born to an APPPS1-21 tg father ( $w^{\left.t^{t}{ }^{w t}\right)}$ or mother ( $w^{\mathrm{wttg}}$ ) and transgenic APPPS1-21 offspring.

(A-C) Number of significant differentially spliced genes in DG from males (A) and females (B) and CA1 from male (C) of wtwtwt, $w^{t^{t g w t}}$ and $w^{t^{w t t g}}$ mice compared to APPPS1-21 transgenic mice. Red columns represent significant alternative inclusion exons; blue columns represent significant alternative exclusion exons and greys are the sums of previous two. Genes, padj $\leq 0.05$; $\log _{2} F C \pm 0.25$. For DG males: $\operatorname{tg} n=4$; $w^{t^{\text {tgwt }}} n=7$; $w^{\text {twtg }} n=5$ and $w^{\text {wtwt }} n=5$; for DG females tg $n=7$; $w^{\text {tgwt }} n=5$; $w^{\text {wttg }} n=4$ and $w^{t^{\text {wtwt }}} n=5$ and for CA1 males tg $n=6$; $w^{t^{\text {tgwt }} n=7 ; ~ w t w t t g ~} n=8$ and $w^{\text {twtwt }} n=6$.

I next focused the studies on alternative exon usage in wt mice born to tg fathers, because not many spliced events were observed in wtwttg mice. Comparing the CA1 and DG regions among each other in $w^{t^{\text {tgwt }}}$ mice, I did not identify many common spliced genes between DG from males and females and CA1 from males, although several genes with alternative exon usage were common between DG from females and males (1172) (Figure 4.2.32C). Common genes between DG males and females represent $66.7 \%$ of the total genes with different spliced events in DG from males and $21.4 \%$ in DG from females (Figure $4.2 .32 \mathrm{~A}-\mathrm{C}$ ).

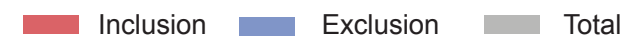

A

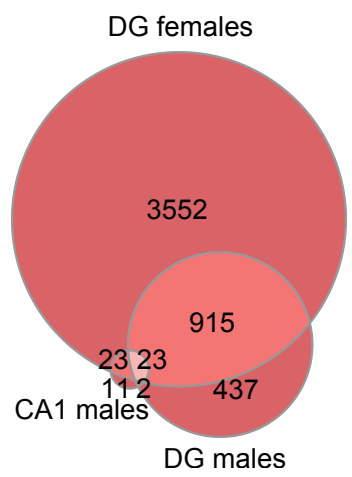

B

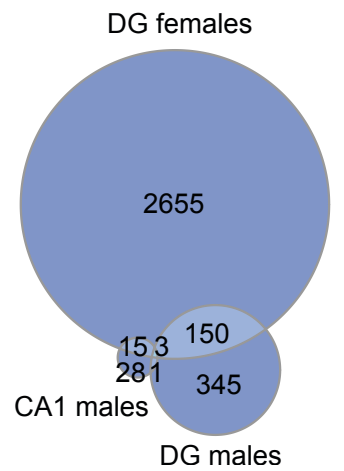

C

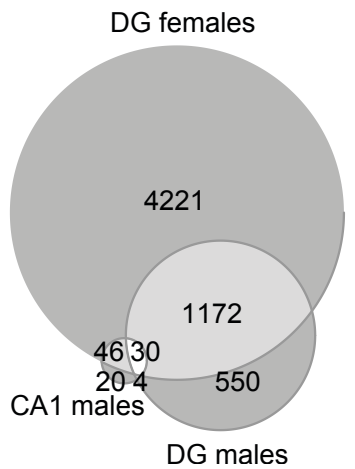


Figure 4.2.32. A large number of identical genes showed alternative exon usage in CA1 and DG regions of wild-type mice born to APPPS1-21 transgenic fathers.

(A-C) Venn diagrams illustrating overlap of spliced genes in DG from males and females and CA1 from males in wtgwt vs. wtwtwt comparison. Red color represents significant alternative inclusion exons; blue represents significant alternative exclusion exons and greys are the sums of previous two. Genes, padj $\leq$ 0.05; $\log _{2} F C \pm 0.25$. For DG males: $w^{\text {tgwt }} n=7$; $w^{\text {wttg }} n=5$ and $w^{\text {wtwt }} n=5$; for DG females: $w^{t^{\text {tgwt }}} n=5$; $w t^{\text {wttg }}$ $n=4$ and $w^{t^{w t w t}} n=5$ and for CA1 males: $w^{t^{\text {tgwt }}} n=7 ; w^{\text {wttg }} n=8$ and $w^{t^{w t w t}} n=6$.

4.2.4.2. Functional pathways linked to alternative exons in wild-type mice born to an APPPS1-21 transgenic father or mother

In order to define biological processes affected by genes that had an alternative exon usage in wt mice born to an APPPS1-21 tg father (wtgwt) compared to control wt mice, I screened the pathways linked to these genes using Cytoscape/ClueGO (Bindea et al., 2009; Shannon et al., 2003). I also established the splicing similarities between wtgwt and APPPS1-21 tg mice. Almost all pathways identified in DG and CA1 regions of $w^{t}{ }^{\text {tgwt }}$ mice were connected to inclusion events (Figure 4.2.33A-C). Analyzing only the top 20 most significant biological or molecular functions in CA1 and DG of wtgwt mice, I recognized that a vast number of them were connected with nervous system development ( 9 for DGm, 1 for DGf and 0 for CA1), signaling receptor activity (2 for DGm, 5 for DGf and 1 for CA1), metabolic process (0 for DGm, 3 for DGf and 0 for CA1), cellular component organization ( 0 for DGm, 3 for DGf and 0 for CA1) and cell and neuronal projection (3 for DGm, 2 for DGf and 0 for CA1) (Figure 4.2.33A-C). Almost all global categories were also affected in APPPS1-21 tg mice, as previously reported in figure 4.1.14 and 16. 

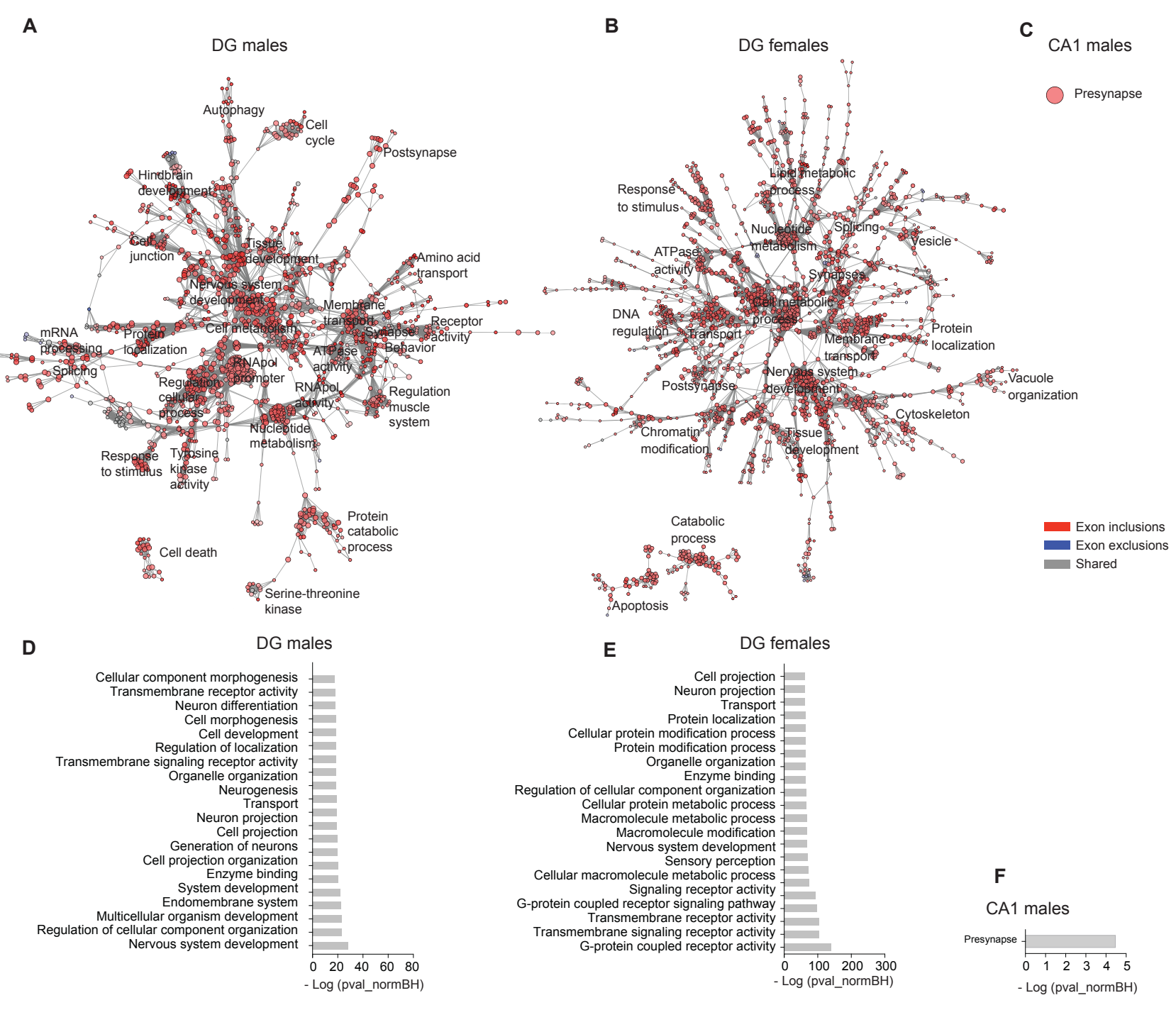

Figure 4.2.33. Several functions were affected by alternative exon usage in genes of wild-type mice born to APPPS1-21 transgenic fathers.

(A-C) Network of functional categories (biological process, molecular function and cellular component ontologies) of significant differentially spliced genes in CA1 and DG regions of wt mice born to APPPS1-21 $\operatorname{tg}$ fathers. The size of the nodes correlates inversely with statistical significance. Categories with a red color spectrum correspond to pathways associated with genes with inclusion of exons in wtgwt mice and with blue correspond to exclusion of exons. Categories that shared genes with inclusion and exclusion of exons are represented in grey. Genes, padj $\leq 0.05$; $\log _{2} \mathrm{FC} \pm 0.25$ and pathways, pval $<0.05$. (D-F) Top 20 significant functional pathways ranked by -log pval after Benjamini-Hochberg correction of significant differentially expressed genes in brain regions of $w^{t^{\text {tgwt }}} v s$. $w^{\text {wtwt }}$ mice. For DG males: $w^{\text {tgwt }} n=7$ and ${ }^{\text {twtwt }}$ $n=5$; for DG females $w^{\text {tgwt }} n=5$ and $w^{t^{w t w t}} n=5$ and for CA1 males $w^{t^{\text {tgwt }}} n=7$ and $w^{\text {twtt }} n=6$.

These results suggest that non-genetic factors transmitted from APPPS1-21 transgenic fathers promote a strong alteration of the transcriptome and alternative exon usage in both offspring, wild-type and transgenic mice. Besides, these results provide evidence for a substantial variation of alternative exon usage between the two wild-type groups, namely control wild-type (wtwtwt) mice and those originating from a transgenic father (wtgwt). 
To verify the similarities in terms of genes and pathways affected by alternative exon usage between $t g$ and $w^{\text {tgwt, }}$ I identified the number of common genes, and the pathways associated with them, both of them were compared to wt control mice. In DG from wt ${ }^{\text {tgwt }}$ males around $50 \%$ of genes with an alternative exon usage were also altered in tg mice (Figure 4.2.34A-C). From DG females, almost $75 \%$ of the genes were commonly affected (Figure 4.2.34D-F) and in CA1 from males around $60 \%$ of the genes (Figure $4.2 .34 \mathrm{G}-\mathrm{I}$ ). These results indicate a clear relation between $w^{\text {tgwt }}$ and tg mice regarding changes in exon usage, which is stronger than the connection concerning differentially expressed genes (Figure 4.2.23).

$$
\text { Inclusion }=\text { Exclusion } \square \text { Total }
$$

DG males

A

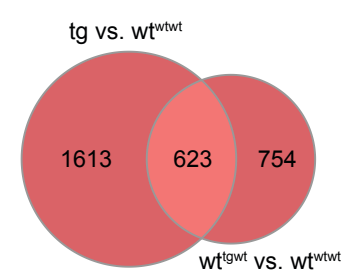

DG females

D

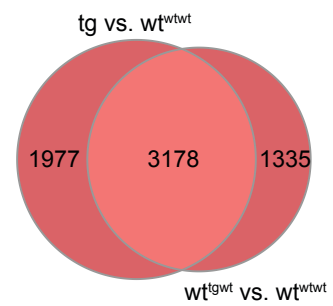

CA1 males

G

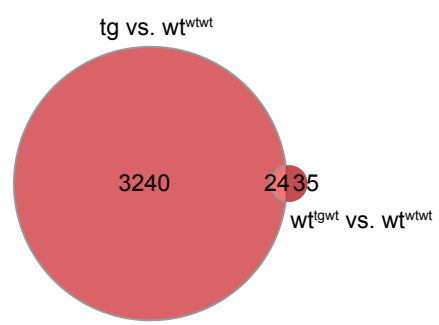

B

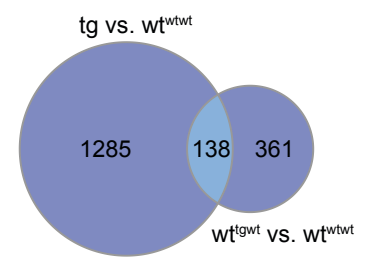

$\mathrm{E}$

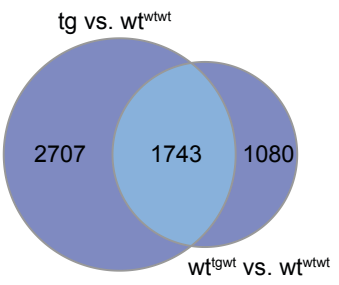

H

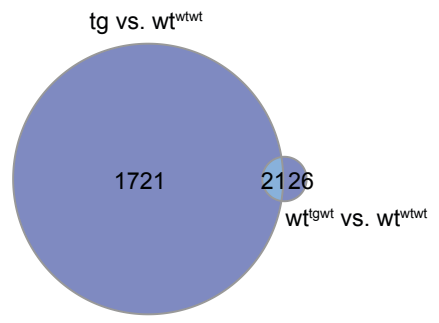

C

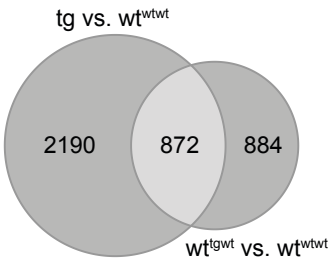

$\mathbf{F}$

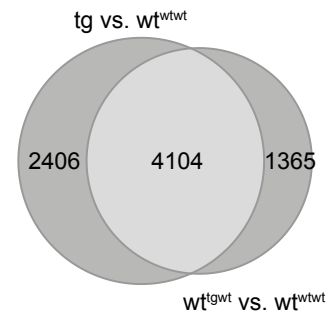

Figure 4.2.34. A large number of differentially spliced genes overlap between wt mice born to APPPS1-21 tg fathers and APPPS1-21 tg mice when both were compared to wt control mice (wtwtwt).

(A-I) Venn diagrams illustrating overlap of differentially spliced genes in $t g v s$. wtwtwt and $w^{\text {tgwt }} v s$. wtwtwt mice. Red color represents significant alternative inclusion exons; blue represents significant alternative exclusion exons and greys are the sums of previous two. Genes, padj $\leq 0.05 ; \log _{2} \mathrm{FC} \pm 0.25$ and pathways, pval $<0.05$. For DG males: $\operatorname{tg} n=4$; wt $n=7$ and $w^{t w t w t} n=5$; for $D G$ females $\operatorname{tg} n=7$; t $^{\text {tgwt }} n=5$

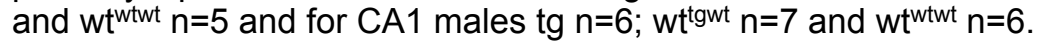


Also, I identified the overlap of differentially spliced and expressed genes in wt ${ }^{\text {tgwt }}$ mice (38 for DGm, 355 for DGf and 0 for CA1m) (Figure 4.2.35A-C). Only a $25 \%$ of differentially expressed genes in $D G$ from males also showed an alternative exon usage (Figure 4.2.35A). This was around $41 \%$ in DG from females (Figure 4.2.35B). And in CA1 differentially expressed genes did not show alteration of the spliced events (Figure 4.2.35C). These numbers differ from the number of common genes identified in transgenic mice, where there was an overlap of around $30 \%$ in all three regions (Figure 4.1.48A-I).
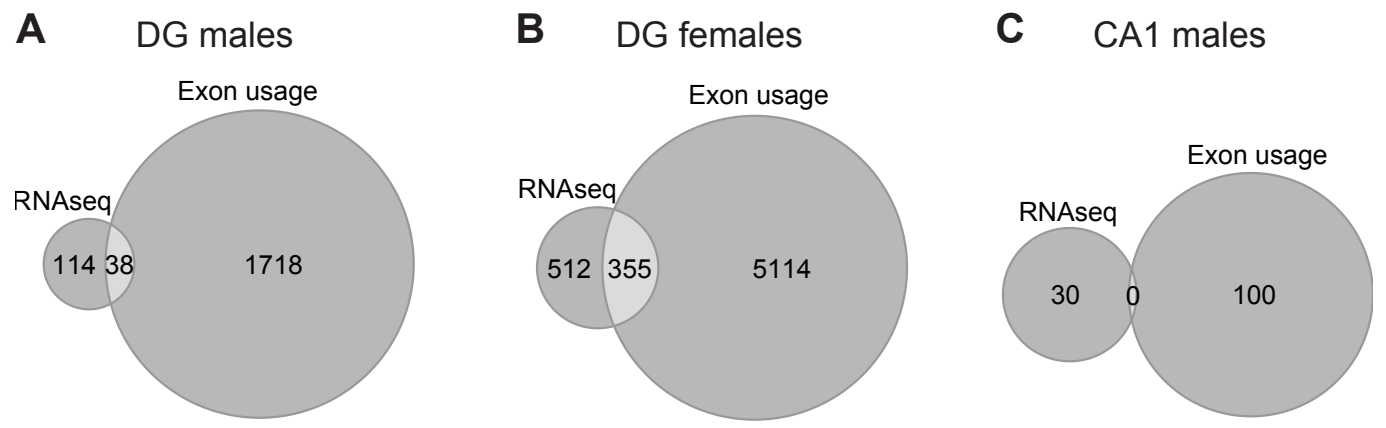

Figure 4.2.35. Subregion variability regarding common genes affected by differentially expressed and spliced genes in wt mice born to APPPS1-21 tg fathers (wt ${ }^{\text {tgwt}}$ ) when were compared to wt control mice (wtwtwt).

(A-C) Venn diagrams illustrating overlap of genes differentially expressed and differentially spliced genes in wtgwt vs. wtwtwt mice. Genes, padj $\leq 0.05 ; \log _{2} \mathrm{FC} \pm 0.25$ and pathways, pval $<0.05$. For DG males: $w^{t^{t g w t}} n=7$ and $w^{t^{w t w t}} n=5$; for DG females $w^{t^{t g w t}} n=5$ and $w^{t^{w t w t}} n=5$ and for CA 1 males $w^{\text {tgwt }} n=7$ and wtwtwt $\mathrm{n}=6$.

Interestingly, differentially expressed genes that had an alternative exon usage in DG from male and female $w^{\text {tgwt }}$ mice were related to similar pathways as those from genes differentially expressed and spliced in APPPS1-21 tg mice (Figure 4.1.16). These included pathways linked to nervous system development, synapse, transmembrane regulation, chromatin modification and RNA metabolic process (Figure 4.2.36). 

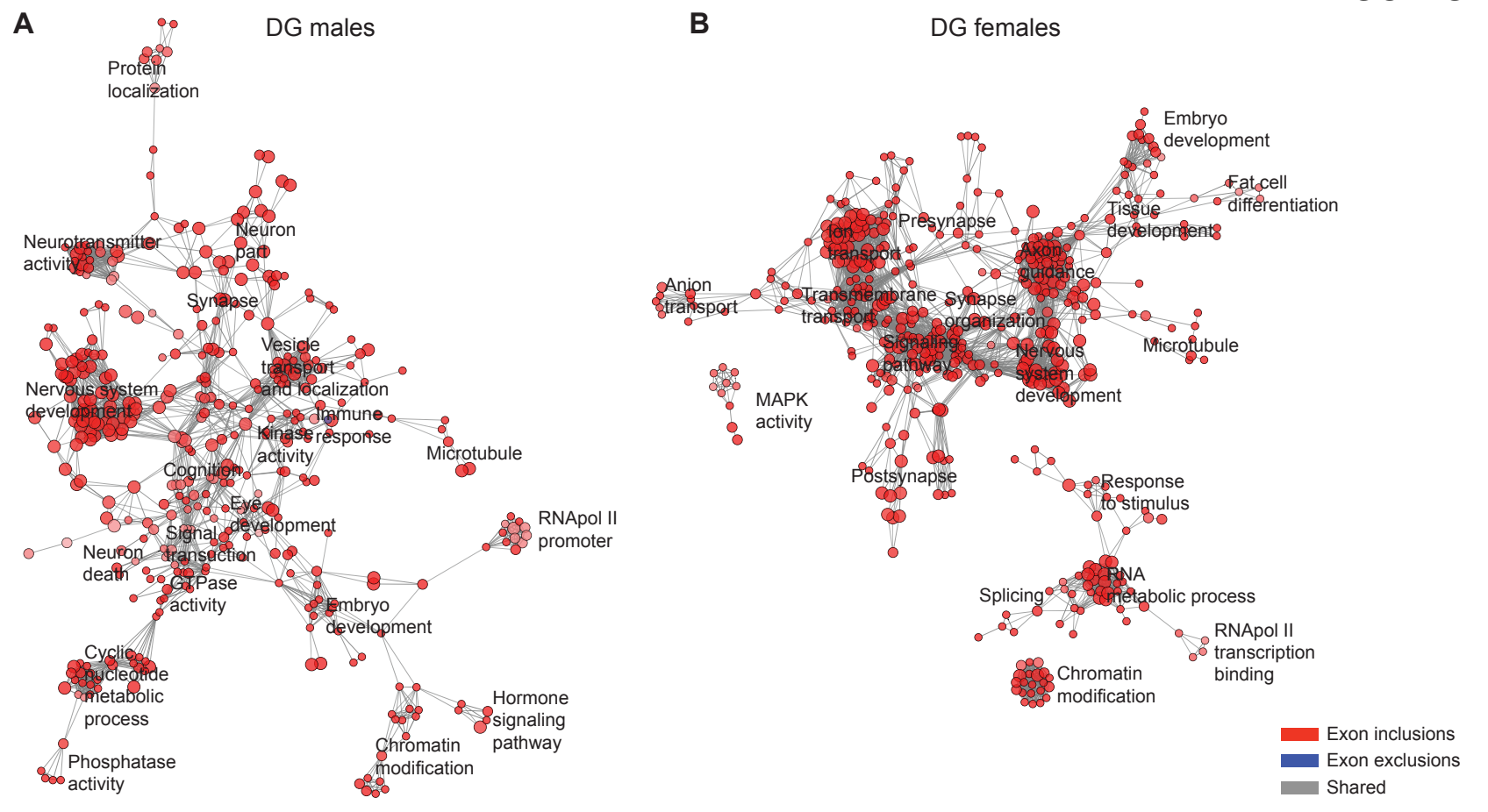

Figure 4.2.36. Several networks were affected by differentially expressed and spliced genes in DG region of wild-type mice born to APPPS1-21 transgenic mice.

(A-B) Network of functional categories (biological process, molecular function and cellular component ontologies) of significant differentially expressed and spliced genes in DG of wt $\mathrm{t}^{\mathrm{tgt}}$ mice. The size of the nodes correlates inversely with statistical significance. Categories with a red color spectrum correspond to pathways associated with genes with inclusion of exons and up-regulated genes in wtgwt mice and blue correspond to exclusion of exons and down-regulated genes. Genes, padj $\leq 0.05$; $\log _{2} \mathrm{FC} \pm 0.25$ and

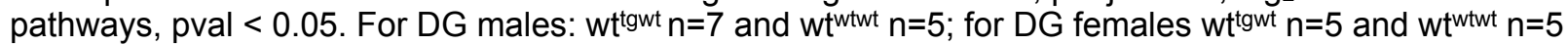
and for CA1 males wt ${ }^{\text {tgwt }} n=7$ and $w^{\text {wtwt }} n=6$.

\subsubsection{Non-genetic inheritance of APPPS1-21 traits}

Previously, I described the cognitive performance, the transcriptome and the splicing events from wt and tg mice born to APPPS1-21 transgenic fathers or mothers. I observed that wt mice born to tg mothers (wtwttg) did not show many differences compared to wt control mice. In contrast, wt mice born to tg fathers ( $w^{\text {tgwt }}$ ) showed a strong variability compared to wt control mice ( $w^{\text {wtwt }}$ ) and strongly resembled APPPS1-21 tg mice. Thus, I hypothesize that these phenotypes were likely mediated via sperm transmission of specific non-genetic factors. Several non-genetic factors of transmission, such as histone modifications, DNA methylation or noncoding RNAs (ncRNAs), were described in the last years (Denham et al., 2015; Gapp et al., 2014; Terashima et al., 2015). The injection of small RNAs from traumatized males into naïve oocytes induced a stress-like phenotype to the offspring (Gapp et al., 2014). Taking into account the important role that small RNAs have in intergenerational inheritance, here I focused specifically on small RNAs. 
4.2.5.1.SmallRNAs as a mechanism of transmission of APPPS1-21 traits

I screened sperm's RNA from APPPS1-21 transgenic fathers of earlier described offspring and compared it to wild-type fathers. From the total of small RNAs only a few small nucleolar RNAs (snoRNAs), Piwi-interacting RNAs (piRNAs) and one novel microRNAs (miRNAs) showed a significant increase in transgenic mice (Figure 4.2.37A-B). I checked whether these specific small RNAs were previously found on mouse sperm samples and interestingly all of them were previously isolated from sperm samples of C57BL/6J mouse line (http://spermbase.org/) (Schuster et al., 2016).

I identified miR-6240 and its immature form as being significantly differentially expressed in APPPS1-21 transgenic fathers. This miRNA was not previously linked to any mechanism or pathology, so it could be a potential target for further investigations. Besides, I found differential expression of different forms of snoRNAs and piRNAs in APPPS1-21 $\mathrm{tg}$ vs. wild-type fathers. To my knowledge, snoRNAs and piRNAs were not previously linked to intergenerational inheritance of phenotypic changes and might be new mechanisms of non-genetic transmission to the next generation. Snord15b-201, snord111-201, snord60-201, snord96a-201, snord47-201 (U47) were enriched in the RNA of APPPS1-21 transgenic fathers, however not much is known about their functions. It is known that snord47 was associated with tumorigenesis in glioblastoma (Xu et al., 2017). The piRNA_000578, piRNA_018323 and piRNA_003040 were up-regulated in APPPS1-21 tg RNA sperm (Figure 4.2.37B); however little is known about their functions. All three piRNAs were previously identified in the cerebellum of a mouse model of Rett syndrome (Saxena, Tang, \& Carninci, 2012).

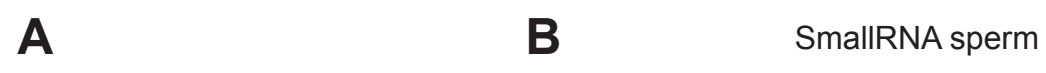

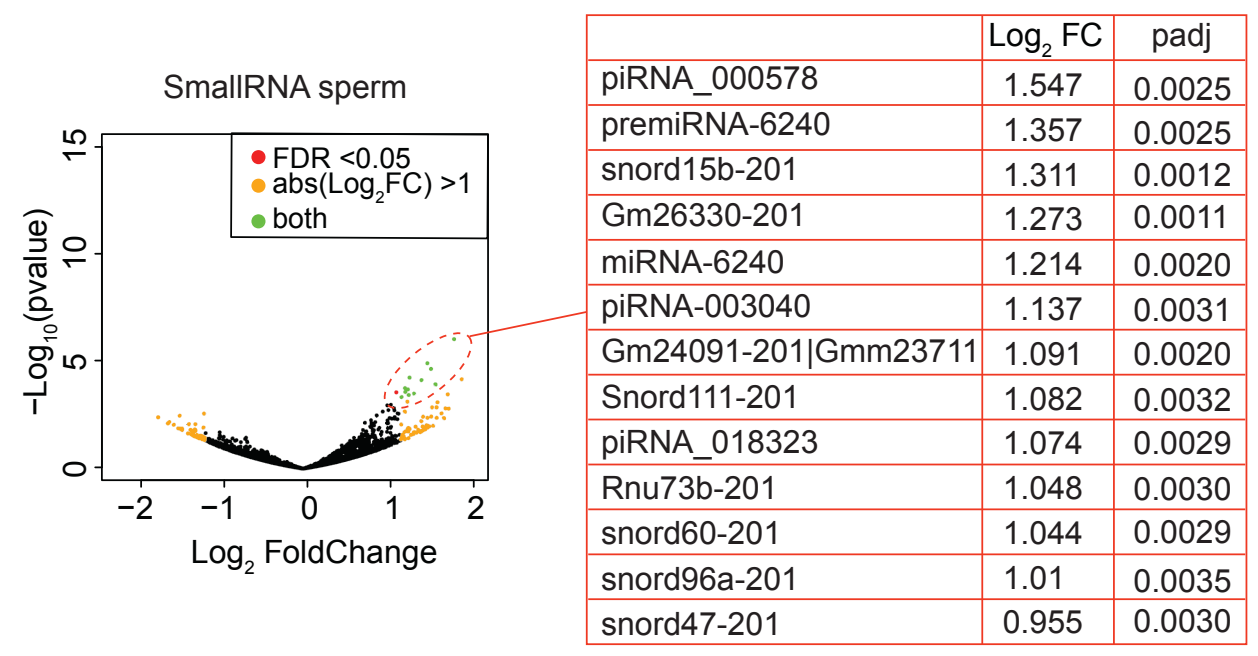


Figure 4.2.37. Expression of small RNAs that could play an important role in the altered phenotype, transcriptome and splicing in wild-type and transgenic offspring of APPPS1-21 transgenic fathers. (A) Volcano plot illustrating the proportion of differentially expressed small RNAs between tg and wild-type fathers. Yellow dots represent small RNAs with $\log _{2} \mathrm{FC} \pm 1$; red dots with a padj < 0.05 ; green dots represent small RNAs that fulfilled both criteria and black dots represent the small RNAs that did not fulfill any of the criteria defined. (B) List of significant differentially expressed small RNAs with their specific padj and $\log _{2} \mathrm{FC}$ values.

To the best of my knowledge, this is the first study that identified potential non-genetic factors for the transmission of a cognitive deficit phenotype caused by genetic mutations (in my case specifically, APP and PS1) in fathers. However, further research is necessary to confirm the direct correlation between these small RNAs and the inheritance observed in wild-type mice born to APPPS1-21 transgenic fathers.

\subsubsection{APP expression in wt mice born to APP fathers}

In addition, I speculated with the idea that APP per se might be transmitted by leakage through the sperm of $\operatorname{tg}$ fathers. While this should not be the case since the promoter of the double mutation, Thy 1 , is specific for neurons, I tested this experimentally. No differential expression of the human APP in sperm was observed between groups (Figure 4.1.57A), discarding the APP leakage as a factor. Besides, quantifying the expression of the human APP in CA1 region, I conclude that the expression of APP is specific for transgenic brain regions.

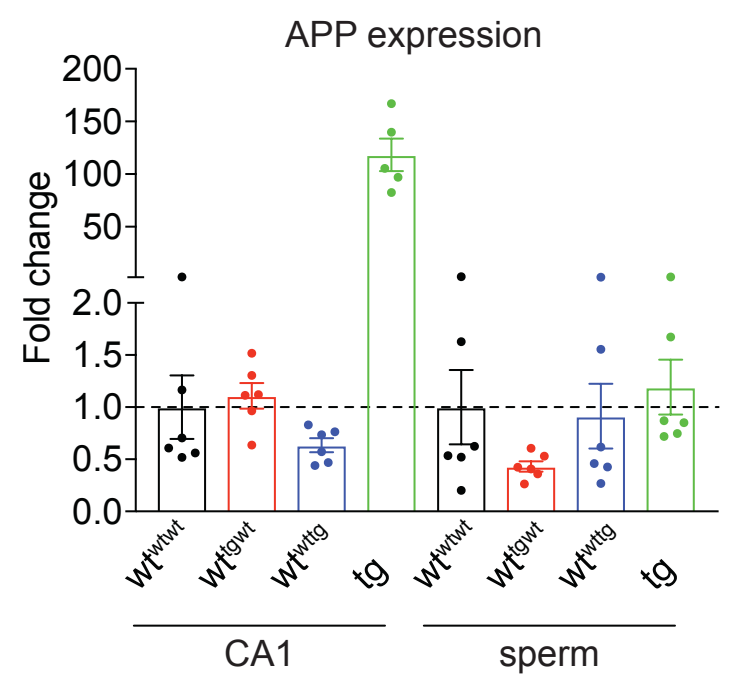

Figure 4.2.38. No expression of APP in sperm of APPPS1-21 transgenic fathers. qPCR quantification of APP gene in the mRNA of CA1 brain subregion and sperm. Error bars indicated SEM. 


\subsection{Inter-individual differences in cognitive performance and linked molecular pathways in aging mice}

Previously I have established the cognitive performance, transcriptome and exon usage of wildtype and transgenic mice born to APPPS1-21 transgenic parents. APPPS1-21 transgenic mice rapidly developed the pathology of Alzheimer's disease (AD) and showed spatial impairments at 3 months of age that were worsened at 9 months of age. The major risk to develop AD is aging. Here, I point the question of whether there could be specific gene expression changes exclusively related to aged individuals with cognitive impairments. For that, I chose to work on an aging mouse model because it is well established that inter-individual variability increases significantly with age, especially in terms of cognitive performance. This cognitive performance inter-individual variability would allow me to preselect aged mice with cognitive decline (Koivisto et al., 1995; Montesanto et al., 2012). It has repeatedly been demonstrated that environmental factors can profoundly impact the organism's behavior, especially in behavioral tasks (HolmHansen et al., 2016; D. Smith et al., 2015). In mice, it is very common to observe inter-individual variability in genetically identical mice. There is solid evidence pointing to epigenetic mechanisms as being involved in this individual variability, and the readout from these assays is often transcriptomic changes.

\subsubsection{Increased variability in cognitive performance in aging mice: choosing good vs. bad learners}

In order to assess the inter-individual variability in cognitive performance and identify specifically mice with cognitive impairments, I tested C57BL/6J wild-type mice at 17 months of age in two spatial memory tasks: Morris water maze and Barnes maze. I also recorded the exploratory and motor abilities in the open field task (Figure 4.3.1A).

To define bad vs. good learners, I carefully examined individual MWM performance for each mouse used in the test. Mice were trained for 9 days and tested at day 10 (probe test, PT). During the learning phase, all averaged mice learned the task and required less than 25 seconds to find the platform in the last days of training (Figure 4.3.1B). However, wt mice at 17 months of age required significantly longer times to find the platform compared to a 3 months of age wt control mice (Figure 4.3.1B). When mice were plotted individually, a greater variability 
between old mice was observed (Figure 4.3.1C). The distribution of the individual data points in the latency time was clearly a lot more widespread in the old mice than that of young mice.

A

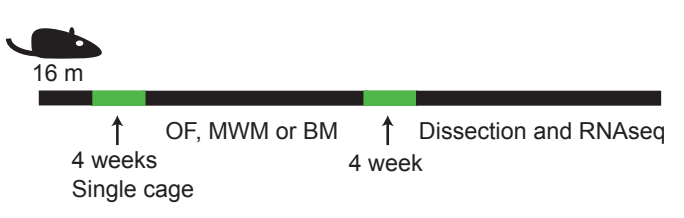

B

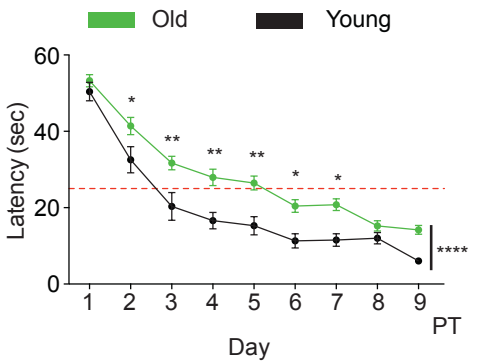

C

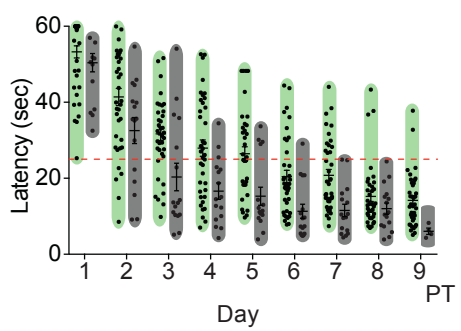

Figure 4.3.1. Increase inter-individual variability in cognitive performance in aging mice.

(A) Experimental design. (B) Old $(17 \mathrm{~m})$ and young $(3 \mathrm{~m})$ group-average time and (C) individual time to find the target platform on each consecutive training day (Day 1-9). Two-way ANOVA after Sidak correction, ${ }^{*} \mathrm{P}$ $<0.05 ;{ }^{* *} \mathrm{P}<0.01 ;{ }^{* *} \mathrm{P}<0.001$. The dashed line represents the chance level of $25 \%$. Error bars indicated SEM.

In the PT, mice at 17 months of age showed greater inter-individual differences in their memory for the platform. Some did not learn the task (bad performers), whereas others (good performers) remembered the exact position of the platform (Figure 4.3.2A). Bad performers moved randomly into all four quadrants, while good performers spent the majority of the test time in the target quadrant (Figure 4.3.1B-D). There is a correlation $\left(r^{2}=0.5802\right)$ between the time a mouse spent in the target quadrant and the number of times that it crosses the platform, another indication of the strong memory of the platform position (Figure 4.3.1E-F). To study the molecular differences between bad (impaired cognition) and good (intact cognition) performers, I empirically set the threshold at the top/bottom 8 out of $\sim 40$ mice and assigned the best 8 and worst 8 to the good and bad group, respectively. 
A

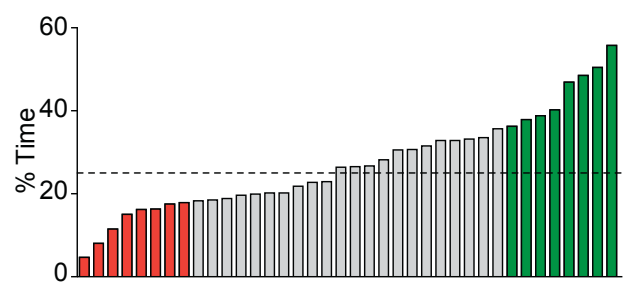

C

Bad Middle Good
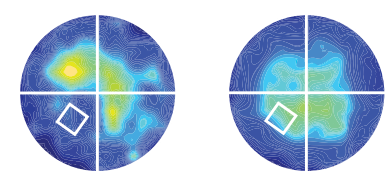

D

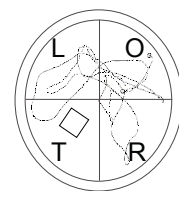

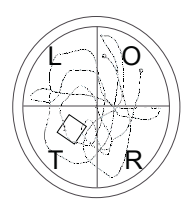

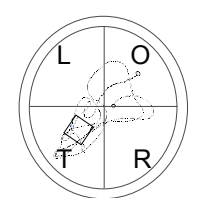

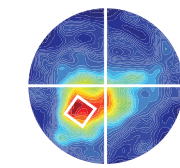

B

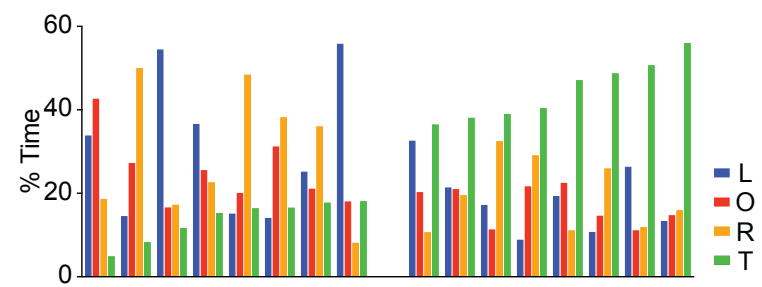

E

$\mathbf{F}$

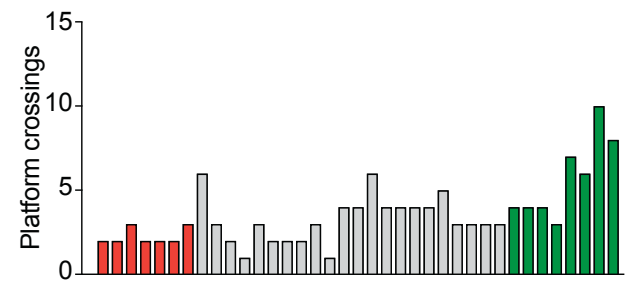

Figure 4.3.2. Establishment of good and bad performers.

(A) Percentage of time spent on the target quadrant vs. other three quadrants. (B) Percentage of time spent on the target quadrant vs. other three quadrants during probe trial and ranked by percentage of time during the probe trial. (C) Heatmap occupancy plots are showing a typical probe trial for a bad, middle and good performer (left to the right, respectively). (D) Representative tracings from the probe trial of one bad, middle and good performer mouse. (E) Number of platform crossings ranked by percentage of time during MWM probe trial. (F) Correlation between platform crossings and time spent in the target quadrant during the probe trial. Linear regression. ${ }^{* * * *} P<0.0001$. Bad performers are defined with red color, middle performers with grey and good performers with green.

To verify that motor abilities are not the cause of this variability, I checked the swim speed of individual mice and sorted them by time spent in the target quadrant (Figure 4.3.3A). There was no correlation $\left(r^{2}=0.0896\right)$ between speed and performance (Figure 4.3.3B). Also, there were no significant differences in swimming speeds between good and bad performers (Figure 4.3.1C), indicating that motor impairments do not cause the performance differences. 
A

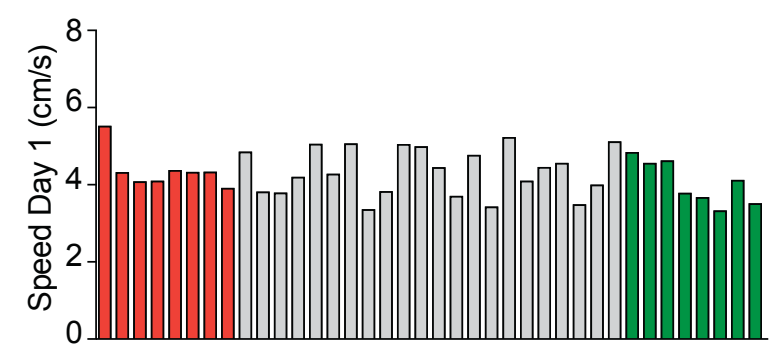

B

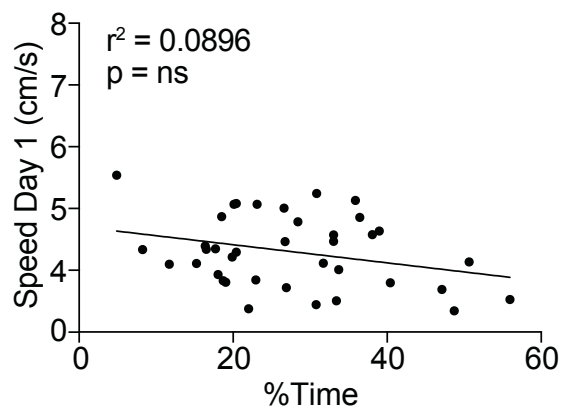

C

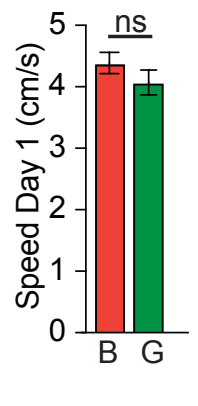

Figure 4.3.3. No motor variability between good and bad performers.

(A) Individual average speed during the first day of training ranked by percentage of time spent in the target quadrant. (B) Correlation between average speed during first day of training and percentage of time during the probe trial. Linear regression, $n s=$ no significant differences. (C) Average speed during the first day training from good and bad performers. Student's two-tailed T-test, ns = no significant differences. Error bars indicated SEM. Bad performers are defined with red color, middle performers with grey and good performers with green.

To verify whether anxiety levels played a role in good and bad performance, I subjected mice to the open field test. While mice showed inter-individual differences in exploratory abilities, with some mice spending more time in the center and others near the walls, I did not observe any difference between our previously defined good vs. bad learners (Figure 4.3.4A, green and red bars, respectively). Anxiety levels did not correlate $\left(r^{2}=0.0035\right)$ with performance in the MWM (Figure 4.3.1B). Thus, neither the motor ability nor anxiety levels affected (or influenced, decide what you like better) the variability in performance observed in the Morris water maze.

A

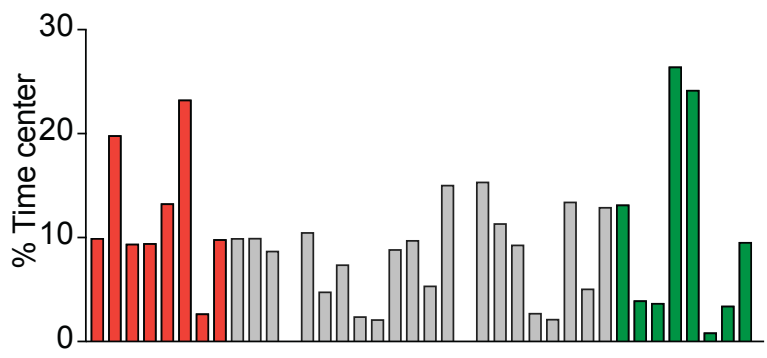

B

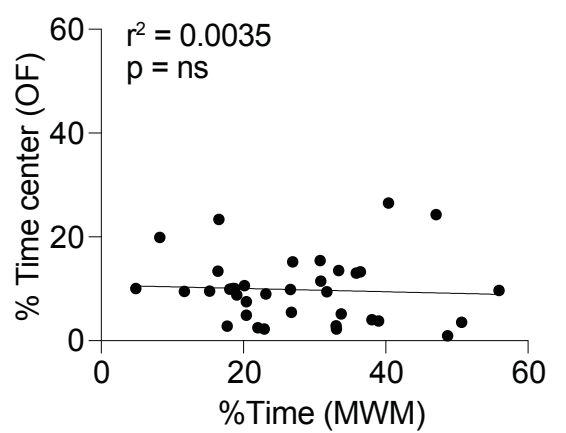

C

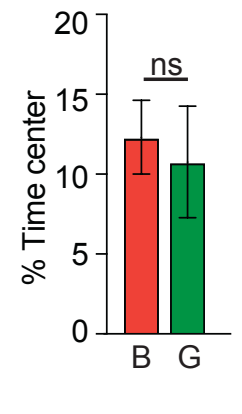


Figure 4.3.4. No changes in motor and abilities and anxiety levels between good and bad performers during the open field test.

(A) Percentage of time spent in the central area of the field ranked by the percentage of time spent in the target quadrant during Morris water maze (MWM) probe trial (PT). (B) Correlation between percentages of time spent in the central area and percentages of time target quadrant during MWM PT. Linear regression. ns = no significant differences. (C) The average percentage of time spent in the central area from bad and good performers. Student's two-tailed T-test, ns = no significant differences. Error bars indicated SEM.

In order to corroborate that I can in fact separate good from bad performers in a spatial memory task, I tested a second cohort of mice in a different spatial memory test, namely the Barnes maze (BM). This learning task consists of placing a mouse in the center of a circular light surface with 20 possible escape holes symmetrically distributed around the circumference of the maze. Only one of the holes has an escape box below, and the mouse needs to explore the maze surface until it eventually finds the escape box and memorizes its spatial location. I conducted this test this test in a separate cohort of 17 month-old mice. Besides, this group was also tested in the open field.

During the first day of the BM, mice were habituated to the new environment. Moreover, during the next 3 days, they were trained to find the dark escape box. The probe test was performed on the 10th day, after 8 days of training (Figure 4.3.5A). During the probe trial, mice at 17 months of age showed a robust inter-individual variability, with some mice spending most of the time around the escape hole (good performers), and others moving randomly around the surface or staying close to other random holes (bad performers) (Figure 4.3.5B-C). As in my previous Morris water maze experiment, I chose the extremes of the learning curve (in this case the top and bottom 7) as my experimental groups (Figure 4.3.5B).

A

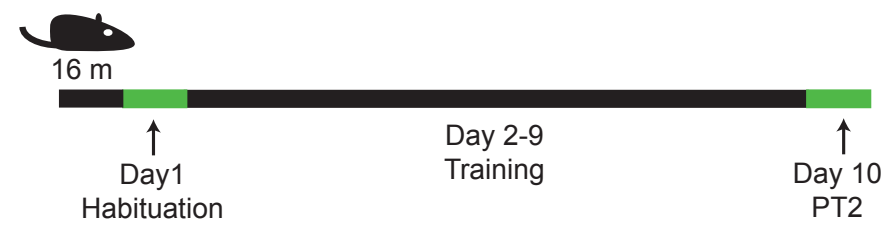

B

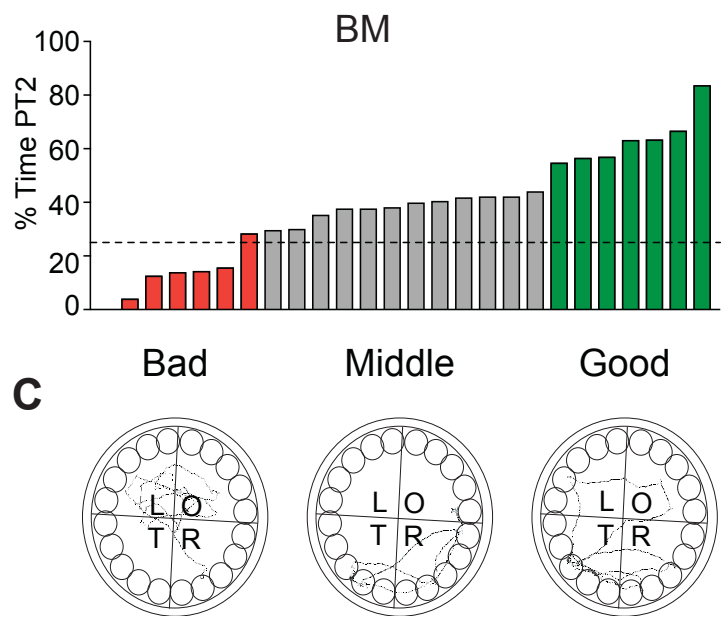


Figure 4.3.5. Establishment of good and bad performers based on escape latency (or performance) in the Barnes maze (BM).

(A) Experimental design. (B) Percentage of time spent in the target quadrant during the Barnes maze (BM) probe trial after 8 days of training. (C) Representative tracings from the probe trial of a bad, middle and good performer mouse. Bad performers are represented with red bars, middle performers with grey and good performers with green.

Similar to my previous results, I did not observe any correlation $\left(r^{2}=0.0015\right)$ between performance in the BM probe trial and speed of the mice. This cohort did not show any motor ability changes between performers either (Figure 4.3.6A-C). This means that this variability concerning learning does not correspond with motor impairments.

A

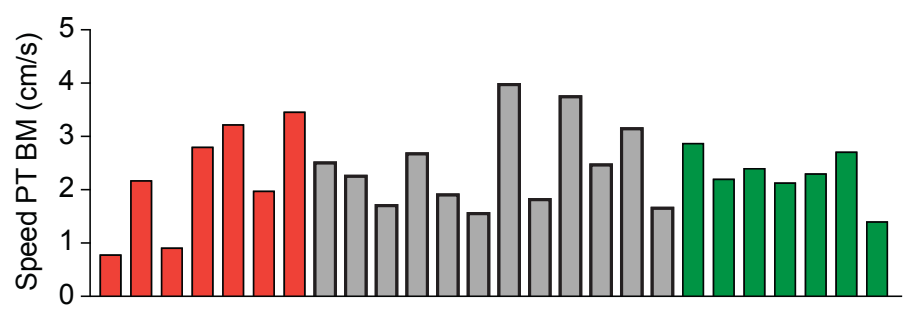

B

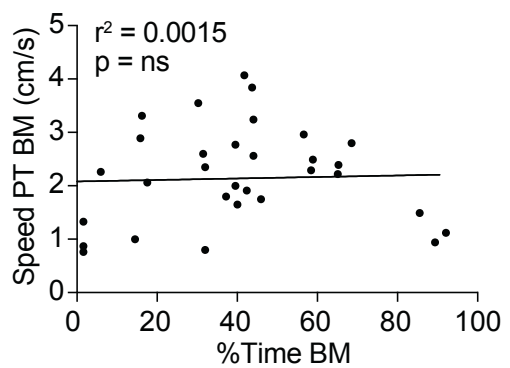

C

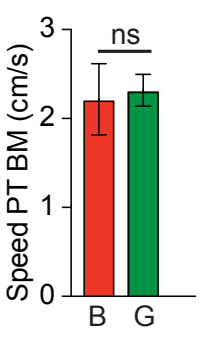

Figure 4.3.6. No motor variability between good and bad performers.

(A) Individual average speed in Barnes Maze (BM) probe trial ranked by percentage of time spent in the target quadrant. (B) Correlation between average speed during the first day of training and percentage of time during the probe trial. Linear regression. ns = no significant differences. (C) Average speed during PT between good and bad performers. Bad performers are defined with red color, middle performers with grey and good performers with green. Student's two-tailed T-test, ns = no significant differences. Error bars indicated SEM.

This observation was further validated in this cohort using the open field test. As expected, some mice spent more time in the center while others preferred the periphery, corresponding to different levels of anxiety (Figure 4.3.7A). However, this variability did not correlate $\left(r^{2}=0.0011\right)$ with their performance in the BM (Figure 4.3.7B-C), again arguing against motor or anxietyrelated factors affecting spatial learning and memory in these mice.

A

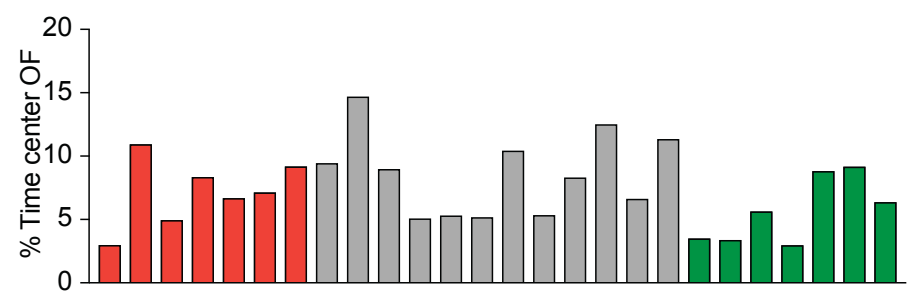

B

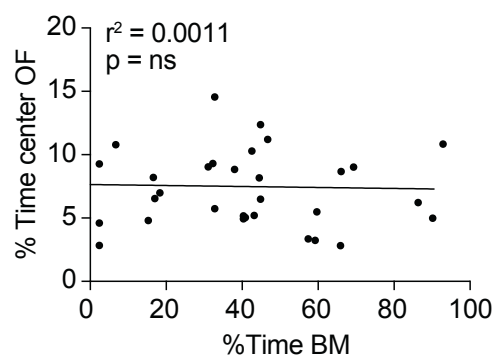

C

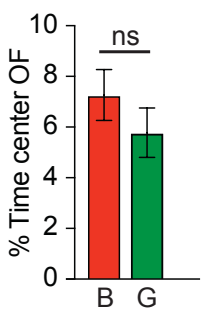


Figure 4.3.7. No anxiety variability between defined good and bad performers during the open field test.

(A) Percentage of time spent in the central area of the open field ranked by the percentage of time spent in the target quadrant during Barnes maze (BM) probe trial (PT) (B) Correlation between percentage of time spent in the central area and percentage of time target quadrant during BM PT. Linear regression. ns = no significant differences. (C) The average percentage of time spent in the central area from bad and good performers. Student's two-tailed T-test, ns = no significant differences. Error bars indicated SEM.

These data suggest that aged wild-type mice raised under identical conditions develop distinct abilities to learn and memorize a task, such as the MWM or the BM tasks.

\subsubsection{Transcriptional profiling and functional pathways analysis of good and bad performers}

To gain a better understanding of the molecular mechanisms associated with aged wild-type mice with cognitive decline, I analyzed the spatial-related brain regions from these bad performers and compared to good performers (intact cognition). I studied the transcriptome of all three hippocampal subregions, Cornu Ammonis 1 and 3 (CA1 and CA3) and Dentate Gyrus (DG) from good and bad performers of the first cohort of mice. Due to time limitations, only the transcriptomic profile of the first cohort was evaluated.

Surprisingly, a little number of genes in CA1, CA3 and DG (maximum 16 in CA3) were significantly different (padj $\leq 0.05$ and $\log _{2} \mathrm{FC} \pm 0.25$ ) between good and bad performers (Fig. 4.3.8A-C). Thus, I decided to evaluate the signaling pathways differentially affected between good and bad performers, using Gene Set Enrichment Analysis (GSEA) (Mootha et al., 2003; Subramanian et al., 2005). GSEA analysis allows studying the whole dataset functions without restricting the study to only genes with a specific threshold.

- FDR $<0.05 \bullet \operatorname{abs}(\operatorname{LogFC})>1 \quad$ both
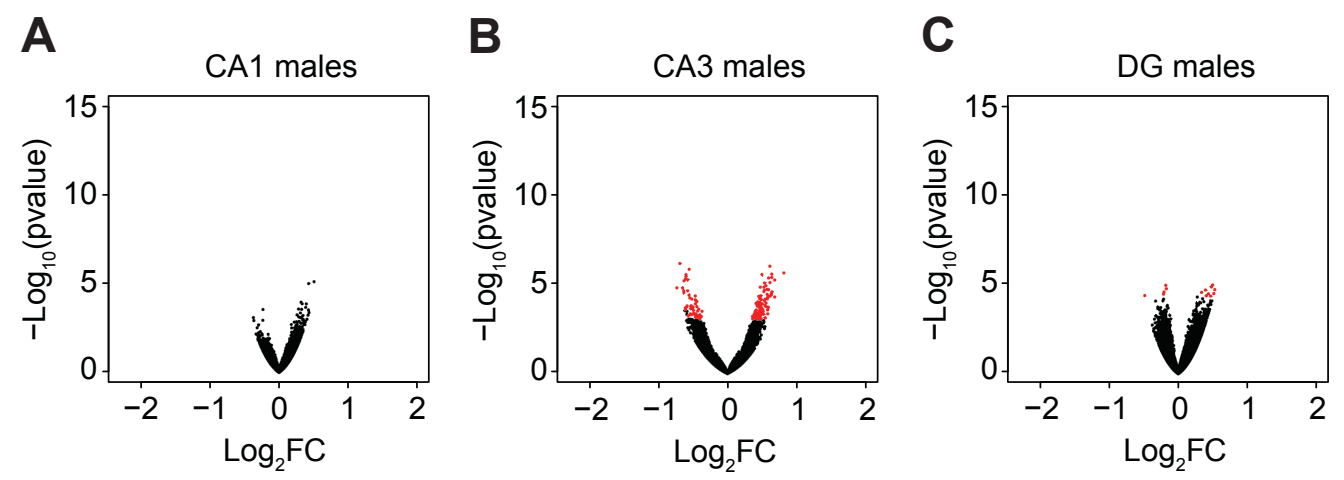
Figure 4.3.8. Changes in transcriptional profile in brain subregions of bad performers compared to good performers.

(A-C) Number of significant differentially expressed genes between good and bad performers in (A) CA1, (B) CA3 and (C) DG. Statistical difference of FDR and $\log _{2} F C$ values are indicated by red dots, and only one parameter is indicated by green dots and none of them by black dots. For CA1 subregion: good $n=7$ and bad $n=7$; for DG good $n=7$ and bad $n=8$ and for CA3 good $n=3$ and bad $n=4$.

In order to evaluate the cellular state of mice from the same line and at the same age but only differing in spatial cognitive abilities, I ran the full list of normalized counts from RNA sequencing of good (intact cognition) and bad (cognitive decline) performers through GSEA. To obtain a first idea of the mechanisms involved, I selected the top 50 most significant pathways (nominal pval $\leq 0.05$; FDR $\leq 0.1$ ) from each category (biological process, molecular function and cellular component) ranked by Normalized Enrichment Score (NES). The NES indicates the level to which a group of genes is represented in the upper or lower part of the ranked list of genes in the expression dataset 13. Positive pathways are enriched in good performer mice, whereas negative pathways are enriched in bad performers (Figure 4.3.9-11). From these top 50 pathways, I screened in detail which broader term could be used to summarize the pathways. This allowed me to identify the most important mechanisms that could promote these differences in spatial performance.

The main significant functional changes were observed in CA1 and DG regions of aged mice (Figure 4.3.9A-H). Analyzing the broader biological processes associated with the top 50 preselected pathways, I discovered that similar functions were over and underrepresented in CA1 and DG subregions of bad performers (aged mice with cognitive impairments) (Figure 4.3.9A-F). Genes related to mitochondrial activity, respiratory chain, general and RNA metabolic processes, translation, protein localization and ribosome biogenesis and assembly were downregulated in CA1 and DG regions of aged mice with cognitive impairments (Figure 4.3.9B and E). On the contrary, up-regulated genes in CA1 and DG regions of bad performers (impaired cognition) were linked to kidney tissue, vessel and embryo development and cellular component organization (Figure 4.3.9C and F). Specifically, in the DG region of bad performers, pathways linked to response to a stimulus, coagulation, immune system, apoptotic and metabolic processes were enriched (Figure 4.3.9K-L). In the CA3 region of mice with cognitive impairments, genes related to immune system, metabolic and apoptotic processes were underrepresented (Figure 4.3.9G-H). 
A

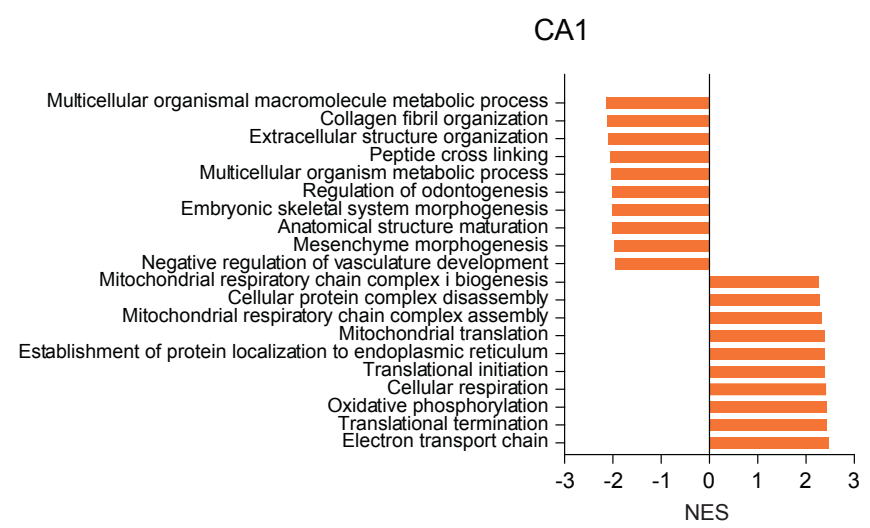

D

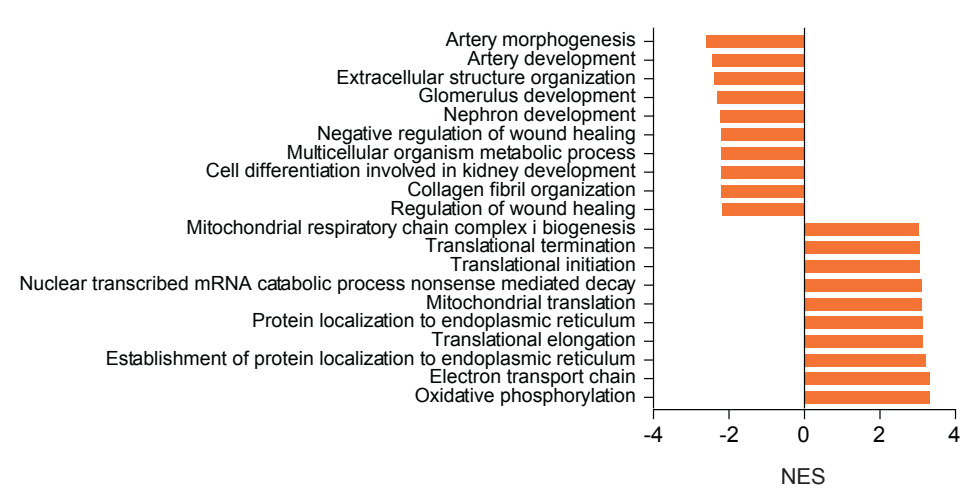

G

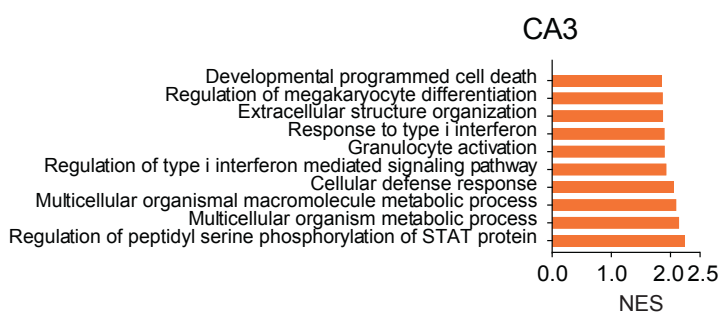

B

Good performers
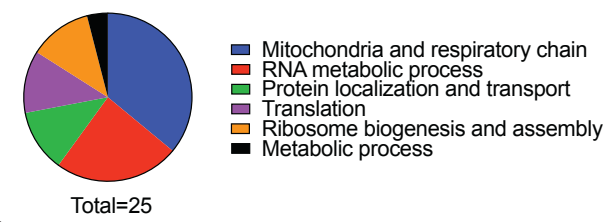

C Bad performers

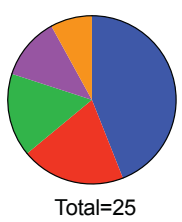

Tissue development

ㅁ Embryo development

Metabolic process

Cellular component organization

E

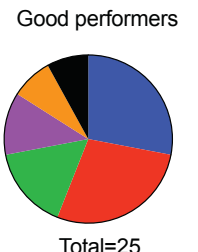

Mitochondria and respiratory chain

RNA metabolic proces

Protein localization and transport Translation

- Metabolic process

F Bad performers

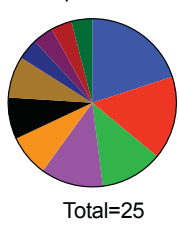

Kidney development
Artery and vessel development
Tissue development
Response to stimulus
Metabolic process
Ceellular component organization
Coagulation
Apoptotic process
Immune system
Vesicle mediated transport
$\square$ Signal transduction

$\mathbf{H}$

Good performers

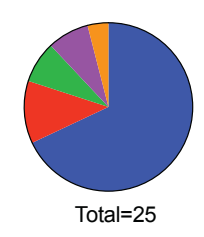

Immune system

Metabolism process

ㅁ Structural organization
글

Figure 4.3.9. Biological processes are differentially affected in good and bad performer mice.

(A, D and G) Top 10 preselected biological processes over or underrepresented within the whole dataset of genes in "good" and "bad" performers in brain subregions: CA1 (A), DG (D) and CA3 (G). NES= Normalized Enrichment Score. Positive NES means overrepresentation in "good" performers, whereas negative NES means overrepresentation in "bad" performers. (B, C, E, F and H) Broader term summary of the top 25 overrepresented (B, E and $\mathbf{H}$ ) and 25 underrepresented ( $C$ and $\mathbf{F}$ ) pathways in "good" performers in the above mentioned brain subregions. Nominal pval $\leq 0.05$; FDR $\leq 0.25$. For CA1 subregion: $\operatorname{good} n=7$ and bad $n=7$; for $D G \operatorname{good} n=7$ and bad $n=8$ and for $C A 3 \operatorname{good} n=3$ and bad $n=$ 4.

Interestingly, genes with the top 50 significant molecular function ontologies altered in hippocampal regions of bad performers compared to good performers were in agreement with my observations in the biological process ontology (Figure 4.3.9 and 10). Categories such as catalytic activity, transport and channel activity linked to mitochondrial complex or genes 
associated with protein biogenesis such as transcription regulatory activity and translation were down-regulated in CA1 and DG of bad performers (Figure 4.3.10A-F). In CA3 region of bad performers, only genes related to protein binding and structural molecular activity were enriched (Figure 4.3.10G-H).

A

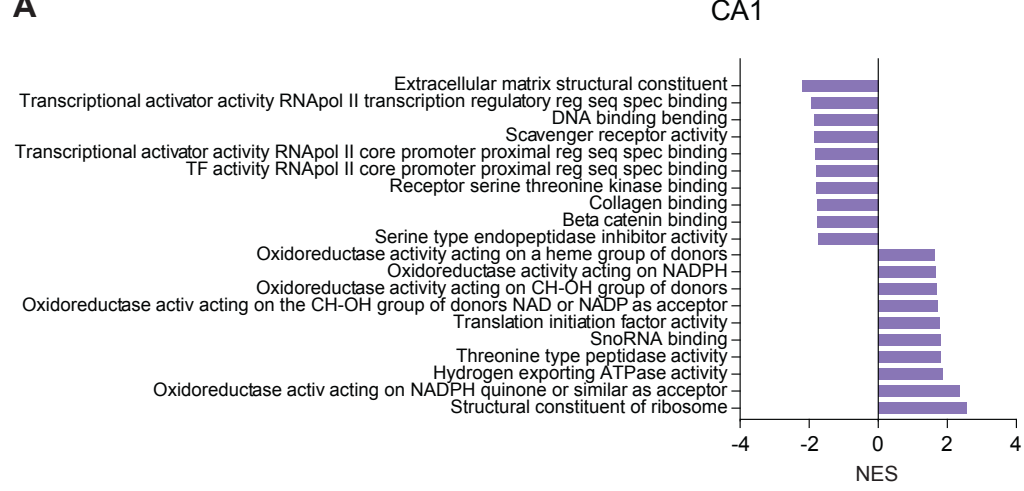

D

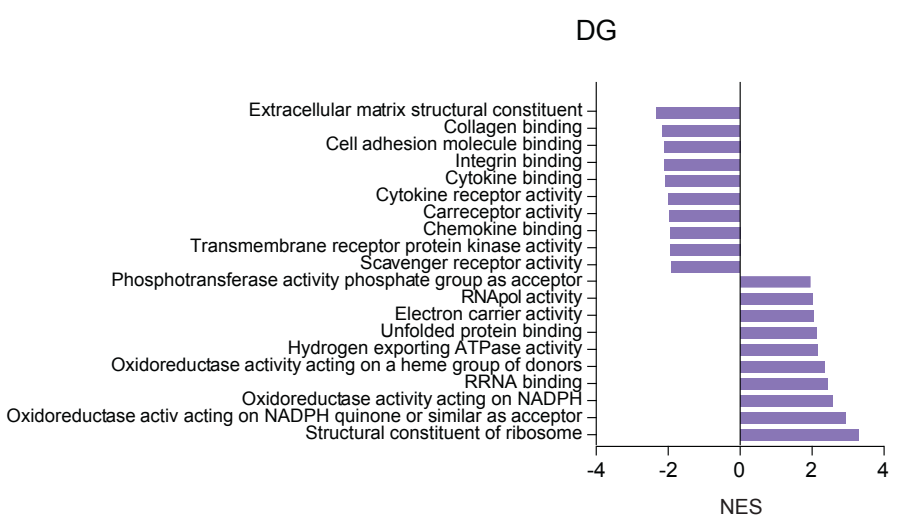

G
CA3

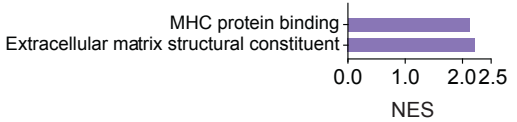

B

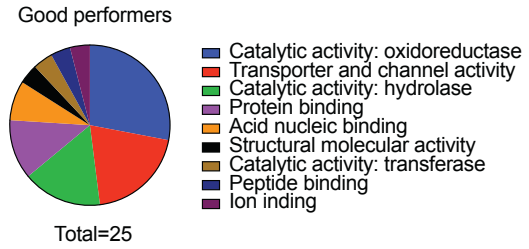

C Bad performers
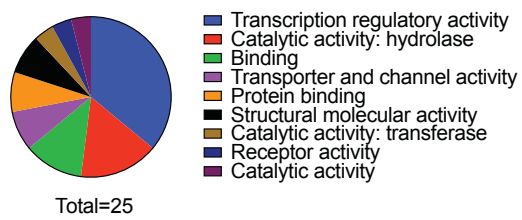

E

Good performers

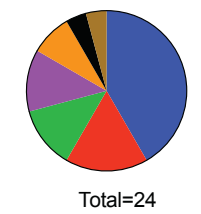

Catalytic activity: hydrolase Catalytic activity: oxidoreductase Protein binding Transport Acid nucleic binding Catalytic activity: Transferase

F Bad performers

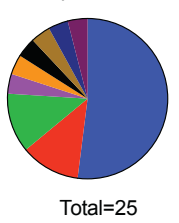

$\square$ Protein binding

Catalytic activity: hydrolase $\checkmark$ Catalytic activity: oxidoreductase $\square$ Transport - Structural molecular activity $\square$ Binding Lipid binding

Total $=25$

H

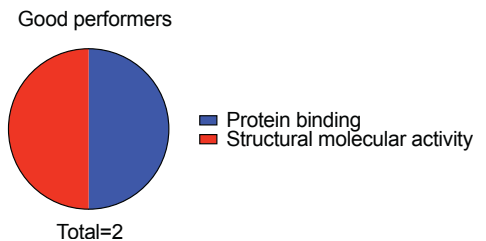

Figure 4.3.10. Molecular functions were differentially affected in good and bad performer mice. (A, D and G) Top 10 preselected biological processes over or underrepresented within the whole dataset of genes in good and bad performers in the brain subregions: CA1 (A), DG (D) and CA3 (G). NES= Normalized Enrichment Score. Positive NES means overrepresentation in good performers, whereas negative NES means overrepresentation in bad performers. (B, C, E, F and H) Broader term summary of the top 25 overrepresented (B, E and $\mathbf{H}$ ) and 25 underrepresented ( $\mathbf{C}$ and $\mathbf{F}$ ) pathways in good performers in the above mentioned brain subregions. Nominal pval $\leq 0.05$; FDR $\leq 0.25$. For CA1 subregion: $\operatorname{good} n=7$ and bad $n=7$; for $D G \operatorname{good} n=7$ and bad $n=8$ and for CA3 good $n=3$ and bad $n=$ 4. 
Lastly, the cellular structures altered in CA1 and DG regions of bad performers correspond to components implicated in previously described biological processes such as mitochondria activity, protein biogenesis, metabolic process and protein localization and transport. For example, genes linked to mitochondria, ribosome, secretory vesicle, cytoskeletal part and extracellular regions were altered in these regions (Figure 4.3.11A-O).

A

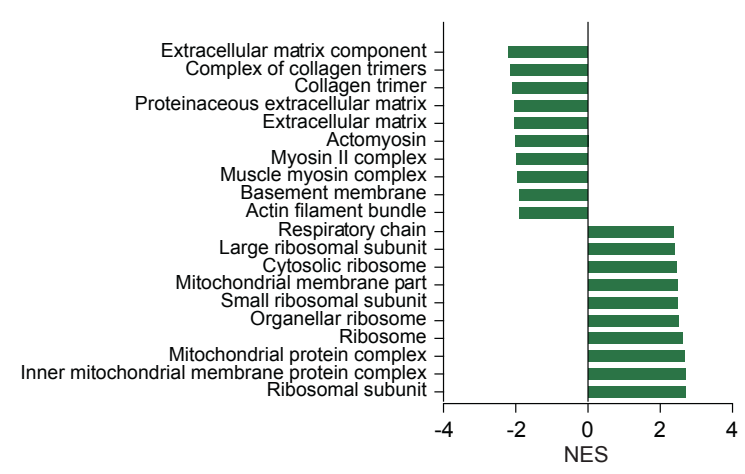

D

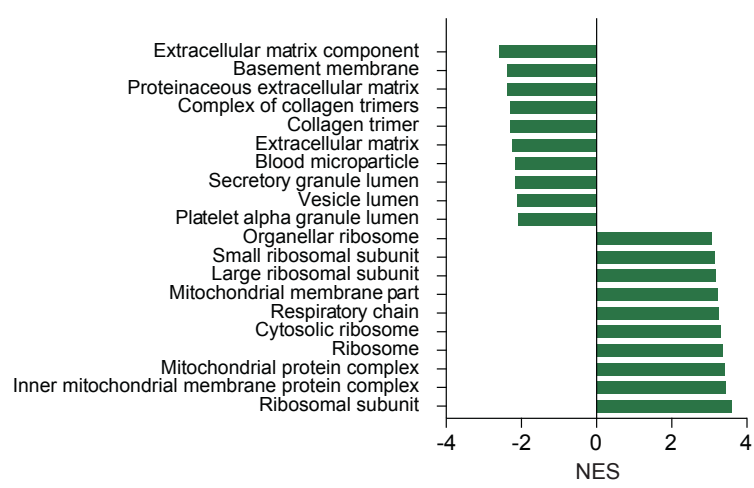

G

CA3

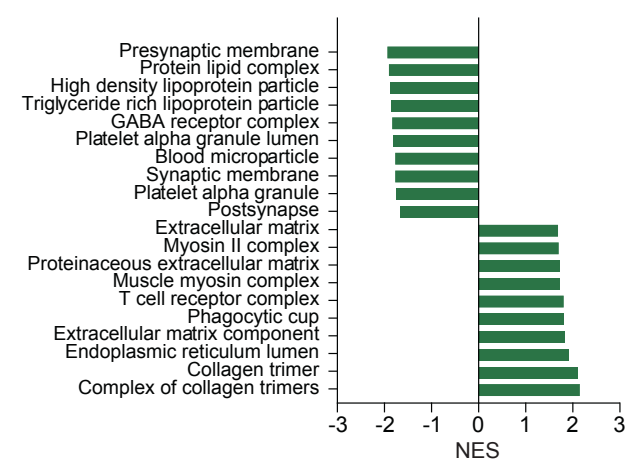

B

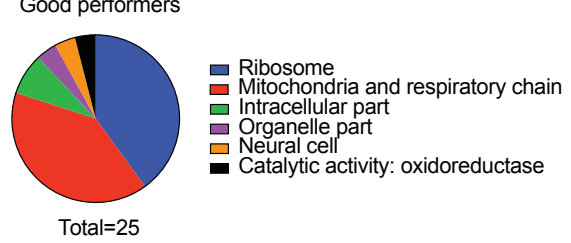

C

Bad performers
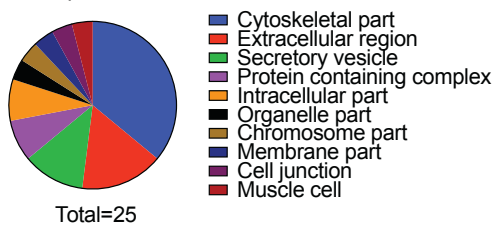

E

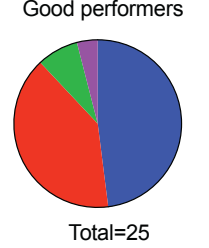

$\square$ Mitochondria and respiratory chain $\square$ Ribosome

$\square$ Splicing
$\square$ Neural cell

F Bad performers

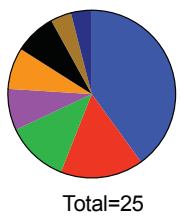

Extracellular region

Secretory vesicle

Intracellular part

Muscle cell

- Cellular component

Organelle part

H Good performers

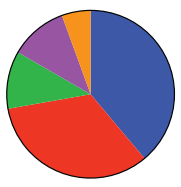

Cytoskeletal part

- Cell projection

Protein containing complex
Macromolecular complex

Total $=18$

I Bad performers

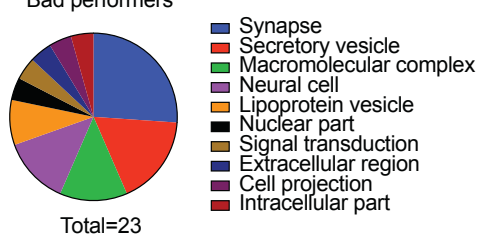


Figure 4.3.11. Cellular components are differentially affected in good and bad performer mice. (A, D and G) Top 10 preselected biological processes over or underrepresented within the whole dataset of genes in good and bad performers in the brain subregions: CA1 (A), DG (D) and CA3 (G). NES= Normalized Enrichment Score. Positive NES means overrepresentation in good performers, whereas negative NES means overrepresentation in "bad" performers. (B, C, E, F, H and I) Broader term summary of the top 25 overrepresented (B, E and $\mathbf{H})$ and 25 underrepresented (C, F and I) pathways in "good" performers in the above mentioned brain subregions. Nominal pval $\leq 0.05$; FDR $\leq 0.25$. For CA1 subregion: $\operatorname{good} n=7$ and bad $n=7$; for $D G \operatorname{good} n=7$ and bad $n=8$ and for CA $3 \operatorname{good} n=3$ and bad $n=$ 4.

In summary, the majority of pathways deregulated in the hippocampal regions of aged mice with cognitive impairments were linked to development, mitochondria activity, respiratory chain complex, RNA metabolic process, ribosome biogenesis and assembly, translation, protein localization, immune system, apoptotic processes, coagulation and response to a stimulus. Of note, pathways linked to mitochondria, respiratory chain, ribosome biogenesis, RNA metabolism, translation and protein localization were as well underrepresented in APPPS1-21 transgenic mice (Figure 4.1.8 and 11), which showed as well spatial cognitive deficits. This suggests that an alteration of pathways linked to energy metabolism (mitochondria activity, respiratory chain complex) and protein biogenesis (ribosome biogenesis, RNA metabolism, translation and protein localization) has an impact on spatial cognitive abilities in aged wild-type mice. Alteration of energy metabolism pathways in aged-mice has been previously described. However, I identified for the first time that these dysfunctions were specific to aged-mice those present cognitive impairments. Besides, pathways linked to development were increased in old performers and APPPS1-21 transgenic mice. However, APPPS1-21 transgenic mice showed mainly altered pathways connected to nervous system development, and bad performers show and upregulation of genes linked to kidney, bone, vessels and artery development.

\subsubsection{Blood biomarkers of "good" and "bad" performers}

Based on my previous results, I was able to identify the altered pathways related to aged mice with distinct cognitive abilities. Next, I wondered whether I could identify a biomarker that allowed me to predict the cognitive abilities of aged mice. To this end, I analyzed the expression of small RNAs in the blood from good (intact cognition) and bad (impaired cognition) performers. Analyzing the global distribution of blood samples using Principal Component Analysis (PCA), I identified certain separation between good and bad performers (Figure 4.3.12A). Interestingly, a group of small RNAs reached statistical significance (Padj $\leq 0.05, \log _{2} F C \pm 0.5$ ) in bad compared to good performers (Figure 4.3.12A). The majority of down-regulated small RNAs in bad performers corresponded to microRNAs (miRNAs), 17 microRNAs from 19 small RNAs, the 
rest were Piwi-interacting RNAs (piRNAs). I investigated the validated target mRNAs from these down-regulated small RNAs in the blood of bad performers and discovered that were related to nervous system development, catabolic processes and ossification (Figure 4.3.12C). Moreover, two microRNAs were up-regulated in the blood of the bad performers, miR-423-3p and miR-9769-3p. These up-regulated miRNAs target mRNAs involved in immune system development and response to cytokine (Figure 4.2.12C). Investigating the specific function of the deregulated miRNAs, I identified some potential candidates for detecting the cognitive abilities of aged mice. For example, human miR-501-3p was down-regulated in the serum of Alzheimer's disease (AD) patients and up-regulated in the brain of the same donors (N. Hara et al., 2017). It also played an important role in working memory in a mouse model of vascular cognitive impairment (Toyama et al., 2018). Moreover, miRNA-100-5p was identified in a mouse model of AD, APP/PS1, targeting mTOR and inducing changes in neuronal survival (Ye et al., 2015) and miRNA-146a-5p was associated with inflammatory signaling in neurodegenerative diseases (Alexandrov, Dua, \& Lukiw, 2014). MiRNA-664-3p expression in the spleen tissue of 6 wild-type mouse strains was related to pathways that play an important role in longevity, such as FOXO, mTOR and MAPK (B. P. Lee et al., 2017). These results suggest that some of these miRNAs could be further investigated as biomarkers of cognitive abilities.

A

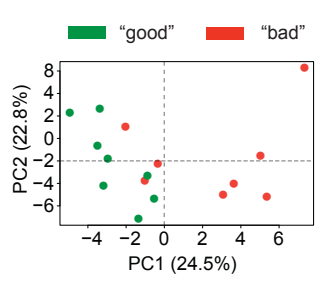

B

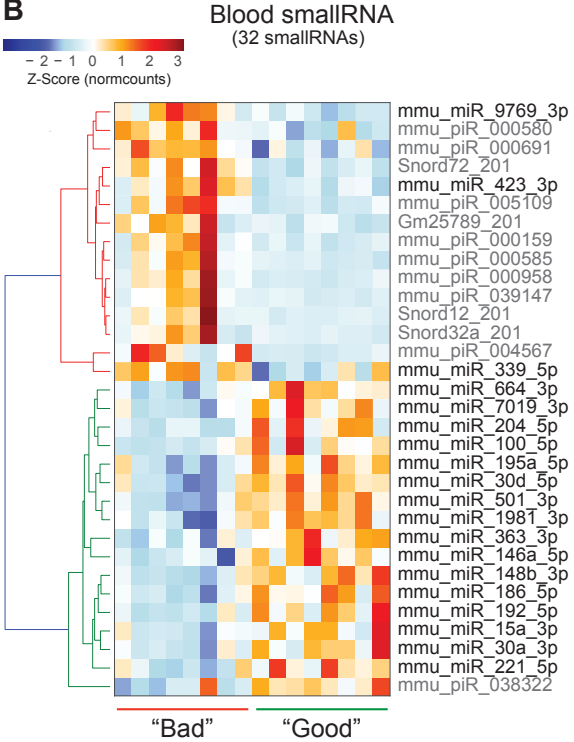

C

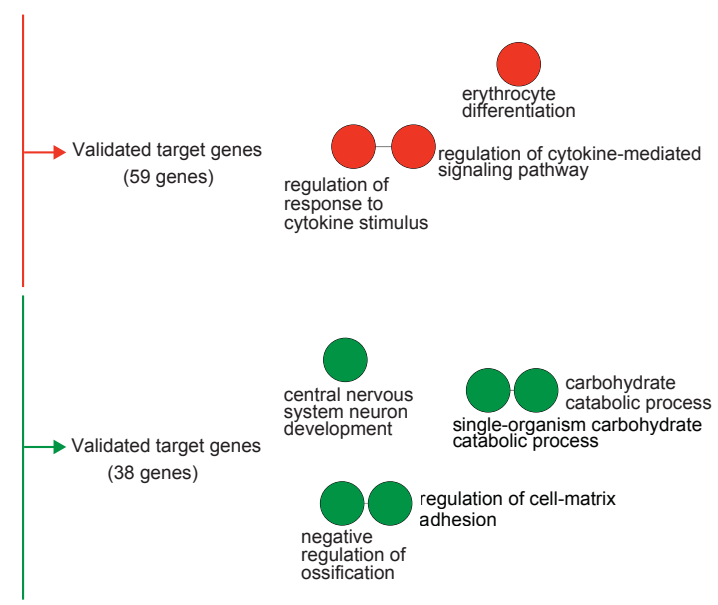

Figure 4.3.12. Blood small RNAs used as biomarkers of good and bad learners.

(A) Principal Component Analysis (PCA) plots for comparison of good and bad performers (B) Heatmaps of differentially expressed small RNAs in the blood of good vs. bad. High expression is indicated by the red color spectrum and low expression by the blue color spectrum. Padj $\leq 0.05, \log _{2} \mathrm{FC} \pm 0.5$. (C) Network of functional categories (biological process, molecular function and cellular component ontologies) of validated target genes. Categories with a red color correspond to target-genes associated to miRNAs that were up-regulated in good performers and red up-regulated in bad performers. MiRNAs, padj $\leq 0.05$; $\log _{2} F C \pm 0.5$ and pathways, pval $<0.05$. For good performers $n=8$ and bad $n=8$. 
DISCUSSION 


\section{DISCUSSION}

\subsection{The early stage of Alzheimer's disease in APPPS1-21 mouse model}

Alzheimer's disease (AD) is a neurodegenerative disease and the most common form of dementia. Over the last few decades, it has become clear that $A D$ is a multifactorial disease ( $K$. Iqbal \& Grundke-lqbal, 2010; Iturria-Medina et al., 2016; Talwar et al., 2017). Aside from treatments that slow down specific symptoms from the pathology, no cure exists for $A D$. The inefficiency of treatments is probably due to the late diagnosis of the disease. Thus, in recent years, several studies have focused their investigations on the understanding of the molecular and phenotypic changes associated with the early stages of pathology (Filippi et al., 2018; Gatta et al., 2014; Parra-Damas et al., 2014). These studies have evaluated the early pathology from different fields; one of them was gene expression analysis (Blalock et al., 2004; Gatta et al., 2014; Parra-Damas et al., 2014). Alzheimer's disease has a genetic component. Genetic factors have been intensively investigated for years, and several genes have been associated with $A D$ forms. Specific mutations in the amyloid precursor protein (APP), presenilin 1 (PSEN1) and PSEN2 induce the familial form of AD (Goate, 2006; Levy-Lahad et al., 1995; Sherrington et al., 1995). The expression of the isoform of apolipoprotein, ApoeE4, increases the risk for familial and sporadic forms of AD (Strittmatter et al., 1993). Despite the exhaustive investigations, the genetic impact is complex, and the results are still controversial, and more research is still necessary to understand the genetic influence at early AD stages (Blalock et al., 2004; Gatta et al., 2014; Parra-Damas et al., 2014). In this study, I described the cognitive impairments and gene expression changes in a well-established mouse model of $A D$ at an early $A D$ stage ( 3 months of age). 


\subsubsection{Spatial cognitive deficits in APPPS1-21 transgenic mice are detectable at $3 \mathrm{~m}$}

Alzheimer's disease is characterized by gradual loss of memory. The most widely used AD mouse models show memory impairments at different time points (Arendash et al., 2001; Chapman et al., 1999; Govindarajan et al., 2013; Kelly et al., 2003; Lalonde, Lewis, Strazielle, Kim, \& Fukuchi, 2003; Radde et al., 2006; Serneels et al., 2009) and in some cases before the accumulation of plaques (Dineley, Xia, Bui, Sweatt, \& Zheng, 2002; Dodart et al., 1999; Oddo et al., 2003; Saura et al., 2005). The APPPS1-21 mouse model is a well-characterized model. This line has been reported to have a significant increase of soluble A 342 levels and deposition of amyloid plaques in different brain regions (Radde et al., 2006). At 3 months of age, this line had deposits of amyloid plaques affecting only the neocortex and DG region of the hippocampus, whereas at 6 months of age plaques appeared in the CA1 hippocampal region, striatum, thalamus and brainstem (Radde et al., 2006). Moreover, this line showed cognitive deficits for specific behavioral tasks from 7 months of age (Govindarajan et al., 2013; Radde et al., 2006; Serneels et al., 2009),19. For example, it was reported that these mice showed impairments in reversal learning of a food-rewarded four-arm spatial maze at 8-months of age (Radde et al., 2006). Additionally, APPPS1-21 mice exhibited spatial learning and memory deficits in Morris water maze (MWM) at 7-8 months and alterations of associative memory in the fear conditioning (FC) paradigm at 12 months of age (Govindarajan et al., 2013; Serneels et al., 2009; Zovoilis et al., 2011). Although the APPPS1-21 mouse line has been characterized at AD late stages, it is still not reported whether these mice show behavioral alterations early on. To the best of my knowledge, this present work is the first characterization of the behavior of the APPPS1-21 mouse line at 3 months of age and I found that APPPS1-21 transgenic mice presented cognitive deficits at such an early stage. However, these deficits were restricted to spatial learning and memory task, assessed using MWM, as shown in figure 4.1.3 and 4.1.4 (D'Hooge \& De Deyn, 2001). In contrast to the cognitive deficits I found in this line, another model of AD known as APP/PS1 (APP K670N/M671L and PS1 M146L) did not exhibit spatial memory deficits or hippocampal plaques at 3 months of age (Sadowski et al., 2004; Yu et al., 2009). These studies and my data suggest a direct connection between cognitive deficits and deposition of $A \beta$ plaques in the hippocampus (Gengler et al., 2010; Radde et al., 2006). However, such connection is still not clear, since some studies have found other AD mouse models showing cognitive deficits before the deposition of plaques (Oddo et al., 2003; Saura et al., 2005; Van Dam et al., 2003). These studies suggested an association between cognitive deficits and the increase of soluble A $\beta$ (Oddo et al., 2003; Saura et al., 2005; Van Dam et al., 2003). One 
example was the APP23 mouse line, which showed spatial cognitive deficits in the MWM task without amyloid deposition at 3 months of age, but a direct correlation with soluble $A \beta$ levels (Van Dam et al., 2003). In APPPS1-21 line, the spatial cognitive deficits at 3 months of age could potentially be induced firstly by an increased $A \beta 42$ soluble form and then by the deposition of $A \beta$ plaques. Motor impairments or increase anxiety could also lead to a detrimental performance in the MWM. However, APPPS1-21 mice did not differ in the travel speed in the MWM or show increased anxiety in the open field test (OF), compared to wild-type controls, strongly suggesting that the poor performance of these mice in the MWM was indeed due to a cognitive impairment (Figure 4.1.2 and 4.1.6). APPPS1-21 mice tended to show a more "reckless" behavior in the open field, spending more time in open spaces, even at 3 months of age (Figure 4.1.2). This reduction of anxiety was not previously observed in APPPS1-21 transgenic mice at 5 or 8 months of age (Radde et al., 2006). One explanation could be that this transgenic line shows a reduction of anxiety levels at early ages but it is then normalized in the late adulthood. In associative tasks with a spatial component, such as the fear conditioning test, 3-month old APPPS1-21 transgenic mice were not affected (Figure 4.16). This might indicate that at this early stage of the pathology tasks that engage more brain areas and are part of the innate response to peril, like the fear conditioning test, are less sensitive to the initial cellular and molecular hallmarks of the disease. In contrast, refined tasks, such as spatial navigation, that may be more fragile in nature, start to show the first signs of the underlying degeneration. These findings indicate that the APPPS1-21 mouse model rapidly develops the pathology of Alzheimer's disease, showing cognitive deficits already at 3 months of age.

\subsubsection{Age-progressive decline of spatial memory and motor abilities}

Aging is one of the major risk factors for Alzheimer's disease, and the probability of suffering from dementia is higher at old stages (Guerreiro \& Bras, 2015). Here, I confirmed the progressive nature of learning and memory decline in the APPPS1-21 mouse model of AD. Hippocampus-dependent memory was worse at 9 months compared to 3 months of age in APPPS1-21 transgenic mice. In the present study, none of the transgenic (tg) groups, those born to an APPPS1-21 $\mathrm{tg}$ father or mother (tg ${ }^{\text {tgwt }}$ or $\operatorname{tg}^{\text {wttg }}$ respectively), acquired the task or learned in the Morris water maze (MWM) (Figure 4.2.9 and 4.2.10). The observed severe impairment at 9 months of age is in line with the reported exponential increase of $A \beta 42$ levels in the mouse brain and the spread of amyloid plaques covering the entire neocortex and almost the whole hippocampus (Radde et al., 2006). These results are consistent with previous observations of progressive cognitive impairment also of different AD mouse models, although the impairments 
were exhibited at different ages and for different performance tasks (Arendash et al., 2001; Chapman et al., 1999; Kelly et al., 2003; Sadowski et al., 2004; Van Dam et al., 2003). Specifically, the gradual spatial decline was observed in APPK670N/M671L/PS1M146L from 8 to 22 months of age (Sadowski et al., 2004) and APP23 from 3 to 25 months (Kelly et al., 2003; Van Dam et al., 2003).

Notably, APPPS1-21 tg offspring born to an APPPS1-21 tg father seemed to show more severe impairments than APPPS1-21 tg mice born to an APPPS1-21 tg mother (Figure4.2.10). This can be in part a consequence of the worsening of their motor abilities at 9 months of age during the open field (OF) and the MWM tasks (Figure 4.2.8 and 4.2.11). It is known that aging also promotes motor disabilities (Wirths \& Bayer, 2008), although none were demonstrated before for this model 18. Additionally, none of the transgenic groups of mice showed alterations in basal anxiety levels at 9 months of age as shown in figure 4.2.8. Several AD mouse models, including ours, showed associative memory impairments at old stages (Cheng, Logge, Low, Garner, \& Karl, 2013; Corcoran, Lu, Turner, \& Maren, 2002; Jacobsen et al., 2006; Kilgore et al., 2010; Knafo et al., 2009). The time point of this decline varies between models. APPSwe/PS1 $\mathrm{E} 9$ line showed associative memory deficits already at 6 months (Kilgore et al., 2010), whereas APP/ PS1 exhibited impairments at 12-14 months of age (Knafo et al., 2009).

Previous studies of the AD mouse model used for this study have shown that APPPS1-21 transgenic mice exhibited associative memory decline, but no motor impairments at 8 and 12 months of age (Govindarajan et al., 2013; Zovoilis et al., 2011). In the present study, APPPS1-21 transgenic mice at 9 months of age showed a significant decrease of the average motion during the training day and an increase of freezing behavior after 24 hours of contextual fear conditioning (Figure 4.2.12). There are two possible meanings for these findings. The first is that APPPS1-21 transgenic groups showed a better associative memory compared to control and their 3 months age counterparts. Secondly is that the decrease of mobility is translated as freezing episodes, giving a large number of percentage of freezing at the end of the test. Since APPPS1-21 transgenic mice showed motor impairments in open field, Morris water maze and average motion during the training day and previous studies confirmed the associative memory impairments at this age; I assumed that the percentage of freezing is an artifact from their motor impairments. In the future, I will need to change the analyses and extract more sensitive parameters in order to use and evaluate APPPS1-21 mice in fear conditioning. 


\subsubsection{No gender differences in behavioral phenotypes of offspring APPPS1-21 mice}

Several controversial results have been observed about gender effects in behavior experiments of mice from different strains (Bridgewater et al., 2017; Chow, Epp, Lieblich, Barha, \& Galea, 2013; Frick, Burlingame, Arters, \& Berger-Sweeney, 2000; Johnston \& File, 1991; King et al., 1999; Kreiner, Chmielarz, Roman, \& Nalepa, 2013; Rummel, Epp, \& Galea, 2010; Voikar, Koks, Vasar, \& Rauvala, 2001). Moreover, not many studies showed a comparable behavior analysis between males and females from an AD mouse model (King et al., 1999; Pistell, Zhu, \& Ingram, 2008). In the present study, no significant gender-specific differences were observed in all parameters of Morris water maze, open field (OF) and fear conditioning test (FC) (Appendix figure 1-9). Differences were only restricted to particular groups and specific parameters in the open field and fear conditioning tasks. Specifically, wild-type and transgenic females born to an APPPS1-21 transgenic father exhibited significantly faster speeds than corresponding males in the OF and during the training day of FC (Appendix figure 1 and 5). Previous studies found that female mice from a wild-type strain showed more activity and faster speeds in the OF and MWM tests than males from the same strain (Frick et al., 2000; Tucker, Fu, \& McCabe, 2016). At 9 months of age, only wild-type mice born to an APPPS1-21 transgenic mother showed higher anxiety levels than females (Appendix 9). In contrast, in the MWM task, there was no difference between males and females within the group they belonged to (either experimental or control) at 3 and 9 months of age (Appendix 2, 3, 7 and 8). One possible reason is that males and females from this mouse model of $A D$ showed similar levels of $A \beta$ and amyloid deposition at least at early stages (Radde et al., 2006).

Sex differences in an AD mouse model have only been reported to date by Smith et al. (1999) in the APPswe mouse line (King et al., 1999). They identified behavior differences between males and females at 3 and 9 months of age. Females showed impairments in the MWM retention and circular platform performance at 3 months of age. Males showed increased activity in the open field and the Y-maze at 3 months of age. And at 9 months of age, males and females exhibited difficulties to detect a visible platform in the water maze task (King et al., 1999). It is known that the sex-specific differences vary by strain (Voikar et al., 2001). Thus, it is not a surprise to observe different results between APPPS1-21 and APPswe mouse lines. It is crucial to study sex-specific behavior and molecular differences in order to understand in detail the pathology and possible treatment responses. However, in the past not many studies evaluated the behavior abilities of both genders. 


\subsubsection{Transcriptional profiling and pathway analysis in APPPS1-21 transgenic mice at $3 \mathrm{~m}$}

Alzheimer's Disease $(A D)$ has a strong genetic component, several studies have reported genetic mutations causing AD (Roses et al., 2010; W. Shao, Peng, \& Wang, 2017) and also the whole transcriptome changes at late stages of the pathology (Annese et al., 2018; Twine et al., 2011). However, few studies evaluated the entire transcriptome at an early AD stage using the next-generation sequencing (Gatta et al., 2014; Parra-Damas et al., 2014). In the present study, I analyzed the transcriptomic state of 2 hippocampal subregions (CA1 and DG) from 3 month-old APPPS1-21 transgenic mice as a readout of the cellular state at an early AD stage. Previous studies identified distinct dysregulation of the transcriptome at different stages of the pathology in different AD mouse lines (Bossers et al., 2010; Cribbs et al., 2012; Gatta et al., 2014; J. A. Miller et al., 2008; Podtelezhnikov et al., 2011). At early stages, the majority of changes in gene expression were considered to be part of a compensatory network pursuing the recovery of the physiological state (Bossers et al., 2010; Gatta et al., 2014). At late stages, the alteration of the transcriptome was mainly associated with the advanced progression of neurodegeneration pathology (Bossers et al., 2010; Cribbs et al., 2012; Gatta et al., 2014; J. A. Miller et al., 2008; Podtelezhnikov et al., 2011). During the progression of AD, different studies identified several changes in gene expression in different brain areas involved in synaptic function, neuronal death and autophagy, mitochondrial function, inflammatory response, intracellular calcium homeostasis, dendritic spine formation, lipid metabolism, cellular adhesion, transport, signaling pathways and cognitive decline (Blalock et al., 2004; Bossers et al., 2010; Gatta et al., 2014). The model used in this study, APPPS1-21 mice, showed some of these transcriptomic changes in the hippocampal subregions at 3 months of age (Figure 4.1.7). To gain a better understanding of whether amyloid deposition impacted transcription and to what extent, the transcriptome of CA1 was also evaluated, a region not affected by plaques at 3 months of age in this AD model (Radde et al., 2006). Notably, the transcriptome of the hippocampal subregion (DG) affected by amyloid plaques was strongly altered compared to the hippocampal subregion without plaques (CA1) as shown in figure 4.1.7. These data suggest that amyloid plaque deposition promotes changes in the transcription activity, but a more in-depth analysis would be needed to determine whether amyloid plaques are the consequence of pathology or the protective response to soluble A $\beta$ oligomer forms (Erten-Lyons et al., 2009; Sloane et al., 1997; Treusch, Cyr, \& Lindquist, 2009). From these changes, genes overrepresented in the transgenic CA1 region participated in pathways involved in: synaptic activity, cognition, cell-cell adhesion and lipid 
metabolism (Figure 4.1.8). In contrast, genes linked to RNA metabolic processes, ribosome biogenesis and mitochondrial activity were underrepresented in the CA1 of transgenic mice (Figure 4.1.8). Furthermore, in the DG, functional gene analyses indicated that genes related to synaptic activity, nervous system development, metabolic process, cell adhesion and signal transduction were overrepresented in DG of males and females (Figure 4.1.8). In contrast, transgenic mice showed a reduction of expression of genes involved in energy metabolism and protein biogenesis. These categories were similarly deregulated in DG from males and females, despite the low percentage of commonly affected genes. The alteration of similar functional networks is in agreement with the fact that both genders showed similar performance abilities, and $A \beta$ levels and amyloid deposition at early stages (Radde et al., 2006).

Since the most relevant alterations in gene expression were related to synaptic activity, neurogenesis, lipid metabolism, energy metabolism and protein biogenesis, I discussed these mechanisms in further detail.

\subsubsection{Up-regulation of genes involved in synaptic pathways}

Aging and late stages of Alzheimer's disease have been associated with synaptic dysfunction and reduction of genes linked to synaptic activities (Annese et al., 2018; Scheff \& Price, 2006). In contrast, in the present study genes related to synaptic activities were up-regulated in CA1 and DG hippocampal subregions of APPPS1-21 mice at early stages of the pathology. Genes such as Slc17a6, Pde11a, Igsf9b, Sv2c and Ston2, which were involved in synaptic vesicle, exocytosis of neurotransmitter and synaptic development were up-regulated in APPPS1-21 mice (Bai, Xu, Collins, \& Ghishan, 2001; Fawcett et al., 2000; Janz \& Sudhof, 1999; Maritzen, Podufall, \& Haucke, 2010; Woo et al., 2013) (Appendix table 3). In line with the increase of synaptic gene transcripts, a previous study found that at early Braak stages (I and II), levels of genes linked to synaptic activity and plasticity were increased in the hippocampus of 3xTg-AD female mice and prefrontal cortex of humans (Bossers et al., 2010; Gatta et al., 2014). Upregulation of genes related to synapse was associated with protective activities against $A \beta$ neurotoxicity (Haberman, Quigley, \& Gallagher, 2012; Kaur \& Levy, 2012; Mitchell et al., 2009). Furthermore, recent findings using different stimulation protocols confirmed the increased of hippocampal synaptic excitability in 3xTgAD mouse from 4 to 6 months (K. E. Davis, Fox, \& Gigg, 2014). This enhancement of genes associated with synaptic activities seemed to be accompanied by an increase of hippocampal and cortex activities (Busche et al., 2008; K. E. Davis et al., 2014; Palop et al., 2007; Saura, Parra-Damas, \& Enriquez-Barreto, 2015). Changes in synaptic activity at early $A D$ stages might be part of a complex mechanism that tries to maintain normal synaptic activity by counteracting the neuronal dysfunction. 
5.1.4.2.Up-regulation of genes involved in nervous system development pathways

The Dentate Gyrus (DG) subregion plays a critical role in neurogenesis (Bonaguidi et al., 2011; Cameron \& McKay, 2001; van Praag et al., 2002). Thus, it is not surprising that the majority of deregulated genes in the DG of APPPS1-21 transgenic mice were linked to neurogenesis, such as central nervous system differentiation, neuron projection morphogenesis, dendrite morphogenesis and regulation of neuron migration (Figure 4.1.11). Interestingly, I observed an increase of transcripts linked to nervous system development in transgenic mice that showed spatial cognitive deficits. Not many genetic studies of AD have described alterations of genes related to neurogenesis at early stages of the pathology (Bossers et al., 2010; Gatta et al., 2014). However, several studies have found changes in neurogenesis response in AD mouse models or human postmortem AD brains (Clelland et al., 2009; Haughey et al., 2002; Sun et al., 2009; Woitke et al., 2017). Some findings corroborated an increase of neurogenesis response in AD mouse models or human AD brains (Q. Chen et al., 2008; K. Jin, Galvan, et al., 2004; K. Jin, Peel, et al., 2004; Lopez-Toledano \& Shelanski, 2007; Yu et al., 2009), whereas others studies suggested no effect or a decrease of neurogenesis activity (Haughey et al., 2002; J. J. Rodriguez et al., 2008; Sun et al., 2009; R. Wang, Dineley, Sweatt, \& Zheng, 2004). My data is in line with a previous study that showed an increase of proliferation and neuronal differentiation in the hippocampus of J20 mice (a mouse model of AD) at 3 months of age. Since the oligomeric $A \beta$ form was also increased, they claimed that the enhanced neurogenesis is induced by $A \beta$ (Lopez-Toledano \& Shelanski, 2007). In contrast, previous findings showed a reduction of proliferation and survival of neural progenitor cells in the DG of different AD mouse models at late AD stages (12-14 months) (Haughey et al., 2002). Moreover, a previous genetic study identified an up-regulation of genes related to nervous system development at early stages of the pathology (Braak I-II) and a reduction of these pathways at late stages (Braak V-VI) (Bossers et al., 2010). Considering these studies and my data, I detected that there is a significant difference between neurogenesis response at early and late AD stages. The majority of the studies at early $A D$ stages identified an enhancement or non-altered neurogenesis response (Bossers et al., 2010; Q. Chen et al., 2008; K. Jin, Galvan, et al., 2004; Lopez-Toledano \& Shelanski, 2007), whereas several studies performed at old stages observed impaired neurogenesis (Bossers et al., 2010; Donovan et al., 2006; Haughey et al., 2002). In summary, my data and previous literature suggest that neurogenesis response plays an important role during $A D$ progression, which differs between early and late AD stages. These results indicate that the up-regulation of neurogenesis-related genes and consequently response at early stages might be part of a compensatory network, which tries to delay the progression of the disease. 
5.1.4.3.Up-regulation of genes involved in lipid metabolism pathways

The role of lipids in neurodegenerative diseases has been intensively studied in the last decades (Bales, 2010; Hamilton et al., 2015; Koudinov \& Berezov, 2005; Q. Liu \& Zhang, 2014; Mills et al., 2013). However, not many studies described the role of dysregulated genes related to lipid metabolism at early AD stages (Barbash et al., 2017; Blalock et al., 2004; Podtelezhnikov et al., 2011). In the present study, I observed that genes linked to lipid metabolic processes were exclusively increased in the CA1 of APPPS1-21 transgenic mice at early AD stages (Figure 4.1.8). These genes were linked to positive regulation of triglyceride metabolic process, regulation of lipid catabolic process and steroid metabolic process. Recent findings also observed this enhancement of genes linked to lipid metabolism in the prefrontal cortex and hippocampus of human postmortem samples at early stages of AD (Blalock et al., 2003; Blalock et al., 2004; Podtelezhnikov et al., 2011). These studies suggest that the up-regulation of these genes play a role in protective mechanisms. This data suggests that there is an activation of different types of compensatory mechanisms that try to delay the pathology.

\subsubsection{Down-regulation of genes involved in energy metabolism}

Mitochondrial impairments have been involved in several brain disorders, such as Down syndrome, autism, Parkinson and Alzheimer's disease (Bhat et al., 2015; Lin \& Beal, 2006; Nunnari \& Suomalainen, 2012; Valenti, de Bari, De Filippis, Henrion-Caude, \& Vacca, 2014). Previous studies have shown that transcripts associated with energy metabolism were downregulated in mouse and human brain regions at advanced stages of $A D$ and during normal aging (Annese et al., 2018; Berchtold et al., 2008; Fang et al., 2016; Gatta et al., 2014; Leshchyns'ka \& Sytnyk, 2016; Liang et al., 2008; J. A. Miller et al., 2008; Sekar et al., 2015; Valla, Berndt, \& Gonzalez-Lima, 2001). Damage to mitochondrial DNA leads to a reduction of mitochondrial gene expression and therefore to a decrease in mitochondrial proteins increasing the proportion of reactive oxygen. This increase of oxidative stress and mitochondrial dysfunction triggers neurodegeneration (Beal, 2005; Bhat et al., 2015; Di Carlo, Giacomazza, Picone, Nuzzo, \& San Biagio, 2012; Lin \& Beal, 2006; Swerdlow, Burns, \& Khan, 2014; Trushina \& McMurray, 2007). Furthermore, other studies suggested that mitochondria processes could lead to and promote several AD pathological processes, such as altering APP expression, APP processing, A $\beta$ accumulation and inducing inflammation (Ichimura, Parthasarathi, Quadri, Issekutz, \& Bhattacharya, 2003; Swerdlow et al., 2014). In contrast to previous findings that observed mitochondrial alterations at late AD stages, APPPS1-21 transgenic mice showed a decrease of 
transcripts related to energy metabolism already at an early AD stage (3 months of age) (Figure 4.1.8 and 4.1.11). Pathways linked to mitochondrial function, respiratory chain complex, ATPase activity and ion transport were strongly reduced in APPPS1-21 mice at 3 months of age.

Examples of genes down-regulated in the DG of APPPS1-21 mice included Cox4i1 (cytochrome C oxidase subunit in mitochondrial electron transport (Lomax, Hewett-Emmett, Yang, \& Grossman, 1992)), Ndufa1 and Ndufa3 (subunits of the mitochondrial membrane respiratory chain NADH dehydrogenase (Janz \& Sudhof, 1999; Loeffen et al., 1998)) and Mrp/20 (mitochondrial ribosomal protein (Kenmochi et al., 2001)) (Appendix table 3). The majority of studies at early stages have not identified down-regulation of genes related to mitochondria activity (Gatta et al., 2014; Parra-Damas et al., 2014). One prominent difference compared to the mouse AD lines (3xTg-AD and APPSw,Ind) used in these two studies is that APPPS1-21 mice showed cognitive deficits at 3 months of age (Gatta et al., 2014; Parra-Damas et al., 2014). Moreover, mitochondrial dysfunction has been associated with cognitive decline (Finsterer, 2012; Y. Hara et al., 2014; Khacho et al., 2017; McEwen, 2012; McManus, Murphy, \& Franklin, 2011; Valenti et al., 2014). In summary, my data strongly suggest that there is a correlation between alteration of energy metabolism pathways and the onset of decline in cognitive abilities.

\subsubsection{Down-regulation of genes involved in protein biogenesis}

Similar to mitochondrial dysfunctions, ribosome and translation alterations have been reported for late AD stages or with aging (Berchtold et al., 2008; Ding, Markesbery, Chen, Li, \& Keller, 2005; Hernandez-Ortega, Garcia-Esparcia, Gil, Lucas, \& Ferrer, 2016; Langstrom, Anderson, Lindroos, Winblad, \& Wallace, 1989; X. Li et al., 2004). A study observed dysfunction of protein synthesis specifically in the posterior cingulate of AD postmortem human brain (Berchtold et al., 2008). In the present study, I identified that genes associated with protein synthesis were downregulated in APPPS1-21 transgenic mice at early AD stage, such as genes related to ribosomerelated processes, translation and protein localization and transport (Figure 4.1.8 and 4.1.11). In line with my data, a previous finding described a substantial decrease in polyribosomes activities, altered tRNAs forms and levels of rRNA processing in cortical brain regions of AD subjects at early stages (Ding et al., 2005). This previous study suggested that the impairment of protein synthesis was one of the earliest alterations in AD pathology (Ding et al., 2005). Moreover, a previous research detected a reduction in gene expression and protein levels linked to the process of protein synthesis in human brain tissue at early stages of AD (Braak I-II) (Hernandez-Ortega et al., 2016). The precise mechanisms by which genes related to protein synthesis are altered remain to be identified. One possibility is that part of these changes is affected by oxidative stress causing mitochondrial genetic dysfunction. 
In line with previous studies using human AD samples (Hernandez-Ortega et al., 2016), I identified a reduction of genes involved in protein synthesis at early AD stage. APPPS1-21 mice showed a decrease of catabolic and anabolic processes already at 3 months of age. Considering that APPPS1-21 mice showed certain features at early stages typical of late stages, such as cognitive deficits or reduction of genes related to energy metabolism and protein biosynthesis, I conclude that this transgenic model has an aggressive AD pathology. Moreover, I was also able to identify specific genetic changes that are more likely to be part of a compensatory network, such as up-regulation of genes involved in synaptic activities and neurogenesis.

\subsubsection{Alternative exon usage and pathway analysis in APPPS1-21 transgenic mice at $3 \mathrm{~m}$}

Different exon usage of specific genes has been reported in various Alzheimer's disease (AD) postmortem brain tissues and in AD mouse models at different stages and cognitive decline (Beffert et al., 2005; Hinrich et al., 2016; M. K. Lai et al., 2014; Mazin et al., 2013; Stilling et al., 2014; Tollervey et al., 2011; Twine et al., 2011). APPPS1-21 transgenic mice showed changes in expression of genes involved in splicing processes, such as U12 type spliceosomal complex, spliceosomal tri snRNP complex, catalytic step 2 spliceosome and spliceosomal complex. Moreover, it is known that several genes directly associated with $A D$ show different exon usages, such as amyloid precursor protein (App), apolipoprotein $\mathrm{E}$ (Apoe) (Beffert et al., 2005; Twine et al., 2011), microtubule-associated protein tau (Mapt) (Goedert \& Jakes, 1990) or presenilin 1 (Ps1) (De Jonghe et al., 1999). I was able to identify different exon usage in CA1 and DG of APPPS1-21 transgenic mice of App, Ps1 and Mapt (Appendix table 1).

Furthermore, I identified massive changes in differentially exon usage in the DG and the CA1 brain subregions of APPPS1-21 transgenic mice, specifically exon inclusions (Figure 4.1.13). The number of genes affected for alternative exon usage was larger than for expression levels and covered the majority of biological processes in CA1 and DG hippocampal subregions of APPPS1-21 mice. There is no agreement of whether these two processes of regulation, gene expression and mRNA processing, altered similar or distinct networks (Stilling et al., 2014; Tollervey et al., 2011). Some studies detected that these mechanisms changed different pathways 118, others suggested that these processes affected similar genes 117 . In this study, despite the seemingly small overlap among the genes differentially expressed and differentially "spliced" (alternative exon usage) (30\%), these two regulatory mechanisms affected similar global categories (Figure 4.1.15). These functions affected: tissue and nervous system 
development, synaptic activity, RNA metabolic process, energy metabolism, microtubule and cytoskeleton organization, cell metabolic process, chromatin modification, cell death, transmembrane transport and response to a stimulus (Figure 4.1.16). The role of some of these functions in early $A D$ stages was already described in the previous section. The categories affected by both regulatory mechanisms suggest that APPPS1-21 transgenic mice at early stages try to return to a homeostatic state by modifying the expression of specific genes and also altering the mRNA processing of specific genes.

Furthermore, alternative exon usage has been detected in interesting candidate genes that were previously associated with memory process and neurodegenerative pathologies (Appendix table 4). The alteration of exon usage of these genes maybe plays an essential role in the progression of $A D$ pathology. Further investigations are necessary in order to confirm the association of this alternative exon usage forms and $A D$ pathology. For example, I identified in the DG from males: Slc6a17, Dlgap4, S/c6a9 and Dock3. Solute carrier family 6 member 17 (S/c6a17) was involved in the vesicular transport for neurotransmitters and amino acids in synaptic transmission (Hoglund, Adzic, Scicluna, Lindblom, \& Fredriksson, 2005). A specific mutation in this gene promoted the loss of dendritic spines, mental disabilities and behavior problems ( $Z$. Iqbal et al., 2015). DLG associated protein 4 (Dlgap4) encodes a protein member of PSD95-interacting proteins and participates in the molecular synapse organization and neuronal cell signaling (Fernandez et al., 2009; Minocherhomji et al., 2014). The family of PSD95 regulates neuronal plasticity and is essential to keep physiological learning and memory abilities (Carlisle, Fink, Grant, \& O'Dell, 2008; Migaud et al., 1998). Solute carrier family 6 member 9 (S/c6a9) encodes the glycine transporter 1 GlyT1 and the inhibition of this transporter enhances the function of NMDA receptors and improves memory retention (Tsai et al., 2004). Dedicator of cytokinesis 3 (Dock3) coding protein is part of a family of guanine nucleotide exchange factors (GEFs) and participates in cell-adhesion and a presenilin-binding protein (Nagase et al., 1997). It has been associated with $A D$ by the interaction with presenilin and the ability to stimulate Tau/MAPT phosphorylation (Namekata, Kimura, Kawamura, Harada, \& Harada, 2014). And, it is also implicated in various neurological disorders (de Silva et al., 2003; Namekata, Kimura, Harada, et al., 2014).

Moreover, in the DG from female APPPS1-21 mice showed differentially exon usage in genes including, Nrxn3, Dnm3 and Kdm5a genes. Neurexin 3 protein (Nrxn3) has been involved in synaptic cell adhesion and presynaptic voltage-gated calcium signaling (Missler et al., 2003). The interaction of neurexin with neuroligin has been related to AD progression (Sindi, Tannenberg, \& Dodd, 2014). Dynamin 3 (Dnm3) encodes for a member of GTP-binding proteins that is involved in cytoskeleton remodeling and clathrin-mediated synaptic vesicle endocytosis (Ferguson \& De Camilli, 2012; Raimondi et al., 2011). The family of dynamin has been 
associated with different neurodegenerative pathologies, such as Parkinson's disease or AD (Aidaralieva et al., 2008; Gonzalez-Jamett et al., 2014; Trinh et al., 2016). Lysine (K)-specific demethylase $5 \mathrm{~A}(K d m 5 a)$ gene encodes for a histone demethylase that demethylates the Lys-4 of histone H3 (H3K4) (G. B. Shao et al., 2014). Dysregulation of H3K4 methylation has been involved in several pathologies including autism, intellectual disability or schizophrenia (Shen, Shulha, Weng, \& Akbarian, 2014).

For example, in the CA1 from male APPPS1-21 mice, some interesting candidate genes were differentially spliced, such as the Nrxn3, Frmd4a or Reln genes. FERM domain containing 4A (Frmd4a) encodes for a protein that participates in the cell structure, transport and signaling. The expression of this gene was reduced in brains of Alzheimer's disease patients, promoting high levels of Tau secretion (Lambert et al., 2013). The reelin (Reln) gene encodes for reelin, which is a glycoprotein that participates in synaptogenesis and neural migration (Dazzo et al., 2015). It has been related to several pathologies, such as Alzheimer's disease, epilepsy or schizophrenia (Lane-Donovan et al., 2015; Negron-Oyarzo, Lara-Vasquez, Palacios-Garcia, Fuentealba, \& Aboitiz, 2016; Zaki et al., 2007).

These previous findings suggest that these genes can play an essential role in the pathology of Alzheimer's disease and it is, therefore, important to evaluate the impact that alternative exon forms of these genes could have in the pathology. 


\title{
5.2.Paternal line-specific inheritance of cognitive deficits in APPPS1-21 mice via non-genetic mechanisms
}

\author{
5.2.1.Spatial cognitive behavior in wild-type mice born to one \\ APPPS1-21 parent
}

Internal conditions are modified during the course of life of an individual by interaction with external environmental factors. Changes in epigenetic factors mainly drive the interaction between these two conditions. External factors can promote reversible or permanent changes of the epigenome of an individual, inducing phenotypic changes (Ho, Tang, Belmonte de Frausto, \& Prins, 2006; Martinez et al., 2009; Vucetic, Kimmel, \& Reyes, 2011; Waterland \& Jirtle, 2003). Multiple of these modifications can be transmitted to the next generations, stimulating changes in their internal conditions, which can be temporal or permanent (Anway et al., 2005; Byrnes, 2005; Carone et al., 2010; Chamorro-Garcia et al., 2013; de Castro Barbosa et al., 2016b; Dias \& Ressler, 2014b; Dunn \& Bale, 2009; Gapp et al., 2014; Ng et al., 2010; Ost et al., 2014; Ribeiro et al., 2017; Schmauss et al., 2014; Vassoler et al., 2013; Wei et al., 2014; S. Zhang et al., 2017). Some studies reported intergenerational or transgenerational inheritance as a protective mechanism, such as improving cognitive performance and synaptic plasticity by paternal spatial training or prevention of obesity in rat offspring when mothers exercise at low intensity during pregnancy (Ribeiro et al., 2017; Vassoler et al., 2013; S. Zhang et al., 2017). However, in some other cases, this transmission of information became a disadvantage for the next generation. For example, exposure of at least one parent to early traumas, diabetes, highfatty diets, morphine or opioids led to negative consequences to their offspring (Anway et al., 2005; Byrnes, 2005; Chamorro-Garcia et al., 2013; de Castro Barbosa et al., 2016b; Dunn \& Bale, 2009; Gapp et al., 2014; Ng et al., 2010; Ost et al., 2014; Schmauss et al., 2014; Wei et al., 2014). These studies were based on the internalization of external factors and posterior transmission of this information to their offspring. Additionally to external factors, there is a strong association between the genotype and the epigenome (Ladd-Acosta \& Fallin, 2016; M. P. Lee, 2012). Here, I described for the first time the behavioral repercussion of the parental origin of the APP and PS1 transgenes. In this study, I used the APPPS1-21 transgenic mouse to describe the potential impact of the parental origin of the APP (human APP with K670N and M671L mutations) and PS1 (human PS1 with L166P mutation) transgenes. These transgenes 
promote phenotypic alterations in the transgenic mice, including cognitive impairments and cerebral amyloidosis (Radde et al., 2006).

APPPS1-21 transgenic offspring (tg) born to tg fathers (tg ${ }^{\text {tgwt }}$ ) or tg mothers (tg ${ }^{\text {wtg }}$ ) showed spatial learning and memory impairments at 3 and 9 months of age. However, tg $^{\text {tgwt }}$ exhibited more severe deficits during acquisition and learning in the Morris water maze (MWM), a spatial memory task, than tgwttg at 3 and 9 months (Figure 4.1.4, 4.1.5, 4.2.9 and 4.2.10). The learning deficits were not due to distinct motor abilities since both groups showed similar traveling speeds during the open field and Morris water maze tasks at 3 months of age (Figure 4.2.2 and 4.2.5). This data suggests that the origin of the transgene has an impact on the behavioral phenotype of the next generation. Previous studies have shown a direct connection between behavioral changes in the offspring and paternal epigenetic transmission of information related to a particular form of stress (Gapp et al., 2014; Rodgers et al., 2015).

In order to confirm the possible implication of non-genetic factors associated with the APP and PS1 transgenes from the mother or father, I investigated the behavioral phenotype of wild-type mice born to one APPPS1-21 transgenic parent.

The behavioral study of these wild-type mice confirmed a certain form of non-genetic transmission associated with the parental origin of the transgene. Wild-type mice born to APPPS1-21 transgenic fathers showed difficulties in learning a spatial task (the MWM) already at 3 months of age (Figure 4.2.3 and 4.2.4). On the contrary, wild-type mice born to APPPS1-21 transgenic mothers learned the spatial task similar to the control group (wild-type mice born to two wild-type parents) (Figure 4.2.3 and 4.2.4). Interestingly, this transmission was specific for a spatial learning and memory task, such as the MWM. Other abilities, such as associative skills were not affected. As shown in the fear conditioning test (Figure 4.2.6). In contrast to other studies, I found that this kind of transmission altered in the same manner the behavior of both genders in the offspring (Appendix figure 1-9) (Alter et al., 2009; de Castro Barbosa et al., 2016b; Vassoler et al., 2013).

Analyzing wild-type mice born to an APPPS1-21 transgenic father or mother at 9 months of age, I observed consistent results with the data obtained at 3 months of age. Wild-type mice born to APPPS1-21 transgenic fathers also showed impairments in spatial learning and memory abilities at 9 months of age. In contrast, wild-type mice born to transgenic mothers ( $w t^{t}{ }^{w t g}$ ) still learned the task and performed as well as the wild-type control mice born to wild-type parents. As shown in the Morris water maze test (Figure 4.2.3 and 4.2.4). These results suggest that intergenerational transmission of a specific behavior is quite stable and persists over time. Notably and similar to what I observed with transgenic and wild-type mice at 3 months of age, no remarkable cognitive differences were visible between wild-type female and male mice born to a transgenic father or mother at 9 months of age (Appendix figure 6-9). Regarding motor abilities, only $w^{\text {tgwt }}$ mice 
showed a significant reduction in swimming speed during the MWM and open field tasks at 9 months of age, a decline that was similar to their co-offspring transgenic group (tgtgwt) (Figure 4.2.10).

Most importantly, these results suggest gender-specific mechanisms that have a negative impact on their offspring in the case of paternal transmission. While it is difficult to pinpoint what those factors may be, based on the published literature, there might be gender-specific epigenetic changes that interplay with the APP and PS1 transgenes, which can be transmitted differently to the next-generation 85,118,(Brykczynska et al., 2010; de Castro Barbosa et al., 2016b; X. Feng \& Guang, 2013; Gapp et al., 2014; Hammoud et al., 2009; Rodgers et al., 2013; Siklenka et al., 2015).

The fact that identical wild-type mice born to the same strain presented different behavioral abilities pose a big issue for future mating designs. And breeding schemes need to be carefully controlled for at least this line in the future. Parameters such as the origin of the transgene, the selection of the control group, the establishment of specific environmental conditions and refreshing of mouse colonies have to be properly defined. It is essential to select an appropriate control group, which should not interfere with the experimental data. For that, it is necessary to evaluate first whether the wild-type littermate from a transgenic line is suitable or not to be used for the study. Moreover, the observation behavior differences between wild-type mice emphasize the importance to investigate in further detail the form of non-genetic inheritance associated with a transgene in this and other mouse lines. It is crucial to evaluate the impact of epigenetic marks in order to predict behavioral phenotypes, maintain colony stability and to obtain reproducible results. The main challenge to assess epigenetic marks is their continuous change during the lifetime of an individual in response to the external conditions. Thus, it is essential to design statistical tools to predict and evaluate the level of epigenetic contribution to the phenotype changes, using regularized regression, non-parametric methods or machine learning (de los Campos et al., 2009; Gonzalez-Recio, 2011; Gonzalez-Recio et al., 2008; Slatkin, 2009; Tal, Kisdi, \& Jablonka, 2010; Varona et al., 2015). For that, it is also necessary to improve the accuracy and efficiency of epigenome-wide mapping and to develop new next-generation sequencing equipment, which allows for sequencing in large scale at an affordable cost. 


\subsubsection{Transcriptional profiling and pathway analysis in APPPS1-21 wild-type mice at $3 \mathrm{~m}$}

In my study, I observed that wild-type mice born to an APPPS1-21 transgenic father showed different spatial cognitive abilities at 3 and 9 months of age. These phenotypic changes suggest the existence of some transmission of gender-specific epigenetic factors through the germ-line. These transmitted epigenetic factors and environmental factors can modify the epigenetic program, which together with the genomic program, play an essential role during development (Skinner, 2011b; I. C. Weaver, Meaney, \& Szyf, 2006). In some specific adult cells and tissues, these epigenetic persists into adulthood, promoting an altered expression pattern of genes and the phenotype, or even increasing the probability to develop a pathology (Skinner, 2011a, 2011b; Skinner, Manikkam, \& Guerrero-Bosagna, 2010). Here, I analyzed the transcriptome of 2 hippocampal regions implicated in spatial memory of wild-type mice born to an APPPS1-21 transgenic father or mother at 3 months of age. I identified changes in the expression pattern of genes in the CA1 and DG of wild-type mice born to one APPPS1-21 transgenic parent. Notable, the transcriptomic profile from wild-type mice born to an APPPS1-21 transgenic mother (wtwttg) was almost not affected. Only a few genes showed significant differential expression in CA1 and DG of wtwttg mice. Whereas, wild-type mice born to an APPPS1-21 tg father (wt ${ }^{\text {tgwt) }}$ showed differential expression of several genes. Both groups of mice were compared to wild-type mice born to wild-type parents ( $w^{t^{\text {wtwt }}}$ ), as shown in figure 4.2.21. Thus, I did not further investigate in detail the pathways related to the transcriptome of wt mice born to APPPS1-21 tg mothers (wt ${ }^{\text {wttg }}$. These transcriptomic differences between two identical wild-type mice reaffirm the idea that APPPS1-21 transgenic mothers and fathers transmit different epigenetic factors to the offspring, promoting distinct alterations to the adult cellular state. In this study, I did not address how these epigenetic factors from the germline escape the reprogramming process or how these epigenetic marks persist into adulthood. The mechanisms remain unknown, and further investigations are necessary.

In the present study, I evaluated in detail the transcriptome of CA1 and DG hippocampal subregions of wild-type mice born to APPPS1-21 transgenic fathers at 3 months of age. To note, there was a tendency to up-regulated genes in CA1 and DG of wild-type mice born to APPPS1-21 transgenic fathers ( $w^{\text {tgwt }}$ ) (Figure 4.2.21). The DG from male and female wt ${ }^{\text {tgwt }}$ mice showed more transcriptomic differences than the CA1 of wt ${ }^{\text {tgwt }}$ (Figure 4.2.21). Despite the little number of shared genes between regions and genders, in global these regions and genders showed distinct transcriptome profile suggesting that the transmitted epigenetic factors induce changes in different tissue in a gender-specific way. In line with my data, previous 
transgenerational transcriptome findings identified tissue-specific and sex-specific changes in gene expression in various tissues of F3 generation rats from grandparents treated with vinclozolin or fed with high-fat diets (Alm, de Castro Barbosa, Barres, Krook, \& Zierath, 2017; Skinner, Manikkam, Haque, Zhang, \& Savenkova, 2012). In contrast to this tissue-specific alterations, paternal high-fat diets altered similar gene expression and pathways changes from retroperitoneal adipose and islet tissues ( $\mathrm{Ng}$ et al., 2014). Thus, in future studies will be interesting to evaluate how different is the transcriptome from other brain regions and other tissues of wild-type mice born to one APPPS1-21 transgenic parent. It is important to investigate the level of gene expression changes promoted by intergenerational epigenetic factors, in order to predict other phenotypic changes and discover potential reversal mechanisms. Although CA1 and DG hippocampal subregions of wild-type mice born to an APPPS1-21 transgenic father ( $w^{\text {tgwt}}$ ) showed differential expression of genes, alteration of common functional categories was identified in these brain regions. Notable, several of the up-regulated genes in wtgwt hippocampal subregions were involved in pathways previously categorized for APPPS1-21 transgenic mice such as synapse, nervous system development, cell-cell adhesion, signal transduction and lipid metabolism (exclusively in CA1 from males) (Figure 4.2.24). Moreover, genes connected with transcription process, mitochondrial functions, protein localization, cellular metabolic and RNA metabolic process (DG from males) were underrepresented in wild-type and transgenic mice born to APPPS1-21 transgenic fathers (Figure 4.2.24). Since the majority of these pathways were previously described in detail, I did not explain them here.

Taken together, this data indicates that non-genetic transmission from transgenic APPPS1-21 fathers has a strong repercussion in their offspring, altering the behavioral phenotype and the cellular state of wild-type and transgenic offspring. Wild-type mice born to APPPS1-21 tg fathers (wt ${ }^{\text {tgwt }}$ ) exhibited similar altered pathways than APPPS1-21 transgenic mice at 3 months of age. It will be necessary for future research to evaluate whether the transcriptomic state detected in wild-type mice born to APPPS1-21 transgenic fathers at 3 months of age is stable or change along the course of aging. Overall, I identified for the first time transcriptomic changes associated with the origin of the APP and PS1 transgene. 


\subsubsection{Alternative exon usage and pathway analysis in APPPS1-21 wild-type mice at $3 \mathrm{~m}$}

In addition to the transcriptomic changes, wild-type mice born to APPPS1-21 tg fathers (wt ${ }^{\text {tgwt}}$ ) exhibited a massive alteration of the alternative exon usage in CA1 and DG regions, with an increased tendency of exon inclusion events, as shown in the figure 4.2.30. To my knowledge, the present study is the first to identify alterations of expressed exons associated with an intergenerational inheritance process. In line with transcriptomic data, CA1 or DG brain regions of wild-type mice born to APPPS1-21 transgenic mothers ( $\left.w^{t^{w t t g}}\right)$ showed few changes in alternative exon usage compared to control wild-type mice, as displayed in the figure 4.2.30. The precise mechanisms by which paternal or maternal intergenerational epigenetic factors promote exon usage changes in specific genes remain to be elucidated. One explanation could be that the germ-line epigenome alters the physiological mRNA processing mechanism during development promoting a different exon usage of particular genes, which could persist in specific tissues into adulthood. An experiment that could proportionate some evidence of the alteration of the mRNA processing process would be to investigate the alternative exon usage of wild-type mice born to APPPS1-21 transgenic mice in early life.

Furthermore, similar to APPPS1-21 tG mice, DG of male and female mice born to APPPS1-21 transgenic fathers ( $w^{t^{\text {tgwt }}}$ ) showed an aberrant modification of the alternative exon usage events (Figure 4.2.30). In contrast, few changes in alternative exon usage of genes were detected in CA1 region of $w^{t g w t}$ mice, which were associated with presynaptic pathways (Figure 4.2.30). Some interesting genes that were previously related to memory process or Alzheimer's disease were identified in the most significant "spliced" genes (top 10, ranked by $\log _{2} \mathrm{FC}$ ) in each region of wt ${ }^{\text {tgwt }}$ mice (Appendix table 5). For example in DG males, Ptpru, Wdr13 and Sbf1 genes had an alternative exon usage. WD repeat domain 13 (Wdr13) encodes a protein of a family involved in cell cycle regulation, transcription process, chromatin organization and protein trafficking (Singh et al., 2012). Deletion of Wdr13 gene promotes better performance of a spatial cognitive task and up-regulation of synaptic proteins (Mitra et al., 2016). Protein tyrosine phosphatase receptor type $U$ (Ptprul) and set binding factor 1 (Sbf1) encode for members of a family of protein tyrosine phosphatase (PTP) that played a role in cell growth, mitosis, differentiation and synaptogenesis. This family has been associated with several pathologies including Alzheimer disease (AD), Parkinson's disease (PD) and multiple sclerosis (MS) (W. J. Hendriks et al., 2013). In DG from female wttgwt mice, several interesting genes showed alternative exon usages, such as Nrxn3, Hook3 and Pms2. Neurexin 3 (Nrxn3) encodes for a protein involved in presynaptic voltage-gated calcium signaling and synaptic cell adhesion (Missler et al., 2003). The interaction 
of neurexin with neuroligin has been related to AD progression (Sindi et al., 2014). Hook microtubule tethering protein 3 (Hook3) encodes for a member of the microtubule-binding protein family, which was linked to AD (Herrmann et al., 2015; Walenta, Didier, Liu, \& Kramer, 2001) as well. PMS1 homolog 2 (Pms2) encodes for a member of a mismatch repair system. Dysfunction of DNA repair process has been associated with neurodegeneration (Baker et al., 1995; Jeppesen, Bohr, \& Stevnsner, 2011).

For example, in the CA1 brain region of wt ${ }^{\text {tgwt }}$ mice, Gabra2 and Agpat1 genes had an alternative exon usage. Gamma-aminobutyric acid (GABA) A receptor (Gabra2) encode for a GABA-A receptor. Expression of this receptor was altered in AD and aged brains (Rissman, De Blas, \& Armstrong, 2007). 1-acylglycerol-3-phosphate O-acyltransferase 1 (Agpat1) encodes for an acyltransferase that has been found as a new candidate for AD (Sherva et al., 2011). Moreover, about $33 \%$ of significant alternative exon usage overlapped with differentially expressed genes in DG males and 70\% in DG females of wild-type mice born to APPPS1-21 transgenic fathers ( $w^{t^{t g w t}}$ ) (Figure 4.2.35). Differentially expressed genes in CA1 region of wtgwt mice did not present changes in the exon usage. In contrast, differentially expressed and spliced genes in DG region of male and female $w^{\text {tgwt }}$ mice were involved in nervous system development, synapse, transmembrane transport, microtubule modification, chromatin modification and RNA metabolic process (Figure 4.2.36).

These results support the idea that some non-genetic factors in paternal germ-line induce behavior, transcriptome and alternative exon usage changes in the wild-type mice born to APPPS1-21 transgenic fathers. Furthermore, this increases the evidence of the critical role of non-genetic inheritance in offspring mice born to transgenic fathers and the necessity to evaluate this transmission in other transgenic lines.

\subsubsection{Transmission of non-genetic factors by germ-line}

Severe changes in behavioral level, transcriptomic profile and alternative exon usage from wildtype offspring born to APPPS1-21 transgenic fathers strongly suggest a non-genetic transmission of the "information" through the germ cells. The specific mechanism for the maternal or paternal transfer to the offspring and changes assorted to it is currently unknown. It seems that specific epigenetic marks could escape from the reprogramming process and transmit certain information to the next generations (Pasque, Jullien, Miyamoto, Halley-Stott, \& Gurdon, 2011; Skinner, 2011b). Distinct epigenetic marks were described as possible transmitters of intergenerational information through the germline 85,118,(Brykczynska et al., 2010; de Castro Barbosa et al., 2016b; X. Feng \& Guang, 2013; Gapp et al., 2014; Hammoud et 
al., 2009; Rodgers et al., 2013; Siklenka et al., 2015). Since the transmission of cognitive deficits was only detected between APPPS1-21 transgenic fathers and their offspring, I assumed that some non-genetic factors would be transmitted through the sperm. Previous studies have demonstrated the role of non-coding RNAs (ncRNAs) for certain forms of intergenerational transmission of stress (Gapp et al., 2014; Rodgers et al., 2015) or environmental enrichment (Benito et al., 2018). In this study, I observed that intergenerational inheritance of a cognitive phenotype induced by APP and PS1 transgene involved changes in sperm small RNAs. The sperm RNA of APPPS1-21 transgenic fathers showed an overexpression of specific small RNAs, which corresponded to different small nuclear RNAs (snoRNAs) (snord15b-201, snord111-201, snord60-201, snord96a-201, snord47-201 (U47)), piwi-interacting RNAs (piRNA_000578, piRNA_018323 and piRNA_003040) and one novel microRNA (miRNA-6240) (Figure 4.2.37). These small RNAs have been previously identified in RNA isolated from sperm (http:// spermbase.org/). Although these small RNAs have been identified previously in the sperm, not much is known about their functions. Snord47 was involved in tumorigenesis suppression (Xu et al., 2017); all three piRNAs were previously identified in the cerebellum of the Mecp2 knockout mouse (model of Rett Syndrome) (Saxena et al., 2012) and piRNA_003040 was highly expressed in the renal cell carcinoma (Y. Li et al., 2015).

It is not the first time that small RNAs were suggested for intergenerational or transgenerational transmission. Previously, some miRNAs (Benito et al., 2018; Rodgers et al., 2015) and transfer RNAs (tRNAs) (Q. Chen et al., 2016; Sharma et al., 2016) were associated with this kind of transmission. Thus, miRNA-6240 could be a potential candidate for intergenerational transmission of a cognitive phenotype induced by an APP and PS1 transgene.

Furthermore, it is the first time to my knowledge that snoRNAs were associated with intergenerational inheritance. SnoRNAs are small structures of RNAs that in the past were only linked to the process of ribosome biogenesis (rRNA), by guiding site-specific modifications of pre-rRNA (Rogelj, 2006). In the last decade, snoRNAs have been involved in other functions, such as regulation of chromatin structure (Schubert et al., 2012), regulation of splicing (Kishore et al., 2010; Scott et al., 2012; Q. F. Yin et al., 2012), mediators of oxidative stress (Michel et al., 2011; Scott et al., 2012) and precursors of small RNAs with miRNA-like functions (Brameier et al., 2011; Ender et al., 2008; Ono et al., 2011; Scott et al., 2009). Previous findings indicated that fragments of snoRNAs are processed and involved in similar functions than miRNAs (Brameier et al., 2011; Ender et al., 2008; Falaleeva \& Stamm, 2013; Garcia-Lopez et al., 2015; Ono et al., 2011; Scott et al., 2009; Taft et al., 2009). Moreover, snoRNAs were found in the sperm, where these structures showed similar levels of expression than miRNAs in different stages of male germ cell development (Garcia-Lopez et al., 2015). In addition to the involvement of snoRNAs in the process of development, my data suggest a potential role of the snoRNAs in 
intergenerational transmission, directly or as a precursor form of specific miRNAs. Further experiments are necessary to confirm this new role of snoRNAs.

Moreover, piRNAs are small structures (20-30 nucleotides) highly expressed in the germline of several species (Fu \& Wang, 2014; Klattenhoff \& Theurkauf, 2008). The primary function of these small RNAs is to silence the transposable elements during germ cell development in order to maintain the integrity of the genome (A. A. Aravin, Hannon, \& Brennecke, 2007; Fu \& Wang, 2014; Ghildiyal \& Zamore, 2009; Grivna, Beyret, Wang, \& Lin, 2006; Siomi, Sato, Pezic, \& Aravin, 2011). These structures also have been related to the silence of imprinted genes and regulation of translation (Gou et al., 2015; Lim, Tao, \& Kai, 2009; Morazzani, Wiley, Murreddu, Adelman, \& Myles, 2012; Rouget et al., 2010; Watanabe et al., 2011). Few studies have described the potential role of piRNAs in intergenerational inheritance (Ashe et al., 2012; Brennecke et al., 2008; Le Thomas et al., 2014). Some studies in Drosophila suggest that the process of piRNA biogenesis was regulated by specific transgenerational inherited maternal piRNAs (Grentzinger et al., 2012; Le Thomas et al., 2014). Besides, the alteration of piRNA pathways led to DNA damage and meiotic arrest (Carmell et al., 2007; Kuramochi-Miyagawa et al., 2004; Shoji et al., 2009). In the present study, specific piRNAs were overexpressed in the sperm of APPPS1-21 transgenic fathers, which means that the population of piRNAs transmitted varies. Since most of the piRNAs map unique sites in the genome and are necessary for silencing homolog transposable elements (TE) (Brennecke et al., 2007; Gainetdinov, Skvortsova, Kondratieva, Funikov, \& Azhikina, 2017; Klattenhoff et al., 2009), it appears logical to think that alterations of the sperm piRNA population might promote a distinct silencing of TE, altering the development process. Moreover, some piRNAs have been involved in posttranscriptional regulation of mRNAs (Gou et al., 2015; Lim et al., 2009; Morazzani et al., 2012; Rouget et al., 2010; Watanabe et al., 2011). Specific piRNAs interact with the embryonic posterior morphogen Nanos promoting deadenylation and mRNA decay in Drosophila embryos (Rouget et al., 2010). I speculate that different piRNAs populations regulate differently the expression of specific genes inducing changes in cognitive skills of APPPS1-21 offspring cognitive skills. An experiment that could provide new information about the role of these specific piRNAs in intergenerational transmission would be the following: the injection of antisense inhibitors of the single piRNAs detected in the sperm of APPPS1-21 tg mice in fertilized oocytes and the study of the development process and the behavior phenotype at adult age. It's early to indicate a direct association between these small RNA structures and previously described changes in the cognition abilities and the cellular state of wild-type mice born to APPPS1-21 transgenic fathers. Further investigations need to be performed. One interesting future experiment would be to microinject the combination of the overexpressed small RNAs in the sperm of APPPS1-21 transgenic fathers into wild-type fertilized oocytes and study the 
cognitive abilities of the offspring (Benito et al., 2018; Rodgers et al., 2015). A previous study showed that the injection of a combination of miRNAs obtained from stressed father sperm promoted a similar stress phenotype than the paternal stressed offspring (Rodgers et al., 2015). It will thus be interesting to observe whether the microinjection of a specific combination of small RNAs from APPPS1-21 transgenic fathers would promote similar cognitive response and transcriptomic changes in the injected offspring than the APPPS1-21 offspring. Additionally, it would be important to study the effect of single piRNA and snoRNA microinjection. The specific molecular mechanism by which the sperm small RNAs may communicate the cognitive phenotype to an adult offspring is not clear. One could think that these small RNAs initiate a cascade of changes that stimulate different epigenetic or gene expression patterns during development promoting alterations of learning skills into adult offspring (Rodgers et al., 2015; Skinner, 2011b). Previous data suggested that specific patterns of DNA methylation in the male germline were maintained in the brain of the offspring (Franklin et al., 2010).

Moreover, the intergenerational inheritance of cognitive deficits induced by a genetic transgene probable also involves another kind of epigenetic factors, such as DNA methylation of histone modifications. These epigenetic factors have been implicated in distinct forms of intergenerational inheritance (Brykczynska et al., 2010; Guerrero-Bosagna et al., 2012; Hammoud et al., 2009; Siklenka et al., 2015; Wei et al., 2014). Thus, it is important to investigate in the future the role of these epigenetic factors in the transmission of the cognitive phenotype associated with the APP and PS1 transgene.

Other interesting questions that need to be answered are: how APP and PS1 double mutation explicitly affects the non-genetic factors that are transmitted in the germline and when it happens? How alteration in the germline remains stable over the time? How epigenetic information affects specific cells and tissues from the organism and not others? And finally, how phenotypic alteration stays over the time? 


\subsection{Inter-individual differences in cognitive performance and associated molecular pathways in aging mice}

\subsubsection{Inter-individual differences in cognitive performance}

Life expectancy is globally increasing and hence, age-related diseases, such as neurodegenerative diseases. Aging is the major risk factor for developing Alzheimer's disease. Thus, it is crucial to properly understand age-related diseases and process of aging per se. Aging is a complex process that represents a progressive accumulation of molecular damage over the time, and therefore it is difficult to study. One of the reasons is that promotes multiple changes at different times and affects distinct manner on each individual, organ or cell type (Chahal \& Drake, 2007; Denic, Glassock, \& Rule, 2016; Gallagher et al., 2006; LessardBeaudoin et al., 2016; Stark, Yassa, \& Stark, 2010). This might because numerous factors have a repercussion to the process of aging, such as genetic and epigenetic factors or external environmental conditions (Bellizzi et al., 2005; Gami \& Wolkow, 2006; Harrison et al., 2009; C. S. Kim, Park, \& Kim, 2017; Oh et al., 2018; v et al., 2006).

It was demonstrated that environmental factors could profoundly impact the process of aging, promoting a distinct severity level of aging and inducing more or less severe consequences (Harrison et al., 2009; Solon-Biet et al., 2014; Strasser, Volaklis, Fuchs, \& Burtscher, 2018). One of the acute effects of aging is cognitive impairment. Cognitive abilities vary between individuals during all life, but the inter-individual variability increases significantly with age (Gallagher et al., 2006; Koivisto et al., 1995; Stark et al., 2010). Young mice show less variability in a specific cognitive task than old mice. Thus, I used mice at late middle age, 17 months of age, in order to evaluate their variability in cognitive abilities. Observing the averaged mice, the old mice showed more difficulties in learning the task than young mice (3 months of age). Evaluating individual behaviors, I discovered that there was a substantial variation in cognitive abilities during the acquisition and learning of a spatial memory task, even these mice grew in same environmental conditions. Some mice learned the spatial task ("good" performers), whereas others they did not learn spatial task ("bad" performers). This high variability was observed in two different spatial tasks, Morris water maze and Barnes maze, and none of the cases were correlated to motor abilities or anxiety levels (Figures4.3.2 and 4.3.5). This data confirms that old mice show higher cognitive variability for a specific spatial task than young mice. This variance existed even they were mice from the same line, age and grew in same conditions. 


\subsubsection{Differences in the transcriptional profiling and functional pathways of aged "good" and "bad" performers}

Aging is triggered by several molecular changes, and the analysis of the transcriptome allows to obtain a first overview of these changes. Previous studies identified specific pathways that commonly change with aging in different aged species (from yeast to humans). These pathways were involved in protein metabolism, immune system, energy metabolism, growth factor signaling, gene expression and mRNA processing (Frenk \& Houseley, 2018). The strong alteration of the transcriptome was in agreement with several theories arguing that during aging there is an increase of senescence and inflammation increase of senescence and inflammation, reduction of respiratory chain activity, dysfunction of mitochondria and decrease of ribosome biogenesis (Bratic \& Larsson, 2013). Several of these studies have investigated the molecular state of old mice comparing them to young individuals or assessing them at different ages (Bors et al., 2018; de Magalhaes et al., 2009; Glass et al., 2013; Verbitsky et al., 2004; Yang et al., 2015). Here, I analyzed transcriptome changes by comparing aged-individuals of the same age with different cognitive abilities, good (intact cognition) and bad performers (impaired cognition). Since the spatial memory explicitly involved the hippocampus (Logue, Paylor, \& Wehner, 1997), I investigated the transcriptomic profile and functional pathways of the hippocampus subregions (CA1, CA3 and DG). A little number of statistical transcriptomic differences was identified between "good" and "bad" performers in all hippocampal regions (Figure 4.3.8). Analyzing the gene set enrichment of these performers, I detected several functional changes in all the hippocampus subregions, as shown in figures 4.3.9-11).

In CA1 and DG subregions of bad performers, I was able to identify the down-regulation of genes defined as the hallmarks of aging (Frenk \& Houseley, 2018), such as genes related to mitochondria activity, protein synthesis machinery and RNA metabolic process. The downregulation of genes related to mitochondria function and ribosome biogenesis has been highly characterized in several tissues from different aged-species 277,280-285. In contrast, I did not report a clear increase expression in CA1 and DG regions of bad performers of others hallmarks of aging, which were the immune system, inflammatory response and heat shock factors (C. K. Lee et al., 2000; T. Lu et al., 2004; Peters et al., 2015). The absence of altered immune and inflammatory pathways in CA1 and DG regions of bad performers might be to the fact that genes associated with these processes were altered in both age-groups, good and bad performers, and were not dependent on cognitive abilities. Thus, I was not able to identify these categories of genes in CA1 and DG regions when I compared bad to good performers. Only the CA3 region 
of bad performers showed an alteration of genes related to inflammatory response, immune system and apoptotic process.

In summary, aged-mice showed a high cognitive inter-individual variability, which allowed me to identify good (intact cognition) and bad (impaired cognition) performers. In this approach, I was able to detect differentially expressed genes that were previously linked to age process. However, to the best of my knowledge, this work is the first to show that aging genes are specifically connected to aged-mice with severe cognitive impairments.

\subsubsection{Detection of biomarkers to screen cognitive abilities}

Several environmental factors influence the process of aging (Bellizzi et al., 2005; Gami \& Wolkow, 2006; Harrison et al., 2009; C. S. Kim et al., 2017; Oh et al., 2018; v et al., 2006). Here, I observed that those identical mice at the same age and grown in the same environmental conditions showed different cognitive abilities. Even these external conditions were well controlled; probably uncontrolled factors still stimulated the cognitive variability between individuals.

Moreover, several studies aimed to identify markers that allow the early detection of distinct pathologies (Hampel et al., 2010; Shaw, Korecka, Clark, Lee, \& Trojanowski, 2007). Here, I wondered whether there would be a specific biomarker in the blood to detect in non-invasive manner the cognitive abilities of aged-individuals, specifically aged-mice. Several studies on the identification of biomarkers of aged-cognitive decline or cognitive deficits associated with Alzheimer's disease have been focused on imaging (PET amyloid imaging or structural MRI) and CSF measurements (Sonnen et al., 2008; Wicklund \& Petersen, 2013). Some studies proof that epigenetic factors or non-coding RNAs were useful markers for the diagnosis of specific diseases (Lopez et al., 2015; Rao et al., 2013; Zhao, Bhattacharjee, Dua, Alexandrov, \& Lukiw, 2015). Thus, I evaluated the expression of small RNAs in the blood of mice with intact (good performers) and impaired cognition (bad performers). I was able to identify some small RNAs that were statistically different in the blood of good and bad performers, as shown in figure 4.3.12. To note, down-regulated small RNAs in bad performers target genes related to nervous system development, catabolic processes and ossification, whereas enriched small RNAs in bad performers target genes linked to immune system development and response to cytokines (Figure 4.3.12). From these small RNAs I could identify potential candidates for the detection of cognition abilities. I detected different expression levels between good and bad performers of miRNA-501-3p, miRNA-100-5p, miRNA-664-3p and miRNA-146-5p (Figure 4.3.12). These miRNAs were down-regulated specifically in aged-mice with cognitive deficits (bad performers). 
All of them have previously been involved in interesting pathways or pathologies. The expression of miRNA501-3p was down-regulated in the serum of AD patients and up-regulated in the brain of the same patients (N. Hara et al., 2017). MiRNA-100-5p bound to mTOR transcript promoting changes in neuronal survival in the cortex of AD mouse model (Ye et al., 2015). MicroRNA-664-3p has also been related to mTOR pathways and involved in longevity processes (B. P. Lee et al., 2017). The expression of miRNA146a-5p was implicated in inflammatory signaling in neurodegenerative pathologies.

Although these are preliminary results and further research is necessary, I was able to identify small RNAs in blood that correlate with cognitive abilities, intact and impaired cognition. 
SUMMARY 


\section{SUMMARY}

Alzheimer's disease (AD) is a neurodegenerative disease and the most common form of dementia. The number of AD cases increases exponentially every year (Prince et al., 2015). Although $A D$ has been investigated for a long time, the precise molecular mechanisms involved remain to be elucidated, especially at early stages of the pathology. Furthermore, a cure does not exist to date. The treatments currently available are administered too late and are only temporarily effective for certain dementia-associated symptoms. These difficulties to discover an effective treatment are probably due to the complexity of the pathological process. There is emerging evidence that $A D$ pathology is affected by the combination of several factors, such as genetic and environmental factors (W. Shao et al., 2017; A. R. Smith, Mill, Smith, \& Lunnon, 2016). Changes in gene expression have an impact on the progression of the pathology (Bossers et al., 2010; J. Lee et al., 2018; Parra-Damas et al., 2014). Thus, it is necessary to investigate the role of gene expression at early stages in the pathology in order to diagnose and begin treatment earlier. Several of the new drugs are firstly tested in AD mouse models. It is therefore of great importance to define in detail transcriptional profile changes in these models. Here, I provided new insights into changes in gene expression at different contexts of cognitive deficits.

First, I assessed the behavioral phenotype, transcriptional state and alternative exon usage related to early AD stages in APPPS1-21 mice, a well-established model of AD-like pathology. The present results indicate that APPPS1-21 transgenic males and females develop an undistinguishable rapid progression of $A D$, showing cognitive impairments restricted to spatial memory at 3 months of age. Cognitive deficits declined with age, and mice performed worse in spatial learning and memory test at 9 months of age, compared to their 3-month old counterparts. Moreover, APPPS1-21 hippocampal subregions (CA1 and DG) showed genderand subregion-specific changes in gene expression and alternative exon usage already at 3 months of age. Despite the fact that APPPS1-21 hippocampal subregions and genders showed transcriptomic differences at the individual level, similar global biological processes were affected in these hippocampal subregions. The findings of this study reveal that two global processes occur in the APPPS1-21 hippocampal subregions at 3 months of age. First, there is an activation of genes involved in compensatory mechanisms, presumably as an attempt to return to a non-pathological status (increased of synaptic activity and neurogenesis). Second, and due to the fast progression of the disease in this model, there is a deregulation of pathways 
typically altered in late AD stages, such as energy metabolism and protein synthesis. Restoring the mitochondrial and ribosomal functions might be a promising therapeutic strategy to treat the AD-related memory decline.

In this study I also identified for the first time a gender-specific transmission to F1 offspring of epigenetic factors induced by the paternal origin in the APPPS1-21 mouse model, promoting cognitive and genetic changes to F1. There is increasing evidence that epigenetic information complements the genetic inheritance from parents to the offspring (Blake \& Watson, 2016; Zeybel et al., 2012). The described findings uniquely revealed that wild-type offspring of APPPS1-21 transgenic fathers, but not mothers, showed spatial cognitive impairments, transcriptomic changes and alternative exon usage at 3 and 9 months of age (only behavior). These changes were associated with specific expression of small RNAs in the sperm. It is interesting to note that the majority of small RNAs expressed in APPPS1-21 transgenic fathers were small nuclear RNAs (snoRNAs) and Piwi-interacting RNAs (piRNAs). Hence, it is the first time to my knowledge that snoRNAs and piRNAs are described as potential transmitters of intergenerational inheritance. These data provide evidence for the existence of new factors that contribute to the phenotype of wild-type and transgenic mice born to APPPS1-21 transgenic parents. This novel information is crucial to take into account when analyzing the behavioral phenotype and for future colony breeding strategies of this mouse model and potentially also other transgenic lines. It will be essential in the future to complement the genetic information with epigenetic information in animal breeding.

Finally, I identified genes and pathways altered exclusively in aged mice that showed cognitive deficits but not in aged mice with intact cognitive abilities. Aging is the major risk for developing Alzheimer's disease (AD). Thus, it is logical to think that aged mice with cognitive deficits will share more molecular alterations with $A D$ mice than aged-mice with intact cognitive abilities. I demonstrated that old mice (17 months of age) showed higher inter-individual variability in performance than young animals. From these individuals, I preselect aged mice with and without cognitive deficits. Interestingly, hippocampal brain subregions of cognitive impaired aged-mice showed deregulation of biological processes previously defined as hallmarks of aging, such as energy metabolism, protein synthesis and RNA metabolism. The present data indicate that deregulation of these pathways affects specifically aged mice with cognitive deficits. Moreover, I identified specific small RNAs expressed in the blood that correlate with agecognitive variability. These findings suggest that they can be used as biomarkers for the evaluation of cognitive abilities. 
In this thesis, I described several molecular pathways related to cognitive decline and potential candidates for future treatments of cognitive deficits associated with aging or Alzheimer's disease. Moreover, I detected potential biomarkers in the blood to evaluate the cognitive abilities in mice in a non-invasive manner.

Furthermore, I identified for the first time novel forms of small RNAs that are associated with the transmission of behavioral and molecular changes in wild-type offspring induced by the transgenic parental genotype in a gender-specific manner. This present study raises new issues to consider when mating transgenic mice, defining an appropriate control group and interpreting behavioral results.

Figure 6.1. Summary of the data described in the three approaches from this thesis.

(A) Changes in cognition, transcription and exon usage in an early AD stage in an APPPS1-21 mouse model. (B) Gender-specific transmission of epigenetic factors by the paternal origin in the APPPS1-21 mouse model, promoting cognitive and genetic changes to F1. (C) Inter-individual variability in aged mice and gene expression changes associated with aged mice that presented cognitive deficits. Identification of potential biomarkers for detecting cognitive abilities. 


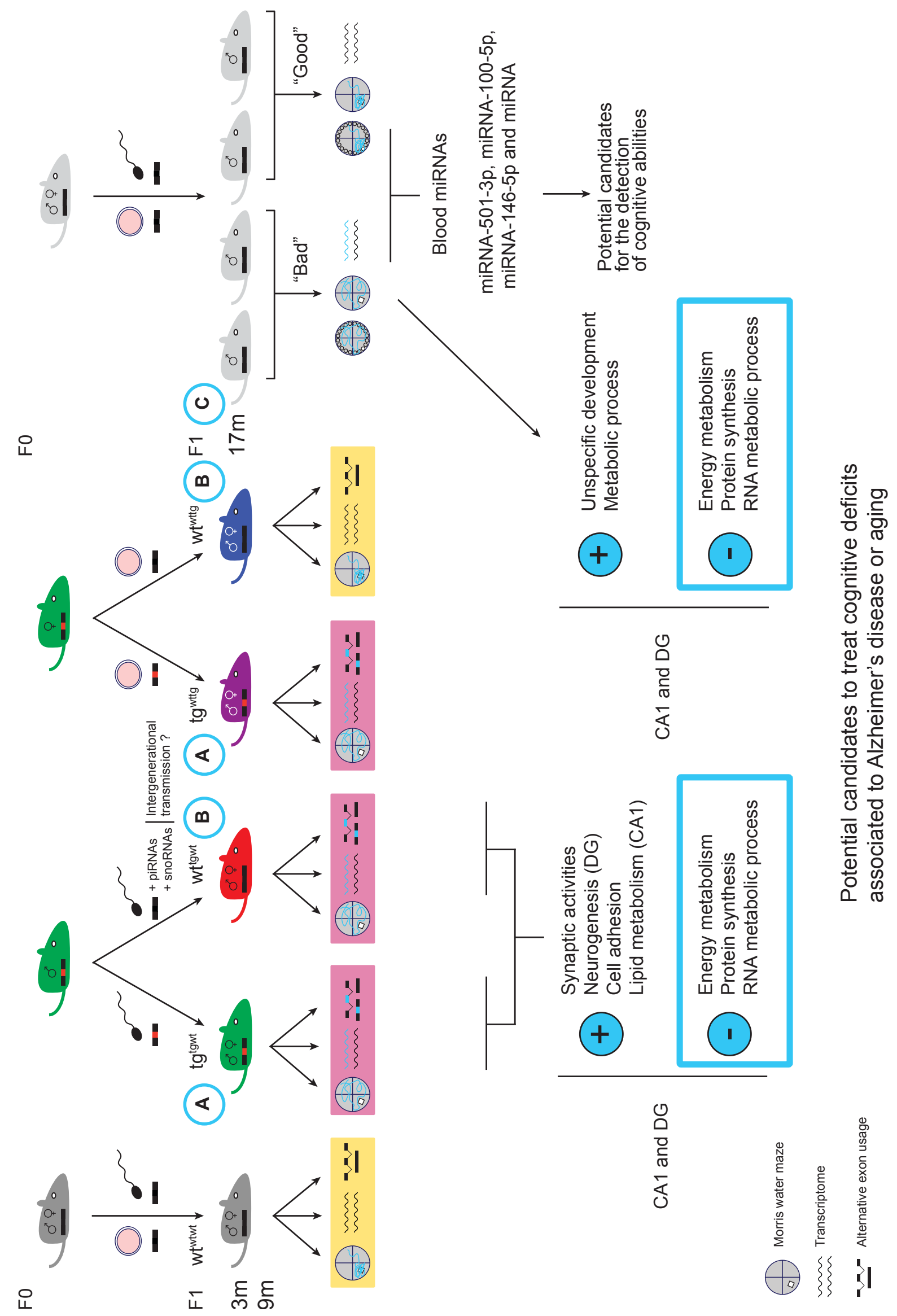


APPENDIX 


\section{APPENDIX}

Appendix figure 1. Male and female wt and tg mice born to APPPS1-21 transgenic fathers or mothers did not show motor abilities and anxiety level differences, except for APPPS1-21 $\mathbf{t g}$ females that showed an slight increase in their motor abilities.

Open field test in male and female wild-type and transgenic mice born to a transgenic father ( $\mathrm{wt}^{\mathrm{tgwt}} \mathrm{M}$ $(n=17)$ and $F(n=15)$ and tgtgwt $M(n=13)$ and $F(n=14))$ or mother (wtwttg $M(n=17)$ and $F(n=15)$ and tgwtg $M$ $(n=7)$ and $F(n=9))$ and control wild-type mice ( $w^{\text {twtt }} M(n=18)$ and $F(n=16)$ ) at 3 months of age. (A and B) Total traveled distance (A) and average speed (B) during the open field test. (C, D and E) Percentage of time (C) and distance (D) spent in the central area of the field and number of visits to the center (E). M $=$ male. $\mathrm{F}=$ female. Student's two-tailed $\mathrm{T}$ test, $\mathrm{ns}=$ no significant differences; ${ }^{* *} \mathrm{P}<0.01 ;{ }^{* * *} \mathrm{P}<0.001$; ${ }^{* * * *} \mathrm{P}<0.0001$. Error bars indicated SEM.
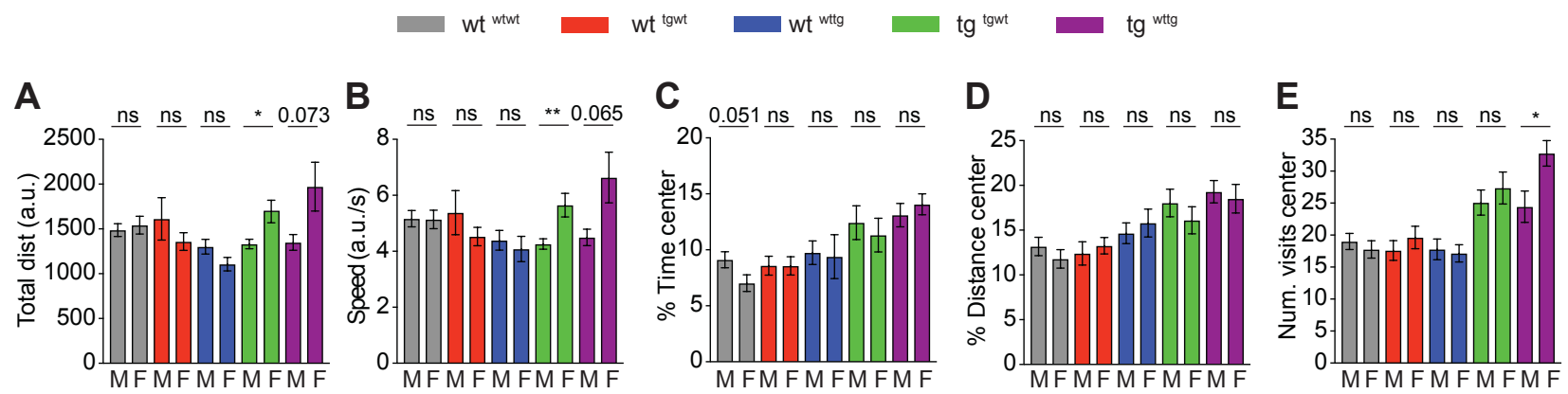

Appendix figure 2. Male and female wt and tg mice born to APPPS1-21 transgenic fathers or mothers did not show differences in learning a spatial task at 3 months of age.

Morris water maze (MWM) in male and female wild-type and transgenic mice born to a transgenic father (wtgwt $M(n=18)$ and $F(n=12)$ and tg tgwt $M(n=13)$ and $F(n=12))$ or mother (wt ${ }^{\text {tttg }} M(n=11)$ and $F(n=7)$ and tg ${ }^{\text {wttg }} M(n=10)$ and $\left.F(n=7)\right)$ and control wild-type mice ( t $^{\text {wtwt }} M(n=16)$ and $\left.F(n=14)\right)$ at 3 months of age. (A, C, E, G and I) male vs. female group-average time to find the target platform on each consecutive training day (Day 1-8). Two-way ANOVA after Sidak correction, ${ }^{*} P<0.05 ;{ }^{* *} P<0.01$. The dashed line represents chance level of $25 \%$. First probe trial (PT1, day 6) and second probe trial (PT2, day 10). (B, D, $\mathbf{F}, \mathbf{H}$ and $\mathbf{J}$ ) Comparison of male and females of each group equivalents analyzing the areas under the learning curves $(A \cup C)$ of escape latency for each group. $M=$ male. $F=$ female. Student's two-tailed $T$ test, ns $=$ no significant differences; ${ }^{* *} \mathrm{P}<0.05 ;{ }^{* *} \mathrm{P}<0.01 ;{ }^{* * *} \mathrm{P}<0.001 ;{ }^{* * *} \mathrm{P}<0.0001$. Error bars indicated SEM. 

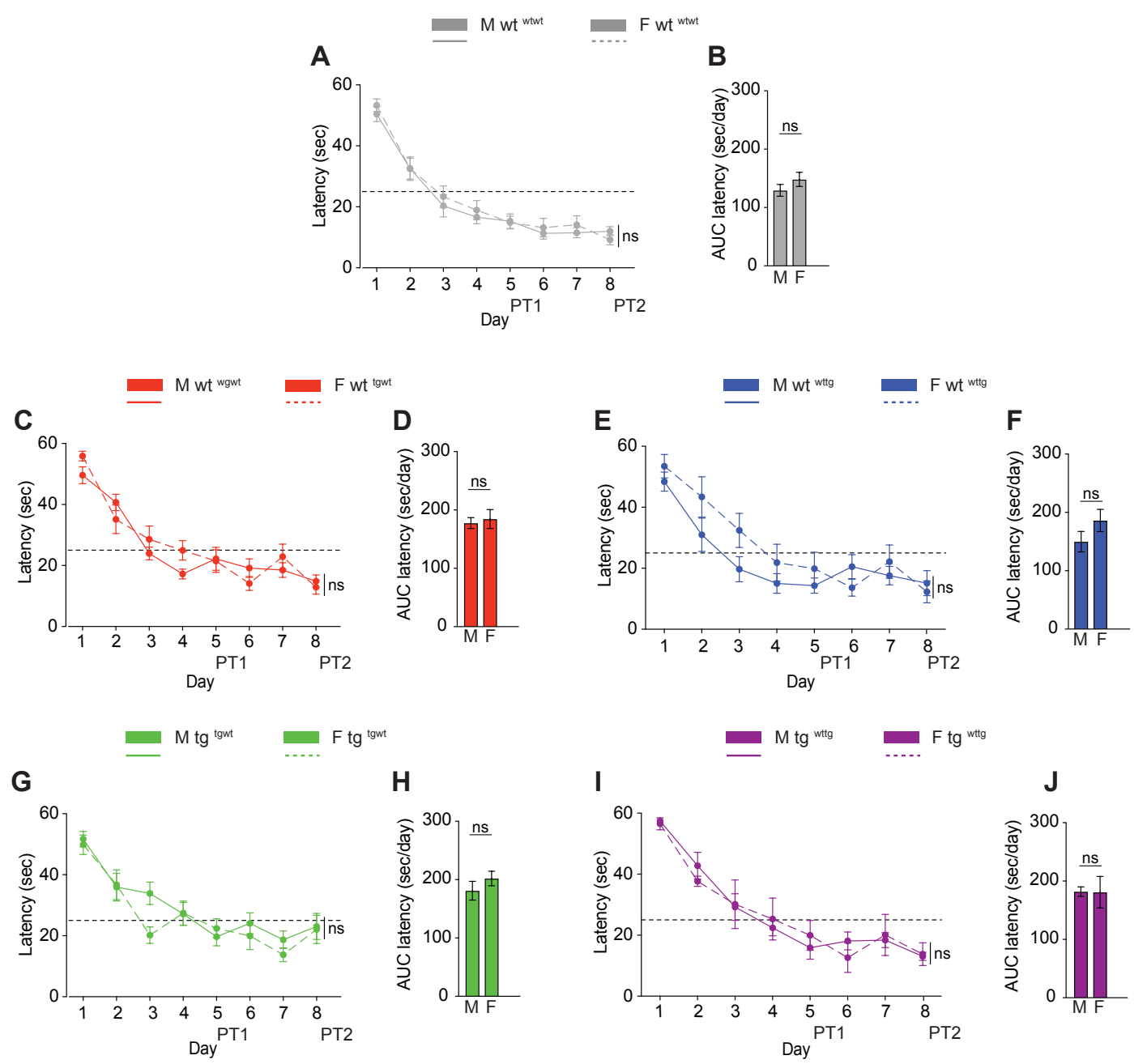

\section{Appendix figure 3. Male and female wt and tg mice born to APPPS1-21 transgenic fathers or mothers did not show differences in spatial memory at $\mathbf{3}$ months of age.}

Morris water maze (MWM) in male and female wild-type and transgenic mice born to a transgenic father (wtgwt $M(n=18)$ and $F(n=12)$ and tgtgwt $M(n=13)$ and $F(n=12))$ or mother ( $w^{\text {twttg }} M(n=11)$ and $F(n=7)$ and tgwttg $M(n=10)$ and $F(n=7))$ and control wild-type mice ( t $^{\text {wtwt }} M(n=16)$ and $\left.F(n=14)\right)$ at 3 months of age. (A-L) Percentage of time (A and C) and distance (B and D) spent on the target quadrant between male (M) and female (F) mice during first probe trial (PT1, day 6) (A and B) and second probe trial (PT2, day 10) $(C$ and $D) . M=$ male and $F=$ female. Student's two-tailed $T$ test, $n s=$ no significant differences; ${ }^{*} P$ $0.05 ;{ }^{* *} P<0.01$. The dashed line represents chance level of $25 \%$.

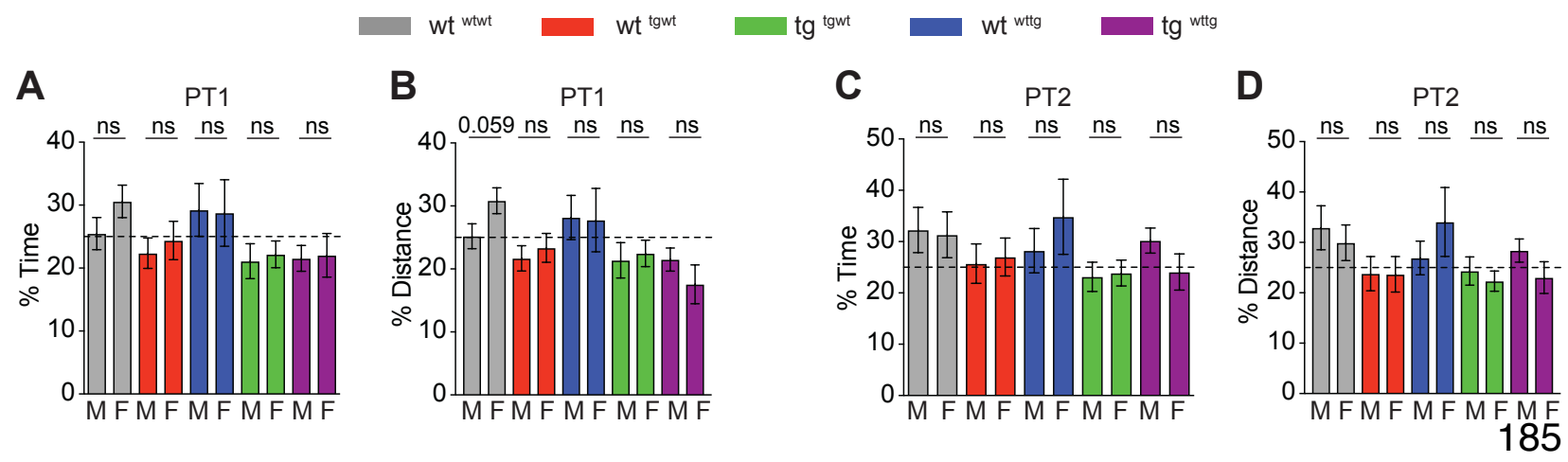


Appendix Figure 4. Male and female wt and tg mice born to APPPS1-21 transgenic fathers or mothers did not show differences in fear conditioning at 3 months of age.

Fear conditioning (FC) in male and female wild-type and transgenic mice born to a transgenic father (wtgwt $M(n=20)$ and $F(n=15)$ and $\operatorname{tg}^{\text {tgwt }} M(n=14)$ and $\left.F(n=14)\right)$ or mother ( $w^{\text {wttg }} M(n=17)$ and $F(n=7)$ and tg ${ }^{\text {wttg }}$ $M(n=10)$ and $F(n=9))$ and control wild-type mice (wtwtwt $M(n=18)$ and $F(n=16))$ at 3 months of age. (A) Average of motion during training day. (B and $\mathbf{C}$ ) Percentage of time (B) and time (C) freezing during training (first column) and testing day (second column). $\mathrm{M}=$ male. $\mathrm{F}=$ female. Student's two-tailed T test, ns $=$ no significant differences; ${ }^{*} \mathrm{P}<0.05 ;{ }^{* *} \mathrm{P}<0.01$. Error bars indicated SEM.

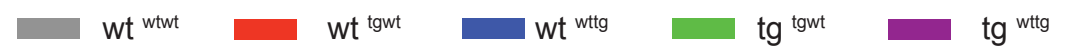

A

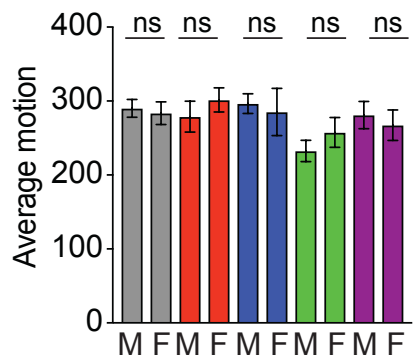

B

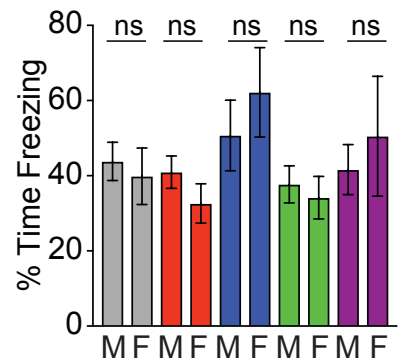

C

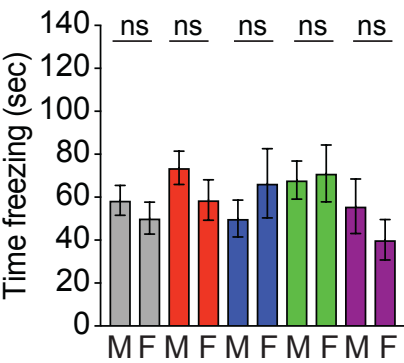

Appendix Figure 5. Male and female wt and tg mice born to APPPS1-21 transgenic fathers or mothers did not show differences in motor abilities and anxiety level at 9 months of age.

Open field test in male and female wild-type and transgenic mice born to a transgenic father ( $w t^{\text {tgwt }} M$ $(n=18)$ and $F(n=13)$ and tg tgwt $M(n=14)$ and $F(n=8))$ or mother ( $w^{\text {twtg }} M(n=5)$ and $F(n=2)$ and tgwttg $M$ $(n=1)$ and $F(n=3)$ ) and control wild-type mice ( $w^{\text {twtwt }} M(n=16)$ and $F(n=15)$ ) at 9 months of age. (A and B) Total traveled distance (A) and average speed (B) during the open field test. (C, D and E) Percentage of time (C) and distance (D) spent in the central area of the field and number of visits to the center (E). M $=$ male. $\mathrm{F}=$ female. Student's two-tailed $\mathrm{T}$ test, $\mathrm{ns}=$ no significant differences; ${ }^{* *} \mathrm{P}<0.01 ;{ }^{* * *} \mathrm{P}<0.001$; ${ }^{* * * *} \mathrm{P}<0.0001$. Error bars indicated SEM.

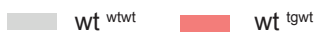

A

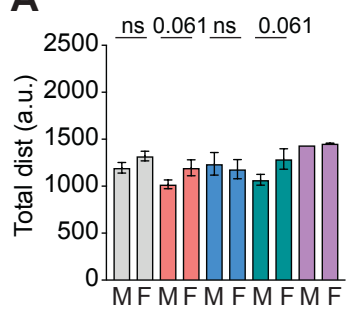

B

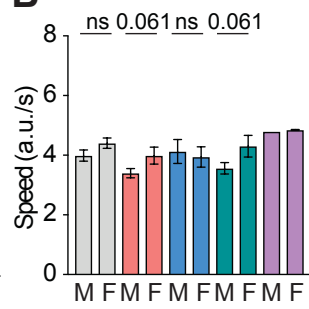

C

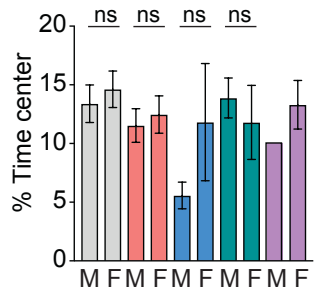

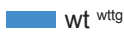

$\operatorname{tg}{ }^{\operatorname{tg} w}$

D

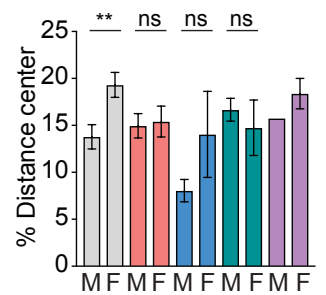

E

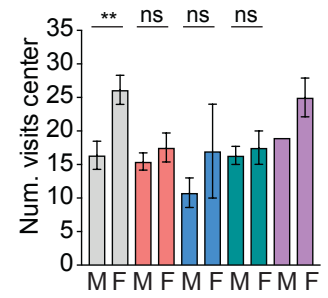


Appendix Figure 6. Male and female wt and tg mice born to APPPS1-21 transgenic fathers or mothers did not show differences in learning a spatial task at 9 months of age.

Morris water maze (MWM) in male and female wild-type and transgenic mice born to a transgenic father (wtgwt $M(n=16)$ and $F(n=10)$ and tg tgwt $M(n=13)$ and $F(n=7))$ or mother (wtwttg $M(n=5)$ and $F(n=1)$ and tg ${ }^{\text {wttg }} M(n=1)$ and $\left.F(n=3)\right)$ and control wild-type mice ( $w^{\text {twtwt }} M(n=14)$ and $\left.F(n=12)\right)$ at 9 months of age. (A, C, E, G and I) male vs. female group-average time to find the target platform on each consecutive training day (Day 1-8). Two-way ANOVA after Sidak correction, ${ }^{*} P<0.05 ;{ }^{* *} P<0.01$. The dashed line represents chance level of $25 \%$. First probe trial (PT1, day 6) and second probe trial (PT2, day 10). (B, D, $\mathbf{F}, \mathbf{H}$ and $\mathbf{J}$ ) Comparison of male and females of each group equivalents analyzing the areas under the learning curves $(A \cup C)$ of escape latency for each group. $M=$ male. $F=$ female. Student's two-tailed T test, ns = no significant differences; ${ }^{* *} \mathrm{P}<0.05 ;{ }^{* *} \mathrm{P}<0.01 ;{ }^{* * *} \mathrm{P}<0.001 ;{ }^{* * *} \mathrm{P}<0.0001$. Error bars indicated SEM.
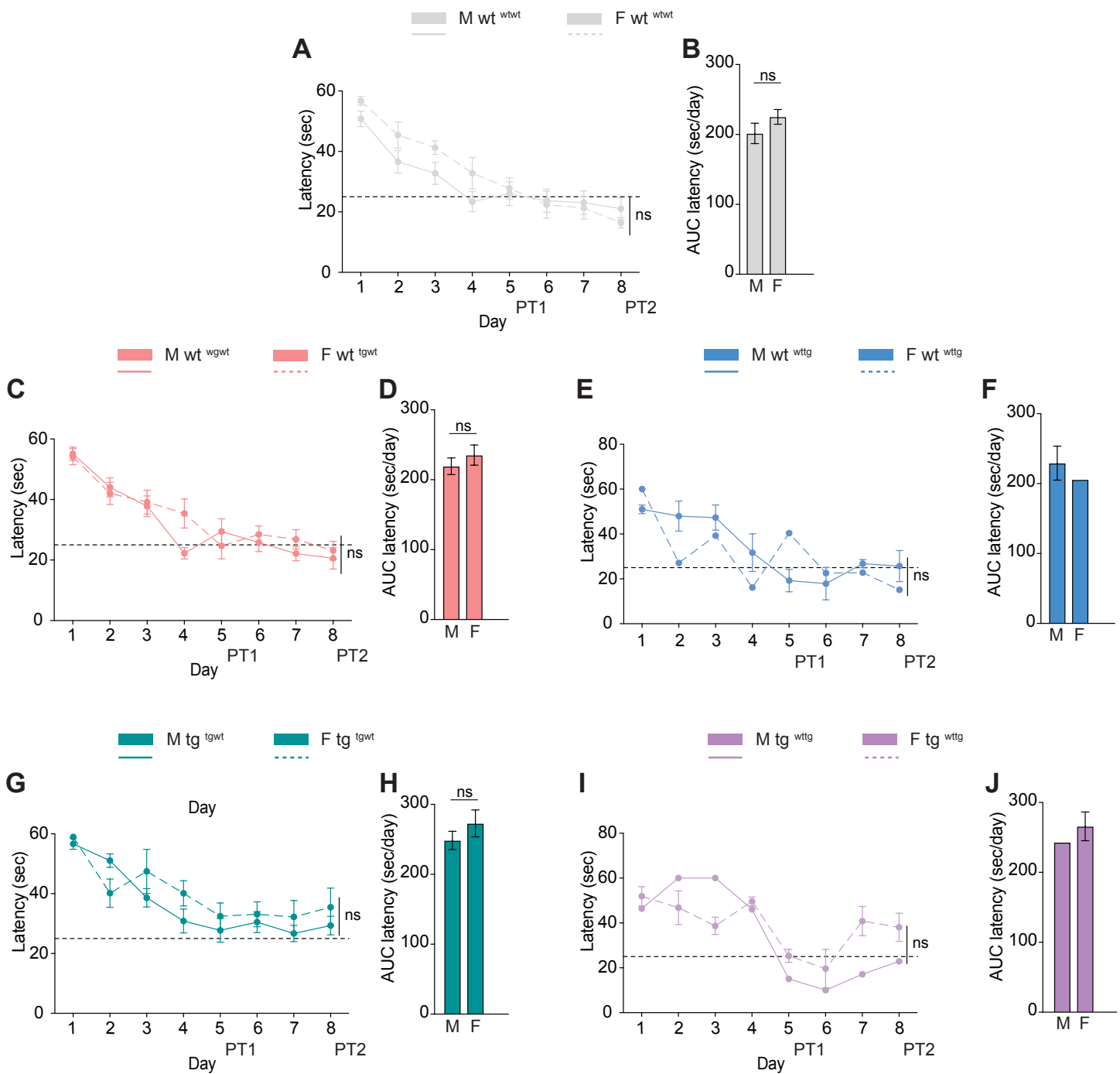
Appendix figure 7. Male and female wt and tg mice born to APPPS1-21 transgenic fathers or mothers did not show differences in spatial memory at 9 months of age.

Morris water maze (MWM) in male and female wild-type and transgenic mice born to a transgenic father ( $w^{\text {tgwt }} M(n=16)$ and $F(n=10)$ and $\operatorname{tg}^{\text {tgwt }} M(n=13)$ and $\left.F(n=7)\right)$ or mother $\left(w^{\text {wttg }} M(n=5)\right.$ and $F(n=1)$ and $\operatorname{tg}^{\text {wttg }} M(n=1)$ and $\left.F(n=3)\right)$ and control wild-type mice ( $w^{\text {twtwt }} M(n=14)$ and $\left.F(n=12)\right)$ at 9 months of age. (A-L) Percentage of time (A and C) and distance (B and D) spent on the target quadrant between male (M) and female (F) mice during first probe trial (PT1, day 6) (A and B) and second probe trial (PT2, day 10) $(C$ and $D) . M=$ male and $F=$ female. Student's two-tailed $T$ test, ns = no significant differences; ${ }^{*}$ < $0.05 ;{ }^{* \star} P<0.01$. The dashed line represents chance level of $25 \%$.

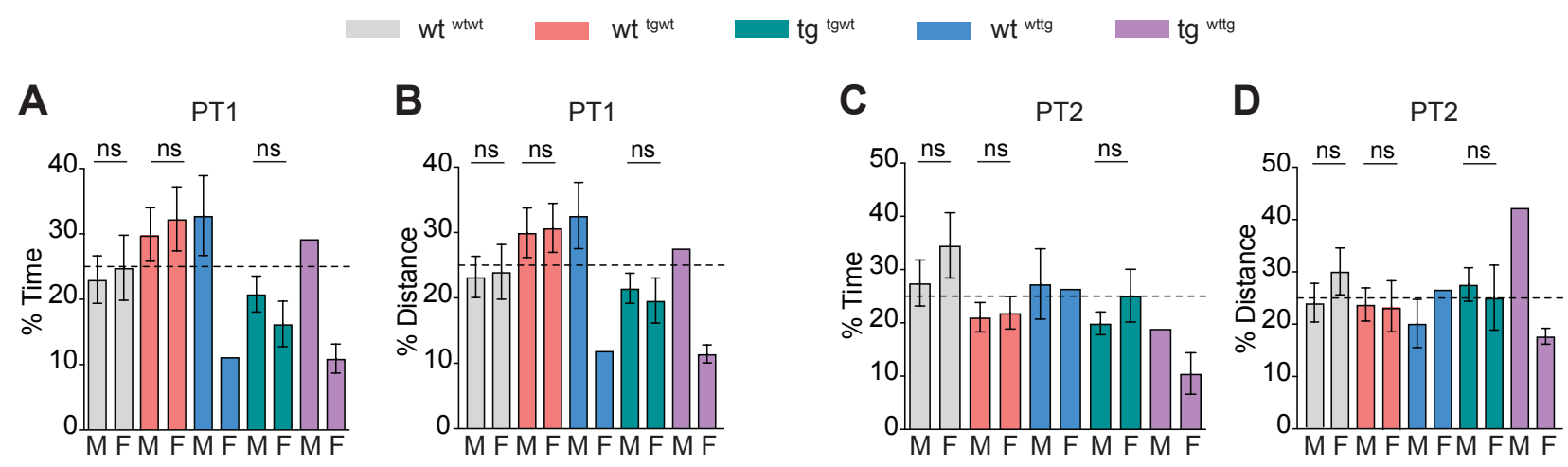

Appendix figure 8. Male and female wt and tg mice born to APPPS1-21 transgenic fathers or mothers did not show differences in fear conditioning at 9 months of age.

Fear conditioning (FC) in male and female wild-type and transgenic mice born to a transgenic father (wtgwt $M(n=19)$ and $F(n=12)$ and $\operatorname{tg}^{\text {tgwt }} M(n=12)$ and $\left.F(n=6)\right)$ or mother $\left(w^{\text {wttg }} M(n=5)\right.$ and $F(n=2)$ and tg ${ }^{\text {wttg }} M$ $(n=1)$ and $F(n=3))$ and control wild-type mice $\left(w^{\text {wtwt }} M(n=14)\right.$ and $\left.F(n=15)\right)$ at 3 months of age. (A) Average of motion during training day. (B and $\mathbf{C}$ ) Percentage of time (B) and time (C) freezing during training (first column) and testing day (second column). $\mathrm{M}=$ male. $\mathrm{F}=$ female. Student's two-tailed $\mathrm{T}$ test, ns $=$ no significant differences; ${ }^{*} \mathrm{P}<0.05 ;{ }^{* *} \mathrm{P}<0.01$. Error bars indicated SEM.
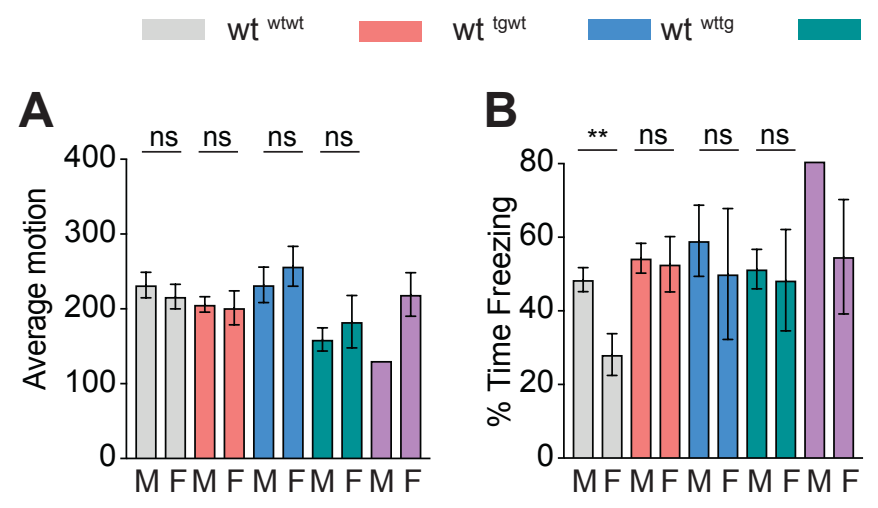

$\operatorname{tg}$ tgwt $\quad \operatorname{tg}^{w}{ }^{w}$

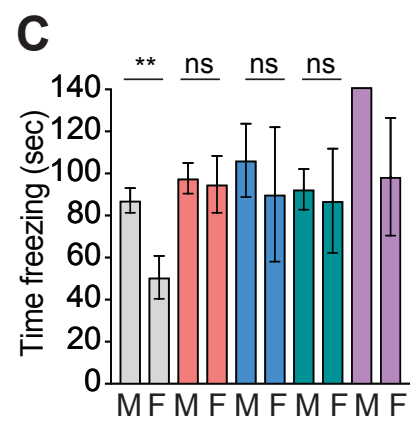


Appendix table 1. Significant alternative exon usage of App, Ps1 and Mapt genes from male and female DG and male CA1 of APPPS1-21 transgenic mice compared to control wild-types (wtwtwt). Exon usage, padj $\leq 0.05, \log _{2} F C \pm 0.25$. For $D G$ males: tg $n=4$ and $w^{w t w t} n=5$; for $D G$ females $\operatorname{tg} n=7$ and $w^{t w t w t} n=5$ and for CA1 males tg $n=6$ and $w^{t w t w t} n=6$.

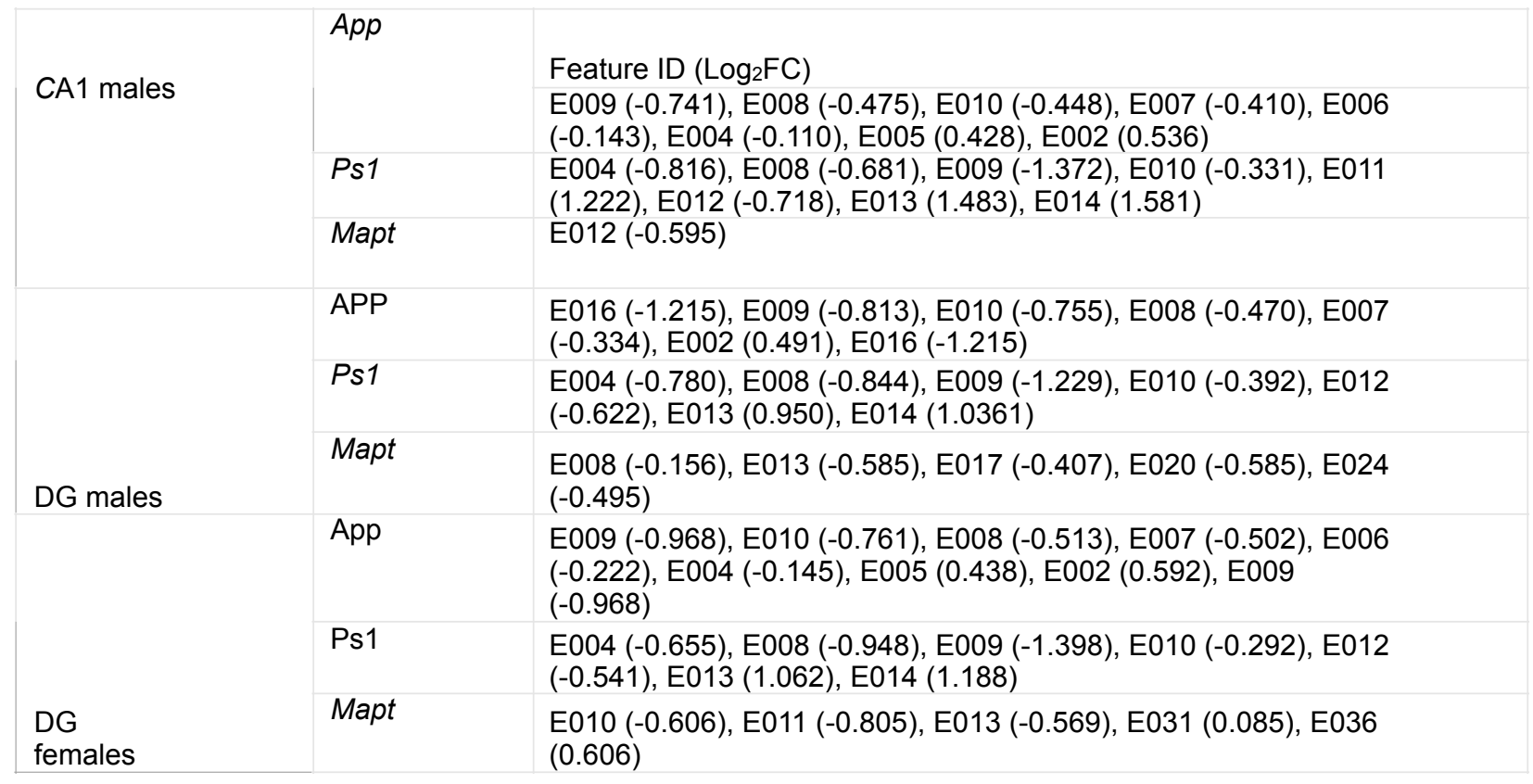

Appendix table 2. Variability between $\operatorname{tg}$ mice born to an APPPS1-21 $\mathrm{tg}$ father or mother and between APPPS1-21 tg mice together and control wild-type ( $\left.w^{\text {wtwt }}\right)$ mice.

Percentage of gene expression compared to the total transcriptome analyzed (48710 mRNAs) in male and female DG and male CA1 in tg vs. $w^{\text {wtwt }}$ and in tg ${ }^{\text {tgwt }}$ vs. tg $^{\text {wtg }}$ comparison. Padj $\leq 0.05, \log _{2} F C \pm 0.25$. For DG males: transgenic ( $\operatorname{tg}^{\text {tgwt }}$ and $\left.\operatorname{tg}^{\text {wttg}}\right) n=1,3$ and $w^{\text {twtwt }} n=5$; for DG females transgenic ( $\operatorname{tg}^{\text {tgwt }}$ and tg ${ }^{\text {wttg}}$ ) $n=3,4$ and $w^{\text {twtwt }} n=5$ and for CA1 males transgenic (tgtgwt and tgwttg) $n=3,4$ and $w^{\text {twtwt }} n=6$.

\begin{tabular}{|c|c|c|}
\hline $\begin{array}{c}\text { Total } \\
\text { transcriptome } \\
\text { (48710 mRNAs) }\end{array}$ & $\begin{array}{c}\% \text { DE } \\
\text { mRNAs in } \\
\text { tg vs. wtwtwt }\end{array}$ & $\begin{array}{c}\% \text { DE mRNAs } \\
\text { in } \\
\text { tg }^{\text {tgwt }} \text { vs. } \operatorname{tg}^{\text {wttg }}\end{array}$ \\
\hline DG males & 0.4 & 2.8 \\
\hline DG females & 0.006 & 3.3 \\
\hline CA1 males & 0.05 & 0.11 \\
\hline
\end{tabular}


Appendix table 3. Top differentially expressed genes in male and female DG and male CA1 of APPPS1-21 transgenic mice.

Genes, padj $\leq 0.05$; $\log _{2} F C \pm 0.25$ and pathways, pval $<0.05$. (D-F) Top 20 significant functional pathways ranked by -log pval after Benjamini-Hochberg correction of significant differentially expressed genes in brain subregions of wt ${ }^{\text {tgwt }} v s$. wt ${ }^{\text {twt }}$ mice. Genes, padj $\leq 0.05$; $\log _{2} \mathrm{FC} \pm 0.25$ and pathways, pval $<0.05$. For DG males: transgenic tg $n=4$ and $w^{w t w t} n=5$; for DG females tg $n=7$ and $w^{t^{w t w t}} n=5$ and for CA1 males transgenic tg $n=6$ and $w^{t w t w t} n=6$.

\begin{tabular}{|c|c|c|}
\hline \multirow{3}{*}{$\begin{array}{l}\text { CA1 } \\
\text { males }\end{array}$} & & Gene name $\left(\log _{2} F C\right)$ \\
\hline & Up & $\begin{array}{l}\text { Kcnk9 (1.321), Slc17a6 (1.227), Dcc (1.205), Gpr165 (1.187), } \\
\text { Plcxd3 (1.185), Gh (1.173), Pde11a (1.165), Lancl3 (1.155), } \\
\text { Cntnap3 (1.137), Igsf9b (1.135), Xkr4 (1.118), Pcsk1 (1.116), } \\
\text { Lmbrd2 (1.098), Ppargc1b (1.056), Tenm3 (1.052), Ston2 (0.899), }\end{array}$ \\
\hline & $\begin{array}{l}\text { Do } \\
\text { wn }\end{array}$ & $\begin{array}{l}\text { Cox4 (-0.470), Mrpl20 (-0.450), Ndufa3 (-0.542), Clec2d (-0.757), } \\
\text { Adam8 (-0.769), Slc38a5 (-0.771), Ovgp1 (-0.798), Mir703 (-0.815), } \\
\text { Gm44090 (-0.819), Plekhg4 (-0.962), Gm44891-(0.999), } \\
\text { Gm44677(-1.095), Gm44242(-1.148) }\end{array}$ \\
\hline \multirow[t]{2}{*}{$\begin{array}{l}\mathrm{DG} \\
\text { males }\end{array}$} & Up & $\begin{array}{l}\text { Cst7 (1.302), Erich5(1.158), Gm44724 (1.145), Ncam2 (1.021), } \\
\text { Thy1 (1.017), Ston2 (0.952), Ptchd1 (0.941), Hist2h ( } 40.935), \\
\text { Trpm3 (0.9110), Pcdhgb1 (0.903), St3gal1 (0.902),Unc13c (0.891), } \\
\text { Sv2c (0.845) }\end{array}$ \\
\hline & $\begin{array}{l}\text { Do } \\
\text { wn }\end{array}$ & $\begin{array}{l}\text { Mrpl2 (-0.389), Ndufa1 (-0.397), Ndufa3 (-0.445), Daw1 } \\
\text { (-1.051),Dnah10 (-1.058), Folr1 (-1.060), 2410004P03Rik (-1.060), } \\
\text { Cfap43 (-1.060), Ak7 (-1.070),Ttll6 (-1.076), Crocc2 (-1.128), } \\
\text { Ccdc108 (-1.145),Calml4 (-1.239), }\end{array}$ \\
\hline \multirow[t]{2}{*}{$\begin{array}{l}\text { DG } \\
\text { femal } \\
\text { es }\end{array}$} & Up & $\begin{array}{l}\text { Thy1 (1.230), Psen1 (0.746), App (0.601), Tenm3 (0.482), Pcdhgb1 } \\
\text { (0.477), Kcnh5 (0.457), Pcdhga6 (0.436), Kcnk9 (0.425), Igsf9b } \\
(0.420), \text { Klf12 (0.412),Cdh6 (0.410), Hs6st3 (0.403), }\end{array}$ \\
\hline & $\begin{array}{l}\text { Do } \\
\text { wn }\end{array}$ & $\begin{array}{l}\text { Gm44891 (-0.444), A230103L15Rik (-0.447), Gm37999 (-0.449), } \\
\text { Gm42970 (-0.451), Gm42769 (-0.453), Gm44677 } \\
(-0.455), G m 44799(-0.479), G m 44559(-0.498), G m 44562 \\
(-0.505), m t-T p(-0.513)\end{array}$ \\
\hline
\end{tabular}


Appendix table 4. Top 10 genes that had a significant alternative exon usage in male and female DG and male CA1 of APPPS1-21 transgenic mice.

Top 10 significant differentially spliced genes in in male and female DG and male CA1 of wtg ${ }^{\text {tgwt }}$ mice compared to $w^{\text {twtwt }}$ mice ranked by $\log _{2} F C$. Genes, padj $\leq 0.05$; $\log _{2} F C \pm 0.25$. For DG males: $w^{\text {tgwt }} n=7$

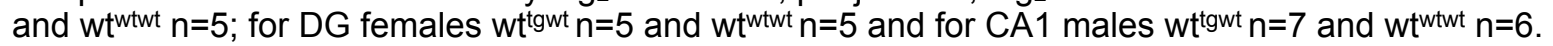

\begin{tabular}{|c|c|c|}
\hline & & Gene name $\left(\log _{2} F C\right)$ \\
\hline \multirow[t]{2}{*}{$\begin{array}{l}\text { CA1 males } \\
\text { APPPS1-21 } \\
\text { Tg }\end{array}$} & Inclusion & $\begin{array}{l}\text { Rapgef4 (2.978),Csmd1 (2.194),Slc6a9 (2.165), Dock3 } \\
\text { (2.057),Babam2 (2.056), Cdk16 (1.948),Npff (1.843), } \\
\text { ()Atp6ap1 (1.815),Limk1 (1.769), }\end{array}$ \\
\hline & Exclusion & $\begin{array}{l}\text { Gabbr1 (-1.840), Ptbp2 (-1.845),Mcm3ap (-1.900), } \\
\text { Nrxn1 (-1.924), Arhgap4 (-1.925), Ptprg (-1.972), Digap4 } \\
\text { (-1.990),Gpr83 (-2.079), Slc6a17 (-2.260), }\end{array}$ \\
\hline \multirow[t]{2}{*}{$\begin{array}{l}\text { DG males } \\
\text { APPPS1-21 } \\
\text { Tg }\end{array}$} & Inclusion & $\begin{array}{l}\text { Mir8108 (2.233),Dnm3 (2.192),Mtif2 (2.114), Lama4 } \\
\text { (2.107),Nrxn3 (2.044),Mapk1ip1I (2.018),Dennd4c } \\
\text { (2.008),Synj1 (1.973),Myof (1.954),Synj1 (1.954), }\end{array}$ \\
\hline & Exclusion & $\begin{array}{l}\text { Arcn1 (-2.993), Xpr1 (-3.003), Cbl (-3.035), Tbc1d8b } \\
(-3.133), \text { Stra6(-3.195), lars2 (-3.338), Cacna1b } \\
(-3.409), \text { Man2a2 (-3.598), Gnai3 (-3.810), Kdm5a } \\
(-4.178),\end{array}$ \\
\hline \multirow[t]{2}{*}{$\begin{array}{l}\text { DG females } \\
\text { APPPS1-21 } \\
\text { Tg }\end{array}$} & Inclusion & $\begin{array}{l}\text { Nrxn3 (3.097), Frmd4a (2.255), Psen1 (1.581), Epha7 } \\
\text { (1.552), Tom1l2 (1.516), Psen1 ()1.483, Vwf (1.390), } \\
\text { Cbl (1.287), Nsf (1.237), Psen1 (1.222) }\end{array}$ \\
\hline & Exclusion & $\begin{array}{l}\text { Atp1a2 (-1.280), Map1a (-1.282), Ptprz1 (-1.285), } \\
\text { Farp1 (-1.348), Cfh (-1.361), Psen1 (-1.372), Nr4a1 } \\
(-1.372), \text { Rbm6 (-1.382), Reln (-1.394), Farp1 (-1.465), }\end{array}$ \\
\hline
\end{tabular}

Appendix table 5. Top 10 genes that had a significant alternative exon usage in male and female DG and male CA1 of wild-type mice born to APPPS1-21 transgenic fathers.

Top 10 significant differentially spliced genes in in male and female DG and male CA1 of wtg ${ }^{\text {tgwt }}$ mice compared to $w^{\text {twtwt }}$ mice ranked by $\log _{2} F C$. Genes, padj $\leq 0.05$; $\log _{2} F C \pm 0.25$. For DG males: $w^{\text {tgwt }} n=7$

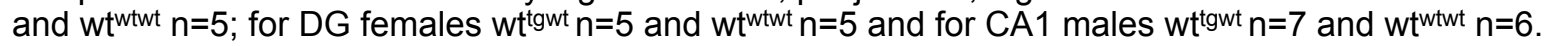

\begin{tabular}{|c|c|c|}
\hline & & Gene name $\left(\log _{2} F C\right)$ \\
\hline \multirow[t]{2}{*}{$\begin{array}{l}\text { CA1 } \\
\text { males } \\
\text { Wttgwt }\end{array}$} & Inclusion & $\begin{array}{l}\text { Cd200 (1.927), Gabrb3 (1.894),4930447C04Rik (1.598), } \\
\text { Hace1 (1.575),Tut4 (1.401),Rnf213 (1.335),A830018L16Rik } \\
\text { (1.320),Spock3 (1.278), Tmem237 (1.273),C530008M17Rik } \\
(1.262),\end{array}$ \\
\hline & Exclusion & $\begin{array}{l}\text { Wdr13 (-1.674),Stambp (-1.686),Tns1 (-1.690),Sbf1 } \\
\text { (-1.690),Irak2 (-1.705),Ptpru (-1.714),Rab10os } \\
\text { (-1.717),Atp6v0e2 (-1.717), Rit1 (-1.765),Zfp354c (-2.253), }\end{array}$ \\
\hline \multirow[t]{2}{*}{$\begin{array}{l}\text { DG } \\
\text { males } \\
\text { Wtgwt }\end{array}$} & Inclusion & $\begin{array}{l}\text { Hook3 (2.870),Mir8108 (2.509),Ppfia1 (2.456), Nrxn3 } \\
\text { (2.290),Pms2 (2.249),Unc80 (2.183),Scn2a } \\
\text { (2.115),Dennd1b (2.105),Stxbp1 (2.103),Fank1 (2.066), }\end{array}$ \\
\hline & Exclusion & $\begin{array}{l}\text { Srrm4 (-2.741), Maz (-2.758), Crim1 (-2.781), Gabrg1 } \\
\text { (-2.787),Paf1 (-2.787),Tnks1bp1 (-2.868), Tbc1d8b } \\
\text { (-3.424),Wnk2 lars2 (-3.449), lars2 (-3.528), }\end{array}$ \\
\hline $\begin{array}{l}\text { DG } \\
\text { female } \\
\text { s }\end{array}$ & Inclusion & $\begin{array}{l}\text { Dlgap1 (0.654),Brat1 (0.648),Fbrsl1 (0.624), Rabl6 } \\
(0.584), \text { Htt (0.559),Gabra2 (0.556),Angptl4 (0.550),Smc6 } \\
(0.515), \text { Hyou1 (0.513),Rps4x (0.512), }\end{array}$ \\
\hline Wttgwt & Exclusion & $\begin{array}{l}\text { Arhgap23 (-0.571),Agpat1 (-0.594), Crym (-0.596), Ppdpf } \\
\text { (-0.667), Lmo4 (-0.687),Arhgef4 (-0.694),Dynll1 } \\
(-0.835), \text { Dnm1 (-0.835), Lmo4 (-1.152) }\end{array}$ \\
\hline
\end{tabular}




\section{REFERENCES}




\section{REFERENCES}

Abel, T., Nguyen, P. V., Barad, M., Deuel, T. A., Kandel, E. R., \& Bourtchouladze, R. (1997). Genetic demonstration of a role for PKA in the late phase of LTP and in hippocampus-based long-term memory. Cell, 88(5), 615-626.

Aggleton, J. P., O'Mara, S. M., Vann, S. D., Wright, N. F., Tsanov, M., \& Erichsen, J. T. (2010). Hippocampal-anterior thalamic pathways for memory: uncovering a network of direct and indirect actions. Eur J Neurosci, 31(12), 2292-2307. doi: 10.1111/j.1460-9568.2010.07251.x

Aidaralieva, N. J., Kamino, K., Kimura, R., Yamamoto, M., Morihara, T., Kazui, H., . . Takeda, M. (2008). Dynamin 2 gene is a novel susceptibility gene for late-onset Alzheimer disease in non-APOE-epsilon4 carriers. $J$ Hum Genet, 53(4), 296-302. doi: 10.1007/s10038-008-0251-9

Alam, S., Suzuki, H., \& Tsukahara, T. (2014). Alternative splicing regulation of APP exon 7 by RBFox proteins. Neurochem Int, 78, 7-17. doi: 10.1016/j.neuint.2014.08.001

Albert, M., Duffy, F. H., \& Naeser, M. (1987). Nonlinear changes in cognition with age and their neuropsychologic correlates. Can J Psychol, 41(2), 141-157.

Alexandrov, P. N., Dua, P., \& Lukiw, W. J. (2014). Up-Regulation of miRNA-146a in Progressive, Age-Related Inflammatory Neurodegenerative Disorders of the Human CNS. Front Neurol, 5, 181. doi: 10.3389/fneur.2014.00181

Allen, B., Ingram, E., Takao, M., Smith, M. J., Jakes, R., Virdee, K., . . Goedert, M. (2002). Abundant tau filaments and nonapoptotic neurodegeneration in transgenic mice expressing human P301S tau protein. J Neurosci, 22(21), 9340-9351.

Allfrey, V. G., Faulkner, R., \& Mirsky, A. E. (1964). Acetylation and Methylation of Histones and Their Possible Role in the Regulation of Rna Synthesis. Proc Natl Acad Sci U S A, 51, 786-794.

Alm, P. S., de Castro Barbosa, T., Barres, R., Krook, A., \& Zierath, J. R. (2017). Grandpaternal-induced transgenerational dietary reprogramming of the unfolded protein response in skeletal muscle. Mol Metab, 6(7), 621-630. doi: 10.1016/j.molmet.2017.05.009

Alter, M. D., Gilani, A. I., Champagne, F. A., Curley, J. P., Turner, J. B., \& Hen, R. (2009). Paternal transmission of complex phenotypes in inbred mice. Biol Psychiatry, 66(11), 1061-1066. doi: 10.1016/j.biopsych.2009.05.026

Alzheimer, A., Stelzmann, R. A., Schnitzlein, H. N., \& Murtagh, F. R. (1995). An English translation of Alzheimer's 1907 paper, "Uber eine eigenartige Erkankung der Hirnrinde". Clin Anat, 8(6), 429-431. doi: 10.1002/ca.980080612

Amaral, D. G., \& Witter, M. P. (1989). The three-dimensional organization of the hippocampal formation: a review of anatomical data. Neuroscience, 31(3), 571-591.

Anagnostaras, S. G., Maren, S., \& Fanselow, M. S. (1999). Temporally graded retrograde amnesia of contextual fear after hippocampal damage in rats: within-subjects examination. $J$ Neurosci, 19(3), 1106-1114.

Anders, S., Reyes, A., \& Huber, W. (2012). Detecting differential usage of exons from RNA-seq data. Genome Res, 22(10), 2008-2017. doi: 10.1101/gr.133744.111

Andorfer, C., Kress, Y., Espinoza, M., de Silva, R., Tucker, K. L., Barde, Y. A., . . Davies, P. (2003). Hyperphosphorylation and aggregation of tau in mice expressing normal human tau isoforms. J Neurochem, 86(3), 582-590.

Andreadis, A., Brown, W. M., \& Kosik, K. S. (1992). Structure and novel exons of the human tau gene. Biochemistry, 31(43), 10626-10633.

Andrews-Hanna, J. R., Snyder, A. Z., Vincent, J. L., Lustig, C., Head, D., Raichle, M. E., \& Buckner, R. L. (2007). Disruption of large-scale brain systems in advanced aging. Neuron, 56(5), 924-935. doi: 10.1016/j.neuron. 2007.10.038

Annese, A., Manzari, C., Lionetti, C., Picardi, E., Horner, D. S., Chiara, M., . . D'Erchia, A. M. (2018). Whole transcriptome profiling of Late-Onset Alzheimer's Disease patients provides insights into the molecular changes involved in the disease. Sci Rep, 8(1), 4282. doi: 10.1038/s41598-018-22701-2

Anway, M. D., Cupp, A. S., Uzumcu, M., \& Skinner, M. K. (2005). Epigenetic transgenerational actions of endocrine disruptors and male fertility. Science, 308(5727), 1466-1469. doi: 10.1126/science.1108190

Appleton, J. P., Scutt, P., Sprigg, N., \& Bath, P. M. (2017). Hypercholesterolaemia and vascular dementia. Clin Sci (Lond), 131(14), 1561-1578. doi: 10.1042/CS20160382

Aravin, A. A., Hannon, G. J., \& Brennecke, J. (2007). The Piwi-piRNA pathway provides an adaptive defense in the transposon arms race. Science, 318(5851), 761-764. doi: 10.1126/science.1146484

Aravin, A. A., Lagos-Quintana, M., Yalcin, A., Zavolan, M., Marks, D., Snyder, B., . . Tuschl, T. (2003). The small RNA profile during Drosophila melanogaster development. Dev Cell, 5(2), 337-350.

Aravin, A. A., Naumova, N. M., Tulin, A. V., Vagin, V. V., Rozovsky, Y. M., \& Gvozdev, V. A. (2001). Double-stranded RNAmediated silencing of genomic tandem repeats and transposable elements in the $D$. melanogaster germline. Curr Biol, 11(13), 1017-1027.

Aravin, A. A., Sachidanandam, R., Bourc'his, D., Schaefer, C., Pezic, D., Toth, K. F., . . Hannon, G. J. (2008). A piRNA pathway primed by individual transposons is linked to de novo DNA methylation in mice. Mol Cell, 31(6), 785-799. doi: 10.1016/j.molcel.2008.09.003

Aravin, A. A., Sachidanandam, R., Girard, A., Fejes-Toth, K., \& Hannon, G. J. (2007). Developmentally regulated piRNA clusters implicate MILI in transposon control. Science, 316(5825), 744-747. doi: 10.1126/science.1142612

Aravin, A., Gaidatzis, D., Pfeffer, S., Lagos-Quintana, M., Landgraf, P., lovino, N., . . Tuschl, T. (2006). A novel class of small RNAs bind to MILI protein in mouse testes. Nature, 442(7099), 203-207. doi: 10.1038/nature04916

Arendash, G. W., King, D. L., Gordon, M. N., Morgan, D., Hatcher, J. M., Hope, C. E., \& Diamond, D. M. (2001). Progressive, age-related behavioral impairments in transgenic mice carrying both mutant amyloid precursor protein and presenilin-1 transgenes. Brain Res, 891(1-2), 42-53.

Arlt, S., Buchert, R., Spies, L., Eichenlaub, M., Lehmbeck, J. T., \& Jahn, H. (2013). Association between fully automated MRI-based volumetry of different brain regions and neuropsychological test performance in patients with amnestic mild cognitive impairment and Alzheimer's disease. Eur Arch Psychiatry Clin Neurosci, 263(4), 335-344. doi: 10.1007/s00406-012-0350-7 
Armisen, J., Gilchrist, M. J., Wilczynska, A., Standart, N., \& Miska, E. A. (2009). Abundant and dynamically expressed miRNAs, piRNAs, and other small RNAs in the vertebrate Xenopus tropicalis. Genome Res, 19(10), 1766-1775. doi: 10.1101/gr.093054.109

Arroyo, J. D., Chevillet, J. R., Kroh, E. M., Ruf, I. K., Pritchard, C. C., Gibson, D. F., . . Tewari, M. (2011). Argonaute2 complexes carry a population of circulating microRNAs independent of vesicles in human plasma. Proc Natl Acad Sci U S A, 108(12), 5003-5008. doi: 10.1073/pnas.1019055108

Ashe, A., Sapetschnig, A., Weick, E. M., Mitchell, J., Bagijn, M. P., Cording, A. C., . . Miska, E. A. (2012). piRNAs can trigger a multigenerational epigenetic memory in the germline of C. elegans. Cell, 150(1), 88-99. doi: 10.1016/ j.cell.2012.06.018

Atallah, H. E., Frank, M. J., \& O'Reilly, R. C. (2004). Hippocampus, cortex, and basal ganglia: insights from computational models of complementary learning systems. Neurobiol Learn Mem, 82(3), 253-267. doi: 10.1016/j.nlm. 2004.06.004

Atkinson, R. C. (1967). Human memory : a proposed system and its control processes. [Stanford, Calif.]: [Institute for Mathematical Studies in Social Sciences].

Attar, A., Liu, T., Chan, W. T., Hayes, J., Nejad, M., Lei, K., \& Bitan, G. (2013). A shortened Barnes maze protocol reveals memory deficits at 4-months of age in the triple-transgenic mouse model of Alzheimer's disease. PLoS One, 8(11), e80355. doi: 10.1371/journal.pone.0080355

Baddeley, A. (2010). Working memory. Curr Biol, 20(4), R136-140. doi: 10.1016/j.cub.2009.12.014

Bai, L., Xu, H., Collins, J. F., \& Ghishan, F. K. (2001). Molecular and functional analysis of a novel neuronal vesicular glutamate transporter. J Biol Chem, 276(39), 36764-36769. doi: 10.1074/jbc.M104578200

Bailer, A. J. (1988). Testing for the equality of area under the curves when using destructive measurement techniques. $J$ Pharmacokinet Biopharm, 16(3), 303-309.

Bak, M., Silahtaroglu, A., Moller, M., Christensen, M., Rath, M. F., Skryabin, B., . . Kauppinen, S. (2008). MicroRNA expression in the adult mouse central nervous system. RNA, 14(3), 432-444. doi: 10.1261/rna.783108

Baker, S. M., Bronner, C. E., Zhang, L., Plug, A. W., Robatzek, M., Warren, G., . . Liskay, R. M. (1995). Male mice defective in the DNA mismatch repair gene PMS2 exhibit abnormal chromosome synapsis in meiosis. Cell, 82(2), 309-319.

Bales, K. R. (2010). Brain lipid metabolism, apolipoprotein E and the pathophysiology of Alzheimer's disease. Neuropharmacology, 59(4-5), 295-302. doi: 10.1016/j.neuropharm.2010.01.005

Bales, K. R., Verina, T., Dodel, R. C., Du, Y., Altstiel, L., Bender, M., . . Paul, S. M. (1997). Lack of apolipoprotein E dramatically reduces amyloid beta-peptide deposition. Nat Genet, 17(3), 263-264. doi: 10.1038/ng1197-263

Balhorn, R., Brewer, L., \& Corzett, M. (2000). DNA condensation by protamine and arginine-rich peptides: analysis of toroid stability using single DNA molecules. Mol Reprod Dev, 56(2 Suppl), 230-234. doi: 10.1002/ (SICl)1098-2795(200006)56:2+<230::AID-MRD3>3.0.CO;2-V

Barbash, S., Garfinkel, B. P., Maoz, R., Simchovitz, A., Nadorp, B., Guffanti, A., . . Soreq, H. (2017). Alzheimer's brains show inter-related changes in RNA and lipid metabolism. Neurobiol Dis, 106, 1-13. doi: 10.1016/j.nbd. 2017.06.008

Barnes, C. A. (1979). Memory deficits associated with senescence: a neurophysiological and behavioral study in the rat. $J$ Comp Physiol Psychol, 93(1), 74-104.

Barnes, D. E., \& Yaffe, K. (2011). The projected effect of risk factor reduction on Alzheimer's disease prevalence. Lancet Neurol, 10(9), 819-828. doi: 10.1016/S1474-4422(11)70072-2

Bartolomei, M. S., \& Ferguson-Smith, A. C. (2011). Mammalian genomic imprinting. Cold Spring Harb Perspect Biol, 3(7). doi: $10.1101 /$ cshperspect.a002592

Bartzokis, G., Cummings, J. L., Sultzer, D., Henderson, V. W., Nuechterlein, K. H., \& Mintz, J. (2003). White matter structural integrity in healthy aging adults and patients with Alzheimer disease: a magnetic resonance imaging study. Arch Neurol, 60(3), 393-398.

Bateman, R. J., Xiong, C., Benzinger, T. L., Fagan, A. M., Goate, A., Fox, N. C., . . Dominantly Inherited Alzheimer, Network. (2012). Clinical and biomarker changes in dominantly inherited Alzheimer's disease. N Engl J Med, 367(9), 795-804. doi: 10.1056/NEJMoa1202753

Batista, P. J., Ruby, J. G., Claycomb, J. M., Chiang, R., Fahlgren, N., Kasschau, K. D., . . Mello, C. C. (2008). PRG-1 and 21U-RNAs interact to form the piRNA complex required for fertility in C. elegans. Mol Cell, 31(1), 67-78. doi: 10.1016/j.molcel.2008.06.002

Beal, M. F. (2005). Mitochondria take center stage in aging and neurodegeneration. Ann Neurol, 58(4), 495-505. doi: 10.1002/ana.20624

Beffert, U., Weeber, E. J., Durudas, A., Qiu, S., Masiulis, I., Sweatt, J. D., . . Herz, J. (2005). Modulation of synaptic plasticity and memory by Reelin involves differential splicing of the lipoprotein receptor Apoer2. Neuron, 47(4), 567-579. doi: 10.1016/j.neuron.2005.07.007

Beher, D., Hesse, L., Masters, C. L., \& Multhaup, G. (1996). Regulation of amyloid protein precursor (APP) binding to collagen and mapping of the binding sites on APP and collagen type I. J Biol Chem, 271(3), 1613-1620.

Belgard, T. G., Marques, A. C., Oliver, P. L., Abaan, H. O., Sirey, T. M., Hoerder-Suabedissen, A., . . Ponting, C. P. (2011). A transcriptomic atlas of mouse neocortical layers. Neuron, 71(4), 605-616. doi: 10.1016/j.neuron.2011.06.039

Bell, K. F., Zheng, L., Fahrenholz, F., \& Cuello, A. C. (2008). ADAM-10 over-expression increases cortical synaptogenesis. Neurobiol Aging, 29(4), 554-565. doi: 10.1016/j.neurobiolaging.2006.11.004

Bellizzi, D., Rose, G., Cavalcante, P., Covello, G., Dato, S., De Rango, F., . . . De Benedictis, G. (2005). A novel VNTR enhancer within the SIRT3 gene, a human homologue of SIR2, is associated with survival at oldest ages. Genomics, 85(2), 258-263. doi: 10.1016/j.ygeno.2004.11.003

Ben Maamar, M., Sadler-Riggleman, I., Beck, D., \& Skinner, M. K. (2018). Epigenetic Transgenerational Inheritance of Altered Sperm Histone Retention Sites. Sci Rep, 8(1), 5308. doi: 10.1038/s41598-018-23612-y 
Benito, E., Kerimoglu, C., Ramachandran, B., Pena-Centeno, T., Jain, G., Stilling, R. M., . . Fischer, A. (2018). RNADependent Intergenerational Inheritance of Enhanced Synaptic Plasticity after Environmental Enrichment. Cell Rep, 23(2), 546-554. doi: 10.1016/j.celrep.2018.03.059

Benito, E., Urbanke, H., Ramachandran, B., Barth, J., Halder, R., Awasthi, A., . . Fischer, A. (2015). HDAC inhibitordependent transcriptome and memory reinstatement in cognitive decline models. J Clin Invest, 125(9), 3572-3584. doi: $10.1172 / \mathrm{JCl} 79942$

Berchtold, N. C., Cribbs, D. H., Coleman, P. D., Rogers, J., Head, E., Kim, R., . . Cotman, C. W. (2008). Gene expression changes in the course of normal brain aging are sexually dimorphic. Proc Natl Acad Sci U S A, 105(40), 15605-15610. doi: 10.1073/pnas.0806883105

Bernard, D., Prasanth, K. V., Tripathi, V., Colasse, S., Nakamura, T., Xuan, Z., . . Bessis, A. (2010). A long nuclearretained non-coding RNA regulates synaptogenesis by modulating gene expression. EMBO J, 29(18), 3082-3093. doi: 10.1038/emboj.2010.199

Bhat, A. H., Dar, K. B., Anees, S., Zargar, M. A., Masood, A., Sofi, M. A., \& Ganie, S. A. (2015). Oxidative stress, mitochondrial dysfunction and neurodegenerative diseases; a mechanistic insight. Biomed Pharmacother, 74, 101-110. doi: 10.1016/j.biopha.2015.07.025

Bindea, G., Mlecnik, B., Hackl, H., Charoentong, P., Tosolini, M., Kirilovsky, A., . . Galon, J. (2009). ClueGO: a Cytoscape plug-in to decipher functionally grouped gene ontology and pathway annotation networks. Bioinformatics, 25(8), 1091-1093. doi: 10.1093/bioinformatics/btp101

Binder, J. R., \& Desai, R. H. (2011). The neurobiology of semantic memory. Trends Cogn Sci, 15(11), 527-536. doi: 10.1016/j.tics.2011.10.001

Bird, A. (2007). Perceptions of epigenetics. Nature, 447(7143), 396-398. doi: 10.1038/nature05913

Blake, G. E., \& Watson, E. D. (2016). Unravelling the complex mechanisms of transgenerational epigenetic inheritance. Curr Opin Chem Biol, 33, 101-107. doi: 10.1016/j.cbpa.2016.06.008

Blalock, E. M., Chen, K. C., Sharrow, K., Herman, J. P., Porter, N. M., Foster, T. C., \& Landfield, P. W. (2003). Gene microarrays in hippocampal aging: statistical profiling identifies novel processes correlated with cognitive impairment. J Neurosci, 23(9), 3807-3819.

Blalock, E. M., Geddes, J. W., Chen, K. C., Porter, N. M., Markesbery, W. R., \& Landfield, P. W. (2004). Incipient Alzheimer's disease: microarray correlation analyses reveal major transcriptional and tumor suppressor responses. Proc Natl Acad Sci U S A, 101(7), 2173-2178. doi: 10.1073/pnas.0308512100

Bliss, T. V., \& Lomo, T. (1973). Long-lasting potentiation of synaptic transmission in the dentate area of the anaesthetized rabbit following stimulation of the perforant path. J Physiol, 232(2), 331-356.

Blurton-Jones, M., \& Laferla, F. M. (2006). Pathways by which Abeta facilitates tau pathology. Curr Alzheimer Res, 3(5), 437-448.

Bohacek, J., \& Mansuy, I. M. (2017). A guide to designing germline-dependent epigenetic inheritance experiments in mammals. Nat Methods, 14(3), 243-249. doi: 10.1038/nmeth.4181

Bonaguidi, M. A., Wheeler, M. A., Shapiro, J. S., Stadel, R. P., Sun, G. J., Ming, G. L., \& Song, H. (2011). In vivo clonal analysis reveals self-renewing and multipotent adult neural stem cell characteristics. Cell, 145(7), 1142-1155. doi: 10.1016/j.cell.2011.05.024

Bonner-Jackson, A., Mahmoud, S., Miller, J., \& Banks, S. J. (2015). Verbal and non-verbal memory and hippocampal volumes in a memory clinic population. Alzheimers Res Ther, 7(1), 61. doi: 10.1186/s13195-015-0147-9

Bors, L., Toth, K., Toth, E. Z., Bajza, A., Csorba, A., Szigeti, K., . . Erdo, F. (2018). Structural and functional characterization of age-associated changes at the blood-brain barrier. A comparative in vivo study in young adult and middle aged Wistar rats. Brain Res Bull. doi: 10.1016/j.brainresbull.2018.03.001

Bossers, K., Wirz, K. T., Meerhoff, G. F., Essing, A. H., van Dongen, J. W., Houba, P., . . Swaab, D. F. (2010). Concerted changes in transcripts in the prefrontal cortex precede neuropathology in Alzheimer's disease. Brain, 133(Pt 12), 3699-3723. doi: 10.1093/brain/awq258

Braak, H., Alafuzoff, I., Arzberger, T., Kretzschmar, H., \& Del Tredici, K. (2006). Staging of Alzheimer disease-associated neurofibrillary pathology using paraffin sections and immunocytochemistry. Acta Neuropathol, 112(4), 389-404. doi: 10.1007/s00401-006-0127-z

Braak, H., \& Braak, E. (1991). Neuropathological stageing of Alzheimer-related changes. Acta Neuropathol, 82(4), 239-259.

Bradbury, E. M., Inglis, R. J., Matthews, H. R., \& Sarner, N. (1973). Phosphorylation of very-lysine-rich histone in Physarum polycephalum. Correlation with chromosome condensation. Eur J Biochem, 33(1), 131-139.

Bradshaw, E. M., Chibnik, L. B., Keenan, B. T., Ottoboni, L., Raj, T., Tang, A., . . De Jager, P. L. (2013). CD33 Alzheimer's disease locus: altered monocyte function and amyloid biology. Nat Neurosci, 16(7), 848-850. doi: 10.1038/nn. 3435

Brameier, M., Herwig, A., Reinhardt, R., Walter, L., \& Gruber, J. (2011). Human box C/D snoRNAs with miRNA like functions: expanding the range of regulatory RNAs. Nucleic Acids Res, 39(2), 675-686. doi: 10.1093/nar/gkq776

Brashers-Krug, T., Shadmehr, R., \& Bizzi, E. (1996). Consolidation in human motor memory. Nature, 382(6588), $252-255$. doi: $10.1038 / 382252 \mathrm{a} 0$

Bratic, A., \& Larsson, N. G. (2013). The role of mitochondria in aging. J Clin Invest, 123(3), 951-957. doi: 10.1172/ $\mathrm{JCl} 64125$

Brem, A. K., Ran, K., \& Pascual-Leone, A. (2013). Learning and memory. Handb Clin Neurol, 116, 693-737. doi: 10.1016/ B978-0-444-53497-2.00055-3

Brennecke, J., Aravin, A. A., Stark, A., Dus, M., Kellis, M., Sachidanandam, R., \& Hannon, G. J. (2007). Discrete small RNA-generating loci as master regulators of transposon activity in Drosophila. Cell, 128(6), 1089-1103. doi: 10.1016/j.cell.2007.01.043

Brennecke, J., Malone, C. D., Aravin, A. A., Sachidanandam, R., Stark, A., \& Hannon, G. J. (2008). An epigenetic role for maternally inherited piRNAs in transposon silencing. Science, 322(5906), 1387-1392. doi: 10.1126/science. 1165171 
Bridgewater, L. C., Zhang, C., Wu, Y., Hu, W., Zhang, Q., Wang, J., . . Zhao, L. (2017). Gender-based differences in host behavior and gut microbiota composition in response to high fat diet and stress in a mouse model. Sci Rep, 7(1), 10776. doi: 10.1038/s41598-017-11069-4

Broadbent, N. J., Squire, L. R., \& Clark, R. E. (2006). Reversible hippocampal lesions disrupt water maze performance during both recent and remote memory tests. Learn Mem, 13(2), 187-191. doi: 10.1101//m.134706

Brunden, K. R., Trojanowski, J. Q., \& Lee, V. M. (2009). Advances in tau-focused drug discovery for Alzheimer's disease and related tauopathies. Nat Rev Drug Discov, 8(10), 783-793. doi: 10.1038/nrd2959

Brykczynska, U., Hisano, M., Erkek, S., Ramos, L., Oakeley, E. J., Roloff, T. C., . . Peters, A. H. (2010). Repressive and active histone methylation mark distinct promoters in human and mouse spermatozoa. Nat Struct Mol Biol, 17(6), 679-687. doi: 10.1038/nsmb.1821

Buratti, E., Brindisi, A., Pagani, F., \& Baralle, F. E. (2004). Nuclear factor TDP-43 binds to the polymorphic TG repeats in CFTR intron 8 and causes skipping of exon 9: a functional link with disease penetrance. Am J Hum Genet, 74(6), 1322-1325. doi: 10.1086/420978

Burke, S. N., \& Barnes, C. A. (2006). Neural plasticity in the ageing brain. Nat Rev Neurosci, 7(1), 30-40. doi: 10.1038/ nrn1809

Burtner, C. R., \& Kennedy, B. K. (2010). Progeria syndromes and ageing: what is the connection? Nat Rev Mol Cell Biol, 11(8), 567-578. doi: 10.1038/nrm2944

Busch, H., Reddy, R., Rothblum, L., \& Choi, Y. C. (1982). SnRNAs, SnRNPs, and RNA processing. Annu Rev Biochem, 51, 617-654. doi: 10.1146/annurev.bi.51.070182.003153

Busche, M. A., Eichhoff, G., Adelsberger, H., Abramowski, D., Wiederhold, K. H., Haass, C., ... Garaschuk, O. (2008). Clusters of hyperactive neurons near amyloid plaques in a mouse model of Alzheimer's disease. Science, 321(5896), 1686-1689. doi: 10.1126/science.1162844

Butcher, S. P., Davis, S., \& Morris, R. G. (1990). A dose-related impairment of spatial learning by the NMDA receptor antagonist, 2-amino-5-phosphonovalerate (AP5). Eur Neuropsychopharmacol, 1(1), 15-20.

Byrnes, E. M. (2005). Transgenerational consequences of adolescent morphine exposure in female rats: effects on anxiety-like behaviors and morphine sensitization in adult offspring. Psychopharmacology (Berl), 182(4), 537-544. doi: 10.1007/s00213-005-0122-4

Cadenas, E., \& Davies, K. J. (2000). Mitochondrial free radical generation, oxidative stress, and aging. Free Radic Biol Med, 29(3-4), 222-230.

Cameron, H. A., \& McKay, R. D. (2001). Adult neurogenesis produces a large pool of new granule cells in the dentate gyrus. J Comp Neurol, 435(4), 406-417.

Cao, X., \& Sudhof, T. C. (2001). A transcriptionally [correction of transcriptively] active complex of APP with Fe65 and histone acetyltransferase Tip60. Science, 293(5527), 115-120. doi: 10.1126/science.1058783

Carlisle, H. J., Fink, A. E., Grant, S. G., \& O'Dell, T. J. (2008). Opposing effects of PSD-93 and PSD-95 on long-term potentiation and spike timing-dependent plasticity. J Physiol, 586(24), 5885-5900. doi: 10.1113/jphysiol. 2008.163469

Carmell, M. A., Girard, A., van de Kant, H. J., Bourc'his, D., Bestor, T. H., de Rooij, D. G., \& Hannon, G. J. (2007). MIWI2 is essential for spermatogenesis and repression of transposons in the mouse male germline. Dev Cell, 12(4), 503-514. doi: 10.1016/j.devcel.2007.03.001

Carninci, P., Kasukawa, T., Katayama, S., Gough, J., Frith, M. C., Maeda, N., . . Genome Science, Group. (2005). The transcriptional landscape of the mammalian genome. Science, 309(5740), 1559-1563. doi: 10.1126/science. 1112014

Carone, B. R., Fauquier, L., Habib, N., Shea, J. M., Hart, C. E., Li, R., . . Rando, O. J. (2010). Paternally induced transgenerational environmental reprogramming of metabolic gene expression in mammals. Cell, 143(7), 1084-1096. doi: 10.1016/j.cell.2010.12.008

Casellas, J. (2011). Inbred mouse strains and genetic stability: a review. Animal, 5(1), 1-7. doi: $10.1017 /$ S1751731110001667

Cavaille, J., Buiting, K., Kiefmann, M., Lalande, M., Brannan, C. I., Horsthemke, B., . . Huttenhofer, A. (2000). Identification of brain-specific and imprinted small nucleolar RNA genes exhibiting an unusual genomic organization. Proc Natl Acad Sci U S A, 97(26), 14311-14316. doi: 10.1073/pnas.250426397

Cavaille, J., Nicoloso, M., \& Bachellerie, J. P. (1996). Targeted ribose methylation of RNA in vivo directed by tailored antisense RNA guides. Nature, 383(6602), 732-735. doi: 10.1038/383732a0

Chahal, H. S., \& Drake, W. M. (2007). The endocrine system and ageing. J Pathol, 211(2), 173-180. doi: 10.1002/path. 2110

Chai, W. J., Abd Hamid, A. I., \& Abdullah, J. M. (2018). Working Memory From the Psychological and Neurosciences Perspectives: A Review. Front Psychol, 9, 401. doi: 10.3389/fpsyg.2018.00401

Chamorro-Garcia, R., Sahu, M., Abbey, R. J., Laude, J., Pham, N., \& Blumberg, B. (2013). Transgenerational inheritance of increased fat depot size, stem cell reprogramming, and hepatic steatosis elicited by prenatal exposure to the obesogen tributyltin in mice. Environ Health Perspect, 121(3), 359-366. doi: 10.1289/ehp.1205701

Champagne, F. A. (2008). Epigenetic mechanisms and the transgenerational effects of maternal care. Front Neuroendocrinol, 29(3), 386-397. doi: 10.1016/j.yfrne.2008.03.003

Champagne, F. A., Weaver, I. C., Diorio, J., Dymov, S., Szyf, M., \& Meaney, M. J. (2006). Maternal care associated with methylation of the estrogen receptor-alpha1b promoter and estrogen receptor-alpha expression in the medial preoptic area of female offspring. Endocrinology, 147(6), 2909-2915. doi: 10.1210/en.2005-1119

Chapman, P. F., White, G. L., Jones, M. W., Cooper-Blacketer, D., Marshall, V. J., Irizarry, M., . . Hsiao, K. K. (1999). Impaired synaptic plasticity and learning in aged amyloid precursor protein transgenic mice. Nat Neurosci, 2(3), 271-276. doi: 10.1038/6374

Chen, M., \& Zhang, L. (2011). Epigenetic mechanisms in developmental programming of adult disease. Drug Discov Today, 16(23-24), 1007-1018. doi: 10.1016/j.drudis.2011.09.008

Chen, Q., Nakajima, A., Choi, S. H., Xiong, X., Sisodia, S. S., \& Tang, Y. P. (2008). Adult neurogenesis is functionally associated with AD-like neurodegeneration. Neurobiol Dis, 29(2), 316-326. doi: 10.1016/j.nbd.2007.09.005 
Chen, Q., Yan, M., Cao, Z., Li, X., Zhang, Y., Shi, J., . . Zhou, Q. (2016). Sperm tsRNAs contribute to intergenerational inheritance of an acquired metabolic disorder. Science, 351(6271), 397-400. doi: 10.1126/science.aad7977

Chen, X., Ba, Y., Ma, L., Cai, X., Yin, Y., Wang, K., . . Zhang, C. Y. (2008). Characterization of microRNAs in serum: a novel class of biomarkers for diagnosis of cancer and other diseases. Cell Res, 18(10), 997-1006. doi: 10.1038/ cr.2008.282

Cheng, D., Logge, W., Low, J. K., Garner, B., \& Karl, T. (2013). Novel behavioural characteristics of the APP(Swe)/ PS1DeltaE9 transgenic mouse model of Alzheimer's disease. Behav Brain Res, 245, 120-127. doi: 10.1016/ j.bbr.2013.02.008

Chow, C., Epp, J. R., Lieblich, S. E., Barha, C. K., \& Galea, L. A. (2013). Sex differences in neurogenesis and activation of new neurons in response to spatial learning and memory. Psychoneuroendocrinology, 38(8), 1236-1250. doi: 10.1016/j.psyneuen.2012.11.007

Chung, H. Y., Kim, H. J., Kim, J. W., \& Yu, B. P. (2001). The inflammation hypothesis of aging: molecular modulation by calorie restriction. Ann N Y Acad Sci, 928, 327-335.

Cleary, J. P., Walsh, D. M., Hofmeister, J. J., Shankar, G. M., Kuskowski, M. A., Selkoe, D. J., \& Ashe, K. H. (2005). Natural oligomers of the amyloid-beta protein specifically disrupt cognitive function. Nat Neurosci, 8(1), 79-84. doi: $10.1038 / \mathrm{nn} 1372$

Clelland, C. D., Choi, M., Romberg, C., Clemenson, G. D., Jr., Fragniere, A., Tyers, P., . . Bussey, T. J. (2009). A functional role for adult hippocampal neurogenesis in spatial pattern separation. Science, 325(5937), 210-213. doi: 10.1126/science.1173215

Cohen, N. J., Eichenbaum, H., Deacedo, B. S., \& Corkin, S. (1985). Different memory systems underlying acquisition of procedural and declarative knowledge. Ann N Y Acad Sci, 444, 54-71.

Cohen, N. J., \& Squire, L. R. (1980). Preserved learning and retention of pattern-analyzing skill in amnesia: dissociation of knowing how and knowing that. Science, 210(4466), 207-210.

Cohen, S. J., Punt, C. J., lannotti, N., Saidman, B. H., Sabbath, K. D., Gabrail, N. Y., . . Meropol, N. J. (2008). Relationship of circulating tumor cells to tumor response, progression-free survival, and overall survival in patients with metastatic colorectal cancer. J Clin Oncol, 26(19), 3213-3221. doi: 10.1200/JCO.2007.15.8923

Conway, M. A., \& Pleydell-Pearce, C. W. (2000). The construction of autobiographical memories in the self-memory system. Psychol Rev, 107(2), 261-288.

Corcoran, K. A., Lu, Y., Turner, R. S., \& Maren, S. (2002). Overexpression of hAPPswe impairs rewarded alternation and contextual fear conditioning in a transgenic mouse model of Alzheimer's disease. Learn Mem, 9(5), 243-252. doi: $10.1101 / \mathrm{Im} .51002$

Corder, E. H., Saunders, A. M., Strittmatter, W. J., Schmechel, D. E., Gaskell, P. C., Small, G. W., . . Pericak-Vance, M. A. (1993). Gene dose of apolipoprotein E type 4 allele and the risk of Alzheimer's disease in late onset families. Science, 261(5123), 921-923.

Cossetti, C., Lugini, L., Astrologo, L., Saggio, I., Fais, S., \& Spadafora, C. (2014). Soma-to-germline transmission of RNA in mice xenografted with human tumour cells: possible transport by exosomes. PLoS One, 9(7), e101629. doi: 10.1371/journal.pone.0101629

Cowan, N. (2008). What are the differences between long-term, short-term, and working memory? Prog Brain Res, 169, 323-338. doi: 10.1016/S0079-6123(07)00020-9

Craik, F. I., Moscovitch, M., \& McDowd, J. M. (1994). Contributions of surface and conceptual information to performance on implicit and explicit memory tasks. J Exp Psychol Learn Mem Cogn, 20(4), 864-875.

Credendino, S. C., Lewin, N., de Oliveira, M., Basu, S., D'Andrea, B., Amendola, E., . . De Vita, G. (2017). Tissue- and Cell Type-Specific Expression of the Long Noncoding RNA KIhl14-AS in Mouse. Int J Genomics, 2017, 9769171. doi: $10.1155 / 2017 / 9769171$

Cribbs, D. H., Berchtold, N. C., Perreau, V., Coleman, P. D., Rogers, J., Tenner, A. J., \& Cotman, C. W. (2012). Extensive innate immune gene activation accompanies brain aging, increasing vulnerability to cognitive decline and neurodegeneration: a microarray study. J Neuroinflammation, 9, 179. doi: 10.1186/1742-2094-9-179

Crosio, C., Heitz, E., Allis, C. D., Borrelli, E., \& Sassone-Corsi, P. (2003). Chromatin remodeling and neuronal response: multiple signaling pathways induce specific histone $\mathrm{H} 3$ modifications and early gene expression in hippocampal neurons. J Cell Sci, 116(Pt 24), 4905-4914. doi: 10.1242/jcs.00804

D'Hooge, R., \& De Deyn, P. P. (2001). Applications of the Morris water maze in the study of learning and memory. Brain Res Brain Res Rev, 36(1), 60-90.

Danchin, E., Charmantier, A., Champagne, F. A., Mesoudi, A., Pujol, B., \& Blanchet, S. (2011). Beyond DNA: integrating inclusive inheritance into an extended theory of evolution. Nat Rev Genet, 12(7), 475-486. doi: 10.1038/nrg3028

Davis, H. P., \& Squire, L. R. (1984). Protein synthesis and memory: a review. Psychol Bull, 96(3), 518-559.

Davis, K. E., Fox, S., \& Gigg, J. (2014). Increased hippocampal excitability in the 3xTgAD mouse model for Alzheimer's disease in vivo. PLoS One, 9(3), e91203. doi: 10.1371/journal.pone.0091203

Dazzo, E., Fanciulli, M., Serioli, E., Minervini, G., Pulitano, P., Binelli, S., . . . Nobile, C. (2015). Heterozygous reelin mutations cause autosomal-dominant lateral temporal epilepsy. Am J Hum Genet, 96(6), 992-1000. doi: 10.1016/j.ajhg.2015.04.020

de Castro Barbosa, T., Ingerslev, L. R., Alm, P. S., Versteyhe, S., Massart, J., Rasmussen, M., ... Barres, R. (2016a). High-fat diet reprograms the epigenome of rat spermatozoa and transgenerationally affects metabolism of the offspring. Mol Metab, 5(3), 184-197. doi: 10.1016/j.molmet.2015.12.002

de Castro Barbosa, T., Ingerslev, L. R., Alm, P. S., Versteyhe, S., Massart, J., Rasmussen, M., . . Barres, R. (2016b). High-fat diet reprograms the epigenome of rat spermatozoa and transgenerationally affects metabolism of the offspring. Mol Metab, 5(3), 184-197. doi: 10.1016/j.molmet.2015.12.002

De Jonghe, C., Cruts, M., Rogaeva, E. A., Tysoe, C., Singleton, A., Vanderstichele, H., ... Van Broeckhoven, C. (1999). Aberrant splicing in the presenilin-1 intron 4 mutation causes presenile Alzheimer's disease by increased Abeta42 secretion. Hum Mol Genet, 8(8), 1529-1540.

de los Campos, G., Naya, H., Gianola, D., Crossa, J., Legarra, A., Manfredi, E., . . . Cotes, J. M. (2009). Predicting quantitative traits with regression models for dense molecular markers and pedigree. Genetics, 182(1), 375-385. doi: $10.1534 /$ genetics.109.101501 
de Magalhaes, J. P., Curado, J., \& Church, G. M. (2009). Meta-analysis of age-related gene expression profiles identifies common signatures of aging. Bioinformatics, 25(7), 875-881. doi: 10.1093/bioinformatics/btp073

de Silva, M. G., Elliott, K., Dahl, H. H., Fitzpatrick, E., Wilcox, S., Delatycki, M., . . Forrest, S. (2003). Disruption of a novel member of a sodium/hydrogen exchanger family and DOCK3 is associated with an attention deficit hyperactivity disorder-like phenotype. J Med Genet, 40(10), 733-740.

Denham, J., O'Brien, B. J., Harvey, J. T., \& Charchar, F. J. (2015). Genome-wide sperm DNA methylation changes after 3 months of exercise training in humans. Epigenomics, 7(5), 717-731. doi: 10.2217/epi.15.29

Denic, A., Glassock, R. J., \& Rule, A. D. (2016). Structural and Functional Changes With the Aging Kidney. Adv Chronic Kidney Dis, 23(1), 19-28. doi: 10.1053/j.ackd.2015.08.004

Di Carlo, M., Giacomazza, D., Picone, P., Nuzzo, D., \& San Biagio, P. L. (2012). Are oxidative stress and mitochondrial dysfunction the key players in the neurodegenerative diseases? Free Radic Res, 46(11), 1327-1338. doi: $10.3109 / 10715762.2012 .714466$

Dias, B. G., \& Ressler, K. J. (2014a). Experimental evidence needed to demonstrate inter- and trans-generational effects of ancestral experiences in mammals. Bioessays, 36(10), 919-923. doi: 10.1002/bies.201400105

Dias, B. G., \& Ressler, K. J. (2014b). Parental olfactory experience influences behavior and neural structure in subsequent generations. Nat Neurosci, 17(1), 89-96. doi: 10.1038/nn.3594

Dietz, D. M., Laplant, Q., Watts, E. L., Hodes, G. E., Russo, S. J., Feng, J., . . Nestler, E. J. (2011). Paternal transmission of stress-induced pathologies. Biol Psychiatry, 70(5), 408-414. doi: 10.1016/j.biopsych.2011.05.005

Dineley, K. T., Xia, X., Bui, D., Sweatt, J. D., \& Zheng, H. (2002). Accelerated plaque accumulation, associative learning deficits, and up-regulation of alpha 7 nicotinic receptor protein in transgenic mice co-expressing mutant human presenilin 1 and amyloid precursor proteins. J Biol Chem, 277(25), 22768-22780. doi: 10.1074/jbc.M200164200

Ding, Q., Markesbery, W. R., Chen, Q., Li, F., \& Keller, J. N. (2005). Ribosome dysfunction is an early event in Alzheimer's disease. J Neurosci, 25(40), 9171-9175. doi: 10.1523/JNEUROSCI.3040-05.2005

Disset, A., Bourgeois, C. F., Benmalek, N., Claustres, M., Stevenin, J., \& Tuffery-Giraud, S. (2006). An exon skippingassociated nonsense mutation in the dystrophin gene uncovers a complex interplay between multiple antagonistic splicing elements. Hum Mol Genet, 15(6), 999-1013. doi: 10.1093/hmg/ddl015

Djebali, S., Davis, C. A., Merkel, A., Dobin, A., Lassmann, T., Mortazavi, A., . . Gingeras, T. R. (2012). Landscape of transcription in human cells. Nature, 489(7414), 101-108. doi: 10.1038/nature11233

Dodart, J. C., Meziane, H., Mathis, C., Bales, K. R., Paul, S. M., \& Ungerer, A. (1999). Behavioral disturbances in transgenic mice overexpressing the V717F beta-amyloid precursor protein. Behav Neurosci, 113(5), 982-990.

Dolci, G. A. M., Damanti, S., Scortichini, V., Galli, A., Rossi, P. D., Abbate, C., . . . Marcucci, M. (2017). Alzheimer's Disease Diagnosis: Discrepancy between Clinical, Neuroimaging, and Cerebrospinal Fluid Biomarkers Criteria in an Italian Cohort of Geriatric Outpatients: A Retrospective Cross-sectional Study. Front Med (Lausanne), 4, 203. doi: 10.3389/fmed.2017.00203

Dompierre, J. P., Godin, J. D., Charrin, B. C., Cordelieres, F. P., King, S. J., Humbert, S., \& Saudou, F. (2007). Histone deacetylase 6 inhibition compensates for the transport deficit in Huntington's disease by increasing tubulin acetylation. J Neurosci, 27(13), 3571-3583. doi: 10.1523/JNEUROSCI.0037-07.2007

Donovan, M. H., Yazdani, U., Norris, R. D., Games, D., German, D. C., \& Eisch, A. J. (2006). Decreased adult hippocampal neurogenesis in the PDAPP mouse model of Alzheimer's disease. $J$ Comp Neurol, 495(1), 70-83. doi: $10.1002 /$ cne. 20840

Doonan, R., McElwee, J. J., Matthijssens, F., Walker, G. A., Houthoofd, K., Back, P., . . Gems, D. (2008). Against the oxidative damage theory of aging: superoxide dismutases protect against oxidative stress but have little or no effect on life span in Caenorhabditis elegans. Genes Dev, 22(23), 3236-3241. doi: 10.1101/gad.504808

Doyon, J., Bellec, P., Amsel, R., Penhune, V., Monchi, O., Carrier, J., . . Benali, H. (2009). Contributions of the basal ganglia and functionally related brain structures to motor learning. Behav Brain Res, 199(1), 61-75. doi: 10.1016/ j.bbr.2008.11.012

Duce, J. A., Tsatsanis, A., Cater, M. A., James, S. A., Robb, E., Wikhe, K., . . Bush, A. I. (2010). Iron-export ferroxidase activity of beta-amyloid precursor protein is inhibited by zinc in Alzheimer's disease. Cell, 142(6), 857-867. doi: 10.1016/j.cell.2010.08.014

Dudai, Y. (2004). The neurobiology of consolidations, or, how stable is the engram? Annu Rev Psychol, 55, 51-86. doi: 10.1146/annurev.psych.55.090902.142050

Dudek, S. M., \& Bear, M. F. (1992). Homosynaptic long-term depression in area CA1 of hippocampus and effects of Nmethyl-D-aspartate receptor blockade. Proc Natl Acad Sci U S A, 89(10), 4363-4367.

Duff, K., Eckman, C., Zehr, C., Yu, X., Prada, C. M., Perez-tur, J., . . Younkin, S. (1996). Increased amyloid-beta42(43) in brains of mice expressing mutant presenilin 1. Nature, 383(6602), 710-713. doi: 10.1038/383710a0

Dunn, G. A., \& Bale, T. L. (2009). Maternal high-fat diet promotes body length increases and insulin insensitivity in secondgeneration mice. Endocrinology, 150(11), 4999-5009. doi: 10.1210/en.2009-0500

Dupret, D., Fabre, A., Dobrossy, M. D., Panatier, A., Rodriguez, J. J., Lamarque, S., . . Abrous, D. N. (2007). Spatial learning depends on both the addition and removal of new hippocampal neurons. PLoS Biol, 5(8), e214. doi: 10.1371/journal.pbio.0050214

Eichenbaum, H. (2001). The hippocampus and declarative memory: cognitive mechanisms and neural codes. Behav Brain Res, 127(1-2), 199-207.

Eichenbaum, H., \& Cohen, N.J. (2004). From Conditioning to Conscious Recollection: Memory Systems of the Brain: Oxford University Press.

Ekstrom, A. D., Kahana, M. J., Caplan, J. B., Fields, T. A., Isham, E. A., Newman, E. L., \& Fried, I. (2003). Cellular networks underlying human spatial navigation. Nature, 425(6954), 184-188. doi: 10.1038/nature01964

Ender, C., Krek, A., Friedlander, M. R., Beitzinger, M., Weinmann, L., Chen, W., . . Meister, G. (2008). A human snoRNA with microRNA-like functions. Mol Cell, 32(4), 519-528. doi: 10.1016/j.molcel.2008.10.017

Erten-Lyons, D., Woltjer, R. L., Dodge, H., Nixon, R., Vorobik, R., Calvert, J. F., . . . Kaye, J. (2009). Factors associated with resistance to dementia despite high Alzheimer disease pathology. Neurology, 72(4), 354-360. doi: 10.1212/01.wnl.0000341273.18141.64

Esteller, M. (2011). Non-coding RNAs in human disease. Nat Rev Genet, 12(12), 861-874. doi: 10.1038/nrg3074 
Faggioli, F., Wang, T., Vijg, J., \& Montagna, C. (2012). Chromosome-specific accumulation of aneuploidy in the aging mouse brain. Hum Mol Genet, 21(24), 5246-5253. doi: 10.1093/hmg/dds375

Falaleeva, M., \& Stamm, S. (2013). Processing of snoRNAs as a new source of regulatory non-coding RNAs: snoRNA fragments form a new class of functional RNAs. Bioessays, 35(1), 46-54. doi: 10.1002/bies.201200117

Fang, D., Zhang, Z., Li, H., Yu, Q., Douglas, J. T., Bratasz, A., . . Yan, S. S. (2016). Increased Electron Paramagnetic Resonance Signal Correlates with Mitochondrial Dysfunction and Oxidative Stress in an Alzheimer's disease Mouse Brain. J Alzheimers Dis, 51(2), 571-580. doi: 10.3233/JAD-150917

Farris, W., Mansourian, S., Chang, Y., Lindsley, L., Eckman, E. A., Frosch, M. P., . . Guenette, S. (2003). Insulindegrading enzyme regulates the levels of insulin, amyloid beta-protein, and the beta-amyloid precursor protein intracellular domain in vivo. Proc Natl Acad Sci U S A, 100(7), 4162-4167. doi: 10.1073/pnas.0230450100

Fawcett, L., Baxendale, R., Stacey, P., McGrouther, C., Harrow, I., Soderling, S., . . Phillips, S. C. (2000). Molecular cloning and characterization of a distinct human phosphodiesterase gene family: PDE11A. Proc Natl Acad Sci U $S$ A, 97(7), 3702-3707. doi: 10.1073/pnas.050585197

Feng, S., Jacobsen, S. E., \& Reik, W. (2010). Epigenetic reprogramming in plant and animal development. Science, 330(6004), 622-627. doi: 10.1126/science.1190614

Feng, X., \& Guang, S. (2013). Small RNAs, RNAi and the inheritance of gene silencing in Caenorhabditis elegans. $J$ Genet Genomics, 40(4), 153-160. doi: 10.1016/j.jgg.2012.12.007

Ferguson, S. M., \& De Camilli, P. (2012). Dynamin, a membrane-remodelling GTPase. Nat Rev Mol Cell Biol, 13(2), $75-88$. doi: $10.1038 / \mathrm{nrm} 3266$

Fernandez, E., Collins, M. O., Uren, R. T., Kopanitsa, M. V., Komiyama, N. H., Croning, M. D., . . Grant, S. G. (2009). Targeted tandem affinity purification of PSD-95 recovers core postsynaptic complexes and schizophrenia susceptibility proteins. Mol Syst Biol, 5, 269. doi: 10.1038/msb.2009.27

Filippi, M., Basaia, S., Canu, E., Imperiale, F., Magnani, G., Falautano, M., . . . Agosta, F. (2018). Changes in functional and structural brain connectome along the Alzheimer's disease continuum. Mol Psychiatry. doi: 10.1038/ s41380-018-0067-8

Finsterer, J. (2012). Cognitive dysfunction in mitochondrial disorders. Acta Neurol Scand, 126(1), 1-11. doi: 10.1111/j. 1600-0404.2012.01649.x

Fischer, A., Sananbenesi, F., Mungenast, A., \& Tsai, L. H. (2010). Targeting the correct HDAC(s) to treat cognitive disorders. Trends Pharmacol Sci, 31(12), 605-617. doi: 10.1016/j.tips.2010.09.003

Fischer, A., Sananbenesi, F., Wang, X., Dobbin, M., \& Tsai, L. H. (2007). Recovery of learning and memory is associated with chromatin remodelling. Nature, 447(7141), 178-182. doi: 10.1038/nature05772

Fivush, R. (2011). The development of autobiographical memory. Annu Rev Psychol, 62, 559-582. doi: 10.1146/ annurev.psych.121208.131702

Fox, N. C., Warrington, E. K., Freeborough, P. A., Hartikainen, P., Kennedy, A. M., Stevens, J. M., \& Rossor, M. N. (1996). Presymptomatic hippocampal atrophy in Alzheimer's disease. A longitudinal MRI study. Brain, 119 ( Pt 6), 2001-2007.

Fraga, M. F., \& Esteller, M. (2007). Epigenetics and aging: the targets and the marks. Trends Genet, 23(8), 413-418. doi: 10.1016/j.tig.2007.05.008

Franceschi, C. (1989). Cell proliferation, cell death and aging. Aging (Milano), 1(1), 3-15.

Franceschi, C., \& Campisi, J. (2014). Chronic inflammation (inflammaging) and its potential contribution to age-associated diseases. J Gerontol A Biol Sci Med Sci, 69 Suppl 1, S4-9. doi: 10.1093/gerona/glu057

Frank, L. M., Brown, E. N., \& Wilson, M. (2000). Trajectory encoding in the hippocampus and entorhinal cortex. Neuron, 27(1), 169-178.

Franklin, T. B., Russig, H., Weiss, I. C., Graff, J., Linder, N., Michalon, A., . . Mansuy, I. M. (2010). Epigenetic transmission of the impact of early stress across generations. Biol Psychiatry, 68(5), 408-415. doi: 10.1016/j.biopsych. 2010.05.036

Fraser, H. B., Khaitovich, P., Plotkin, J. B., Paabo, S., \& Eisen, M. B. (2005). Aging and gene expression in the primate brain. PLoS Biol, 3(9), e274. doi: 10.1371/journal.pbio.0030274

Frenk, S., \& Houseley, J. (2018). Gene expression hallmarks of cellular ageing. Biogerontology. doi: 10.1007/ s10522-018-9750-Z

Frey, U., Huang, Y. Y., \& Kandel, E. R. (1993). Effects of cAMP simulate a late stage of LTP in hippocampal CA1 neurons. Science, 260(5114), 1661-1664.

Frick, K. M., Burlingame, L. A., Arters, J. A., \& Berger-Sweeney, J. (2000). Reference memory, anxiety and estrous cyclicity in C57BL/6NIA mice are affected by age and sex. Neuroscience, 95(1), 293-307.

Frisoni, G. B., Fox, N. C., Jack, C. R., Jr., Scheltens, P., \& Thompson, P. M. (2010). The clinical use of structural MRI in Alzheimer disease. Nat Rev Neurol, 6(2), 67-77. doi: 10.1038/nrneurol.2009.215

Fu, Q., \& Wang, P. J. (2014). Mammalian piRNAs: Biogenesis, function, and mysteries. Spermatogenesis, 4, e27889. doi: 10.4161/spmg.27889

Fyhn, M., Molden, S., Witter, M. P., Moser, E. I., \& Moser, M. B. (2004). Spatial representation in the entorhinal cortex. Science, 305(5688), 1258-1264. doi: 10.1126/science.1099901

Gagnon, R. C., \& Peterson, J. J. (1998). Estimation of confidence intervals for area under the curve from destructively obtained pharmacokinetic data. J Pharmacokinet Biopharm, 26(1), 87-102.

Gainetdinov, I., Skvortsova, Y., Kondratieva, S., Funikov, S., \& Azhikina, T. (2017). Two modes of targeting transposable elements by piRNA pathway in human testis. RNA, 23(11), 1614-1625. doi: 10.1261/rna.060939.117

Gallagher, M., Colantuoni, C., Eichenbaum, H., Haberman, R. P., Rapp, P. R., Tanila, H., \& Wilson, I. A. (2006). Individual differences in neurocognitive aging of the medial temporal lobe. Age (Dordr), 28(3), 221-233. doi: 10.1007/ s11357-006-9017-5

Gami, M. S., \& Wolkow, C. A. (2006). Studies of Caenorhabditis elegans DAF-2/insulin signaling reveal targets for pharmacological manipulation of lifespan. Aging Cell, 5(1), 31-37. doi: 10.1111/j.1474-9726.2006.00188.x

Gangwar, R. S., Rajagopalan, S., Natarajan, R., \& Deiuliis, J. A. (2018). Noncoding RNAs in Cardiovascular Disease: Pathological Relevance and Emerging Role as Biomarkers and Therapeutics. Am J Hypertens, 31(2), 150-165. doi: 10.1093/ajh/hpx197 
Ganot, P., Bortolin, M. L., \& Kiss, T. (1997). Site-specific pseudouridine formation in preribosomal RNA is guided by small nucleolar RNAs. Cell, 89(5), 799-809.

Gapp, K., Jawaid, A., Sarkies, P., Bohacek, J., Pelczar, P., Prados, J., . . Mansuy, I. M. (2014). Implication of sperm RNAs in transgenerational inheritance of the effects of early trauma in mice. Nat Neurosci, 17(5), 667-669. doi: $10.1038 / \mathrm{nn} .3695$

Garcia-Lopez, J., Alonso, L., Cardenas, D. B., Artaza-Alvarez, H., Hourcade Jde, D., Martinez, S., . . Del Mazo, J. (2015). Diversity and functional convergence of small noncoding RNAs in male germ cell differentiation and fertilization. RNA, 21(5), 946-962. doi: 10.1261/rna.048215.114

Gatta, V., D'Aurora, M., Granzotto, A., Stuppia, L., \& Sensi, S. L. (2014). Early and sustained altered expression of agingrelated genes in young 3xTg-AD mice. Cell Death Dis, 5, e1054. doi: 10.1038/cddis.2014.11

Gazzaniga, M.S., Ivry, R.B., \& Mangun, G.R. (2002). Cognitive Neuroscience: The Biology of the Mind: Norton.

Gengler, S., Hamilton, A., \& Holscher, C. (2010). Synaptic plasticity in the hippocampus of a APP/PS1 mouse model of Alzheimer's disease is impaired in old but not young mice. PLoS One, 5(3), e9764. doi: 10.1371/journal.pone. 0009764

Gerra, G., Zaimovic, A., Garofano, L., Ciusa, F., Moi, G., Avanzini, P., . . . Donnini, C. (2007). Perceived parenting behavior in the childhood of cocaine users: relationship with genotype and personality traits. Am J Med Genet $B$ Neuropsychiatr Genet, 144B(1), 52-57. doi: 10.1002/ajmg.b.30388

Ghildiyal, M., \& Zamore, P. D. (2009). Small silencing RNAs: an expanding universe. Nat Rev Genet, 10(2), 94-108. doi: $10.1038 / \mathrm{nrg} 2504$

Glass, D., Vinuela, A., Davies, M. N., Ramasamy, A., Parts, L., Knowles, D., . . Spector, T. D. (2013). Gene expression changes with age in skin, adipose tissue, blood and brain. Genome Biol, 14(7), R75. doi: 10.1186/gb-2013-14-7r75

Goate, A. (2006). Segregation of a missense mutation in the amyloid beta-protein precursor gene with familial Alzheimer's disease. J Alzheimers Dis, 9(3 Suppl), 341-347.

Goate, A., Chartier-Harlin, M. C., Mullan, M., Brown, J., Crawford, F., Fidani, L., . . et al. (1991). Segregation of a missense mutation in the amyloid precursor protein gene with familial Alzheimer's disease. Nature, 349(6311), 704-706. doi: 10.1038/349704a0

Goedert, M., \& Jakes, R. (1990). Expression of separate isoforms of human tau protein: correlation with the tau pattern in brain and effects on tubulin polymerization. EMBO J, 9(13), 4225-4230.

Goedert, M., Wischik, C. M., Crowther, R. A., Walker, J. E., \& Klug, A. (1988). Cloning and sequencing of the cDNA encoding a core protein of the paired helical filament of Alzheimer disease: identification as the microtubuleassociated protein tau. Proc Natl Acad Sci U S A, 85(11), 4051-4055.

Goldgaber, D., Lerman, M. I., McBride, O. W., Saffiotti, U., \& Gajdusek, D. C. (1987). Characterization and chromosomal localization of a cDNA encoding brain amyloid of Alzheimer's disease. Science, 235(4791), 877-880.

Goldknopf, I. L., Taylor, C. W., Baum, R. M., Yeoman, L. C., Olson, M. O., Prestayko, A. W., \& Busch, H. (1975). Isolation and characterization of protein A24, a "histone-like" non-histone chromosomal protein. J Biol Chem, 250(18), 7182-7187.

Gong, B., Cao, Z., Zheng, P., Vitolo, O. V., Liu, S., Staniszewski, A., . . Arancio, O. (2006). Ubiquitin hydrolase Uch-L1 rescues beta-amyloid-induced decreases in synaptic function and contextual memory. Cell, 126(4), 775-788. doi: 10.1016/j.cell.2006.06.046

Gonzalez, A., Lovic, V., Ward, G. R., Wainwright, P. E., \& Fleming, A. S. (2001). Intergenerational effects of complete maternal deprivation and replacement stimulation on maternal behavior and emotionality in female rats. Dev Psychobiol, 38(1), 11-32.

Gonzalez-Jamett, A. M., Haro-Acuna, V., Momboisse, F., Caviedes, P., Bevilacqua, J. A., \& Cardenas, A. M. (2014). Dynamin-2 in nervous system disorders. J Neurochem, 128(2), 210-223. doi: 10.1111/jnc.12455

Gonzalez-Recio, O. (2011). Epigenetics: a new challenge in the post-genomic era of livestock. Front Genet, 2, 106. doi: 10.3389/fgene.2011.00106

Gonzalez-Recio, O., Gianola, D., Long, N., Weigel, K. A., Rosa, G. J., \& Avendano, S. (2008). Nonparametric methods for incorporating genomic information into genetic evaluations: an application to mortality in broilers. Genetics, 178(4), 2305-2313. doi: 10.1534/genetics.107.084293

Goode, B. L., Chau, M., Denis, P. E., \& Feinstein, S. C. (2000). Structural and functional differences between 3-repeat and 4-repeat tau isoforms. Implications for normal tau function and the onset of neurodegenetative disease. J Biol Chem, 275(49), 38182-38189. doi: 10.1074/jbc.M007489200

Goodrich, R., Johnson, G., \& Krawetz, S. A. (2007). The preparation of human spermatozoal RNA for clinical analysis. Arch Androl, 53(3), 161-167. doi: 10.1080/01485010701216526

Gou, L. T., Dai, P., Yang, J. H., Xue, Y., Hu, Y. P., Zhou, Y., . . Liu, M. F. (2015). Pachytene piRNAs instruct massive mRNA elimination during late spermiogenesis. Cell Res, 25(2), 266. doi: 10.1038/cr.2015.14

Govindarajan, N., Agis-Balboa, R. C., Walter, J., Sananbenesi, F., \& Fischer, A. (2011). Sodium butyrate improves memory function in an Alzheimer's disease mouse model when administered at an advanced stage of disease progression. J Alzheimers Dis, 26(1), 187-197. doi: 10.3233/JAD-2011-110080

Govindarajan, N., Rao, P., Burkhardt, S., Sananbenesi, F., Schluter, O. M., Bradke, F., . . Fischer, A. (2013). Reducing HDAC6 ameliorates cognitive deficits in a mouse model for Alzheimer's disease. EMBO Mol Med, 5(1), 52-63. doi: 10.1002/emmm.201201923

Graf, P., \& Schacter, D. L. (1985). Implicit and explicit memory for new associations in normal and amnesic subjects. $J$ Exp Psychol Learn Mem Cogn, 11(3), 501-518.

Green, D. R., Galluzzi, L., \& Kroemer, G. (2011). Mitochondria and the autophagy-inflammation-cell death axis in organismal aging. Science, 333(6046), 1109-1112. doi: 10.1126/science.1201940

Grentzinger, T., Armenise, C., Brun, C., Mugat, B., Serrano, V., Pelisson, A., \& Chambeyron, S. (2012). piRNA-mediated transgenerational inheritance of an acquired trait. Genome Res, 22(10), 1877-1888. doi: 10.1101/gr.136614.111

Griesemer, J. (2002). What is "epi" about epigenetics? Ann N Y Acad Sci, 981, 97-110.

Grivna, S. T., Beyret, E., Wang, Z., \& Lin, H. (2006). A novel class of small RNAs in mouse spermatogenic cells. Genes Dev, 20(13), 1709-1714. doi: 10.1101/gad.1434406 
Grundke-lqbal, I., Iqbal, K., Tung, Y. C., Quinlan, M., Wisniewski, H. M., \& Binder, L. I. (1986). Abnormal phosphorylation of the microtubule-associated protein tau (tau) in Alzheimer cytoskeletal pathology. Proc Natl Acad Sci U S A, 83(13), 4913-4917.

Guan, Z., Giustetto, M., Lomvardas, S., Kim, J. H., Miniaci, M. C., Schwartz, J. H., . . Kandel, E. R. (2002). Integration of long-term-memory-related synaptic plasticity involves bidirectional regulation of gene expression and chromatin structure. Cell, 111(4), 483-493.

Guerreiro, R., \& Bras, J. (2015). The age factor in Alzheimer's disease. Genome Med, 7, 106. doi: 10.1186/ s13073-015-0232-5

Guerreiro, R., Wojtas, A., Bras, J., Carrasquillo, M., Rogaeva, E., Majounie, E., . . Alzheimer Genetic Analysis, Group. (2013). TREM2 variants in Alzheimer's disease. N Engl J Med, 368(2), 117-127. doi: 10.1056/NEJMoa1211851

Guerrero-Bosagna, C., Covert, T. R., Haque, M. M., Settles, M., Nilsson, E. E., Anway, M. D., \& Skinner, M. K. (2012). Epigenetic transgenerational inheritance of vinclozolin induced mouse adult onset disease and associated sperm epigenome biomarkers. Reprod Toxicol, 34(4), 694-707. doi: 10.1016/j.reprotox.2012.09.005

Gupta, A. S., van der Meer, M. A., Touretzky, D. S., \& Redish, A. D. (2010). Hippocampal replay is not a simple function of experience. Neuron, 65(5), 695-705. doi: 10.1016/j.neuron.2010.01.034

Haass, C., Hung, A. Y., Schlossmacher, M. G., Teplow, D. B., \& Selkoe, D. J. (1993). beta-Amyloid peptide and a 3-kDa fragment are derived by distinct cellular mechanisms. J Biol Chem, 268(5), 3021-3024.

Haass, C., \& Selkoe, D. J. (2007). Soluble protein oligomers in neurodegeneration: lessons from the Alzheimer's amyloid beta-peptide. Nat Rev Mol Cell Biol, 8(2), 101-112. doi: 10.1038/nrm2101

Haberman, R. P., Quigley, C. K., \& Gallagher, M. (2012). Characterization of CpG island DNA methylation of impairmentrelated genes in a rat model of cognitive aging. Epigenetics, 7(9), 1008-1019. doi: 10.4161/epi.21291

Hahn, S. (2004). Structure and mechanism of the RNA polymerase II transcription machinery. Nat Struct Mol Biol, 11(5), 394-403. doi: $10.1038 / \mathrm{nsmb} 763$

Hajkova, P. (2011). Epigenetic reprogramming in the germline: towards the ground state of the epigenome. Philos Trans $R$ Soc Lond B Biol Sci, 366(1575), 2266-2273. doi: 10.1098/rstb.2011.0042

Hamatani, T. (2012). Human spermatozoal RNAs. Fertil Steril, 97(2), 275-281. doi: 10.1016/j.fertnstert.2011.12.035

Hamilton, L. K., Dufresne, M., Joppe, S. E., Petryszyn, S., Aumont, A., Calon, F., . . Fernandes, K. J. (2015). Aberrant Lipid Metabolism in the Forebrain Niche Suppresses Adult Neural Stem Cell Proliferation in an Animal Model of Alzheimer's Disease. Cell Stem Cell, 17(4), 397-411. doi: 10.1016/j.stem.2015.08.001

Hammoud, S. S., Nix, D. A., Zhang, H., Purwar, J., Carrell, D. T., \& Cairns, B. R. (2009). Distinctive chromatin in human sperm packages genes for embryo development. Nature, 460(7254), 473-478. doi: 10.1038/nature08162

Hamos, J. E., DeGennaro, L. J., \& Drachman, D. A. (1989). Synaptic loss in Alzheimer's disease and other dementias. Neurology, 39(3), 355-361.

Hampel, H., Frank, R., Broich, K., Teipel, S. J., Katz, R. G., Hardy, J., . . Blennow, K. (2010). Biomarkers for Alzheimer's disease: academic, industry and regulatory perspectives. Nat Rev Drug Discov, 9(7), 560-574. doi: 10.1038/ $\operatorname{nrd} 3115$

Hampel, H., Vergallo, A., Aguilar, L. F., Benda, N., Broich, K., Cuello, A. C., . . Alzheimer Precision Medicine, Initiative. (2018). Precision pharmacology for Alzheimer's disease. Pharmacol Res. doi: 10.1016/j.phrs.2018.02.014

Hampsey, M. (1998). Molecular genetics of the RNA polymerase II general transcriptional machinery. Microbiol Mol Biol Rev, 62(2), 465-503.

Han, S., \& Brunet, A. (2012). Histone methylation makes its mark on longevity. Trends Cell Biol, 22(1), 42-49. doi: 10.1016/ j.tcb.2011.11.001

Hara, N., Kikuchi, M., Miyashita, A., Hatsuta, H., Saito, Y., Kasuga, K., . . Kuwano, R. (2017). Serum microRNA miR-501-3p as a potential biomarker related to the progression of Alzheimer's disease. Acta Neuropathol Commun, 5(1), 10. doi: 10.1186/s40478-017-0414-z

Hara, Y., Yuk, F., Puri, R., Janssen, W. G., Rapp, P. R., \& Morrison, J. H. (2014). Presynaptic mitochondrial morphology in monkey prefrontal cortex correlates with working memory and is improved with estrogen treatment. Proc Natl Acad Sci U S A, 111(1), 486-491. doi: 10.1073/pnas.1311310110

Hardy, J., \& Selkoe, D. J. (2002). The amyloid hypothesis of Alzheimer's disease: progress and problems on the road to therapeutics. Science, 297(5580), 353-356. doi: 10.1126/science.1072994

Harman, D. (1965). The Free Radical Theory of Aging: Effect of Age on Serum Copper Levels. J Gerontol, 20, 151-153.

Harman, D. (2003). The free radical theory of aging. Antioxid Redox Signal, 5(5), 557-561. doi: $10.1089 / 152308603770310202$

Harold, D., Abraham, R., Hollingworth, P., Sims, R., Gerrish, A., Hamshere, M. L., . . Williams, J. (2009). Genome-wide association study identifies variants at CLU and PICALM associated with Alzheimer's disease. Nat Genet, 41(10), 1088-1093. doi: 10.1038/ng.440

Harries, L. W., Hernandez, D., Henley, W., Wood, A. R., Holly, A. C., Bradley-Smith, R. M., . . Melzer, D. (2011). Human aging is characterized by focused changes in gene expression and deregulation of alternative splicing. Aging Cell, 10(5), 868-878. doi: 10.1111/j.1474-9726.2011.00726.x

Harrison, D. E., Strong, R., Sharp, Z. D., Nelson, J. F., Astle, C. M., Flurkey, K., . . Miller, R. A. (2009). Rapamycin fed late in life extends lifespan in genetically heterogeneous mice. Nature, 460(7253), 392-395. doi: 10.1038/ nature 08221

Haughey, N. J., Nath, A., Chan, S. L., Borchard, A. C., Rao, M. S., \& Mattson, M. P. (2002). Disruption of neurogenesis by amyloid beta-peptide, and perturbed neural progenitor cell homeostasis, in models of Alzheimer's disease. $J$ Neurochem, 83(6), 1509-1524.

He, M., Liu, Y., Wang, X., Zhang, M. Q., Hannon, G. J., \& Huang, Z. J. (2012). Cell-type-based analysis of microRNA profiles in the mouse brain. Neuron, 73(1), 35-48. doi: 10.1016/j.neuron.2011.11.010

Head, E., Mehta, R., Hartley, J., Kameka, M., Cummings, B. J., Cotman, C. W., . . Milgram, N. W. (1995). Spatial learning and memory as a function of age in the dog. Behav Neurosci, 109(5), 851-858.

Hebb, D.O. (1949). The Organization of Behavior: A Neuropsychological Theory: Wiley. 
Hebert, S. S., Papadopoulou, A. S., Smith, P., Galas, M. C., Planel, E., Silahtaroglu, A. N., . . De Strooper, B. (2010). Genetic ablation of Dicer in adult forebrain neurons results in abnormal tau hyperphosphorylation and neurodegeneration. Hum Mol Genet, 19(20), 3959-3969. doi: 10.1093/hmg/ddq311

Hedden, T., \& Gabrieli, J. D. (2004). Insights into the ageing mind: a view from cognitive neuroscience. Nat Rev Neurosci, 5(2), 87-96. doi: 10.1038/nrn1323

Heintz, C., Doktor, T. K., Lanjuin, A., Escoubas, C., Zhang, Y., Weir, H. J., . . . Mair, W. B. (2017). Splicing factor 1 modulates dietary restriction and TORC1 pathway longevity in C. elegans. Nature, 541(7635), 102-106. doi: 10.1038/nature20789

Hekimi, S., Lapointe, J., \& Wen, Y. (2011). Taking a "good" look at free radicals in the aging process. Trends Cell Biol, 21(10), 569-576. doi: 10.1016/j.tcb.2011.06.008

Hendriks, L., van Duijn, C. M., Cras, P., Cruts, M., Van Hul, W., van Harskamp, F., . . et al. (1992). Presenile dementia and cerebral haemorrhage linked to a mutation at codon 692 of the beta-amyloid precursor protein gene. Nat Genet, 1(3), 218-221. doi: 10.1038/ng0692-218

Hendriks, W. J., Elson, A., Harroch, S., Pulido, R., Stoker, A., \& den Hertog, J. (2013). Protein tyrosine phosphatases in health and disease. FEBS J, 280(2), 708-730. doi: 10.1111/febs.12000

Herman, J. J., \& Sultan, S. E. (2016). DNA methylation mediates genetic variation for adaptive transgenerational plasticity. Proc Biol Sci, 283(1838). doi: 10.1098/rspb.2016.0988

Hernandez-Ortega, K., Garcia-Esparcia, P., Gil, L., Lucas, J. J., \& Ferrer, I. (2016). Altered Machinery of Protein Synthesis in Alzheimer's: From the Nucleolus to the Ribosome. Brain Pathol, 26(5), 593-605. doi: 10.1111/bpa.12335

Herrmann, L., Wiegmann, C., Arsalan-Werner, A., Hilbrich, I., Jager, C., Flach, K., . . Holzer, M. (2015). Hook proteins: association with Alzheimer pathology and regulatory role of hook3 in amyloid beta generation. PLoS One, 10(3), e0119423. doi: 10.1371/journal.pone.0119423

Hikosaka, O., Nakamura, K., Sakai, K., \& Nakahara, H. (2002). Central mechanisms of motor skill learning. Curr Opin Neurobiol, 12(2), 217-222

Hill, A. S., Sahay, A., \& Hen, R. (2015). Increasing Adult Hippocampal Neurogenesis is Sufficient to Reduce Anxiety and Depression-Like Behaviors. Neuropsychopharmacology, 40(10), 2368-2378. doi: 10.1038/npp.2015.85

Hinrich, A. J., Jodelka, F. M., Chang, J. L., Brutman, D., Bruno, A. M., Briggs, C. A., . . Hastings, M. L. (2016). Therapeutic correction of ApoER2 splicing in Alzheimer's disease mice using antisense oligonucleotides. EMBO Mol Med, 8(4), 328-345. doi: 10.15252/emmm.201505846

Hippius, H., \& Neundorfer, G. (2003). The discovery of Alzheimer's disease. Dialogues Clin Neurosci, 5(1), 101-108.

Ho, S. M., Tang, W. Y., Belmonte de Frausto, J., \& Prins, G. S. (2006). Developmental exposure to estradiol and bisphenol A increases susceptibility to prostate carcinogenesis and epigenetically regulates phosphodiesterase type 4 variant 4. Cancer Res, 66(11), 5624-5632. doi: 10.1158/0008-5472.CAN-06-0516

Hoeijmakers, J. H. (2009). DNA damage, aging, and cancer. N Engl J Med, 361(15), 1475-1485. doi: 10.1056/ NEJMra0804615

Hoglund, P. J., Adzic, D., Scicluna, S. J., Lindblom, J., \& Fredriksson, R. (2005). The repertoire of solute carriers of family 6: identification of new human and rodent genes. Biochem Biophys Res Commun, 336(1), 175-189. doi: 10.1016/j.bbrc.2005.08.048

Holcomb, L., Gordon, M. N., McGowan, E., Yu, X., Benkovic, S., Jantzen, P., . . Duff, K. (1998). Accelerated Alzheimertype phenotype in transgenic mice carrying both mutant amyloid precursor protein and presenilin 1 transgenes. Nat Med, 4(1), 97-100.

Holliday, R. (1987). DNA methylation and epigenetic defects in carcinogenesis. Mutat Res, 181(2), 215-217.

Hollingworth, P., Harold, D., Sims, R., Gerrish, A., Lambert, J. C., Carrasquillo, M. M., . . Williams, J. (2011). Common variants at ABCA7, MS4A6A/MS4A4E, EPHA1, CD33 and CD2AP are associated with Alzheimer's disease. Nat Genet, 43(5), 429-435. doi: 10.1038/ng.803

Holm-Hansen, S., Low, J. K., Zieba, J., Gjedde, A., Bergersen, L. H., \& Karl, T. (2016). Behavioural effects of high fat diet in a mutant mouse model for the schizophrenia risk gene neuregulin 1. Genes Brain Behav, 15(3), 295-304. doi: $10.1111 / \mathrm{gbb} .12267$

Honda, K., Smith, M. A., Zhu, X., Baus, D., Merrick, W. C., Tartakoff, A. M., . . Perry, G. (2005). Ribosomal RNA in Alzheimer disease is oxidized by bound redox-active iron. J Biol Chem, 280(22), 20978-20986. doi: 10.1074/ jbc.M500526200

Hort, J., O'Brien, J. T., Gainotti, G., Pirttila, T., Popescu, B. O., Rektorova, I., . . Dementia, Efns Scientist Panel on. (2010). EFNS guidelines for the diagnosis and management of Alzheimer's disease. Eur J Neurol, 17(10), 1236-1248. doi: 10.1111/j.1468-1331.2010.03040.x

Hsiao, K., Chapman, P., Nilsen, S., Eckman, C., Harigaya, Y., Younkin, S., . . Cole, G. (1996). Correlative memory deficits, Abeta elevation, and amyloid plaques in transgenic mice. Science, 274(5284), 99-102.

Huttenhofer, A., Kiefmann, M., Meier-Ewert, S., O'Brien, J., Lehrach, H., Bachellerie, J. P., \& Brosius, J. (2001). RNomics: an experimental approach that identifies 201 candidates for novel, small, non-messenger RNAs in mouse. EMBO J, 20(11), 2943-2953. doi: 10.1093/emboj/20.11.2943

Hyun, D. H., Emerson, S. S., Jo, D. G., Mattson, M. P., \& de Cabo, R. (2006). Calorie restriction up-regulates the plasma membrane redox system in brain cells and suppresses oxidative stress during aging. Proc Natl Acad Sci U S A, 103(52), 19908-19912. doi: 10.1073/pnas.0608008103

ladevaia, V., Liu, R., \& Proud, C. G. (2014). mTORC1 signaling controls multiple steps in ribosome biogenesis. Semin Cell Dev Biol, 36, 113-120. doi: 10.1016/j.semcdb.2014.08.004

Ichimura, H., Parthasarathi, K., Quadri, S., Issekutz, A. C., \& Bhattacharya, J. (2003). Mechano-oxidative coupling by mitochondria induces proinflammatory responses in lung venular capillaries. J Clin Invest, 111(5), 691-699. doi: $10.1172 / \mathrm{JCl} 17271$

Ihara, Y., Nukina, N., Miura, R., \& Ogawara, M. (1986). Phosphorylated tau protein is integrated into paired helical filaments in Alzheimer's disease. J Biochem, 99(6), 1807-1810.

Iqbal, K., \& Grundke-lqbal, I. (2010). Alzheimer's disease, a multifactorial disorder seeking multitherapies. Alzheimers Dement, 6(5), 420-424. doi: 10.1016/j.jalz.2010.04.006 
Iqbal, Z., Willemsen, M. H., Papon, M. A., Musante, L., Benevento, M., Hu, H., . . van Bokhoven, H. (2015). Homozygous SLC6A17 mutations cause autosomal-recessive intellectual disability with progressive tremor, speech impairment, and behavioral problems. Am J Hum Genet, 96(3), 386-396. doi: 10.1016/j.ajhg.2015.01.010

Iturria-Medina, Y., Sotero, R. C., Toussaint, P. J., Mateos-Perez, J. M., Evans, A. C., \& Alzheimer's Disease Neuroimaging, Initiative. (2016). Early role of vascular dysregulation on late-onset Alzheimer's disease based on multifactorial data-driven analysis. Nat Commun, 7, 11934. doi: 10.1038/ncomms11934

Jablonka, E., \& Raz, G. (2009). Transgenerational epigenetic inheritance: prevalence, mechanisms, and implications for the study of heredity and evolution. Q Rev Biol, 84(2), 131-176.

Jack, C. R., Jr., Shiung, M. M., Gunter, J. L., O'Brien, P. C., Weigand, S. D., Knopman, D. S., . . Petersen, R. C. (2004). Comparison of different MRI brain atrophy rate measures with clinical disease progression in AD. Neurology, 62(4), 591-600.

Jacob, F., \& Monod, J. (1961). Genetic regulatory mechanisms in the synthesis of proteins. J Mol Biol, 3, 318-356.

Jacobsen, J. S., Wu, C. C., Redwine, J. M., Comery, T. A., Arias, R., Bowlby, M., . . Bloom, F. E. (2006). Early-onset behavioral and synaptic deficits in a mouse model of Alzheimer's disease. Proc Natl Acad Sci U S A, 103(13), 5161-5166. doi: 10.1073/pnas.0600948103

Jacoby, L. L., \& Dallas, M. (1981). On the relationship between autobiographical memory and perceptual learning. J Exp Psychol Gen, 110(3), 306-340.

Jaki, Thomas, \& Wolfsegger, Martin J. (2009). A Theoretical Framework for Estimation of AUCs in Complete and Incomplete Sampling Designs. Statistics in Biopharmaceutical Research, 1(2), 176-184. doi: 10.1198/sbr. 2009.0025

James, William. (1950). The principles of psychology. [New York]: Dover Publications.

Jansen-Durr, P., \& Osiewacz, H. D. (2002). Healthy ageing: a question of stress, damage and repair. Meeting on mechanisms of biological ageing. EMBO Rep, 3(12), 1127-1132. doi: 10.1093/embo-reports/kvf247

Janssens, G. E., Meinema, A. C., Gonzalez, J., Wolters, J. C., Schmidt, A., Guryev, V., . . Heinemann, M. (2015). Protein biogenesis machinery is a driver of replicative aging in yeast. Elife, 4, e08527. doi: 10.7554/eLife.08527

Janz, R., \& Sudhof, T. C. (1999). SV2C is a synaptic vesicle protein with an unusually restricted localization: anatomy of a synaptic vesicle protein family. Neuroscience, 94(4), 1279-1290.

Jarrett, J. T., Berger, E. P., \& Lansbury, P. T., Jr. (1993). The carboxy terminus of the beta amyloid protein is critical for the seeding of amyloid formation: implications for the pathogenesis of Alzheimer's disease. Biochemistry, 32(18), 4693-4697.

Jenkins, T. G., James, E. R., Alonso, D. F., Hoidal, J. R., Murphy, P. J., Hotaling, J. M., . . Aston, K. I. (2017). Cigarette smoking significantly alters sperm DNA methylation patterns. Andrology, 5(6), 1089-1099. doi: 10.1111/andr. 12416

Jensen, M. T., Mottin, M. D., Cracchiolo, J. R., Leighty, R. E., \& Arendash, G. W. (2005). Lifelong immunization with human beta-amyloid (1-42) protects Alzheimer's transgenic mice against cognitive impairment throughout aging. Neuroscience, 130(3), 667-684. doi: 10.1016/j.neuroscience.2004.09.055

Jeppesen, D. K., Bohr, V. A., \& Stevnsner, T. (2011). DNA repair deficiency in neurodegeneration. Prog Neurobiol, 94(2), 166-200. doi: 10.1016/j.pneurobio.2011.04.013

Jiang, C. H., Tsien, J. Z., Schultz, P. G., \& Hu, Y. (2001). The effects of aging on gene expression in the hypothalamus and cortex of mice. Proc Natl Acad Sci U S A, 98(4), 1930-1934. doi: 10.1073/pnas.98.4.1930

Jin, B., Li, Y., \& Robertson, K. D. (2011). DNA methylation: superior or subordinate in the epigenetic hierarchy? Genes Cancer, 2(6), 607-617. doi: 10.1177/1947601910393957

Jin, J., \& Maren, S. (2015). Prefrontal-Hippocampal Interactions in Memory and Emotion. Front Syst Neurosci, 9, 170. doi: $10.3389 /$ fnsys.2015.00170

Jin, K., Galvan, V., Xie, L., Mao, X. O., Gorostiza, O. F., Bredesen, D. E., \& Greenberg, D. A. (2004). Enhanced neurogenesis in Alzheimer's disease transgenic (PDGF-APPSw,Ind) mice. Proc Natl Acad Sci U S A, 101(36), 13363-13367. doi: 10.1073/pnas.0403678101

Jin, K., Peel, A. L., Mao, X. O., Xie, L., Cottrell, B. A., Henshall, D. C., \& Greenberg, D. A. (2004). Increased hippocampal neurogenesis in Alzheimer's disease. Proc Natl Acad Sci U S A, 101(1), 343-347. doi: 10.1073/pnas. 2634794100

Johnson, G. D., Lalancette, C., Linnemann, A. K., Leduc, F., Boissonneault, G., \& Krawetz, S. A. (2011). The sperm nucleus: chromatin, RNA, and the nuclear matrix. Reproduction, 141(1), 21-36. doi: 10.1530/REP-10-0322

Johnson, S. C., Rabinovitch, P. S., \& Kaeberlein, M. (2013). mTOR is a key modulator of ageing and age-related disease. Nature, 493(7432), 338-345. doi: 10.1038/nature11861

Johnston, A. L., \& File, S. E. (1991). Sex differences in animal tests of anxiety. Physiol Behav, 49(2), 245-250.

Jonides, J., Lewis, R. L., Nee, D. E., Lustig, C. A., Berman, M. G., \& Moore, K. S. (2008). The mind and brain of short-term memory. Annu Rev Psychol, 59, 193-224. doi: 10.1146/annurev.psych.59.103006.093615

Jorjani, H., Kehr, S., Jedlinski, D. J., Gumienny, R., Hertel, J., Stadler, P. F., . . G Gruber, A. R. (2016). An updated human snoRNAome. Nucleic Acids Res, 44(11), 5068-5082. doi: 10.1093/nar/gkw386

Kamei, Y., Tamada, Y., Nakayama, Y., Fukusaki, E., \& Mukai, Y. (2014). Changes in transcription and metabolism during the early stage of replicative cellular senescence in budding yeast. J Biol Chem, 289(46), 32081-32093. doi: 10.1074/jbc.M114.600528

Kamino, K., Orr, H. T., Payami, H., Wijsman, E. M., Alonso, M. E., Pulst, S. M., . . et al. (1992). Linkage and mutational analysis of familial Alzheimer disease kindreds for the APP gene region. Am J Hum Genet, 51(5), 998-1014.

Kandel, E. R. (2001). The molecular biology of memory storage: a dialogue between genes and synapses. Science, 294(5544), 1030-1038. doi: 10.1126/science.1067020

Kandel, E. R., Dudai, Y., \& Mayford, M. R. (2014). The molecular and systems biology of memory. Cell, 157(1), 163-186. doi: 10.1016/j.cell.2014.03.001

Kandel, Eric R. (2013). Principles of neural science.

Kang, J., Lemaire, H. G., Unterbeck, A., Salbaum, J. M., Masters, C. L., Grzeschik, K. H., . . Muller-Hill, B. (1987). The precursor of Alzheimer's disease amyloid A4 protein resembles a cell-surface receptor. Nature, 325(6106), 733-736. doi: 10.1038/325733a0 
Katsel, P., Li, C., \& Haroutunian, V. (2007). Gene expression alterations in the sphingolipid metabolism pathways during progression of dementia and Alzheimer's disease: a shift toward ceramide accumulation at the earliest recognizable stages of Alzheimer's disease? Neurochem Res, 32(4-5), 845-856. doi: 10.1007/s11064-007-9297$\mathrm{x}$

Katzman, R., \& Saitoh, T. (1991). Advances in Alzheimer's disease. FASEB J, 5(3), 278-286.

Kaur, G., \& Levy, E. (2012). Cystatin C in Alzheimer's disease. Front Mol Neurosci, 5, 79. doi: 10.3389/fnmol.2012.00079

Kawasaki, H., \& Taira, K. (2003). Functional analysis of microRNAs during the retinoic acid-induced neuronal differentiation of human NT2 cells. Nucleic Acids Res Supp/(3), 243-244.

Kelly, P. H., Bondolfi, L., Hunziker, D., Schlecht, H. P., Carver, K., Maguire, E., . . Sommer, B. (2003). Progressive agerelated impairment of cognitive behavior in APP23 transgenic mice. Neurobiol Aging, 24(2), 365-378.

Kenmochi, N., Suzuki, T., Uechi, T., Magoori, M., Kuniba, M., Higa, S., . . Tanaka, T. (2001). The human mitochondrial ribosomal protein genes: mapping of 54 genes to the chromosomes and implications for human disorders. Genomics, 77(1-2), 65-70. doi: 10.1006/geno.2001.6622

Kentros, C. G., Agnihotri, N. T., Streater, S., Hawkins, R. D., \& Kandel, E. R. (2004). Increased attention to spatial context increases both place field stability and spatial memory. Neuron, 42(2), 283-295.

Khacho, M., Clark, A., Svoboda, D. S., MacLaurin, J. G., Lagace, D. C., Park, D. S., \& Slack, R. S. (2017). Mitochondrial dysfunction underlies cognitive defects as a result of neural stem cell depletion and impaired neurogenesis. Hum Mol Genet, 26(17), 3327-3341. doi: 10.1093/hmg/ddx217

Khor, V. K., Tong, M. H., Qian, Y., \& Song, W. C. (2008). Gender-specific expression and mechanism of regulation of estrogen sulfotransferase in adipose tissues of the mouse. Endocrinology, 149(11), 5440-5448. doi: 10.1210/en. 2008-0271

Kikusui, T., Isaka, Y., \& Mori, Y. (2005). Early weaning deprives mouse pups of maternal care and decreases their maternal behavior in adulthood. Behav Brain Res, 162(2), 200-206. doi: 10.1016/j.bbr.2005.03.013

Kilgore, M., Miller, C. A., Fass, D. M., Hennig, K. M., Haggarty, S. J., Sweatt, J. D., \& Rumbaugh, G. (2010). Inhibitors of class 1 histone deacetylases reverse contextual memory deficits in a mouse model of Alzheimer's disease. Neuropsychopharmacology, 35(4), 870-880. doi: 10.1038/npp.2009.197

Kim, C. S., Park, S., \& Kim, J. (2017). The role of glycation in the pathogenesis of aging and its prevention through herbal products and physical exercise. J Exerc Nutrition Biochem, 21(3), 55-61. doi: 10.20463/jenb.2017.0027

Kim, Y. K. (2015). Extracellular microRNAs as Biomarkers in Human Disease. Chonnam Med J, 51(2), 51-57. doi: 10.4068/ cmj.2015.51.2.51

King, D. L., Arendash, G. W., Crawford, F., Sterk, T., Menendez, J., \& Mullan, M. J. (1999). Progressive and genderdependent cognitive impairment in the APP(SW) transgenic mouse model for Alzheimer's disease. Behav Brain Res, 103(2), 145-162.

Kirkwood, T. B. (2005). Understanding the odd science of aging. Cell, 120(4), 437-447. doi: 10.1016/j.cell.2005.01.027

Kirkwood, T. B., Boys, R. J., Gillespie, C. S., Proctor, C. J., Shanley, D. P., \& Wilkinson, D. J. (2003). Towards an e-biology of ageing: integrating theory and data. Nat Rev Mol Cell Biol, 4(3), 243-249. doi: 10.1038/nrm1051

Kishore, S., Khanna, A., Zhang, Z., Hui, J., Balwierz, P. J., Stefan, M., . . Stamm, S. (2010). The snoRNA MBII-52 (SNORD 115) is processed into smaller RNAs and regulates alternative splicing. Hum Mol Genet, 19(7), 1153-1164. doi: 10.1093/hmg/ddp585

Klattenhoff, C., \& Theurkauf, W. (2008). Biogenesis and germline functions of piRNAs. Development, 135(1), 3-9. doi: $10.1242 / \mathrm{dev} .006486$

Klattenhoff, C., Xi, H., Li, C., Lee, S., Xu, J., Khurana, J. S., . . Theurkauf, W. E. (2009). The Drosophila HP1 homolog Rhino is required for transposon silencing and piRNA production by dual-strand clusters. Cell, 138(6), 1137-1149. doi: 10.1016/j.cell.2009.07.014

Knafo, S., Venero, C., Merino-Serrais, P., Fernaud-Espinosa, I., Gonzalez-Soriano, J., Ferrer, I., . . DeFelipe, J. (2009). Morphological alterations to neurons of the amygdala and impaired fear conditioning in a transgenic mouse model of Alzheimer's disease. J Pathol, 219(1), 41-51. doi: 10.1002/path.2565

Kobayashi, H., Sakurai, T., Imai, M., Takahashi, N., Fukuda, A., Yayoi, O., . . Kono, T. (2012). Contribution of intragenic DNA methylation in mouse gametic DNA methylomes to establish oocyte-specific heritable marks. PLoS Genet, 8(1), e1002440. doi: 10.1371/journal.pgen.1002440

Koivisto, K., Reinikainen, K. J., Hanninen, T., Vanhanen, M., Helkala, E. L., Mykkanen, L., . . Riekkinen, P. J., Sr. (1995). Prevalence of age-associated memory impairment in a randomly selected population from eastern Finland. Neurology, 45(4), 741-747.

Konopka, W., Kiryk, A., Novak, M., Herwerth, M., Parkitna, J. R., Wawrzyniak, M., . . Schutz, G. (2010). MicroRNA loss enhances learning and memory in mice. $J$ Neurosci, 30(44), 14835-14842. doi: 10.1523/JNEUROSCl. 3030-10.2010

Kosik, K. S., Joachim, C. L., \& Selkoe, D. J. (1986). Microtubule-associated protein tau (tau) is a major antigenic component of paired helical filaments in Alzheimer disease. Proc Natl Acad Sci U S A, 83(11), 4044-4048.

Koudinov, A. R., \& Berezov, T. T. (2005). Cholesterol, statins, and Alzheimer disease. PLoS Med, 2(3), e81; author reply e85. doi: 10.1371/journal.pmed.0020081

Kowald, A., \& Kirkwood, T. B. (1996). A network theory of ageing: the interactions of defective mitochondria, aberrant proteins, free radicals and scavengers in the ageing process. Mutat Res, 316(5-6), 209-236.

Krawetz, S. A. (2005). Paternal contribution: new insights and future challenges. Nat Rev Genet, 6(8), 633-642. doi: $10.1038 /$ nrg1654

Krawetz, S. A., Kruger, A., Lalancette, C., Tagett, R., Anton, E., Draghici, S., \& Diamond, M. P. (2011). A survey of small RNAs in human sperm. Hum Reprod, 26(12), 3401-3412. doi: 10.1093/humrep/der329

Kreiner, G., Chmielarz, P., Roman, A., \& Nalepa, I. (2013). Gender differences in genetic mouse models evaluated for depressive-like and antidepressant behavior. Pharmacol Rep, 65(6), 1580-1590.

Krichevsky, A. M., Sonntag, K. C., Isacson, O., \& Kosik, K. S. (2006). Specific microRNAs modulate embryonic stem cellderived neurogenesis. Stem Cells, 24(4), 857-864. doi: 10.1634/stemcells.2005-0441

Kropff, E., Yang, S. M., \& Schinder, A. F. (2015). Dynamic role of adult-born dentate granule cells in memory processing. Curr Opin Neurobiol, 35, 21-26. doi: 10.1016/j.conb.2015.06.002 
Kumar, A., Gibbs, J. R., Beilina, A., Dillman, A., Kumaran, R., Trabzuni, D., . . Cookson, M. R. (2013). Age-associated changes in gene expression in human brain and isolated neurons. Neurobiol Aging, 34(4), 1199-1209. doi: 10.1016/j.neurobiolaging.2012.10.021

Kummer, M. P., \& Heneka, M. T. (2014). Truncated and modified amyloid-beta species. Alzheimers Res Ther, 6(3), 28. doi: 10.1186/alzrt258

Kuramochi-Miyagawa, S., Kimura, T., Ijiri, T. W., Isobe, T., Asada, N., Fujita, Y., . . Nakano, T. (2004). Mili, a mammalian member of piwi family gene, is essential for spermatogenesis. Development, 131(4), 839-849. doi: 10.1242/dev. 00973

Kuret, J., Congdon, E. E., Li, G., Yin, H., Yu, X., \& Zhong, Q. (2005). Evaluating triggers and enhancers of tau fibrillization. Microsc Res Tech, 67(3-4), 141-155. doi: 10.1002/jemt.20187

Ladd-Acosta, C., \& Fallin, M. D. (2016). The role of epigenetics in genetic and environmental epidemiology. Epigenomics, 8(2), 271-283. doi: 10.2217/epi.15.102

Lagos-Quintana, M., Rauhut, R., Lendeckel, W., \& Tuschl, T. (2001). Identification of novel genes coding for small expressed RNAs. Science, 294(5543), 853-858. doi: 10.1126/science.1064921

Lai, M. K., Esiri, M. M., \& Tan, M. G. (2014). Genome-wide profiling of alternative splicing in Alzheimer's disease. Genom Data, 2, 290-292. doi: 10.1016/j.gdata.2014.09.002

Lai, Z. C., Moss, M. B., Killiany, R. J., Rosene, D. L., \& Herndon, J. G. (1995). Executive system dysfunction in the aged monkey: spatial and object reversal learning. Neurobiol Aging, 16(6), 947-954.

Lalonde, R., Lewis, T. L., Strazielle, C., Kim, H., \& Fukuchi, K. (2003). Transgenic mice expressing the betaAPP695SWE mutation: effects on exploratory activity, anxiety, and motor coordination. Brain Res, 977(1), 38-45.

Lamarck, Jean-Baptiste Pierre Antoine de. (1809). Philosophie zoologique, ou exposition des considËrations relatives $\ddagger$ l'histoire naturelle des animaux; $¥$ la diversitE de leur organisation et des facultÉs qu'ils en obtiennent; aux causes physiques qui maintiennent en eux la vie et donnent lieu aux mouvements qu'ils exËcutent; enfin, $\neq$ celles qui produisent, les unes le sentiment, et les autres l'intelligence de ceux qui en sont douĖs T. 1 T. 1. Paris: Germer BaillËre.

Lambert, J. C., Grenier-Boley, B., Harold, D., Zelenika, D., Chouraki, V., Kamatani, Y., . . Amouyel, P. (2013). Genomewide haplotype association study identifies the FRMD4A gene as a risk locus for Alzheimer's disease. Mol Psychiatry, 18(4), 461-470. doi: 10.1038/mp.2012.14

Lambert, J. C., Heath, S., Even, G., Campion, D., Sleegers, K., Hiltunen, M., . . Amouyel, P. (2009). Genome-wide association study identifies variants at CLU and CR1 associated with Alzheimer's disease. Nat Genet, 41(10), 1094-1099. doi: 10.1038/ng.439

Lamming, D. W. (2016). Inhibition of the Mechanistic Target of Rapamycin (mTOR)-Rapamycin and Beyond. Cold Spring Harb Perspect Med, 6(5). doi: 10.1101/cshperspect.a025924

Lane, C. A., Hardy, J., \& Schott, J. M. (2017). Alzheimer's disease. Eur J Neurol. doi: 10.1111/ene.13439

Lane-Donovan, C., Philips, G. T., Wasser, C. R., Durakoglugil, M. S., Masiulis, I., Upadhaya, A., . . Herz, J. (2015). Reelin protects against amyloid beta toxicity in vivo. Sci Signal, 8(384), ra67. doi: 10.1126/scisignal.aaa6674

Langstrom, N. S., Anderson, J. P., Lindroos, H. G., Winblad, B., \& Wallace, W. C. (1989). Alzheimer's disease-associated reduction of polysomal mRNA translation. Brain Res Mol Brain Res, 5(4), 259-269.

Lau, N. C., Seto, A. G., Kim, J., Kuramochi-Miyagawa, S., Nakano, T., Bartel, D. P., \& Kingston, R. E. (2006). Characterization of the piRNA complex from rat testes. Science, 313(5785), 363-367. doi: 10.1126/science. 1130164

Lazarov, O., \& Hollands, C. (2016). Hippocampal neurogenesis: Learning to remember. Prog Neurobiol, 138-140, 1-18. doi: 10.1016/j.pneurobio.2015.12.006

Le Thomas, A., Stuwe, E., Li, S., Du, J., Marinov, G., Rozhkov, N., . . Aravin, A. A. (2014). Transgenerationally inherited piRNAs trigger piRNA biogenesis by changing the chromatin of piRNA clusters and inducing precursor processing. Genes Dev, 28(15), 1667-1680. doi: 10.1101/gad.245514.114

Lechner, H. A., Squire, L. R., \& Byrne, J. H. (1999). 100 years of consolidation--remembering Muller and Pilzecker. Learn Mem, 6(2), 77-87.

LeDoux, J. (2003). The emotional brain, fear, and the amygdala. Cell Mol Neurobiol, 23(4-5), 727-738.

Lee, A. K., \& Wilson, M. A. (2002). Memory of sequential experience in the hippocampus during slow wave sleep. Neuron, 36(6), 1183-1194.

Lee, B. P., Buric, I., George-Pandeth, A., Flurkey, K., Harrison, D. E., Yuan, R., . . Harries, L. W. (2017). MicroRNAs miR-203-3p, miR-664-3p and miR-708-5p are associated with median strain lifespan in mice. Sci Rep, 7, 44620. doi: $10.1038 /$ srep44620

Lee, C. K., Weindruch, R., \& Prolla, T. A. (2000). Gene-expression profile of the ageing brain in mice. Nat Genet, 25(3), 294-297. doi: 10.1038/77046

Lee, C. Y., \& Landreth, G. E. (2010). The role of microglia in amyloid clearance from the AD brain. J Neural Transm (Vienna), 117(8), 949-960. doi: 10.1007/s00702-010-0433-4

Lee, E. J., Banerjee, S., Zhou, H., Jammalamadaka, A., Arcila, M., Manjunath, B. S., \& Kosik, K. S. (2011). Identification of piRNAs in the central nervous system. RNA, 17(6), 1090-1099. doi: 10.1261/rna.2565011

Lee, J., Kim, Y., Liu, T., Hwang, Y. J., Hyeon, S. J., Im, H., . . Ryu, H. (2018). SIRT3 deregulation is linked to mitochondrial dysfunction in Alzheimer's disease. Aging Cell, 17(1). doi: 10.1111/acel.12679

Lee, M. P. (2012). Allele-specific gene expression and epigenetic modifications and their application to understanding inheritance and cancer. Biochim Biophys Acta, 1819(7), 739-742. doi: 10.1016/j.bbagrm.2012.02.007

Lee, R. C., Feinbaum, R. L., \& Ambros, V. (1993). The C. elegans heterochronic gene lin-4 encodes small RNAs with antisense complementarity to lin-14. Cell, 75(5), 843-854.

Lee, S. H., Kwak, C., Shim, J., Kim, J. E., Choi, S. L., Kim, H. F., . . Kaang, B. K. (2012). A cellular model of memory reconsolidation involves reactivation-induced destabilization and restabilization at the sensorimotor synapse in Aplysia. Proc Natl Acad Sci U S A, 109(35), 14200-14205. doi: 10.1073/pnas.1211997109

Lee, T. I., \& Young, R. A. (2000). Transcription of eukaryotic protein-coding genes. Annu Rev Genet, 34, 77-137. doi: 10.1146/annurev.genet.34.1.77 
Leshchyns'ka, I., \& Sytnyk, V. (2016). Synaptic Cell Adhesion Molecules in Alzheimer's Disease. Neural Plast, 2016, 6427537. doi: 10.1155/2016/6427537

Lessard-Beaudoin, M., Laroche, M., Loudghi, A., Demers, M. J., Denault, J. B., Grenier, G., . . Graham, R. K. (2016). Organ-specific alteration in caspase expression and STK3 proteolysis during the aging process. Neurobiol Aging, 47, 50-62. doi: 10.1016/j.neurobiolaging.2016.07.003

Levy, E., Carman, M. D., Fernandez-Madrid, I. J., Power, M. D., Lieberburg, I., van Duinen, S. G., . . Frangione, B. (1990). Mutation of the Alzheimer's disease amyloid gene in hereditary cerebral hemorrhage, Dutch type. Science, 248(4959), 1124-1126.

Levy-Lahad, E., Wasco, W., Poorkaj, P., Romano, D. M., Oshima, J., Pettingell, W. H., . . et al. (1995). Candidate gene for the chromosome 1 familial Alzheimer's disease locus. Science, 269(5226), 973-977.

Li, X., An, W. L., Alafuzoff, I., Soininen, H., Winblad, B., \& Pei, J. J. (2004). Phosphorylated eukaryotic translation factor 4E is elevated in Alzheimer brain. Neuroreport, 15(14), 2237-2240.

Li, Y., Wu, X., Gao, H., Jin, J. M., Li, A. X., Kim, Y. S., . . Wu, H. (2015). Piwi-Interacting RNAs (piRNAs) Are Dysregulated in Renal Cell Carcinoma and Associated with Tumor Metastasis and Cancer-Specific Survival. Mol Med, 21, 381-388. doi: 10.2119/molmed.2014.00203

Liang, W. S., Reiman, E. M., Valla, J., Dunckley, T., Beach, T. G., Grover, A., . . Stephan, D. A. (2008). Alzheimer's disease is associated with reduced expression of energy metabolism genes in posterior cingulate neurons. Proc Natl Acad Sci U S A, 105(11), 4441-4446. doi: 10.1073/pnas.0709259105

Lim, A. K., Tao, L., \& Kai, T. (2009). piRNAs mediate posttranscriptional retroelement silencing and localization to pi-bodies in the Drosophila germline. J Cell Biol, 186(3), 333-342. doi: 10.1083/jcb.200904063

Lin, M. T., \& Beal, M. F. (2006). Mitochondrial dysfunction and oxidative stress in neurodegenerative diseases. Nature, 443(7113), 787-795. doi: 10.1038/nature05292

Lin, M. T., Simon, D. K., Ahn, C. H., Kim, L. M., \& Beal, M. F. (2002). High aggregate burden of somatic mtDNA point mutations in aging and Alzheimer's disease brain. Hum Mol Genet, 11(2), 133-145.

Linnane, A. W., Marzuki, S., Ozawa, T., \& Tanaka, M. (1989). Mitochondrial DNA mutations as an important contributor to ageing and degenerative diseases. Lancet, 1(8639), 642-645.

Liu, Q., \& Zhang, J. (2014). Lipid metabolism in Alzheimer's disease. Neurosci Bull, 30(2), 331-345. doi: 10.1007/ s12264-013-1410-3

Liu, X., Erikson, C., \& Brun, A. (1996). Cortical synaptic changes and gliosis in normal aging, Alzheimer's disease and frontal lobe degeneration. Dementia, 7(3), 128-134.

Livak, K. J., \& Schmittgen, T. D. (2001). Analysis of relative gene expression data using real-time quantitative PCR and the 2(-Delta Delta C(T)) Method. Methods, 25(4), 402-408. doi: 10.1006/meth.2001.1262

Loeffen, J. L., Triepels, R. H., van den Heuvel, L. P., Schuelke, M., Buskens, C. A., Smeets, R. J., . . Smeitink, J. A. (1998). cDNA of eight nuclear encoded subunits of NADH:ubiquinone oxidoreductase: human complex I cDNA characterization completed. Biochem Biophys Res Commun, 253(2), 415-422. doi: 10.1006/bbrc.1998.9786

Loerch, P. M., Lu, T., Dakin, K. A., Vann, J. M., Isaacs, A., Geula, C., . . Yankner, B. A. (2008). Evolution of the aging brain transcriptome and synaptic regulation. PLoS One, 3(10), e3329. doi: 10.1371/journal.pone.0003329

Logan, J. M., Sanders, A. L., Snyder, A. Z., Morris, J. C., \& Buckner, R. L. (2002). Under-recruitment and nonselective recruitment: dissociable neural mechanisms associated with aging. Neuron, 33(5), 827-840.

Logue, S. F., Paylor, R., \& Wehner, J. M. (1997). Hippocampal lesions cause learning deficits in inbred mice in the Morris water maze and conditioned-fear task. Behav Neurosci, 111(1), 104-113.

Lomax, M. I., Hewett-Emmett, D., Yang, T. L., \& Grossman, L. I. (1992). Rapid evolution of the human gene for cytochrome c oxidase subunit IV. Proc Natl Acad Sci U S A, 89(12), 5266-5270.

Lomo, T. (1966). Frequency potentiation of excitatory synaptic activity in dentate area of hippocampal formation. Acta Physiol. Scand., 68, 277.

Lopez, J. P., Diallo, A., Cruceanu, C., Fiori, L. M., Laboissiere, S., Guillet, I., . . Ernst, C. (2015). Biomarker discovery: quantification of microRNAs and other small non-coding RNAs using next generation sequencing. BMC Med Genomics, 8, 35. doi: 10.1186/s12920-015-0109-x

Lopez-Toledano, M. A., \& Shelanski, M. L. (2007). Increased neurogenesis in young transgenic mice overexpressing human APP(Sw, Ind). J Alzheimers Dis, 12(3), 229-240.

Love, M. I., Huber, W., \& Anders, S. (2014). Moderated estimation of fold change and dispersion for RNA-seq data with DESeq2. Genome Biol, 15(12), 550. doi: 10.1186/s13059-014-0550-8

Lovic, V., Gonzalez, A., \& Fleming, A. S. (2001). Maternally separated rats show deficits in maternal care in adulthood. Dev Psychobiol, 39(1), 19-33.

Lu, M., \& Kosik, K. S. (2001). Competition for microtubule-binding with dual expression of tau missense and splice isoforms. Mol Biol Cell, 12(1), 171-184.

Lu, T., Pan, Y., Kao, S. Y., Li, C., Kohane, I., Chan, J., \& Yankner, B. A. (2004). Gene regulation and DNA damage in the ageing human brain. Nature, 429(6994), 883-891. doi: 10.1038/nature02661

Ludwig, N., Leidinger, P., Becker, K., Backes, C., Fehlmann, T., Pallasch, C., . . Keller, A. (2016). Distribution of miRNA expression across human tissues. Nucleic Acids Res, 44(8), 3865-3877. doi: 10.1093/nar/gkw116

Luger, K., Mader, A. W., Richmond, R. K., Sargent, D. F., \& Richmond, T. J. (1997). Crystal structure of the nucleosome core particle at 2.8 A resolution. Nature, 389(6648), 251-260. doi: 10.1038/38444

Lukiw, W. J. (2012). Evolution and complexity of micro RNA in the human brain. Front Genet, 3, 166. doi: 10.3389/fgene. 2012.00166

Ma, X., Zhan, G., Sleumer, M. C., Chen, S., Liu, W., Zhang, M. Q., \& Liu, X. (2016). Analysis of C. elegans muscle transcriptome using trans-splicing-based RNA tagging (SRT). Nucleic Acids Res, 44(21), e156. doi: 10.1093/nar/ gkw734

Madathil, S. A., Rousseau, M. C., Allison, P., Netuveli, G., Humphris, G. M., Varghese, I., . . Nicolau, B. (2015). Maternal and paternal contribution to intergenerational psychosocial transmission of paan chewing. Community Dent Oral Epidemiol, 43(4), 289-297. doi: 10.1111/cdoe.12153

Madison, D. V., Malenka, R. C., \& Nicoll, R. A. (1991). Mechanisms underlying long-term potentiation of synaptic transmission. Annu Rev Neurosci, 14, 379-397. doi: 10.1146/annurev.ne.14.030191.002115 
Maestripieri, D. (2005). Early experience affects the intergenerational transmission of infant abuse in rhesus monkeys. Proc Natl Acad Sci U S A, 102(27), 9726-9729. doi: 10.1073/pnas.0504122102

Malanga, C. J., Pejchal, M., \& Kosofsky, B. E. (2007). Prenatal exposure to cocaine alters the development of conditioned place-preference to cocaine in adult mice. Pharmacol Biochem Behav, 87(4), 462-471. doi: 10.1016/j.pbb. 2007.06.002

Malenka, R. C., \& Bear, M. F. (2004). LTP and LTD: an embarrassment of riches. Neuron, 44(1), 5-21. doi: 10.1016/ j.neuron.2004.09.012

Malenka, R. C., \& Nicoll, R. A. (1999). Long-term potentiation--a decade of progress? Science, 285(5435), 1870-1874.

Mandelkow, E. M., \& Mandelkow, E. (2012). Biochemistry and cell biology of tau protein in neurofibrillary degeneration. Cold Spring Harb Perspect Med, 2(7), a006247. doi: 10.1101/cshperspect.a006247

Manoli, D. S., \& Tollkuhn, J. (2018). Gene regulatory mechanisms underlying sex differences in brain development and psychiatric disease. Ann N Y Acad Sci. doi: 10.1111/nyas.13564

Maritzen, T., Podufall, J., \& Haucke, V. (2010). Stonins--specialized adaptors for synaptic vesicle recycling and beyond? Traffic, 11(1), 8-15. doi: 10.1111/j.1600-0854.2009.00971.x

Markesbery, W. R. (1997). Oxidative stress hypothesis in Alzheimer's disease. Free Radic Biol Med, 23(1), 134-147.

Martin-Ruiz, C., Dickinson, H. O., Keys, B., Rowan, E., Kenny, R. A., \& Von Zglinicki, T. (2006). Telomere length predicts poststroke mortality, dementia, and cognitive decline. Ann Neurol, 60(2), 174-180. doi: 10.1002/ana.20869

Martinez, R., Martin-Subero, J. I., Rohde, V., Kirsch, M., Alaminos, M., Fernandez, A. F., . . Esteller, M. (2009). A microarray-based DNA methylation study of glioblastoma multiforme. Epigenetics, 4(4), 255-264.

Martinez-Jimenez, C. P., Eling, N., Chen, H. C., Vallejos, C. A., Kolodziejczyk, A. A., Connor, F., . . Odom, D. T. (2017). Aging increases cell-to-cell transcriptional variability upon immune stimulation. Science, 355(6332), 1433-1436. doi: 10.1126/science.aah4115

Masters, C. L., Simms, G., Weinman, N. A., Multhaup, G., McDonald, B. L., \& Beyreuther, K. (1985). Amyloid plaque core protein in Alzheimer disease and Down syndrome. Proc Natl Acad Sci U S A, 82(12), 4245-4249.

Mastroeni, D., Grover, A., Delvaux, E., Whiteside, C., Coleman, P. D., \& Rogers, J. (2010). Epigenetic changes in Alzheimer's disease: decrements in DNA methylation. Neurobiol Aging, 31(12), 2025-2037. doi: 10.1016/ j.neurobiolaging.2008.12.005

Matigian, N., Abrahamsen, G., Sutharsan, R., Cook, A. L., Vitale, A. M., Nouwens, A., . . Mackay-Sim, A. (2010). Diseasespecific, neurosphere-derived cells as models for brain disorders. Dis Model Mech, 3(11-12), 785-798. doi: 10.1242/dmm.005447

Mattick, J. S., \& Makunin, I. V. (2005). Small regulatory RNAs in mammals. Hum Mol Genet, 14 Spec No 1, R121-132. doi: $10.1093 / \mathrm{hmg} / \mathrm{ddi} 101$

Mazin, P., Xiong, J., Liu, X., Yan, Z., Zhang, X., Li, M., . . Khaitovich, P. (2013). Widespread splicing changes in human brain development and aging. Mol Syst Biol, 9, 633. doi: 10.1038/msb.2012.67

McCarroll, S. A., Murphy, C. T., Zou, S., Pletcher, S. D., Chin, C. S., Jan, Y. N., . . Li, H. (2004). Comparing genomic expression patterns across species identifies shared transcriptional profile in aging. Nat Genet, 36(2), 197-204. doi: 10.1038/ng1291

McEwen, B. S. (2012). Brain on stress: how the social environment gets under the skin. Proc Natl Acad Sci U S A, 109 Suppl 2, 17180-17185. doi: 10.1073/pnas.1121254109

McGaugh, J. L. (2000). Memory--a century of consolidation. Science, 287(5451), 248-251.

McKhann, G., Drachman, D., Folstein, M., Katzman, R., Price, D., \& Stadlan, E. M. (1984). Clinical diagnosis of Alzheimer's disease: report of the NINCDS-ADRDA Work Group under the auspices of Department of Health and Human Services Task Force on Alzheimer's Disease. Neurology, 34(7), 939-944.

McKhann, G. M., Knopman, D. S., Chertkow, H., Hyman, B. T., Jack, C. R., Jr., Kawas, C. H., . . Phelps, C. H. (2011). The diagnosis of dementia due to Alzheimer's disease: recommendations from the National Institute on AgingAlzheimer's Association workgroups on diagnostic guidelines for Alzheimer's disease. Alzheimers Dement, 7(3), 263-269. doi: 10.1016/j.jalz.2011.03.005

McManus, M. J., Murphy, M. P., \& Franklin, J. L. (2011). The mitochondria-targeted antioxidant MitoQ prevents loss of spatial memory retention and early neuropathology in a transgenic mouse model of Alzheimer's disease. $J$ Neurosci, 31(44), 15703-15715. doi: 10.1523/JNEUROSCI.0552-11.2011

Meaney, M. J. (2001). Maternal care, gene expression, and the transmission of individual differences in stress reactivity across generations. Annu Rev Neurosci, 24, 1161-1192. doi: 10.1146/annurev.neuro.24.1.1161

Mecocci, P., MacGarvey, U., Kaufman, A. E., Koontz, D., Shoffner, J. M., Wallace, D. C., \& Beal, M. F. (1993). Oxidative damage to mitochondrial DNA shows marked age-dependent increases in human brain. Ann Neurol, 34(4), 609-616. doi: 10.1002/ana.410340416

Mehler, M. F., \& Mattick, J. S. (2006). Non-coding RNAs in the nervous system. J Physiol, 575(Pt 2), 333-341. doi: 10.1113/jphysiol.2006.113191

Melton, A. W. (1963). Implications of short-term memory for a general theory of memory. Science, 140(3562), 82-86. doi: 10.1126/science.140.3562.82

Mercer, T. R., Dinger, M. E., Sunkin, S. M., Mehler, M. F., \& Mattick, J. S. (2008). Specific expression of long noncoding RNAs in the mouse brain. Proc Natl Acad Sci U S A, 105(2), 716-721. doi: 10.1073/pnas.0706729105

Meshorer, E., \& Soreq, H. (2002). Pre-mRNA splicing modulations in senescence. Aging Cell, 1(1), 10-16.

Mesquita, A., Weinberger, M., Silva, A., Sampaio-Marques, B., Almeida, B., Leao, C., . . L Ludovico, P. (2010). Caloric restriction or catalase inactivation extends yeast chronological lifespan by inducing $\mathrm{H} 2 \mathrm{O} 2$ and superoxide dismutase activity. Proc Natl Acad Sci U S A, 107(34), 15123-15128. doi: 10.1073/pnas.1004432107

Michel, C. I., Holley, C. L., Scruggs, B. S., Sidhu, R., Brookheart, R. T., Listenberger, L. L., . . . Schaffer, J. E. (2011). Small nucleolar RNAs U32a, U33, and U35a are critical mediators of metabolic stress. Cell Metab, 14(1), 33-44. doi: 10.1016/j.cmet.2011.04.009

Mielke, M. M., Vemuri, P., \& Rocca, W. A. (2014). Clinical epidemiology of Alzheimer's disease: assessing sex and gender differences. Clin Epidemiol, 6, 37-48. doi: 10.2147/CLEP.S37929

Mietelska-Porowska, A., Wasik, U., Goras, M., Filipek, A., \& Niewiadomska, G. (2014). Tau protein modifications and interactions: their role in function and dysfunction. Int J Mol Sci, 15(3), 4671-4713. doi: 10.3390/ijms15034671 
Migaud, M., Charlesworth, P., Dempster, M., Webster, L. C., Watabe, A. M., Makhinson, M., ... Grant, S. G. (1998). Enhanced long-term potentiation and impaired learning in mice with mutant postsynaptic density-95 protein. Nature, 396(6710), 433-439. doi: 10.1038/24790

Miller, G. A. (1956). The magical number seven plus or minus two: some limits on our capacity for processing information. Psychol Rev, 63(2), 81-97.

Miller, J. A., Oldham, M. C., \& Geschwind, D. H. (2008). A systems level analysis of transcriptional changes in Alzheimer's disease and normal aging. J Neurosci, 28(6), 1410-1420. doi: 10.1523/JNEUROSCI.4098-07.2008

Mills, J. D., Nalpathamkalam, T., Jacobs, H. I., Janitz, C., Merico, D., Hu, P., \& Janitz, M. (2013). RNA-Seq analysis of the parietal cortex in Alzheimer's disease reveals alternatively spliced isoforms related to lipid metabolism. Neurosci Lett, 536, 90-95. doi: 10.1016/j.neulet.2012.12.042

Ming, G. L., \& Song, H. (2011). Adult neurogenesis in the mammalian brain: significant answers and significant questions. Neuron, 70(4), 687-702. doi: 10.1016/j.neuron.2011.05.001

Minocherhomji, S., Hansen, C., Kim, H. G., Mang, Y., Bak, M., Guldberg, P., . . Silahtaroglu, A. (2014). Epigenetic remodelling and dysregulation of DLGAP4 is linked with early-onset cerebellar ataxia. Hum Mol Genet, 23(23), 6163-6176. doi: 10.1093/hmg/ddu337

Mirza, N., Pollock, K., Hoelzinger, D. B., Dominguez, A. L., \& Lustgarten, J. (2011). Comparative kinetic analyses of gene profiles of naive CD4+ and CD8+ T cells from young and old animals reveal novel age-related alterations. Aging Cell, 10(5), 853-867. doi: 10.1111/j.1474-9726.2011.00730.x

Missler, M., Zhang, W., Rohlmann, A., Kattenstroth, G., Hammer, R. E., Gottmann, K., \& Sudhof, T. C. (2003). Alphaneurexins couple Ca2+ channels to synaptic vesicle exocytosis. Nature, 423(6943), 939-948. doi: 10.1038/ nature 01755

Mitchell, J. C., Ariff, B. B., Yates, D. M., Lau, K. F., Perkinton, M. S., Rogelj, B., . . McLoughlin, D. M. (2009). X11beta rescues memory and long-term potentiation deficits in Alzheimer's disease APPswe Tg2576 mice. Hum Mol Genet, 18(23), 4492-4500. doi: 10.1093/hmg/ddp408

Mitra, S., Sameer Kumar, G. S., Tiwari, V., Lakshmi, B. J., Thakur, S. S., \& Kumar, S. (2016). Implication of Genetic Deletion of Wdr13 in Mice: Mild Anxiety, Better Performance in Spatial Memory Task, with Upregulation of Multiple Synaptic Proteins. Front Mol Neurosci, 9, 73. doi: 10.3389/fnmol.2016.00073

Molecular classification of Alzheimer's disease. (1991). Lancet, 337(8753), 1342-1343.

Montesanto, A., Dato, S., Bellizzi, D., Rose, G., \& Passarino, G. (2012). Epidemiological, genetic and epigenetic aspects of the research on healthy ageing and longevity. Immun Ageing, 9(1), 6. doi: 10.1186/1742-4933-9-6

Moonga, I., Niccolini, F., Wilson, H., Pagano, G., Politis, M., \& Alzheimer's Disease Neuroimaging, Initiative. (2017). Hypertension is associated with worse cognitive function and hippocampal hypometabolism in Alzheimer's disease. Eur J Neurol, 24(9), 1173-1182. doi: 10.1111/ene.13374

Mootha, V. K., Lindgren, C. M., Eriksson, K. F., Subramanian, A., Sihag, S., Lehar, J., . . Groop, L. C. (2003). PGC-1alpha-responsive genes involved in oxidative phosphorylation are coordinately downregulated in human diabetes. Nat Genet, 34(3), 267-273. doi: 10.1038/ng1180

Morazzani, E. M., Wiley, M. R., Murreddu, M. G., Adelman, Z. N., \& Myles, K. M. (2012). Production of virus-derived pingpong-dependent piRNA-like small RNAs in the mosquito soma. PLoS Pathog, 8(1), e1002470. doi: 10.1371/ journal.ppat.1002470

Mori, C., Spooner, E. T., Wisniewsk, K. E., Wisniewski, T. M., Yamaguch, H., Saido, T. C., . . Lemere, C. A. (2002). Intraneuronal Abeta42 accumulation in Down syndrome brain. Amyloid, 9(2), 88-102.

Morris, K. V., Chan, S. W., Jacobsen, S. E., \& Looney, D. J. (2004). Small interfering RNA-induced transcriptional gene silencing in human cells. Science, 305(5688), 1289-1292. doi: 10.1126/science.1101372

Morris, R. (1984). Developments of a water-maze procedure for studying spatial learning in the rat. $J$ Neurosci Methods, 11(1), 47-60.

Moser, E. I., Kropff, E., \& Moser, M. B. (2008). Place cells, grid cells, and the brain's spatial representation system. Annu Rev Neurosci, 31, 69-89. doi: 10.1146/annurev.neuro.31.061307.090723

Mullan, M., Crawford, F., Axelman, K., Houlden, H., Lilius, L., Winblad, B., \& Lannfelt, L. (1992). A pathogenic mutation for probable Alzheimer's disease in the APP gene at the N-terminus of beta-amyloid. Nat Genet, 1(5), 345-347. doi: 10.1038/ng0892-345

Murray, K. (1964). The Occurrence of Epsilon-N-Methyl Lysine in Histones. Biochemistry, 3, 10-15.

Muzzio, I. A., Kentros, C., \& Kandel, E. (2009). What is remembered? Role of attention on the encoding and retrieval of hippocampal representations. J Physiol, 587(Pt 12), 2837-2854. doi: 10.1113/jphysiol.2009.172445

Nagase, T., Ishikawa, K., Nakajima, D., Ohira, M., Seki, N., Miyajima, N., . . Ohara, O. (1997). Prediction of the coding sequences of unidentified human genes. VII. The complete sequences of 100 new cDNA clones from brain which can code for large proteins in vitro. DNA Res, 4(2), 141-150.

Namekata, K., Kimura, A., Harada, C., Yoshida, H., Matsumoto, Y., \& Harada, T. (2014). Dock3 protects myelin in the cuprizone model for demyelination. Cell Death Dis, 5, e1395. doi: 10.1038/cddis.2014.357

Namekata, K., Kimura, A., Kawamura, K., Harada, C., \& Harada, T. (2014). Dock GEFs and their therapeutic potential: neuroprotection and axon regeneration. Prog Retin Eye Res, 43, 1-16. doi: 10.1016/j.preteyeres.2014.06.005

Neaves, William B. (2002). Genomes, 2nd ed. T.A. Brown. Oxford, United Kingdom: Wiley-Liss, 2002, 600 pp., $\$ 97.50$, cloth. ISBN 0-471-25046-5. Clinical Chemistry, 48(12), 2300-2300.

Negron-Oyarzo, I., Lara-Vasquez, A., Palacios-Garcia, I., Fuentealba, P., \& Aboitiz, F. (2016). Schizophrenia and reelin: a model based on prenatal stress to study epigenetics, brain development and behavior. Biol Res, 49, 16. doi: 10.1186/s40659-016-0076-5

Neumann, M., Sampathu, D. M., Kwong, L. K., Truax, A. C., Micsenyi, M. C., Chou, T. T., . . Lee, V. M. (2006). Ubiquitinated TDP-43 in frontotemporal lobar degeneration and amyotrophic lateral sclerosis. Science, 314(5796), 130-133. doi: 10.1126/science.1134108

Neves, G., Cooke, S. F., \& Bliss, T. V. (2008). Synaptic plasticity, memory and the hippocampus: a neural network approach to causality. Nat Rev Neurosci, 9(1), 65-75. doi: 10.1038/nrn2303 
Newbold, R. R., Padilla-Banks, E., \& Jefferson, W. N. (2006). Adverse effects of the model environmental estrogen diethylstilbestrol are transmitted to subsequent generations. Endocrinology, 147(6 Suppl), S11-17. doi: 10.1210/ en.2005-1164

Ng, S. F., Lin, R. C., Laybutt, D. R., Barres, R., Owens, J. A., \& Morris, M. J. (2010). Chronic high-fat diet in fathers programs beta-cell dysfunction in female rat offspring. Nature, 467(7318), 963-966. doi: 10.1038/nature09491

Ng, S. F., Lin, R. C., Maloney, C. A., Youngson, N. A., Owens, J. A., \& Morris, M. J. (2014). Paternal high-fat diet consumption induces common changes in the transcriptomes of retroperitoneal adipose and pancreatic islet tissues in female rat offspring. FASEB J, 28(4), 1830-1841. doi: 10.1096/fj.13-244046

Niblock, M., \& Gallo, J. M. (2012). Tau alternative splicing in familial and sporadic tauopathies. Biochem Soc Trans, 40(4), 677-680. doi: 10.1042/BST20120091

Noorlander, C. W., Visser, G. H., Ramakers, G. M., Nikkels, P. G., \& de Graan, P. N. (2008). Prenatal corticosteroid exposure affects hippocampal plasticity and reduces lifespan. Dev Neurobiol, 68(2), 237-246. doi: 10.1002/dneu. 20583

Nunnari, J., \& Suomalainen, A. (2012). Mitochondria: in sickness and in health. Cell, 148(6), 1145-1159. doi: 10.1016/j.cell. 2012.02.035

O'Keefe, J. (1976). Place units in the hippocampus of the freely moving rat. Exp Neurol, 51(1), 78-109.

O'Keefe, J., \& Dostrovsky, J. (1971). The hippocampus as a spatial map. Preliminary evidence from unit activity in the freely-moving rat. Brain Res, 34(1), 171-175.

O'Reilly, R. C., \& Norman, K. A. (2002). Hippocampal and neocortical contributions to memory: advances in the complementary learning systems framework. Trends Cogn Sci, 6(12), 505-510.

Oakley, H., Cole, S. L., Logan, S., Maus, E., Shao, P., Craft, J., . . Vassar, R. (2006). Intraneuronal beta-amyloid aggregates, neurodegeneration, and neuron loss in transgenic mice with five familial Alzheimer's disease mutations: potential factors in amyloid plaque formation. J Neurosci, 26(40), 10129-10140. doi: 10.1523/ JNEUROSCI.1202-06.2006

Oberg, A. L., \& Mahoney, D. W. (2007). Linear mixed effects models. Methods Mol Biol, 404, 213-234. doi: 10.1007/978-1-59745-530-5 11

Oddo, S., Caccamo, A., Shepherd, J. D., Murphy, M. P., Golde, T. E., Kayed, R., . . LaFerla, F. M. (2003). Tripletransgenic model of Alzheimer's disease with plaques and tangles: intracellular Abeta and synaptic dysfunction. Neuron, 39(3), 409-421.

Oddo, S., Caccamo, A., Smith, I. F., Green, K. N., \& LaFerla, F. M. (2006). A dynamic relationship between intracellular and extracellular pools of Abeta. Am J Pathol, 168(1), 184-194.

Oddo, S., Caccamo, A., Tran, L., Lambert, M. P., Glabe, C. G., Klein, W. L., \& LaFerla, F. M. (2006). Temporal profile of amyloid-beta (Abeta) oligomerization in an in vivo model of Alzheimer disease. A link between Abeta and tau pathology. J Biol Chem, 281(3), 1599-1604. doi: 10.1074/jbc.M507892200

Oh, G., Ebrahimi, S., Carlucci, M., Zhang, A., Nair, A., Groot, D. E., . . . Petronis, A. (2018). Cytosine modifications exhibit circadian oscillations that are involved in epigenetic diversity and aging. Nat Commun, 9(1), 644. doi: 10.1038/ s41467-018-03073-7

Okano, M., Xie, S., \& Li, E. (1998). Cloning and characterization of a family of novel mammalian DNA (cytosine-5) methyltransferases. Nat Genet, 19(3), 219-220. doi: 10.1038/890

Olton, D. S., Walker, J. A., \& Gage, F. H. (1978). Hippocampal connections and spatial discrimination. Brain Res, 139(2), 295-308.

Ono, M., Scott, M. S., Yamada, K., Avolio, F., Barton, G. J., \& Lamond, A. I. (2011). Identification of human miRNA precursors that resemble box C/D snoRNAs. Nucleic Acids Res, 39(9), 3879-3891. doi: 10.1093/nar/gkq1355

Onuki, Y., Van Someren, E. J., De Zeeuw, C. I., \& Van der Werf, Y. D. (2015). Hippocampal-cerebellar interaction during spatio-temporal prediction. Cereb Cortex, 25(2), 313-321. doi: 10.1093/cercor/bht221

Osorio, F. G., Barcena, C., Soria-Valles, C., Ramsay, A. J., de Carlos, F., Cobo, J., . . Lopez-Otin, C. (2012). Nuclear lamina defects cause ATM-dependent NF-kappaB activation and link accelerated aging to a systemic inflammatory response. Genes Dev, 26(20), 2311-2324. doi: 10.1101/gad.197954.112

Ost, A., Lempradl, A., Casas, E., Weigert, M., Tiko, T., Deniz, M., . . Pospisilik, J. A. (2014). Paternal diet defines offspring chromatin state and intergenerational obesity. Cell, 159(6), 1352-1364. doi: 10.1016/j.cell.2014.11.005

Padurariu, M., Ciobica, A., Mavroudis, I., Fotiou, D., \& Baloyannis, S. (2012). Hippocampal neuronal loss in the CA1 and CA3 areas of Alzheimer's disease patients. Psychiatr Danub, 24(2), 152-158.

Palop, J. J., Chin, J., Roberson, E. D., Wang, J., Thwin, M. T., Bien-Ly, N., . . Mucke, L. (2007). Aberrant excitatory neuronal activity and compensatory remodeling of inhibitory hippocampal circuits in mouse models of Alzheimer's disease. Neuron, 55(5), 697-711. doi: 10.1016/j.neuron.2007.07.025

Paoloni-Giacobino, A., \& Chaillet, J. R. (2006). The role of DMDs in the maintenance of epigenetic states. Cytogenet Genome Res, 113(1-4), 116-121. doi: 10.1159/000090822

Parra-Damas, A., Valero, J., Chen, M., Espana, J., Martin, E., Ferrer, I., . . Saura, C. A. (2014). Crtc1 activates a transcriptional program deregulated at early Alzheimer's disease-related stages. J Neurosci, 34(17), 5776-5787. doi: 10.1523/JNEUROSCI.5288-13.2014

Pasinetti, G. M. (2001). Use of cDNA microarray in the search for molecular markers involved in the onset of Alzheimer's disease dementia. J Neurosci Res, 65(6), 471-476. doi: 10.1002/jnr.1176

Pasque, V., Jullien, J., Miyamoto, K., Halley-Stott, R. P., \& Gurdon, J. B. (2011). Epigenetic factors influencing resistance to nuclear reprogramming. Trends Genet, 27(12), 516-525. doi: 10.1016/j.tig.2011.08.002

Pearson, B. E., \& Choi, T. K. (1993). Expression of the human beta-amyloid precursor protein gene from a yeast artificial chromosome in transgenic mice. Proc Natl Acad Sci U S A, 90(22), 10578-10582.

Penna, I., Vassallo, I., Nizzari, M., Russo, D., Costa, D., Menichini, P., . . Pagano, A. (2013). A novel snRNA-like transcript affects amyloidogenesis and cell cycle progression through perturbation of Fe65L1 (APBB2) alternative splicing. Biochim Biophys Acta, 1833(6), 1511-1526. doi: 10.1016/j.bbamcr.2013.02.020

Persson, J., Sylvester, C. Y., Nelson, J. K., Welsh, K. M., Jonides, J., \& Reuter-Lorenz, P. A. (2004). Selection requirements during verb generation: differential recruitment in older and younger adults. Neuroimage, 23(4), 1382-1390. doi: 10.1016/j.neuroimage.2004.08.004 
Peters, M. J., Joehanes, R., Pilling, L. C., Schurmann, C., Conneely, K. N., Powell, J., . . Johnson, A. D. (2015). The transcriptional landscape of age in human peripheral blood. Nat Commun, 6, 8570. doi: 10.1038/ncomms9570

Petersen, R. C., Smith, G., Kokmen, E., Ivnik, R. J., \& Tangalos, E. G. (1992). Memory function in normal aging. Neurology, 42(2), 396-401.

Pistell, P. J., Zhu, M., \& Ingram, D. K. (2008). Acquisition of conditioned taste aversion is impaired in the amyloid precursor protein/presenilin 1 mouse model of Alzheimer's disease. Neuroscience, 152(3), 594-600. doi: 10.1016/ j.neuroscience.2008.01.025

Plagemann, A. (2005). Perinatal programming and functional teratogenesis: impact on body weight regulation and obesity. Physiol Behav, 86(5), 661-668. doi: 10.1016/j.physbeh.2005.08.065

Pletcher, S. D., Macdonald, S. J., Marguerie, R., Certa, U., Stearns, S. C., Goldstein, D. B., \& Partridge, L. (2002). Genome-wide transcript profiles in aging and calorically restricted Drosophila melanogaster. Curr Biol, 12(9), 712-723.

Podtelezhnikov, A. A., Tanis, K. Q., Nebozhyn, M., Ray, W. J., Stone, D. J., \& Loboda, A. P. (2011). Molecular insights into the pathogenesis of Alzheimer's disease and its relationship to normal aging. PLoS One, 6(12), e29610. doi: 10.1371/journal.pone.0029610

Poldrack, R. A., Clark, J., Pare-Blagoev, E. J., Shohamy, D., Creso Moyano, J., Myers, C., \& Gluck, M. A. (2001). Interactive memory systems in the human brain. Nature, 414(6863), 546-550. doi: 10.1038/35107080

Prince, Martin James, Wimo, Anders, Guerchet, Maelenn Mari, Ali, Gemma Claire, Wu, Yu-Tzu, \& Prina, Matthew. (2015). World Alzheimer Report 2015 - The Global Impact of Dementia. London: Alzheimer's Disease International.

Profenno, L. A., Porsteinsson, A. P., \& Faraone, S. V. (2010). Meta-analysis of Alzheimer's disease risk with obesity, diabetes, and related disorders. Biol Psychiatry, 67(6), 505-512. doi: 10.1016/j.biopsych.2009.02.013

Ptashne, M. (2007). On the use of the word 'epigenetic'. Curr Biol, 17(7), R233-236. doi: 10.1016/j.cub.2007.02.030

Puzzo, D., \& Arancio, O. (2013). Amyloid-beta peptide: Dr. Jekyll or Mr. Hyde? J Alzheimers Dis, 33 Suppl 1, S111-120. doi: 10.3233/JAD-2012-129033

Qian, W., \& Liu, F. (2014). Regulation of alternative splicing of tau exon 10. Neurosci Bull, 30(2), 367-377. doi: 10.1007/ s12264-013-1411-2

Qiu, W., Guo, X., Lin, X., Yang, Q., Zhang, W., Zhang, Y., . . Luo, X. (2017). Transcriptome-wide piRNA profiling in human brains of Alzheimer's disease. Neurobiol Aging, 57, 170-177. doi: 10.1016/j.neurobiolaging.2017.05.020

Quon, D., Wang, Y., Catalano, R., Scardina, J. M., Murakami, K., \& Cordell, B. (1991). Formation of beta-amyloid protein deposits in brains of transgenic mice. Nature, 352(6332), 239-241. doi: 10.1038/352239a0

Qureshi, I. A., \& Mehler, M. F. (2012). Emerging roles of non-coding RNAs in brain evolution, development, plasticity and disease. Nat Rev Neurosci, 13(8), 528-541. doi: 10.1038/nrn3234

Qureshi, I. A., \& Mehler, M. F. (2013). Long non-coding RNAs: novel targets for nervous system disease diagnosis and therapy. Neurotherapeutics, 10(4), 632-646. doi: 10.1007/s13311-013-0199-0

Qvarnstrom, A., \& Price, T. D. (2001). Maternal effects, paternal effects and sexual selection. Trends Ecol Evol, 16(2), 95-100.

R., Morris. (1981). Spatial localization does not require the presence of local cues. Learn Motiv., 12, 239-260.

Radde, R., Bolmont, T., Kaeser, S. A., Coomaraswamy, J., Lindau, D., Stoltze, L., . . . Jucker, M. (2006). Abeta42-driven cerebral amyloidosis in transgenic mice reveals early and robust pathology. EMBO Rep, 7(9), 940-946. doi: 10.1038/sj.embor.7400784

Radford, E. J., Ito, M., Shi, H., Corish, J. A., Yamazawa, K., Isganaitis, E., . . Ferguson-Smith, A. C. (2014). In utero effects. In utero undernourishment perturbs the adult sperm methylome and intergenerational metabolism. Science, 345(6198), 1255903. doi: 10.1126/science.1255903

Raimondi, A., Ferguson, S. M., Lou, X., Armbruster, M., Paradise, S., Giovedi, S., . . De Camilli, P. (2011). Overlapping role of dynamin isoforms in synaptic vesicle endocytosis. Neuron, 70(6), 1100-1114. doi: 10.1016/j.neuron. 2011.04.031

Rani, N., Nowakowski, T. J., Zhou, H., Godshalk, S. E., Lisi, V., Kriegstein, A. R., \& Kosik, K. S. (2016). A Primate IncRNA Mediates Notch Signaling during Neuronal Development by Sequestering miRNA. Neuron, 90(6), 1174-1188. doi: 10.1016/j.neuron.2016.05.005

Rao, P., Benito, E., \& Fischer, A. (2013). MicroRNAs as biomarkers for CNS disease. Front Mol Neurosci, 6, 39. doi: 10.3389/fnmol.2013.00039

Rapoport, M., Dawson, H. N., Binder, L. I., Vitek, M. P., \& Ferreira, A. (2002). Tau is essential to beta -amyloid-induced neurotoxicity. Proc Natl Acad Sci U S A, 99(9), 6364-6369. doi: 10.1073/pnas.092136199

Reik, W., \& Walter, J. (2001). Genomic imprinting: parental influence on the genome. Nat Rev Genet, 2(1), 21-32. doi: $10.1038 / 35047554$

Reisberg, B., Ferris, S. H., de Leon, M. J., \& Crook, T. (1988). Global Deterioration Scale (GDS). Psychopharmacol Bull, 24(4), 661-663.

Ribeiro, T. A., Tofolo, L. P., Martins, I. P., Pavanello, A., de Oliveira, J. C., Prates, K. V., . . de Freitas Mathias, P. C. (2017). Maternal low intensity physical exercise prevents obesity in offspring rats exposed to early overnutrition. Sci Rep, 7(1), 7634. doi: 10.1038/s41598-017-07395-2

Rissman, R. A., De Blas, A. L., \& Armstrong, D. M. (2007). GABA(A) receptors in aging and Alzheimer's disease. J Neurochem, 103(4), 1285-1292. doi: 10.1111/j.1471-4159.2007.04832.x

Roberts, R.B. (1958). Microsomal particles and protein synthesis.

Rodgers, A. B., Morgan, C. P., Bronson, S. L., Revello, S., \& Bale, T. L. (2013). Paternal stress exposure alters sperm microRNA content and reprograms offspring HPA stress axis regulation. J Neurosci, 33(21), 9003-9012. doi: 10.1523/JNEUROSCI.0914-13.2013

Rodgers, A. B., Morgan, C. P., Leu, N. A., \& Bale, T. L. (2015). Transgenerational epigenetic programming via sperm microRNA recapitulates effects of paternal stress. Proc Natl Acad Sci U S A, 112(44), 13699-13704. doi: $10.1073 /$ pnas. 1508347112

Rodriguez, J. J., Jones, V. C., Tabuchi, M., Allan, S. M., Knight, E. M., LaFerla, F. M., . . Verkhratsky, A. (2008). Impaired adult neurogenesis in the dentate gyrus of a triple transgenic mouse model of Alzheimer's disease. PLoS One, 3(8), e2935. doi: 10.1371/journal.pone.0002935 
Rodriguez, S. A., Grochova, D., McKenna, T., Borate, B., Trivedi, N. S., Erdos, M. R., \& Eriksson, M. (2016). Global genome splicing analysis reveals an increased number of alternatively spliced genes with aging. Aging Cell, 15(2), 267-278. doi: 10.1111/acel.12433

Rogaev, E. I., Sherrington, R., Rogaeva, E. A., Levesque, G., Ikeda, M., Liang, Y., . . et al. (1995). Familial Alzheimer's disease in kindreds with missense mutations in a gene on chromosome 1 related to the Alzheimer's disease type 3 gene. Nature, 376(6543), 775-778. doi: 10.1038/376775a0

Rogan, M. T., Leon, K. S., Perez, D. L., \& Kandel, E. R. (2005). Distinct neural signatures for safety and danger in the amygdala and striatum of the mouse. Neuron, 46(2), 309-320. doi: 10.1016/j.neuron.2005.02.017

Rogelj, B. (2006). Brain-specific small nucleolar RNAs. J Mol Neurosci, 28(2), 103-109. doi: 10.1385/JMN:28:2:103

Rogelj, B., Hartmann, C. E., Yeo, C. H., Hunt, S. P., \& Giese, K. P. (2003). Contextual fear conditioning regulates the expression of brain-specific small nucleolar RNAs in hippocampus. Eur J Neurosci, 18(11), 3089-3096.

Rohe, M., Carlo, A. S., Breyhan, H., Sporbert, A., Militz, D., Schmidt, V., . . . Andersen, O. M. (2008). Sortilin-related receptor with A-type repeats (SORLA) affects the amyloid precursor protein-dependent stimulation of ERK signaling and adult neurogenesis. J Biol Chem, 283(21), 14826-14834. doi: 10.1074/jbc.M710574200

Rolland, Y., Abellan van Kan, G., \& Vellas, B. (2008). Physical activity and Alzheimer's disease: from prevention to therapeutic perspectives. J Am Med Dir Assoc, 9(6), 390-405. doi: 10.1016/j.jamda.2008.02.007

Roses, A. D., Lutz, M. W., Amrine-Madsen, H., Saunders, A. M., Crenshaw, D. G., Sundseth, S. S., . . Reiman, E. M. (2010). A TOMM40 variable-length polymorphism predicts the age of late-onset Alzheimer's disease. Pharmacogenomics J, 10(5), 375-384. doi: 10.1038/tpj.2009.69

Roth, T. L., Lubin, F. D., Funk, A. J., \& Sweatt, J. D. (2009). Lasting epigenetic influence of early-life adversity on the BDNF gene. Biol Psychiatry, 65(9), 760-769. doi: 10.1016/j.biopsych.2008.11.028

Rouget, C., Papin, C., Boureux, A., Meunier, A. C., Franco, B., Robine, N., . . Simonelig, M. (2010). Maternal mRNA deadenylation and decay by the piRNA pathway in the early Drosophila embryo. Nature, 467(7319), 1128-1132. doi: 10.1038/nature09465

Routtenberg, A., \& Rekart, J. L. (2005). Post-translational protein modification as the substrate for long-lasting memory. Trends Neurosci, 28(1), 12-19. doi: 10.1016/j.tins.2004.11.006

Roy, S., Zhang, B., Lee, V. M., \& Trojanowski, J. Q. (2005). Axonal transport defects: a common theme in neurodegenerative diseases. Acta Neuropathol, 109(1), 5-13. doi: 10.1007/s00401-004-0952-x

Rummel, J., Epp, J. R., \& Galea, L. A. (2010). Estradiol does not influence strategy choice but place strategy choice is associated with increased cell proliferation in the hippocampus of female rats. Horm Behav, 58(4), 582-590. doi: 10.1016/j.yhbeh.2010.07.009

Russo, V. E. A., Martienssen, Robert A., \& Riggs, Arthur D. (1996). Epigenetic mechanisms of gene regulation.

Russo, Vincenzo E. A., Martienssen, Robert A., \& Riggs, Arthur D. (1996). Epigenetic mechanisms of gene regulation. New York: Cold Spring Harbor Laboratory Press.

Sadowski, M., Pankiewicz, J., Scholtzova, H., Ji, Y., Quartermain, D., Jensen, C. H., . . Wisniewski, T. (2004). Amyloidbeta deposition is associated with decreased hippocampal glucose metabolism and spatial memory impairment in APP/PS1 mice. J Neuropathol Exp Neurol, 63(5), 418-428.

Sah, S. K., Lee, C., Jang, J. H., \& Park, G. H. (2017). Effect of high-fat diet on cognitive impairment in triple-transgenic mice model of Alzheimer's disease. Biochem Biophys Res Commun, 493(1), 731-736. doi: 10.1016/j.bbrc. 2017.08.122

Sahay, A., \& Hen, R. (2007). Adult hippocampal neurogenesis in depression. Nat Neurosci, 10(9), 1110-1115. doi: 10.1038/ nn1969

Saito, E. K., Diaz, N., Chung, J., \& McMurtray, A. (2017). Smoking history and Alzheimer's disease risk in a communitybased clinic population. J Educ Health Promot, 6, 24. doi: 10.4103/jehp.jehp 4515

Saleem, A. B., Ayaz, A., Jeffery, K. J., Harris, K. D., \& Carandini, M. (2013). Integration of visual motion and locomotion in mouse visual cortex. Nat Neurosci, 16(12), 1864-1869. doi: 10.1038/nn.3567

Salminen, A., Kaarniranta, K., \& Kauppinen, A. (2012). Inflammaging: disturbed interplay between autophagy and inflammasomes. Aging (Albany NY), 4(3), 166-175. doi: 10.18632/aging.100444

Salta, E., \& De Strooper, B. (2017). Noncoding RNAs in neurodegeneration. Nat Rev Neurosci, 18(10), 627-640. doi: 10.1038/nrn.2017.90

Sandhu, F. A., Salim, M., \& Zain, S. B. (1991). Expression of the human beta-amyloid protein of Alzheimer's disease specifically in the brains of transgenic mice. J Biol Chem, 266(32), 21331-21334.

Saura, C. A., Chen, G., Malkani, S., Choi, S. Y., Takahashi, R. H., Zhang, D., . . Shen, J. (2005). Conditional inactivation of presenilin 1 prevents amyloid accumulation and temporarily rescues contextual and spatial working memory impairments in amyloid precursor protein transgenic mice. J Neurosci, 25(29), 6755-6764. doi: 10.1523/ JNEUROSCI.1247-05.2005

Saura, C. A., Parra-Damas, A., \& Enriquez-Barreto, L. (2015). Gene expression parallels synaptic excitability and plasticity changes in Alzheimer's disease. Front Cell Neurosci, 9, 318. doi: 10.3389/fncel.2015.00318

Saxena, A., Tang, D., \& Carninci, P. (2012). piRNAs warrant investigation in Rett Syndrome: an omics perspective. Dis Markers, 33(5), 261-275. doi: 10.3233/DMA-2012-0932

Scheff, S. W., \& Price, D. A. (2006). Alzheimer's disease-related alterations in synaptic density: neocortex and hippocampus. J Alzheimers Dis, 9(3 Suppl), 101-115.

Scheff, S. W., Price, D. A., Schmitt, F. A., \& Mufson, E. J. (2006). Hippocampal synaptic loss in early Alzheimer's disease and mild cognitive impairment. Neurobiol Aging, 27(10), 1372-1384. doi: 10.1016/j.neurobiolaging.2005.09.012

Scheuner, D., Eckman, C., Jensen, M., Song, X., Citron, M., Suzuki, N., . . Younkin, S. (1996). Secreted amyloid betaprotein similar to that in the senile plaques of Alzheimer's disease is increased in vivo by the presenilin 1 and 2 and APP mutations linked to familial Alzheimer's disease. Nat Med, 2(8), 864-870.

Schmauss, C., Lee-McDermott, Z., \& Medina, L. R. (2014). Trans-generational effects of early life stress: the role of maternal behavior. Sci Rep, 4, 4873. doi: 10.1038/srep04873 
Schubert, T., Pusch, M. C., Diermeier, S., Benes, V., Kremmer, E., Imhof, A., \& Langst, G. (2012). Df31 protein and snoRNAs maintain accessible higher-order structures of chromatin. Mol Cell, 48(3), 434-444. doi: 10.1016/ j.molcel.2012.08.021

Schuster, A., Tang, C., Xie, Y., Ortogero, N., Yuan, S., \& Yan, W. (2016). SpermBase: A Database for Sperm-Borne RNA Contents. Biol Reprod, 95(5), 99. doi: 10.1095/biolreprod.116.142190

Scott, M. S., Avolio, F., Ono, M., Lamond, A. I., \& Barton, G. J. (2009). Human miRNA precursors with box H/ACA snoRNA features. PLoS Comput Biol, 5(9), e1000507. doi: 10.1371/journal.pcbi.1000507

Scott, M. S., Ono, M., Yamada, K., Endo, A., Barton, G. J., \& Lamond, A. I. (2012). Human box C/D snoRNA processing conservation across multiple cell types. Nucleic Acids Res, 40(8), 3676-3688. doi: 10.1093/nar/gkr1233

Scoville, W. B., \& Milner, B. (1957). Loss of recent memory after bilateral hippocampal lesions. J Neurol Neurosurg Psychiatry, 20(1), 11-21.

Sebestyen, E., Zawisza, M., \& Eyras, E. (2015). Detection of recurrent alternative splicing switches in tumor samples reveals novel signatures of cancer. Nucleic Acids Res, 43(3), 1345-1356. doi: 10.1093/nar/gku1392

Sekar, S., McDonald, J., Cuyugan, L., Aldrich, J., Kurdoglu, A., Adkins, J., . . Liang, W. S. (2015). Alzheimer's disease is associated with altered expression of genes involved in immune response and mitochondrial processes in astrocytes. Neurobiol Aging, 36(2), 583-591. doi: 10.1016/j.neurobiolaging.2014.09.027

Selkoe, D. J., \& Hardy, J. (2016). The amyloid hypothesis of Alzheimer's disease at 25 years. EMBO Mol Med, 8(6), 595-608. doi: 10.15252/emmm.201606210

Semon, R.W. (1911). Die Mneme als erhaltendes Prinzip im Wechsel des organischen Geschehens: Engelmann.

Sen, D., Majumder, A., Arora, V., Yadu, N., \& Chakrabarti, R. (2017). Taming Alzheimer's disease: New perspectives, newer horizons. Iran J Neurol, 16(3), 146-155.

Serneels, L., Van Biervliet, J., Craessaerts, K., Dejaegere, T., Horre, K., Van Houtvin, T., . . De Strooper, B. (2009). gamma-Secretase heterogeneity in the Aph1 subunit: relevance for Alzheimer's disease. Science, 324(5927), 639-642. doi: 10.1126/science.1171176

Serpell, L. C. (2000). Alzheimer's amyloid fibrils: structure and assembly. Biochim Biophys Acta, 1502(1), 16-30.

Seshadri, S., Fitzpatrick, A. L., Ikram, M. A., DeStefano, A. L., Gudnason, V., Boada, M., . . Consortium, Eadi. (2010). Genome-wide analysis of genetic loci associated with Alzheimer disease. JAMA, 303(18), 1832-1840. doi: 10.1001/jama.2010.574

Sha, K. (2008). A mechanistic view of genomic imprinting. Annu Rev Genomics Hum Genet, 9, 197-216. doi: 10.1146/ annurev.genom.122007.110031

Shannon, P., Markiel, A., Ozier, O., Baliga, N. S., Wang, J. T., Ramage, D., . . I Ideker, T. (2003). Cytoscape: a software environment for integrated models of biomolecular interaction networks. Genome Res, 13(11), 2498-2504. doi: 10.1101/gr.1239303

Shao, G. B., Chen, J. C., Zhang, L. P., Huang, P., Lu, H. Y., Jin, J., . . Sang, J. R. (2014). Dynamic patterns of histone H3 lysine 4 methyltransferases and demethylases during mouse preimplantation development. In Vitro Cell Dev Biol Anim, 50(7), 603-613. doi: 10.1007/s11626-014-9741-6

Shao, W., Peng, D., \& Wang, X. (2017). Genetics of Alzheimer's disease: From pathogenesis to clinical usage. J Clin Neurosci, 45, 1-8. doi: 10.1016/j.jocn.2017.06.074

Sharma, U., Conine, C. C., Shea, J. M., Boskovic, A., Derr, A. G., Bing, X. Y., . . Rando, O. J. (2016). Biogenesis and function of tRNA fragments during sperm maturation and fertilization in mammals. Science, 351(6271), 391-396. doi: $10.1126 /$ science.aad6780

Sharp, E. S., \& Gatz, M. (2011). Relationship between education and dementia: an updated systematic review. Alzheimer Dis Assoc Disord, 25(4), 289-304. doi: 10.1097/WAD.0b013e318211c83c

Shaw, L. M., Korecka, M., Clark, C. M., Lee, V. M., \& Trojanowski, J. Q. (2007). Biomarkers of neurodegeneration for diagnosis and monitoring therapeutics. Nat Rev Drug Discov, 6(4), 295-303. doi: 10.1038/nrd2176

Shen, E., Shulha, H., Weng, Z., \& Akbarian, S. (2014). Regulation of histone H3K4 methylation in brain development and disease. Philos Trans R Soc Lond B Biol Sci, 369(1652). doi: 10.1098/rstb.2013.0514

Sherrington, R., Rogaev, E. I., Liang, Y., Rogaeva, E. A., Levesque, G., Ikeda, M., . . St George-Hyslop, P. H. (1995). Cloning of a gene bearing missense mutations in early-onset familial Alzheimer's disease. Nature, 375(6534), 754-760. doi: $10.1038 / 375754 a 0$

Sherva, R., Baldwin, C. T., Inzelberg, R., Vardarajan, B., Cupples, L. A., Lunetta, K., . . Farrer, L. A. (2011). Identification of novel candidate genes for Alzheimer's disease by autozygosity mapping using genome wide SNP data. $J$ Alzheimers Dis, 23(2), 349-359. doi: 10.3233/JAD-2010-100714

Shibata, M., Nakao, H., Kiyonari, H., Abe, T., \& Aizawa, S. (2011). MicroRNA-9 regulates neurogenesis in mouse telencephalon by targeting multiple transcription factors. J Neurosci, 31(9), 3407-3422. doi: 10.1523/ JNEUROSCI.5085-10.2011

Shoji, M., Tanaka, T., Hosokawa, M., Reuter, M., Stark, A., Kato, Y., . . Chuma, S. (2009). The TDRD9-MIWI2 complex is essential for piRNA-mediated retrotransposon silencing in the mouse male germline. Dev Cell, 17(6), 775-787. doi: 10.1016/j.devcel.2009.10.012

Shu, L., Sun, W., Li, L., Xu, Z., Lin, L., Xie, P., . . Li, X. (2016). Genome-wide alteration of 5-hydroxymenthylcytosine in a mouse model of Alzheimer's disease. BMC Genomics, 17, 381. doi: 10.1186/s12864-016-2731-1

Siklenka, K., Erkek, S., Godmann, M., Lambrot, R., McGraw, S., Lafleur, C., . . Kimmins, S. (2015). Disruption of histone methylation in developing sperm impairs offspring health transgenerationally. Science, 350(6261), aab2006. doi: 10.1126/science.aab2006

Sindi, I. A., Tannenberg, R. K., \& Dodd, P. R. (2014). Role for the neurexin-neuroligin complex in Alzheimer's disease. Neurobiol Aging, 35(4), 746-756. doi: 10.1016/j.neurobiolaging.2013.09.032

Singh, V. P., Lakshmi, B. J., Singh, S., Shah, V., Goel, S., Sarathi, D. P., \& Kumar, S. (2012). Lack of Wdr13 gene in mice leads to enhanced pancreatic beta cell proliferation, hyperinsulinemia and mild obesity. PLoS One, 7(6), e38685. doi: 10.1371 /journal.pone.0038685

Siomi, M. C., Sato, K., Pezic, D., \& Aravin, A. A. (2011). PIWI-interacting small RNAs: the vanguard of genome defence. Nat Rev Mol Cell Biol, 12(4), 246-258. doi: 10.1038/nrm3089 
Skaggs, W. E., \& McNaughton, B. L. (1996). Replay of neuronal firing sequences in rat hippocampus during sleep following spatial experience. Science, 271(5257), 1870-1873.

Skinner, M. K. (2008). What is an epigenetic transgenerational phenotype? F3 or F2. Reprod Toxicol, 25(1), 2-6. doi: 10.1016/j.reprotox.2007.09.001

Skinner, M. K. (2011a). Environmental epigenetic transgenerational inheritance and somatic epigenetic mitotic stability. Epigenetics, 6(7), 838-842.

Skinner, M. K. (2011b). Role of epigenetics in developmental biology and transgenerational inheritance. Birth Defects Res C Embryo Today, 93(1), 51-55. doi: 10.1002/bdrc.20199

Skinner, M. K., Anway, M. D., Savenkova, M. I., Gore, A. C., \& Crews, D. (2008). Transgenerational epigenetic programming of the brain transcriptome and anxiety behavior. PLoS One, 3(11), e3745. doi: 10.1371/ journal.pone.0003745

Skinner, M. K., Manikkam, M., \& Guerrero-Bosagna, C. (2010). Epigenetic transgenerational actions of environmental factors in disease etiology. Trends Endocrinol Metab, 21(4), 214-222. doi: 10.1016/j.tem.2009.12.007

Skinner, M. K., Manikkam, M., Haque, M. M., Zhang, B., \& Savenkova, M. I. (2012). Epigenetic transgenerational inheritance of somatic transcriptomes and epigenetic control regions. Genome Biol, 13(10), R91. doi: 10.1186/ gb-2012-13-10-r91

Slatkin, M. (2009). Epigenetic inheritance and the missing heritability problem. Genetics, 182(3), 845-850. doi: 10.1534/ genetics.109.102798

Sloane, J. A., Pietropaolo, M. F., Rosene, D. L., Moss, M. B., Peters, A., Kemper, T., \& Abraham, C. R. (1997). Lack of correlation between plaque burden and cognition in the aged monkey. Acta Neuropathol, 94(5), 471-478.

Small, S. A., Tsai, W. Y., DeLaPaz, R., Mayeux, R., \& Stern, Y. (2002). Imaging hippocampal function across the human life span: is memory decline normal or not? Ann Neurol, 51(3), 290-295.

Smirnova, L., Grafe, A., Seiler, A., Schumacher, S., Nitsch, R., \& Wulczyn, F. G. (2005). Regulation of miRNA expression during neural cell specification. Eur J Neurosci, 21(6), 1469-1477. doi: 10.1111/j.1460-9568.2005.03978.x

Smith, Adam R. , Mill, Jonathan , Smith, Rebecca G. , \& Lunnon, Katie. (2016). Elucidating novel dysfunctional pathways in Alzheimer's disease by integrating loci identified in genetic and epigenetic studies. Neuroepigenetics, 6 .

Smith, C. W., \& Valcarcel, J. (2000). Alternative pre-mRNA splicing: the logic of combinatorial control. Trends Biochem Sci, 25(8), 381-388.

Smith, D., Aherrera, A., Lopez, A., Neptune, E., Winickoff, J. P., Klein, J. D., . . McGrath-Morrow, S. A. (2015). Adult Behavior in Male Mice Exposed to E-Cigarette Nicotine Vapors during Late Prenatal and Early Postnatal Life. PLoS One, 10(9), e0137953. doi: 10.1371/journal.pone.0137953

Soba, P., Eggert, S., Wagner, K., Zentgraf, H., Siehl, K., Kreger, S., . . Beyreuther, K. (2005). Homo- and heterodimerization of APP family members promotes intercellular adhesion. EMBO J, 24(20), 3624-3634. doi: 10.1038/sj.emboj.7600824

Soderling, T. R., \& Derkach, V. A. (2000). Postsynaptic protein phosphorylation and LTP. Trends Neurosci, 23(2), 75-80.

Solon-Biet, S. M., McMahon, A. C., Ballard, J. W., Ruohonen, K., Wu, L. E., Cogger, V. C., . . Simpson, S. J. (2014). The ratio of macronutrients, not caloric intake, dictates cardiometabolic health, aging, and longevity in ad libitum-fed mice. Cell Metab, 19(3), 418-430. doi: 10.1016/j.cmet.2014.02.009

Sonnen, J. A., Montine, K. S., Quinn, J. F., Kaye, J. A., Breitner, J. C., \& Montine, T. J. (2008). Biomarkers for cognitive impairment and dementia in elderly people. Lancet Neurol, 7(8), 704-714. doi: 10.1016/S1474-4422(08)70162-5

Spear, N.E., \& Riccio, D.C. (1994). Memory: phenomena and principles: Allyn and Bacon.

Sperling, R., \& Johnson, K. (2013). Biomarkers of Alzheimer disease: current and future applications to diagnostic criteria. Continuum (Minneap Minn), 19(2 Dementia), 325-338. doi: 10.1212/01.CON.0000429181.60095.99

Squire, L. R. (1986). Mechanisms of memory. Science, 232(4758), 1612-1619.

Squire, L. R. (1987). The organization and neural substrates of human memory. Int J Neurol, 21-22, 218-222.

Squire, L. R., \& Cave, C. B. (1991). The hippocampus, memory, and space. Hippocampus, 1(3), 269-271. doi: 10.1002/ hipo.450010313

Squire, L. R., Stark, C. E., \& Clark, R. E. (2004). The medial temporal lobe. Annu Rev Neurosci, 27, 279-306. doi: 10.1146/ annurev.neuro.27.070203.144130

Sridhar, P., Gan, H. H., \& Schlick, T. (2008). A computational screen for C/D box snoRNAs in the human genomic region associated with Prader-Willi and Angelman syndromes. J Biomed Sci, 15(6), 697-705. doi: 10.1007/ s11373-008-9271-X

Stark, S. M., Yassa, M. A., \& Stark, C. E. (2010). Individual differences in spatial pattern separation performance associated with healthy aging in humans. Learn Mem, 17(6), 284-288. doi: 10.1101//m.1768110

Steinbusch, M. M., Fang, Y., Milner, P. I., Clegg, P. D., Young, D. A., Welting, T. J., \& Peffers, M. J. (2017). Serum snoRNAs as biomarkers for joint ageing and post traumatic osteoarthritis. Sci Rep, 7, 43558. doi: 10.1038/ srep43558

Stern, David G. (1991). Models of memory: Wittgenstein and cognitive science. PHILOSOPHICAL PSYCHOLOGY, VOL. 4, NO. 2.

Sternberg, Robert J., \& Sternberg, Karin. (2017). Cognitive psychology.

Stilling, R. M., Benito, E., Gertig, M., Barth, J., Capece, V., Burkhardt, S., . . Fischer, A. (2014). De-regulation of gene expression and alternative splicing affects distinct cellular pathways in the aging hippocampus. Front Cell Neurosci, 8, 373. doi: 10.3389/fncel.2014.00373

Strasser, B., Volaklis, K., Fuchs, D., \& Burtscher, M. (2018). Role of Dietary Protein and Muscular Fitness on Longevity and Aging. Aging Dis, 9(1), 119-132. doi: 10.14336/AD.2017.0202

Strittmatter, W. J., Saunders, A. M., Schmechel, D., Pericak-Vance, M., Enghild, J., Salvesen, G. S., \& Roses, A. D. (1993). Apolipoprotein $\mathrm{E}$ : high-avidity binding to beta-amyloid and increased frequency of type 4 allele in late-onset familial Alzheimer disease. Proc Natl Acad Sci U S A, 90(5), 1977-1981.

Subramanian, A., Tamayo, P., Mootha, V. K., Mukherjee, S., Ebert, B. L., Gillette, M. A., . . Mesirov, J. P. (2005). Gene set enrichment analysis: a knowledge-based approach for interpreting genome-wide expression profiles. Proc Natl Acad Sci U S A, 102(43), 15545-15550. doi: 10.1073/pnas.0506580102 
Suh, N., \& Blelloch, R. (2011). Small RNAs in early mammalian development: from gametes to gastrulation. Development, 138(9), 1653-1661. doi: 10.1242/dev.056234

Sun, B., Halabisky, B., Zhou, Y., Palop, J. J., Yu, G., Mucke, L., \& Gan, L. (2009). Imbalance between GABAergic and Glutamatergic Transmission Impairs Adult Neurogenesis in an Animal Model of Alzheimer's Disease. Cell Stem Cell, 5(6), 624-633. doi: 10.1016/j.stem.2009.10.003

Suzuki, W. A. (2009). Perception and the medial temporal lobe: evaluating the current evidence. Neuron, 61(5), 657-666. doi: 10.1016/j.neuron.2009.02.008

Swerdlow, R. H., Burns, J. M., \& Khan, S. M. (2014). The Alzheimer's disease mitochondrial cascade hypothesis: progress and perspectives. Biochim Biophys Acta, 1842(8), 1219-1231. doi: 10.1016/j.bbadis.2013.09.010

Taft, R. J., Glazov, E. A., Lassmann, T., Hayashizaki, Y., Carninci, P., \& Mattick, J. S. (2009). Small RNAs derived from snoRNAs. RNA, 15(7), 1233-1240. doi: 10.1261/rna.1528909

Takami, M., Nagashima, Y., Sano, Y., Ishihara, S., Morishima-Kawashima, M., Funamoto, S., \& Ihara, Y. (2009). gammaSecretase: successive tripeptide and tetrapeptide release from the transmembrane domain of beta-carboxyl terminal fragment. J Neurosci, 29(41), 13042-13052. doi: 10.1523/JNEUROSCI.2362-09.2009

Tal, O., Kisdi, E., \& Jablonka, E. (2010). Epigenetic contribution to covariance between relatives. Genetics, 184(4), 1037-1050. doi: 10.1534/genetics.109.112466

Talwar, P., Grover, S., Sinha, J., Chandna, P., Agarwal, R., Kushwaha, S., \& Kukreti, R. (2017). Multifactorial Analysis of a Biomarker Pool for Alzheimer Disease Risk in a North Indian Population. Dement Geriatr Cogn Disord, 44(1-2), 25-34. doi: 10.1159/000477206

Tan, J. Y., Vance, K. W., Varela, M. A., Sirey, T., Watson, L. M., Curtis, H. J., . . Marques, A. C. (2014). Cross-talking noncoding RNAs contribute to cell-specific neurodegeneration in SCA7. Nat Struct Mol Biol, 21(11), 955-961. doi: 10.1038/nsmb.2902

Tanner, K. G., Trievel, R. C., Kuo, M. H., Howard, R. M., Berger, S. L., Allis, C. D., . . Denu, J. M. (1999). Catalytic mechanism and function of invariant glutamic acid 173 from the histone acetyltransferase GCN5 transcriptional coactivator. J Biol Chem, 274(26), 18157-18160.

Tanzi, R. E., Gusella, J. F., Watkins, P. C., Bruns, G. A., St George-Hyslop, P., Van Keuren, M. L., . . Neve, R. L. (1987). Amyloid beta protein gene: cDNA, mRNA distribution, and genetic linkage near the Alzheimer locus. Science, 235(4791), 880-884.

Terashima, M., Barbour, S., Ren, J., Yu, W., Han, Y., \& Muegge, K. (2015). Effect of high fat diet on paternal sperm histone distribution and male offspring liver gene expression. Epigenetics, 10(9), 861-871. doi: $10.1080 / 15592294.2015 .1075691$

Tognini, P., Putignano, E., Coatti, A., \& Pizzorusso, T. (2011). Experience-dependent expression of miR-132 regulates ocular dominance plasticity. Nat Neurosci, 14(10), 1237-1239. doi: 10.1038/nn.2920

Tollervey, J. R., Wang, Z., Hortobagyi, T., Witten, J. T., Zarnack, K., Kayikci, M., . . Ule, J. (2011). Analysis of alternative splicing associated with aging and neurodegeneration in the human brain. Genome Res, 21(10), 1572-1582. doi: 10.1101/gr.122226.111

Toyama, K., Spin, J. M., Deng, A. C., Huang, T. T., Wei, K., Wagenhauser, M. U., . . Tsao, P. S. (2018). MicroRNAMediated Therapy Modulating Blood-Brain Barrier Disruption Improves Vascular Cognitive Impairment. Arterioscler Thromb Vasc Biol. doi: 10.1161/ATVBAHA.118.310822

Treusch, S., Cyr, D. M., \& Lindquist, S. (2009). Amyloid deposits: protection against toxic protein species? Cell Cycle, 8(11), 1668-1674. doi: 10.4161/cc.8.11.8503

Trifunovic, A., Wredenberg, A., Falkenberg, M., Spelbrink, J. N., Rovio, A. T., Bruder, C. E., . . Larsson, N. G. (2004). Premature ageing in mice expressing defective mitochondrial DNA polymerase. Nature, 429(6990), 417-423. doi: 10.1038/nature02517

Trinh, J., Gustavsson, E. K., Vilarino-Guell, C., Bortnick, S., Latourelle, J., McKenzie, M. B., . . Farrer, M. J. (2016). DNM3 and genetic modifiers of age of onset in LRRK2 Gly2019Ser parkinsonism: a genome-wide linkage and association study. Lancet Neurol, 15(12), 1248-1256. doi: 10.1016/S1474-4422(16)30203-4

Trojanowski, J. Q., Smith, A. B., Huryn, D., \& Lee, V. M. (2005). Microtubule-stabilising drugs for therapy of Alzheimer's disease and other neurodegenerative disorders with axonal transport impairments. Expert Opin Pharmacother, 6(5), 683-686. doi: 10.1517/14656566.6.5.683

Trushina, E., \& McMurray, C. T. (2007). Oxidative stress and mitochondrial dysfunction in neurodegenerative diseases. Neuroscience, 145(4), 1233-1248. doi: 10.1016/j.neuroscience.2006.10.056

Tsai, G., Ralph-Williams, R. J., Martina, M., Bergeron, R., Berger-Sweeney, J., Dunham, K. S., . . Coyle, J. T. (2004). Gene knockout of glycine transporter 1: characterization of the behavioral phenotype. Proc Natl Acad Sci U S A, 101(22), 8485-8490. doi: 10.1073/pnas.0402662101

Tsankova, N. M., Kumar, A., \& Nestler, E. J. (2004). Histone modifications at gene promoter regions in rat hippocampus after acute and chronic electroconvulsive seizures. J Neurosci, 24(24), 5603-5610. doi: 10.1523/JNEUROSCl. 0589-04.2004

Tucker, L. B., Fu, A. H., \& McCabe, J. T. (2016). Performance of Male and Female C57BL/6J Mice on Motor and Cognitive Tasks Commonly Used in Pre-Clinical Traumatic Brain Injury Research. J Neurotrauma, 33(9), 880-894. doi: 10.1089/neu.2015.3977

Tulving, E. (1985). How many memory systems are there? American Psychologist, 40, 385-398.

Tulving, E., \& Thomson, D. M. (1973). Encoding specificity and retrieval processes in episodic memory. Psychol Rev.

Tulving, Endel. (1985). Elements of episodic memory. Oxford: Clarendon.

Turchinovich, A., Weiz, L., Langheinz, A., \& Burwinkel, B. (2011). Characterization of extracellular circulating microRNA Nucleic Acids Res, 39(16), 7223-7233. doi: 10.1093/nar/gkr254

Twine, N. A., Janitz, K., Wilkins, M. R., \& Janitz, M. (2011). Whole transcriptome sequencing reveals gene expression and splicing differences in brain regions affected by Alzheimer's disease. PLoS One, 6(1), e16266. doi: 10.1371/ journal.pone.0016266

v, B. Hjelmborg J., lachine, I., Skytthe, A., Vaupel, J. W., McGue, M., Koskenvuo, M., . . Christensen, K. (2006). Genetic influence on human lifespan and longevity. Hum Genet, 119(3), 312-321. doi: 10.1007/s00439-006-0144-y 
Valadi, H., Ekstrom, K., Bossios, A., Sjostrand, M., Lee, J. J., \& Lotvall, J. O. (2007). Exosome-mediated transfer of mRNAs and microRNAs is a novel mechanism of genetic exchange between cells. Nat Cell Biol, 9(6), 654-659. doi: $10.1038 / \mathrm{ncb} 1596$

Valenti, D., de Bari, L., De Filippis, B., Henrion-Caude, A., \& Vacca, R. A. (2014). Mitochondrial dysfunction as a central actor in intellectual disability-related diseases: an overview of Down syndrome, autism, Fragile $X$ and Rett syndrome. Neurosci Biobehav Rev, 46 Pt 2, 202-217. doi: 10.1016/j.neubiorev.2014.01.012

Valla, J., Berndt, J. D., \& Gonzalez-Lima, F. (2001). Energy hypometabolism in posterior cingulate cortex of Alzheimer's patients: superficial laminar cytochrome oxidase associated with disease duration. J Neurosci, 21(13), 4923-4930.

Van Dam, D., D'Hooge, R., Staufenbiel, M., Van Ginneken, C., Van Meir, F., \& De Deyn, P. P. (2003). Age-dependent cognitive decline in the APP23 model precedes amyloid deposition. Eur J Neurosci, 17(2), 388-396.

van Praag, H., Schinder, A. F., Christie, B. R., Toni, N., Palmer, T. D., \& Gage, F. H. (2002). Functional neurogenesis in the adult hippocampus. Nature, 415(6875), 1030-1034. doi: 10.1038/4151030a

Van Remmen, H., Ikeno, Y., Hamilton, M., Pahlavani, M., Wolf, N., Thorpe, S. R., . . Richardson, A. (2003). Life-long reduction in MnSOD activity results in increased DNA damage and higher incidence of cancer but does not accelerate aging. Physiol Genomics, 16(1), 29-37. doi: 10.1152/physiolgenomics.00122.2003

Van Speybroeck, L. (2002). From epigenesis to epigenetics: the case of C. H. Waddington. Ann N Y Acad Sci, 981, 61-81.

Varona, L., Munilla, S., Mouresan, E. F., Gonzalez-Rodriguez, A., Moreno, C., \& Altarriba, J. (2015). A Bayesian model for the analysis of transgenerational epigenetic variation. G3 (Bethesda), 5(4), 477-485. doi: 10.1534/ g3.115.016725

Vassoler, F. M., White, S. L., Schmidt, H. D., Sadri-Vakili, G., \& Pierce, R. C. (2013). Epigenetic inheritance of a cocaineresistance phenotype. Nat Neurosci, 16(1), 42-47. doi: 10.1038/nn.3280

Verbitsky, M., Yonan, A. L., Malleret, G., Kandel, E. R., Gilliam, T. C., \& Pavlidis, P. (2004). Altered hippocampal transcript profile accompanies an age-related spatial memory deficit in mice. Learn Mem, 11(3), 253-260. doi: 10.1101/Im. 68204

Verdin, E., \& Ott, M. (2015). 50 years of protein acetylation: from gene regulation to epigenetics, metabolism and beyond. Nat Rev Mol Cell Biol, 16(4), 258-264. doi: 10.1038/nrm3931

Vermulst, M., Wanagat, J., Kujoth, G. C., Bielas, J. H., Rabinovitch, P. S., Prolla, T. A., \& Loeb, L. A. (2008). DNA deletions and clonal mutations drive premature aging in mitochondrial mutator mice. Nat Genet, 40(4), 392-394. doi: $10.1038 / \mathrm{ng} .95$

Vickers, K. C., Palmisano, B. T., Shoucri, B. M., Shamburek, R. D., \& Remaley, A. T. (2011). MicroRNAs are transported in plasma and delivered to recipient cells by high-density lipoproteins. Nat Cell Biol, 13(4), 423-433. doi: 10.1038/ ncb2210

Vijg, J., \& Campisi, J. (2008). Puzzles, promises and a cure for ageing. Nature, 454(7208), 1065-1071. doi: 10.1038/ nature 07216

Vina, J., \& Lloret, A. (2010). Why women have more Alzheimer's disease than men: gender and mitochondrial toxicity of amyloid-beta peptide. J Alzheimers Dis, 20 Suppl 2, S527-533. doi: 10.3233/JAD-2010-100501

Voikar, V., Koks, S., Vasar, E., \& Rauvala, H. (2001). Strain and gender differences in the behavior of mouse lines commonly used in transgenic studies. Physiol Behav, 72(1-2), 271-281.

Vojtech, L., Woo, S., Hughes, S., Levy, C., Ballweber, L., Sauteraud, R. P., . . Hladik, F. (2014). Exosomes in human semen carry a distinctive repertoire of small non-coding RNAs with potential regulatory functions. Nucleic Acids Res, 42(11), 7290-7304. doi: 10.1093/nar/gku347

Vucetic, Z., Kimmel, J., \& Reyes, T. M. (2011). Chronic high-fat diet drives postnatal epigenetic regulation of mu-opioid receptor in the brain. Neuropsychopharmacology, 36(6), 1199-1206. doi: 10.1038/npp.2011.4

Waddington, C. H. (1939). Preliminary Notes on the Development of the Wings in Normal and Mutant Strains of Drosophila. Proc Natl Acad Sci U S A, 25(7), 299-307.

Waddington, C. H. (1957). The strategy of genes. London: Allen \& Unwin.

Waddington, C. H. (1959). Canalization of development and genetic assimilation of acquired characters. Nature, 183(4676), 1654-1655

Waddington, C. H. (2012). The Epigenotype. International Journal of Epidemiology, 41(1), 10-13. doi: 10.1093/ije/dyr184

Waddington, C.H. (1957). The strategy of the genes: a discussion of some aspects of theoretical biology: Allen \& Unwin.

Wahlsten, D., Bachmanov, A., Finn, D. A., \& Crabbe, J. C. (2006). Stability of inbred mouse strain differences in behavior and brain size between laboratories and across decades. Proc Natl Acad Sci U S A, 103(44), 16364-16369. doi: 10.1073/pnas.0605342103

Walenta, J. H., Didier, A. J., Liu, X., \& Kramer, H. (2001). The Golgi-associated hook3 protein is a member of a novel family of microtubule-binding proteins. J Cell Biol, 152(5), 923-934.

Wang, E. T., Sandberg, R., Luo, S., Khrebtukova, I., Zhang, L., Mayr, C., . . Burge, C. B. (2008). Alternative isoform regulation in human tissue transcriptomes. Nature, 456(7221), 470-476. doi: 10.1038/nature07509

Wang, R., Dineley, K. T., Sweatt, J. D., \& Zheng, H. (2004). Presenilin 1 familial Alzheimer's disease mutation leads to defective associative learning and impaired adult neurogenesis. Neuroscience, 126(2), 305-312. doi: 10.1016/ j.neuroscience.2004.03.048

Wang, W., Kwon, E. J., \& Tsai, L. H. (2012). MicroRNAs in learning, memory, and neurological diseases. Learn Mem, 19(9), 359-368. doi: 10.1101//m.026492.112

Wang, W., Nitulescu, I., Lewis, J. S., Lemos, J. C., Bamford, I. J., Posielski, N. M., . . Bamford, N. S. (2013). Overinhibition of corticostriatal activity following prenatal cocaine exposure. Ann Neurol, 73(3), 355-369. doi: 10.1002/ana.23805

Ward, W. S., \& Coffey, D. S. (1991). DNA packaging and organization in mammalian spermatozoa: comparison with somatic cells. Biol Reprod, 44(4), 569-574.

Watanabe, T., Tomizawa, S., Mitsuya, K., Totoki, Y., Yamamoto, Y., Kuramochi-Miyagawa, S., . . Sasaki, H. (2011). Role for piRNAs and noncoding RNA in de novo DNA methylation of the imprinted mouse Rasgrf1 locus. Science, 332(6031), 848-852. doi: 10.1126/science.1203919 
Waterland, R. A., \& Jirtle, R. L. (2003). Transposable elements: targets for early nutritional effects on epigenetic gene regulation. Mol Cell Biol, 23(15), 5293-5300.

Weaver, I. C. (2007). Epigenetic programming by maternal behavior and pharmacological intervention. Nature versus nurture: let's call the whole thing off. Epigenetics, 2(1), 22-28.

Weaver, I. C., Cervoni, N., Champagne, F. A., D'Alessio, A. C., Sharma, S., Seckl, J. R., . . Meaney, M. J. (2004). Epigenetic programming by maternal behavior. Nat Neurosci, 7(8), 847-854. doi: 10.1038/nn1276

Weaver, I. C., Champagne, F. A., Brown, S. E., Dymov, S., Sharma, S., Meaney, M. J., \& Szyf, M. (2005). Reversal of maternal programming of stress responses in adult offspring through methyl supplementation: altering epigenetic marking later in life. J Neurosci, 25(47), 11045-11054. doi: 10.1523/JNEUROSCI.3652-05.2005

Weaver, I. C., Meaney, M. J., \& Szyf, M. (2006). Maternal care effects on the hippocampal transcriptome and anxietymediated behaviors in the offspring that are reversible in adulthood. Proc Natl Acad Sci U S A, 103(9), 3480-3485. doi: 10.1073/pnas.0507526103

Weaver, J. R., Susiarjo, M., \& Bartolomei, M. S. (2009). Imprinting and epigenetic changes in the early embryo. Mamm Genome, 20(9-10), 532-543. doi: 10.1007/s00335-009-9225-2

Wei, Y., Yang, C. R., Wei, Y. P., Zhao, Z. A., Hou, Y., Schatten, H., \& Sun, Q. Y. (2014). Paternally induced transgenerational inheritance of susceptibility to diabetes in mammals. Proc Natl Acad Sci U S A, 111(5), 1873-1878. doi: 10.1073/pnas. 1321195111

Weinberg, R. A., \& Penman, S. (1968). Small molecular weight monodisperse nuclear RNA. J Mol Biol, 38(3), 289-304.

Weinert, B. T., \& Timiras, P. S. (2003). Invited review: Theories of aging. J Appl Physiol (1985), 95(4), 1706-1716. doi: 10.1152/japplphysiol.00288.2003

Weingarten, M. D., Lockwood, A. H., Hwo, S. Y., \& Kirschner, M. W. (1975). A protein factor essential for microtubule assembly. Proc Natl Acad Sci U S A, 72(5), 1858-1862.

Weinstein, J. D. (2018). A new direction for Alzheimer's research. Neural Regen Res, 13(2), 190-193. doi: $10.4103 / 1673-5374.226381$

Weiskopf, D., Weinberger, B., \& Grubeck-Loebenstein, B. (2009). The aging of the immune system. Transpl Int, 22(11), 1041-1050. doi: 10.1111/j.1432-2277.2009.00927.x

Whitaker, K. L., Jarvis, M. J., Beeken, R. J., Boniface, D., \& Wardle, J. (2010). Comparing maternal and paternal intergenerational transmission of obesity risk in a large population-based sample. Am J Clin Nutr, 91(6), 1560-1567. doi: 10.3945/ajen.2009.28838

Wicklund, M., \& Petersen, R. C. (2013). Emerging biomarkers in cognition. Clin Geriatr Med, 29(4), 809-828. doi: 10.1016/ j.cger.2013.07.006

Wiley, C. D., Flynn, J. M., Morrissey, C., Lebofsky, R., Shuga, J., Dong, X., . . Campisi, J. (2017). Analysis of individual cells identifies cell-to-cell variability following induction of cellular senescence. Aging Cell, 16(5), 1043-1050. doi: 10.1111/acel.12632

Wirths, O., \& Bayer, T. A. (2008). Motor impairment in Alzheimer's disease and transgenic Alzheimer's disease mouse models. Genes Brain Behav, 7 Suppl 1, 1-5. doi: 10.1111/j.1601-183X.2007.00373.x

Witter, M. P., Groenewegen, H. J., Lopes da Silva, F. H., \& Lohman, A. H. (1989). Functional organization of the extrinsic and intrinsic circuitry of the parahippocampal region. Prog Neurobiol, 33(3), 161-253.

Wixted, J. T., Goldinger, S. D., Squire, L. R., Kuhn, J. R., Papesh, M. H., Smith, K. A., . . Steinmetz, P. N. (2018). Coding of episodic memory in the human hippocampus. Proc Natl Acad Sci U S A, 115(5), 1093-1098. doi: 10.1073/ pnas. 1716443115

Woitke, F., Ceanga, M., Rudolph, M., Niv, F., Witte, O. W., Redecker, C., . . Keiner, S. (2017). Adult hippocampal neurogenesis poststroke: More new granule cells but aberrant morphology and impaired spatial memory. PLoS One, 12(9), e0183463. doi: 10.1371/journal.pone.0183463

Woo, J., Kwon, S. K., Nam, J., Choi, S., Takahashi, H., Krueger, D., . . Kim, E. (2013). The adhesion protein IgSF9b is coupled to neuroligin 2 via S-SCAM to promote inhibitory synapse development. J Cell Biol, 201(6), 929-944. doi: 10.1083/jcb.201209132

Xu, B., Ye, M. H., Lv, S. G., Wang, Q. X., Wu, M. J., Xiao, B., . . Zhu, X. G. (2017). SNORD47, a box C/D snoRNA, suppresses tumorigenesis in glioblastoma. Oncotarget, 8(27), 43953-43966. doi: 10.18632/oncotarget.16693

Yan, Z., Hu, H. Y., Jiang, X., Maierhofer, V., Neb, E., He, L., . . Khaitovich, P. (2011). Widespread expression of piRNAlike molecules in somatic tissues. Nucleic Acids Res, 39(15), 6596-6607. doi: 10.1093/nar/gkr298

Yang, J., Huang, T., Petralia, F., Long, Q., Zhang, B., Argmann, C., . . Tu, Z. (2015). Synchronized age-related gene expression changes across multiple tissues in human and the link to complex diseases. Sci Rep, 5, 15145. doi: 10.1038/srep15145

Ye, X., Luo, H., Chen, Y., Wu, Q., Xiong, Y., Zhu, J., . . Wan, J. (2015). MicroRNAs 99b-5p/100-5p Regulated by Endoplasmic Reticulum Stress are Involved in Abeta-Induced Pathologies. Front Aging Neurosci, 7, 210. doi: 10.3389/fnagi.2015.00210

Yin, Q. F., Yang, L., Zhang, Y., Xiang, J. F., Wu, Y. W., Carmichael, G. G., \& Chen, L. L. (2012). Long noncoding RNAs with snoRNA ends. Mol Cell, 48(2), 219-230. doi: 10.1016/j.molcel.2012.07.033

Yin, Y. I., Bassit, B., Zhu, L., Yang, X., Wang, C., \& Li, Y. M. (2007). \{gamma\}-Secretase Substrate Concentration Modulates the Abeta42/Abeta40 Ratio: IMPLICATIONS FOR ALZHEIMER DISEASE. J Biol Chem, 282(32), 23639-23644. doi: 10.1074/jbc.M704601200

Younger, S. T., \& Corey, D. R. (2011). Transcriptional gene silencing in mammalian cells by miRNA mimics that target gene promoters. Nucleic Acids Res, 39(13), 5682-5691. doi: 10.1093/nar/gkr155

Youngson, N. A., \& Whitelaw, E. (2008). Transgenerational epigenetic effects. Annu Rev Genomics Hum Genet, 9 , 233-257. doi: 10.1146/annurev.genom.9.081307.164445

Yu, Y., He, J., Zhang, Y., Luo, H., Zhu, S., Yang, Y., . . Li, X. M. (2009). Increased hippocampal neurogenesis in the progressive stage of Alzheimer's disease phenotype in an APP/PS1 double transgenic mouse model. Hippocampus, 19(12), 1247-1253. doi: 10.1002/hipo.20587

Zaki, M., Shehab, M., El-Aleem, A. A., Abdel-Salam, G., Koeller, H. B., Ilkin, Y., . . Gleeson, J. G. (2007). Identification of a novel recessive RELN mutation using a homozygous balanced reciprocal translocation. Am J Med Genet $A$, 143A(9), 939-944. doi: 10.1002/ajmg.a.31667 


\section{REFERENCES}

Zelinski, E. M., \& Burnight, K. P. (1997). Sixteen-year longitudinal and time lag changes in memory and cognition in older adults. Psychol Aging, 12(3), 503-513.

Zeybel, M., Hardy, T., Wong, Y. K., Mathers, J. C., Fox, C. R., Gackowska, A., . . Mann, J. (2012). Multigenerational epigenetic adaptation of the hepatic wound-healing response. Nat Med, 18(9), 1369-1377. doi: 10.1038/nm.2893

Zhang, S., Li, X., Wang, Z., Liu, Y., Gao, Y., Tan, L., . . Wang, J. Z. (2017). Paternal spatial training enhances offspring's cognitive performance and synaptic plasticity in wild-type but not improve memory deficit in Alzheimer's mice. Sci Rep, 7(1), 1521. doi: 10.1038/s41598-017-01811-3

Zhang, W., Li, J., Suzuki, K., Qu, J., Wang, P., Zhou, J., . . Belmonte, J. C. (2015). Aging stem cells. A Werner syndrome stem cell model unveils heterochromatin alterations as a driver of human aging. Science, 348(6239), 1160-1163. doi: 10.1126/science.aaa1356

Zhang, X. Y., Yang, Z. L., Lu, G. M., Yang, G. F., \& Zhang, L. J. (2017). PET/MR Imaging: New Frontier in Alzheimer's Disease and Other Dementias. Front Mol Neurosci, 10, 343. doi: 10.3389/fnmol.2017.00343

Zhang, Y., Ikeno, Y., Qi, W., Chaudhuri, A., Li, Y., Bokov, A., . . Van Remmen, H. (2009). Mice deficient in both Mn superoxide dismutase and glutathione peroxidase- 1 have increased oxidative damage and a greater incidence of pathology but no reduction in longevity. J Gerontol A Biol Sci Med Sci, 64(12), 1212-1220. doi: 10.1093/ gerona/glp132

Zhao, Y., Bhattacharjee, S., Dua, P., Alexandrov, P. N., \& Lukiw, W. J. (2015). microRNA-Based Biomarkers and the Diagnosis of Alzheimer's Disease. Front Neurol, 6, 162. doi: 10.3389/fneur.2015.00162

Zheng, K., \& Wang, P. J. (2012). Blockade of pachytene piRNA biogenesis reveals a novel requirement for maintaining post-meiotic germline genome integrity. PLoS Genet, 8(11), e1003038. doi: 10.1371/journal.pgen.1003038

Zou, S., Meadows, S., Sharp, L., Jan, L. Y., \& Jan, Y. N. (2000). Genome-wide study of aging and oxidative stress response in Drosophila melanogaster. Proc Natl Acad Sci U S A, 97(25), 13726-13731. doi: 10.1073/pnas. 260496697

Zovoilis, A., Agbemenyah, H. Y., Agis-Balboa, R. C., Stilling, R. M., Edbauer, D., Rao, P., . . Fischer, A. (2011).

microRNA-34c is a novel target to treat dementias. EMBO J, 30(20), 4299-4308. doi: 10.1038/emboj.2011.327 


\section{ACKNOWLEDGMENT}

First of all I would like to express my gratitude to the Prof. Dr. Fischer. It was definitely a nice opportunity to do the PhD in this lab and with group of incredible people that works and worked here. Thanks to belief in me that day with a Skype call. Thanks for your patience and to support me. I have really enjoyed this time in the institute, it was a great experience and I learnt a lot about of science, but also about of life.

I really appreciated the support that l've received from the members of my committee, Tiago Outeiro and Thomas Dresbach. Thanks for your advises and help. It was really easy the communication and you were there when I need you. Thanks, that makes every meeting and very interaction really really easy. My thankfulness also goes to Michael Hörner for his support in the organizational issues and also all the GGNB office, for their help, kindness and mainly patience.

Regarding my colleagues, my experience here would not be the same without them. When I passed for really extremely bad days, my mood changed to happiness every time I went out out from my "bunker" and I interacted with some of them in the kitchen, corridor, ..... I was super lucky to be part of such a nice international environment and learnt about other cultures and other ways to life and do science. I really want to the thank all the team, especially, I want to thank Tea, Ana, Rashi and Eva for their help at the beginning, during and at the end of my PhD. I could not finish this thesis without you. Thanks to help me in the lab, but also thanks to spend hours and hours reading and reviewing my thesis. Thanks to help me whenever I need you! I also want to thanks Susi for her analysis work and for her kindness! You are incredible women!

I would like also want to mention Gaurav, Tona and Vincenzo. I really appreciate all the bioinformatician work you did for my data. It was really easy to learn and work with you. Moreover, the lab would not be the same without my big brothers, Hendrik, Henning and Christian, it was a pleasure to be with you in the lab, working in the lab, going to the Mensa or having a BBQ together, even when you annoying me a bit. Thanks to accept my craziness! As I told you several times, I will miss you a lot. I also appreciate the work that Danniel and Ulrike have done are doing everyday, you are incredible. Thanks for your administrative support and for your patience. Thanks to the rest of my colleagues, I could write a thesis only writing my thankfulness. 
I also want to thank my real and my second family here in Göttingen. Thanks to be there when I need you and to support me all the time. The life in Göttingen was one of the best experience in my life and was thanks to all of you. I also want to thanks my former and current flatmates in Göttingen, I was really lucky to share part of my life with you and that we perfectly understand each other in the daily life, specially I appreciate your support during these last weeks of my PhD. It was really easy to life with all of you, thanks Johannes, Wilken, Martina, Patrick and Jan. I would also like to thanks Johannes, Svenja and Jannes to be there and worried for my status.

Me gustaría dedicarle unas palabras a Evita. Primero de todo gracias por creer en mi en la primera llamada por Skype que tuvimos, la recuerdo perfectamente. Te admiro muchísimo por todo lo que haces y te agradezco muchísimo todo lo que has hecho por mi, desde el principio al final, tanto a nivel personal como en el trabajo, te debo el resultado de la tesis a ti. Eres una ...... crack. A nivel personal, ya sabes que tú e Iran habéis sido mis mamis en Göttingen y me he sentido muy arropada por vosotras, sois increíbles. Gracias por hacerme ver la realidad cuando estaba a punto de tocar fondo.

Quiero agradecer también la paciencia, cariño y la ayuda que he recibido de mi segunda familia en Göttingen, ONG. He tenido la suerte de conoceros y compartir parte de mi vida con vosotros. Gracias por quitarme una sonrisa cuando mas estrellada y agobiada estaba por el doctorado. En especial quiero agradecer la ayuda y soporte que he recibido de mis chicas, las poquianchis. Sois increíbles, os debo muchísimo y sobretodo haber salido viva de este doctorado, sin vuestra paciencia y sin vuestras palabras no lo habría logrado.

También quiero agradecerle a Diego todo el amor que he recibido desde la distancia de él y sobretodo por toda la santa paciencia que ha tenido y que tiene conmigo.

Moltes gràcies a la meva familia per també la paciència que han tingut amb mi i el suport que m'han donat des de la distància.

I can only say thanks, Göttingen would not be the same without all of you. All you made my life especial and magic every day. 
